\title{
A LONGITUDINAL INVESTIGATION OF NON-SUICIDAL SELF-INJURY AND PERFECTIONISM IN A SAMPLE OF NEW ZEALAND ADOLESCENTS
}

MADELEINE ROSE BROCKLESBY

A thesis submitted to Victoria University of Wellington

in fulfilment of the requirements for the degree of

Doctor of Philosophy

Victoria University of Wellington

2017 


\begin{abstract}
Non-Suicidal Self-Injury (NSSI) is defined as the intentional, direct injury to body tissue, undertaken without suicidal intent, and for a purpose that is not socially or culturally sanctioned (International Society for the Study of Self-Injury, 2007; Klonsky \& Muehlenkamp, 2007; Muehlenkamp, 2014). NSSI is prevalent in adolescent samples worldwide (Muehlenkamp, Claes, Havertape, \& Plener, 2012; Swannell, Martin, Page, Hasking, \& St John, 2014) and is typically considered a marker of wider distress. NSSI in adolescents has been associated with numerous poor mental health outcomes, including depression, anxiety, substance abuse, eating disorders, and attempted and completed suicide (Asarnow et al., 2011; Brunner et al., 2014; Claes, Soenens, Vansteenkiste, \& Vandereycken, 2012; Fox et al., 2015; Glenn \& Klonsky, 2011; Jacobson \& Gould, 2007).

In addition, research has demonstrated that perfectionism, defined as the setting of excessively high standards of performance (Frost, Marten, Lahart, \& Rosenblate, 1990), is also commonly associated with substantial distress. Unfortunately, perfectionism in adolescents is thought to be on the rise (see Flett \& Hewitt, 2014; Portesova \& Urbanek, 2013) with many adolescents reporting multi-sourced and relentless pressure to perform highly and adhere to societal ideals. As such, individuals are setting excessively high goals for themselves, and increasingly worrying about the consequences of less than perfect performance. Similar to the research pertaining to NSSI, perfectionism has been shown to also be associated with a raft of poor outcomes including depression, anxiety, eating disorders, and general psychological distress (e.g., Boone, Braet, Vandereycken, \& Claes, 2013; Claes et al., 2012; DiBartolo et al., 2007; Lombardo, Mallia, Battagliese, Grano, \& Violani, 2013; Vartanian \& Grisham, 2011).

Research has shown an association between NSSI and perfectionism (e.g., Hoff \& Muehlenkamp, 2009; O’Connor, Rasmussen, \& Hawton, 2010). However, this literature is currently very limited and the relationship between NSSI and perfectionism is not well understood. In light of this, I set out to thoroughly explore if, and how, NSSI and perfectionism are related in New Zealand adolescents. Moreover, I aimed to gain insight into the mechanisms that could underpin such relationships.

Of the four studies conducted, the first and second studies established a foundation for my research. Specifically, Study 1 meta-analyses synthesised data from 118 studies investigating the relationship between perfectionism and adaptive and maladaptive outcomes. These analyses demonstrated a robust relationship between negative perfectionism and
\end{abstract}


maladaptive outcomes. More specifically, they revealed a significant, positive summary correlation for the relationship between negative perfectionism and self-injurious thoughts and behaviours.

Study 2 aimed to define the most appropriate conceptualisation of perfectionism for research with New Zealand adolescents. This involved investigating the psychometric properties and factor structure of the Frost Multidimensional Perfectionism Scale (Frost et al., 1990) in 930 adolescents with a mean age of 14 years old. A hierarchical structure with two overarching components (positive perfectionism, negative perfectionism), comprised of four second-level components (concerns and doubts, parental pressure, personal standards, and organisation) was identified and adopted for all following research.

Studies 3 and 4 investigated the cross-sectional and longitudinal relationships between NSSI and perfectionism. Study 3a specifically aimed to ascertain whether negative and positive perfectionism are associated with NSSI in New Zealand adolescents, based on survey data from 930 adolescents in their second year of high school. As expected, negative perfectionism was significantly associated with NSSI in females, however this relationship did not hold for males. On the other hand, positive perfectionism was associated with less engagement in NSSI in both males and females. This suggested that negative perfectionism may represent a risk factor for NSSI, while positive perfectionism may buffer against risk of NSSI. In addition, Study $3 b$ investigated the relationships between perfectionism and the functions of NSSI, indicating that self-punishment functions are particularly relevant for perfectionistic adolescents. As the final component of the cross-sectional analyses, Study $3 \mathrm{c}$ illustrated that the association between perfectionism and NSSI is more accurately captured when the interaction between positive and negative perfectionism is also considered.

Study 4 involved the examination of this relationship over time. To do so, another wave of data was collected, resulting in data matched across two times points for 608 adolescents. Longitudinal analyses demonstrated that negative perfectionism prospectively predicted NSSI one year later in females only. Moreover, again for only females, positive perfectionism predicted an increase in negative perfectionism over time. No significant longitudinal relationships were demonstrated for male adolescents.

The ultimate aim of this research was to provide clinicians, school staff and parents with the information required to effectively identify at-risk adolescents, and thereby prevent the onset of NSSI and its vast associated negative outcomes. This research suggests that perfectionism is one such risk factor to be aware of. As such, it is argued that targeted prevention and intervention strategies for perfectionism will help prevent the onset and 
maintenance of NSSI in females, and are also likely to be of benefit to the wider mental wellbeing of New Zealand adolescents. 


\section{ACKNOWLEDGEMENTS}

To my supervisor, Professor Marc Wilson, I feel incredibly lucky that you took me on as an honours student, supported me through my nearly Masters, and then encouraged me to do a $\mathrm{PhD}$ when it wasn't even on my radar. Even more, I appreciated your ongoing encouragement and explicit confidence in my ability to complete each stage. I am extremely grateful for all your support, vast knowledge, and advice. I have appreciated the many opportunities you have provided for me to connect with school staff, guidance counsellors and students, as well numerous opportunities to present my research to various audiences. It was these experiences that gave me the motivation to do my best and kept feeding my interest in finding out more. Finally, thank you for reminding me that I am doing well when the selfdoubt and dare I say it...perfectionism frequently kicked in.

To the Youth Wellbeing Study Team; Jess, Angelique, Kealagh, Tahlia, and Gloria. What a great team! Your support went way beyond that directly relevant to the research. In particular, thank you to my two office mates, Kealagh who put up with endless statistics questions and allowed me to use her brain like an encyclopaedia, and Jess who was always happy to provide clinical advice. We also mustn’t underestimate how much the weekly meeting influenced my week (thanks for the coffee Marc!), they will be missed. I must also mention those who came before me, in particular Emma, Maddie, Robyn, and Lynne who along with those mentioned above put in the hard yards to develop the Youth Wellbeing Study and form relationships with all of those who made it possible for this research to happen.

I would like to thank the many guidance counsellors, school staff, and students involved in the Youth Wellbeing Study, many of whom likely don't realise the true value of their contribution. A special thank you to the students for their honesty and willingness to complete a massive survey multiple times. Without you, we would not have such a good understanding of young people's needs. Thank you to the NZ Health Research Council for funding the research, and Victoria University for supporting me with a PhD scholarship.

To my clinical class, you are all amazing friends whom have provided incredible amounts of support over the past four years. I am grateful that I got the chance to know you, and am certain that you will go on to positively contribute to lives of both future clients, and others who are lucky enough to cross your paths.

Mum and Dad, you set me up wonderfully to pursue my interests and reach my goals. You taught me to do my best and supported me in all the decisions that have led to this point. 
Thank you for all of your practical support over the years (and I am sure the years to come), and thank you for encouraging me to do a $\mathrm{PhD}$ when I wasn't sure. I am extremely lucky to have such a wonderful family (you too Seb and Soph!).

Finally, to Mike. Thank you for supporting me on this journey and not only being willing to stay put for four years but also graciously tolerating the often very limited hours I spent outside of the university buildings. Your day to day support has kept me sane and allowed me to achieve at a level I didn't think was possible. I will forever be grateful for the many outdoors adventures during which time out from my studies was compulsory, the never-ending supply of coffees (I wouldn't want to know how many), your encouragement to take up biking and then your time spent constantly improving my bikes for me, and finally the frequent reminders that there is a world outside clinical/PhD land. 


\section{TABLE OF CONTENTS}

ABSTRACT

ACKNOWLEDGEMENTS vi

LIST OF TABLES Xiv

LIST OF FIGURES X xvi

FOREWORD $\quad$ xx

INTRODUCTION 1

Research Overview 3

CHAPTER ONE: NON-SUICIDAL SELF-INJURY 5

Defining Non-Suicidal Self-Injury $\quad 6$

A Brief History of NSSI Research

NSSI Behaviours and Descriptive Characteristics $\quad 8$

NSSI in Different Populations 9

Risk and Protective Factors for NSSI 13

Functions of NSSI $\quad 15$

Theoretical Models of NSSI $\quad 18$

NSSI and Suicidal Thoughts and Behaviours $\quad 20$

Chapter Summary 22

CHAPTER TWO: PERFECTIONISM 25

Theories of Perfectionism $\quad 27$

The Development of Perfectionism 31

Changing Societal Influences on Perfectionism 33

Perfectionism in Different Populations $\quad 35$

Perfectionism and Mental Health and Wellbeing Outcomes 38

Chapter Summary 39

CHAPTER THREE: PERFECTIONISM AND NSSI

Perfectionism and NSSI Prevalence, and Severity $\quad 41$

Perfectionism and the Functions of NSSI 45

Perfectionism and Theoretical Models of NSSI 46

RESEARCH QUESTIONS $\quad 48$

CHAPTER FOUR: THE RELATIONSHIP BETWEEN PERFECTIONISM AND

MENTAL HEALTH AND WELLBEING OUTCOMES

Challenges in Understanding Existing Perfectionism Literature 49

Study One: Systematic Review and Meta-Analyses of the Relationship between

Positive and Negative Perfectionism, and Mental Health and Wellbeing Outcomes 51

Advantages of Meta-Analyses

Study 1a: Meta-analyses of Positive and Negative Perfectionism and Their

Relationship with Positive and Negative Outcomes $\quad 53$

Method 53

Inclusion/Exclusion Strategy 53

Information Sources and Search Strategy $\quad 54$

Measure of Perfectionism $\quad 56$ 
Outcome Variables 56

Data-Synthesis $\quad 57$

Data Synthesis Model $\quad 57$

Moderator Analyses $\quad 59$

Assessing for Publication Bias $\quad 59$

Results 61

Results of Article Search $\quad 61$

Negative Perfectionism and Depression $\quad 61$

Positive Perfectionism and Depression $\quad 65$

Specific Outcome Results Summary $\quad 67$

Perfectionism and Demographic Variables $\quad 75$

Negative Perfectionism and Maladaptive and Adaptive Outcomes $\quad 75$

Positive Perfectionism and Maladaptive and Adaptive Outcomes 81

Negative and Positive Perfectionism 85

$\begin{array}{ll}\text { Summary and Conclusions } & 87\end{array}$

CHAPTER FIVE: PERFECTIONISM, SELF-INJURY, AND SUICIDAL BEHAVIOURS

Perfectionism and Suicide Literature Review $\quad 92$

Study 1b: Meta-Analysis of the Relationship between Positive and Negative Perfectionism and Self-Injurious and Suicidal Behaviours 95

$\begin{array}{ll}\text { Method } & 96\end{array}$

Inclusion/Exclusion Strategy 96

Information Sources and Search Strategy 97

Measure of Perfectionism $\quad 99$

Self-Injury/Suicide Variables $\quad 99$

$\begin{array}{lr}\text { Data-Synthesis } & 101\end{array}$

$\begin{array}{lr}\text { Moderator Analyses } & 101\end{array}$

$\begin{array}{ll}\text { Assessing for Publication Bias } & 102\end{array}$

$\begin{array}{ll}\text { Results } & 102\end{array}$

Perfectionism and Self-Injurious and Suicidal Thoughts and Behaviours 102

$\begin{array}{ll}\text { Negative Perfectionism and Self-Injury } & 106\end{array}$

Positive Perfectionism and Self-Injury 107

$\begin{array}{ll}\text { Negative Perfectionism and Suicidal Thoughts and Behaviours } & 109\end{array}$

Positive Perfectionism and Suicidal Thoughts and Behaviours 112

Summary and Conclusions $\quad 113$

Combined Study 1 Discussion $\quad 115$

Strengths 116

Limitations 116

$\begin{array}{ll}\text { Conclusion } & 117\end{array}$

CHAPTER SIX: PERFECTIONISM IN A NEW ZEALAND ADOLESCENT SAMPLE

Study Two: Using the Frost Multidimensional Perfectionism Scale in a Sample of New Zealand Community Adolescents

Measuring Perfectionism 
The Frost Multidimensional Perfectionism Scale $\quad 120$

The Structure of Positive and Negative Perfectionism in Adolescents 124

$\begin{array}{ll}\text { Method } & 125\end{array}$

Participants 126

$\begin{array}{ll}\text { Measures } & 128\end{array}$

Results 131

Confirmatory Factor Analysis 131

Exploratory Factor Analysis 133

Model Fit across Sex 136

Summary and Conclusions $\quad 139$

First Level Structure 139

Overarching Positive and Negative Components 139

$\begin{array}{ll}\text { Strengths } & 140\end{array}$

Limitations $\quad 141$

Conclusions 141

CHAPTER SEVEN: THE RELATIONSHIP BETWEEN NSSI AND

PERFECTIONISM IN NEW ZEALAND ADOLESCENTS 143

Study Three: Non-Suicidal Self-Injury, its Functions, and Perfectionism in a Sample of New Zealand Adolescents $\quad 144$

Method for Study 3a, 3b and 3c 144

$\begin{array}{ll}\text { Participants } & 144\end{array}$

$\begin{array}{ll}\text { Measures } & 145\end{array}$

Procedure $\quad 151$

Study 3a Results $\quad 152$

Descriptive Statistics $\quad 152$

Perfectionism and NSSI 156

$\begin{array}{lr}\text { Moderator analyses } & 158\end{array}$

Summary of Results 161

Study 3b: Examining the Relationship between Perfectionism and the Functions of NSSI

Theoretical Predictions on the Relationship between Perfectionism and NSSI

Empirical Research on Perfectionism and NSSI Functions 163

Results 165

$\begin{array}{ll}\text { Summary of Results } & 168\end{array}$

Study 3c: Two-Component, 2x2, and Tripartite Models of Perfectionism 168

$\begin{array}{ll}\text { Results } & 169\end{array}$

Summary of Results 173

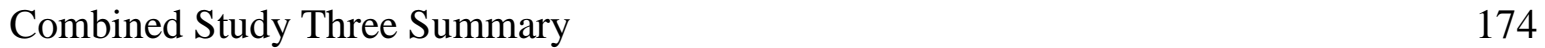

$\begin{array}{ll}\text { Perfectionism and NSSI } & 174\end{array}$

Perfectionism and the Functions of Self-Injury 175 
The Combined Effect of Positive and Negative Perfectionism in Relation to

Self-Injury

$\begin{array}{ll}\text { Strengths } & 179\end{array}$

$\begin{array}{lr}\text { Limitations } & 180\end{array}$

$\begin{array}{ll}\text { Conclusions } & 181\end{array}$

CHAPTER EIGHT: LONGITUDINAL INVESTIGATION OF PERFECTIONISM AND NON-SUICIDAL SELF-INJURY $\quad 183$

Longitudinal Research on Self-Injury $\quad 183$

Longitudinal Research on Perfectionism 185

Perfectionism and Self-Injurious and Suicidal Thoughts and Behaviours 186

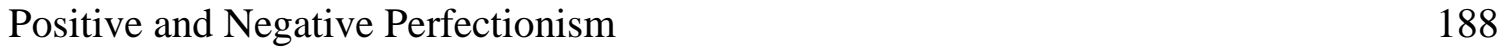

Study Four: Examining the Longitudinal Relationship between Negative Perfectionism, Positive Perfectionism and NSSI in New Zealand Adolescents $\quad 188$

$\begin{array}{ll}\text { Method } & 189\end{array}$

$\begin{array}{lr}\text { Participants } & 189\end{array}$

$\begin{array}{lr}\text { Measures } & 189\end{array}$

Reliability analyses $\quad 193$

Participant attrition 193

$\begin{array}{ll}\text { Procedure } & 193\end{array}$

$\begin{array}{ll}\text { Results } & 195\end{array}$

Descriptive Statistics for the Longitudinal Matched Sample 195

Perfectionism and NSSI across T1 and T2 199

Cross-Lagged Panel Correlations 203

$\begin{array}{ll}\text { Summary and Conclusions } & 206\end{array}$

Negative Perfectionism and NSSI 206

Positive Perfectionism and NSSI 207

Positive and Negative Perfectionism 208

$\begin{array}{ll}\text { Conclusion } & 208\end{array}$

CHAPTER NINE: DISCUSSION AND CONCLUSIONS 211

Summary of the Research $\quad 211$

Positive and Negative Perfectionism and Mental Health and Wellbeing 211

Perfectionism in NZ Adolescents 213

Non-Suicidal Self-Injury in NZ Adolescents 216

Empirical Findings for the Relationship between Perfectionism and NSSI in NZ

$\begin{array}{ll}\text { Adolescents } & 218\end{array}$

Perfectionism and the Theoretical Models of NSSI 219

Sex Differences in the Relationship between Perfectionism and NSSI 224

Other Contributing Factors $\quad 226$

Summary of Theoretical Implications 228

$\begin{array}{ll}\text { Clinical Implications } & 228\end{array}$

Applications of the Research $\quad 229$

$\begin{array}{ll}\text { Strengths } & 231\end{array}$

Limitations of this Research 233

Future Directions $\quad 235$

$\begin{array}{ll}\text { Finally } & 237\end{array}$ 


\section{APPENDICES}

$\begin{array}{llr}\text { APPENDIX A. } & \text { Acronym glossary }\end{array}$

$\begin{array}{llr}\text { APPENDIX B. } & \text { Study 1a forest plots } & 292\end{array}$

$\begin{array}{lll}\text { APPENDIX C. } & \text { Study } 1 \mathrm{~b} \text { forest plots } & 301\end{array}$

APPENDIX D. Study 2 MIMIC analyses complete model including path 303 values

Supplementary material (e.g., details of the studies included in meta-analyses, results of all moderator analyses, consent forms, copies of the survey) can be viewed at https://www.dropbox.com/sh/oggqp5h4z617znv/AACGbtc31EsecpQrCTCsecefa?dl=0 


\section{LIST OF TABLES}

Table 1. Frost, Heimberg, Holt, Mattia and Neubauer's (1993) Two Component

Model of Perfectionism

Table 2. Moderator Categories and Specific Variables for Study 1a

Table 3. Summary of Negative Perfectionism Meta-Analyses Results

Table 4. Summary of Positive Perfectionism Meta-Analyses Results

73

Table 5. Summary of Positive Perfectionism and Negative Perfectionism with

Demographic Variables Meta-Analytic Results

Table 6. Summary of Positive Perfectionism and Negative Perfectionism with Adaptive and Maladaptive Outcomes Meta-Analytic Results

Table 7. Subscales of Existing Perfectionism Scales Assigned to

Table 8. Moderator Categories and Specific Variables for Study $1 \mathrm{~b}$

Table 9. Summary of Meta-Analytic Results for the Relationship between

Perfectionism and Self-Injurious and Suicidal Thoughts and

Behaviours

Table 10. Goodness of fit Statistics for Frost Multidimensional Perfectionism

Scale Factor Structures

Table 11. Results of Parallel Analysis used to Determine the most Appropriate Number of Components, within the FMPS, for a sample of New Zealand Adolescents

Table 12. Result of FMPS PCA; Factor Structure and Item Loadings

Table 13. Result of FMPS Second-order PCA; Factor Structure and Item Loadings

Table 14. Two Factor Model of the Functions of NSSI

Table 15. Cronbach's Alpha Reliability Coefficients and Mean Inter-Item Correlations for the FMPS, DSHI-s, and ISAS

Table 16. NSSI Prevalence, Methods, and Functions Frequencies and ChiSquared Analyses across Males and Females.

Table 17. Means, Standard Deviations, and Pearson's Correlation Coefficients for the Relationship between the Components of Perfectionism

Table 18. Pearson's Correlation Coefficients for the Relationship between Perfectionism and NSSI

Table 19. Summary of Multiple Regression of NSSI Predicted by Sex, Negative Perfectionism and Sex-Negative Perfectionism Interaction

Table 20. Summary of Multiple Regression of NSSI Predicted by Sex, Positive Perfectionism and Sex-Positive Perfectionism Interaction

Table 21. NSSI Function Frequencies and Chi-Squared Analyses across Sex

Table 22. Pearson's Correlation Coefficients for NSSI Individual Functions, Functional Categories and Perfectionism

Table 23. Summary of Multiple Regression of NSSI Predicted by Positive Perfectionism, Negative Perfectionism and a Positive PerfectionismNegative Perfectionism Interaction in the Total Sample 
Table 24. Summary of Multiple Regression of NSSI Predicted by Positive

Perfectionism, Negative Perfectionism and a Positive Perfectionism-

Negative Perfectionism Interaction in Males only

Table 25. Summary of Multiple Regression of NSSI Predicted by Positive

Perfectionism, Negative Perfectionism and a Positive Perfectionism-

Negative Perfectionism Interaction in Females only

Table 26. FMPS-22 Factor Structure based on Cox et al., 2002 and Results of Study 2

Table 27. Cronbach's Alpha Reliability Coefficients for the FMPS, DSHI-s, and ISAS across T1 and T2 for the Longitudinal Matched Sample only

Table 28. Change in NSSI Prevalence for T1 and T2

Table 29. NSSI Prevalence and Chi-Squared Analyses across Sex for T1 and T2 197

Table 30. Endorsement of Perfectionism, NSSI, and Functions at T1 and T2 - 198 total and Split by Sex

Table 31. Pearson's Correlation Coefficients for the Relationship between Positive Perfectionism, Negative Perfectionism, and NSSI at T1 and T2 for both Males and Females

Table 32. Correlations between Perfectionism and NSSI at T1 and T2 


\section{LIST OF FIGURES}

Figure 1. Nock and Favazza's (2009) classification of self-injurious 5 thoughts and behaviours

Figure 2. $\quad$ Four-Functional Model (FFM) of NSSI 16

Figure 3. The Experiential Avoidance Model (EAM) of Deliberate Self-Harm 19

Figure 4. Nock's Integrated Theoretical Model of Self-Injury 20

Figure 5. Shafran et al.,'s (2016) cognitive-behavioural model of negative 28 perfectionism

Figure 6. Alden, Ryder and Melling's (2002) Two Component Model 30

Figure 7. Tripartite Model as proposed by Rice and Ashby (2007), and 30 Stoeber and Otto (2006)

Figure 8. Gaudreau and Thompson's (2010) 2x2 Model 30

Figure 9. Inclusion/exclusion process for Study 1a 55

Figure 10. Forest plot with effect sizes for negative perfectionism and $\quad 62$ depression

Figure 11. Funnel plot of the standard error by Fischer's $Z$ for the association 64 between negative perfectionism and depression

Figure 12. Forest plot with effect sizes for positive perfectionism and $\quad 65$ depression

Figure 13. Funnel plot of the standard error by Fischer's $Z$ for the association between positive perfectionism and depression

Figure 14. Forest plot with effect sizes for negative perfectionism and maladaptive outcomes

Figure 15. Forest plot with effect sizes for negative perfectionism and adaptive outcomes

Figure 16. Funnel plot of the standard error by Fischer's $Z$ for the association between negative perfectionism and maladaptive outcomes

Figure 17. Funnel plot of the standard error by Fischer's $Z$ for the association between negative perfectionism and adaptive outcomes

Figure 18. Forest plot with effect sizes for positive perfectionism and maladaptive outcomes

Figure 19. Forest plot with effect sizes for positive perfectionism and adaptive outcomes

Figure 20. Funnel plot of the standard error by Fischer's $\mathrm{Z}$ for the association between positive perfectionism and maladaptive outcomes

Figure 21. Funnel plot of the standard error by Fischer's $Z$ for the association between positive perfectionism and adaptive outcomes

Figure 22. Forest plot with effect sizes for negative perfectionism and positive perfectionism

Figure 23. Funnel plot of the standard error by Fischer's $Z$ for the association between negative perfectionism and positive perfectionism

Figure 24. Perfectionism Social Disconnection Model (Hewitt et al., 2006)

Figure 25. Inclusion/exclusion process for Study $1 \mathrm{~b}$ 
Figure 26. Forest plot with effect sizes for negative perfectionism and self-

injurious thoughts and behaviour

Figure 27. Funnel plot of the standard error by Fischer's $\mathrm{Z}$ for the association

between negative perfectionism and self-injurious thoughts and behaviour

Figure 28. Forest plot with effect sizes for positive perfectionism and selfinjurious thoughts and behaviour

Figure 29. Funnel plot of the standard error by Fischer's $Z$ for the association between positive perfectionism and self-injurious thoughts and behaviour

Figure 30. Forest plot with effect sizes for negative perfectionism and suicidal thoughts and behaviour

Figure 31. Funnel plot of the standard error by Fischer's $Z$ for the association between negative perfectionism and suicidal thoughts and behaviour

Figure 32. Forest plot with effect sizes for positive perfectionism and suicidal thoughts and behaviour

Figure 33. Funnel plot of the standard error by Fischer's $Z$ for the association between positive perfectionism and suicidal thoughts and behaviour

Figure 34. The Frost Multidimensional Perfectionism Scale as presented in YWS Wave Two survey

Figure 35. The Suicidal Behaviours Questionnaire as presented in YWS Wave Two survey

Figure 36. Four factor, hierarchical FMPS model resulting from PCA analyses

Figure 37. MIMIC analyses: Four factor, hierarchical FMPS model with sex as a covariate

Figure 38. DSHI-s as presented in YWS Wave Two survey

Figure 39. ISAS as presented in YWS Wave Two survey (Part one)

Figure 40. ISAS as presented in YWS Wave Two survey (Part two)

Figure 41. Self-injury (DSHI-s) screening question, and branching instructions as presented in YWS Wave Two survey

Figure 42. Mean FMPS negative and positive perfectionism scores among males and females

Figure 43. Mean FMPS subscale scores among males and females

Figure 44. Graphical depiction of a moderation analysis

Figure 45. Moderation of negative perfectionism and sex against NSSI

Figure 46. Moderation of negative perfectionism and positive perfectionism against NSSI for male participants.

Figure 47. Moderation of negative perfectionism and positive perfectionism against NSSI for female participants

Figure 48. 22-item FMPS

Figure 49. DSHI-s used in T2 survey

Figure 50. Proposed cross lagged panel correlation model to test for the relationship between perfectionism and NSSI over time 
Figure 51. Cross lagged panel correlation models testing for significant relationships between positive perfectionism, negative perfectionism and NSSI over time

Figure 52. Experiential Avoidance Model (EAM) modified to include hypothesised influence of negative perfectionism 


\section{FOREWORD}

This research was conducted within the Youth Wellbeing Study, a longitudinal research project, funded by the New Zealand Health Research Council, investigating the nature of NSSI in NZ adolescents. In addition to in-depth information about NSSI, detailed information on the overall wellbeing of NZ adolescents was collected. The YWS utilised a wide range of methods including survey based quantitative research, qualitative research (e.g. interviews with adolescents and the adults who support them) and research specifically with rangatahi Māori. Moreover, the YWS has resulted in the development of presentations and resources (e.g., graphic novels, pamphlets) to aid understanding of adolescent wellbeing and self-injury ${ }^{1}$.

My research took place within the YWS longitudinal survey which was administered to over 1000 adolescents each year from 2013 to 2016. I joined the Youth Wellbeing Study team in 2014 during the second wave of the survey, and have since been involved in survey design, survey administration, data entry, data management, survey archiving, community and workshop presentations, and the development of psychoeducation resources. During this time I was especially involved in the collection and management of the data used in both the cross-sectional and longitudinal aspects of my research. Specifically, my research involved analyses of data from waves two and three of the longitudinal survey to investigate the relationship between perfectionism and NSSI in NZ adolescents.

\footnotetext{
${ }^{1}$ For further information about the Youth Wellbeing Study, and access to the research outputs, please see www.youthwellbeingstudy.wordpress.com
} 


\section{INTRODUCTION}

Every year, thousands of people present to New Zealand (NZ) emergency departments with deliberate, self-inflicted injuries (Hatcher, Sharon, \& Collins, 2009). Approximately 3,000 of these individuals require a hospital admission of more than one day (Ministry of Health, 2015). Many more will access medical care that does not lead to hospital admission, and $85 \%$ of the total self-injuring population will never seek medical attention for their injuries (Garisch, 2010) or disclose their self-injury to a mental health professional (e.g., only $8.9 \%$ disclosure rate; Whitlock et al., 2011). Further, community-based research has demonstrated that when confidentially surveyed, up to $50 \%$ of NZ 16 to 18 year-olds report previous self-injury (Garisch \& Wilson, 2015). Relatedly, national statistics show that over 500 people take their lives through suicide each year (Ministry of Health, 2015). Statistics such as these have resulted in the NZ Ministry of Health (2015) recognising self-injury and related suicidal behaviours as a public health issue requiring substantial attention from policy makers, researchers, and health professionals.

Self-injurious behaviours are also widespread and problematic on a global scale. To illustrate, reviews of international research have indicated that approximately 16 to $18 \%$ of adolescents have engaged in self-injury at least once in their lifetime (Muehlenkamp et al., 2012; Swannell et al., 2014). Individuals who engage in self-injury are at significantly higher risk for negative outcomes such as anxiety, depression, borderline personality disorder, substance abuse, and attempted and completed suicide (Asarnow et al., 2011; Brunner et al., 2014; Fox et al., 2015; Glenn \& Klonsky, 2011; Hawton, Saunders, \& O’Connor, 2012; Jacobson \& Gould, 2007; Prinstein, 2008). To illustrate, almost nine out of ten adolescents engaging in self-injury also met criteria for a DSM-IV diagnosis (Diagnostic and Statistical Manual of Mental Disorders - Fourth Edition; American Psychiatric Association, 2000). Consequently, associated medical needs and mental health difficulties place strain on the individual's own psychological, social, and financial resources, and the resources of their families, peers, wider social network and national health care systems (Arbuthnott \& Lewis, 2015; Byrne et al., 2008; O’Dea \& Tucker, 2005). The high prevalence rates illustrated above, coupled with the many possible negative outcomes, form a strong case for research focusing on self-injury.

While raw statistics on self-injury and associated psychopathologies are a cause for concern, they reveal little about the risk factors and psychological or social mechanisms that promote and maintain self-injurious behaviours (Nock, 2010). Without this knowledge, 
researchers, clinicians, families, peers and school staff, are poorly equipped to identify those at risk of engaging in self-injury, and prevent these individuals adopting and relying on selfinjury as a coping strategy. Therefore, international research is currently focusing on uncovering the aetiology, risk factors and possible motivations for engaging in self-injury, which will in turn inform the effective identification of at risk individuals and enable communities to address this phenomenon (Nock \& Cha, 2009).

Perfectionism, typically characterised by the setting of especially high standards for one's performance and little tolerance for mistakes (Frost et al., 1990), is one such psychological construct that has previously been identified in relation to self-injury (e.g., Hoff \& Muehlenkamp, 2009; Nock \& Prinstein, 2005). Currently, however, the relationship between perfectionism and self-injury has received very little research attention. On the other hand, perfectionism shares many mental health correlates with self-injury. For example, selfinjury and perfectionism have both been associated with eating disorders, depression, anxiety, and obsessive compulsive disorder (Boone et al., 2013; Claes et al., 2012; DiBartolo et al., 2007; Lombardo et al., 2013; Vartanian \& Grisham, 2011).

Prevalence data suggest that perfectionism may characterise up to one third of children and adolescents in community settings, and likely more in clinical populations (Flett $\&$ Hewitt, 2014; Hawkins, Watt, \& Sinclair, 2006; Parker, 1997; Portesova \& Urbanek, 2013). Further, some researchers propose that with the increasingly competitive nature of education and employment, perfectionism in young populations may be increasing (Headmasters' and Headmistresses' Conference, 2015; Portesova \& Urbanek, 2013). Flett and Hewitt (2014) even go so far as to propose that we are in the midst of a perfectionism "epidemic" (p. 901). With our current knowledge of perfectionism's many mental health correlates and 'comorbidities' this could equate to potentially wide-spread and serious mental health consequences, and subsequently poses the question of whether perfectionism is currently receiving the much needed recognition and attention from school staff, researchers, and clinicians that it requires.

I hope this brief outline indicates that perfectionism and self-injury are a concern for individuals, communities and societies across the globe. With this in mind, my research was aimed at better understanding the nature of both self-injury and perfectionism, and the relationship between them. 


\section{Research Overview}

This thesis presents multiple meta-anlayses of research on perfectionism and a range of mental health variables including self-injury (Study 1), a psychometric study establishing the utility of the Frost Multidimensional Perfectionism Scale (FMPS) in New Zealand adolescent populations (Study 2), followed by two studies investigating the relationship between perfectionism and self-injury in an adolescent sample, first cross-sectionally (Study 3), and then longitudinally (Study 4). 


\section{CHAPTER ONE: NON-SUICIDAL SELF-INJURY}

While self-injurious behaviours refer to a broad category of self-directed and intentionally harmful actions (Nock, Cha, \& Dour, 2011), these behaviours can be further classified based on more specific details of each behaviour. To illustrate, Nock and Favazza (2009) developed a simple classification system that differentiated self-injurious acts based on intent, thoughts or behaviour, lethality, and severity (see Figure 1).

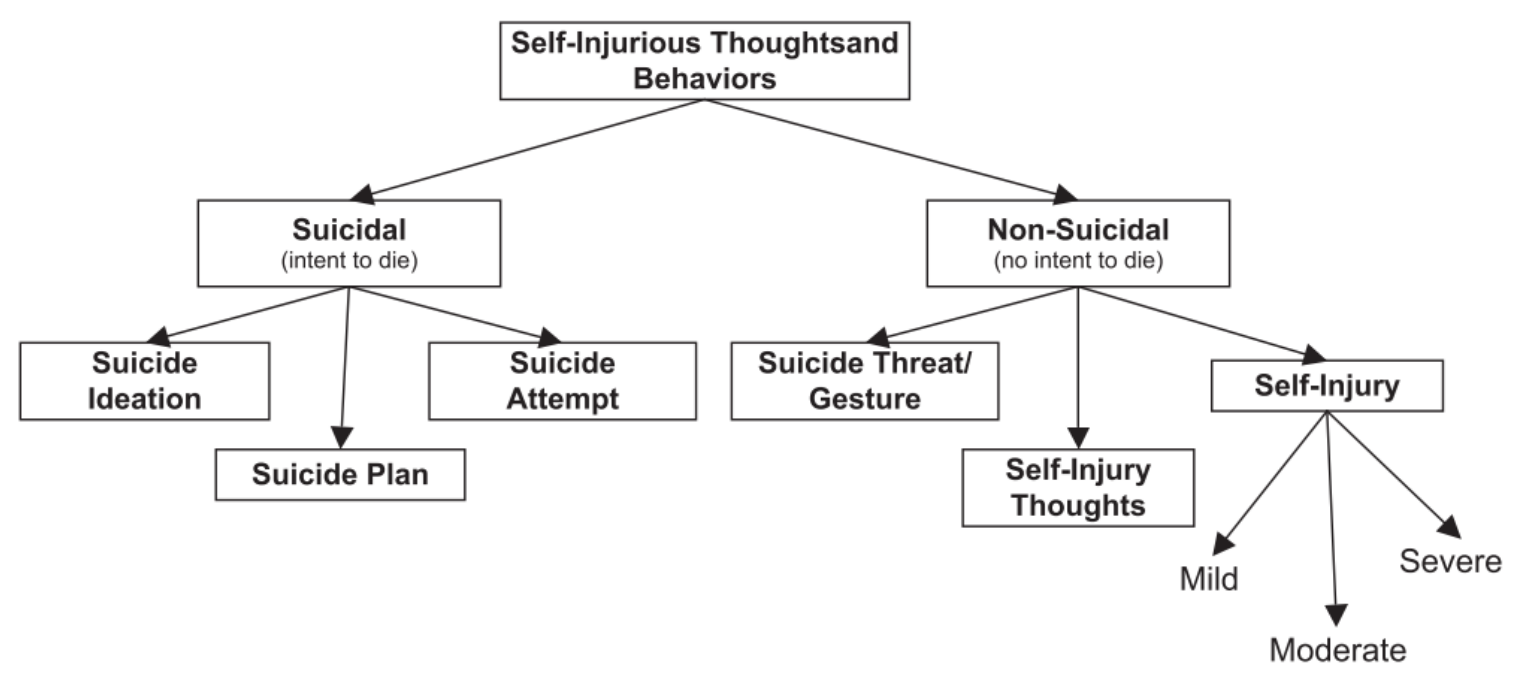

Figure 1: Nock and Favazza's (2009) classification of self-injurious thoughts and behaviours (p. 11)

The first, and perhaps most commonly overlooked, distinction is made between behaviours with and without suicidal intent. Self-injury with suicide intent (i.e., an unsuccessful, interrupted or discontinued suicide attempt) and self-injury without suicide intent undoubtedly have similarities in presentation, and share risk factors and correlates. Moreover, they can be hard to distinguish due to their common co-occurrence and intertwined nature (Muehlenkamp, 2014). However, when differentiated, research suggests that individuals who engage in self-injury without suicide intent have markedly different cognitions regarding the act of self-injury, and motivations in relation to their expectations of the consequences of their behaviour, than those intending to complete suicide (Joiner, Ribeiro, \& Silva, 2012; Muehlenkamp, 2014; Muehlenkamp \& Gutierrez, 2004; Muehlenkamp \& Kerr, 2010a; Nock et al., 2006). Moreover non-suicidal self-injury, when compared to suicidal behaviour often differs in regard to severity, course, appropriate treatment approach, and treatment response (for further information see Muehlenkamp, 2014). Therefore, researchers maintain that studies differentiating acts based on intent are 
better able to inform valid theoretical models and can be used to develop more targeted and thus, effective intervention strategies (e.g., Jacobson, Muehlenkamp, Miller, \& Turner, 2008; Joiner et al., 2012; Muehlenkamp, 2014; Nock, 2010). Consequently, this research will focus specifically on Non-Suicidal Self-Injury (NSSI). That is, self-injury with no intent of attempting suicide (Klonsky \& Muehlenkamp, 2007).

\section{Defining Non-Suicidal Self-Injury}

Non-Suicidal Self-Injury (NSSI) ${ }^{2}$ is defined as culturally inappropriate, intentional and self-directed, tissue-damaging behaviour which occurs in the absence of suicidal intent (International Society for the Study of Self-Injury, 2007; Klonsky \& Muehlenkamp, 2007; Muehlenkamp, 2014). This definition includes behaviours such as cutting, scratching, burning, and punching or banging one's head enough to cause bruising (for a full list of NSSI behaviours assessed in this thesis see Figure 38, p. 147). This definition of NSSI does not include behaviours that do not cause direct damage to body tissue, such as disordered eating (e.g., restricted eating, starvation or binging), substance abuse or overdose, or risky behaviours that do not result in tissue damage (e.g., careless driving, risky sexual behaviour, aggression towards others). NSSI also does not include culturally sanctioned behaviours such as tattooing, body art, piercing, or self-injury as part of a cultural ritual or consistent with ingroup membership. Finally NSSI, under this definition, does not include stereotypic acts of self-injury often seen in individuals with developmental disorders or intellectual disabilities (see Oliver \& Richards, 2010).

Other terminology used for self-injurious and suicidal thoughts and behaviours. A variety of other terms for self-injury are often used by other researchers. In addition to NSSI, self-injury, some of these include; deliberate self-harm (DSH), self-mutilation, selfharm, self-destructive behaviour, parasuicide, or self-inflicted injury. Whilst some terms are quite clear cut and self-explanatory (e.g., suicide attempt, NSSI), others are comparatively ambiguous (e.g., parasuicide, DSH). As a result it is often difficult to interpret research using such terms. Within this thesis, a variety of terms will be referred to when describing and referring to the findings of past research, and therefore, a brief description of common terminology can be found below. However, it is important to note that researchers have often used terms interchangeably, or have deviated from the original definitions of the terminology used. Therefore, when use of terminology and definitions are incongruent, or especially ambiguous, I will endeavour to highlight the author's own definition of the behaviour

\footnotetext{
${ }^{2}$ An acronym glossary is provided in Appendix A
} 
described when explaining their results.

'Deliberate Self-Harm' (DSH) is one of the most commonly used terms in this field of research. DSH refers to any direct, tissue damaging, self-injurious behaviour, regardless of intent. Research on DSH, although not ideal, can be used to predict patterns of NSSI (Muehlenkamp et al., 2012). Likewise, 'self-destructive behaviours', 'self-inflicted injury' and 'self-mutilation' usually refer to self-injury without specifying intent, but include a wider range of behaviours, not limited to those that cause direct body tissue damage (e.g., risky behaviours, substance use, restricted eating; Aldridge et al., 2014). Finally, 'parasuicide' is used to describe behaviours that appear to be suicidal but are not undertaken with serious suicidal intent (Holding, Buglass, Duffy, \& Kreitman, 1977). However, it seems that the term 'parasuicide' is sometimes misunderstood and has been used to refer to suicidal behaviours in some research (e.g., Hunter \& O’Connor, 2003). To reiterate, in contrast to the terms described above, NSSI comprises only of behaviours that are engaged in without intent to die, and that cause direct harm to body tissue (International Society for the Study of Self-Injury, 2007). Unless specifically stated, the terms 'self-injury' and 'NSSI' will be used interchangeably throughout this thesis. Therefore, unless qualified as occurring with suicidal intent, or referred to as DSH (intent not specified), self-injury refers to harm without suicidal intent.

\section{A Brief History of NSSI Research}

Although research began to differentiate between NSSI and suicidal behaviour some time ago (see for example, Muehlenkamp, 2006; Muehlenkamp \& Gutierrez, 2004; Nock et al., 2006), NSSI has only recently become a commonly recognised and used term. To illustrate, a search of PsychInfo using the search terms "Non-Suicidal Self-Injury" showed a substantial increase in published articles over the past 10-15 years (66 published articles regarding NSSI prior to 2010, compared to 366 published between 2010 and 2015). Increased interest in NSSI, and the subsequent increase in research, has led to a shift in researchers' and clinicians' understanding and conceptualisations of the behaviour. Most notably, historical understandings of NSSI were almost exclusively pertaining to self-injury as a 'symptom' of Borderline Personality Disorder (BPD; e.g., Glenn \& Klonsky, 2013; Zetterqvist, 2015). However, research has since highlighted that many individuals who engage in self-injurious behaviours have only a few or no symptoms of BPD. For example, some research suggests that $70-80 \%$ of individuals with a history of self-injury do not meet BPD criteria (Aldridge et al., 2014; In-Albon, Burli, Ruf, \& Schmid, 2013), and conversely BPD patients do not all 
engage in self-injury (e.g., Sher \& Stanley, 2009). As a result, recent research has been extended beyond BPD samples to include the investigation of NSSI in community samples (e.g., Muehlenkamp et al., 2012; Swannell et al., 2014).

As a further illustration of the substantial progress made by researchers and clinicians in understanding NSSI, 'Non-Suicidal Self-Injury Disorder' features in the Diagnostic and Statistical Manual of Mental Disorders - Fifth Edition (DSM-5; American Psychiatric Association, 2013) as its own standalone condition for further study (see Glenn \& Klonsky, 2013; Muehlenkamp \& Brausch, 2016; Wilkinson, 2013; Zetterqvist, 2015 for in depth discussions of the proposed criteria). Very briefly, NSSI disorder requires the individual to have engaged in NSSI on at least five different days in the past year, with the expectation that self-injury will modify their experience of emotions or cognitions. In addition, the individual has to experience interpersonal difficulties or a negative emotional or cognitive state prior to their self-injury, or have regular thoughts about self-injury.

\section{NSSI Behaviours and Descriptive Characteristics}

This recent research expansion has resulted in a large body of knowledge about common presentations of NSSI. For example, research has demonstrated a core set of NSSI behaviours that occur across both community and clinical samples. In research using behavioural checklists, cutting is typically the most common behaviour reported (e.g., Aldridge et al., 2014; Klonsky, 2007; Lewis \& Arbuthnott, 2012), and is arguably classed as the most prototypical or stereotypical self-injurious behaviour. In fact, some research uses only a single question with a probe for cutting as an assessment for self-injury history (e.g., O’Connor, Rasmussen, Miles, \& Hawton, 2009, "Have you ever deliberately taken an overdose or tried to harm yourself in some other way [such as cut yourself]?") or has specifically investigated self-injurious cutting (e.g., Kumar, Pepe, \& Steer, 2004; Miskey, Hill, \& Heulsman, 2012). Other common self-injurious behaviours include banging one's head, picking at wounds, and burning or scratching skin (Aldridge et al., 2014; Rodham \& Hawton, 2009).

Further research has demonstrated that the majority of individuals with a history of self-injury have typically engaged in multiple forms of NSSI (e.g., Gratz, 2001; Robertson, Miskey, Mitchell, Nelson-Gray, et al., 2013) and this number of methods of self-injury could be an effective indicator of risk of psychopathology (Nock et al., 2006). For example, Robertson et al., (2013) found that in a sample of university students, participants had, on average, experimented with three different methods of self-injury. Moreover, the site of self- 
injury may be an effective indicator of the individual's overall level of psychopathology (Walsh, 2012). Self-injury is most commonly located on an individual's arms, followed by their wrists, hands, and thighs (Hasking et al., 2010; Whitlock et al., 2011), however injuries on the head, face and neck, and injuries on genitals and breasts may be indicative of particularly severe mental health difficulties (Walsh, 2012).

\section{NSSI in Different Populations}

The research outlined above has provided a basic description of common NSSI profiles. However, beyond this there are some key differences in the prevalence rates and characteristics of NSSI in specific populations. These differences will be highlighted below.

NSSI in clinical and non-clinical/community populations. Despite recent research broadening into non-clinical, community samples, it is important to note that there is still a clear difference in NSSI prevalence rates amongst clinical and non-clinical samples. For example, Briere and Gil (1998) report that $21 \%$ of their adult inpatient sample had deliberately hurt themselves within the last six months, compared to only $6 \%$ of their general sample. Moreover, others have reported particularly high rates of NSSI in hospitalised patients. For example, Klonsky, May, and Glenn (2013) found that 59\% of their adolescent inpatient sample reported engaging in NSSI. In another study, $58 \%$ of adolescents hospitalised in psychiatric units reported self-injury (Wolff et al., 2014).

With regard to psychopathology, self-injury is most often investigated in groups of BPD patients, and as expected high rates of self-injury are demonstrated. For example, Chapman, Specht and Cellucci (2005) found that 73\% of their sample of BPD patients reported a history of self-injury. Self-injury is also common for those diagnosed with depression or those who are at acute risk of suicide (e.g., Groschwitz et al., 2015; Wilkinson, Kelvin, Roberts, Dubicka, \& Goodyer, 2011), and is especially prevalent in eating disorder patients (e.g., Cucchi et al., 2016).

Whilst these prevalence rates are alarming and may tempt researchers to focus their research on self-injury in clinical populations, this should not lead to assumptions that selfinjury is uncommon in community populations. As mentioned above, meta-analyses of selfinjury in community populations have indicated that almost one in five (or more) individuals have a history of self-injury (e.g., Muehlenkamp et al., 2012; Swannell et al., 2014). Further, some individual studies estimate greater prevalence with results indicating that up to $20 \%$ (Lundh, Wångby-Lundh, \& Bjärehed, 2011) of community adolescent populations not only have a history of NSSI but have engaged in self-injury five or more times, thus meeting one 
of the proposed criteria for a DSM-5 NSSI diagnosis (also see Brunner et al., 2014; Gratz, 2001; Howe-Martin, Murrell, \& Guarnaccia, 2012; Manca, Presaghi, \& Cerutti, 2014; Zetterqvist, Lundh, Dahlström, \& Svedin, 2013 for prevalence rates of repetitive self-injury in community samples). Therefore, although NSSI is less common in community samples than in clinical samples, it is important that NSSI in community populations is not overlooked.

NSSI in adolescents. NSSI can clearly be seen across the lifespan (Whitlock \& Selekman, 2014), however research often highlights the high prevalence and concerning nature of NSSI in adolescents (e.g., Muehlenkamp, Williams, Gutierrez, \& Claes, 2009). Some researchers have suggested that the prevalence of NSSI in adolescents has been gradually increasing over recent years (e.g., Miller, Muehlenkamp, \& Jacobson, 2009), whilst others argue that prevalence rates have stabilised (Muehlenkamp et al., 2012; Swannell et al., 2014). Moreover, with an average onset of NSSI between 12-14 years (Jacobson \& Gould, 2007; Klonsky \& Muehlenkamp, 2007; Nock et al., 2006), it is likely that many adults who engage in NSSI began doing so in their adolescent years. Hence, NSSI research with adolescents is likely to be particularly fruitful.

Research has been conducted with a wide variety of adolescent samples (community vs. clinical, male vs. female, local vs. international, etc.), and has demonstrated consistently high rates of NSSI amongst this age group. One study found that almost one in two (46.5\%) of 633 United States community adolescents had engaged in NSSI within the previous 12months (Lloyd-Richardson, Perrine, Dierker, \& Kelley, 2007). Moreover, 60\% of these adolescents were classified as engaging in moderate or severe NSSI. Similarly, in an Australian community sample of 393 13-18 year olds, one third had engaged in NSSI within the previous 12-months (Hasking et al., 2010). Further, Bjärehed and Lundh (2008) discovered that $40 \%$ of the Swedish 14 years olds surveyed had engaged in at least one form of NSSI in the previous six months. Overall, in an international review of NSSI prevalence information among non-clinical populations, Swannell and colleagues (2014) reported that on average, $17 \%$ of adolescents had engaged in NSSI at least once in their lifetime. ${ }^{3}$ These rates are compared to $13 \%$ of young adults and $6 \%$ of adults.

In a first NZ study, Garisch and Wilson (2015) investigated NSSI among 1162 Wellington adolescents and found that $49 \%$ reported one or more episodes of NSSI in their

\footnotetext{
3 Swannell et al.,'s (2014) estimates are likely lower than those mentioned above due to the inclusion of studies that used a single item to capture history of self-injury. See p. 145 for a discussion of how the scale used to capture self-injurious thoughts and behaviours can influence prevalence estimates.
} 
lifetime. With the exception of this research, local NSSI data is scarce (but see Fitzgerald \& Curtis, 2015), therefore research on DSH is employed to help estimate the NSSI prevalence rate among NZ samples. For example, in a sample of clients of a South Auckland Child and Family Mental Health Service 48\% reported that they had previously engaged in DSH (Fortune, Seymour, \& Lambie, 2005). Moreover, Lucassen and colleagues' (2011) used data from a national population-based youth health and wellbeing survey (Youth 2007) and demonstrated that $21 \%$ of the 9,107 adolescents surveyed reported having engaged in DSH within the last year. These statistics paint a concerning picture for adolescents both locally and internationally.

NSSI in males and females. Historically NSSI has often been assumed to be a predominantly female phenomenon (e.g., Taylor, 2003; Whitlock et al., 2011). Unfortunately, this may have led to a bias toward female only samples in the investigation of NSSI and the relationship between NSSI and sex remaining understudied and misunderstood (Whitlock et al., 2011). Contrary to popular belief, some research has shown that the prevalence rates for NSSI in males and females may actually be comparable (Marshall, Tilton-Weaver, \& Stattin, 2013; Muehlenkamp \& Gutierrez, 2004; Plener, Libal, Keller, Fegert, \& Muehlenkamp, 2009; Taliaferro \& Muehlenkamp, 2014; Tanner, Hasking, \& Martin, 2013). For example, Garisch and Wilson (2015) reported no significant difference in prevalence rates of NSSI across sex in NZ adolescents, as did Hilt, Nock, Lloyd-Richardson and Prinstein (2008) in their longitudinal study of NSSI in American adolescents. In a review of research investigating adolescent NSSI, three out of six studies reporting sex found no sex differences, while three found NSSI to be more common in females (Jacobson \& Gould, 2007). There is also evidence that in some samples NSSI may be more common amongst males (Chinese adolescent sample; Barrocas, Giletta, Hankin, Prinstein, \& Abela, 2014). Following this ongoing debate, a recent meta-analysis including 116 studies concluded that, NSSI is indeed slightly more common in females. However, the authors concluded that with an effect size considered small for epidemiological studies $(\mathrm{OR}=1.50)$, this simply reinforces the importance of routinely assessing for, and researching, NSSI in both male and female populations (Bresin \& Schoenleber, 2015).

Research moving beyond pure prevalence statistics suggests that there may be key sex differences in the more specific details of NSSI and, dependant on the method of data collection, male and females disclosure of NSSI behaviours may be quite different (Bresin \& Schoenleber, 2015; Whitlock et al., 2011). These sex differences include onset, frequency, methods, and functions of NSSI; differences in help seeking behaviour, and the social 
acceptance of engaging in NSSI (Whitlock et al., 2011).

With regard to onset, it has been consistently identified that females begin to engage in NSSI earlier than males (Andover, Primack, Gibb, \& Pepper, 2010; Hawton, Bergen, et al., 2012; Nixon, Cloutier, \& Aggarwal, 2002). Therefore, although lifetime prevalence appears to be quite similar across males and females, the younger the sample, the more it may appear that more females engage in NSSI. Concerning the method of self-injury, Bresin and Schoenleber's (2015) meta-analysis found that females are more likely to use methods that produce blood, such as cutting and scratching. Garisch (2010) found similar results with NZ adolescents, namely that females were more likely to engage in more prototypical methods, such as cutting and self-scratching until scarring or bleeding, whereas males use other, less recognised, methods of DSH such as rubbing sand paper and dripping acid on their skin. Moreover, sex differences with regard to methods of self-injury have been reported in numerous other studies (e.g., females more likely to cut themselves, Brunner et al., 2014; Lundh, Karim, \& Quilisch, 2007; males more likely to engage in self-hitting behaviours; Latimer, Meade, \& Tennant, 2013). In terms of the data collection method used, some studies may not recognise or measure methods of self-injury more common to males (e.g., banging one's head, self-hitting) and therefore may be biased towards identifying females who engage in NSSI.

Lastly, in terms of help-seeking behaviour, some research suggests that females are more likely than males to seek assistance for their self-inflicted injuries. For example, Whitlock and colleagues (2011) found that females were not only more likely to seek medical attention for the injuries caused, they were also more likely to have sought psychological therapy for any reason. Locally, the Ministry of Health (2015) reported that, every year, more than twice as many females than males are hospitalised for DSH. In line with this, previous research has suggested that differences in help-seeking and prevalence of males and females presenting to emergency care centres could be partly due to increased societal awareness of female NSSI, and the behaviour being perceived as more socially 'acceptable' in females (Muehlenkamp \& Gutierrez, 2004; Taylor, 2003). This could have potentially skewed hospital statistics such as those presented by the Ministry of Health, and those described above.

Overall, sex differences are common across many facets of NSSI behaviour and although one meta-analysis suggested that females engage in slightly more NSSI, these results may be confounded by the data collection methods used (e.g., investigating forms of NSSI more likely to be seen in females such as cutting), differences in help seeking 
behaviours, and less societal awareness and acceptance of NSSI in males. Therefore, research should continue to investigate NSSI across both sexes whilst also considering that risk and protective factors, the presentation of NSSI, methods used and functions endorsed, and helpseeking patterns may substantially differ across male and female populations.

NSSI in different cultures and countries. The study of self-injury has been dominated by research in North America, Europe, and Australia. As a result, our current understandings are largely based on the presentation of self-injury in Western populations, and typically in majority groups. Research in Asia, Africa, and South America, and research with minority and some indigenous populations (e.g., Māori, Indian Americans, Australian and Canadian Aboriginal populations) is extremely limited. Therefore, it is important to note that Western understandings may not automatically be directly and meaningfully applied to individuals of non-Western, or minority cultures. At a very rudimentary level, cross-cultural research has highlighted that even the simple definitions and understandings of self-injury can vary greatly across cultures (e.g., Cwik et al., 2011; McAllister, 2003). Of direct relevance to this research, NZ research with rangatahi Māori (Māori youth) has demonstrated that typical definitions of NSSI do not incorporate all behaviours that Māori would classify as directly harmful. For example, harm to wairua (spiritual aspect of wellbeing) is considered injurious in a similar way to direct harm to body tissue (Kingi, 2015). In addition to differences across culture, difference in self-injury correlates have been demonstrated across countries. For example, Brunner and colleagues (2014) have demonstrated that whilst family environment was an important correlate of DSH in Germany, risky behaviours and psychopathology was especially related to DSH in Irish adolescents.

In summary, this research and most other self-injury research focuses on Western conceptualisations of NSSI and broader Western understandings of psychological wellbeing and mental health. Unfortunately, there is limited capacity within this research to investigate cultural differences, however this is a very important direction for future research. In the meantime, findings and discussion of Western research should be interpreted with caution especially when considering generalisation to non-western populations. Moreover, crosscountry research (e.g., Brunner et al., 2014) highlights the importance of having local research to inform prevention and intervention strategies and understand the relationships between self-injury, common risk factors, and the overall wellbeing of local adolescents.

\section{Risk and Protective Factors for NSSI}

Research over the past 10-15 years has highlighted numerous factors associated with 
increased risk for self-injury. First, adolescents are at greater risk for self-injury than children, young adults or older adults (e.g., Swannell et al., 2014; Ministry of Health, 2015). Also with regard to demographic characteristics, females are slightly more likely to engage in self-injury than males (Bresin \& Schoenleber, 2015). Some research has also shown that minority populations also appear to be at greater risk of self-injury, and that this is especially so for indigenous (e.g., Bhui, McKenzie, \& Rasul, 2007; Cwik et al., 2011; Nada-Raja, Skegg, Langley, Morrison, \& Sowerby, 2004; Smith, Chesin, \& Jeglic, 2014) and sexual minority populations (Batejan, Jarvi, \& Swenson, 2015; Lucassen et al., 2011).

With regard to psychological factors, emotion regulation difficulties are considered a core feature of self-injury. To briefly explain, leading theories of self-injury posit that most often individuals will self-injure to modify their emotional experience (i.e., to distract themselves or release the tension associated with unmanageable negative emotions: Klonsky \& Glenn, 2009). These theories are based on substantial empirical research indicating that individuals who self-injure typically have difficulty managing emotions (e.g., Gratz, Conrad, \& Roemer, 2002; Vieira, Ramalho, Brandão, Saraiva, \& Gonçalves, 2016; Voon, Hasking, \& Martin, 2014). Research also suggests that this population may experience emotions as especially distressing and with greater intensity (Nock, Wedig, Holmberg, \& Hooley, 2008), and also may have a lower distress tolerance (Nock \& Mendes, 2008) than non-self-injuring populations. The relationship between emotion regulation and self-injury will be explored in depth at various stages throughout this thesis.

Psychological disorders are also commonly associated with NSSI. As previously outlined (see p. 7) self-injury was originally thought to primarily occur in clinical populations (Glenn \& Klonsky, 2013; Zetterqvist, 2015). Whilst self-injury is now acknowledged in much wider populations, its significance to BPD populations remains. Depression and anxiety are also commonly noted as risk factors for self-injurious behaviours (e.g., Hawton \& James, 2005; Hawton, Saunders, et al., 2012; Jacobson \& Gould, 2007). In particular, self-injury is often conceptualised as a method for managing the distress associated with high levels of anxiety, persistent low mood, or the feelings of numbness often associated with depression. Moreover, empirical evidence consistently supports the co-occurrence of self-injury and depression (e.g., Asarnow et al., 2011; Bentley, Cassiello-Robbins, Vittorio, Sauer-Zavala, \& Barlow, 2015; Brunner et al., 2014; Nock et al., 2006), and self-injury and anxiety (e.g., Bentley et al., 2015; Brunner et al., 2014; Selby, Bender, Gordon, Nock, \& Joiner, 2012) in a wide range of samples.

Eating disorders and self-injury also commonly co-occur. In fact, a meta-analysis and 
systematic review of research on self-injury in eating disorder patients showed that $22 \%$ and $32 \%$ of anorexia nervosa and bulimia nervosa patients respectively had histories of NSSI (Cucchi et al., 2016). These rates, especially amongst bulimia nervosa patients, are higher than average NSSI prevalence rates among community samples (Swannell et al., 2014). Researchers who have investigated the relationship between NSSI and eating disorder pathology commonly suggest that both behaviours are related to difficulties in emotion regulation (Vieira et al., 2016) and may in fact both serve emotion regulation functions (Jeppson, Richards, Hardman, \& Granley, 2003; Peyerl, 2010; Wedig, 2014).

Finally, some contextual and event-based factors have shown to increase risk for selfinjury. First, Brown (2015) conducted a meta-analysis of all research examining the relationship between bullying and NSSI. She reported that although relatively small $(r=.26)$, bullying was significantly associated with increased risk of self-injury. Second, research investigating deprivation, often via social economic status, has demonstrated that adolescents whose parents consistently reported low income during their childhood, were subsequently 1.5 times more likely to report self-injury during their adolescence than adolescents whose parents never reported low income (Page et al., 2014). The risk factors presented above are only a snapshot of the many factors that can place an individual at increased risk for selfinjury (for a review see Fox et al., 2015).

Conversely, research has highlighted some factors that are associated with less engagement in NSSI. One particularly protective factor appears to be a strong attachment to family and peers, and associated perceptions of interpersonal support (e.g., Bureau et al., 2010; Tatnell, Kelada, Hasking, \& Martin, 2014; Wichstrøm, 2009). For example, Claes, Luyckx, Baetens, Van de Ven and Witteman (2015) found that adolescents who experienced bullying or victimisation were less likely to go on to engage in NSSI when they perceived their parents to be supportive. Lastly, high self-esteem (Garisch \& Wilson, 2015) has been shown to buffer against risk of NSSI in NZ adolescents, as well as buffer against risk of repetitive DSH in adults (McAuliffe et al., 2005).

\section{Functions of NSSI}

Thus far I have detailed research on NSSI prevalence, some of its correlates and particular at-risk populations. However, a major missing piece of the puzzle is why adolescents engage in NSSI. To answer the question of why, some researchers have taken a functional approach. This has broadened understanding of NSSI, and has provided insight into how the behaviour is being maintained. Consequently, targets for treatment are identified 
(Heath, Toste, \& MacPhee, 2014; Lloyd-Richardson, Nock, \& Prinstein, 2009; Stanley, Fineran, \& Brodsky, 2014).

Based on their early functions research Nock and Prinstein (2004) outlined the Four Functional Model (FFM) of NSSI which proposes that the functions of NSSI can first be grouped according to whether the behaviour is internally (automatic reinforcement) or externally reinforced (social reinforcement), and then by whether the reinforcement is negative (removal of negative stimulus) or positive (elicits a positive stimulus). Therefore, the functional categories include; automatic positive reinforcement, automatic negative reinforcement, social positive reinforcement and social negative reinforcement (see Figure 2). Nock and Prinstein reported that their sample of psychiatric adolescent inpatients were more likely to endorse automatic negative reinforcement functions than functions from the other three categories. In particular, over half of the adolescents said that they engaged in NSSI 'to stop bad feelings' (automatic negative reinforcement). Moreover, automatic positive reinforcement functions were endorsed more than either of the social functional categories. This suggests that, contrary to public opinion, NSSI is more likely to be serving an automatic and primarily emotion regulating function, than the attention seeking or social functions that are often assumed (Klonsky, Victor, \& Saffer, 2007).

\begin{tabular}{c|c|c|}
\multicolumn{2}{c}{ Positive Reinforcement } & Negative Reinforcement \\
\cline { 2 - 3 } Automatic & $\frac{\text { Automatic Positive Reinforcement }}{\text { e.g., 'to punish yourself' }}$ & $\frac{\text { Automatic Negative Reinforcement }}{\text { e.g., 'to stop bad feelings' }}$ \\
\cline { 2 - 3 } Social & $\begin{array}{c}\text { e.g., 'to get a reaction from someone, even } \\
\text { if it is negative' }\end{array}$ & $\begin{array}{c}\text { Social Negative Reinforcement } \\
\text { e.g., 'to avoid being with people' }\end{array}$ \\
\hline
\end{tabular}

Figure 2: Four-Functional Model (FFM) of NSSI (Nock \& Prinstein, 2004)

Nock, Prinstein and Sterba (2009) later published another study assessing the functions of self-injury with a real-time method. They used an ecological momentary assessment method, whereby 30 adolescents and young adults carried around an electronic device which prompted them to complete a series of questions about their self-injurious thoughts and behaviours in the period since their previous entry. Participants were prompted to respond twice daily for two weeks. Nock et al., reported that participants most commonly 
described automatic negative reinforcement (64\% of self-injury episodes), followed by automatic positive reinforcement (25\%), social negative reinforcement (15\%), and social positive reinforcement (4\%). Consistent with their previous research (Nock \& Prinstein, 2005), participants most commonly reported using NSSI to escape from negative affective and/or cognitive states (Nock et al., 2009). This suggests that on the day of self-injury, rather than days or months following the act of self-injury (as is most commonly captured with survey measures), automatic negative reinforcement functions remained the most salient to these adolescents and young adults.

Furthermore, in a review of research exploring the functions of NSSI, Klonsky (2007) identified seven specific functions that have consistently been endorsed by subsequent study participants. These included affect regulation, anti-dissociation, anti-suicide, interpersonal boundaries, interpersonal influence, self-punishment, and sensation seeking. Consistent with Nock and Prinstein's (2004) findings, in all the studies using self-report, affect regulation functions were the most endorsed ( $>50 \%$ of participants endorsed affect regulation; Brown, Comtois, \& Linehan, 2002; Laye-Gindhu \& Schonert-Reichl, 2005; Nock \& Prinstein, 2004). Self-punishment functions were also frequently reported. Additionally, the pattern of reported functions was broadly consistent regardless of the sample demographics (e.g., adolescent vs. adult; community vs. clinical).

A couple of years later, Klonsky and Glenn (2009) developed the Inventory of Statements about Self-Injury (ISAS), a self-report measure capturing both NSSI behaviours and functions. They included the seven functions identified by Klonsky's (2007) review and six other functions developed from the anecdotal reports of researchers and clinicians. The extra functions include self-care, peer bonding, toughness, marking of distress, revenge and autonomy. In a sample of university students with a history of NSSI, Klonsky and Glenn again illustrated that affect regulation and self-punishment were the two most commonly endorsed functions. Factor analysis revealed two broad factors: intrapersonal and interpersonal. Linking this research back to the FFM, Klonsky and Glenn's intrapersonal factor corresponds to the automatic positive and automatic negative reinforcement categories. Conversely, the interpersonal factor corresponds to the social positive and social negative reinforcement components of the FFM. At this higher level of analysis, intrapersonal reasons for engaging in NSSI (e.g., affect regulation, self-punishment) were reported significantly more often than interpersonal reasons (e.g., interpersonal influence, peer bonding), thus reflecting previous findings outlined above.

Finally, some research suggests that there may be differences in the patterns of 
endorsed functions of NSSI for females and males. For example, Klonsky and Glenn (2009) reported that females endorsed intrapersonal functions significantly more than males, while there was no sex difference for interpersonal functions. This pattern has also been found in a community sample of adolescents (Zetterqvist et al., 2013). At a finer level, Klonsky and Glenn found that males endorsed the sensation seeking function significantly more than females. In addition, Lloyd-Richardson and colleagues (2007) found that adolescent girls were more likely than adolescent boys to report NSSI to self-punish, and boys were more likely than girls to report NSSI to make others angry. Finally, Laye-Gindhu and SchonertReichl (2005) demonstrated that adolescent girls reported NSSI due to feelings of despair, negative feelings about the self, and loneliness, whereas adolescent boys reported NSSI out of boredom, or to communicate or influence others. Others have found no significant sex differences in the endorsement of specific functions (e.g., Leong, Wu, \& Poon, 2014) or broader intrapersonal/interpersonal categories (Lloyd-Richardson et al., 2007).

\section{Theoretical Models of NSSI}

Once the common functions of NSSI were identified, researchers combined them with identified risk factors and correlates of NSSI to propose theoretical models that consider both the development and maintenance of the behaviour. For example, Chapman, Gratz and Brown (2006) proposed the Experiential Avoidance Model (EAM; see Figure 3), to explain the reinforcement contingencies involved in the maintenance of NSSI for intrapersonal reasons and, in particular, affect regulation functions.

The main premise of the EAM is that NSSI is negatively reinforced (aversive stimulus is removed and the individual is rewarded; Mazur, 2002) through avoidance of negative emotional states and other negative internal experiences. Chapman and colleagues argue that each time the individual feels emotional relief following an act of NSSI, the behaviour becomes a little more ingrained as an automatic avoidance strategy. This reinforcement then raises the prospect of NSSI becoming the primary short-term coping strategy for aversive internal states. Thus, Chapman and colleagues describe the experiential avoidance cycle of NSSI as a vicious, self-perpetuating, and potentially dangerous spiral. 


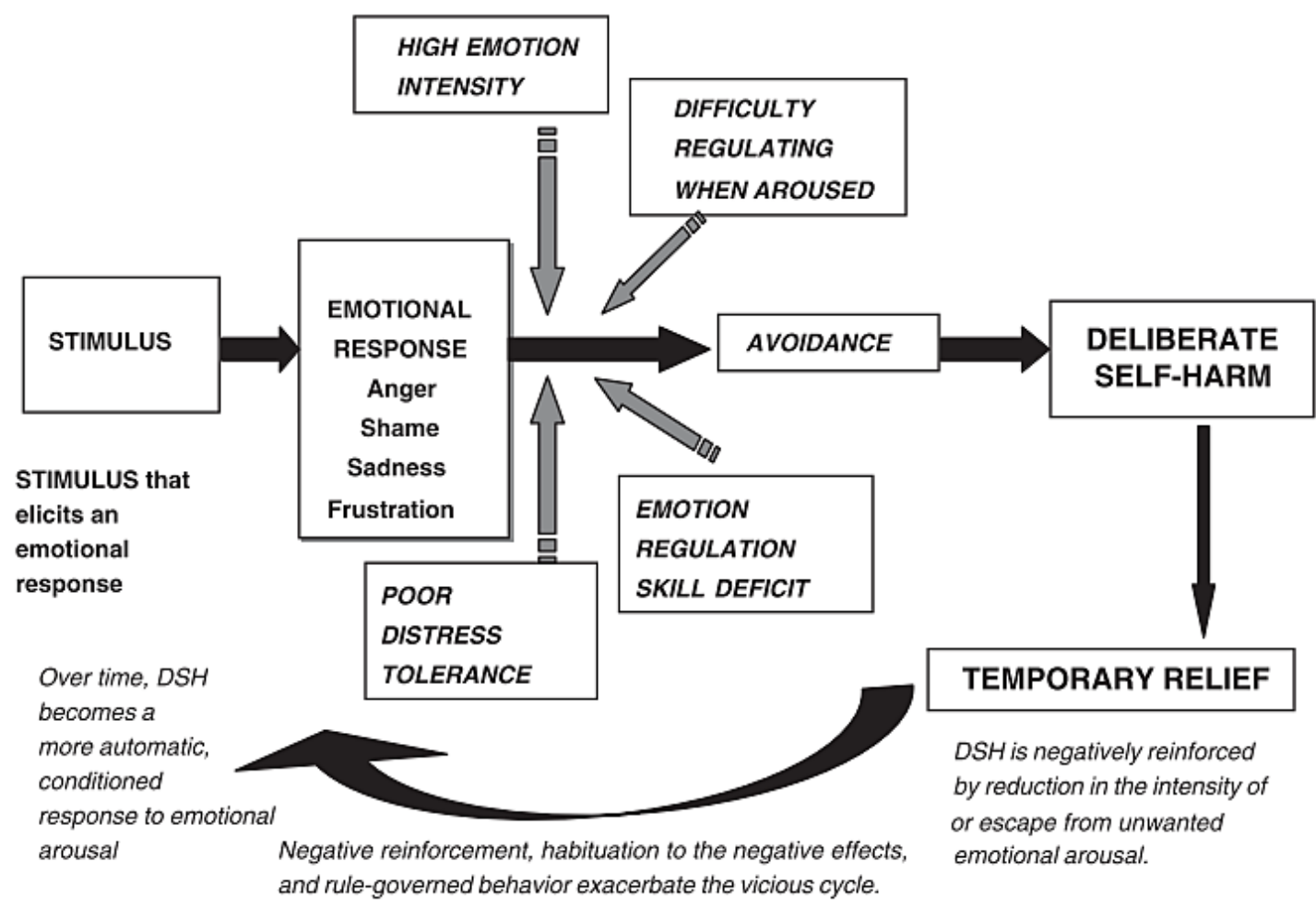

Figure 3: The Experiential Avoidance Model (EAM) of Deliberate Self-Harm (Chapman et al., 2006, p. 373)

Nock (2010) proposed a similar model, namely the Integrated Theoretical Model of Self-injury (ITMS; Figure 4). In the ITMS, NSSI is reinforced either through its affect regulation function, or the resulting regulation of a social situation. Nock's ITMS integrates the FFM. For example, with regard to the regulation of affect, negative reinforcement will likely involve relief from intense negative emotion, whereas positive reinforcement could be feeling generation. On the other hand, social negative reinforcement could be the removal of expectations of others, and social positive reinforcement may be an increase in support from others.

In addition, the ITMS includes distal risk factors for general psychopathology (e.g., childhood abuse, family criticism), that in combination with interpersonal vulnerabilities (e.g., poor social problem-solving, poor communication skills) and intrapersonal vulnerabilities (e.g., poor distress tolerance) can decrease an individual's ability to cope with high social demands or over/under arousal of emotion (Nock, 2010). Nock then proposes that the resulting distress, coupled with more specific NSSI vulnerability factors (e.g., access to means, high criticism from others increasing tendency to self-punish), increases the chance of NSSI becoming the primary coping method. 


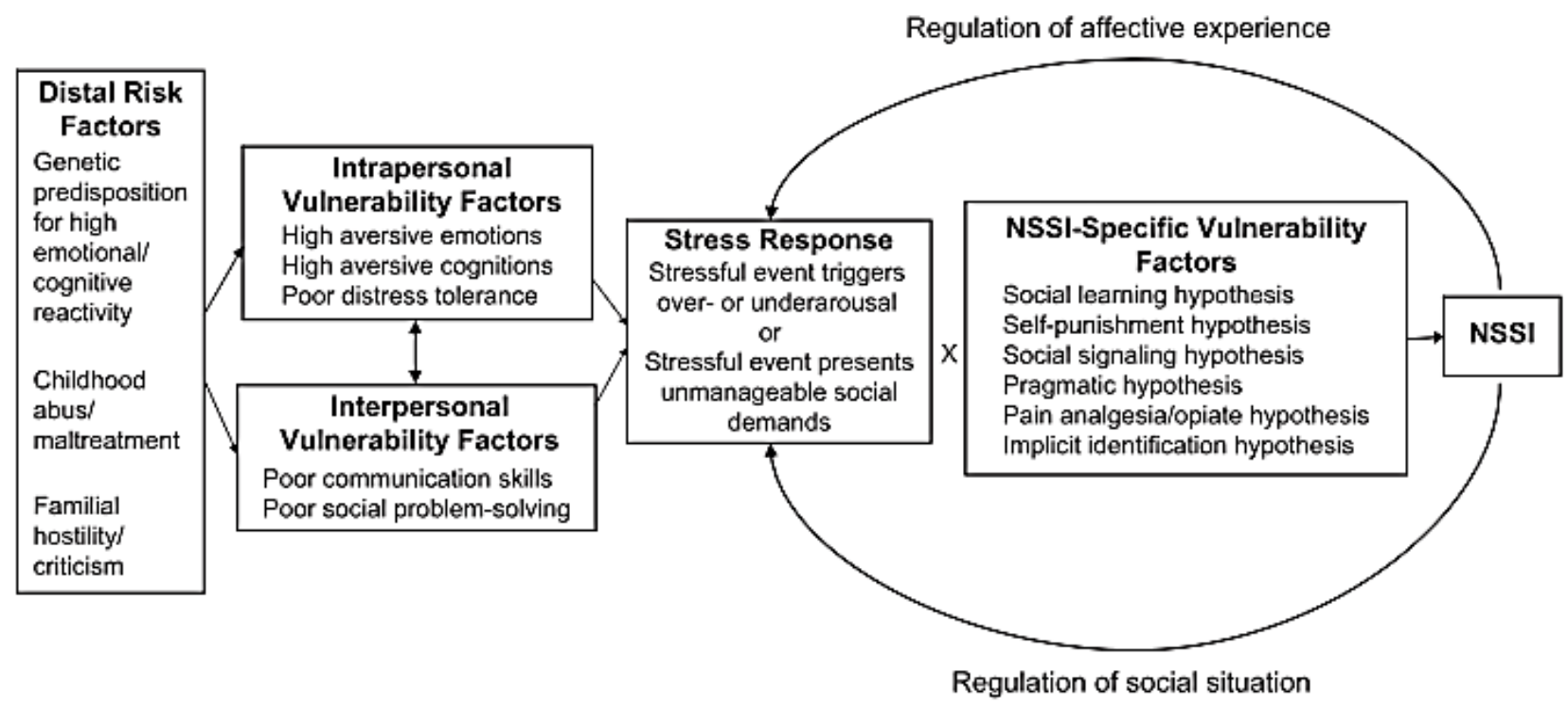

Figure 4: Nock’s (2010) Integrated Theoretical Model of Self-Injury (p. 10)

Nock's (2010) ITMS also highlights the importance of understanding the distal factors that could contribute to an individual experiencing a situation as unmanageable, either intrapersonally or interpersonally, thereby placing them at risk of NSSI. From previous research we can propose that some of these factors may include sexual and emotional abuse, impulsivity, depression, low self-esteem, alexithymia and bullying (Andrews, Martin, Hasking, \& Page, 2013; Barrocas et al., 2014; Di Pierro, Sarno, Perego, Gallucci, \& Madeddu, 2012; Garisch \& Wilson, 2010; Glassman, Weierich, Hooley, Deliberto, \& Nock, 2007; Jacobson \& Gould, 2007). In addition, perfectionism may be another factor that is implicated in the development of NSSI (Hoff \& Muehlenkamp, 2009; O'Connor et al., 2010). This will be explored in great detail shortly.

As the final component of this chapter I will briefly digress to explore the relationship between NSSI and suicidality. As will become apparent, a thorough assessment of an individual's NSSI behaviours can provide valuable information about their concurrent risk of suicide. Therefore it is important to properly understand how information about both nonsuicidal and suicidal behaviours is important in forming a comprehensive understanding of an individual's wellbeing.

\section{NSSI and Suicidal Thoughts and Behaviours}

Whilst, it is clear that NSSI and suicidal self-injury are distinct behaviours (see p. 5) and therefore it remains important to differentiate between them when conducting research, there is a substantial body of research demonstrating that NSSI and suicidal behaviours are 
closely related. For example, Arsanow and colleagues (2011) reported that in a sample of adolescents with a DSM-IV diagnosis of depression, $37 \%$ of participants with a history of NSSI had also engaged suicidal self-injury, compared to only $15 \%$ of their non-NSSI group. Likewise, Glenn and Klonsky (2009) found that within their sample of young adults with a history of self-injury, $47 \%$ had experienced suicidal ideation, $37 \%$ had previously had a plan for suicide, $9 \%$ had attempted suicide once, and 10\% had attempted suicide multiple times. Conversely, in their control sample of young adults with no history of self-injury, only $10 \%$ had experienced suicidal ideation, $6 \%$ had previously had a plan for suicide, $1 \%$ had attempted suicide once, and less than $1 \%$ had attempted suicide multiple times. Both of these studies highlight the common existence of suicidal thoughts and behaviours in individuals with a history of NSSI (also see Hamza, Stewart, \& Willoughby, 2012; Nada-Raja et al., 2004; Whitlock et al., 2013).

Beyond a simple co-occurrence, the characteristics of an individual's NSSI profile (e.g., frequency, severity, methods) can be used to predict their risk of past, concurrent, and future suicidal ideation and behaviours. For example, Prinstein et al., (2008) found that greater frequency of self-injury in a sample of adolescent inpatients was associated with more frequent suicidal ideation prior to treatment, and also predicted ongoing and sustained suicidal ideation following a treatment programme (also see Victor \& Klonsky, 2014; Whitlock et al., 2013). Moreover, Tang and colleagues (2011) reported that severe forms of NSSI, such as cutting and burning, were associated with a greater likelihood of a history of suicide attempts than less severe self-injury. These findings are further supported by Lloyd-Richardson and colleagues (2007) who found that methods such as cutting or carving the skin were more strongly associated with suicidal behaviours than pulling hair, picking at wounds, biting or hitting oneself, and inserting objects under the skin. Moreover, using meta-analytic techniques Victor and Klonsky (2014) demonstrated that the number of methods of self-injury an individual has engaged in was the second strongest predictor of suicide attempts, only exceeded by suicidal ideation. Taken together, these findings suggest that a thorough assessment of an individual's self-injury profile can be clinically relevant to their suicide risk assessment.

With regard to the temporal relationship between self-injury and suicide, longitudinal research has shown that NSSI typically predicts suicide attempts, rather than suicide attempts predicting NSSI (e.g., Asarnow et al., 2011; Prinstein et al., 2008; Wilkinson et al., 2011). Moreover, data suggests a history of NSSI is in fact one of the strongest predictors of subsequent suicidal behaviour (Franklin et al., 2016). What is more, NSSI has significantly 
predicted suicidality even when controlling for baseline suicidal thoughts and behaviours (Hamza \& Willoughby, 2016).

In light of the empirical data, Joiner (2005) proposed the Interpersonal-Psychological Theory of Suicidal Behaviour which argues that an individual with a history of self-injury is at higher risk of future suicide through the acquisition of capability to fatally harm themselves through the practice of self-injury. Specifically, Joiner notes that self-injury increases an individual's pain tolerance, and decreases their fear of hurting themselves, thus decreasing barriers to suicide. In addition, he suggests that individuals with a substantial history of selfinjury have likely experienced the relief, albeit often short-lived, that self-injury can provide. Therefore, they may believe that in order to feel sustained relief, or escape, they must complete suicide (Joiner, 2005). Whitlock and colleagues (2013) provide support for NSSI as a gateway to suicide in a sample of 1,467 university students. They demonstrated that suicidal thoughts and behaviours were particularly prominent in students who had a history of more than 20 acts of NSSI, and suggested that NSSI provides an opportunity to habituate to suicidal acts over time.

Before concluding this chapter, it is important to reiterate that although self-injury and suicide are strongly associated, these phenomena are more appropriately researched as distinct but overlapping phenomena. However, if the direct relationship between NSSI and future suicidal behaviour is as prominent as research suggests, targeting NSSI may subsequently reduce an individual's risk of future suicidal behaviour. In summary, NSSI research may also indirectly be utilised in the reduction or prevention of death by suicide for many at risk individuals.

\section{Chapter Summary}

Overall this chapter has outlined the importance of investigating NSSI, particularly in adolescent populations. Moreover, common characteristics of NSSI were explored alongside theories about why an individual begins engaging in NSSI, and why they continue to use selfinjury over time. The relationship between NSSI and suicidal thoughts and behaviours was discussed, with an emphasis on the importance of understanding how the two sets of behaviours are related. Finally, throughout the chapter reference was made to important risk factors for the development of self-injurious behaviours.

While the specific details remain unclear it has been suggested that perfectionism is another factor that is related to self-injurious behaviours (e.g., Hoff \& Muehlenkamp, 2009; O'Connor et al., 2010). Therefore, this thesis will now progress to explore the nature of 
perfectionism, and I shall argue that perfectionism may be incorporated into both the EAM and ITMS models of self-injury. Prior to this, the next part of the thesis will provide a comprehensive overview of the nature of perfectionism. 


\section{CHAPTER TWO: PERFECTIONISM}

Perfectionism is defined as "the setting of excessively high standards of performance" (Frost et al., 1990, p. 450). In many situations this can be a positive quality, and may contribute to high levels of motivation and achievement (Hamachek, 1978). More often, however, research has shown that perfectionism is an underlying cognitive vulnerability which, when coupled with adverse environmental circumstances, can result in psychological distress and maladaptive coping behaviours (e.g., Ashby \& Kottman, 1996; Dunkley, Blankstein, Halsall, Williams, \& Winkworth, 2000; O'Connor et al., 2010; Richardson \& Rice, 2015). To further elaborate, Flett and Hewitt (2016) recently summed up perfectionism as "an achievement-based personality construct, rooted in goal attainment, and specific reactions and responses when the person who so desperately strives for perfection almost inevitably falls short of the cherished goal" (p. 615). This highlights the vulnerable situation that many perfectionists face, whereby their unrealistic goals and need to strive for perfection in turn almost guarantee their failure to achieve to such standards.

This contemporary understanding of perfectionism owes much to the highly influential contribution of Frost and colleagues (1990) who effectively consolidated previously conflicting and ambiguous ideas of what perfectionism might entail. First they identified the key components of perfectionism captured in previous measures (e.g., The Burns Perfectionism Scale [BPS]; Burns, 1980; subscales within the Eating Disorders Inventory [EDI]; Garner, Olmsted, \& Polivy, 1983; The Dysfunctional Attitudes Scale [DAS]; Weissman \& Beck, 1978; The Irrational Beliefs Test [IBT]; Jones, 1969) and, second, developed the first multidimensional perfectionism measure, the Frost Multidimensional Perfectionism Scale (FMPS). The FMPS is comprised of six subscales; Concern over Mistakes (CM; where even slight mistakes are perceived as complete failures), Doubts about Actions (DA; feeling that a task is never successfully completed), Parental Criticism (PC; the perception that anything less than perfection will result in disapproval), Parental Expectations (PE; feeling that parental expectations are at an ever-increasing high level), Personal Standards (PS; setting of challenging goals for oneself), and Organisation (O; 'having a strong preference for order and neatness).

Subsequent research using the FMPS has demonstrated that the six subscales differentially relate to other positive and negative traits and behaviours. For example, Organisation has been related to positive achievement striving and good work habits (Frost et al., 1990). Conversely, the components tapping into negative self-evaluation, namely CM and 
DA, have been associated with psychopathological symptoms and disorders such as depression, anxiety disorders, obsessive compulsive disorder, eating disorders, substance abuse, stress, and suicide ideation and attempts (Antony et al., 1998; Bieling, Israeli, \& Antony, 2004; Claes et al., 2012; Dickie, Wilson, McDowall, \& Surgenor, 2012; Frost et al., 1990; Hamilton \& Schweitzer, 2000; Handley, Egan, Kane, \& Rees, 2014; Libby, Reynolds, Derisley, \& Clark, 2004).

Around the same time that Frost and colleagues (1990) developed the FMPS, Hewitt and Flett (1991) introduced their own Multidimensional Perfectionism Scale (HFMPS). Like Frost et al., Hewitt and Flett combined existing conceptualisations of perfectionism and introduced their own perspective of the key components of perfectionism. This resulted in three subscales; Self-Orientated Perfectionism (SOP), Other-Orientated Perfectionism (OOP) and Socially-Prescribed Perfectionism (SPP). In comparison to the FMPS, the HFMPS takes an interpersonal focus with Self-Orientated Perfectionism and Other-Orientated Perfectionism referring to having overly-high expectations of oneself and others respectively, and SociallyPrescribed Perfectionism capturing feelings that others are judgmental and have high expectations of you (Hewitt \& Flett, 1991).

Research with the HFMPS has since shown that SPP is primarily related to negative outcomes such as interpersonal and achievement hopelessness (Blankstein, Lumley, \& Crawford, 2007; Blankstein \& Winkworth, 2004), maladaptive cognitions (Blankstein \& Winkworth, 2004), suicidal ideation (Blankstein et al., 2007; Izadi, 2014), stress (Chang, 2000; Short \& Mazmanian, 2013), and shame (Casale, Fioravanti, Flett, \& Hewitt, 2014; Chen, Hewitt, \& Flett, 2015; Izadi, 2014). Alternatively, Self-Orientated Perfectionism has been positively associated with self-esteem (Klibert, Langhinrichsen-Rohling, \& Saito, 2005), self-control (Flett, Hewitt, Blankstein, \& O’Brien, 1991; Klibert et al., 2005) and problemsolving (Blankstein et al., 2007). This again suggests that perfectionism can be both adaptive and maladaptive

A couple of years later, Frost, Heimberg, Holt, Mattia and Neubauer (1993) aimed to consolidate the research that had resulted from the FMPS and HFMPS, and combine the unique focuses and benefits of each scale. To do so they used factor analysis to investigate the degree of overlap between the six subscales from the FMPS and the three subscales of the HFMPS. Consequently, a two-factor perfectionism model that split the FMPS and HFMPS subscales across Maladaptive Evaluative Concerns and Positive Achievement Striving was presented (Table 1). 
Table 1

Frost, Heimberg, Holt, Mattia and Neubauer (1993) Two Component Model of Perfectionism

Maladaptive Evaluative Concerns

Parental Criticism (PC; FMPS)

Parental Expectations (PE; FMPS)

Concern Over Mistakes (CM; FMPS)

Doubts about Actions (DA; FMPS)

Socially-Prescribed Perfectionism (SPP; HFMPS)
Positive Achievement Striving

Personal Standards (PS; FMPS)

Organisation (O; FMPS)

Self-Oriented Perfectionism (SOP; HFMPS)

Other-Oriented Perfectionism (OOP; HFMPS)

While the overarching consensus is that one component represents negative aspects of perfectionism and one represents positive aspects of perfectionism, this distinction has been referred to in various ways; for example, neurotic or normal (Hamachek, 1978), satisfied or dissatisfied (Owens \& Slade, 1987), passive or active (Lynd-Stevenson \& Hearne, 1999), maladaptive or adaptive (Kawamura, Hunt, Frost, \& DiBartolo, 2001), personal standards or self-critical (Dunkley, Sanislow, Grilo, \& McGlashan, 2006), ego-dystonic or ego-syntonic (Stairs, Smith, Zapolski, Combs, \& Settles, 2012), and maladaptive evaluative concerns or pure personal standards (DiBartolo, Frost, Chang, LaSota, \& Grills, 2004).

With specific reference to Frost and colleagues' (1993) study (see Table 1) negative perfectionism, termed Maladaptive Evaluative Concerns, is comprised of CM, DA, PC, PE, and SPP. On the other hand, positive perfectionism, termed Positive Achievement Striving, comprises Organisation, PS, SOP, and OOP (Frost et al., 1993; Terry-Short, Owens, Slade, \& Dewey, 1995). For the sake of simplicity, the two components above will be referred to as positive and negative perfectionism ${ }^{4}$ throughout this thesis regardless of the labels assigned to them in each individual article.

\section{Theories of Perfectionism}

Shafran and colleagues (2016) have recently incorporated varying perspectives of negative perfectionism into a model that concisely demonstrates the possible mechanisms contributing to its detrimental effect on individuals (see Figure 5). First, they highlight that for a negative perfectionist, self-worth is highly dependent on their perceived achievement. Moreover, they argue that this is compounded by a negative interpretation bias that persists regardless of the actual outcome or standard of achievement (Shafran et al., 2016).

\footnotetext{
4 The original use of positive and negative perfectionism pertained to reinforcement theory and was used to describe whether someone was avoiding negative outcomes (negative perfectionism) or working towards positive outcomes (positive perfectionism) (Slade \& Owens, 1998). For ease of understanding, throughout this thesis positive and negative will be used in a much broader sense to represents aspects of perfectionism that are typically considered to be related to adaptive outcomes (positive perfectionism) and components that are typically considered to be related to maladaptive outcomes (negative perfectionism).
} 


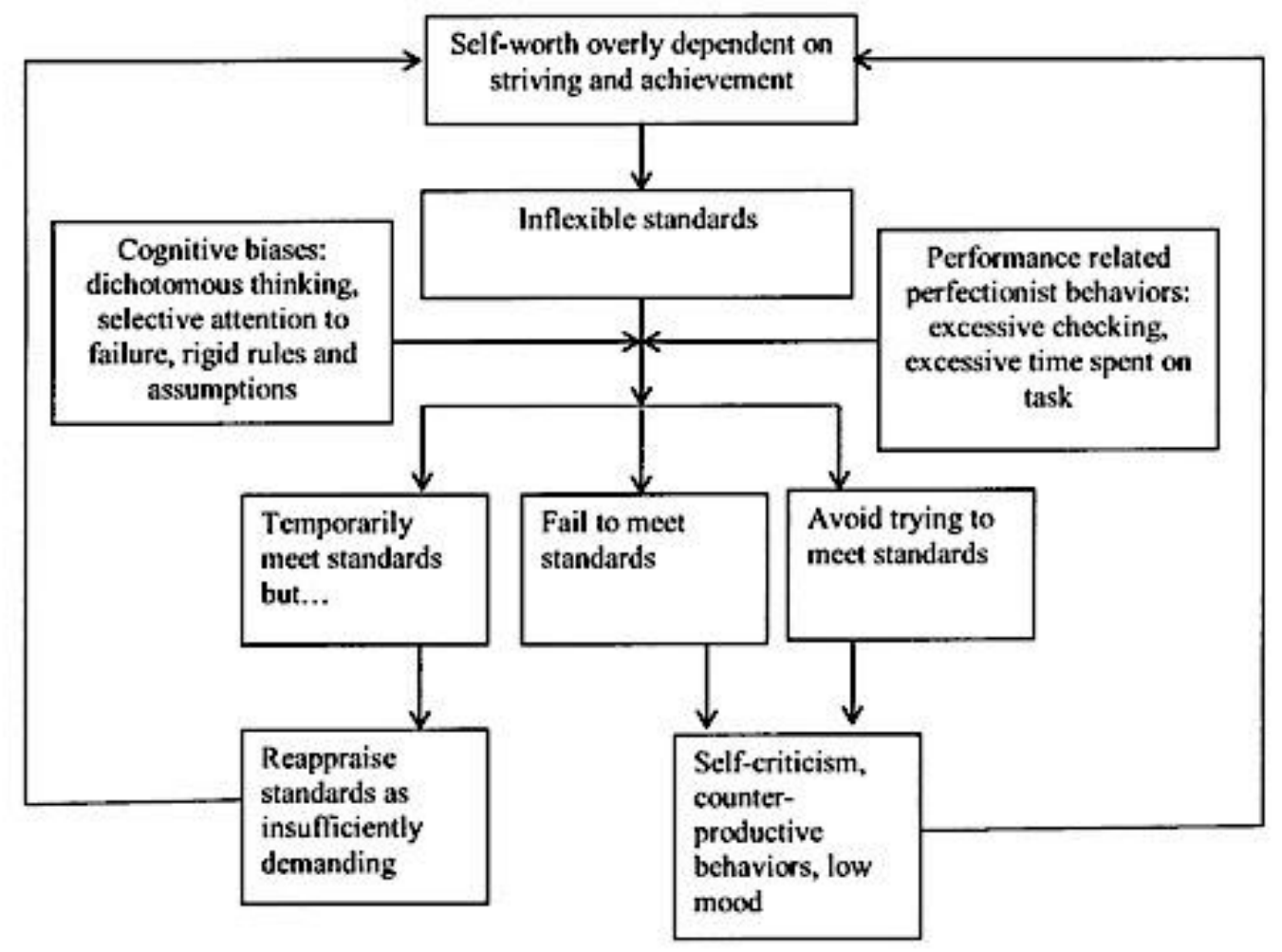

Figure 5. Shafran et al.,'s (2016, p. 161) cognitive-behavioural model of negative perfectionism

Within this model, Shafran and colleagues (2016) have incorporated both behavioural and cognitive perspectives. To further explain, behaviourists have argued that negative perfectionism is an avoidance strategy with the primary goal to avoid negative evaluation from the self, or others (Slade \& Owens, 1998). Alternatively, from a cognitive perspective, negative perfectionism is characterised by rigid, dichotomised thinking and inflexible standards (e.g., Brown \& Beck, 2002; Frost et al., 1993; Shafran, Coughtrey, \& Kothari, 2016; Terry-Short et al., 1995). When evaluating their own performance, a negative perfectionist is especially aware of mistakes, and has strict rules about how a task must be done (Shafran et al., 2016). For a negative perfectionist, failures to reach a goal are interpreted as a sign of negative self-worth and an indication of inadequacy (Frost et al., 1993; TerryShort et al., 1995), and perceived failures are routinely coupled with increased concern about negative evaluation from others (Flett, Hewitt, \& De Rosa, 1996). Similar to that proposed by behavioural researchers, if a negative perfectionist does reach their goal, they experience relief associated with avoided failure, rather than satisfaction with their success (Kelly, 2015).

Whilst Shafran and colleagues (2016) only considered negative perfectionism, others have applied their understanding to positive perfectionism too. For example, Slade and Owen 
(1998), argue that in contrast to the avoidance associated with negative perfectionism, positive perfectionism is motivated by approach goals whereby actions are undertaken with a goal of personal success. Therefore, when the goals are met, positive perfectionism is positively reinforced with a sense of achievement. Alternatively, from a cognitive perspective positive perfectionism is characterised by striving high standards in a flexible manner (Hamachek, 1978). Therefore, failure to achieve a goal is acceptable and does not significantly impact upon the individual' s sense of worth. Rather, a positive perfectionist is likely to re-evaluate their standards and try again. In addition, consistent with behavioural theories, when a positive perfectionist succeeds, they report gaining a sense of satisfaction from their achievement (Gaudreau \& Thompson, 2010).

Based on these ideas, it would be easy to assume that those displaying positive perfectionism are well-adapted and successful individuals, whereas those high on negative perfectionism are maladapted and psychologically distressed. In this case, one would be able to look at an individual's level of positive or negative perfectionism (or both) and make predictions about their psychological health and wellbeing with regard to a range of mental health outcomes. However, most perfectionism researchers suggest this is an oversimplification (e.g., Gaudreau \& Thompson, 2010; Slade \& Owens, 1998). Importantly, many argue that negative and positive perfectionism do not occur in isolation of each other (e.g., Alden, Ryder, \& Mellings, 2002; Gaudreau \& Thompson, 2010; Slade \& Owens, 1998). Rather, it is the combination of specific components of perfectionism that determine whether an individual can be considered positively perfectionistic, negatively perfectionistic, or both, and then how perfectionism subsequently impacts upon their wellbeing (Sironic \& Reeve, 2015). Currently, there are three competing conceptualisations regarding the interaction of positive and negative perfectionism in relation to adaptive and maladaptive outcomes. These will be presented below.

Two Component Model. First, Alden, Ryder and Melling (2002) considered both negative and positive perfectionism and hypothesised the combinations of perfectionism that are more likely to negatively impact individuals than others (see Figure 6). Specifically, they propose that individuals high on both positive and negative perfectionism are true perfectionists and will be the most maladjusted, and experience more negative outcomes. In addition, they argue that individuals who are high on positive perfectionism but low on negative are the most well adapted (Achievement Orientation). In between the two extremes, they propose that individuals low on both forms of perfectionism are self-accepting, and 
individuals low on positive perfectionism but high on negative perfectionism are selfdepreciating. Self-accepted individuals are deemed to be more well-adjusted than selfdepreciating individuals (Alden et al., 2002).

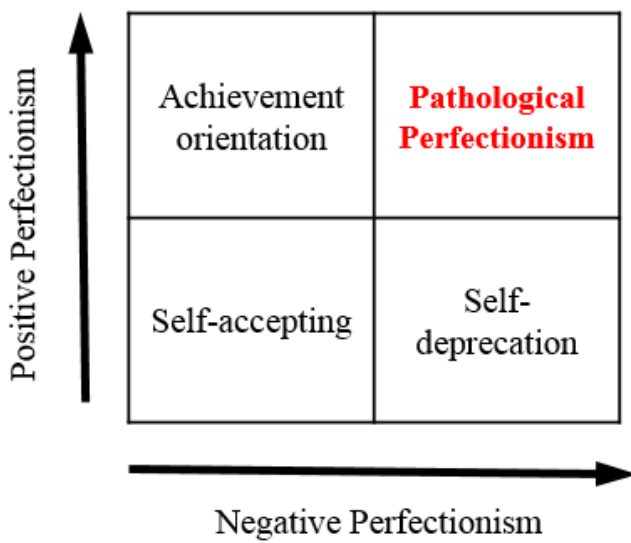

Figure 6. Alden, Ryder and Melling's (2002) Two Component Model

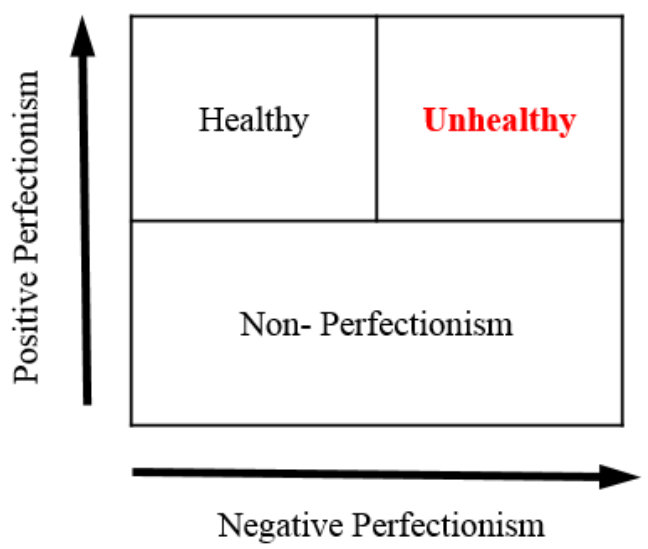

Figure 7. Tripartite Model as proposed by Rice \& Ashby (2007), and Stoeber and Otto (2006)

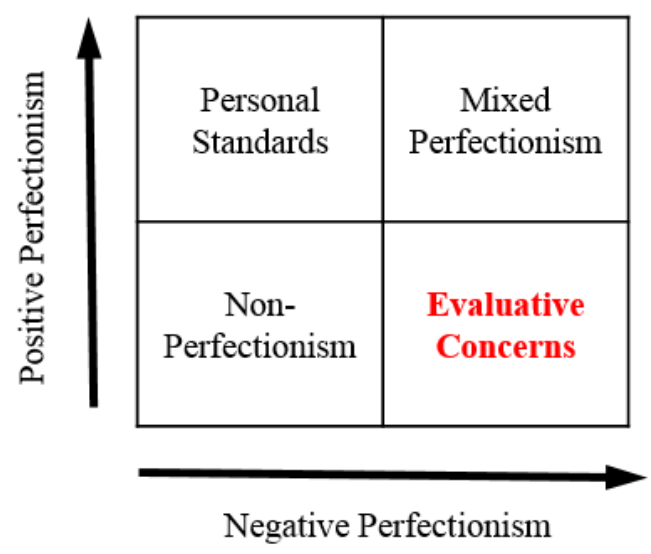

Figure 8. Gaudreau and Thompson's (2010) $2 \times 2$ model

Note: the figures above have been developed based on the theories outlined in each paper. The red represents the combination of perfectionism hypothesised to be associated with the most maladaptive outcomes.

Tripartite Model. Alternatively, the tripartite model of perfectionism (Rice \& Ashby, 2007; Stoeber \& Otto, 2006) classifies three types of individuals; healthy perfectionists (characterised by positive perfectionism but not negative perfectionism), unhealthy perfectionists (positive perfectionism and negative perfectionism), and non-perfectionists. Thus, this model proposes that a perfectionist is characterised by the existence of positive perfectionism with the effect on their wellbeing determined by their concurrent level of negative perfectionism (see Figure 7). Similar to the two component model, the tripartite model proposes that the most maladaptive perfectionist is one who is both positively and 
negatively perfectionistic (Unhealthy Perfectionism). Conversely, an individual high on positive perfectionism but without the maladaptive influence of negative perfectionism is named a Healthy Perfectionist, and an individual low on both forms of perfectionism is a Non-Perfectionist (Rice \& Ashby, 2007; Stoeber \& Otto, 2006). It must be noted that this model does not attend to negatively but not positively perfectionistic individuals which, as will be seen below, is the group identified by the next model $(2 \times 2)$ as the most maladaptive (Gaudreau \& Thompson, 2010).

$2 \times 2$ Model. Finally, and similar to the two-component model, Gaudreau and Thompson (2010) propose four types of individuals (see Figure 8). However, in contrast with both the two-component and tripartite models, they argue that the most maladaptive perfectionist is one characterised by negative but not positive perfectionism. Specifically, they propose that positive perfectionism acts as a buffer against the detrimental effects of negative perfectionism. Thus, as opposed to an individual displaying both positive and negative perfectionism, who may be considered especially perfectionistic (Mixed Perfectionism), someone high on negative perfectionism but without the protective buffer of positive perfectionism is left more vulnerable to negative outcomes (Evaluative Concerns Perfectionism). In line with the Tripartite Model, an individual low on both positive and negative perfectionism is a non-perfectionist.

Despite the contrasting views presented above, there appears to be a consensus that at a broad level perfectionism has both positive and negative components. However, the $2 \times 2$ (Gaudreau \& Thompson, 2010), tripartite (Rice \& Ashby, 2007; Stoeber \& Otto, 2006) and two-component (Alden et al., 2002) models of perfectionism suggest that in order to accurately predict the overall effect of perfectionism on an individual, parallel consideration of both positive and negative perfectionism is required.

\section{The Development of Perfectionism}

As alluded to above, and as will become clear throughout this thesis, perfectionism has the potential to significantly impact upon an individual's wellbeing. Therefore, there is a need for research specifically investigating the development of perfectionism, including how to promote the more positive components of perfectionism, and prevent or reduce the development of negative perfectionism. Although overall very limited, existing research on the development of perfectionism has primarily referenced the role of families, and in particular on parent characteristics and parenting styles that promote perfectionistic qualities in their children (e.g., Frost, Lahart, \& Rosenblate, 1991; for a review see Flett, Hewitt, 
Oliver, \& Macdonald, 2002). Others have drawn attention to a broader set of factors, including the influence of teachers and peers, as well as societal and cultural expectations (Flett et al., 2002). This research will only touch on the development of perfectionism, mainly addressing characteristics or behaviours that may give rise to perfectionistic tendencies. Therefore, this section is provided to outline and highlight factors external to the individual that may have influenced the development of perfectionistic beliefs. It is hoped that a summary of this research will allow a more comprehensive and complete picture of the nature of perfectionism.

The majority of research on the etiology of perfectionism proposes that perfectionistic parents are likely to promote perfectionism in their children (e.g., Frost et al., 1991; Morris \& Lomax, 2014). For example, Frost and colleagues (1991) investigated the possibility that perfectionism develops as a result of demanding and perfectionistic parents. In their study with parents and their daughters, they found that mothers' perfectionism and mothers' harshness, both self-rated and daughter-rated, were associated with daughters' perfectionism. In addition, fathers' harshness, as rated by their daughters, was associated with their daughters' perfectionism. Although this research is cross-sectional and cannot be used to infer causality, it suggests that a causal relationship between mothers' and daughters' perfectionism may be a viable possibility (Frost et al., 1991). More recent research has supported similar intergenerational transmissions of perfectionism (e.g., Appleton, Hall, \& Hill, 2010; Chang \& Sanna, 2001), and suggested that this relationship is especially prominent among children and parents of the same sex (Vieth \& Trull, 1999).

In a review of the research, Flett and colleagues (2002) proposed four possible mechanisms through which a child may develop similar perfectionistic tendencies to their parents. First, they proposed that perfectionism is transmitted between parent and child through processes of modelling, whereby the child witnesses perfectionistic behaviours in their parents and subsequently adopts them as their own. Second, perfectionism may develop as a result of high parental expectations placed upon the child (captured in the FMPS through the PE subscale). In this case, parent's approval is often dependant on achievement and the child is likely to develop a sense of contingent self-worth. Third, perfectionism could form as a reaction to harsh parenting, in an attempt to gain some control over a potentially chaotic environment and reduce opportunities for criticism from family members (captured in the FMPS through the PC subscale). Finally, Flett and colleagues proposed that perfectionism in children may be a result of anxious parents who are highly attuned to mistakes and signs of imperfection. As a result, the child's attention is frequently drawn to the possible negative 
consequences of their actions, including perceived negative evaluation from others. Overall, it can be argued that perfectionism results from perfectionistic or anxious parents, combined with parenting styles characterised by excessive expectations, indirect criticism, and approval that is either absent, inconsistent, or conditional (Flett, Hewitt, Oliver, \& Macdonald, 2002; Shafran \& Mansell, 2001).

Unfortunately, there is little research investigating the development of perfectionism outside the family environment. This may in part be attributable to Frost and colleagues' (1990) focus on the influence of parents in the FMPS. Conversely, in the HFMPS, Hewitt and Flett (1991) took a much wider approach, considering the influence of all others. Originally the term 'others' was used in a very general sense, however in subsequent research they have specified that perceived pressure may come from parents, peers, teachers, and the wider cultural and societal environment (e.g., portrayal of the perfect body image in the media; Flett et al., 2002).

With this in mind, Perera and Chang (2015) investigated the source of perceived expectations in European American and Asian American undergraduates. Specifically, they focused on the role of teachers, peers, friends, siblings, romantic partners, and an individual's culture. Consistent with the FMPS and the research presented above, they reported that parental expectations remained the most important contributor to negative perfectionism, especially for European American students. In addition, European American students perceived greater expectations from their teachers, whilst Asian American students perceived greater expectations from their peers. This may fit with Flett and colleague's (2002) theory that in Western, individualist cultures, pressure is more likely to be associated with academic and personal achievement (hence the importance of teachers), whereas in collectivist cultures the expectations of others within one's social group or peers may be more salient. Overall, Perera and Chang clearly demonstrate that students perceive high expectations from a much wider group of others than just their parents and highlighted some key cultural differences in the nature of negative perfectionism (as measured by SPP). Thus, the development of perfectionism should be considered within the context of an individual's broad social environment, including the expectations and social responsibilities specific to their cultural, peer, and family environments.

\section{Changing Societal Influences on Perfectionism}

At an even broader level, recent research suggests that over the last 10-20 years expectations for young people's performance have changed substantially. Carol Dweck has 
led research investigating these changes in relation to children's perceived self-efficacy (e.g., Diener \& Dweck, 1978; Dweck \& Molden, 2005; Haimovitz \& Dweck, 2016). Across multiple studies she reports an increase in children avoiding activities that they may fail at, noting that they have become especially worried about even slight possibilities of failure (Dweck, 2007; Yeager \& Dweck, 2012). Dweck also discusses parental expectations and notes that parents have begun to go to extreme lengths to make sure their children do not fail (e.g., by completing their assignments for them) but, in the process, have negatively impacted their child's self-confidence. In addition, she argues that society often models to children that they must always do their best and that if you are intelligent this will happen easily (Dweck, 2007; see also Thompson, 2004). Although Dweck does not explicitly relate her research to perfectionism, preferring to focus on perceptions of intelligence and mind-sets associated with achievement motivation, it is clear that these parental expectations and behaviours are a combination well suited to promoting perfectionism in children and adolescents.

Perfectionism and social media. In addition, it is important to consider the everchanging nature and pervasiveness of multiple sources of perceived evaluation and high expectations. Whilst adolescents previously experienced social evaluation face-to-face, and mostly at school or when socialising with peers, they are now subject to continuous evaluation and social comparisons through social media (Headmasters' and Headmistresses' Conference [HMC], 2015). As a result, not only are perceived expectations relentless, they are typically much more diverse in their source than they used to be. This is supported by local and international experiences of adolescents, teachers, parents, and clinicians, who commonly attribute increases in adolescent distress to the rapid development of social media and the online world (Education Review Office [ERO], 2015; HMC, 2015; Wilksch, Durbridge, \& Wade, 2008; Youth Wellbeing Study, 2015).

Some perfectionism researchers focus on 'Perfectionistic Self-Presentation' which specifically captures the need to appear perfect, rather than the need to be perfect (e.g., Hassan, Flett, Ganguli, \& Hewitt, 2014; Hellmann, 2016; Nepon, Flett, \& Hewitt, 2016). Perfectionistic self-presentation is especially relevant in the context of social media, given that most social media allows individual control of what is and what is not posted online. Hellman (2016) notes that this increased control may place added pressure on perfectionists to constantly filter what is posted online and use computer image enhancement and modification tools to remove any perceived blemishes. With regard to mental health outcomes, Hellman demonstrated that perfectionistic self-presentation and non-display of imperfection on social media were associated with increased depression, stress, and anxiety. 
Perfectionism and academic achievement. It appears that adolescents are not only facing increased pressure within their social networks, they are also having to manage high expectations within structured environments such as their schools. To illustrate, the recent NZ Education Review Office report (ERO; 2015), Wellbeing for Young People's Success at Secondary School, highlighted the intensive, demanding, and relentless nature of assessment within NZ's school achievement system. They expressed concern that constant evaluation of students performance, all of which could be perceived to, and can in fact have, an impact on their future career pathways, was increasing student anxiety. Moreover, they noted that students "were highly motivated and held extremely high expectations for success, which was negatively affecting their wellbeing” (ERO, 2015, p. 27).

An increase in school-based pressure has also been noted by academic researchers. For example, Putwain (2008) argued that, to the detriment of students, schooling has become increasingly assessment driven, whilst Johnson, Panagioti, Bass, Ramsey and Harrison (2017) highlight worries about failing to succeed contributing to an overall increase in student distress. Finally, the HMC (2015) in the United Kingdom noted that adolescents are facing increased pressure to achieve due to the increasingly difficult challenge of being accepted into universities. Overall, it can also be argued that this pressure within the school system is likely to promote perfectionistic cognitions and behaviours and contribute to poor mental health outcomes.

Increased parental protection and pressure, greater use of social media, and the relentless focus on achievement evaluation in schools, are all likely to have contributed to a rise of perfectionism in young people. Flett et al., (2002) note that similar concerns are seen in adults, perhaps with regard to their career and family, however, achievement pressure, social expectations, and the perceived importance of upkeep of appearance remain most prominent in adolescents. Research on perfectionism in adolescents is further outlined below, along with a brief discussion of differences in perfectionism across males and females, and in different cultural groups.

\section{Perfectionism in Different Populations}

As with many psychological phenomena, perfectionism may differ in its salience and influence in individuals of differing ages, sex, and ethnic backgrounds. However, the existing literature is somewhat limited with regard to identifying important population differences. As noted in relation to NSSI, recognising key demographic differences or particular at risk populations is essential in developing effective prevention and intervention programmes for 
perfectionistic individuals.

Perfectionism and age. There is little evidence to suggest any consistent patterns of difference in the presentation of perfectionism in individuals of different ages. In fact, some literature suggests that perfectionism is stable across the lifespan and presents similarly in childhood and adolescence as it does in adults (e.g., Asseraf \& Vaillancourt, 2014; Nilsson, Sundbom, \& Hagglof, 2008; O’Connor, Dixon, \& Rasmussen, 2009; Rice \& Aldea, 2006). However, from a developmental perspective childhood and adolescence are particularly important life stages with regard to the onset of problematic perfectionistic thoughts and tendencies and thus the development of maladaptive outcomes often linked to perfectionism (Flett et al., 2002; also see below for the relationship between perfectionism and mental health outcomes).

Keeping in mind previous discussions about factors associated with the development of perfectionism (see p. 31-33), researchers suggest that these factors are particularly prominent during early adolescence. Specifically, researchers have highlighted a developmentally normative increase in self-consciousness that typically occurs during early adolescence (Damian et al., 2013; Flett et al., 2002; Morris \& Lomax, 2014) and that may have an influence on the development of perfectionism. For example, Rankin, Lane, Gibbons and Gerrard (2004) note that self-consciousness and the frequency of social comparisons are at their highest in early adolescent years. This could translate to concern regarding one's appearance, achievement, and social skills in relation to that of their peers. In light of this, adolescents will be particularly motivated to present themselves in a positive light, and avoid making mistakes in front of their peers and important others. Researchers also argue that adolescents are particularly sensitive to feedback from others due to their ongoing navigation of identity formation, establishing where they fit with regard to others, and developing friend groups with like-minded peers (O'Connor et al., 2010; Rankin et al., 2004; Steinberg \& Morris, 2001). In light of this it makes sense to focus research on adolescents, who are within a period of increased risk for the development of perfectionism and subsequent maladaptive cognitions, beliefs and behaviours.

Perfectionism in males and females. There is some empirical evidence that males and females typically score differently on aspects of perfectionism, and that they experience different maladaptive and adaptive psychological phenomena as a result of similar perfectionistic tendencies (Blankstein, Dunkley, \& Wilson, 2008; Blankstein \& Winkworth, 2004; Klibert et al., 2005). At the subscale level, Parker and Stumpf (1995) found that females scored significantly higher on the Organisation subscale of the FMPS, suggesting higher 
positive perfectionism. Likewise, Stallman and Hurst (2011) illustrated that females score significant higher on both Organisation and PS. Some studies have also reported no sex differences at the subscale level (e.g., Parker \& Adkins, 1995; Rice \& Mirzadeh, 2000).

Rather than differences at the subscale level it is more common for researchers to report sex differences at the outcome level. For example, Blankstein and Winkworth (2004) described the association between negative perfectionism (SPP) and dysphoria as stronger in males than in females. Additionally, Blankstein et al., (2008) reported that positive perfectionism was negatively associated with self-esteem but only in females. Moreover, Klibert and colleagues' (2005) illustrated that while negative perfectionism (SPP) was positively correlated with procrastination in males, it was related to higher levels of guilt in females. Finally, Kawamura and Frost (2004) demonstrated that perfectionism was related to greater self-concealment in females, but not in males.

Others have described sex differences in the association between perfectionism and psychological disorders. For example, Ferreiro, Seoane and Senra (2012) found that perfectionism was a predictor of disordered eating in adolescent females but not adolescent males. Similarly, Shanmungam and Davies (2015) reported that among their athlete sample, negative perfectionism (CPQ; Riley, Lee, Cooper, Fairburn, \& Shafran, 2007) was only associated with eating psychopathology in females. Finally, Klibert, Lamis, Naufel, Yancey, and Lohr (2015) reported that negative perfectionism (SPP) was directly associated with generalised anxiety symptoms in females, however for males the relationship was indirect through adherence to threat and control schemas.

Overall, this suggests that perfectionism does not consistently affect males and females. This highlights the importance of considering sex when conducting perfectionism research, and moreover having accessible sex specific research that can guide clinical practice when working with perfectionistic clients.

Perfectionism in different cultural groups. Perfectionism, as defined in this thesis, is based on Western perspectives of psychology, therefore research investigating perfectionism across cultures must be interpreted with caution. For example, Tachi, KusanoSchwarz, Murakami, Kobayashi, and Miyake (2007) investigated the nature of perfectionism in a sample of Japanese women diagnosed with eating disorders. They reported that perfectionism, as measured by the EDI-II, was significantly lower in their sample than in equivalent studies with Western participants. They suggested that this difference could indicate a discrepancy between the perceived value of academic and occupational accomplishment, or substantial differences in how psychopathology manifests across cultures. 
With regard to the investigation of perfectionism across cultures, other researchers have noted variability in perfectionism across different ethnic groups within Western societies. For example, Castro and Rice (2003) investigated perfectionism using the FMPS in Caucasian Americans, Asian Americans and African Americans. They reported that Asian Americans scored significantly higher than both other groups on CM, DA, PC and PE, and suggested that Asian students received considerably more pressure from their parents to achieve to a high standard. Interestingly, Asian Americans did not score any higher on indicators of resulting distress.

Overall, cross-cultural research on perfectionism is limited. As with cultural differences in NSSI, it is important to recognise that the specific manifestations of perfectionism may differ across culture and therefore requires an openness from researchers and clinicians to be flexible and incorporate cultural understandings into research and clinical practice.

\section{Perfectionism and Mental Health and Wellbeing Outcomes}

Whilst being a positive perfectionist may be beneficial, research convincingly demonstrates associations between negative perfectionism and poor mental health. Some of the research pertaining to outcomes associated with positive perfectionism will be presented below, followed by a brief summary of research on negative perfectionism ${ }^{5}$.

As has been outlined above, most perfectionism researchers argue for a differentiation between positive and negative perfectionism (e.g., Slade \& Owens, 1998; Stoeber \& Otto, 2006). Specifically with regard to psychopathology, research has shown that positive perfectionism is negatively associated with depression (e.g., DiBartolo, Li, \& Frost, 2008; Steffen, 2014; Stoeber \& Rambow, 2007), and anxiety (e.g., Steffen, 2014; Weiner \& Carton, 2012) especially when negative perfectionism is partialed out. That is, positive perfectionism may protect against the development of such disorders. However, somewhat countering the prevailing view of positive perfectionism as adaptive, it has also been associated with greater risk for eating disorders (e.g., Boone et al., 2012; Dickie et al., 2012; Malkina-Pykh, 2012), and OCD (e.g., Norman, Davies, Nicholson, Cortese, \& Malla, 1998; Wu \& Cortesi, 2009).

Research in an adolescent samples has demonstrated associations between negative perfectionism and depression (e.g., Flett, Coulter, Hewitt, \& Nepon, 2011; Huggins, Davis, Rooney, \& Kane, 2008; Soenens et al., 2008; Soenens, Vansteenkiste, Luyten, Duriez, \&

\footnotetext{
${ }^{5}$ For an in depth discussion about the adaptive and maladaptive correlates and outcomes of perfectionism see Chapters 4 and 5
} 
Goossens, 2005), anxiety (e.g., Burton et al., 2013; Hewitt et al., 2002; Vansteenkiste et al., 2010), and eating disorders (e.g., Boone, Soenens, Braet, \& Goossens, 2010; Soenens, Vansteenkiste, et al., 2008). Moreover, negative perfectionism is commonly associated with OCD (e.g., Khawaja \& Armstrong, 2005; Norman et al., 1998), stress (e.g., Chang, Banks, \& Watkins, 2004; DiBartolo et al., 2007), suicidal thoughts and behaviours (e.g., Caelian, 2005; Enns, Cox, \& Inayatulla, 2003; Hewitt, Caelian, Chen, \& Flett, 2014) and, as will be discussed in great depth, self-injury (e.g., Hoff \& Muehlenkamp, 2009; O’Connor et al., 2010).

Overall, research on negative perfectionism has suggested that addressing perfectionism may indeed be a very fruitful point of focus for individuals struggling with a wide range of mental health difficulties. Specifically, Nehmy and Wade (2015) have described perfectionism as a trans-diagnostic process that contributes to many facets of psychopathology (the trans-diagnostic approach is also noted by Egan, Wade, \& Shafran, 2011).

\section{Chapter Summary}

This chapter has outlined the dominant conceptualisations of perfectionism, including the clear differentiation between positive and negative perfectionism. Leading models of how positive and negative perfectionism, both individually and in combination, can impact upon an individual's wellbeing were also presented. Theories about the development of perfectionism were discussed, followed by a summary of research investigating perfectionism in different demographic groups. Finally, a brief outline of some common associations between positive and negative perfectionism and adaptive and maladaptive outcomes were presented.

In sum, further research is required to clarify the nature of perfectionism in different populations (sex, culture, age groups). However, it is evident that for some, perfectionism, and specifically negative perfectionism, can have distressing and damaging consequences. As outlined in the previous section, adolescent NSSI is another phenomenon that can have serious detrimental effects for adolescents, their family and peers (Jacobson \& Gould, 2007; Klonsky, 2007; Swannell et al., 2014). Research suggests that these two phenomena could be linked, with NSSI being one of the more detrimental correlates or outcomes of perfectionism (e.g., Hoff \& Muehlenkamp, 2009; O’Connor et al., 2010). Moreover, further investigation is required to identify a potentially more complex relationship, with perfectionism not only acting as a risk factor for engagement in NSSI, but also as a key maintaining factor. 
Therefore, research examining perfectionism and NSSI will be outlined below. 


\section{CHAPTER THREE: PERFECTIONISM AND NSSI}

The sheer quantity of research surrounding both perfectionism and NSSI individually has grown exponentially in recent years, yet very few researchers have investigated the relationship between the two phenomena. Of the limited research that has been reported, the results are somewhat inconclusive and many questions remain (e.g., Hoff \& Muehlenkamp, 2009; Hunter \& O' Connor, 2003; Izadi, 2014; Miskey et al., 2012). This section will examine existing perfectionism and NSSI research; first highlighting research exploring the direct relationship between perfectionism and NSSI, and; second, looking at research on perfectionism and the functions of NSSI. Finally, the relationship between perfectionism and NSSI will be explored in the context of key models of self-injury. Overall, the aim of this chapter is to provide some background information, and set the scene for the first empirical components of this thesis.

\section{Perfectionism and NSSI Prevalence, and Severity}

Perfectionism and self-injury in adults. Of the few studies investigating the relationship between perfectionism and self-injury, research with adult samples has established the foundation for further research in differing populations. As will be evidenced below, such research has typically indicated significant relationships between negative perfectionism and self-injurious thoughts and behaviours.

To begin, Claes and colleagues (2012) recognised that ED patients typically have both elevated levels of self-reported perfectionism and greater rates of NSSI than patients with other disorders or non-clinical samples. Therefore, they set out to test whether there is a relationship between the two phenomena. In their sample of eating disorder patients, they found that negative perfectionism (as measured by CM + DA, FMPS) was higher in the group with a history of NSSI, than the group with no history of NSSI. There was no difference between the groups on positive perfectionism, therefore suggesting that positive perfectionism is not significantly related to NSSI engagement in their sample. It is important to note that through comparing a group with a history of NSSI to a non-NSSI group, Claes and colleagues (2012) were effectively investigating the relationship between perfectionism and the occurrence of at least one episode of NSSI. Thus, their research suggests that negative perfectionism increases risk for at least a single episode of NSSI, however does not reveal anything about the relationship between perfectionism and the severity or frequency of NSSI. 
Hoff and Muehlenkamp (2009) also reported results consistent with the twodimension conceptualisation of perfectionism (Frost et al., 1993). Among a sample of undergraduate students, they examined multiple forms of NSSI using the DSHI (Lundh et al., 2007) and perfectionism as measured by the FMPS (Frost et al., 1990). Hoff and Muehlenkamp described that students who reported NSSI scored higher on the CM and PC subscales, and lower on the Organisation subscale. This suggests that compared to those with no history of self-injury, students who have engaged in NSSI score higher on negative perfectionism and lower on positive perfectionism (Frost et al., 1993). Further, this could be indicative of negative perfectionism as a vulnerability factor for engaging in NSSI, and positive perfectionism as a buffer against NSSI risk. Through Hoff and Muehlenkamp's specific focus on NSSI, rather than self-injury without differentiation based on suicide intent, and their consideration of the multidimensional nature of perfectionism, this study provides more detailed insight into the relationship between the two phenomena. However, their primarily Caucasian undergraduate sample limits generalisation of findings to a more diverse adult community, clinical, or adolescent populations (Henrich, Heine, \& Norenzayan, 2010).

Miskey, Hill, and Huelsman (2012) also investigated perfectionism and NSSI in adults. They used the Perfectionism Inventory (Hill et al., 2004) and the Deliberate Self Harm Inventory (DSHI; Lundh et al., 2007), with a specific focus on non-suicidal cutting (NScutting) rather than the wide range of NSSI behaviours. Overall, in their sample of undergraduate students with a history of NS-cutting, they reported that greater perfectionistic rumination (a PI subscale, typically subsumed under negative perfectionism) was associated with greater duration of NS-cutting (the total number of months individuals reported engaging in NS-cutting). They also reported that higher CM and greater Need for Approval scores were significantly associated with later onset of NS-cutting. These findings can be summarised to indicate that negative perfectionism is associated with later onset and longer engagement in NSSI. In addition, although non-significant, Miskey and colleagues (2012) noted a trending, almost significant, negative relationship between CM and NS-cutting frequency. Again with regard to NS-cutting frequency, they also reported a trending, positive relationship with Organisation. That is, negative perfectionism could be related to less frequent cutting, and positive perfectionism to more frequent cutting. Whilst the results pertaining to perfectionistic rumination support the notion of positive and negative perfectionism, all latter findings are inconsistent with the two-dimension adaptivemaladaptive conceptualisation, and inconsistent with Hoff and Muehlenkamp's findings (2009). Under this conceptualisation, negative perfectionism would be associated with more 
frequent, earlier onset, and greater duration NSSI, and a similar but mirrored effect would be seen for positive perfectionism.

Miskey and colleagues' (2012) unexpected results could partly be due to their sample of only individuals with a history of NS-cutting. In contrast to Claes and colleagues (2012) research, which involved a sample of both individuals with a history of NSSI and individuals with no history of NSSI, and therefore was investigating NSSI occurrence, the use of a NScutting only sample effectively investigates the relationship between perfectionism and NSSI severity. Therefore, the incongruent results may suggest the relationship between perfectionism and having a history of NSSI is different to the relationship between perfectionism and NSSI severity, and moreover the specific relationship between perfectionism and cutting only.

Moreover, it is important to note that Miskey and colleagues (2012) argued that they were adding to the literature by specifically focusing on NS-cutting rather than all NSSI behaviours. However, the majority (84\%) of participants who endorsed non-suicidal cutting also endorsed another form of NSSI. Therefore, it is hard to make conclusions about a NScutting relationship and may instead be more indicative of a wider relationship between perfectionism and all NSSI behaviours.

Finally, with regard to NSSI and perfectionism in adult samples, Izadi (2014) examined perfectionism in a sample of 50 adults with a history of NSSI. Izadi's study, unlike many others, required participants to have engaged in NSSI at least five times (consistent with the proposed frequency criterion for NSSI Disorder in the DSM 5; Zetterqvist, 2015). This enabled him to focus on individuals engaging in repetitive self-injurious behaviours rather than those who have tried NSSI once but have not adopted it as a persistent maladaptive coping strategy. Also consistent with the two factor model of perfectionism, but inconsistent with Miskey and colleagues' findings (2012), Izadi reported that individuals scoring high on specific components of negative perfectionism (SPP and PE) engaged in NSSI more frequently. Unexpectedly, other components of negative perfectionism (PC and CM) had no effect on the frequency of NSSI. Overall, Izadi's research demonstrates a relationship between at least some components of negative perfectionism and frequency of NSSI in self-injuring adults. Similar to Miskey and colleagues' (2012) research, this does not provide any information on whether perfectionism can differentiate between people who do and who do not engage in NSSI. However, it does suggest that perfectionism could contribute to the severity of the behaviour in those who have a history of NSSI.

Perfectionism and self-injury in adolescents. Both perfectionism and NSSI are 
highly prevalent in adolescent populations (e.g., Flett \& Hewitt, 2014; Muehlenkamp et al., 2012; Swannell et al., 2014). Moreover, it is clear that both of these phenomena can have severe and long lasting effects on adolescent wellbeing. Unfortunately, to my knowledge, no one has specifically investigated NSSI and a multidimensional conceptualisation of perfectionism in both male and female adolescents. Rather, research has focused on only females (e.g., Luyckx, Gandhi, Bijttebier, \& Claes, 2015), has addressed perfectionism as a one-dimensional construct (e.g., Luyckx et al., 2015), or has not made the distinction between self-injury with and without suicide intent (e.g., O'Connor et al., 2010).

For example, Luyckx and colleagues (2015) investigated NSSI (Self-Injurious Questionnaire-Treatment Related; SIQ- TR; Claes \& Vandereycken, 2007) and a onedimensional conceptualisation of perfectionism (EDI-II; Garner, 1991), in a sample of adolescents diagnosed with an ED or BPD, and a sample of community adolescents. Luyckx and colleagues found that perfectionism was associated with NSSI in both samples.

Moreover, in the community sample, this effect was significant over and above the effects of age, anxiety, depression, identity synthesis, and the Big Five personality factors and in fact perfectionism contributed the most to engagement in NSSI. Unfortunately, Luyckx and colleagues used the EDI-II, therefore their results pertain to a unidimensional conceptualisation of perfectionism. This prevents differentiation between negative and positive perfectionism and instead gives a non-specific account of the relationship between perfectionism and NSSI. Moreover, their use of a female only sample severely limits generalisation to a wider adolescent population. Despite these limitations, their findings provide support for a relationship between perfectionism and NSSI in female adolescents.

O'Connor, and colleagues (2010) explored a similar relationship with male and female adolescents from three Scottish schools. They used longitudinal data to examine the interaction between life stress and DSH, in conjunction with perfectionism. They illustrated that negative perfectionism (SPP; HFMPS) was particularly relevant, such that for those experiencing low levels of life stress, an increase in negative perfectionism significantly increased the probability of engaging in DSH. However, for those experiencing high life stress, perfectionism had little effect on the already high probability of DSH.

O'Connor et al.'s (2010) study is unique due to their use of the Child and Adolescent Perfectionism Scale, an adolescent-specific measure of perfectionism (CAPS; O'Connor, Dixon, et al., 2009). However, their study is limited by the use of a single self-harm question. Thus, O'Connor and colleagues may have underestimated the rate of self-harm in their sample. Another limitation is the absence of differentiation based on suicide intent. As has 
been discussed, investigating self-harm, without differentiating according to intent, may capture two very different groups of adolescents who have markedly different cognitions and motivations (Joiner et al., 2012; Muehlenkamp, 2014; Muehlenkamp \& Kerr, 2010; Nock et al., 2006) and thus this should be taken into account when applying the findings to the relationship between perfectionism and NSSI.

\section{Perfectionism and the Functions of NSSI}

The research presented above suggests that perfectionism is in some way related to NSSI. At this stage, the most plausible scenario is one where negative perfectionism is associated with greater risk or engagement in NSSI. However, there is a need for further research looking at the more intricate details of this relationship. Currently, there is only a small collection of research exploring the mechanisms through which perfectionism could relate to NSSI. Insight into these mechanisms is typically gained through investigation of the functions of NSSI. That is, is perfectionism disproportionately associated with particular reasons for engaging in NSSI, and therefore, do individuals high on perfectionism have unique needs that are met by NSSI? The limited available research is explored below.

First, Nock and Prinstein (2005) investigated the relationship between perfectionism, measured by the CAPS (Flett, Hewitt, Boucher, Davidson, \& Munro, 1997), and the four categories of NSSI functions measured by the FASM (Functional Assessment of SelfMutilation; Lloyd, Kelly, \& Hope, 1997: social positive reinforcement, social negative reinforcement, automatic positive reinforcement, automatic negative reinforcement; see p. 16 for a summary of the four function model). In their adolescent psychiatric sample, negative perfectionism (SPP, HFMPS) was positively associated with both social negative reinforcement functions and social positive reinforcement functions. Nock and Prinstein did not empirically test the relationship with more specific aspects of the functional categories, however hypothesised that adolescents who score high on negative perfectionism could be engaging in NSSI either to reduce the perceived expectations of others (social negative reinforcement), or as an indirect strategy of eliciting support from others (social positive reinforcement).

Second, Claes and colleagues (2012) explored the relationship between perfectionism (FMPS) and three common functions of NSSI (self-punishment, cry for help, self-torture), in a sample of ED patients. They found that negative perfectionism (CM and DA combined) was positively associated with endorsement of all three functions tested. The relationships between negative perfectionism and self-punishment, and self-torture may suggest that 
perfectionism increases risk for NSSI through a need or desire to punish oneself for perceived failures or wrong doings. Together, these studies suggest that individuals who score high on negative perfectionism may be especially likely to use NSSI for self-punishment or to manage their interpersonal relationships.

\section{Perfectionism and Theoretical Models of NSSI}

With reference to leading models of NSSI, there are multiple stages in the development and occurrence of NSSI that perfectionism could play a part. First, in terms of the EAM (see Figure 3, p. 19), perfectionism may be conceptualised as the stimulus that triggers the cycle of self-injury (Chapman et al., 2006). For example, through the very nature of perfectionism, perfectionistic individuals are more likely to interpret their performance on a task as failing to meet their own or others standards, or as a complete failure (Frost et al., 1990). Therefore, perceived failure may be one stimulus that triggers strong negative emotion (e.g., shame, anger, and guilt) for which self-injury may serve as an effective coping strategy. In addition, there is research that suggests individuals high on perfectionism experience negative performance related feedback as significantly more distressing than nonperfectionists (Fedewa, Burns, \& Gomez, 2005). In terms of the EAM, this may mean that the threshold at which the stimulus (e.g., feedback about performance) triggers a strong emotional response is lower in individual's high on perfectionism. Consequently, a perfectionist may be much more likely to experience emotional distress to a level beyond their ability to manage it and therefore may be more likely to need a coping strategy, such as self-injury, to regulate their emotion.

In further support of the EAM (Chapman et al., 2006), some research has demonstrated a link between perfectionism and the propensity for an individual to engage in experiential avoidance. As a reminder, experiential avoidance lies at the heart of the EAM whereby the primary function of self-injury is to avoid the experience of negative emotion. The relationship between perfectionism and experiential avoidance was first demonstrated by Santanello and Gardener (2007) who reported that as an individual's negative perfectionism increased, the tendency to avoid distressing thoughts and feelings also increased. Similarly, Moroz and Dunkley (2015) illustrated that experiential avoidance mediated the association between negative perfectionism and depression, leading the authors to suggest that experiential avoidance may be used to cope with self-criticism and perceived harsh criticism from others. In light of this, perfectionism may be implicated at both the stimulus and avoidance stages of the EAM. 
Alternatively, within Nock's (2010) Integrated Model of Self-Injury (ITMS; see Figure 4, p. 20), perfectionism could be classed as an intrapersonal and/or an interpersonal risk factor for self-injury. Nock describes these as personal characteristics that limit an individual's ability to manage stressful events or experiences and therefore place the individual at greater risk of using maladaptive coping strategies (e.g., self-injury, alcohol and drug use). With regard to perfectionism, the tendency to be very doubtful of one's own standards of performance, and the adoption of highly self-critical interpretations of performance or events could be classed as an intrapersonal vulnerability factor. Similarly, perfectionism could be conceptualised as an interpersonal vulnerability, whereby the individual is reluctant to mobilise support networks due to anxiety about revealing imperfection and subsequent feared negative evaluation from others (Flett, Hewitt, \& Heisel, 2014).

Finally, with regard to the ITMS, perfectionism may be implicated in the NSSIspecific vulnerability factors, factors described by Nock (2010) as those that increase risk that the individual will choose NSSI over another behaviour or coping strategy. One NSSIspecific vulnerability, namely the self-punishment hypothesis, may be especially relevant to perfectionism. Specifically, Nock proposes that some individuals have a particularly selfcritical and self-depreciating cognitive style which can promote maladaptive behaviours, such as NSSI, aimed at punishing oneself for perceived wrong doings. In these instances, NSSI could function as a method of self-punishment to alleviate the feelings of self-hatred and low self-worth that are often associated with negative perfectionism (Tangney, 2002). Claes and colleagues' (2012) research indicating that negative perfectionism was significantly associated with self-punishment and self-torture functions of NSSI provides some support for the association between perfectionism and the self-punishment NSSI-specific vulnerability factor. 


\section{RESEARCH QUESTIONS}

Overall, there is ongoing debate about the relationships between negative and positive perfectionism, and NSSI. Although some of the existing research provides evidence that perfectionism may be related to NSSI (e.g., Hoff \& Muehlenkamp, 2009; Claes et al., 2012), there is still substantial empirical research needed. Further studies aimed at clarifying the relationship between the two phenomena, followed by research pertaining to how exactly perfectionism may increase risk of NSSI (or vice versa), are needed to better understand perfectionism in relation to risk of NSSI. Prior to this however, there is the broader question regarding clarification of the nature of positive and negative perfectionism, and their relationship with mental health outcomes in general. Moreover, to date no perfectionism research has been conducted with a NZ sample, let alone specifically NZ adolescents. Therefore, it is important to investigate how perfectionism manifests in a local sample. As such, this research will be guided by four main research questions:

1. To what extent are positive and negative perfectionism associated with positive and negative outcomes?

2. Is the Frost Multidimensional Perfectionism Scale an appropriate scale for conducting perfectionism research with New Zealand adolescents?

3. Is there a relationship between perfectionism and NSSI in New Zealand adolescents?

4. If so, can perfectionism prospectively predict risk of NSSI?

This thesis will involve four studies aimed at providing answers to these questions. To begin, an in-depth exploration of perfectionism and associated research relating to mental health and wellbeing outcomes will be conducted (Study 1). This will be followed by an examination of the nature of perfectionism in NZ adolescents (Study 2). Subsequently, the relationship between perfectionism and NSSI will be investigated, both cross-sectionally (Study 3) and longitudinally (Study 4). 


\section{CHAPTER FOUR: THE RELATIONSHIP BETWEEN PERFECTIONISM AND MENTAL HEALTH AND WELLBEING OUTCOMES}

Thus far research has suggested that perfectionism is likely associated with a raft of both adaptive, and maladaptive outcomes (see p. 38). Moreover, longitudinal research implicates perfectionism in the development of many disorders and negative outcomes (e.g., eating disorders, Boone, Vansteenkiste, Soenens, Van der Kaap-Deeder, \& Verstuyf, 2014; depression, Lombardo et al., 2013; anxiety disorders, DiBartolo et al., 2007; suicide, O'Connor et al., 2007). Therefore, it is paramount that researchers, medical practitioners, and clinicians are able to easily source and understand perfectionism research in order to sufficiently comprehend how perfectionism may affect an individual's current and future wellbeing. However, a plethora of perfectionism terms, differing perfectionism measures and competing unidimensional and multidimensional conceptualisations of perfectionism has resulted in a confusing body of literature (Stairs et al., 2012).

\section{Challenges in Understanding Existing Perfectionism Literature}

To review, prior to the boom in perfectionism research beginning in the early 1990's, perfectionism was generally understood as a negative trait, commonly described using terms such as 'neurotic' (Hamachek, 1978), 'clinical' (Shafran, Cooper, \& Fairburn, 2002), 'irrational' (Jones, 1969) and 'dysfunctional' (Weissman \& Beck, 1978). However, key researchers, particularly Hamachek (1978) and Slade (1982), also argued that perfectionistic traits could be a positive quality leading to high levels of motivation and a strong drive to be very successful. Despite this, it took another decade and the development of both the Frost Multidimensional Perfectionism Scale (FMPS; Frost et al., 1990) and Hewitt and Flett's Multidimensional Perfectionism Scale (HFMPS; Hewitt \& Flett, 1991) before the multidimensional conceptualisation of perfectionism really took hold. Thereafter, both the leading view of perfectionism as a maladaptive trait, associated with detrimental outcomes for an individual, and the more optimistic idea of perfectionism underpinning success, were accepted as viable possibilities.

Subsequent research with the FMPS and HFMPS has repeatedly lead to conclusions that there are indeed both adaptive and maladaptive outcomes associated with being highly perfectionistic. For example, negative perfectionism has been related to many psychological disorders, such as eating disorders (e.g., Boone \& Soenens, 2015; Dickie et al., 2012), OCD (e.g., Moretz \& McKay, 2009; Vartanian \& Grisham, 2011), anxiety disorders (e.g., DiBartolo et al., 2007; Khawaja \& Armstrong, 2005), and depression (e.g., Cheng, 2001; 
Lynd-Stevenson \& Hearne, 1999; Yoon \& Lau, 2008) as well as other poor outcomes (e.g., stress, suicidal ideation; Chang et al., 2004; Wimberley \& Stasio, 2013). In addition, some perfectionistic traits have been associated with positive outcomes such as greater motivation (Neumeister, Williams, \& Cross, 2009), higher academic achievement (Bong, Hwang, Noh, \& Kim, 2014) and increased self-esteem (Stumpf \& Parker, 2000).

Once familiar with the myriad of perfectionism terms and measures, it first appears that research differentiating positive and negative perfectionism is relatively clear cut. However, on closer inspection it becomes apparent that there are varied results regarding the relationship between negative perfectionism and various adaptive and maladaptive outcomes. For example, whilst there seems to be a consensus that negative perfectionism is related to greater depression (e.g., Kawamura, Hunt, Frost, \& DiBartolo, 2001; Luyckx, Soenens, Goossens, Beckx, \& Wouters, 2008; Soenens et al., 2005), some researchers report this to be only a weak to moderate relationship (e.g., $r=.27$, Santanello \& Gardner, 2007; $r=.25$, Wirtz et al., 2007) whilst others report it as very strong (e.g., $r=.55$, Lynd-Stevenson $\&$ Hearne, $1999 ; r=.57$, Yoon \& Lau, 2008).

The relationship between positive perfectionism and psychological outcomes is even less clear with most researchers arguing that positive perfectionism is fundamentally positive or adaptive (e.g., Andrews et al., 2014; Shih, 2011; Stoeber \& Otto, 2006), and others finding that it may also place an individual at greater risk for some negative outcomes (e.g., Boone et al., 2013; Malkina-Pykh, 2012; Stöber \& Joormann, 2001). For example, whilst Steffen (2014) reported that positive perfectionism appeared to buffer against depression, DiSchiena, Luminet, Philippot and Douilliez (2012) found no significant relationship with depression, and DiBartolo and colleagues (2007) found that greater positive perfectionism was associated with more depressive symptoms. There also appears to be similar conflicting research regarding positive perfectionism and other negative mental health outcomes such as anxiety and stress.

A few systematic reviews have already been conducted in an attempt to clarify the conflicting perfectionism research (e.g., Gotwals, Stoeber, Dunn, \& Stoll, 2012; O'Connor, 2007; Stoeber \& Otto, 2006). For example, Stoeber and Otto (2006) examined research on positive perfectionism and concluded that positive perfectionism is indeed positive, especially when studies were able to control for negative perfectionism. In another review, O’Connor (2007) concluded that negative perfectionism increases risk for suicidality. Moreover, in two separate reviews of perfectionism and eating disorders, Bardone-Cone and colleagues (2007), and Farstad, McGeown and von Ranson (2016), concluded that both 
negative and positive perfectionism are implicated in the development of eating disorders. However, some considerable gaps in understanding remain.

In conclusion, although researchers and clinicians appear to be in agreement that perfectionism has both positive and negative outcomes, research has not always resulted in consistent findings regarding the magnitude and sometimes even the direction of these relationships. This lack of clarity could be attributed to the wide variety of perfectionism measures used, different compositions of positive and negative perfectionism within those measures, and the persistence of unidimensional perfectionism measures (e.g., EDI-II) leading to results that do not distinguish between positive and negative perfectionism. Overall, it becomes very difficult for an individual who is unfamiliar with perfectionism to easily gain an accurate understanding of the research.

Against such background, Study 1 aims to remedy the noted ambiguity by providing a thorough evaluation and synthesis of existing perfectionism research. This study will address whether the combined available research links positive and negative perfectionism to positive and negative mental health and broader psychological outcomes, and if as expected it does, it will aim to clarify the magnitude and direction of these relationships.

\section{Study One: Systematic Review and Meta-Analyses of the Relationship between Positive and Negative Perfectionism, and Mental Health and Wellbeing Outcomes}

This study will employ meta-analytic techniques to objectively summarise all available research findings on the relationship between positive and negative perfectionism, and mental health and wellbeing outcomes. Very briefly, meta-analysis involves summarising individual effect sizes, from multiple samples, and usually from numerous published articles or papers. All effect sizes concerning a particular relationship (e.g., the relationship between negative perfectionism and depression), are collated and a summary effect is generated. This provides the researcher or clinician with an objective summary of the existing research pertaining to the relationship between two variables (for other common uses of meta-analysis see Borenstein, Hedges, Higgins, \& Rothstein, 2009). Specific meta-analytic techniques will be explored in detail throughout Study 1.

\section{Advantages of Meta-Analyses}

In the absence of published meta-analyses, there are a number of difficulties associated with sourcing and reviewing published articles. First, without a significant time investment, it may only be feasible to examine a small number of individual articles. Unfortunately, this is likely to result in an understanding of only a small snapshot of the total 
research that has been conducted. Further, the research sourced and examined may be biased towards highly cited articles, articles with significant results, and articles that support the researcher's views on a topic (Cooper \& Hedges, 2009). Although it requires substantial initial time investment from a researcher, the systematic review and meta-analytic process is beneficial because it results in a summary resource that can be used by others for quick, easy, and an accurate understanding of the area of interest.

Second, when undertaking a narrative review of existing research, the reader often first looks for whether the relationship between the two variables of interest is significant. If the relationship is significant, the magnitude of the relationship is usually examined. However, if the relationship is non-significant, the reader may erroneously assume that there is no relationship when non-significance may instead be a product of the sample size and a lack of statistical power within the study (Borenstein et al., 2009). In contrast to a narrative review, meta-analysis allows the researcher to combine multiple significant and nonsignificant results, to produce an overall summary effect.

Third, as demonstrated above, there may be multiple studies investigating the same relationship in similar samples which report conflicting results. This leaves the researcher with a decision about how they should interpret differences in results and whether they should place more weight on one study over the other. Alternatively, the researcher may engage in a 'vote-counting' procedure whereby the number of articles that present a significant positive effect are compared to those who present a significant negative effect and those with no significant effect (Borenstein et al., 2009). Eventually the category with the most studies is deemed to represent the true effect. This method does not account for sample or effect size and is therefore considerably flawed. In contrast, meta-analyses take into account all studies regardless of the direction and significance of results. Finally, metaanalytic tools allow assessment of likely impact of publication bias, and investigation of potential moderators of the effect size (e.g., age and sex of sample, scale used to measure the variable of interest).

In sum, meta-analysis provides a method for taking the large quantities of competing and sometimes conflicting results and bringing them together to gain a statistically robust and objective picture of the extant research. Systematically sourcing and reviewing the literature enables the inclusion of as much research as possible, regardless of differing conceptualisations of perfectionism. In addition, research that may be at risk of being overlooked or ignored in narrative reviews, for example research with few citations, or research that contradicts the dominant understanding of the state of the research (e.g., finds 
that positive perfectionism may not be as adaptive as once thought), is less likely to be missed.

\section{Study 1a: Meta-analyses of Positive and Negative Perfectionism and Their Relationship with Positive and Negative Outcomes}

The aim of Study 1a is to gather together all existing empirical perfectionism research and succinctly draw it together to form an inclusive understanding of the current state of perfectionism research. Specifically this will include multiple meta-analyses that clarify the nature of the relationships between positive and negative perfectionism and adaptive and maladaptive outcomes. The systematic review and meta-analyses will result in a resource for clinicians, education professionals, policy makers and researchers to refer to both in their work with perfectionistic individuals and in future research.

\section{Method}

These meta-analyses were informed by the Preferred Reporting Items for Systematic reviews and Meta-Analyses guidelines (PRISMA; Moher, Liberati, Tetzlaff, \& Altman, 2009). As such, all articles considered potentially eligible for inclusion in the meta-analyses were systematically examined as detailed below. In addition, AMSTAR (Shea et al., 2009), a tool for assessing the quality of systematic reviews, was used to guide the review process and inform appropriate steps to conducting the research in a methodologically sound manner (e.g., forming a research question and setting out clear inclusion criteria prior to conducting the review).

\section{Inclusion/Exclusion Strategy}

These meta-analyses focused only on research that used the dominant FMPS (Frost et al., 1990; one of two very frequently used perfectionism scales), in order to eliminate the extra variance that would be added if all perfectionism scales were used. It was also necessary to gather data that made the distinction between positive and negative perfectionism thereby allowing investigation of their unique relationships with various mental health and wellbeing outcomes. Therefore, only studies that identified and provided a composite measure of either negative perfectionism (e.g., combined CM and DA subscales) or positive perfectionism (e.g., combined PS and Organisation subscales) were included. However, given that it is quite common to report PS as the leading or only subscale corresponding to positive perfectionism (e.g., Di Schiena et al., 2012; Kawamura, Hunt, Frost, \& DiBartolo, 2001; Khawaja \& Armstrong, 2005), if a study used a composite scale 
for negative perfectionism, thereby seeming to acknowledge the distinction between positive and negative perfectionism, their data for the PS subscale was automatically considered their equivalent measure of positive perfectionism. Finally, articles that used the FMPS, but combined the items with items from another perfectionism scale (e.g., Longbottom, Robert Grove, \& Dimmock, 2010; Soreni et al., 2014; Zhang \& Cai, 2012) were excluded due to the added variance from the additional scale.

\section{Information Sources and Search Strategy}

A search of PsychINFO database was conducted in January, 2016. The single search term 'perfectionism' was used, whilst limiting the results to English, peer reviewed, scholarly articles, and articles that contained empirical data. In addition, the search was limited to articles published before 2016. A total of 1,864 articles met this criteria.

Of these, three articles were unable to be sourced (Ashby, 1995; De Petrillo, Kaufman, Glass, \& Arnkoff, 2009; Spence \& Robbins, 1992) and one article was incorrectly documented as written in English (Besharat, 2004). Therefore, a total of 1860 articles were evaluated for their eligibility to be included in the meta-analyses (see Figure 9).

As outlined in Figure 9, articles were first included or excluded based on whether they used the FMPS. The FMPS was the most commonly used measure of perfectionism (544 articles), followed by the HFMPS (415 articles). 144 articles did not contain a relevant perfectionism scale and 1085 articles contained data based on various other measures of perfectionism (e.g., EDI-Perfectionism Subscale, Garner et al., 1983; Perfectionistic Cognitions Inventory [PCI], Flett, Hewitt, Blankstein, \& Pickering, 1998; Perfectionistic Self-Presentation Scale [PSPS], Hewitt, Flett, \& Ediger, 1995) ${ }^{6}$. Of the 544 articles that used the FMPS, only 144 gave composite scores for either positive or negative perfectionism. Of these, only 89 used composite measures comprised purely of FMPS items and subscales.

The remaining articles were then examined for effect size details. Of the 89 identified as including a pure FMPS measure of negative perfectionism, positive perfectionism or both, 65 provided appropriate effect size data. ${ }^{7}$ In cases where data was not published in a compatible format ( $n=14$; e.g., data was presented as a partial correlation or as a component of multiple regression analyses) or extra detail about the sample was required $(n=5)$, the

\footnotetext{
${ }^{6}$ Note: Due to some articles containing multiple measures of perfectionism, these numbers do not add to 1860 ${ }^{7}$ Data that crossed two individuals or time points (e.g. mother's perfectionism and their child's anxiety; intervention change data) were excluded. In addition, studies that compared perfectionism across clinical groups (i.e. ED vs. OCD samples), or used cluster analysis to group individuals based on their perfectionism profile were also excluded.
} 
author was contacted, and subsequently the data for seven articles and all requested sample information was provided. Once identified as including relevant effect size data, other study details were recorded. This included sample characteristics (sample size, sex, age,

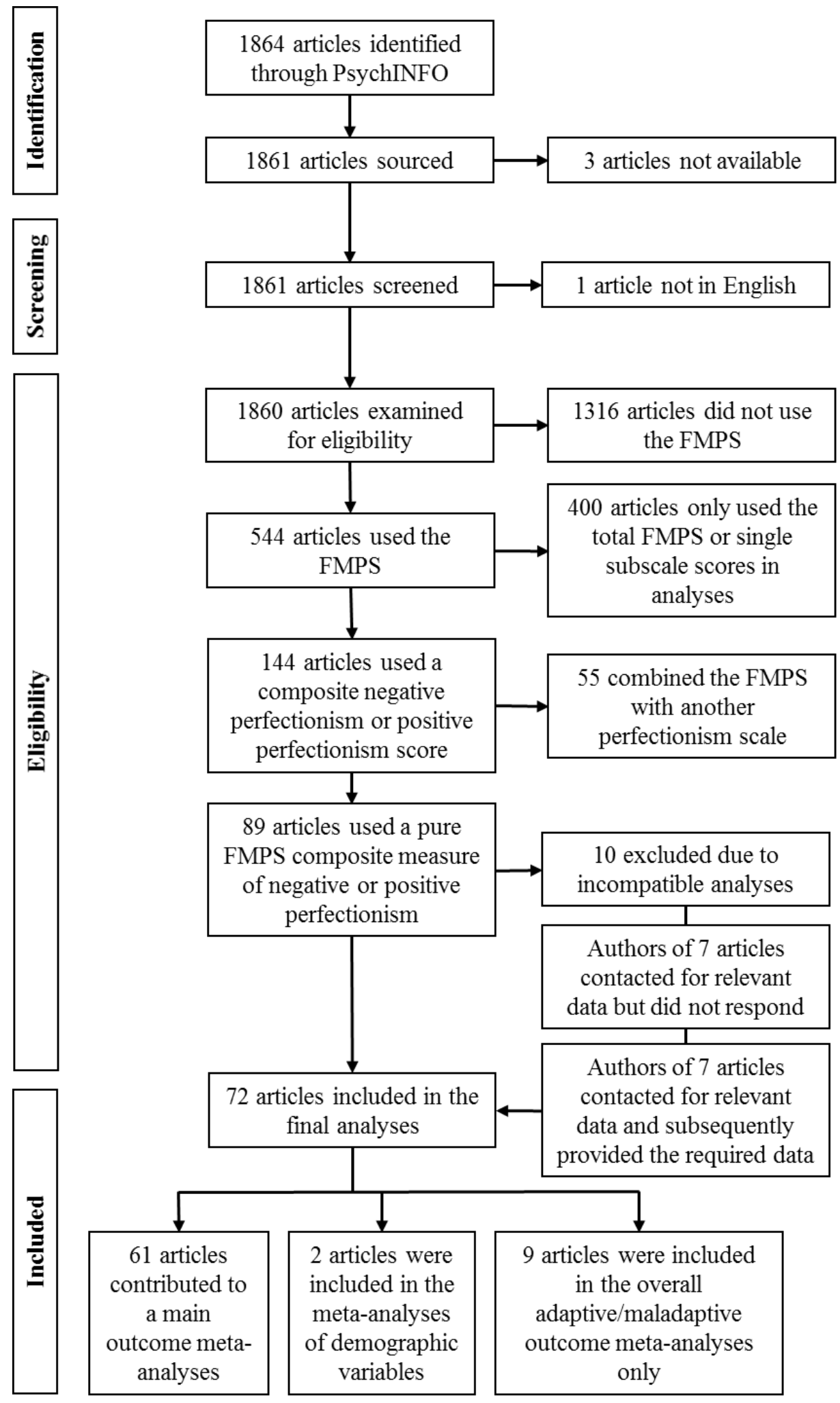

Figure 9. Inclusion/exclusion process for Study 1a 
clinical/non-clinical), the specific FMPS composition used to measure negative and/or positive perfectionism, and the outcome pertaining to the effect size (i.e., depression, anxiety).

Overall, 72 articles provided an effect size relating to a composite measure of either positive or negative perfectionism, or both. Sixty one studies provided data for 10 different specific outcomes (e.g., depression, anxiety), 9 articles did not provide data pertaining to main outcomes but reported results for other positive or negative outcomes (e.g., suicide ideation, academic achievement), one article only provided the relationship between positive and negative perfectionism (Craddock, Church, \& Sands, 2009) and one only provided data on the relationship with age (Craddock, Church, Harrison, \& Sands, 2010).

\section{Measure of Perfectionism}

As outlined above, with the aim of eliminating the variation that would be associated with using multiple measures of perfectionism, these meta-analyses focused only on research that used the FMPS. However, it is important to note that within the FMPS there are numerous combinations of subscales used to measure negative and positive perfectionism. With regard to measuring negative perfectionism, the most common combination of subscales was a composite score including CM and DA, followed by a composite score including CM, DA, PC and PE. In terms of measuring positive perfectionism, the most commonly used subscale was PS, followed by a combination of PS and Organisation. ${ }^{8}$ The manner in which the perfectionism variable was calculated, was then included in moderator analyses exploring differences in the strength of relationships based on the specific perfectionism measure used.

\section{Outcome Variables}

Initially all outcome variables were considered and included for further examination. However, through the process of extracting data for synthesis it became clear that there were a number of outcomes that had been investigated multiple times which therefore warranted their own meta-analysis (outcomes for which the data from four or more samples was available). A cut-off of four studies was decided upon consideration of the limitations associated with small meta-analysis sample sizes (Borenstein et al., 2009). Specifically, Borenstein and colleagues explain that with fewer studies the accuracy of the between-study error is substantially diminished and as a result confidence in the accuracy of the summary

\footnotetext{
${ }^{8}$ Positive perfectionism data from Lynd-Stevenson \& Hearne (1999) was excluded due to the unconventional measure of positive perfectionism used (PS + PE + PC)
} 
point estimate is limited. However, Borenstein and colleagues note that, when there are few studies, there is no ideal way of interpreting the overall group findings. Therefore, metaanalytic techniques may be used but the limitations must be recognised. These limitations will be highlighted in more detail throughout.

Outcomes with sufficient data for their own meta-analysis included: depression, anxiety, eating disorders, obsessive-compulsive disorder, stress, negative coping skills, positive coping skills, self-esteem, psychological distress, life satisfaction, age and sex. The above outcomes, in combination with all other outcomes that did not qualify for their own meta-analysis, were then grouped according to whether they were adaptive (e.g., self-esteem, academic achievement, positive coping skills) or maladaptive (e.g., depression, procrastination, career indecision). These were subsequently included in overarching metaanalyses for the relationship between positive and negative perfectionism and both adaptive and maladaptive outcomes. ${ }^{9}$ Finally a meta-analysis for the relationship between positive and negative perfectionism was conducted.

\section{Data-Synthesis}

The relevant data (usually the sample size and Pearson's correlation coefficient) for each outcome of interest were extracted from all eligible studies. This data was then synthesised using Comprehensive Meta-Analysis (CMA; Borenstein, Hedges, Higgins, \& Rothstein, 2005) with a random effects model (see more detail below). As most studies provided effect sizes in the form of Pearson's correlations, it made logical sense for the summary effect to also be presented as a Pearson's correlation coefficient (Borenstein et al., 2009; Field \& Gillett, 2010). However, in order to do so, the correlations must first be converted to the Fisher's $Z$ which effectively controls for non-normal distributions (as the population mean moves further from zero, the distribution of effect sizes becomes increasingly non-normal; Card, 2012). The summary Fisher's Z value was then converted back to a Pearson's correlation coefficient for final interpretation.

\section{Data Synthesis Model}

When conducting meta-analyses it is important to choose the model of analysis that is most appropriate for the population of interest, and the research being conducted (Field \& Gillett, 2010). Two commonly used approaches are the fixed-effects model and the randomeffects model. Whilst the main assumption for the fixed-effects model is that there is one

\footnotetext{
${ }^{9}$ Some data was excluded from the meta-analyses due to their focus on outcomes that could not easily be classified into adaptive or maladaptive (e.g. personality traits, allocentrism, and social desirability).
} 
'true' effect size and any variance across studies will be due to sampling error, the randomeffects model assumes that there is a distribution of effects and instead works on the premise that the summary effect will be the mean of all the effect sizes. In other words, the random effects model assumes that there is added variance between studies due to sample and study characteristics (Borenstein et al., 2009). Subsequently, in the random effects model, the standard error and confidence intervals are typically wider than when using a fixed effects model and the probability of Type 1 Errors (false positives) is lower (Field \& Gillett, 2010).

In addition, given that fixed effects models assume there is one true effect size, much greater weight is assigned to studies with large samples and greater precision in their estimates (Card, 2012). Conversely, studies with smaller samples are considered less precise and are therefore assigned less weight and contribute less to the overall effect size. However, in the random effects model, the aim is to find the mean of a variety of effects whilst recognising that they will differ according to sample and study characteristics. Therefore, it is assumed that each study contains a unique sample and therefore has a unique contribution regardless of the sample size (e.g., a study with a small sample may be the only study with a clinical sample). To account for this, the weight disparity between large and small studies is lessened.

When deciding between a fixed effects and random effects model it is also important to consider the purpose of the meta-analyses in terms of how the results are likely to be used and what conclusions are likely to be drawn (Card, 2012). Meta-analyses using fixed effects models are limited in that they only allow the researcher to make inferences about the populations on which the meta-analyses were based. Conversely, meta-analyses using a random effects model allow the researcher to make inferences beyond the specific sample measured and generalise the results to a greater variety of populations (Field \& Gillett, 2010).

In light of the above, a random-effects model was used for the meta-analyses in this thesis. As is the case in most psychology research, the data collected is based on a wide variety of samples which differ across multiple sample characteristics. For example, some samples are gathered from university populations whilst other are drawn from adolescent and adult populations. Moreover, some research focuses on samples comprised of individuals with a clinical diagnosis, whilst others are taken from community populations. Sex is also a key characteristic that helps to define some samples in the studies included. Therefore, given the likely variance associated with using a diverse group of samples, a random effects model was deemed most appropriate. 


\section{Moderator Analyses}

Sample and study characteristics were gathered at the data extraction phase and were subsequently tested as potential moderator variables in the final analyses. The moderator variables and their corresponding levels are outlined in Table 2. Demographic moderators were included due to research highlighting individual characteristics as important in relation to the specific nature and effect of perfectionism (e.g., sex, Downey, Reinking, Gibson, Cloud, \& Chang, 2014; sample type, Claes et al., 2012). In addition, method characteristics, such as the perfectionism composite and the specific outcome variable, were deemed important given likely variation in results based on the exact variables being measured. Inclusion of these moderators allowed the relationship of interest to be investigated at a more specific level (e.g., investigating the relationship between Concerns and Doubts and Depression, as opposed to the broader relationship between Negative Perfectionism and Depression). In order for moderator analyses to be conducted, at least two categories of moderator were required (for example, studies with adolescent and adult samples, when testing age as a moderator) in addition to at least two studies within each moderator category (e.g., two adolescent samples and two adult samples). In many cases there were insufficient studies to conduct these analyses (see the online supplementary material ${ }^{10}$ for the eligibility and results of all moderator analyses).

Table 2

Moderator Categories and Specific Variables

\begin{tabular}{ll}
\hline Moderator & Categories of Moderator \\
Sex & male, female, both \\
children, adolescents, university sample, adults \\
clinical, non-clinical, academically talented \\
Sample Type & CM + DA, CM + DA + PC + PE, CM + DA + PC, CM \\
Measure of Negative Perfectionism & + DA + PS, CMD, CMD + PEC, Khawaja and \\
& Armstrong (2005) 11 item scale \\
Measure of Positive Perfectionism & PS, PS + O, PPS (PS excl. items 4 and 6), PSS + O, PS \\
& + PE, Khawaja and Armstrong 6 item scale (2005)
\end{tabular}

\section{Assessing for Publication Bias}

Each meta-analysis was subject to a number of methods for assessing publication bias (e.g., funnel plot, Eggers regression intercept). This is an important step in meta-analyses

\footnotetext{
10 Supplementary material can be viewed at https://www.dropbox.com/sh/oggqp5h4z617znv/AACGbtc3lEsecpQrCTCsecefa?dl=0
} 
because, whilst meta-analytical techniques may be mathematically sound, they only provide an accurate representation of the studies that are identified and included in the analyses (Borenstein et al., 2009). Unfortunately, the meta-analytic process does not account for data that may not have been published. Research shows that studies that find a small effect size or that have non-significant results are much less likely to be put forward, and accepted, for publication (Borenstein et al., 2009). Therefore, it is likely that within the quantum of unpublished data, there is a disproportionate amount of non-significant data, or data yielding relatively small effect sizes. This phenomenon has become known as the 'file drawer' problem, where each researcher has a file drawer containing unpublished, non-significant or small effect-data (Rosenthal, 1979). By contrast, the data included in meta-analyses may be disproportionately weighted towards large studies, large effect sizes and significant results and as a result the meta-analytic summary effect may be overestimated. In the process of conducting the meta-analyses, some researchers attempt to remedy this as much as possible by contacting researchers in the field and asking for any unpublished data that may be pertinent. However, responses can be limited and the 'file drawer' problem remains. Overall, most methods for assessing publication bias assume that as the sample size decreases risk of bias increases (Borenstein et al., 2009).

Multiple methods for identifying bias (e.g., funnel plot, Kendall's tau, Egger's regression intercept) and assessing the impact of bias (Fail-safe N, Duval and Tweedie's Trim and Fill) are outlined within the results of the first set of meta-analyses and then reported in a summary table for each meta-analysis that follows. However, before referring to these results it is important to note that these methods are by no means conclusive and must be interpreted with caution, especially when the sample of studies is small (Sterne \& Harbord, 2004). To reiterate, it is often concluded that there is evidence of bias when there are disproportionately large effect sizes in studies with smaller samples. Whilst this may evidence bias there are multiple other reasons this could occur (for a full review of alternative causes of asymmetric funnel plots and significant tests for bias see Sterne \& Harbord, 2004).

Methods of assessing publication bias are also very limited when there are few samples or when there is little dispersion between the effect sizes (Borenstein et al., 2009). Moreover, even when these conditions are satisfied the tests have relatively low power and may only detect severe publication bias (Card, 2012). Therefore, whilst the methods above can be used to indicate bias, they should always be interpreted with considerable caution. 


\section{Results}

\section{Results of Article Search}

As outlined above, an initial search identified 1,864 articles of which 544 used the FMPS. Sixty five of these provided data for a composite measure of negative or positive perfectionism and a relevant outcome. Multiple attempts were made to contact the authors of 14 articles, and upon the provision of the relevant information, seven of these articles were eligible for inclusion in the analyses. Overall, 10 broad outcomes were identified, each of which were subject to individual meta-analyses with both positive and negative perfectionism. All other outcomes were included in meta-analyses of positive and negative perfectionism and adaptive and maladaptive outcomes. In addition, meta-analyses for sex, age and the relationship between negative and positive perfectionism were conducted.

As an exemplar of the meta-analyses conducted in Study 1, results for the metaanalyses between negative and positive perfectionism and depression are presented in detail below. This includes both forest plots and funnel plots, visually displaying the individual study effects in combination with the summary effect, and any publication bias respectively. For the sake of simplicity and to provide an easy reference, all other meta-analyses results are presented in Tables 3-6, with only key results reported in text. To assist in easy interpretation of the results that follow, an outline of how to interpret forest plots and other key statistics are included in the description of the depression meta-analyses below.

\section{Negative Perfectionism and Depression}

In total, 28 samples from 25 articles were included in a meta-analysis of the relationship between negative perfectionism and depression. Each individual study, as well as the summary effect, is presented in the forest plot below (Figure 10). A summary of the individual study characteristics can be found by following the link to the online supplementary material.

Forest plot. A forest plot is a visual depiction of the individual study effect sizes, their $95 \%$ confidence intervals, as well as an overall summary effect. Although the main outcome of a meta-analysis is the summary effect, the forest plot gives a visual depiction that allows the researcher to keep each individual study and unique effect size in mind (Borenstein et al., 2009). As Lewis and Clarke (2001) state, it enables a researcher to "see the wood [forest] and the trees" (p. 1479). Thus, the forest plot encourages the identification of outliers and visually depicts any heterogeneity between the studies. Within the plot, the effect size for each study is depicted using a black square, with the $95 \%$ confidence intervals 
represented by horizontal lines either side of the square. Shorter lines represent a smaller confidence interval, therefore indicating more precision in the effect size. In addition, square size represents how much weight is given to each study in computing the overall summary effect, with a larger squares indicating more weight than a smaller square. Finally, the summary effect is represented by the diamond at the bottom of the plot. A wide diamond indicates less precision in the summary effect than a relatively narrow one (Borenstein et al., 2009).

\begin{tabular}{lrrrrr} 
Study name & \multicolumn{5}{c}{ Statistics for each study } \\
\cline { 2 - 6 } & \multicolumn{5}{c}{ Lower Upper } \\
& Correlation & limit & limit & Z-Value p-Value \\
Brown \& Kocovski, 2014 & 0.52 & 0.36 & 0.65 & 5.71 & 0.000 \\
Cheng, 2001 & 0.38 & 0.22 & 0.51 & 4.58 & 0.000 \\
Di Schiena et al., 2012 & 0.30 & 0.15 & 0.44 & 3.85 & 0.000 \\
DiBartolo et al., 2007 & 0.51 & 0.43 & 0.58 & 10.36 & 0.000 \\
DiBartolo et al., 2008 & 0.46 & 0.36 & 0.54 & 8.08 & 0.000 \\
Harris et al., 2008 & 0.36 & 0.17 & 0.52 & 3.63 & 0.000 \\
Kawamura et al., 2001 & 0.57 & 0.47 & 0.66 & 9.29 & 0.000 \\
Khawaja \& Armstrong, 2005 & 0.60 & 0.52 & 0.67 & 11.35 & 0.000 \\
Levinson et al., 2013 & 0.47 & 0.34 & 0.58 & 6.31 & 0.000 \\
Lombardo et al., 2013 & 0.44 & 0.38 & 0.49 & 13.49 & 0.000 \\
Luyckx et al., 2008 & 0.43 & 0.31 & 0.54 & 6.32 & 0.000 \\
Lynd-Stevenson \& Hearne, 1999 & 0.55 & 0.42 & 0.66 & 7.29 & 0.000 \\
Norman et al., 1998 & 0.38 & 0.22 & 0.52 & 4.36 & 0.000 \\
Santanello \& Gardener, 2007 & 0.27 & 0.10 & 0.43 & 3.06 & 0.002 \\
Scott et al., 2014 & 0.52 & 0.42 & 0.61 & 8.87 & 0.000 \\
Soenens et al., 2005 & 0.53 & 0.45 & 0.60 & 10.77 & 0.000 \\
Soenens et al., 2005 & 0.37 & 0.27 & 0.46 & 7.11 & 0.000 \\
Soenens et al., 2010 & 0.40 & 0.30 & 0.49 & 7.57 & 0.000 \\
Soenens et al., 2010 & 0.37 & 0.27 & 0.46 & 6.94 & 0.000 \\
Soenens, Luyckx et al., 2008 & 0.44 & 0.38 & 0.50 & 12.26 & 0.000 \\
Steffen, 2014 & 0.51 & 0.44 & 0.57 & 13.04 & 0.000 \\
Steffen, 2014 & 0.55 & 0.48 & 0.61 & 13.58 & 0.000 \\
Stoeber \& Joormann, 2001 & 0.34 & 0.20 & 0.46 & 4.71 & 0.000 \\
Valero et al., 2013 & 0.42 & 0.31 & 0.52 & 6.71 & 0.000 \\
Wimberley \& Stasio, 2013 & 0.65 & 0.53 & 0.75 & 8.13 & 0.000 \\
Wirtz et al., 2007 & 0.25 & -0.03 & 0.49 & 1.74 & 0.082 \\
Wu \& Cortesi, 2009 & 0.30 & 0.21 & 0.38 & 6.59 & 0.000 \\
Yoon \& Lau, 2008 & 0.57 & 0.45 & 0.67 & 7.58 & 0.000 \\
& 0.45 & 0.42 & 0.49 & 21.76 & 0.000
\end{tabular}

Figure 10. Forest plot with effect sizes for negative perfectionism and depression

Figure 10 shows that whilst there was some variance in the effect sizes for the relationship between negative perfectionism and depression, there are no clear outliers. Overall, as represented by the diamond at the bottom of the plot, using a random effects model, the overall summary correlation coefficient was .45 (95\% CI, [.42, .49]), and was 
significantly different to $0(p<.001)$. The individual effect sizes ranged from .25 (Wirtz et al., 2007) to .65 (Wimberley \& Stasio, 2013).

Heterogeneity. In order to assess heterogeneity between the effect sizes, the Qstatistic and $\mathrm{I}^{2}$ were examined. A significant Q-statistic suggests that it is unlikely that all the variability between the studies is due to random error (Borenstein et al., 2009). In addition, the $\mathrm{I}^{2}$ value indicates how much of the variance is due to study and sample characteristics rather than within study random error (Huedo-Medina, Sánchez-Meca, Marín-Martínez, \& Botella, 2006). In this meta-analysis of the relationship between negative perfectionism and depression, the Q-statistic indicated that there was significant heterogeneity $(Q(27)=93.18, p$ $<.001$ ) between the effect sizes. Moreover, the $\mathrm{I}^{2}$ statistic indicated that $71 \%$ of the variance could be attributed to study characteristics $\left(\mathrm{I}^{2}=71.02\right)$. Given significant heterogeneity, moderator analyses were conducted to investigate which samples or which study methods resulted in a significantly stronger or weaker association between negative perfectionism and depression.

Moderator analyses. Moderator analyses demonstrated that there were no significant differences in effect sizes based on the type of sample (clinical or non-clinical), and the age and sex of the sample. However, the composite measure of negative perfectionism (e.g., CM + DA, CM + DA + PC + PE; $Q(2)=9.56, p=.01)$ was identified as significant moderator of the relationship. In particular, the relationship between negative perfectionism and depression was strongest when negative perfectionism was calculated using $\mathrm{CM}+\mathrm{DA}+\mathrm{PC}(n=3, r=$ $.53,95 \% \mathrm{CI},[.49, .57])$, followed by the composite measure including CM + DA + PE + PC $(n=8, r=.46,95 \% \mathrm{CI},[.37, .54])$, and finally a combination of $\mathrm{CM}+\mathrm{DA}(n=17, r=.44$, $95 \%$ CI, $[.40, .48])$.

Assessing for publication bias. The final step in each meta-analysis is to assess publication bias in order to gauge whether the summary effect is a reliable estimate of all research that has been conducted. An assessment of publication bias for the meta-analyses usually requires a combination of subjective judgments and statistical tests that look for the relationship between effect size and standard error for each study included in the metaanalysis.

Funnel plots (see Figure 11 for the funnel plot for the relationship between negative perfectionism and depression) show the distribution of all studies based on each individual effect size (Fisher Z) and its standard error (Borenstein et al., 2009). Thus, the smaller the standard error, or the further up the y-axis the study is plotted, the larger the sample and the smaller the standard error a study has. Moreover, the greater the effect size, the further from 
the centre of the x-axis the study will be plotted. When there is little evidence of publication bias, the studies will be plotted symmetrically around the summary effect (represented by the hollow triangle and the vertical line) and will broadly fit within the shape of an inverted funnel (Sterne, Egger, \& Moher, 2008). Conversely, if publication bias exists, it is expected that the distribution of studies will be biased to one side of the summary effect and will be clustered at the top of the funnel. This indicates that studies with larger samples, thus greater power for finding significant relationships, are more likely to be published (Field \& Gillett, 2010). As can be seen in Figure 11, the observed studies (hollow circles) are distributed relatively symmetrically around the summary effect indicating little evidence of bias.

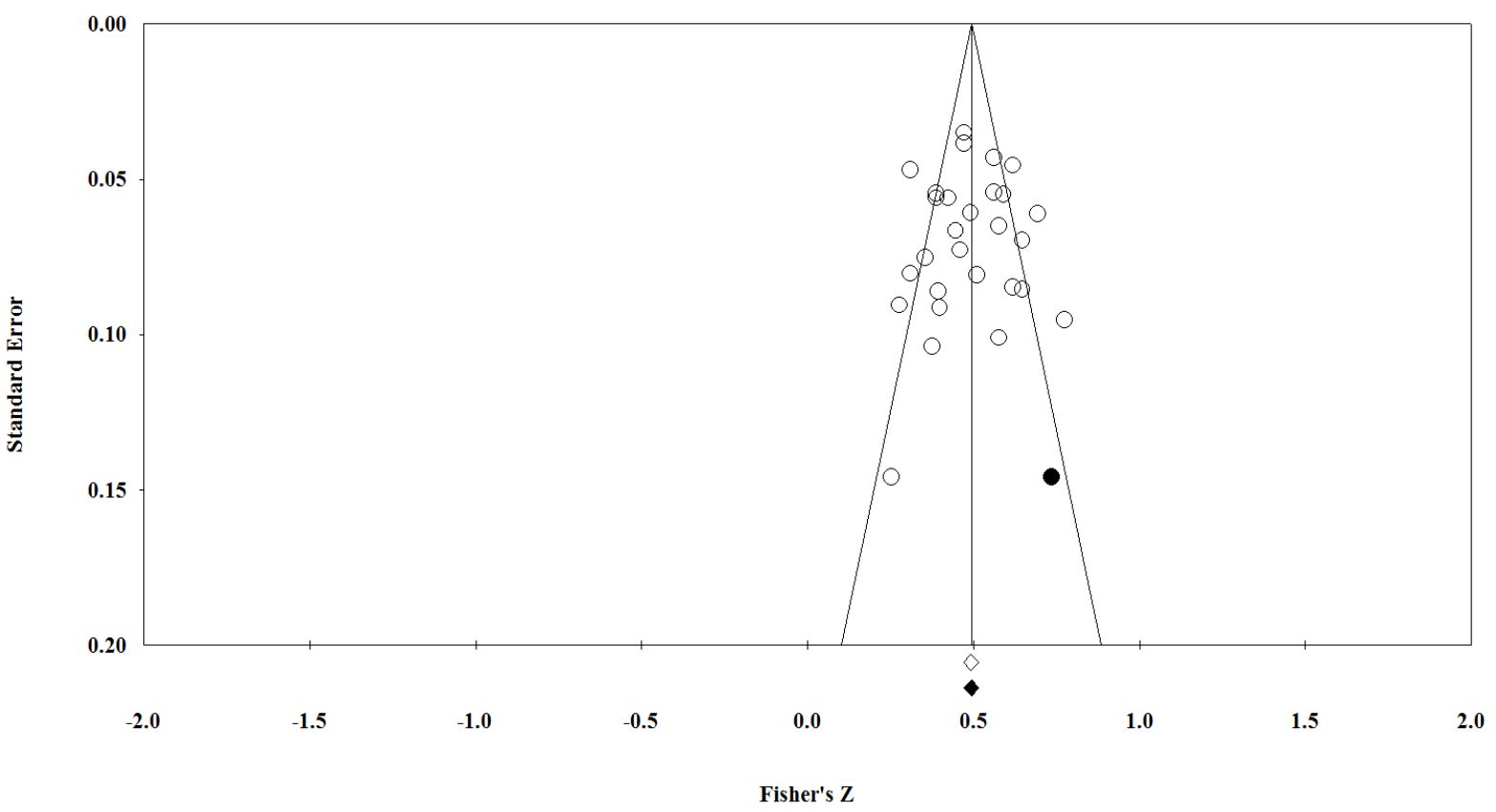

Figure 11. Funnel plot of the standard error by Fischer's Z for the association between negative perfectionism and depression. Hollow circles: observed studies, filled circles: inputted studies

Assessment of the funnel plot symmetry is largely subjective (Sterne et al., 2008), therefore, statistical measures are also often employed. Both Begg and Mazumdar's rank correlation (Kendall's tau; Begg \& Mazumdar, 1994) and Egger's regression intercept (Sterne, Gavaghan, \& Egger, 2000) are considered statistical equivalents of the funnel plot (Card, 2012), also examining the relationship between the effect size and standard error. If either or both of these statistics are significant it is considered additional evidence towards a biased sample of studies. As expected, in the meta-analysis between negative perfectionism and depression both Kendall's tau and Egger's regression intercept were non-significant ( $p=$ 
.75 and $p=.82$ respectively), thus providing further evidence against publication bias.

\section{Positive Perfectionism and Depression}

A total of 19 samples from 17 articles contributed to a meta-analysis of the relationship between positive perfectionism and depression ${ }^{11}$. All 19 samples and their respective effect sizes are presented in the forest plot below (Figure 12). Overall, the summary Pearson's correlation coefficient, using a random effects model, for the relationship between positive perfectionism and depression was .07 (95\% CI, $[.02, .12])$, and was significantly different to $0(p=.01)$. The effect sizes ranged from -.12 (Steffen, 2014; Sample 2) to .25 (Khawaja \& Armstrong, 2005). The significant Q-statistic $(Q(18)=65.74, p<.001)$ indicated significant heterogeneity between the studies and the $\mathrm{I}^{2}$ statistic $\left(\mathrm{I}^{2}=72.62\right)$ suggested that up to $73 \%$ of the variance was due to individual study and sample characteristics. Moderator analyses indicated that there were no significant differences in effect based on the composite of positive perfectionism, or the age and sex of participants. There were insufficient studies in each moderator category to test the sample type as a moderator.

\begin{tabular}{lrrrrr} 
Study name & \multicolumn{5}{c}{ Statistics for each study } \\
\cline { 2 - 6 } & $\begin{array}{c}\text { Lower Upper } \\
\text { Correlation }\end{array}$ & limit & limit & Z-Value p-Value \\
Di Schiena et al., 2012 & -0.02 & -0.18 & 0.14 & -0.25 & 0.803 \\
DiBartolo et al., 2007 & 0.12 & 0.01 & 0.22 & 2.22 & 0.026 \\
DiBartolo et al., 2008 & 0.06 & -0.06 & 0.17 & 0.92 & 0.356 \\
Harris et al., 2008 & 0.15 & -0.05 & 0.34 & 1.46 & 0.145 \\
Kawamura et al., 2001 & 0.21 & 0.08 & 0.34 & 3.06 & 0.002 \\
Khawaja \& Armstrong, 2005 & 0.25 & 0.13 & 0.36 & 4.18 & 0.000 \\
Levinson et al., 2013 & 0.11 & -0.05 & 0.26 & 1.37 & 0.172 \\
Lombardo et al., 2013 & 0.09 & 0.03 & 0.16 & 2.69 & 0.007 \\
Luyckx et al., 2008 & 0.12 & -0.02 & 0.26 & 1.66 & 0.097 \\
Norman et al., 1998 & 0.13 & -0.05 & 0.30 & 1.43 & 0.152 \\
Santanello \& Gardener, 2007 & 0.08 & -0.10 & 0.25 & 0.89 & 0.376 \\
Soenens et al., 2005 & 0.13 & 0.02 & 0.23 & 2.39 & 0.017 \\
Soenens et al., 2005 & 0.04 & -0.07 & 0.15 & 0.73 & 0.464 \\
Soenens, Luyckx et al., 2008 & 0.14 & 0.07 & 0.21 & 3.66 & 0.000 \\
Steffen, 2014 & -0.10 & -0.18 & -0.02 & -2.33 & 0.020 \\
Steffen, 2014 & -0.12 & -0.21 & -0.03 & -2.65 & 0.008 \\
Stoeber \& Joormann, 2001 & 0.09 & -0.06 & 0.23 & 1.20 & 0.230 \\
Wimberley \& Stasio, 2013 & 0.02 & -0.17 & 0.20 & 0.21 & 0.834 \\
Wu \& Cortesi, 2009 & -0.08 & -0.17 & 0.01 & -1.71 & 0.088 \\
& 0.06 & 0.03 & 0.08 & 4.49 & 0.000
\end{tabular}
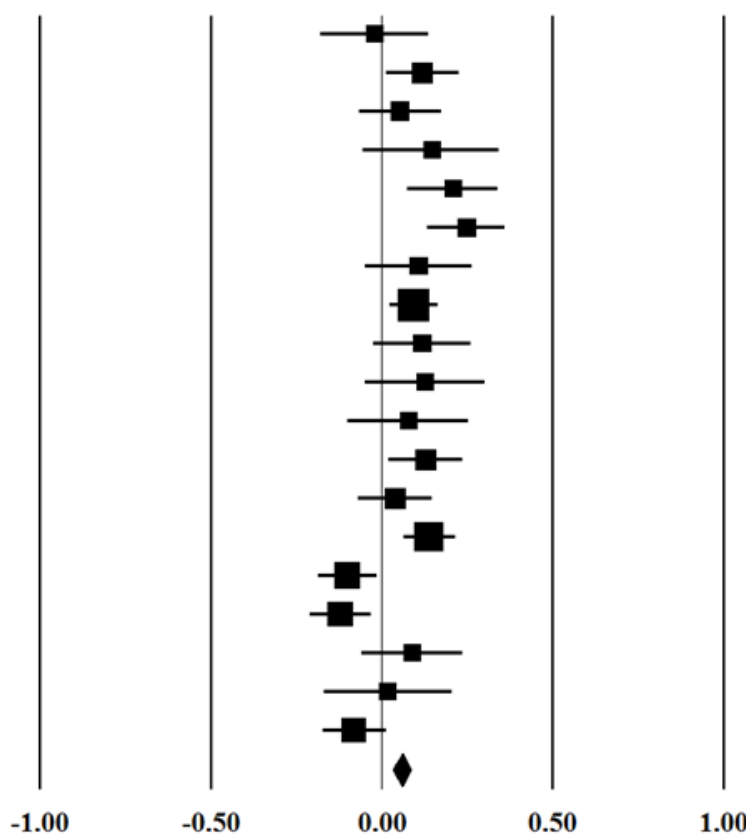

Figure 12. Forest plot with effect sizes for positive perfectionism and depression

\footnotetext{
11 Data from Lynd-Stevenson \& Hearne (1999), was excluded from this meta-analysis due to their unconventional measure of positive perfectionism (PS + PS + PE) which has substantial overlap with common measures of negative perfectionism.
} 
Assessment for publication bias indicated that this sample of studies was biased towards greater positive effect sizes, as demonstrated by Figure 13. That is, researchers may have been more likely to publish their research on positive perfectionism and depression when the association between the two variables was positive. Kendall's tau and Egger's regression intercept were non-significant ( $p=.89$ and $p=.34$ respectively). However, it is important to note that these measures are limited by their relatively low power for detecting bias in metaanalyses containing less than 20 studies (Sterne \& Harbord, 2004). Whilst, Egger's regression intercept is slightly more sensitive than Begg and Mazumdar's rank correlation, neither can be relied upon as a sole measure for accurately identifying bias, especially when there are few studies. With regard to the meta-analyses to follow, whilst these statistics will be presented for all meta-analyses conducted, the fewer studies in the meta-analyses the less weight we can give the measures of publication bias. In particular, following the guidelines of Sterne and colleagues (2000), in meta-analyses with fewer than five studies these tests should only be interpreted as indications of what publication bias might exist rather than evidence that it does.

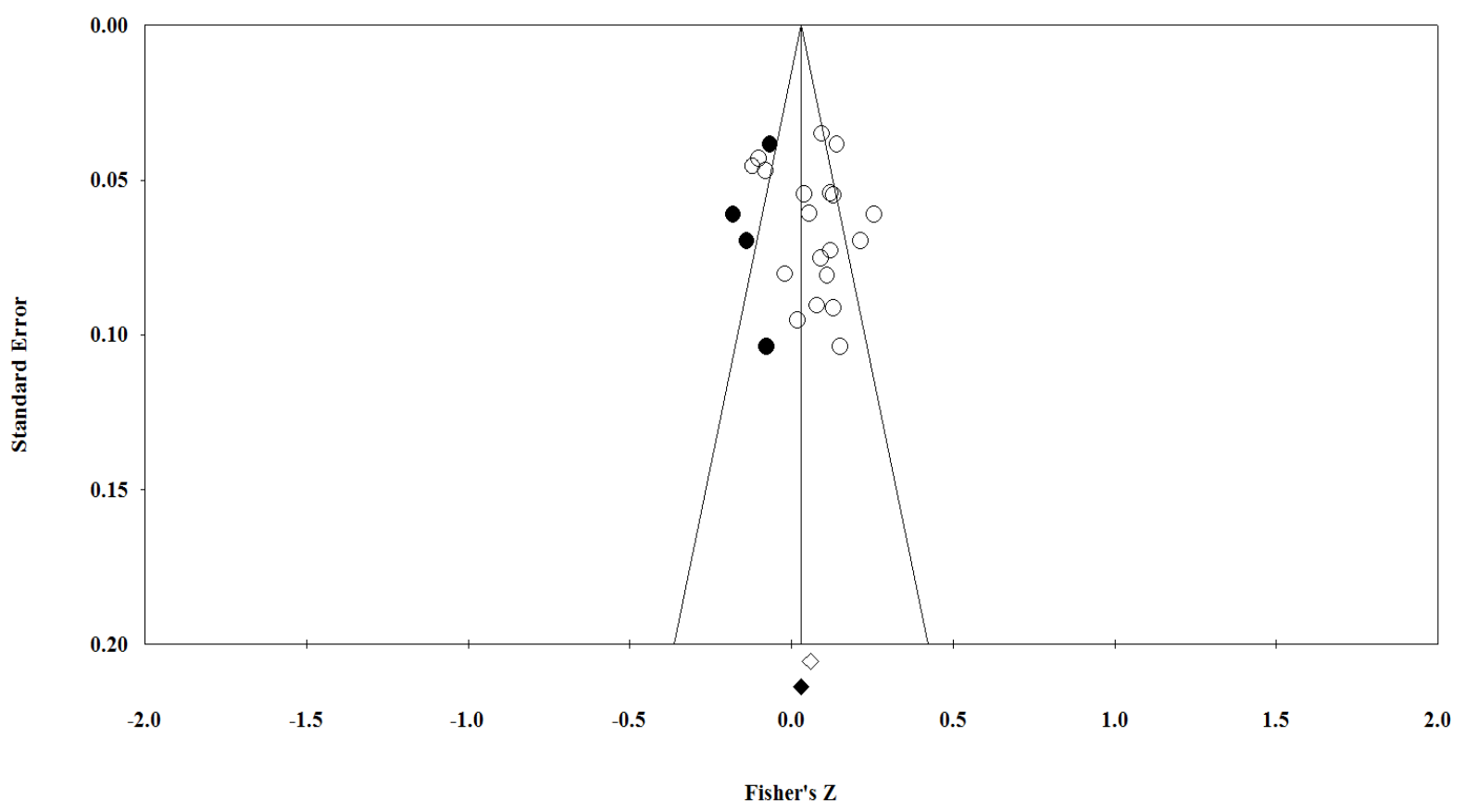

Figure 13. Funnel plot of the standard error by Fischer's $\mathrm{Z}$ for the association between positive perfectionism and depression.

Estimating the effect of publication bias. Given that there was some indication of publication bias, a brief summary of methods that assist in determining how much influence the suspected publication bias is having, is outlined below. Put simply, the aim of these 
analyses is to gauge whether, in the absence of bias, a significant effect would exist or whether the summary effect is solely an artefact of publication bias (Borenstein et al., 2009). First, Orwin's fail-safe N (Orwin, 1983) is used to estimate how many studies with null results would be required to change the summary effect to 'negligible'. For the purpose of these meta-analyses a 'negligible' relationship is below 0.1 , representing a small, usually nonsignificant effect size (Cohen, 1988). However, in cases where the summary effect is already below 0.1, the Classic Fail-Safe N (Rosenthal, 1979) is used. This estimates how many additional studies with a null effect would be needed to nullify the summary effect. In the case of positive perfectionism and depression, the summary effect $(r=.07)$ was already considered negligible, therefore Orwin's Fail Safe N was not applicable. However, the Classic Fail Safe $\mathrm{N}$ estimated that an additional 98 studies would be required to completely nullify the effect.

Finally, Duval and Tweedie's (2000) 'trim and fill' method is used to estimate the effect size in the absence of bias. This method involves inputting studies that mirror observed studies to create a more symmetrical distribution of effect sizes around the summary effect. The observed studies are displayed as hollow circles, whereas the inputted studies are displayed as black circles (see Figure 13). An estimated unbiased summary effect size is then represented by the black triangle (Borenstein et al., 2009). As is illustrated in Figure 13, Duval and Tweedie's trim and fill method resulted in four inputted data points. The adjusted estimate was .04 (95\% CI, [-.01, .09]) which would also be considered a negligible effect with little practical meaning.

Thus far, detailed meta-analysis results for the relationships between both negative perfectionism and depression, and positive perfectionism and depression have been presented. All meta-analytic results for the other nine outcomes are presented in Tables 3-4, followed by a brief in-text summary of key results. Forest plots for all meta-analyses can be found in Appendix B, while a summary table of the studies included across the meta-analyses, and a funnel plot for each individual meta-analysis can be viewed within the online supplementary material.

\section{Specific Outcome Results Summary}

Negative perfectionism. Overall, with regard to maladaptive outcomes, the metaanalyses presented in Table 3 show that negative perfectionism was positively and significantly associated with depression (.45), anxiety (.42), ED symptoms (.40), OCD 
symptoms (.40), stress (.48), psychological distress (.47) and negative coping skills $(.34)^{12}$. Conversely, with regard to adaptive outcomes, negative perfectionism was negatively and significantly associated with self-esteem (-.36) and life satisfaction (-.37). There was no significant relationship between negative perfectionism and positive coping skills $(p=.80)$. This suggests that in general, negative perfectionism is associated with greater maladaptive outcomes and fewer adaptive outcomes.

Significant heterogeneity ( $Q$-statistic) in effect sizes was indicated in all negative perfectionism meta-analyses. The $\mathrm{I}^{2}$ statistic ranged from 66.26 (OCD symptoms) to 91.08 (self-esteem) indicating that up to $91 \%$ of the variance discovered in these meta-analyses was likely 'true' variation (i.e. due to differences in sample and study characteristics). Accordingly, moderator analyses were performed for all meta-analyses. Overall, only sample type and the composite measure of perfectionism were revealed as significant moderators.

First, sample type moderated the relationship between negative perfectionism and anxiety $(Q(1)=4.84, p=.03)$, such that the relationship was significantly stronger in clinical samples $(n=.26 ; r=.43 ; 95 \% \mathrm{CI},[.38, .47])$, than in non-clinical samples $(n=3 ; r=.31$; $95 \%$ CI, $[.21, .40])$. Sample type did not moderate any other negative perfectionism relationship.

Second, the specific negative perfectionism composite significantly moderated the relationships between negative perfectionism and depression (previously outlined, see p. 63), ED symptoms $(Q(2)=62.52, p<.001)$, and self-esteem $(Q(2)=6.49, p=.04)$. With regard to ED symptoms, the relationship with negative perfectionism was strongest when measured using a combination of scales including CM + DA + PS ( $n=3 ; r=.56 ; 95 \% \mathrm{CI},[.52, .59])$, next, a similar combination but without the PS component (a combination of CM + DA; $n=$ $14 ; r=.37 ; 95 \% \mathrm{CI},[.33, .41])$ also rendered a moderate relationship, and finally the relationship between negative perfectionism and ED symptoms was least strong when a combination of CM + DA + PC + PE was used $(n=4 ; r=.32 ; 95 \% \mathrm{CI},[.23, .39])$.

Conversely, the relationship between negative perfectionism and self-esteem was strongest when only CM + DA $(n=3 ; r=-.48 ; 95 \% \mathrm{CI},[-.60,-.34])$ were used, followed by CM + DA $+\mathrm{PE}+\mathrm{PC}(n=2 ; r=-.35 ; 95 \% \mathrm{CI},[-.60,-.04])$, and finally $\mathrm{CM}+\mathrm{DA}+\mathrm{PS}(n=2 ; r=-.28$; $95 \%$ CI, [-.33, -.23]).

\footnotetext{
12 Outcomes presented in order from the meta-analysis with the greatest number of studies to the meta-analysis with the least contributing studies
} 
Table 3

Summary of Negative Perfectionism Meta-Analyses Results ${ }^{13}$

\begin{tabular}{|c|c|c|c|}
\hline Meta-analysis & Summary effect & Heterogeneity & Moderator analysis \\
\hline $\begin{array}{l}\text { Negative perfectionism and } \\
\text { depression }(n=28)^{14}\end{array}$ & $\begin{array}{l}r=.45, p<.001 \\
95 \% \text { CI, }[.42, .49]\end{array}$ & $\begin{array}{l}Q(27)=93.18, p<.001 \\
\mathrm{I}^{2}=71.02\end{array}$ & $\begin{array}{l}\text { No significant } \\
\text { moderators }^{15}\end{array}$ \\
\hline $\begin{array}{l}\text { Negative perfectionism and } \\
\text { anxiety }^{17}(n=29)\end{array}$ & $\begin{array}{l}r=.42, p<.001 \\
95 \% \text { CI, }[.37, .46]\end{array}$ & $\begin{array}{l}Q(28)=135.38, p<.001 \\
\mathrm{I}^{2}=79.32\end{array}$ & $\begin{array}{l}\text { Specific anxiety outcome; } \\
Q(8)=18.44, p=.02\end{array}$ \\
\hline
\end{tabular}

anxiety $^{17}(n=29)$

$95 \%$ CI, $[.37, .46] \quad \mathrm{I}^{2}=79.32$

$Q(8)=18.44, p=.02$

Publication bias

Funnel plot: bias unlikely

Kendall's tau: $p=.75$

Egger's intercept: $p=.82$

Orwin's Fail-safe $\mathrm{N}^{16}$ : 40

Funnel plot: inconclusive

Adjusted estimate: $r=.43,95 \%$ CI, $[.39, .47]$

Kendall's tau: $p=.31$

Egger's intercept: $p=.08$

Orwin's Fail-safe N: 36

Negative perfectionism and $r=.41, p<.001 \quad Q(19)=94.26, p<.001$

eating disorders $(n=20)^{18}$

$95 \%$ CI, $[.35, .46] \quad \mathrm{I}^{2}=79.84$

Perfectionism composite:

$Q(2)=53.90, p<.001 ;$

Funnel plot: bias unlikely

Clinical/Non-clinical:

Kendall's tau: $p=.58$

$\begin{array}{ll}\text { Clinical/Non-clinical: } & \text { Egger's intercept: } p=.17 \\ Q(1)=4.71, p=.03 ; \text { Age: } & \text { Orwin's Fail-safe N: } 26\end{array}$

Negative perfectionism and

$$
r=.40, p<.001 \quad Q(6)=16.99, p<.001
$$

$Q(2)=11.89, p=.01$

obsessive compulsive

$95 \%$ CI, $[.32, .47] \quad \mathrm{I}^{2}=64.69$

No significant moderators

disorder $(n=7)$

Funnel plot: inconclusive

Adjusted estimate: $r=.38,95 \%$ CI, $[.30, .45]$

Kendall's tau: $p=.55$

Egger's intercept: $p=.66$

Orwin's Fail-safe N: 8

${ }^{13}$ Appendix B includes forest plots each individual meta-analysis. A description of all studies included in the meta-analyses, and funnel plots for each meta-analysis can be viewed in the online supplementary material

${ }^{14} \mathrm{~N}$ represents the number of samples included in the meta-analysis, rather than the number of articles

${ }^{15}$ In the case of significant heterogeneity between individual studies, all moderators (see Table 8) were tested, however only significant moderators are reported

${ }^{16}$ In cases where the summary effect already signifies a negligible relationship, the Classic Fail Safe N is used instead to estimate how many additional studies would be required to bring the relationship to 0 . In cases where the relationship is already non-significant, neither Orwin's fail safe N nor the Classic Fail Safe N are appropriate.

17 The relationship between perfectionism and anxiety was tested both with and without Fear of Negative Evaluation due to concerns that perfectionism and FNE are

conceptually similar. However, there was no significant difference therefore Fear of Negative Evaluation was included in the final analyses.

${ }^{18}$ Data on BMI was collected, however BMI in itself is neither an adaptive or maladaptive outcome, therefore it was excluded from all analyses. 
Table 3

Summary of Negative Perfectionism Meta-Analyses Results ${ }^{13}$

\begin{tabular}{|c|c|c|c|c|}
\hline Meta-analysis & Summary effect & Heterogeneity & Moderator analysis & Publication bias \\
\hline $\begin{array}{l}\text { Negative perfectionism and } \\
\text { positive coping }(n=4)^{19}\end{array}$ & $\begin{array}{l}r=.03, p=.69 \\
95 \% \text { CI, [-.11, - } \\
.16]\end{array}$ & $\begin{array}{l}Q(3)=11.59, p=.01 \\
\mathrm{I}^{2}=74.11\end{array}$ & $\begin{array}{l}\text { Specific outcome: } Q(3)= \\
12.59, p=.01\end{array}$ & $\begin{array}{l}\text { Funnel plot: inconclusive } \\
\text { Adjusted estimate: } r=.08,95 \% \text { CI, }[-.07, .23] \\
\text { Kendall's tau: } p=.31 \\
\text { Egger's intercent: } p=32\end{array}$ \\
\hline $\begin{array}{l}\text { Negative perfectionism and } \\
\text { negative coping }(n=9)\end{array}$ & $\begin{array}{l}r=.34, p<.001 \\
95 \% \text { CI, }[.21, .45]\end{array}$ & $\begin{array}{l}Q(7)=57.40, p<.001 \\
\mathrm{I}^{2}=86.06\end{array}$ & No significant moderators & $\begin{array}{l}\text { Funnel plot: bias unlikely } \\
\text { Kendall's tau: } p=.03 \\
\text { Egger's intercept: } p=.01 \\
\text { Orwin's Fail-safe N: } 4\end{array}$ \\
\hline $\begin{array}{l}\text { Negative perfectionism and } \\
\text { psychological distress }(n=8)\end{array}$ & $\begin{array}{l}r=.47, p<.001 \\
95 \% \text { CI, }[.36, .56]\end{array}$ & $\begin{array}{l}Q(7)=56.38, p<.001 \\
\mathrm{I}^{2}=87.58\end{array}$ & $\begin{array}{l}\text { Specific outcome: } Q(5)= \\
16.37, p=.01\end{array}$ & $\begin{array}{l}\text { Funnel plot: bias unlikely } \\
\text { Kendall's tau: } p=1.00 \\
\text { Egger's intercept: } p=.78 \\
\text { Orwin's Fail-safe N: } 13\end{array}$ \\
\hline $\begin{array}{l}\text { Negative perfectionism and } \\
\text { stress }(n=7)\end{array}$ & $\begin{array}{l}r=.48, p<.001 \\
95 \% \text { CI, }[.36, .59]\end{array}$ & $\begin{array}{l}Q(6)=39.61, p<.001 \\
\mathrm{I}^{2}=84.85\end{array}$ & $\begin{array}{l}\text { Specific outcome: } Q(3)= \\
11.41, p=.01\end{array}$ & $\begin{array}{l}\text { Funnel plot: bias unlikely } \\
\text { Kendall's tau: } p=.07 \\
\text { Egger's intercept: } p=.09 \\
\text { Orwin's Fail-safe N: } 14\end{array}$ \\
\hline $\begin{array}{l}\text { Negative perfectionism and } \\
\text { self-esteem }(n=8)\end{array}$ & $\begin{array}{l}r=-.36, p<.001 \\
95 \% \text { CI, [-.45, - } \\
.25]\end{array}$ & $\begin{array}{l}Q(7)=81.41, p<.001 \\
\mathrm{I}^{2}=91.40\end{array}$ & No significant moderators & $\begin{array}{l}\text { Funnel plot: bias unlikely } \\
\text { Kendall's tau: } p=.90 \\
\text { Egger's intercept: } p=.85 \\
\text { Orwin's Fail-safe N: } 20\end{array}$ \\
\hline $\begin{array}{l}\text { Negative perfectionism and } \\
\text { life satisfaction }(n=5)\end{array}$ & $\begin{array}{l}r=-.37, p<.001 \\
95 \% \mathrm{CI},[-.46,- \\
.27]\end{array}$ & $\begin{array}{l}Q(4)=19.45, p<.001 \\
\mathrm{I}^{2}=79.43\end{array}$ & No significant moderators & $\begin{array}{l}\text { Funnel plot: inconclusive } \\
\text { Adjusted estimate: } r=-.36,95 \% \mathrm{CI},[-.39,-.32] \\
\text { Kendall's tau: } p=.14 \\
\text { Egger's intercept: } p=.19 \\
\text { Orwin's Fail-safe N: } 6\end{array}$ \\
\hline
\end{tabular}

${ }^{19}$ Due to low power, the publication bias tests, for meta-analyses with fewer than five studies, should be interpreted with extreme caution (Sterne et al., 2000). 
Positive perfectionism. As seen in Table 4, positive perfectionism was significantly and positively (but weakly) associated with depression ${ }^{20}$ (.07), ED symptoms (.13), and OCD symptoms (.18). With regard to adaptive outcomes, positive perfectionism was significantly and positively associated with life satisfaction (.19). Positive perfectionism was not significantly associated with anxiety, negative coping skills, psychological distress, stress, or self-esteem. This suggests that the relationship between positive perfectionism and adaptive and maladaptive outcomes is largely dependent on the specific outcome being measured. As with the negative perfectionism, most (all but OCD) meta-analyses had significant $Q$-statistics which suggested significant heterogeneity between the effect sizes in each analysis. The $\mathrm{I}^{2}$ statistics ranged from 0 (OCD symptoms) to 92.91 (self-esteem), indicating that up to $93 \%$ of variance in effect size can be attributed to study and sample characteristics.

Moderator analyses were conducted, resulting in only the composition of positive perfectionism being identified as a significant moderator. It is important to note that, due to the relatively small sample sizes in the positive perfectionism meta-analyses, only the eating disorder meta-analysis met the criteria for all of the moderator analyses, and three metaanalyses (OCD symptoms, psychological distress, stress) did not meet the criteria for any moderator analyses (see supplementary online material for a summary of all moderator analyses conducted).

The composition of the scale used to measure perfectionism was a significant moderator in the anxiety $(Q(3)=8.72, p=.03)$, and self-esteem $(Q(1)=5, p=.03)$ metaanalyses. In some cases the specific measure of positive perfectionism was associated with differing relationships between positive perfectionism and the outcome variable. For example, positive perfectionism was only significantly associated with anxiety when PS ( $n=8 ; r=.11$; $95 \%$ CI, $[.00, .22])$ was used alone and non-significant when PPS, PPS + O, or PS + O were used. There was also a significant difference in the relationship between positive perfectionism and self-esteem depending on whether PS + O or PS was used, however in both of these cases the overall relationship remained non-significant.

Publication bias. Each meta-analysis was subject to multiple methods for assessing publication bias. About half of the meta-analyses described above, and presented in Table 4, showed some signs of publication bias. However, in most cases this was minimal or, due to the very small samples, had to be interpreted with extreme caution. In the meta-analysis of the

\footnotetext{
20 These results are in order from the meta-analysis with the largest sample size to the meta-analysis with the smallest sample size
} 
relationship between negative perfectionism and depression, anxiety, ED symptoms, OCD symptoms, and life satisfaction, the funnel plots appeared slightly asymmetric; however neither Egger's regression intercept, or Begg and Mazumdar's rank correlation suggested significant bias. Moreover, in all five meta-analyses Duval and Tweedie's trim and fill method resulted in an estimated effect size extremely close to the original summary effect (see Table 4). Tests of publication bias in the negative perfectionism-positive coping skills meta-analysis also resulted in an asymmetric funnel plot, however both statistical methods were non-significant. The adjusted effect size was greater than the calculated effect which suggested that had there not been publication bias, negative perfectionism may have been more strongly associated with positive coping skills albeit still a relatively negligible association $(r=.08)$.

The meta-analyses of the relationships between positive perfectionism and ED symptoms, OCD symptoms, and life satisfaction showed some signs of bias (see Table 4). Although all funnel plots appeared asymmetric, statistical tests for publication bias were nonsignificant and the adjusted effect size estimates were also close to the original findings (see Table 4). Similarly, the meta-analyses of the relationships between positive perfectionism, depression $^{21}$, stress, and psychological distress produced asymmetric funnel plots but statistically did not appear to be biased (with the exception of a significant Egger's regression intercept, $p=.04$, for the stress meta-analysis). Duval and Tweedie's trim and fill method resulted in adjusted estimates that were greater than the unadjusted results. Specifically, the summary effect of the relationship between positive perfectionism and stress was originally .09 but had an adjusted estimate of .15 (95\% CI, [-.03, .31]). In the same way, the relationship between positive perfectionism and psychological distress was originally .07 and had an adjusted estimate of $.13(95 \%$ CI, $[-.06, .31])$. However, there were only four samples in both the stress and the psychological distress meta-analyses, therefore the interpretation of these statistics is severely limited.

Finally, the funnel plot pertaining to the meta-analysis of the relationship between negative perfectionism and negative coping skills suggested publication bias whereby there were a disproportionate number of studies reporting larger effect sizes. Specifically, both Egger's regression intercept $(p=.01)$ and Begg and Mazumdar's rank correlation $(p=.03)$ were significant. However, Duval and Tweedie's Trim and Fill method did not result in any inputted studies to balance the distribution of effect sizes within the funnel plot, nor did the

${ }^{21}$ See p 65-67 for a summary of positive perfectionism - depression results 
Table 4

Summary of Positive Perfectionism Meta-Analyses Results

\begin{tabular}{llll}
\hline Meta-analysis & $\frac{\text { Summary effect }}{r=.07, p=.01}$ & Heterogeneity & Moderator analysis \\
$\begin{array}{l}\text { Positive perfectionism and } \\
\text { depression }(n=19)\end{array}$ & $95 \%$ CI, $[.02, .12]$ & $\mathrm{I}^{2}=72.62$
\end{tabular}

Publication bias

depression $(n=19)$

$95 \%$ CI, $[.02, .12] \quad \mathrm{I}^{2}=72.62$

Adjusted estimate: $r=.03,95 \%$ CI, $[.01, .05]$

Kendall's tau: $p=.89$

Egger's intercept: $p=.34$

Orwin's Fail-safe N: N/A

Classic Fail-safe N: 98

Positive perfectionism and

anxiety $(n=19)$

Positive perfectionism and

eating disorders $(n=14)$

Positive perfectionism and obsessive compulsive

disorder $(n=4)$

$r=.18, p<.001$

$Q(3)=0.36, p=.95$

$95 \%$ CI, $[.12, .23] \quad \mathrm{I}^{2}=0$

$95 \%$ CI, $[-.03, .12] \quad \mathrm{I}^{2}=82.3$

$\begin{array}{ll}r=.14, p<.001 & Q(13)=23.28, p=.04 \\ 95 \% \text { CI, }[.09, .20] & \mathrm{I}^{2}=44.15\end{array}$

Age: $Q(2)=6.37, p=$

.04; Specific ED outcome:

$Q(3)=11.67, p=.01$

Funnel plot: bias unlikely

Kendall's tau: $p=.92$

Egger's intercept: $p=.15$

Funnel plot: inconclusive

Adjusted estimate: $r=.13,95 \%$ CI, $[.07, .19]$

Kendall's tau: $p=.32$

Egger's intercept: $p=.52$

Classic Fail-safe N: 158

No significant moderators Funnel plot: inconclusive

Adjusted estimate: $r=.17,95 \%$ CI, $[.11, .23]$

Kendall's tau: $p=.50$

Egger's intercept: $p=.45$

Orwin's Fail-safe N: N/A

Classic Fail-safe N: 29

Positive perfectionism and

$r=.16, p=.01$

$Q(27)=8.08, p=.04$

positive coping $(n=4)$
$95 \%$ CI, $[.05, .27] \quad \mathrm{I}^{2}=62.89$
Specific outcome: $Q(3)=$ $8.08, p=.04$

Perfectionism composite:

$Q(2)=8.06, p=.02$
Funnel plot: bias unlikely Kendall's tau: $p=.73$

Egger's intercept: $p=.61$

Orwin's Fail-safe N: N/A

Classic Fail-safe N: 17 
Table 4

Summary of Positive Perfectionism Meta-Analyses Results

\begin{tabular}{|c|c|c|c|c|}
\hline $\begin{array}{l}\text { Meta-analysis } \\
\text { Positive perfectionism and } \\
\text { negative coping }(n=7)\end{array}$ & $\begin{array}{l}\text { Summary effect } \\
r=.03, p=.60 \\
95 \% \text { CI, }[.09, .15]\end{array}$ & $\begin{array}{l}\text { Heterogeneity } \\
Q(6)=26.00, p<.001 \\
\mathrm{I}^{2}=76.93\end{array}$ & $\begin{array}{l}\text { Moderator analysis } \\
\text { No significant moderators }\end{array}$ & $\begin{array}{l}\text { Publication bias } \\
\text { Funnel plot: inconclusive } \\
\text { Adjusted estimate: } r=-.02,95 \% \mathrm{CI},[-.07, \\
.03] \\
\text { Kendall's tau: } p=.04 \\
\text { Egger's intercept: } p=.08\end{array}$ \\
\hline $\begin{array}{l}\text { Positive perfectionism and } \\
\text { psychological distress }(n=4)\end{array}$ & $\begin{array}{l}r=.07, p=.45 \\
95 \% \text { CI, }[-.10, .23]\end{array}$ & $\begin{array}{l}Q(3)=19.78, p<.001 \\
\mathrm{I}^{2}=84.83\end{array}$ & $\begin{array}{l}\text { Specific outcome: } Q(3)= \\
19.78, p^{<.001}\end{array}$ & $\begin{array}{l}\text { Funnel plot: inconclusive } \\
\text { Adjusted estimate: } r=.13,95 \% \text { CI, }[-.06, .31] \\
\text { Kendall's tau: } p=1.00 \\
\text { Egger's intercept: } p=.75\end{array}$ \\
\hline $\begin{array}{l}\text { Positive perfectionism and } \\
\text { stress }(n=4)\end{array}$ & $\begin{array}{l}r=.09, p=.36 \\
95 \% \text { CI, }[-.10, .26]\end{array}$ & $\begin{array}{l}Q(3)=22.26, p<.001 \\
\mathrm{I}^{2}=86.52\end{array}$ & $\begin{array}{l}\text { Perfectionism composite: } \\
Q(2)=22.14, p<.001\end{array}$ & $\begin{array}{l}\text { Funnel plot: inconclusive } \\
\text { Adjusted estimate: } r=.15,95 \% \text { CI, [-.03, .31] } \\
\text { Kendall's tau: } p=.50 \\
\text { Egger's intercept: } p=.04\end{array}$ \\
\hline $\begin{array}{l}\text { Positive perfectionism and } \\
\text { self-esteem }(n=6)\end{array}$ & $\begin{array}{l}r=.07, \mathrm{p}=.34 \\
95 \% \mathrm{CI},[-.08, .22]\end{array}$ & $\begin{array}{l}Q(5)=62.22, p<.001 \\
\mathrm{I}^{2}=91.96\end{array}$ & $\begin{array}{l}\text { Perfectionism composite: } \\
Q(2)=7.82, p=.020\end{array}$ & $\begin{array}{l}\text { Funnel plot: bias unlikely } \\
\text { Kendall's tau: } p=.26 \\
\text { Egger's intercept: } p=.23\end{array}$ \\
\hline $\begin{array}{l}\text { Positive perfectionism and } \\
\text { life satisfaction }(n=4)\end{array}$ & $\begin{array}{l}r=.19, p<.001 \\
95 \% \text { CI, }[.12, .26]\end{array}$ & $\begin{array}{l}Q(3)=4.84, p<.001 \\
\mathrm{I}^{2}=37.98\end{array}$ & No significant moderators & $\begin{array}{l}\text { Funnel plot: inconclusive } \\
\text { Adjusted estimate: } r=.16,95 \% \text { CI, }[.09, .23] \\
\text { Kendall's tau: } p=.37 \\
\text { Egger's intercept: } p=.22 \\
\text { Orwin's Fail-safe N: N/A } \\
\text { Classic Fail-safe N: } 41\end{array}$ \\
\hline
\end{tabular}


analyses result in an adjusted estimate. Overall, although there appears to be some evidence of publication bias in the meta-analysis with negative coping skills, it does not seem to greatly affect the minimal relationship with negative perfectionism.

\section{Perfectionism and Demographic Variables}

Four further meta-analyses were conducted to explore the relationship between perfectionism and age and sex (Table 5). Put simply, neither negative nor positive perfectionism were significantly associated with age or sex. Despite significant heterogeneity within the sex metaanalyses analyses did not reveal any significant moderators ${ }^{22}$. There was little indication of publication bias in the meta-analyses between perfectionism and age and sex (see supplementary online material for relevant funnel plots), however it is important to interpret these tests very cautiously given the small number of samples included in the analyses.

\section{Negative Perfectionism and Maladaptive and Adaptive Outcomes}

A summary meta-analysis of all data pertaining to negative perfectionism and a broad range of maladaptive outcomes was conducted. This included both outcomes that had already been examined in previous meta-analyses, as well as those that addressed various other maladaptive outcomes. In total, 77 samples from 67 articles were included (presented in Figure 14). Overall, using a random effects model, the summary correlation coefficient was $.41(95 \%$ CI, $[.38, .43])$ and was significantly different to $0(p<.001)$. The effect sizes ranged from .17 (Soenens, Vansteenkiste, et al., 2008) to .69 (Clark \& Coker, 2009). Overall, these results suggest that negative perfectionism is associated with increased risk of poorer mental health and wellbeing. Heterogeneity analysis illustrated that there was significant variance between the effect sizes $(Q(76)=391.00, p<.001)$ and that study and sample characteristics could account for up to $81 \%$ of the variance $\left(\mathrm{I}^{2}=80.56\right.$; see Table 6 for a summary of results. Moderator analyses did not indicate any significant differences across most moderators tested (sex, clinical/non-clinical samples, and specific negative perfectionism composition).

However, the age of the sample significantly moderated the overall relationship $(Q(2)=6.52$, $p=.04)$. Specifically, the relationship between negative perfectionism and negative outcomes was stronger in adult $(n=21 ; r=.42 ; 95 \% \mathrm{CI},[.37, .46])$ and university samples $(n=42 ; r=$ $.42 ; 95 \% \mathrm{CI},[.38, .46])$ than it was in adolescent samples ( $n=13 ; r=.35 ; 95 \% \mathrm{CI},[.30, .39])$. Finally, investigation of publication bias indicated no significant bias (see Table 6 and Figure

\footnotetext{
22 Due to at least two moderator categories, each with at least two studies, being required for moderator analyses, across all demographic meta-analyses only two moderator analyses were conducted. See the supplementary online material for all moderator analyses
} 
Table 5

Summary of Positive Perfectionism and Negative Perfectionism with Demographic Variables Meta-Analytic Results

\begin{tabular}{|c|c|c|c|c|}
\hline Meta-analysis & $\underline{\text { Summary effect }}$ & Heterogeneity & Moderator analysis & Publication bias \\
\hline $\begin{array}{l}\text { Negative perfectionism and } \\
\text { age }(n=4)\end{array}$ & $\begin{array}{l}r=.05, p=.32 \\
95 \% \text { CI, }[-.04, .13]\end{array}$ & $\begin{array}{l}Q(4)=6.27, p=.18 \\
\mathrm{I}^{2}=36.15\end{array}$ & $\begin{array}{l}\text { Clinical/Non-clinical: } Q(1)= \\
4.40, p=.036\end{array}$ & $\begin{array}{l}\text { Funnel plot: bias unlikely } \\
\text { Kendall's tau: } p=.81 \\
\text { Egger's intercept: } p=.49\end{array}$ \\
\hline $\begin{array}{l}\text { Negative perfectionism and } \\
\operatorname{sex}^{23}(n=4)\end{array}$ & $\begin{array}{l}r=.01, p=.86 \\
95 \% \mathrm{CI},[-.13, .15]\end{array}$ & $\begin{array}{l}Q(3)=16.04, p=.01 \\
\mathrm{I}^{2}=81.29\end{array}$ & No significant moderators & $\begin{array}{l}\text { Funnel plot: bias unlikely } \\
\text { Kendall's tau: } p=.73 \\
\text { Egger's intercept: } p=.81\end{array}$ \\
\hline $\begin{array}{l}\text { Positive perfectionism and } \\
\text { age }(n=4)\end{array}$ & $\begin{array}{l}r=.05, p=.10 \\
95 \% \text { CI, }[-.01, .12]\end{array}$ & $\begin{array}{l}Q(3)=1.78, p=.62^{24} \\
\mathrm{I}^{2}=0\end{array}$ & No significant moderators & $\begin{array}{l}\text { Funnel plot: bias unlikely } \\
\text { Kendall's tau: } p=.73 \\
\text { Egger's intercept: } p=.78\end{array}$ \\
\hline $\begin{array}{l}\text { Positive perfectionism and } \\
\operatorname{sex}(n=5)\end{array}$ & $\begin{array}{l}r=-.01, p=.89 \\
95 \% \mathrm{CI},[-.17, .15]\end{array}$ & $\begin{array}{l}Q(4)=41.44, p<.001 \\
\mathrm{I}^{2}=90.35\end{array}$ & $\begin{array}{l}\text { Perfectionism composite: } Q(3)= \\
41.35, p<.001\end{array}$ & $\begin{array}{l}\text { Funnel plot: bias unlikely } \\
\text { Kendall's tau: } p=.46 \\
\text { Egger's intercept: } p=.74\end{array}$ \\
\hline
\end{tabular}

${ }^{23}$ Relationship with being male

${ }^{24}$ In this case, a non-significant Q-statistic is likely due to a lack of power (given the small sample size) or large within study error (Borenstein et al., 2009). Therefore, it is not assumed that the non-significant Q-statistic indicates no variance between the individual study effect sizes and moderator analyses were subsequently carried out. 
16). Subsequently, the relationship between negative perfectionism and adaptive or positive outcomes was explored. Thirty samples from 30 articles were included in the meta-analysis (presented in Figure 15 and summarised in the online supplementary material) resulting in a significant negative summary effect ( $r=-.16,95 \%$ CI, [-.23, -.09]; also see Table 6). The individual effect sizes ranged from -0.50 (Soenens et al., 2005) to 0.30 (Harris, Pepper, \& Maack, 2008). In combination with the previous meta-analysis, this suggests that negative perfectionism is associated with not only greater maladaptive outcomes but also fewer adaptive outcomes. As with the meta-analysis above, significant heterogeneity was identified $(Q(29)=496.02, p<.001)$ and the $\mathrm{I}^{2}$ statistic suggested that a large $94 \%$ of the variance could be attributed to individual study characteristics $\left(\mathrm{I}^{2}=94.15\right)$.

Moderator analyses indicated that some of this variance is likely to be a result of the composition of the negative perfectionism scale used $(Q(3)=9.03, p=.03)$. The relationship between negative perfectionism and positive outcomes was only significant when either a combination of CM + DA + PS ( $n=2 ; r=-.28 ; 95 \% \mathrm{CI},[-.33,-.23])$, or a combination of CM $+\mathrm{DA}+\mathrm{PC}+\mathrm{PE}(n=11 ; r=-.20 ; 95 \% \mathrm{CI},[-.30,-.10])$ were used. Combinations of $\mathrm{CM}+$ $\mathrm{DA}(n=13 ; r=-.08 ; 95 \% \mathrm{CI},[-.21, .06], n s)$, and CM + DA + PC $(n=4 ; r=-.24 ; 95 \% \mathrm{CI}$, [$.46,-.01], n s)$ resulted in non-significant summary effects.

Tests for publication bias revealed some conflicting results. On one hand the funnel plot appeared asymmetric whereby there were more studies with effect sizes closer to 0 and disproportionately more studies with large samples, thus smaller error (see Figure 17). This was supported by Duval and Tweedie's trim and fill method inputting an additional three data points and calculating an adjusted estimate of -.20 (95\% CI, [-.27, -.13]). However, on the other hand, neither Kendall's tau ( $p=.34$ ) nor Egger's regression intercept ( $p=.37$ ) were significant. This makes it hard to come to a conclusion about whether bias is present. In cases like this, Borenstein and colleagues (2009) suggest taking an approach where the aim is not to decide whether or not bias is present, but rather to ascertain how much the potential bias would affect the summary correlation coefficient. In this meta-analysis the adjusted effect size (-.20) is not hugely different to original summary effect (-.16). Finally, Orwin's fail safe N indicated that an additional 28 studies of a null or negligible effect would be required to bring the summary effect to a negligible level (0.1). In addition, the classic fail safe $\mathrm{N}$ showed that an additional 2,788 studies with null effects would be required to bring the relationship to 0 . It seems rather unlikely that there would be another 28 sets of unpublished data and even more unlikely that there would be close to 2,800 additional studies. Therefore, this information allows confidence that the effect is unlikely to be solely an artefact of bias. 
Table 6

Summary of positive perfectionism and negative perfectionism with adaptive and maladaptive outcomes meta-analytic results

\begin{tabular}{llll}
\hline Meta-analysis & Summary effect & Heterogeneity & Moderator analysis \\
$\begin{array}{l}\text { Negative perfectionism and } \\
\text { maladaptive outcomes } \\
(n=77)\end{array}$ & $95 \%$ CI, $[.38, .43]$ & $Q(76)=391.00, p<.001$ & Age: $Q(2)=6.52, p=.04$ \\
$\mathrm{I}^{2}=80.56$ &
\end{tabular}

$(n=77)$

Negative perfectionism and positive outcomes $(n=30)$

Positive perfectionism and maladaptive outcomes $(n=46)$

Positive perfectionism and positive outcomes $(n=21)$

Negative perfectionism and positive perfectionism $(n=$ 42)

$$
\begin{array}{lll}
r=-.16, p<.001 & Q(29)=496.02, p<.001 & \text { Perfectionism composite: } Q(3)=9.03, \\
\text { 95\% CI, }[-.23,- & \mathrm{I}^{2}=94.15 & p=.03
\end{array}
$$

$r=.07, p=.01$

$$
95 \% \text { CI, }[.03, .11] \quad \mathrm{I}^{2}=80.10
$$

Ket

$Q(45)=226.09, p<$. 
Study name

Andrews et al., 2014

Boone \& Soenens, 2015

Boone et al., 2010/Boone et al., 2011

Boone et al., 2012

Boone et al., 2013

Boone et al., 2014*

Brown \& Kocovski, 2014

Burton et al., 2013

Chang et al., 2004

Chang et al., 2004

Cheng, 2001

Claes et al., 2012

Clark \& Coker, 2009

Clark \& Coker, 2009

Clark \& Coker, 2009

Dakanalis et al., 2015

Dakanalis, Timko, Clerici et al., 2014

Dakanalis, Timko, Zanetti et al., 2014

Di Schiena et al., 2012

DiBartolo et al., 2007

DiBartolo et al., 2008

Dickie et al., 2012

Dickie et al., 2012

Elllickson-Larew et al., 2013

Harris et al., 2008

Immundsen et al., 2005

James et al., 2015

Jung, 2013

Kawamura \& Frost, 2004

Kawamura et al., 2001

Khawaja \& Armstrong, 2005

Laird, 2011

Lehmann \& Konstam, 2011

Levinson et al., 2013

Levinson et al., 2013

Levinson et al., 2015

Levinson et al., 2015

Lombardo et al., 2013

Luyckx et al., 2008

Lynd-Stevenson \& Hearne, 1999

Malkina-Pykh, 2012

Menatti et al., 2013

Moretz \& McKay, 2009

Norman et al., 1998

Santanello \& Gardener, 2007

Scott et al., 2014

Shih, 2011

Shumaker et al., 2009

Soenens et al., 2005

Soenens et al., 2005

Soenens et al., 2006

Soenens et al., 2006

Soenens et al., 2010

Soenens et al., 2010

Soenens et al., 2010

Soenens, Luyckx et al., 2008

Soenens, Vansteenkiste et al., 2008

Soenens, Vansteenkiste et al., 2008

Soysa \& Weiss, 2014

Steffen, 2014

Steffen, 2014

Stoeber \& Joormann, 2001

Stumpf \& Parker, 2000

Turner \& Turner, 2011

Valero et al., 2013

Vansteenkiste et al., 2010

Vartanian \& Grisham, 2012

Vartanian \& Grisham, 2012

Weiner \& Carton, 2012

Wielkiewicz \& Wonderlich, 2006

Williams \& Cropley, 2014

Wimberley \& Stasio, 2013

Wirtz et al., 2007

Wirtz et al., 2008

Wirtz et al., 2013

Wu \& Cortesi, 2009

Yoon \& Lau, 2008
Statistics for each study

Lower Upper

Correlation limit limit Z-Valuep-Value

$\begin{array}{lllll}0.42 & 0.32 & 0.51 & 7.72 & 0.00\end{array}$

$\begin{array}{lllll}0.45 & 0.19 & 0.65 & 3.23 & 0.001\end{array}$

$\begin{array}{lllll}0.30 & 0.23 & 0.37 & 7.64 & 0.000\end{array}$

$\begin{array}{lllll}0.36 & 0.18 & 0.52 & 3.72 & 0.000\end{array}$

$\begin{array}{lllll}0.50 & 0.32 & 0.64 & 5.06 & 0.000 \\ 0.32 & 0.23 & 0.40 & 7.16 & 0.000\end{array}$

$\begin{array}{lllll}0.32 & 0.23 & 0.40 & 7.16 & 0.000\end{array}$

$\begin{array}{lllll}0.39 & 0.21 & 0.54 & 4.03 & 0.000\end{array}$

$\begin{array}{lllll}0.29 & 0.17 & 0.41 & 4.41 & 0.000\end{array}$

$\begin{array}{lllll}0.32 & 0.17 & 0.46 & 4.00 & 0.000\end{array}$

$\begin{array}{lllll}0.45 & 0.31 & 0.57 & 5.84 & 0.000\end{array}$

$\begin{array}{lllll}0.27 & 0.11 & 0.42 & 3.28 & 0.001\end{array}$

$\begin{array}{lllll}0.16 & -0.05 & 0.35 & 1.50 & 0.133\end{array}$

$\begin{array}{lllll}0.69 & 0.57 & 0.77 & 8.71 & 0.000\end{array}$

$\begin{array}{lllll}0.66 & 0.48 & 0.78 & 5.95 & 0.000\end{array}$

$\begin{array}{lllll}0.52 & 0.28 & 0.69 & 3.92 & 0.000\end{array}$

$\begin{array}{lllll}0.50 & 0.44 & 0.56 & 14.11 & 0.000\end{array}$

$\begin{array}{lllll}0.40 & 0.33 & 0.46 & 10.37 & 0.000\end{array}$

$\begin{array}{lllll}0.55 & 0.48 & 0.61 & 12.37 & 0.000\end{array}$

$\begin{array}{lllll}0.22 & 0.07 & 0.37 & 2.82 & 0.005\end{array}$

$\begin{array}{lllll}0.50 & 0.41 & 0.57 & 10.04 & 0.000\end{array}$

$\begin{array}{lllll}0.45 & 0.35 & 0.54 & 7.93 & 0.000 \\ 0.46 & 0.32 & 0.58 & 5.78 & 0.000\end{array}$

$\begin{array}{lllll}0.46 & 0.32 & 0.58 & 5.78 & 0.000\end{array}$

$\begin{array}{llllll}0.50 & 0.21 & 0.71 & 3.20 & 0.001\end{array}$

$\begin{array}{lllll}0.28 & 0.17 & 0.38 & 4.90 & 0.000\end{array}$

$\begin{array}{lllll}0.50 & 0.33 & 0.63 & 5.25 & 0.000\end{array}$

$\begin{array}{lllll}0.29 & 0.24 & 0.33 & 12.21 & 0.000 \\ 0.60 & 0.53 & 0.66 & 13.36 & 0.000\end{array}$

$\begin{array}{lllll}0.60 & 0.53 & 0.66 & 13.36 & 0.000\end{array}$

$\begin{array}{lllll}0.40 & 0.33 & 0.46 & 10.94 & 0.000\end{array}$

$\begin{array}{lllll}0.54 & 0.41 & 0.65 & 7.19 & 0.000\end{array}$

$\begin{array}{lllll}0.40 & 0.28 & 0.51 & 6.03 & 0.000\end{array}$

$\begin{array}{rrrrr}0.59 & 0.50 & 0.66 & 11.03 & 0.000\end{array}$

$\begin{array}{lllll}0.36 & 0.24 & 0.47 & 5.53 & 0.000\end{array}$

$\begin{array}{lllll}0.52 & 0.46 & 0.59 & 12.78 & 0.000\end{array}$

$\begin{array}{lllll}0.28 & 0.16 & 0.40 & 4.46 & 0.000\end{array}$

$\begin{array}{lllll}0.32 & 0.17 & 0.45 & 4.10 & 0.000\end{array}$

$\begin{array}{lllll}0.30 & 0.16 & 0.42 & 4.05 & 0.000\end{array}$

$\begin{array}{lllll}0.29 & 0.13 & 0.44 & 3.42 & 0.001\end{array}$

$\begin{array}{lllll}0.32 & 0.25 & 0.38 & 9.34 & 0.000\end{array}$

$\begin{array}{lllll}0.43 & 0.31 & 0.54 & 6.32 & 0.000\end{array}$

$\begin{array}{lllll}0.55 & 0.42 & 0.66 & 7.29 & 0.000\end{array}$

$\begin{array}{lllll}0.37 & 0.19 & 0.53 & 3.92 & 0.000\end{array}$

$\begin{array}{lllll}0.34 & 0.20 & 0.47 & 4.55 & 0.000\end{array}$

$\begin{array}{lllll}0.46 & 0.34 & 0.56 & 6.71 & 0.000\end{array}$

$\begin{array}{lllll}0.39 & 0.23 & 0.53 & 4.57 & 0.000\end{array}$

$\begin{array}{lllll}0.31 & 0.14 & 0.46 & 3.49 & 0.000\end{array}$

$\begin{array}{lllll}0.49 & 0.38 & 0.58 & 8.17 & 0.000\end{array}$

$\begin{array}{lllll}0.29 & 0.20 & 0.37 & 6.44 & 0.000\end{array}$

$\begin{array}{lllll}0.33 & 0.16 & 0.47 & 3.79 & 0.000\end{array}$

$\begin{array}{lllll}0.53 & 0.45 & 0.60 & 10.77 & 0.000\end{array}$

$\begin{array}{lllll}0.37 & 0.27 & 0.46 & 7.11 & 0.000\end{array}$

$\begin{array}{lllll}0.33 & 0.25 & 0.41 & 7.43 & 0.000\end{array}$

$\begin{array}{lllll}0.37 & 0.29 & 0.44 & 9.00 & 0.000\end{array}$

$\begin{array}{lllll}0.37 & 0.27 & 0.46 & 6.95 & 0.000\end{array}$

$\begin{array}{lllll}0.39 & 0.29 & 0.48 & 7.35 & 0.000\end{array}$

$\begin{array}{llllll}0.37 & 0.27 & 0.46 & 6.94 & 0.000\end{array}$

$\begin{array}{lllll}0.44 & 0.38 & 0.50 & 12.26 & 0.000\end{array}$

$\begin{array}{lllll}0.17 & -0.09 & 0.40 & 1.26 & 0.208\end{array}$

$\begin{array}{llllll}0.45 & 0.26 & 0.61 & 4.40 & 0.000\end{array}$

$\begin{array}{lllll}0.26 & 0.13 & 0.39 & 3.86 & 0.000\end{array}$

$\begin{array}{lllll}0.56 & 0.50 & 0.62 & 14.73 & 0.000\end{array}$

$\begin{array}{lllll}0.57 & 0.51 & 0.63 & 14.23 & 0.000\end{array}$

$\begin{array}{rrrrr}0.47 & 0.35 & 0.58 & 6.84 & 0.000\end{array}$

$\begin{array}{lllll}0.29 & 0.19 & 0.39 & 5.42 & 0.000\end{array}$

$\begin{array}{lllll}0.36 & 0.27 & 0.44 & 7.50 & 0.000\end{array}$

$\begin{array}{lllll}0.40 & 0.29 & 0.51 & 6.45 & 0.000\end{array}$

$\begin{array}{lllll}0.27 & 0.13 & 0.39 & 3.72 & 0.000\end{array}$

$\begin{array}{lllll}0.45 & 0.31 & 0.57 & 5.71 & 0.000\end{array}$

$\begin{array}{lllll}0.37 & 0.24 & 0.49 & 5.16 & 0.000\end{array}$

$\begin{array}{lllll}0.38 & 0.24 & 0.50 & 5.14 & 0.000\end{array}$

$\begin{array}{lllll}0.05 & -0.06 & 0.15 & 0.87 & 0.385\end{array}$

$\begin{array}{lllll}0.50 & 0.42 & 0.57 & 10.54 & 0.000\end{array}$

$\begin{array}{rrrrr}0.59 & 0.45 & 0.70 & 7.09 & 0.000\end{array}$

$\begin{array}{lllll}0.35 & 0.08 & 0.58 & 2.54 & 0.011\end{array}$

$\begin{array}{lllll}0.31 & 0.05 & 0.53 & 2.33 & 0.020\end{array}$

$\begin{array}{llllll}0.12 & -0.18 & 0.41 & 0.78 & 0.437\end{array}$

$\begin{array}{lllll}0.39 & 0.30 & 0.46 & 8.66 & 0.000\end{array}$

$\begin{array}{lllll}0.57 & 0.45 & 0.67 & 7.58 & 0.000\end{array}$

$\begin{array}{lllll}0.40 & 0.38 & 0.43 & 26.05 & 0.000\end{array}$
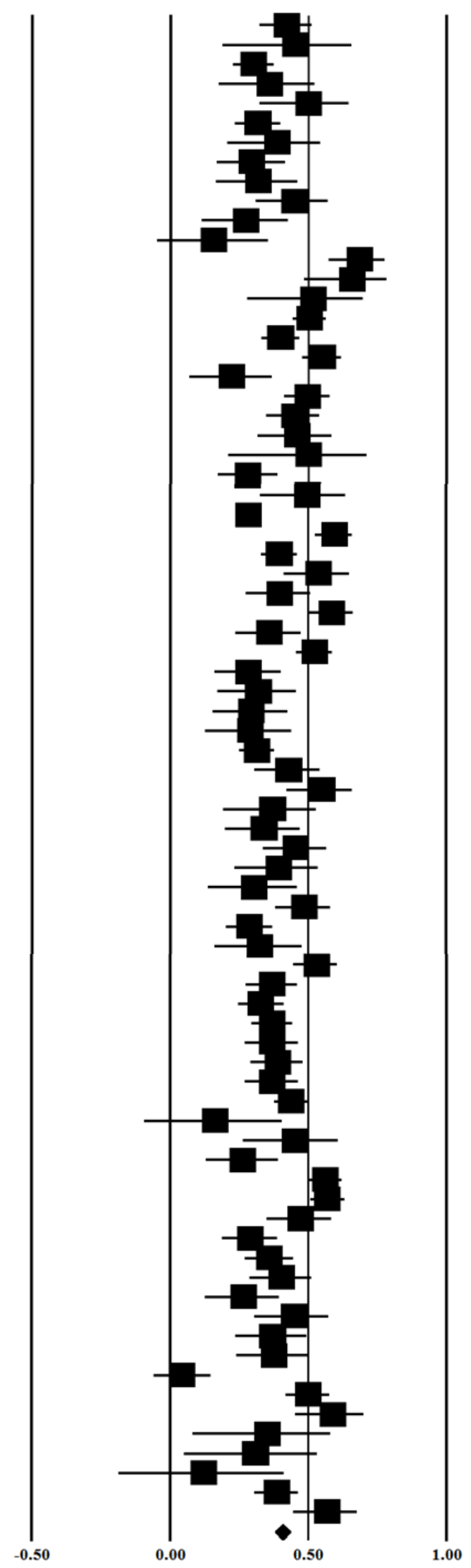

Figure 14. Forest plot with effect sizes for negative perfectionism and maladaptive outcomes 
$\underline{\text { Study name }}$

Burton et al., 2013

Chang et al., 2004

Chang et al., 2004

Dakanalis et al., 2015

Dakanalis, Timko, Clerici et al., 2014

DiBartolo et al., 2008

Harris et al., 2008

Immundsen et al., 2005

James et al., 2015

Jung, 2013

Kawamura \& Frost, 2004

Laird, 2011

Luyckx et al., 2008

Pulford \& Sohal, 2006

Shih, 2011

Shumaker et al., 2009

Soenens et al., 2005

Soenens et al., 2005

Steffen, 2014

Steffen, 2014

Stumpf \& Parker, 2000

Turner \& Turner, 2011

Vansteenkiste et al., 2010

Weiner \& Carton, 2012

Wielkiewicz \& Wonderlich, 2006

Wigert et al., 2012

Williams \& Cropley, 2014

Zeiger-Hill \& Terry, 2007
Boone et al., 2014*
Statistics for each study

Lower Upper
Correlation limit limit Z-Valuep-Value

$\begin{array}{lllll}-0.09 & -0.20 & 0.02 & -1.56 & 0.120\end{array}$

$\begin{array}{lllll}-0.37 & -0.44 & -0.30 & -9.22 & 0.000\end{array}$

$\begin{array}{lllll}0.02 & -0.12 & 0.15 & 0.23 & 0.820\end{array}$

$\begin{array}{llllll}-0.16 & -0.31 & 0.00 & -1.91 & 0.057\end{array}$

$\begin{array}{llllll}-0.37 & -0.50 & -0.22 & -4.66 & 0.000\end{array}$

$\begin{array}{lllll}-0.30 & -0.37 & -0.23 & -8.05 & 0.000\end{array}$

$\begin{array}{llllll}-0.12 & -0.24 & -0.00 & -2.00 & 0.045\end{array}$

$\begin{array}{lllll}0.30 & 0.11 & 0.47 & 2.98 & 0.003\end{array}$

$\begin{array}{lllll}-0.18 & -0.23 & -0.13 & -7.57 & 0.000\end{array}$

$\begin{array}{lllll}-0.14 & -0.24 & -0.04 & -2.74 & 0.006\end{array}$

$\begin{array}{lllll}-0.14 & -0.21 & -0.07 & -3.69 & 0.000\end{array}$

$\begin{array}{llllll}-0.29 & -0.43 & -0.13 & -3.50 & 0.000\end{array}$

$\begin{array}{lllll}-0.01 & -0.14 & 0.12 & -0.15 & 0.883\end{array}$

$\begin{array}{lllll}0.24 & 0.10 & 0.37 & 3.37 & 0.001\end{array}$

$\begin{array}{lllll}-0.21 & -0.39 & -0.02 & -2.14 & 0.033\end{array}$

$\begin{array}{rrrrr}0.12 & 0.03 & 0.20 & 2.57 & 0.010\end{array}$

$\begin{array}{lllll}-0.13 & -0.30 & 0.04 & -1.46 & 0.144\end{array}$

$\begin{array}{lllll}-0.50 & -0.58 & -0.42 & -10.02 & 0.000\end{array}$

$\begin{array}{llllll}-0.35 & -0.44 & -0.25 & -6.69 & 0.000\end{array}$

$\begin{array}{llllll}-0.44 & -0.51 & -0.37 & -10.94 & 0.000\end{array}$

$\begin{array}{lllll}-0.43 & -0.50 & -0.35 & -10.10 & 0.000\end{array}$

$\begin{array}{lllll}-0.49 & -0.54 & -0.44 & -15.10 & 0.000\end{array}$

$\begin{array}{lllll}0.04 & -0.06 & 0.14 & 0.80 & 0.426\end{array}$

$\begin{array}{lllll}-0.05 & -0.19 & 0.09 & -0.68 & 0.495\end{array}$

$\begin{array}{lllll}0.00 & -0.15 & 0.15 & 0.03 & 0.979\end{array}$

$\begin{array}{lllll}-0.04 & -0.14 & 0.06 & -0.72 & 0.470\end{array}$

$\begin{array}{lllll}-0.01 & -0.07 & 0.04 & -0.50 & 0.619\end{array}$

$\begin{array}{lllll}0.04 & -0.07 & 0.14 & 0.69 & 0.489\end{array}$

$\begin{array}{lllll}-0.20 & -0.28 & -0.12 & -4.80 & 0.000\end{array}$

$\begin{array}{lllll}-0.16 & -0.23 & -0.09 & -4.34 & 0.000\end{array}$ $\begin{array}{lllll}-0.26 & -0.33 & -0.18 & -6.53 & 0.000\end{array}$
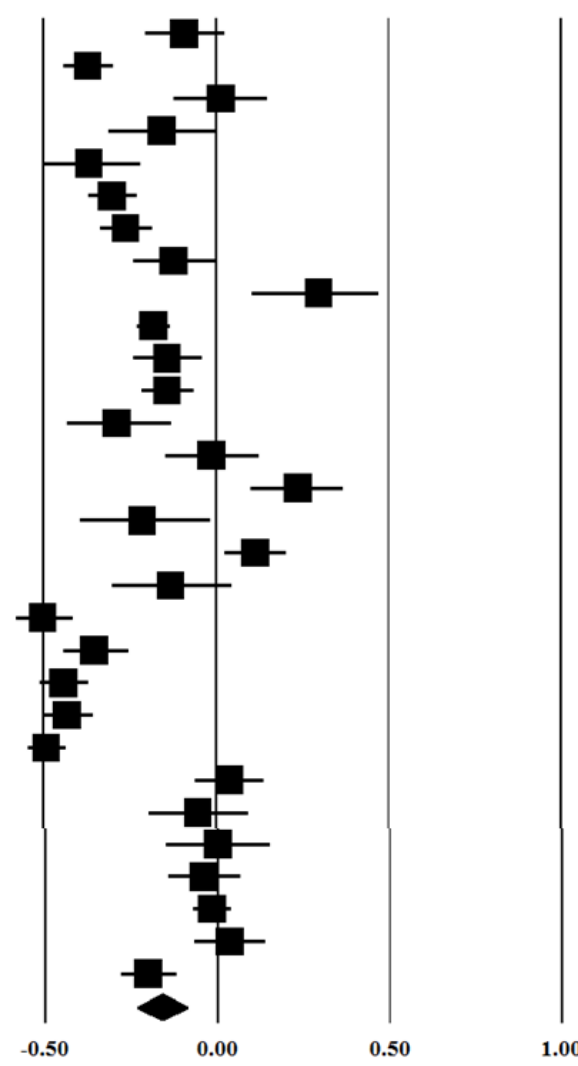

Figure 15. Forest plot with effect sizes for negative perfectionism and adaptive outcomes

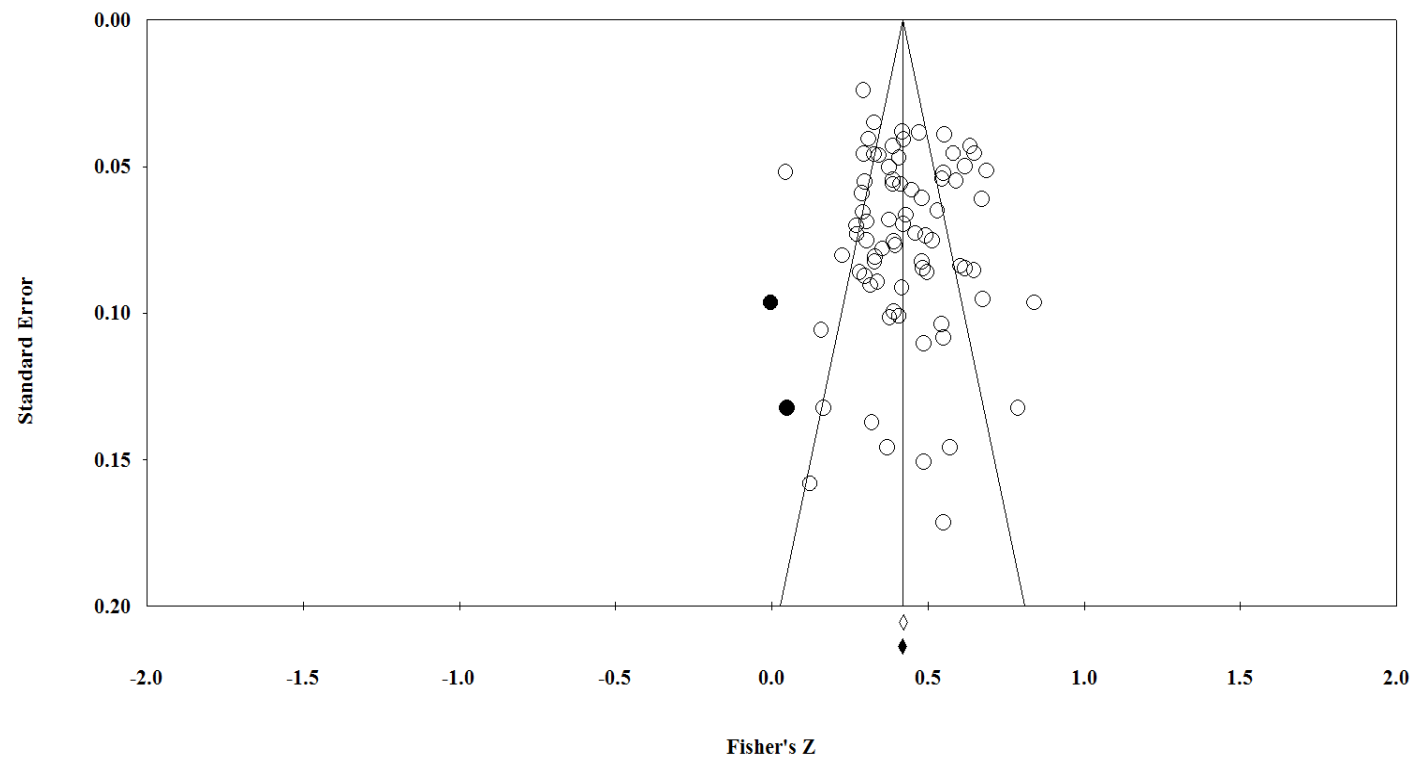

Figure 16. Funnel plot of the standard error by Fischer's $\mathrm{Z}$ for the association between negative perfectionism and maladaptive outcomes 


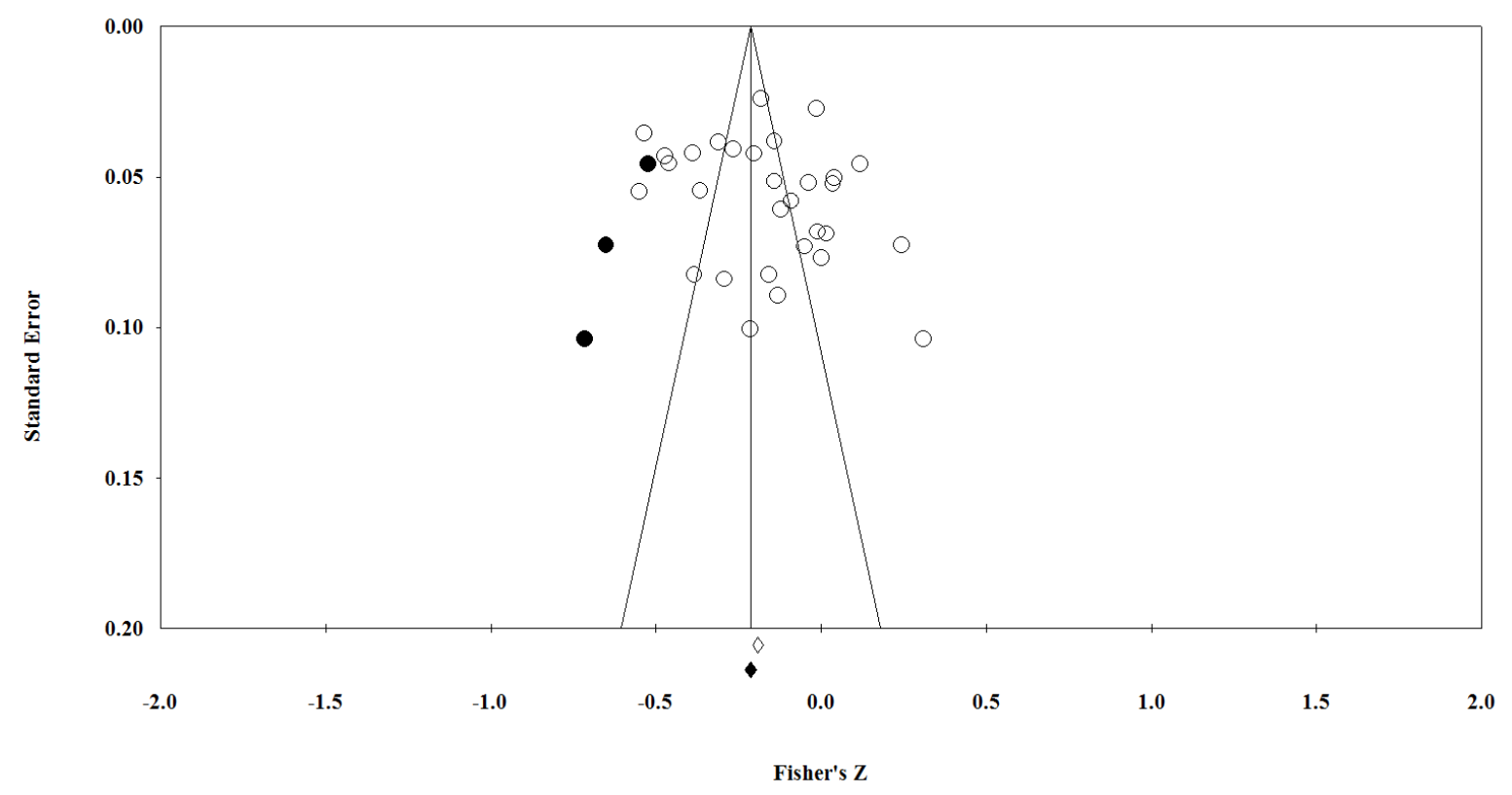

Figure 17. Funnel plot of the standard error by Fischer's $\mathrm{Z}$ for the association between negative perfectionism and adaptive outcomes

\section{Positive Perfectionism and Maladaptive and Adaptive Outcomes}

The relationship between positive perfectionism and adaptive and maladaptive outcomes was examined using the same process as above. The meta-analysis pertaining to positive perfectionism and maladaptive outcomes included 46 samples from 42 articles (Figure 18). Unexpectedly, positive perfectionism was significantly and positively associated with maladaptive outcomes (see Table $6 ; r=.07,95 \% \mathrm{CI},[.03, .11]$ ). Effect sizes ranged from -.22 (Andrews et al., 2014) to .34 (Boone et al., 2013). On face value this suggests that positive perfectionism is associated with increased risk for maladaptive outcomes, albeit a relatively small relationship. Tests for heterogeneity indicated significant variance between the effect sizes $(Q(46)=226.09, p<.001)$, of which approximately $80 \%$ could be attributed to study differences $\left(\mathrm{I}^{2}=80.01\right)$. Moderator analyses identified the composite of positive perfectionism as a significant moderator in the relationship $(Q(3)=20.68 p<.001)$ such that measures including Organisation were negatively, however non-significantly associated with maladaptive outcomes (PS + O: $n=9 ; r=-.06 ; 95 \%$ CI, [-.13, -.01], $n s ;$ PPS + O: $n=3 ; r=-$ $.05 ; 95 \% \mathrm{CI},[-.21,-.12], n s)$, whereas measures excluding $\mathrm{O}$ were positively associated with maladaptive outcomes (PS: $n=28 ; r=.12 ; 95 \%$ CI, $[.08, .16]$; PPS: $n=6 ; r=.09 ; 95 \%$ CI, $[.04, .14])$.

Next, the relationship between positive perfectionism and adaptive outcomes was investigated. This meta-analyses included data from 21 samples presented in 18 articles (Figure 19). Overall, positive perfectionism was related to greater adaptive outcomes (see 
Lower Upper
Correlation limit limit Z-Valuep-Value

Andrews et al., 2014

Boone \& Soenens, 2015

Boone et al., 2010/Boone et al., 2011

Boone et al., 2012

Boone et al., 2013

Boone et al., 2014*

Chang et al., 2004

Chang et al., 2004

Claes et al., 2012

Di Schiena et al., 2012

DiBartolo et al., 2007

DiBartolo et al., 2008

Dickie et al., 2012

Dickie et al., 2012

Harris et al., 2008

Immundsen et al., 2005

Kawamura \& Frost, 2004

Kawamura et al., 2001

Khawaja \& Armstrong, 2005

Lehmann \& Konstam, 2011

Levinson et al., 2013

Levinson et al., 2013

Levinson et al., 2015

Levinson et al., 2015

Lombardo et al., 2013

Luyckx et al., 2008

Malkina-Pykh, 2012

Menatti et al., 2013

Norman et al., 1998

Santanello \& Gardener, 2007

Shih, 2011

Shumaker et al., 2009

Soenens et al., 2005

Soenens et al., 2005

Soenens, Luyckx et al., 2008

Soenens, Vansteenkiste et al., 2008

Soenens, Vansteenkiste et al., 2008

Steffen, 2014

Steffen, 2014

Stoeber \& Joormann, 2001

Stumpf \& Parker, 2000

Vansteenkiste et al., 2010

Weiner \& Carton, 2012

Wielkiewicz \& Wonderlich, 2006

Wimberley \& Stasio, 2013

Wu \& Cortesi, 2009

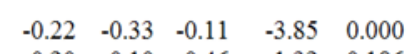

$\begin{array}{lllll}0.20 & -0.10 & 0.46 & 1.32 & 0.186\end{array}$

$\begin{array}{lllll}0.13 & 0.05 & 0.21 & 3.25 & 0.001\end{array}$

$\begin{array}{llllll}0.28 & 0.09 & 0.45 & 2.87 & 0.004\end{array}$

$\begin{array}{lllll}0.34 & 0.14 & 0.51 & 3.26 & 0.001\end{array}$

$\begin{array}{lllll}0.13 & 0.03 & 0.22 & 2.70 & 0.007\end{array}$

$\begin{array}{lllll}-0.10 & -0.26 & 0.06 & -1.26 & 0.207\end{array}$

$\begin{array}{lllll}-0.09 & -0.24 & 0.07 & -1.05 & 0.292\end{array}$

$\begin{array}{lllll}-0.03 & -0.24 & 0.17 & -0.33 & 0.744\end{array}$

$\begin{array}{lllll}0.05 & -0.10 & 0.21 & 0.67 & 0.505\end{array}$

$\begin{array}{lllll}0.14 & 0.03 & 0.24 & 2.56 & 0.011\end{array}$

$\begin{array}{lllll}0.15 & 0.04 & 0.27 & 2.57 & 0.010\end{array}$

$\begin{array}{lllll}0.17 & 0.00 & 0.33 & 1.99 & 0.046\end{array}$

$\begin{array}{lllll}0.32 & -0.00 & 0.58 & 1.93 & 0.053\end{array}$

$\begin{array}{lllll}0.17 & -0.03 & 0.36 & 1.66 & 0.098\end{array}$

$\begin{array}{lllll}0.22 & 0.17 & 0.26 & 9.30 & 0.000\end{array}$

$\begin{array}{lllll}0.10 & -0.07 & 0.25 & 1.14 & 0.256\end{array}$

$\begin{array}{lllll}0.18 & 0.05 & 0.31 & 2.67 & 0.008\end{array}$

$\begin{array}{lllll}0.01 & -0.08 & 0.09 & 0.11 & 0.912\end{array}$

$\begin{array}{lllll}0.02 & -0.11 & 0.15 & 0.31 & 0.760\end{array}$

$\begin{array}{rrrrr}-0.02 & -0.18 & 0.14 & -0.25 & 0.805\end{array}$

$\begin{array}{llllll}-0.05 & -0.20 & 0.10 & -0.67 & 0.504\end{array}$

$\begin{array}{lllll}0.10 & -0.07 & 0.27 & 1.15 & 0.251\end{array}$

$\begin{array}{lllll}0.06 & -0.01 & 0.13 & 1.79 & 0.074\end{array}$

$\begin{array}{lllll}0.12 & -0.02 & 0.26 & 1.66 & 0.097\end{array}$

$\begin{array}{lllll}0.28 & 0.10 & 0.45 & 2.92 & 0.003\end{array}$

$\begin{array}{lllll}0.02 & -0.13 & 0.17 & 0.23 & 0.820\end{array}$

$\begin{array}{lllll}0.15 & -0.03 & 0.32 & 1.68 & 0.093\end{array}$

$\begin{array}{lllll}0.11 & -0.06 & 0.28 & 1.25 & 0.212\end{array}$

$\begin{array}{lllll}-0.12 & -0.20 & -0.03 & -2.56 & 0.011\end{array}$

$\begin{array}{rrrrr}0.08 & -0.09 & 0.25 & 0.93 & 0.350\end{array}$

$\begin{array}{lllll}0.13 & 0.02 & 0.23 & 239 & 0.017\end{array}$

$\begin{array}{lllll}0.04 & -0.07 & 0.15 & 0.73 & 0.464\end{array}$

$\begin{array}{lllll}0.14 & 0.07 & 0.21 & 3.66 & 0.000\end{array}$

$\begin{array}{lllll}-0.02 & -0.28 & 0.23 & -0.18 & 0.860\end{array}$

$\begin{array}{lllll}0.12 & -0.10 & 0.32 & 1.06 & 0.287\end{array}$

$\begin{array}{lllll}-0.12 & -0.20 & -0.04 & -2.80 & 0.005\end{array}$

$\begin{array}{lllll}-0.15 & -0.24 & -0.06 & -3.32 & 0.001\end{array}$

$\begin{array}{lllll}0.13 & -0.01 & 0.27 & 1.77 & 0.076\end{array}$

$\begin{array}{lllll}-0.11 & -0.22 & -0.00 & -2.00 & 0.045\end{array}$

$\begin{array}{lllll}0.13 & -0.01 & 0.27 & 1.79 & 0.073\end{array}$

$\begin{array}{lllll}-0.20 & -0.34 & -0.05 & -2.57 & 0.010\end{array}$

$\begin{array}{lllll}-0.09 & -0.19 & 0.02 & -1.65 & 0.098\end{array}$

$\begin{array}{lllll}0.06 & -0.13 & 0.24 & 0.58 & 0.563\end{array}$

$\begin{array}{lllll}0.13 & 0.04 & 0.22 & 2.80 & 0.005\end{array}$

$\begin{array}{lllll}0.07 & 0.05 & 0.09 & 7.91 & 0.000\end{array}$ $\begin{array}{lllll}0.25 & 0.14 & 0.36 & 4.22 & 0.000\end{array}$

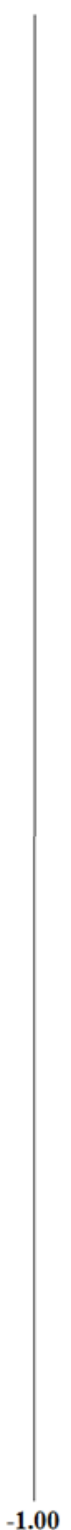

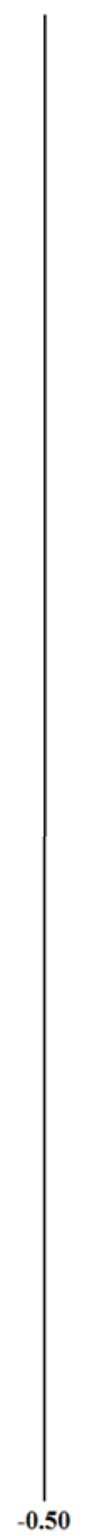

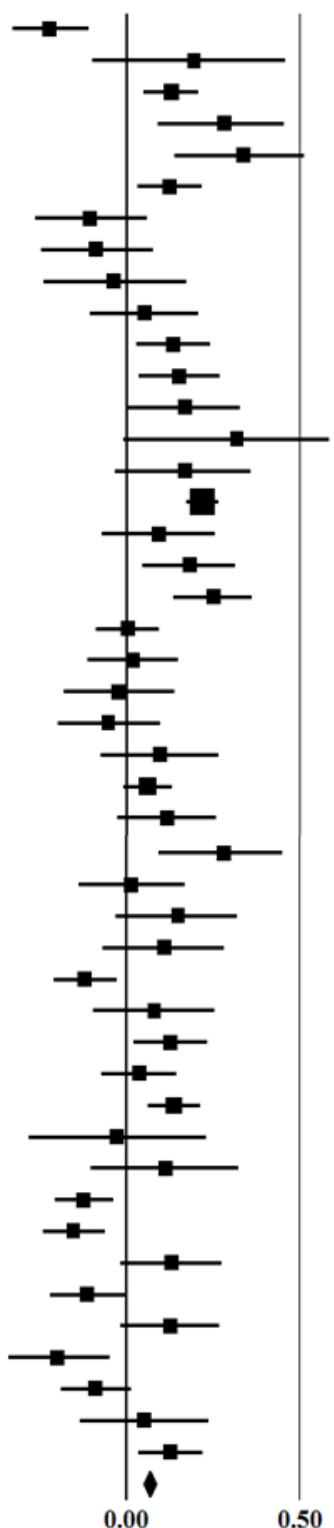

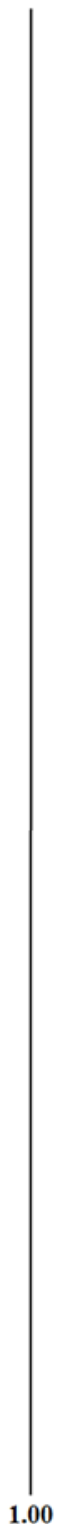

Figure 18. Forest plot with effect sizes for positive perfectionism and maladaptive outcomes

Table 6; $r=.17, p<.001,95 \%$ CI, $[.10, .24]$ ). Effect sizes ranged from -.11 (Soenens et al., 2005 ) to .42 (Andrews et al., 2014). Heterogeneity analyses highlighted significant

differences between the studies with approximately $92 \%$ of the variance attributable to study characteristics $\left(Q(20)=237.54, p<.001 ; \mathrm{I}^{2}=91.58\right)$. As above, the measure of positive perfectionism significantly moderated the relationship between positive perfectionism and adaptive outcomes $(Q(3)=10.90 p=.01)$. Studies using PS $+\mathrm{O}(n=9 ; r=.23 ; 95 \% \mathrm{CI}$, [.10, $.35])$, and PPS $+\mathrm{O}(n=2 ; r=.21 ; 95 \% \mathrm{CI},[.15, .27])$ both rendered weak to moderate associations with adaptive outcomes. However, the relationship between positive perfectionism and adaptive outcomes was non-significant when PPS $(n=2 ; r=.23 ; 95 \%$ CI, 
$[-.01, .45], n s)$, or PS $(n=8 ; r=.06 ; 95 \%$ CI, $[-.02, .42], n s)$ only were used. None of the other moderators tested were significant (sex, age, clinical/non-clinical samples).

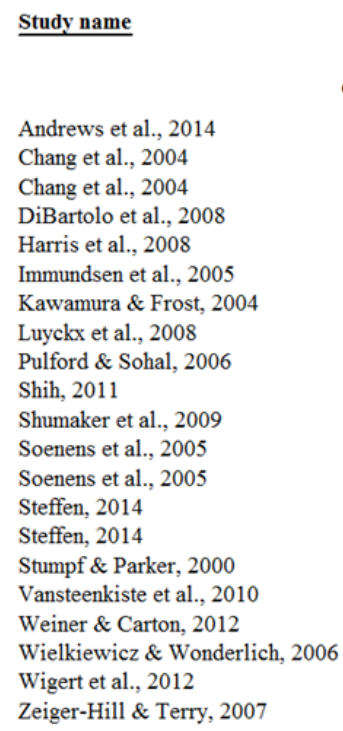

\begin{tabular}{|c|c|c|c|c|}
\hline \multicolumn{4}{|c|}{ Lower Upper } & \\
\hline & limit & & alu & Valne \\
\hline 0.42 & 0.32 & 0.51 & 7.72 & 0.000 \\
\hline 0.10 & -0.07 & 0.25 & 1.16 & 0.247 \\
\hline 0.27 & 0.12 & 0.41 & 3.37 & 0.001 \\
\hline 0.34 & 0.23 & 0.44 & 5.77 & 0.000 \\
\hline 0.09 & -0.11 & 0.29 & 0.87 & 0.384 \\
\hline 0.02 & -0.02 & 0.07 & 1.01 & 0.314 \\
\hline 0.11 & -0.06 & 0.27 & 1.28 & 0.201 \\
\hline 0.06 & -0.08 & 0.20 & 0.83 & 0.408 \\
\hline 0.02 & -0.18 & 0.21 & 0.16 & 0.870 \\
\hline 0.54 & 0.48 & 0.60 & 13.35 & 0.000 \\
\hline 0.10 & -0.07 & 0.2 & 1.12 & 0.262 \\
\hline-0.11 & -0.21 & -0.00 & -2.02 & 0.044 \\
\hline 0.03 & -0.08 & 0.14 & 0.55 & 0.583 \\
\hline 0.19 & 0.11 & 0.27 & 4.46 & 0.000 \\
\hline 0.24 & 0.15 & 0.32 & 5.37 & 0.000 \\
\hline 0.29 & 0.23 & 0.35 & 8.41 & 0.000 \\
\hline 0.11 & -0.03 & 0.25 & 1.49 & 0.136 \\
\hline 0.32 & 0.18 & 0.45 & 4.31 & 0.000 \\
\hline 0.09 & -0.02 & 0.19 & 1.64 & 0.100 \\
\hline 0.09 & 0.03 & 0.14 & 3.22 & 0.001 \\
\hline 0.09 & 0.01 & 0.17 & 2.14 & 0.033 \\
\hline 0.17 & 0.10 & 0.24 & 4.43 & 0.000 \\
\hline
\end{tabular}

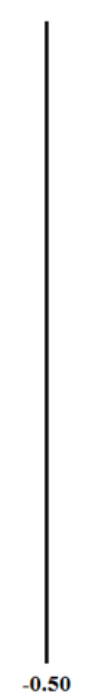

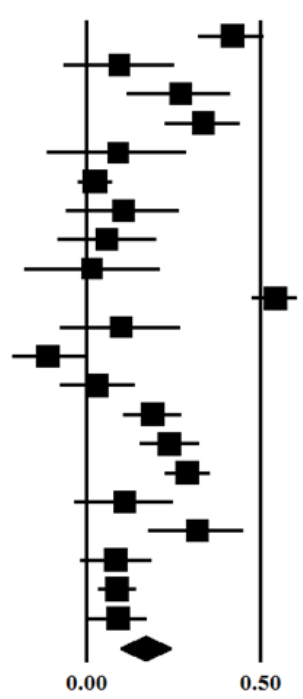

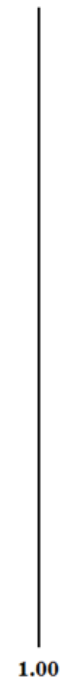

Figure 19. Forest plot with effect sizes for positive perfectionism and adaptive outcomes

Similar to that seen in the negative perfectionism-adaptive outcomes meta-analysis, both of the positive perfectionism meta-analyses produced asymmetric funnel plots that suggested publication bias (see Figures $21 \& 22$ ). However, statistical methods for assessing bias did not support the funnel plot findings in either relationship (see Eggers regression intercept and Kendall's tau statistics in Table 4). Therefore, instead of subjectively deciding whether or not there was evidence of bias, the potential impact on key findings was estimated. With regard to the positive perfectionism-maladaptive outcomes meta-analysis, Duval and Tweedie's trim and fill method resulted in an estimated adjusted effect size of .04 (original summary effect: $r=.07$ ) suggesting that, had all articles and data been sourced, positive perfectionism would be slightly less associated with maladaptive outcomes. However, this did not give significant additional weight to what could already be considered a relatively meaningless relationship. In addition, because the relationship was already considered negligible (criteria of 0.1) Orwin's fail safe N was not applicable. Instead, the Classic Failsafe $\mathrm{N}$ was calculated. As a result is was estimated that a further 515 studies would be required to bring the summary effect to 0 . This suggests that there is likely to be a relationship between positive perfectionism and negative outcomes, however it is at best negligible and more likely non-significant. 


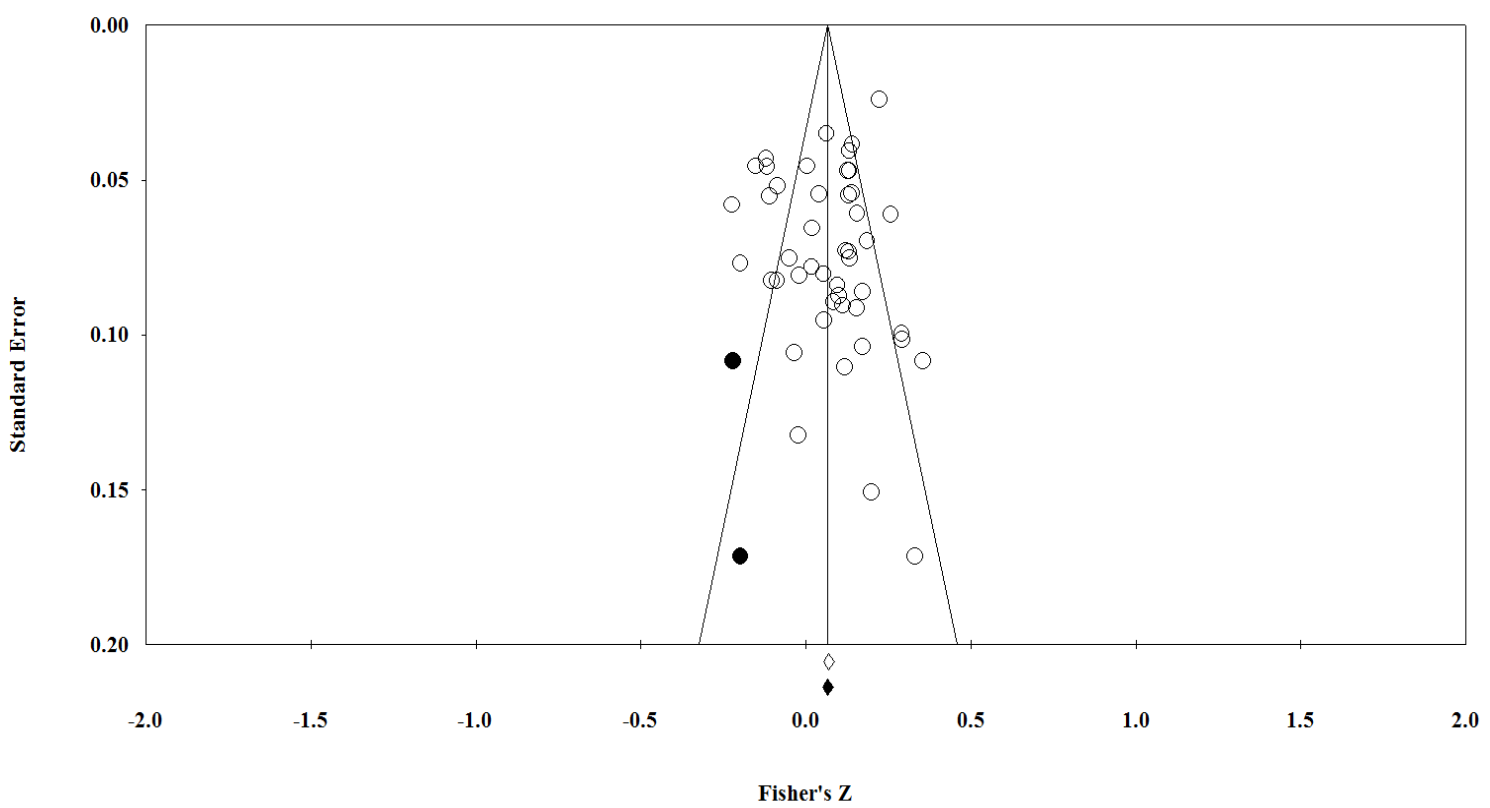

Figure 20. Funnel plot of the standard error by Fischer's $\mathrm{Z}$ for the association between positive perfectionism and maladaptive outcomes

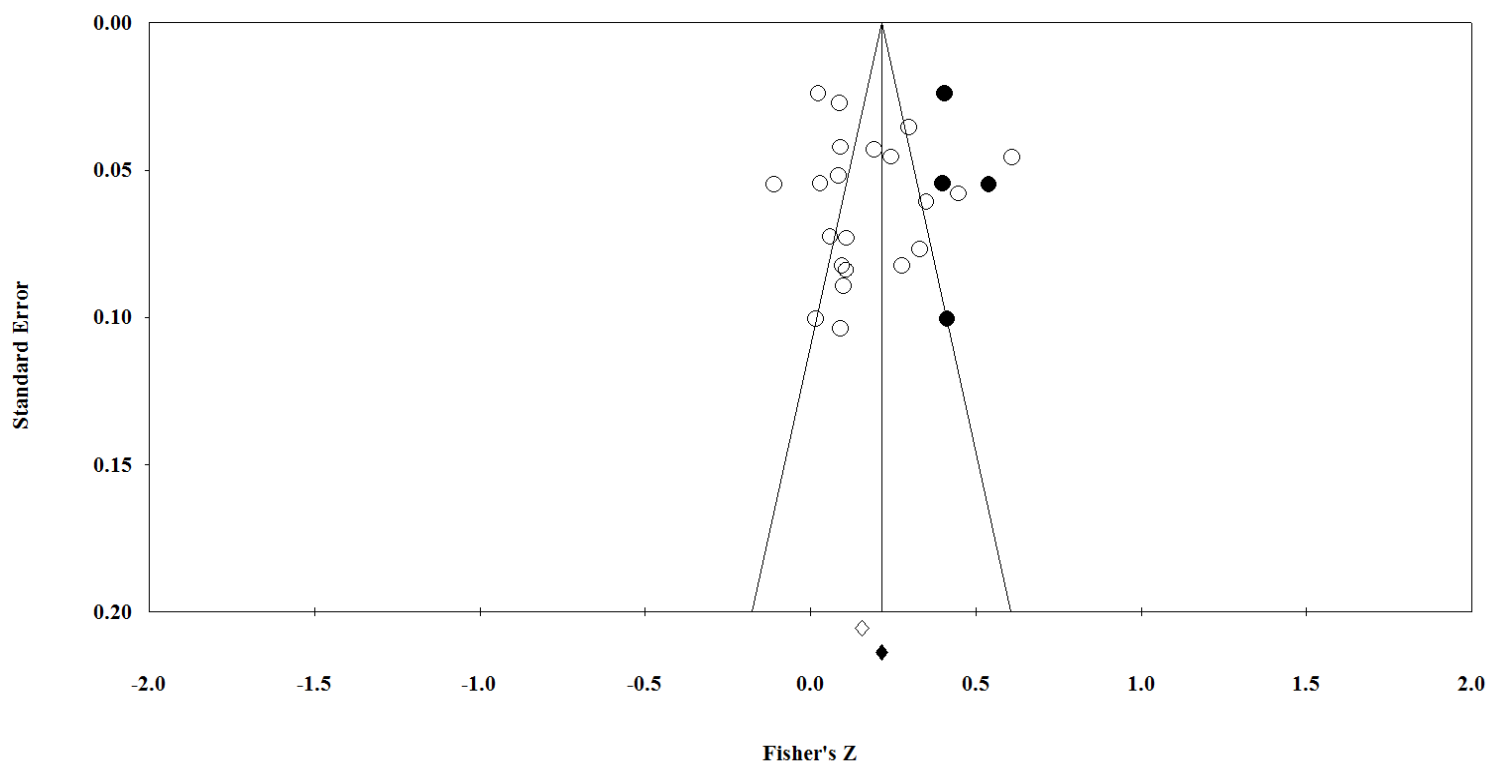

Figure 21. Funnel plot of the standard error by Fischer's Z for the association between positive perfectionism and adaptive outcomes

For the relationship between positive perfectionism and adaptive outcomes the adjusted effect size ( $r=.21)$ was only slightly greater than the calculated summary effect ( $r=$ .17; see Table 4). Therefore, with consideration of possible publication bias the positive relationship between positive perfectionism and adaptive outcomes would most likely remain positive and may even increase in strength. Further, Orwin's fail safe $\mathrm{N}$ estimated that a further 12 studies with negligible or null effect sizes would be required to reduce this relationship to less than .1. Overall, this suggests that the relationship between positive 
perfectionism and adaptive outcomes is more robust than the equivalent relationship with maladaptive outcomes.

\section{Negative and Positive Perfectionism}

Finally, the relationship between negative and positive perfectionism was explored.

Data was gathered from 42 samples within 37 different articles (presented in Figure 22). As expected, negative perfectionism was significantly and positively associated with positive perfectionism (see Table $6 ; r=.41,95 \% \mathrm{CI},[.36, .46]$ ).

$\underline{\text { Study name }}$

Boone et al., 2010/Boone et al., 2011

Boone et al., 2012

Boone et al., 2013

Boone et al., 2014*

Chang et al., 2004

Chang et al., 2004

Claes et al., 2012

Craddock et al., 2009

Di Schiena et al., 2012

DiBartolo et al., 2007

DiBartolo et al., 2008

Harris et al., 2008

Immundsen et al., 2005

Kawamura \& Frost, 2004

Khawaja \& Armstrong, 2005

Lehmann \& Konstam, 2011

Levinson et al., 2013

Levinson et al., 2013

Levinson et al., 2015

Levinson et al., 2015

Levinson et al., 2015

Luyckx et al., 2008

Luyckx et al., 2008

Malkina-Pykh, 2012

Menatti et al., 2013

Shih, 2011

Shumaker \& Rodebaugh, 2009

Soenens et al., 2005

Soenens et al., 2005

Soenens, Luyckx et al., 2008a

Soenens, Vansteenkiste et al., 2008b

Soenens, Vansteenkiste et al., 2008b

Steffen, 2014

Steffen, 2014

Vansteenkiste et al., 2010

Weiner \& Carton, 2012

Wielkiewicz \& Wonderlich, 2006

Wigert et al., 2012

Wimberley \& Stasio, 2013

Wu \& Cortesi, 2009

Ziegler-Hill \& Terry, 2007

\section{Statistics for each study}

Lower Upper

Correlation limit limit Z-Value p-Value

$\begin{array}{lllll}0.250 & 0.141 & 0.353 & 4.402 & 0.000\end{array}$

$\begin{array}{lllll}0.580 & 0.522 & 0.633 & 15.621 & 0.000\end{array}$

$\begin{array}{lllll}0.540 & 0.384 & 0.666 & 5.950 & 0.000\end{array}$

$\begin{array}{lllll}0.560 & 0.493 & 0.620 & 13.454 & 0.000\end{array}$

$\begin{array}{lllll}0.020 & -0.093 & 0.133 & 0.345 & 0.730\end{array}$

$\begin{array}{lllll}0.180 & 0.068 & 0.287 & 3.136 & 0.002\end{array}$

$\begin{array}{lllll}0.611 & 0.467 & 0.723 & 6.815 & 0.000\end{array}$

$\begin{array}{lllll}0.180 & 0.061 & 0.294 & 2.940 & 0.003\end{array}$

$\begin{array}{lllll}0.480 & 0.350 & 0.592 & 6.511 & 0.000\end{array}$

$\begin{array}{lllll}0.360 & 0.264 & 0.449 & 6.939 & 0.000\end{array}$

$\begin{array}{lllll}0.455 & 0.356 & 0.544 & 8.083 & 0.000\end{array}$

$\begin{array}{lllll}0.350 & 0.161 & 0.514 & 3.524 & 0.000\end{array}$

$\begin{array}{lllll}0.530 & 0.495 & 0.563 & 24.560 & 0.000\end{array}$

$\begin{array}{lllll}0.440 & 0.298 & 0.563 & 5.627 & 0.000\end{array}$

$\begin{array}{lllll}0.410 & 0.306 & 0.505 & 7.131 & 0.000\end{array}$

$\begin{array}{lllll}0.240 & 0.154 & 0.322 & 5.379 & 0.000\end{array}$

$\begin{array}{lllll}0.230 & 0.105 & 0.347 & 3.575 & 0.000\end{array}$

$\begin{array}{lllll}0.360 & 0.215 & 0.489 & 4.662 & 0.000\end{array}$

$\begin{array}{lllll}0.270 & 0.129 & 0.400 & 3.683 & 0.000\end{array}$

$\begin{array}{lllll}0.240 & 0.163 & 0.314 & 5.991 & 0.000\end{array}$

$\begin{array}{lllll}0.360 & 0.203 & 0.499 & 4.314 & 0.000\end{array}$

$\begin{array}{lllll}0.580 & 0.494 & 0.655 & 10.682 & 0.000\end{array}$

$\begin{array}{llllll}0.490 & 0.374 & 0.591 & 7.370 & 0.000\end{array}$

$\begin{array}{lllll}0.480 & 0.317 & 0.616 & 5.256 & 0.000\end{array}$

$\begin{array}{lllll}0.410 & 0.272 & 0.531 & 5.458 & 0.000\end{array}$

$\begin{array}{lllll}0.420 & 0.343 & 0.491 & 9.788 & 0.000\end{array}$

$\begin{array}{lllll}0.500 & 0.357 & 0.620 & 6.141 & 0.000\end{array}$

$\begin{array}{lllll}0.490 & 0.404 & 0.567 & 9.782 & 0.000\end{array}$

$\begin{array}{lllll}0.520 & 0.463 & 0.573 & 14.963 & 0.000\end{array}$

$\begin{array}{lllll}0.620 & 0.434 & 0.755 & 5.474 & 0.000\end{array}$

$\begin{array}{llllll}0.440 & 0.250 & 0.597 & 4.276 & 0.000\end{array}$

$\begin{array}{lllll}0.110 & 0.026 & 0.193 & 2.559 & 0.010\end{array}$

$\begin{array}{lllll}0.140 & 0.052 & 0.226 & 3.094 & 0.002\end{array}$

$\begin{array}{lllll}0.620 & 0.524 & 0.701 & 9.914 & 0.000\end{array}$

$\begin{array}{llllll}0.290 & 0.147 & 0.421 & 3.881 & 0.000\end{array}$

$\begin{array}{lllll}0.300 & 0.205 & 0.390 & 5.954 & 0.000\end{array}$

$\begin{array}{lllll}0.610 & 0.575 & 0.643 & 25.883 & 0.000\end{array}$

$\begin{array}{lllll}0.280 & 0.100 & 0.442 & 3.017 & 0.003\end{array}$

$\begin{array}{lllll}0.520 & 0.450 & 0.584 & 12.267 & 0.000\end{array}$

$\begin{array}{llllll}0.250 & 0.171 & 0.326 & 6.044 & 0.000\end{array}$

$\begin{array}{lllll}0.423 & 0.409 & 0.436 & 53.786 & 0.000\end{array}$ $\begin{array}{lllll}0.750 & 0.641 & 0.829 & 8.970 & 0.000\end{array}$

$\begin{array}{lllll}0.480 & 0.393 & 0.558 & 9.572 & 0.000\end{array}$
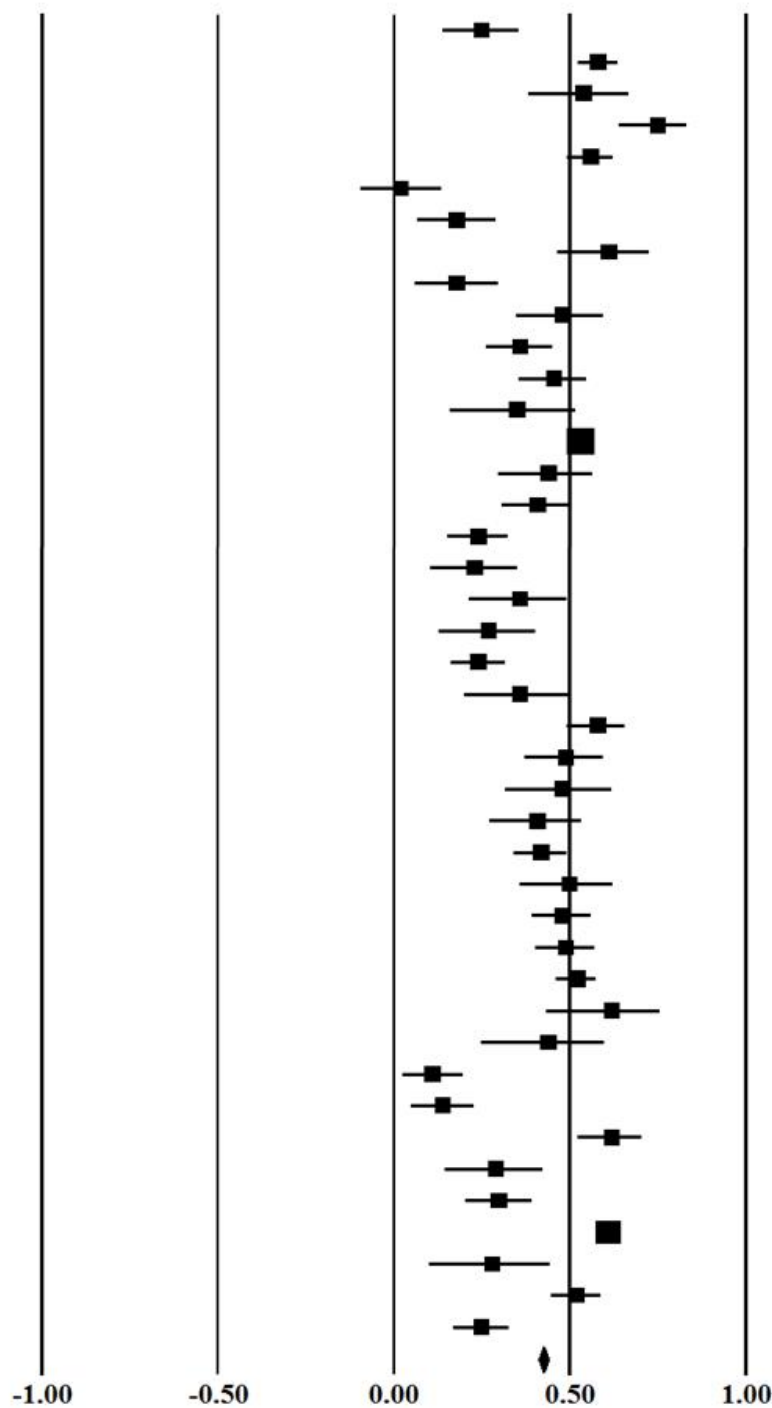

Figure 22. Forest plot with effect sizes for negative perfectionism and positive perfectionism

The individual study correlation coefficients ranged from .11 (Steffen, 2014) to .75

(Boone et al., 2013). This large range was supported by the $\mathrm{Q}$ statistic $(Q(41)=567.65, p<$ 
.001) suggesting significant heterogeneity across the studies. Further, the $\mathrm{I}^{2}$ statistic indicated that approximately $93 \%$ of this variance could be attributed to study characteristics $\left(\mathrm{I}^{2}=\right.$ 92.78) (see Figure 22 for the distribution of effect sizes).

Moderator analysis identified age $(Q(2)=19.08, p<.001)$, sample type $(Q(1)=9.78$, $p=.01)$, and specific positive perfectionism composite $(Q(3)=80.94, p<.001)$ and negative perfectionism composite $(Q(3)=33.86, p<.001)$ as significant moderators. Specifically, the relationship between negative and positive perfectionism was greatest in adolescent samples $(n=8 ; r=.53 ; 95 \%$ CI, $[.49, .57])$, followed by adult samples $(n=5 ; r=.48 ; 95 \%$ CI, [.29, $.64]$ ), and finally university samples ( $n=29 ; r=.36 ; 95 \% \mathrm{CI},[.29, .43])$. With regard to sample type, the relationship was stronger in clinical samples $(n=5 ; r=.60 ; 95 \%$ CI, [.49, $.69])$ than in non-clinical samples $(n=36 ; r=.39 ; 95 \% \mathrm{CI},[.33, .44])$. In addition, the relationship was strongest when positive perfectionism was measured using only PS ( $n=24 ; r$ $=.49 ; 95 \% \mathrm{CI},[.43, .53])$ or PPS $(n=6 ; r=.37 ; .95 \% \mathrm{CI},[.29, .44])$ and weaker when the scale included Organisation (PS + O, $n=9 ; r=.32 ; .95 \% \mathrm{CI},[.16, .46]$; PPS + O, $n=2 ; r=$ $.12 ; 95 \% \mathrm{CI},[.06, .18])$. With regard to the measure of negative perfectionism, the relationship with positive perfectionism was strongest when only $\mathrm{CM}+\mathrm{DA}$ were used $(n=$ $18 ; r=.50 ; 95 \%$ CI, $[.44, .56])$, followed by CM + DA + PS $(n=2 ; r=.49 ; 95 \%$ CI, [.43, $.54]), \mathrm{CM}+\mathrm{DA}+\mathrm{PE}+\mathrm{PC}(n=18 ; r=.36 ; 95 \% \mathrm{CI},[.27, .44])$, and finally weakest when a combination of CM + DA + PC was used $(n=3 ; r=.18 ; 95 \% \mathrm{CI},[.07, .29])$. The funnel plot

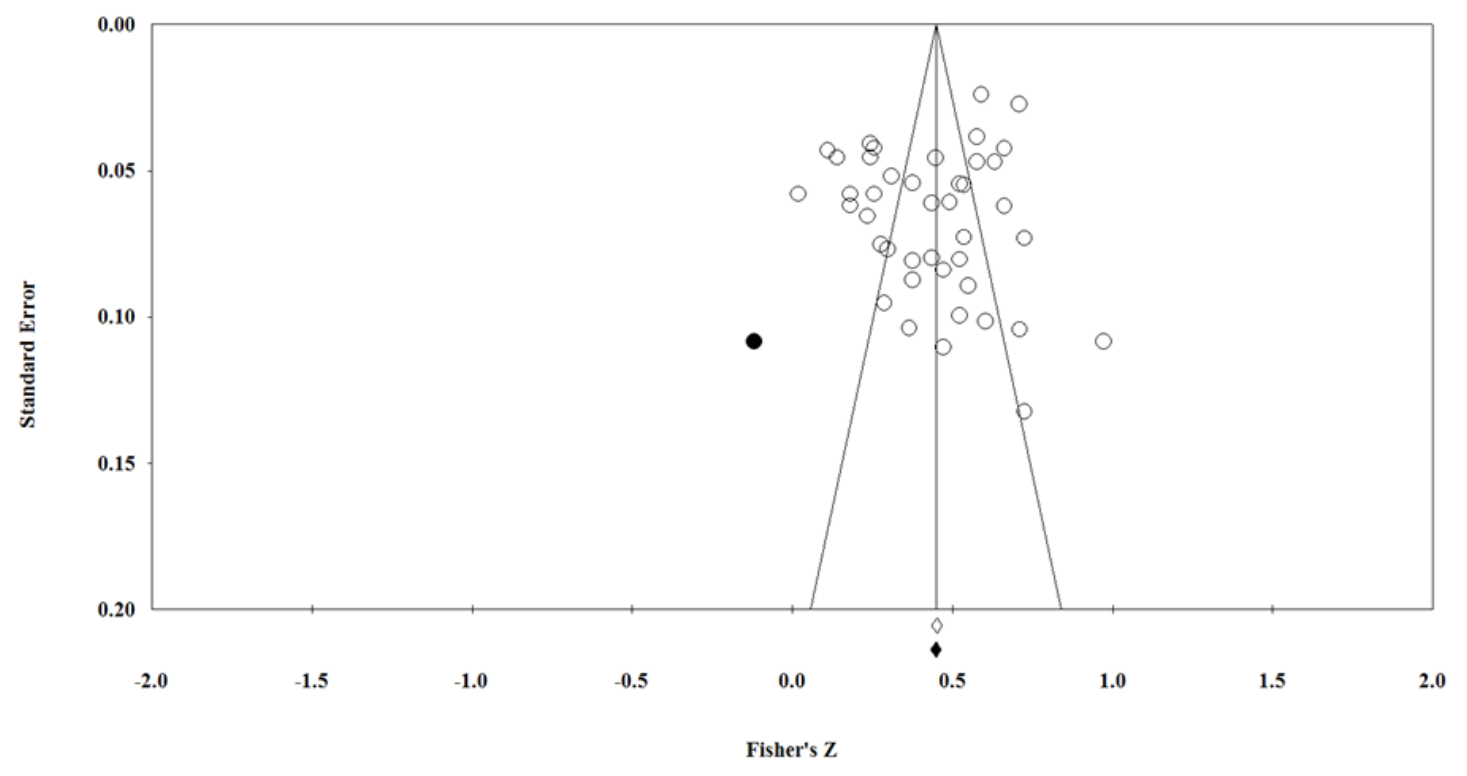

Figure 23. Funnel plot of the standard error by Fischer's Z for the association between negative perfectionism and positive perfectionism 
(see Figure 23), Duval and Tweedie's trim and fill, Egger's regression intercept and Begg and Mazumdar's rank correlation all resulted in no evidence of publication bias. Overall, this demonstrates that an individual scoring high on negative perfectionism is likely to also score high on positive perfectionism (and vice versa).

\section{Summary and Conclusions}

Study 1a examined the relationship between negative and positive perfectionism and adaptive and maladaptive outcomes. The meta-analytic results indicated that negative perfectionism is associated with greater depression, anxiety, ED symptoms, OCD symptoms, stress, psychological distress, and negative coping skills. On the other hand negative perfectionism was associated with lower self-esteem and less life satisfaction. This suggests that negative perfectionism is, on the whole, maladaptive.

Comparatively, the meta-analyses for positive perfectionism produced mixed results. Overall, positive perfectionism was positively associated with greater depression, ED symptoms, and OCD symptoms. With regard to adaptive outcomes, positive perfectionism was only significantly associated with life satisfaction. Therefore, without considering the specific outcome in question, it is difficult to conclusively categorise positive perfectionism as either adaptive or maladaptive.

In addition, whilst in almost all of the negative perfectionism meta-analyses the summary effect was significant (all except negative coping skills), only four out of the nine summary effects pertaining to positive perfectionism were significant. The summary effect for the relationship with depression (.07) was negligible (Cohen, 1988) and all other significant effects were small (below .20). In other words, this suggests that, at most, positive perfectionism contributes to $4 \%$ of the variance in predicting adaptive and maladaptive outcomes. It can then be argued that positive perfectionism has limited utility with regard to predicting overall psychological wellbeing.

Overall, these findings bring to question whether the label 'positive' is appropriate for positive perfectionism given the relationship, albeit small, with some poor mental health outcomes. In contrast, negative perfectionism appears to have a more consistent and greater impact on psychological wellbeing, therefore calling for active prevention and intervention strategies for individuals at risk for, or experiencing, negative perfectionism.

The meta-analyses of the relationship between perfectionism and demographic variables suggested that perfectionism does not increase with age, nor does it differ across sex. However, with regard to age, it is particularly important to note that this finding is based 
on cross-sectional data only. That is, in general older individuals are not more or less perfectionistic their younger counterparts. Unfortunately, this does not indicate whether an individual's perfectionism traits increase or decrease over time.

Finally, the relationship between positive and negative perfectionism was explored. This resulted in evidence for a strong relationship between positive and negative perfectionism. That is, an individual high on negative perfectionism is likely to also be high on positive perfectionism and vice-versa. When only considering the direct relationship between positive and negative perfectionism this seems rather simple; however it becomes somewhat complicated when considering the differential relationships between positive and negative perfectionism and adaptive and maladaptive outcomes. For example, although previous results demonstrated that positive perfectionism was positively associated with some negative outcomes, the statistical procedures used could not take into account the concurrent level of negative perfectionism in the sample involved. With this is mind, it is not beyond the realms of possibility that positive perfectionism appeared to be linked to maladaptive outcomes because the individuals high on positive perfectionism were also high on negative perfectionism and thus were simultaneously experiencing the maladaptive effects of negative perfectionism. This idea has been supported in a review by Stoeber and Otto (2006) who found that positive perfectionism was significantly more adaptive when the effects of negative perfectionism were controlled for.

Altogether, these findings could support the theoretical models of perfectionism present in Chapter 2; namely the two-component, tripartite and 2x2 models (see p. 30) which propose that positive and negative perfectionism interact to influence the likelihood of various adaptive and maladaptive outcomes. More specifically, these results support the leading argument of all three models, that is that negative perfectionism is inherently maladaptive. However, consistent with the conflicts between the three models, there continues to be confusion about the role of positive perfectionism. This again is reflected in the current study results where positive perfectionism can appear to have both a positive and negative influence on various aspects of an individual's wellbeing.

These meta-analyses are limited in a couple of ways. First, given that the function of this study was to set the scene for the main component of this thesis, rather than the primary focus of the thesis itself, these meta-analyses included only research that used the FMPS and differentiated between positive and negative perfectionism. The FMPS is the most widely used measure of perfectionism, and therefore it made logical sense to begin with such research. However, had the nature of positive and negative perfectionism been the sole focus 
of this thesis, a set of meta-analyses that included research using HFMPS and even better, research that used the wide variety of two-dimensional perfectionism scales would have provided a more comprehensive overview of the perfectionism literature. Despite this limitation, with regard to negative perfectionism in particular, there is research that suggests a wider range of scales would not have significantly changed the results. For example, in their meta-analysis of the effectiveness of perfectionism interventions, Lloyd and colleagues (2015) found that there was little heterogeneity in results across research that used the most common FMPS measure of negative perfectionism (CM) and research that used the HFMPS equivalent (SPP). However, they did find significant heterogeneity between studies that used the FMPS (PS) to measure positive perfectionism and research that used the HFMPS equivalent (SOP).

Second, and again due to practical constraints, these meta-analyses are limited by the exclusion of dissertations, theses, unpublished data, and articles not written in English. Some authors were contacted for additional unpublished data, however this only included those who were already being contacted for details regarding their other published data. Despite generally not sourcing unpublished or dissertation data, publication bias analyses were conducted to estimate the likely effect that including unpublished data would have on the summary effects. For the majority of the meta-analyses, no or little signs of publication bias were found. This suggests that sourcing all of the unpublished data would not significantly change the outcomes. Similarly, in terms of the publication language, only articles written in English were included (resulting in the exclusion of approximately 220 from the initial search results). However, research conducted with cross-cultural samples has found only a few differences in perfectionism across diverse samples (e.g., Chang, Yu, \& Lin, 2014; DiBartolo \& Rendón, 2012; Essau, Leung, Conradt, Cheng, \& Wong, 2008; Lee \& Park, 2011). Therefore, had there been the resources to translate and include perfectionism research presented in other languages, it would be expected that the findings would be broadly similar to those reported in English publications.

Overall, this study provides robust evidence for the detrimental impact of negative perfectionism on an individual's psychological wellbeing. Comparatively, there is still some doubt regarding the effect of positive perfectionism on an individual. 


\section{CHAPTER FIVE: PERFECTIONISM, SELF-INJURY, AND SUICIDAL BEHAVIOURS}

Study 1a clarified the differing relationships between positive and negative perfectionism and various mental health related outcomes. However, unfortunately due to both the limited research conducted, and the relatively narrow inclusion criteria set in Study 1a, the meta-analyses conducted thus far shed little further light on the relationship between perfectionism and self-injury. In fact, only one of the included articles reported the relationship between perfectionism and self-injurious behaviour (Claes et al., 2012). Therefore understanding of the relationship between perfectionism and NSSI remains based on the narrative review outlined in Chapter 3. As a brief reminder, this narrative review demonstrated that negative perfectionism was most often positively associated with NSSI, suggesting that negative perfectionism could be one risk factor for self-injury (e.g., Claes et al., 2012, Hoff \& Muehlenkamp, 2009; O’Connor et al., 2010). However, there have been some inconsistent results. For example, Miskey and colleagues (2012) reported that key components of negative perfectionism were unrelated to frequency and duration of nonsuicidal cutting whilst Hoff and Muehlenkamp (2009) reported that their sample with a history of NSSI scored higher on CM than their sample with no history of NSSI. Contrary to both of these findings, Izadi (2014) reported no significant relationship between CM and frequency of NSSI, but reported significant relationships between NSSI and other components of negative perfectionism. These results make it difficult to objectively summarise and form a conclusion about the relationship between perfectionism and NSSI and as such further metaanalyses will be beneficial.

In the absence of substantial perfectionism-self-injury research under the criteria set it Study 1a, it becomes necessary to draw on a wider research base in order to fully understand the extant perfectionism-self-injury literature, and the nature and magnitude of the relationship between the two constructs. Expanding the relevant pool of literature will be done in two ways. First, all research that has investigated perfectionism and self-injury, regardless of the authors' conceptualisation of perfectionism, will be explored (i.e., no longer focusing the discussion on positive or negative perfectionism). Second, research on the relationship between perfectionism and suicidal thoughts and behaviours will also be examined.

There are a number of reasons for expanding the search to included research on suicidal thoughts and behaviours. As previously noted, there is substantial research indicating a strong association between self-injurious and suicidal behaviour (e.g., Asarnow et al., 2011; Prinstein et al., 2008; Wilkinson et al., 2011; also see p. 20-22). Moreover, the relationship 
between various risk factors and NSSI often closely mirrors the relationship between the same risk factor and suicidal thoughts and behaviours (e.g., Muehlenkamp \& Gutierrez, 2004; Wichstrøm, 2009). Therefore, research on perfectionism and suicide may indirectly provide information on what may be expected when investigating perfectionism and NSSI. In addition, self-injury and suicidal behaviours are often intertwined or indistinguishable in research that conflates both self-injurious and suicidal behaviours, or in research that measures self-injury without differentiating based on suicidal intent (e.g., O'Connor et al., 2010; O'Connor, Rasmussen, \& Hawton, 2009). Therefore, by broadening the outcome variable criteria, additional studies that may have an ambiguous definition of self-injury are more likely to be captured. Overall, whilst this thesis is focused on the relationship between perfectionism and self-injury, research investigating suicidal thoughts and behaviours will also be explored.

Consequently, the aim of this chapter is to expand understanding of the relationships between perfectionism, and self-injurious and suicidal thoughts and behaviours. Further metaanalyses with broadened inclusion criteria will be conducted, enabling quantification of the relationship. However, prior to embarking on further analyses, in order to provide additional context for the results to come, a brief review of the literature pertaining to perfectionism and suicide will be presented.

\section{Perfectionism and Suicide Literature Review}

In comparison to the small body of research investigating perfectionism and selfinjury, the research on perfectionism and suicide is well established. For one, O'Connor (2007) published a systematic review of all available perfectionism-suicide research published up until mid-2006. In total, O'Connor sourced 29 published papers from which he concluded that negative perfectionism is associated with increased suicidal thoughts and behaviours. Similar to the results of Study 1a, O'Connor was unable to make a conclusion about the contribution of positive perfectionism to the existence of suicidal thoughts and behaviours and instead suggested that the effect of positive perfectionism depends largely on other characteristics of the individual, current stressors, and the context in which they live.

Recent research has typically supported these findings. For example, Blankstein and colleagues (2007) investigated perfectionism and suicide ideation in a sample of university students. They subsequently concluded that negative perfectionism (measured using SPP, HFMPS) was consistently related to stronger suicidal ideation in both males and females. They proposed that highly perfectionistic individuals can feel a sense of hopelessness and 
pessimism about their future academic and interpersonal success and as a result experience a desire to escape, and thus greater suicide ideation. Also consistent with O'Connor's review, positive perfectionism was not associated with suicide ideation.

A similar relationship between perfectionism and suicide has also been demonstrated in adolescent samples. For example, Hewitt and colleagues (2014) investigated perfectionism (measured using SOP and SPP, CAPS), suicide ideation, and suicide potential (a composite score including anxious-impulsive depression, suicide ideation and acts, and family distress; Child and Adolescent Suicide Potential Index [CASPI]; Pfeffer, Jiang, \& Kakuma, 2000) in depressed adolescent inpatients. Whilst negative perfectionism was not significantly associated with suicidal ideation, it was moderately associated with suicide potential. Interestingly, this relationship remained significant even after controlling for depression, hopelessness and previous suicide attempts. This suggests that negative perfectionism in adolescents has a unique effect on suicide risk, over and above other factors more commonly associated with suicide risk. Again, there was no significant relationship between positive perfectionism and any measures of suicide ideation or suicide potential.

In addition to the empirical studies testing the relationship between perfectionism and suicide, prospective qualitative studies looking at the factors influencing an individual's progression to suicide have also been conducted. In one study, the families of a sample of twenty men who completed suicide were interviewed (Kiamanesh, Dyregrov, Haavind, \& Dieserud, 2014). The men had had no prior suicide attempts or previous involvement in mental health services. The researchers subsequently reported that perfectionism was a factor in just over a quarter of the deaths. More specifically, interviews with the six families of men who were identified as highly perfectionistic resulted in the identification of four perfectionism themes that were said to contribute to their suicide; striving for success, fear of failure, keeping up the facade, and rigidity. The authors highlighted that none of the men appeared to have had any mental illnesses commonly thought to precede suicide (e.g., depression), and suggested that this common belief of mental illness as a prerequisite for suicide needs to be challenged. As a result, Kiamanesh and colleagues proposed that further suicide research that considers factors such as perfectionism would likely highlight a previously unidentified group of at risk individuals.

Following substantial empirical and qualitative research, research on perfectionism and suicide has largely moved to exploring the mechanisms driving the relationship. For example, the Perfectionism Social Disconnection Model (PSDM; Figure 24) is proposed to explain how perfectionism influences suicidality (Hewitt, Flett, Sherry, \& Caelian, 2006). 
Specifically, Hewitt and colleagues argue that negative perfectionism can lead to a profound sense of interpersonal disconnection and hopelessness that can substantially increase their risk of suicide.

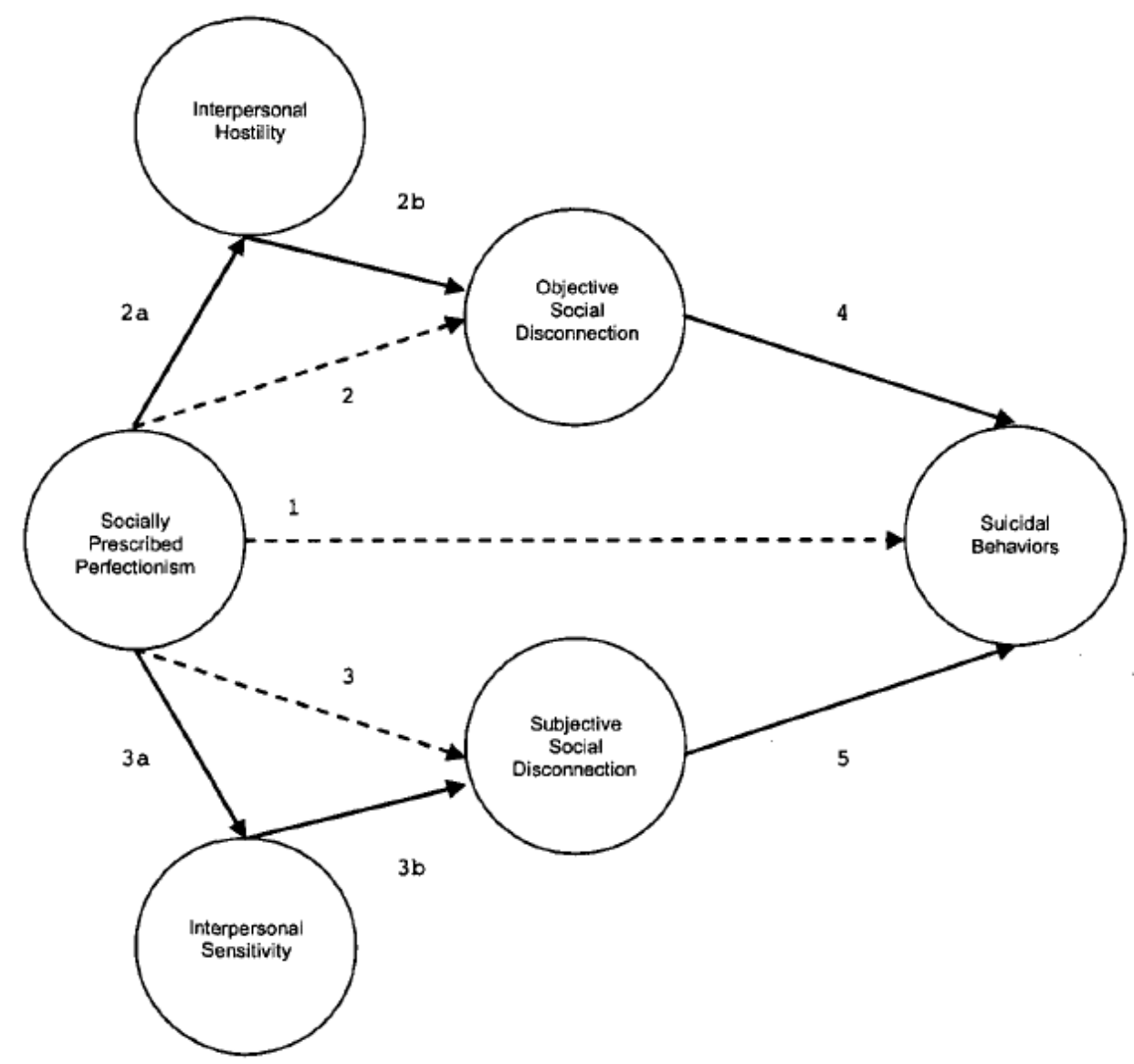

Figure 24. Perfectionism Social Disconnection Model (Hewitt et al., 2006, p. 227)

In more detail, the PSDM posits that negative perfectionism is associated with interpersonal hostility (e.g., resentment, suspicion, irritability) which creates objective disconnection such that the individual may experience relationship conflict, experience a lack of companionship and support from others, and have infrequent social contact. In addition, negative perfectionism is associated with interpersonal sensitivity (fragile inner self, timidity, heightened interpersonal awareness), putting the individual at increased risk for experiencing and interpreting the actions of others in a way that fosters subjective social disconnection. Subjective social disconnection may manifest as feelings of rejection, experiencing a lack of emotional intimacy from others and the perception of others as uncaring. Both objective and subjective disconnection can foster a sense of interpersonal hopelessness and significantly increase risk for suicidality (Hewitt et al., 2006).

The PSDM has been supported in a clinical sample of adolescents (Roxborough et al., 
2012). Roxborough and colleagues found that the relationship between perfectionism and suicide was mediated by social hopelessness and bullying. These factors, together, were taken as evidence for a relationship between perfectionism and social disconnection, which in turn increases suicide risk.

Another theory regarding suicide, Baumeister's (1990) Escape from Self theory, may also be particularly relevant to perfectionistic individuals. Baumeister proposed that suicide is often motivated by a strong desire or need to escape from high self-awareness and distressing emotions. Highly relevant to perfectionism, he proposed a step in the escape theory as "a severe experience where current outcomes (or circumstances) fall far below standards produced either by unrealistically high expectations or by recent problems or setbacks, or by both" (Baumeister, 1990, p. 91). As second and third steps, this experience results in persistent self-criticism, and hyper-awareness of ones perceived inadequacies brought about by comparisons between ideal standards and actual performance. Fourth, the individual experiences strong negative affect. Fifth, they attempt to reach a state of cognitive deconstruction to numb the negative emotions. And finally, if emotional relief is only partially successful, the individual progresses to the sixth step, the search of stronger methods of avoidance, one of which is complete escape through suicide.

Finally, with regard to the relationship between perfectionism and both suicide and self-injury, it is important to note that some researchers in the area appear to have intentionally, or sometimes seemingly unintentionally, conflated the two categories of behaviour. For example, Hunter and O’Connor (2003) investigated parasuicide and perfectionism in a hospital setting and found that negative perfectionism was more pronounced in adults who presented to a hospital emergency department with parasuicidal injuries, than in those receiving treatment for a physical injury, or in community controls.

They concluded that these results suggest an association between negative perfectionism and suicidal behaviours. However, Hunter and O'Connor defined parasuicide as an act of "deliberate self-harm irrespective of intention" (p. 356). As such, it appears that they conflated self-injurious and suicidal behaviours. Overall, this provides further justification for a very broad investigation of perfectionism and self-injury, which would aim to capture DSH research that may be under the guise of suicide research.

\section{Study 1b: Meta-Analysis of the Relationship between Positive and Negative Perfectionism and Self-Injurious and Suicidal Behaviours}

Compared to Study 1a, the focus of Study $1 \mathrm{~b}$ is relatively specific, and given the 
limited research on perfectionism, self-injury, and suicide, the initial search will be restricted in as few ways as practically possible. As explained above, although the primary focus is selfinjury, research on perfectionism and suicide will also be explored.

Based on Study 1a findings and existing perfectionism-self-injury/suicide research, it is expected that negative perfectionism will be positively associated with both self-injury and suicide. With regard to positive perfectionism, Study 1a highlighted that positive perfectionism may not be as adaptive as commonly argued and in some cases may even be associated with maladaptive outcomes. Therefore, investigation of the direction of the relationship between positive perfectionism and self-injurious and suicidal behaviours will be largely exploratory with no specific outcome expected. However, regardless of direction, the magnitude of effect between positive perfectionism and self-injury/suicide is expected to be smaller than the effect for the relationship between negative perfectionism and selfinjury/suicide. At a more specific level, previous research has identified differences in the manifestation of perfectionism and presentation of self-injury and suicide based on demographic factors such as age (e.g., Flett et al., 2002; Swannell et al., 2014), sex (e.g., Blankstein et al., 2008; Klibert et al., 2015; Whitlock et al., 2011), and the sample type (e.g., clinical/non-clinical; Briere \& Gil, 1998). Therefore, it is expected that some of these factors may moderate the relationships between negative and positive perfectionism, and selfinjurious and suicidal behaviours.

\section{Method}

Consistent with Study 1a, these meta-analyses were conducted in line with the PRISMA guidelines (Moher et al., 2009), and informed by the AMSTAR systematic review quality guide (Shea et al., 2009). Accordingly, a thorough search for relevant research was conducted followed by systematic examination of all articles with data relevant to the relationship between perfectionism, self-injury and suicide.

\section{Inclusion/Exclusion Strategy}

Initially, all research that looked at perfectionism, and self-injurious/suicidal thoughts or behaviours was considered for inclusion in these meta-analyses. Unlike Study 1a, the search was not limited to research using the FMPS, nor was it limited to only research that explicitly distinguished between positive and negative perfectionism. However, in order to test the research question, presentation of the data in such a way that it could be classified as tapping into either negative or positive perfectionism was required. The search strategy for the outcome variable was considerably narrower than in Study 1a, with the search limited to 
articles which addressed self-injurious and suicidal thoughts and behaviours.

\section{Information Sources and Search Strategy}

PsychINFO and Web of Science databases were searched in February, 2016. The search keywords were as follows; 'perfectionism' AND 'self-injury' OR 'self-injurious behaviour' OR ‘self-mutilation' OR ‘self-destructive' OR ‘non-suicidal self-injury' OR ‘self-harm' OR ‘self-inflicted wounds' OR ‘suicide’ OR ‘suicidality' OR ‘suicidal ideation' OR ‘suicidal behaviours'. The multitude of keywords for self-injury and suicide were intended to capture the vast variety of terms used in research on self-injurious and suicidal thoughts and behaviour.

First, a search of PsychINFO was conducted, limited to peer reviewed, scholarly articles, that were reported in English, contained empirical data, and were published prior to 2016. Second, a search with very similar criteria (excl. the peer reviewed and scholarly criteria), instead targeting dissertations and conference proceedings was conducted. In total, 76 published articles and 11 dissertations were identified and were subsequently gathered for initial examination. In sourcing these articles, only one dissertation could not be accessed (Dean, 1997), however, an identically titled and authored article has since been published and appears to report the relevant data from the inaccessible thesis (see Figure 25).

Next, a search of Web of Science databases was conducted. This involved conducting a search of all perfectionism literature (again using the keyword 'perfectionism') and then refining the search using the above keywords for self-injurious and suicidal behaviours. This resulted in 153 articles, 76 of which had already been identified in the PsychINFO search. In total, a further $77 \mathrm{Web}$ of Science articles were sourced for detailed examination.

In addition, one extra article was discovered during the literature review stage for this thesis. This article did not appear on either PsychINFO or Web of Science searches however upon review appeared to be very relevant and therefore was included (Miskey et al., 2012). Finally, two of the researchers contacted for further information (see below for detail regarding further contact) provided additional studies containing relevant data. The two additional studies provided were also included in the meta-analyses.

Overall, the initial search process identified 163 articles or dissertations that mentioned both perfectionism and a variation of self-injurious and/or suicidal behaviours (see Figure 25). Of these, 49 articles contained effect size data for the relationship between perfectionism and self-injury/suicide and gave the appropriate sample information. Three articles contained appropriate data however additional sample information was required, and eight articles 
investigated relevant variables however did not report the required data. Altogether, the authors of 11 articles were contacted. Of these, one author provided the study/sample information required and the authors of three articles provided their relevant data. The authors of the remaining seven articles could not be contacted or were unable to provide the requested information.

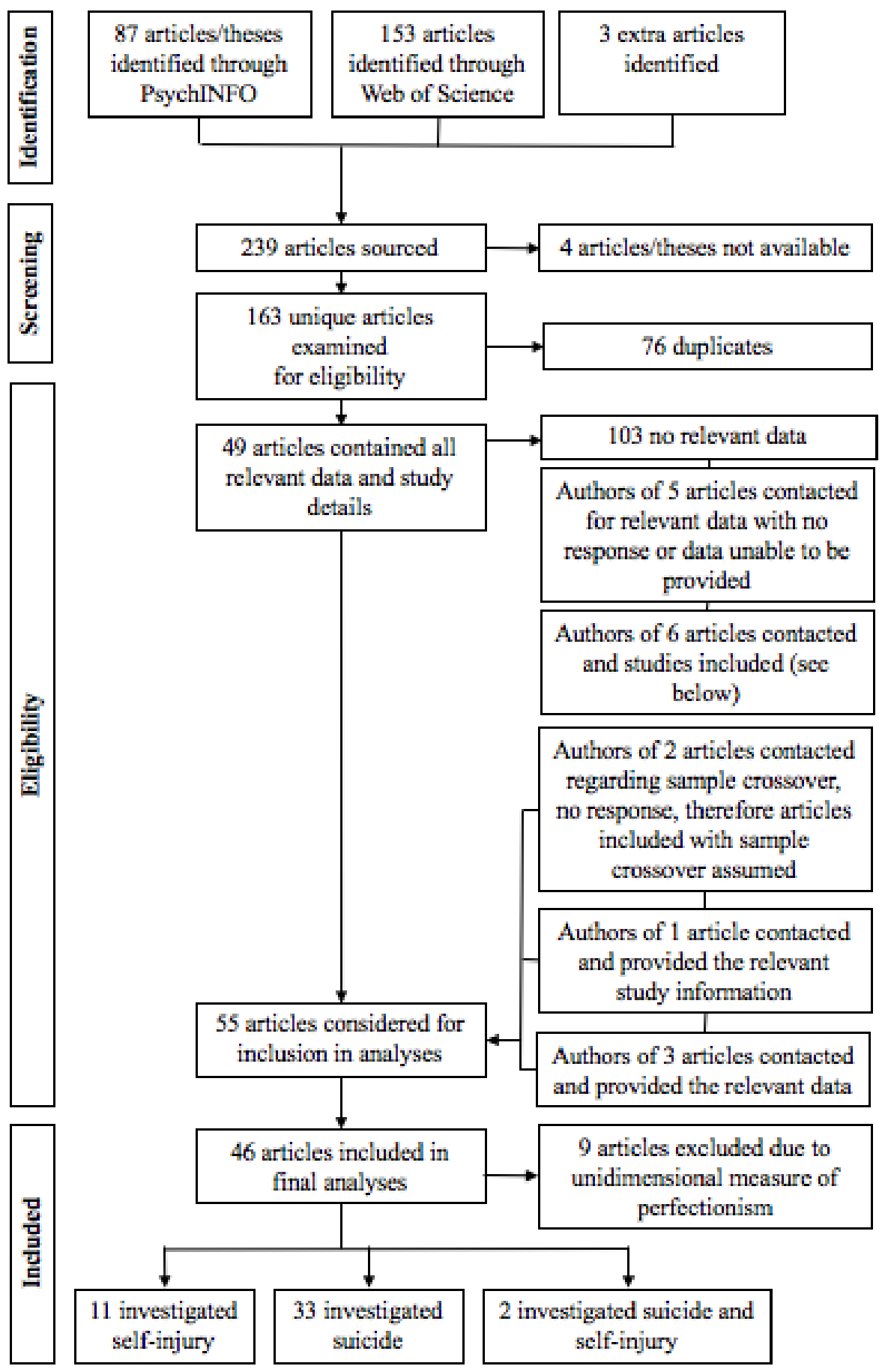

Figure 25. Inclusion/exclusion process for Study $1 \mathrm{~b}$ 
In total, the data from 46 articles were included in the final analyses. Of these, 11 articles contained effect size data for the relationship between perfectionism and self-injurious thoughts or behaviours, 33 contained effect size data for perfectionism and suicidal thoughts or behaviours, and two contained data for perfectionism and both self-injury and suicide related outcomes.

\section{Measure of Perfectionism}

As expected, the FMPS was the most frequently used scale (Frost et al., 1990), followed by the HFMPS (Hewitt \& Flett, 1991), and the Almost Perfect Scale-Revised (APSR; Slaney, Rice, Mobley, Trippi, \& Ashby, 2001). Some unidimensional measures were also used (e.g., Neurotic Perfectionism Questionnaire [NPQ], Mitzman, Slade, \& Dewey, 1994; Personal Style Inventory-II [PSI-II], Robins et al., 1994). Where possible, these scales were assigned to positive or negative perfectionism based on the scale origin and their specific items (see Table 7). For example, the DAS, NPQ, PSI-II and the DSM-IV Diagnostic Interview for Personality Disorders (DIPV-IV; Zanarini, Frankenburg, Sickel, \& Yong, 1996) align well with negative conceptualisations of perfectionism. On the other hand, the EDI perfectionism scale contains four items that fit with negative perfectionism and two items that fit with positive perfectionism (see Joiner \& Schmidt, 1995). Therefore, the EDI-II appears to measure a composite of both positive and negative perfectionism. As such, research that only used the EDI perfectionism scale ( $n=3$; Fujimori et al., 2011; Luyckx et al., 2015; Yamaguchi et al., 2000) could not contribute to answering the research question and were excluded from the analyses.

In addition, some articles presented data gathered using multidimensional perfectionism measures, however only reported total scale scores (e.g., FMPS-Total, HFMPSTotal, APS-R-Total), consequently, and similar to research with the EDI-II, this data was not included. Finally, the PSPS - perfectionistic self-promotion and HFMPS - OOP subscales did not fit with either positive or negative perfectionism and for that reason were also excluded. Given the wide variety of scales used to measure positive and negative perfectionism, the scale used was tested as a moderator of the overall relationship between perfectionism and self-injury/suicide.

\section{Self-Injury/Suicide Variables}

A number of key outcome or group comparison variables were identified. These included: aggression towards self (an experimental proxy for self-injury), deliberate selfharm, non-suicidal self-injury (total and cutting only), past suicidal ideation, current suicidal 
ideation, reasons for living (reverse scored), suicide attempts (both a score on suicide psychological assessment tools and number of suicide attempts), suicide intent, suicide plan, and suicide risk. These were specifically coded as above, as well as broadly coded according to whether the outcome is suicide related (e.g., suicide attempts, suicidal ideation), self-injury with no suicide intent (NSSI), a combination of self-injury and suicide behaviours, or selfinjury without differentiation based on suicide intent (e.g., deliberate self-harm). This distinction also formed an important moderator variable.

Table 7

Subscales of Existing Perfectionism Scales Assigned to Positive/Negative Categories

\begin{tabular}{|c|c|c|}
\hline Negative Perfectionism & Positive Perfectionism & Excluded \\
\hline APS-R - Discrepancy & APS-R - Order & APS-R total score \\
\hline $\begin{array}{l}\text { CAPS - Socially Prescribed } \\
\text { Perfectionism }\end{array}$ & APS-R - High Standards & $\begin{array}{l}\text { EDI- Perfectionism } \\
\text { subscale }\end{array}$ \\
\hline DAS - Perfectionism subscale & $\begin{array}{l}\text { CAPS - Self-Oriented } \\
\text { Perfectionism }\end{array}$ & FMPS total score \\
\hline FAPS-R - Discrepancy & FMPS - Organisation & HFMPS total score \\
\hline FMPS - Concerns over Mistakes & FMPS - Personal Standards & $\begin{array}{l}\text { HFMPS - Other Oriented } \\
\text { Perfectionism }\end{array}$ \\
\hline FMPS - Doubts about Actions & $\begin{array}{l}\text { HFMPS - Self-Oriented } \\
\text { Perfectionism }\end{array}$ & $\begin{array}{l}\text { PSPS - Perfectionistic } \\
\text { Self-Promotion }\end{array}$ \\
\hline FMPS - Parental Criticism & PI- High Standards & \\
\hline FMPS - Parental Expectations & PI - Order & \\
\hline $\begin{array}{l}\text { HFMPS - Socially Prescribed } \\
\text { Perfectionism }\end{array}$ & PI - Planfulness & \\
\hline NPQ total score & PI - Striving & \\
\hline \multicolumn{3}{|l|}{ PI - Concerns over Mistakes } \\
\hline \multicolumn{3}{|l|}{ PI - Need } \\
\hline \multicolumn{3}{|l|}{ PI - Parental Pressure } \\
\hline \multicolumn{3}{|l|}{ PI - Rumination } \\
\hline \multicolumn{3}{|l|}{ PSPS - Non-Disclosure } \\
\hline \multicolumn{3}{|l|}{ PSPS - Non-Display } \\
\hline \multicolumn{3}{|l|}{ S-DIPD-IV } \\
\hline \multicolumn{3}{|c|}{$\begin{array}{l}\text { Note: APS-R - Almost Perfect Scale-Revised, CAPS - Child and Adolescent Perfectionism Scale, DAS - } \\
\text { Dysfunctional Attitudes Scale, FAPS - Family Almost Perfect Scale, EDI - Eating Disorder Inventory, FMPS } \\
\text { - Frost Multidimensional Perfectionism Scale, HFMPS - Hewitt and Flett Multidimensional Perfectionism } \\
\text { Scale, NPQ - Neurotic Perfectionism Questionnaire, PI - Perfectionism Inventory, PSPS - Perfectionistic } \\
\text { Self-Presentation Scale, S-DIPD-IV - Spanish-Diagnostic Interview for DSM-IV Personality Disorders; also } \\
\text { see Appendix A for an acronym glossary }\end{array}$} \\
\hline
\end{tabular}




\section{Data-Synthesis}

The relevant data was extracted from each article and entered into CMA for synthesis. In most cases the data was either presented as a Pearson's correlation coefficient or as group comparisons (categorised based on self-injury or suicide history) of the mean scores on perfectionism measures. For the sake of simplicity the overall summary effect is presented as a Pearson's correlation coefficient. The data was analysed using a random effects model due to the significant variation likely to be associated with individual study and sample characteristics (Field \& Gillet, 2010). These meta-analytic techniques were first conducted as two summary meta-analyses (negative perfectionism and self-injury/suicide; positive perfectionism and self-injury/suicide), allowing statistical comparison of the self-injury and suicide effect sizes. Next, more specific meta-analyses for first negative perfectionism and self-injury, and negative perfectionism and suicide, and then for positive perfectionism and self-injury, and positive perfectionism and suicide were conducted. Overall, this resulted in six distinct meta-analyses.

\section{Table 8}

\begin{tabular}{ll}
\begin{tabular}{l} 
Moderator Categories and Specific Variables for Study $1 \mathrm{~b}$ \\
\hline Moderator
\end{tabular} & $\begin{array}{l}\text { Categories of Moderator } \\
\text { Male, female, both }\end{array}$ \\
Age & $\begin{array}{l}\text { Child/adolescent combination, adolescent, } \\
\text { adolescent/adult combination, adult, university } \\
\text { students, older adults }\end{array}$ \\
Sample Type & $\begin{array}{l}\text { Clinical, non-clinical, combination } \\
\text { Requirement for inclusion in sample, not } \\
\text { specified }\end{array}$ \\
Measure of Negative Perfectionism & $\begin{array}{l}\text { See Table 7 above } \\
\text { Measure of Positive Perfectionism }\end{array}$ \\
Self-Injury/Suicide Classification & $\begin{array}{l}\text { See Table 7 above } \\
\text { Suicidal ideation, suicide attempt, suicide plan, } \\
\text { suicide risk, suicide intent }\end{array}$ \\
Self-Injury/Suicide Specific Outcome & $\begin{array}{l}\text { Aggression towards self, non-suicidal self- } \\
\text { injury, deliberate self-harm, non-suicidal cutting }\end{array}$ \\
\hline
\end{tabular}

\section{Moderator Analyses}

In addition to those already mentioned (e.g., the scale used to measure perfectionism, specific suicide/self-injury outcome), other study details were gathered for moderator analyses. These included sample characteristics such as the sex, and age of the sample, 
whether it was a clinical or non-clinical sample, and whether the sample was made up of individuals with a history of self-injury or suicidal thoughts and behaviours, or it was a more representative sample of the total population. Each moderator category and their corresponding levels are presented in Table 8.

\section{Assessing for Publication Bias}

Consistent with processes used in Study 1a, publication bias was first and foremost assessed with a funnel plot (Borenstein et al., 2009), followed by examining Begg and Mazumdar's rank correlation, Kendall's tau statistic, and Egger's regression intercept (Sterne et al., 2000). In the event of significant publication bias, Orwin's fail safe $\mathrm{N}$ was examined to estimate how many additional null studies would bring the effect size to negligible $(0.10$; Cohen, 1988), and Duval and Tweedie's Trim and Fill method the likely summary effect was recalculated, adjusting for a less biased sample of effect sizes.

\section{Results}

A comprehensive search of both PsychINFO and Web of Science databases resulted in 55 articles that contained data on perfectionism and self-injury or suicide. Following the exclusion of nine of these due to the inability to differentiate positive and negative perfectionism, 46 articles were included in the meta-analyses reported below (refer to Figure 25).

\section{Perfectionism and Self-Injurious and Suicidal Thoughts and Behaviours}

As an exploratory first step, all self-injurious and suicidal outcomes were grouped and analysed together with regard to their relationship with first negative perfectionism, and second positive perfectionism. Within all 46 articles, 52 distinct samples had data pertaining to the relationship between negative perfectionism and self-injury/suicide. However, only 32 of those samples also had data for the relationship between positive perfectionism and selfinjury/suicide.

\section{Negative perfectionism and self-injurious and suicidal thoughts and behaviours.}

The summary Pearson's correlation coefficient, using a random effects model, for the relationship between negative perfectionism and self-injury/suicide was .22 (.19-.26, 95\% CI), and was significantly different to 0 ( $p<.001$; see Table 9 for a summary of all relevant statistics). The effect sizes of individual studies ranged from -.06 (Miskey et al., 2012) to .51 (Hewitt, Newton, Flett, \& Callander, 1997 $)^{25}$. The Q-statistic $(Q(54)=242.85, p<.001)$

\footnotetext{
${ }^{25}$ See Appendix C, Figure 1 for the supporting forest plot and the online supplementary material for a summary of the studies included in this analysis
} 
indicated significant heterogeneity between studies, of which the $\mathrm{I}^{2}$ statistic $\left(\mathrm{I}^{2}=77.76\right)$ suggested up to $78 \%$ was due to individual study and sample characteristics rather than within study random error. The main purpose of this inclusive meta-analysis was to test whether the relationship between negative perfectionism and self-injurious thoughts and behaviours was significantly different to the relationship between negative perfectionism and suicidal thoughts and behaviours, therefore, only the self-injury/suicide distinction was tested as a moderator. This analysis revealed that negative perfectionism was more strongly associated with suicide $(n=38 ; r=.25 ; .21-.29,95 \% \mathrm{CI})$ than self-injury $(n=14 ; r=.14 ; .07-.21,95 \%$ CI; $Q(2)=8.44, p=.02$ ). Accordingly, individual meta-analyses for the relationship between negative perfectionism and self-injurious thoughts and behaviours, and negative perfectionism and suicidal thoughts and behaviours are reported below with greater depth of investigation into moderating factors.

Publication bias analyses were also conducted to ascertain whether the sample of research included was a representative sample of all research conducted. In short, there was some evidence of publication bias with disproportionate group of studies with larger samples and greater effect sizes (see the supplementary online material for the relevant funnel plot). However, there was insufficient evidence to conclude that publication bias impacts upon what seems to be a relatively robust positive relationship between negative perfectionism and selfinjurious/suicidal thoughts and behaviours (see Table 8 for a summary of publication bias statistics).

\section{Positive perfectionism and self-injurious and suicidal thoughts and behaviours.}

The same process was carried out for the relationship between positive perfectionism and selfinjurious/suicidal thoughts and behaviours, resulting in a summary Pearson's correlation coefficient of .02 (-.00-.05, 95\% CI), which was not significantly different to 0 ( $p=.11$; see Table 8 for a summary of all relevant statistics). The individual effect sizes ranged from -.22 (Hoff \& Muehlenkamp, 2009) to .23 (Rasmussen, O’Connor, \& Brodie, 2008) and the Qstatistic indicated significant heterogeneity $(Q(41)=57.19, p=.05)^{26}$. In this case, approximately $28 \%$ of the variance between studies was attributed to study characteristics $\left(\mathrm{I}^{2}\right.$ $=28.30$ ). Given significant heterogeneity, moderator analyses were carried out based on whether the data pertained to self-injury or suicide related outcomes, however no significant

\footnotetext{
${ }^{26}$ See Appendix C, Figure 2 for the supporting forest plot and the online supplementary material for a summary of the studies included in this analysis
} 
Table 9

Summary of Meta-Analytic Results for the Relationship between Perfectionism and Self-Injurious and Suicidal Thoughts and Behaviours

\begin{tabular}{|c|c|c|c|}
\hline Meta-analysis & $\underline{\text { Summary effect }}$ & Heterogeneity & Moderator analysis \\
\hline $\begin{array}{l}\text { Negative perfectionism and } \\
\text { self-injury/suicide } \\
(n=55)\end{array}$ & $\begin{array}{l}r=.22, p<.001 \\
.19-.26,95 \% \mathrm{CI}\end{array}$ & $\begin{array}{l}Q(54)=242.85, p<.001 \\
\mathrm{I}^{2}=77.76\end{array}$ & $\begin{array}{l}\text { Self-injury/suicide distinction: } \\
Q(2)=8.44, p=.02^{27}\end{array}$ \\
\hline
\end{tabular}

$(n=55)$

$$
\begin{array}{ll}
r=.02, p=.11 & Q(41)=54.19, p=.05 \\
-.01-.05,95 \% \mathrm{CI} & \mathrm{I}^{2}=28.30
\end{array}
$$

No significant moderators ${ }^{28}$ self-injury/suicide $(n=42)$ Negative perfectionism and
self-injury $(n=17)$

Positive perfectionism and self-injury $(n=12)$

$$
\begin{array}{ll}
r=.15, p<.001 & Q(16)=83.33, p<.001 \\
.09-.21,95 \% \mathrm{CI} & \mathrm{I}^{2}=80.80
\end{array}
$$

Age $: Q(2)=6.46, p=.04$

$$
r=.00, p=.89 \quad Q(11)=18.40, p=.07
$$
$-.05-.05,95 \% \mathrm{C}$
$Q(11)=18.4$
$I^{2}=40.21$

\section{Publication bias}

Funnel plot: inconclusive

Adjusted estimate: $r=.18 ; .14-.22,95 \%$ CI

Kendall's tau: $p=.70$

Egger's intercept: $p<.001$

Orwin's Fail-safe N: 31

Funnel plot: bias unlikely

Adjusted estimate: $r=.01 ;-.02-.04$,

95\% CI

Kendall's tau: $p=.85$

Egger's intercept: $p=.96$

Orwin's Fail-safe N: N/A

Classic Fail-safe N: 4

Funnel plot: bias unlikely

Adjusted estimate: $r=.15 ; .09-.21,95 \%$

CI

Kendall's tau: $p=.80$

Orwin's Fail-safe N: N/A

Classic Fail-safe N: 399

Funnel plot: bias unlikely Adjusted estimate: $r=-.01 ;-.06-.04$, 95\% CI

Kendall's tau: $p=1.00$

Egger's intercept: $p=.25$

Orwin's Fail-safe N: N/A

Classic Fail-safe N: N/A

${ }^{27}$ Note: only the distinction between self-injurious thoughts and behaviours and suicidal thoughts and behaviours was tested as a moderator in this analysis

${ }^{28}$ As above 
Table 9

Summary of Meta-Analytic Results for the Relationship between Perfectionism and Self-Injurious and Suicidal Thoughts and Behaviours

\begin{tabular}{|c|c|c|c|c|}
\hline $\begin{array}{l}\text { Meta-analysis } \\
\text { Negative perfectionism and } \\
\text { suicide }(n=41)\end{array}$ & $\begin{array}{l}\frac{\text { Summary effect }}{r=.25, p<.001} \\
.21-.28,95 \% \mathrm{CI}\end{array}$ & $\begin{array}{l}\text { Heterogeneity } \\
Q(40)=96.99, p<.001 \\
\mathrm{I}^{2}=58.76\end{array}$ & $\begin{array}{l}\text { Moderator analysis } \\
\text { Specific outcome: } Q(3)=21.99, \\
p<.001 ; \operatorname{Sex}: Q(2)=11.93, p \\
=.01\end{array}$ & $\begin{array}{l}\text { Publication bias } \\
\text { Funnel plot: bias unlikely } \\
\text { Adjusted estimate: } r=.25 ; .21-.28,95 \% \\
\text { CI } \\
\text { Kendall's tau: } p=.65 \\
\text { Egger's intercept: } p=.01 \\
\text { Orwin's Fail-safe N: } 50\end{array}$ \\
\hline $\begin{array}{l}\text { Positive perfectionism and } \\
\text { suicide }(n=31)\end{array}$ & $\begin{array}{l}r=.03, p=.06 \\
-.00-.06,95 \% \mathrm{CI}\end{array}$ & $\begin{array}{l}Q(30)=38.98, p=.13 \\
\mathrm{I}^{2}=23.05\end{array}$ & No significant moderators & $\begin{array}{l}\text { Funnel plot: bias unlikely } \\
\text { Kendall's tau: } p=.84 \\
\text { Egger's intercept: } p=.19 \\
\text { Orwin's Fail-safe N: N/A } \\
\text { Classic Fail-safe N: } 11\end{array}$ \\
\hline
\end{tabular}


differences were found $(Q(2)=1.43, p=.49)$. That is, the relationship between positive perfectionism and self-injury was not significantly different to the relationship between positive perfectionism and suicide. Despite this, due to the importance of the relationship between positive perfectionism and self-injury/suicidal thoughts and behaviours to the research that will follow, a detailed exploration of first the relationship between positive perfectionism and self-injury and second the relationship between positive perfectionism and suicide is reported below. The following section focuses on exploring whether there are any other factors (i.e., age, sex) that influence the relationship. With regard to publication bias, subjective interpretation of the funnel plot and Duval and Tweedie's Trim and Fill method suggested some publication bias, but this was not supported by equivalent statistical tests. Overall, this suggests that the calculated summary effect is likely an accurate representation of the mean of all effect sizes.

\section{Negative Perfectionism and Self-Injury}

In addition to the comparatively broad meta-analyses reported above, more focused meta-analyses were carried out. With regard to the relationship between negative perfectionism and self-injury, data from 17 samples from 15 articles were gathered. Overall, three articles presented data for self-injury without specifying intent, and 11 reported data specific to NSSI. Most research was conducted with mixed sex, non-clinical, university samples. Three samples were comprised of only individuals with a history of self-injury, thus representing a relationship with severity of self-injury rather than simply the existence of selfinjury.

\begin{tabular}{rrrrrr}
\multicolumn{5}{c}{ Lower Upper } \\
Correlation & limit & \multicolumn{4}{c}{ limit } \\
Z-Valuep-Value \\
0.350 & 0.141 & 0.529 & 3.207 & 0.001 \\
0.129 & -0.075 & 0.322 & 1.240 & 0.215 \\
0.101 & -0.036 & 0.234 & 1.442 & 0.149 \\
0.002 & -0.184 & 0.187 & 0.019 & 0.985 \\
0.428 & 0.304 & 0.538 & 6.240 & 0.000 \\
0.364 & 0.095 & 0.583 & 2.617 & 0.009 \\
0.176 & -0.107 & 0.433 & 1.222 & 0.222 \\
0.232 & 0.130 & 0.329 & 4.386 & 0.000 \\
0.187 & -0.046 & 0.400 & 1.573 & 0.116 \\
-0.058 & -0.331 & 0.224 & -0.399 & 0.690 \\
0.076 & -0.077 & 0.225 & 0.972 & 0.331 \\
0.113 & 0.025 & 0.198 & 2.521 & 0.012 \\
0.009 & -0.024 & 0.042 & 0.514 & 0.607 \\
0.175 & 0.109 & 0.239 & 5.177 & 0.000 \\
0.198 & 0.121 & 0.272 & 5.004 & 0.000 \\
0.110 & 0.026 & 0.193 & 2.555 & 0.011 \\
0.075 & -0.014 & 0.163 & 1.657 & 0.098 \\
0.152 & 0.091 & 0.212 & 4.849 & 0.000
\end{tabular}

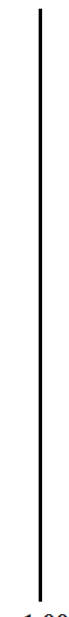

$-1.00$
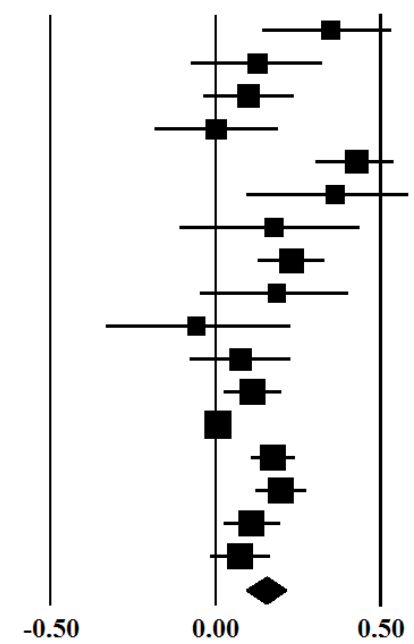

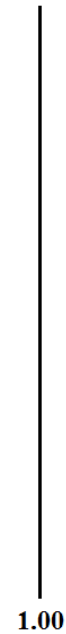

Figure 26. Forest plot with effect sizes for negative perfectionism and self-injurious thoughts and behaviour 
The distribution of individual study effect sizes were first visually examined using a forest plot (see Figure 26) and, second, analysed using a random effects model to produce a summary effect of .15 (.09-.21, 95\% CI), that was significantly different to 0 ( $p<.001$; see Table 8 for a summary of relevant statistics). Effect sizes ranged from -.06 (Miskey et al., 2012) to .43 (Hoff \& Muehlenkamp, 2009). Further, heterogeneity analyses indicated that there was significant heterogeneity between studies $(Q(16)=83.33, p<.001)$, with the $\mathrm{I}^{2}$ statistic $\left(\mathrm{I}^{2}=80.80\right)$ indicating that $81 \%$ of the heterogeneity could be attributed to individual study characteristics rather than random within study error.

Moderator analyses indicated that the relationship between negative perfectionism and self-injury was moderated by age such that adults $(n=4 ; r=.20 ; .08-.31)$ and university students $(n=7 ; r=.20 ; .09-.30)$ exhibited a stronger relationship between the two constructs than adolescents $(n=4 ; r=.07 ; .01-.13)$. However, this relationship was not moderated by sex, sample type (clinical/non-clinical), the specific definition of self-injury (including whether suicide intent was specified), or the scale used to measure negative perfectionism. The relationship between negative perfectionism and self-injury also was not moderated by whether the sample was comprised of only individuals with a history of self-injury or also included individuals with no history of self-injury. Assessment of publication bias resulted in little indication that the sample of studies was biased towards those with large samples and significant effect sizes. This was evidenced by a symmetrical distribution of effect sizes within the funnel plot (see Figure 27) and a non-significant Kendall's tau ( $p=.80)$. Egger's regression intercept $(p=.02)$ was significant, however Duval and Tweedie's Trim and Fill method resulted in no additional inputted effect sizes and no adjustment to the effect size. Despite an already relatively small summary effect (.15), the Classic fail-safe $\mathrm{N}$ indicated that a further 399 studies with non-significant effects would be required to bring the summary effect to $0^{29}$. Overall, this allows confidence that there is indeed a positive relationship between negative perfectionism and self-injurious thoughts and behaviours.

\section{Positive Perfectionism and Self-Injury}

Next, the relationship between positive perfectionism and self-injurious thoughts and behaviours was explored. This meta-analysis included data gathered from 12 samples, presented in 11 articles. Data for NSSI was gathered from seven samples, and data for selfinjury without specification of intent was gathered from the remaining five samples.

\footnotetext{
${ }^{29}$ Orwin's fail safe $\mathrm{N}$ could not be calculated be the fixed effects summary correlation fell below .10 (.09). Therefore, the Classic Fail Safe N is used.
} 


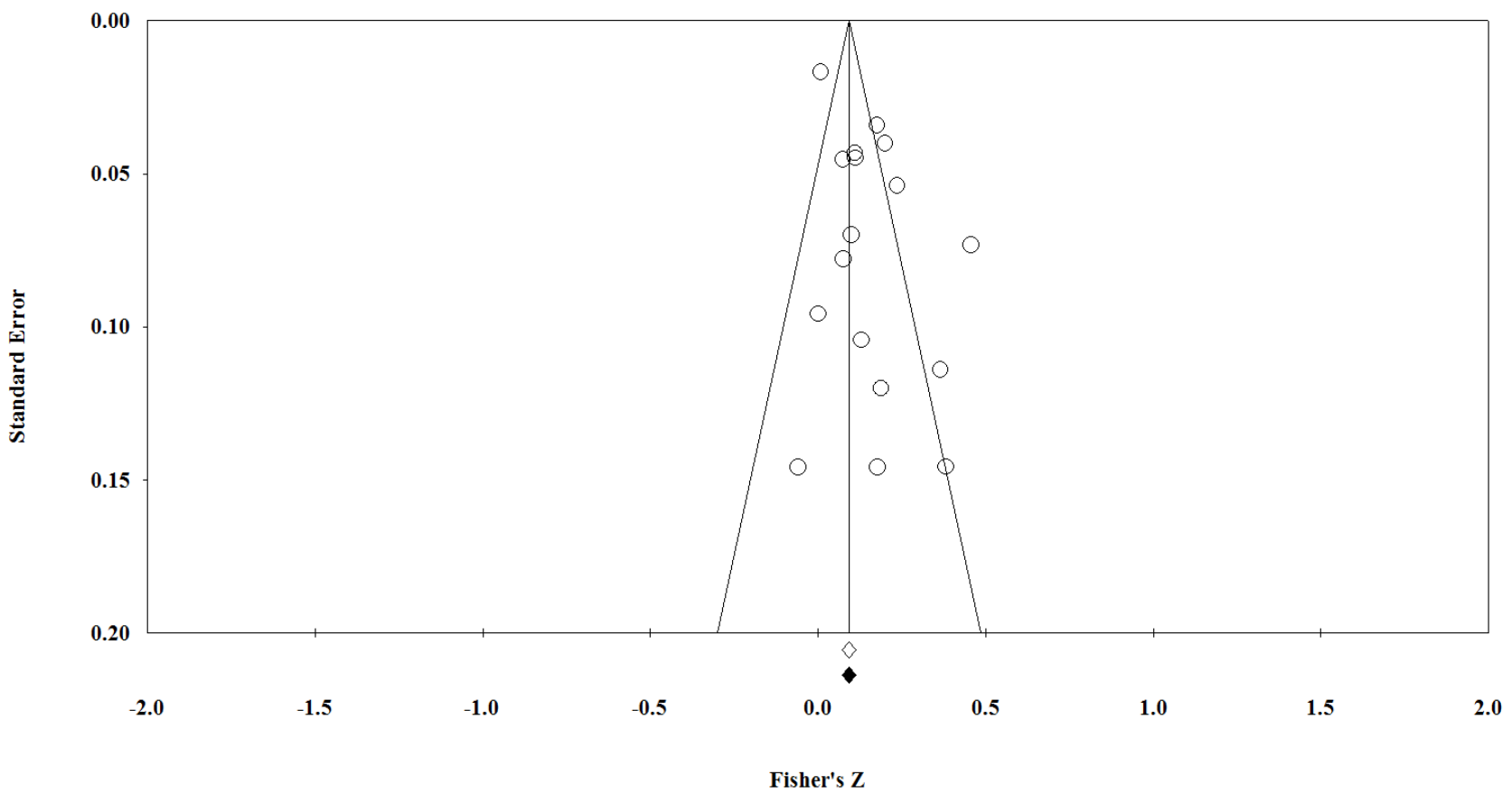

Figure 27. Funnel plot of the standard error by Fischer's $\mathrm{Z}$ for the association between negative perfectionism and self-injurious thoughts and behaviour

Similar to above, half of the data came from mixed sex, non-clinical, university samples. The remaining data comprised of clinical samples (4 samples), and community adolescent samples (2 samples). Only two samples were comprised of only individuals with a history of selfinjury.

\begin{tabular}{rrrrr}
\multicolumn{6}{c}{$\begin{array}{c}\text { Lower } \\
\text { Correlation }\end{array}$} & limit & \multicolumn{4}{c}{ limit } & Z-Valuep-Value \\
0.161 & -0.061 & 0.368 & 1.428 & 0.153 \\
-0.007 & -0.208 & 0.195 & -0.067 & 0.946 \\
-0.007 & -0.143 & 0.130 & -0.095 & 0.924 \\
-0.183 & -0.356 & 0.003 & -1.930 & 0.054 \\
-0.216 & -0.354 & -0.069 & -2.860 & 0.004 \\
-0.010 & -0.296 & 0.278 & -0.066 & 0.947 \\
0.017 & -0.217 & 0.249 & 0.137 & 0.891 \\
0.109 & -0.175 & 0.376 & 0.748 & 0.454 \\
0.028 & -0.124 & 0.179 & 0.358 & 0.720 \\
0.012 & -0.076 & 0.100 & 0.268 & 0.789 \\
0.041 & 0.008 & 0.074 & 2.434 & 0.015 \\
0.047 & -0.031 & 0.125 & 1.180 & 0.238 \\
0.004 & -0.045 & 0.052 & 0.144 & 0.885
\end{tabular}
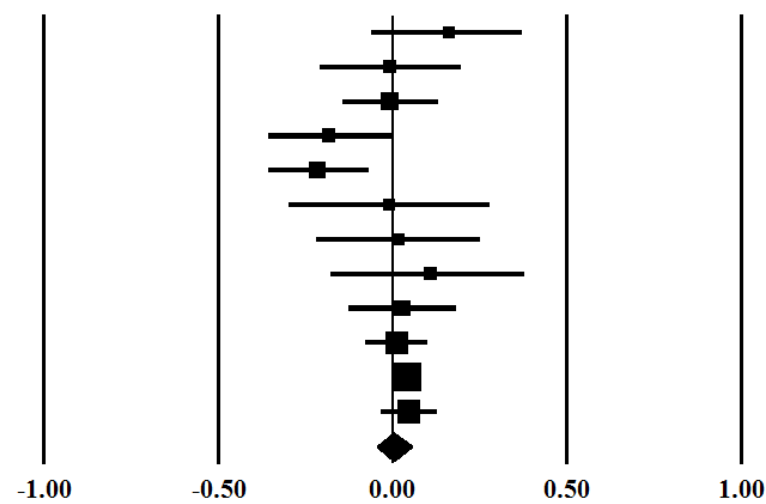

Chester, Merwin \& DeWall, 2015

Claes et al., 2012

Flett, Goldstein, Hewitt \& Wekerle, 2012

Flett, Goldstein, Hewitt \& Wekerle, 2012

Hoff \& Muehlenkamp, 2009

Hunter \& O'Connor, 2003

Kubal, 2005

Miskey, Hill \& Huelsman, 2012

O'Connor et al., 2007

O'Connor, Rasmussen \& Hawton, 2010

O'Connor, Rasmussen \& Hawton, 2014

Smith, Chesin \& Jeglic, 2014

Figure 28. Forest plot with effect sizes for positive perfectionism and self-injurious thoughts and behaviour

Figure 28 shows that there was a relatively narrow distribution of effects (ranging from -.22, Hoff \& Muehlenkamp, 2009, to .16, Chester, Merwin, \& Dewall, 2015), all largely 
centred on 0 (see Table 8 for a summary of relevant statistics). Only two studies (Hoff \& Muehlenkamp, 2009; (O’Connor, Rasmussen, \& Hawton, 2014) reported significant effects ( $p$ $<.05$ ) and one article reported an effect with $p<.10$ (Flett, Goldstein, Hewitt, \& Wekerle, 2012). As a result, the summary effect was .00 (-.05-.05, 95\% CI) and was non-significant ( $p$ $=.89)$. Heterogeneity analyses did not indicate significant between-study differences $(Q(11)$ $=18.40, p=.07$ ) and the relationship was not moderated by the sample characteristics (age, sex, sample type) or study methodology factors tested (scale used for perfectionism, specific component/definition of self-injury).

In assessing for publication bias, the funnel plot (Figure 29) appeared to be slightly weighted towards studies with a positive effect, and as such, Duval and Tweedie's Trim and Fill method resulted in two additional effect sizes and estimated an adjusted effect size of .01 . However, statistical tests of publication bias (Kendall's tau: $p=1.00$; Egger's regression intercept: $p=.25$ ) did not indicate significant bias, allowing confidence that there is no relationship between positive perfectionism and self-injurious thoughts and behaviours.

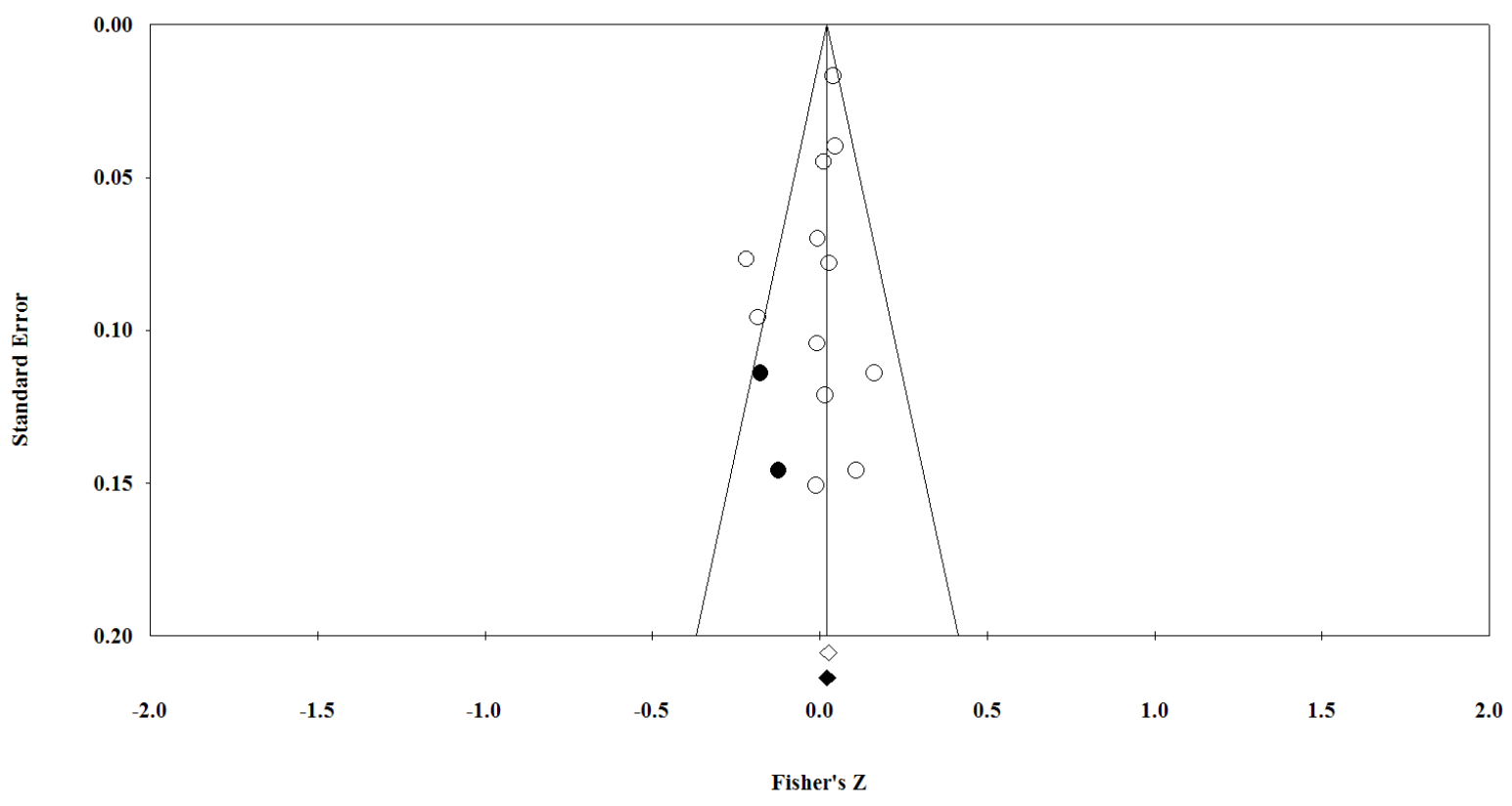

Figure 29. Funnel plot of the standard error by Fischer's $Z$ for the association between positive perfectionism and self-injurious thoughts and behaviour

\section{Negative Perfectionism and Suicidal Thoughts and Behaviours}

The relationship between negative perfectionism and suicidal thoughts and behaviours was investigated in 41 samples, reported in 37 articles. The data mostly pertained to suicidal ideation, followed by suicide risk, suicide attempts, and suicide intent. Some data was based on a combination of the above (e.g., total SBQ-R scores). Approximately half represented 
clinical samples, seven of which used samples of individuals that had previously attempted suicide. Most were mixed sex samples (see the online supplementary material for individual study details).

The resulting aggregate effect size, using a random effects model, was .25 (.21-.28, 95\% CI) and was significantly different to $0(p<.001)$ (Figure 30; Table 8). Individual sample effect sizes ranged from .11 (Rasmussen et al., 2008) to .51 (Hewitt, Norton, Flett, Callander, \& Cowan, 1998). Tests for heterogeneity resulted in a significant Q-statistic $(Q(40)$ $=96.99, p<.001)$ and, of this variation, $59 \%$ could be attributed to sample and study characteristics rather than random error $\left(I^{2}=58.76\right)$.

Study name

Adkins \& Parker, 1996 Ansell et al., 2010

Beevers \& Miller, 2004

Blankstein, Lumley \& Crawford, 2007

Blankstein, Lumley \& Crawford, 2007

Caelian, 2006

Chang, 1998

Chang, 1998

Chang, Watkins \& Banks, 2004

Chang, Watkins \& Banks, 2004

Dean \& Range, 1996*

Dean \& Range, 1999

Enns, Cox \& Inayatulla, 2003

Flamenbaum \& Holden, 2007

Freudenstein et al., 2012

Hamilton \& Schweitzer, 2000

Hewitt, Caelian, Chen \& Flett, 2014

Hewitt, Flett \& Weber, 1994

Hewitt, Flett \& Weber, 1994

Hewitt, Flett, \& Turnbull-Donovan, 1992

Hewitt, Newton, Flett \& Callander, 1997

Hewitt, Newton, Flett \& Callander, 1997

Hewitt, Norton, Flett, Callander \& Cower, 1998

Izadi, 2014

Kirtley, O'Connor \& Carroll, 2015

Klibert et al., 2005

Muyan \& Chang, 2015

O'Connor \& Forgan, 2007

O'Connor et al., 2007

O'Connor et al., 2007

O'Connor, O'Connor \& Marshall, 2007

O'Riley \& Fiske, 2012

O'Riley, 2011

Portzky, van Heeringen \& Vervaet, 2014

Rasmussen et al., 2012

Rasmussen, Elliot \& O'Connor, 2012

Rasmussen, O'Connor \& Brodie, 2008

Rasmussen, O'Connor \& Brodie, 2008

Roxborough et al., 2012

Smith, Chesin \& Jeglic, 2014

Wang, Wong \& Fu, 2013

\begin{tabular}{|ccccc}
\multicolumn{5}{c}{ Statistics for each study } \\
\hline \multicolumn{5}{c}{ Lower Upper } \\
Correlation & limit & limit & Z-Valuep-Value \\
0.215 & 0.044 & 0.374 & 2.450 & 0.014 \\
0.310 & 0.146 & 0.458 & 3.612 & 0.000 \\
0.330 & 0.161 & 0.480 & 3.724 & 0.000 \\
0.420 & 0.275 & 0.546 & 5.316 & 0.000 \\
0.500 & 0.284 & 0.668 & 4.183 & 0.000 \\
0.176 & -0.094 & 0.422 & 1.283 & 0.200 \\
0.418 & 0.217 & 0.585 & 3.879 & 0.000 \\
0.210 & -0.028 & 0.426 & 1.733 & 0.083 \\
0.200 & 0.041 & 0.349 & 2.458 & 0.014 \\
0.400 & 0.256 & 0.527 & 5.136 & 0.000 \\
0.244 & 0.079 & 0.397 & 2.873 & 0.004 \\
0.156 & -0.015 & 0.318 & 1.785 & 0.074 \\
0.320 & 0.105 & 0.506 & 2.872 & 0.004 \\
0.140 & 0.020 & 0.256 & 2.278 & 0.023 \\
0.170 & -0.027 & 0.355 & 1.691 & 0.091 \\
0.117 & 0.018 & 0.213 & 2.322 & 0.020 \\
0.176 & -0.094 & 0.422 & 1.283 & 0.200 \\
0.287 & 0.087 & 0.466 & 2.775 & 0.006 \\
0.284 & 0.135 & 0.421 & 3.664 & 0.000 \\
0.385 & 0.190 & 0.551 & 3.720 & 0.000 \\
0.390 & 0.054 & 0.647 & 2.256 & 0.024 \\
0.510 & 0.202 & 0.726 & 3.082 & 0.002 \\
0.339 & 0.137 & 0.514 & 3.213 & 0.001 \\
0.134 & -0.149 & 0.398 & 0.927 & 0.354 \\
0.189 & 0.086 & 0.289 & 3.561 & 0.000 \\
0.260 & 0.174 & 0.342 & 5.781 & 0.000 \\
0.241 & 0.129 & 0.347 & 4.149 & 0.000 \\
0.270 & 0.152 & 0.380 & 4.395 & 0.000 \\
0.214 & 0.074 & 0.345 & 2.980 & 0.003 \\
0.262 & 0.039 & 0.460 & 2.292 & 0.022 \\
0.390 & 0.269 & 0.499 & 5.939 & 0.000 \\
0.290 & 0.217 & 0.360 & 7.512 & 0.000 \\
0.040 & -0.171 & 0.247 & 0.369 & 0.712 \\
0.067 & 0.015 & 0.118 & 2.538 & 0.011 \\
0.180 & 0.047 & 0.307 & 2.643 & 0.008 \\
0.194 & 0.023 & 0.354 & 2.223 & 0.026 \\
0.236 & -0.195 & 0.591 & 1.076 & 0.282 \\
0.108 & -0.393 & 0.560 & 0.406 & 0.685 \\
0.221 & 0.067 & 0.364 & 2.794 & 0.005 \\
0.250 & 0.177 & 0.321 & 6.507 & 0.000 \\
0.231 & 0.143 & 0.315 & 5.059 & 0.000 \\
0.248 & 0.213 & 0.283 & 13.227 & 0.000 \\
& & & & \\
0 & & &
\end{tabular}

Statistics for each study

on limit limit Z-Valuep-Value

$\begin{array}{llllll}0.330 & 0.161 & 0.480 & 3.724 & 0.000\end{array}$

$\begin{array}{llllll}0.500 & 0.284 & 0.668 & 4.183 & 0.000\end{array}$

$2.458 \quad 0.014$

$1.785 \quad 0.074$

6910.091

$2.775 \quad 0.006$

$\begin{array}{lll}0.385 & 0.190\end{array}$

$\begin{array}{llllll}0.390 & 0.054 & 0.647 & 2.256 & 0.024\end{array}$

$\begin{array}{llllll}0.510 & 0.202 & 0.726 & 3.082 & 0.002\end{array}$

$\begin{array}{lllll}0.339 & 0.137 & 0.514 & 3.213 & 0.001\end{array}$

$\begin{array}{lllll}0.189 & 0.086 & 0.289 & 3.561 & 0.000\end{array}$

$\begin{array}{llllll}0.342 & 5.781 & 0.000\end{array}$

$\begin{array}{llllll}0.270 & 0.152 & 0.380 & 4.395 & 0.000\end{array}$

$\begin{array}{llll}0.074 & 0.345 & 2.980 & 0.003\end{array}$

$\begin{array}{llllll}0.390 & 0.269 & 0.499 & 5.939 & 0.000\end{array}$

$\begin{array}{lllll}0.217 & 0.360 & 7.512 & 0.000\end{array}$

$\begin{array}{lllll}0.067 & 0.015 & 0.118 & 2.538 & 0.011\end{array}$

$0.236-0.195 \quad 0.591-1.076 \quad 0.282$

$\begin{array}{llllll}0.108 & -0.393 & 0.560 & 0.406 & 0.685\end{array}$

$\begin{array}{lllll}0.248 & 0.213 & 0.283 & 13.227 & 0.000\end{array}$
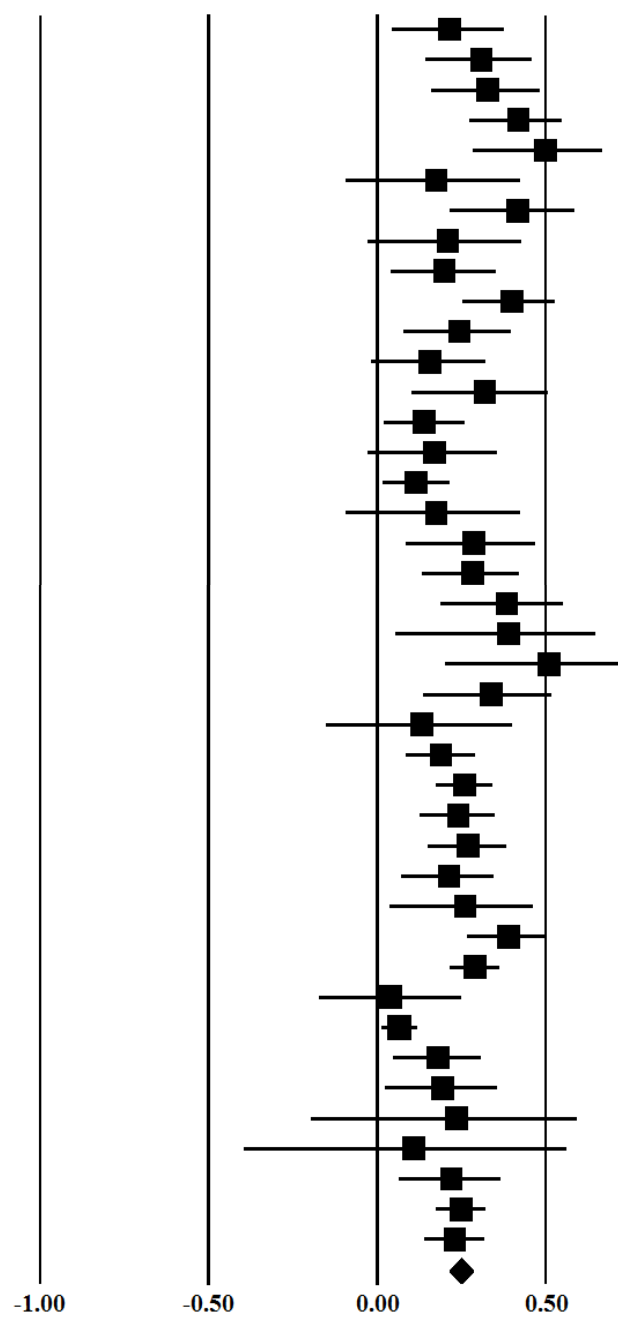

1.00

Figure 30. Forest plot with effect sizes for negative perfectionism and suicidal thoughts and behaviour 
Further analyses revealed that some of this heterogeneity could be a result of the sex of the participants within each sample $(Q(2)=11.93, p=.01)$. More specifically, the relationship between negative perfectionism and suicide was stronger in male-only samples $(n=2 ; r=.50$; $.33-.64,95 \% \mathrm{CI})$, than it was in female-only $(n=4 ; r=.35 ; .23-.46,95 \% \mathrm{CI})$, and combined $(n=35 ; r=.23 ; .20-.26,95 \% \mathrm{CI})$ samples. However, there were only two male-only samples. The first was an adolescent clinical inpatient sample (Hewitt et al., 1997) which given the specificity of the sample could have contributed to the particularly strong effect. However, the second sample was a university, non-clinical sample and did not have any features that could easily explain a significantly greater association between negative perfectionism and suicidal thoughts and behaviours. This may suggest that sex moderates the relationship between negative perfectionism and suicidal thoughts and behaviours, however more research is needed to have confidence in this finding.

The specific suicide related outcome was identified as another moderator of this relationship $(Q(4)=21.93, p<.001)$. In particular, negative perfectionism was more strongly associated with suicidal ideation $(n=23 ; r=.21 ; .16-.26,95 \% \mathrm{CI})$, than suicide risk $(n=7 ; r$ $=.17 ; .11-.22,95 \% \mathrm{CI})$, and past suicide attempts $(n=4 ; r=.05 ; .01-.10,95 \% \mathrm{CI})$. All other moderators tested were non-significant.

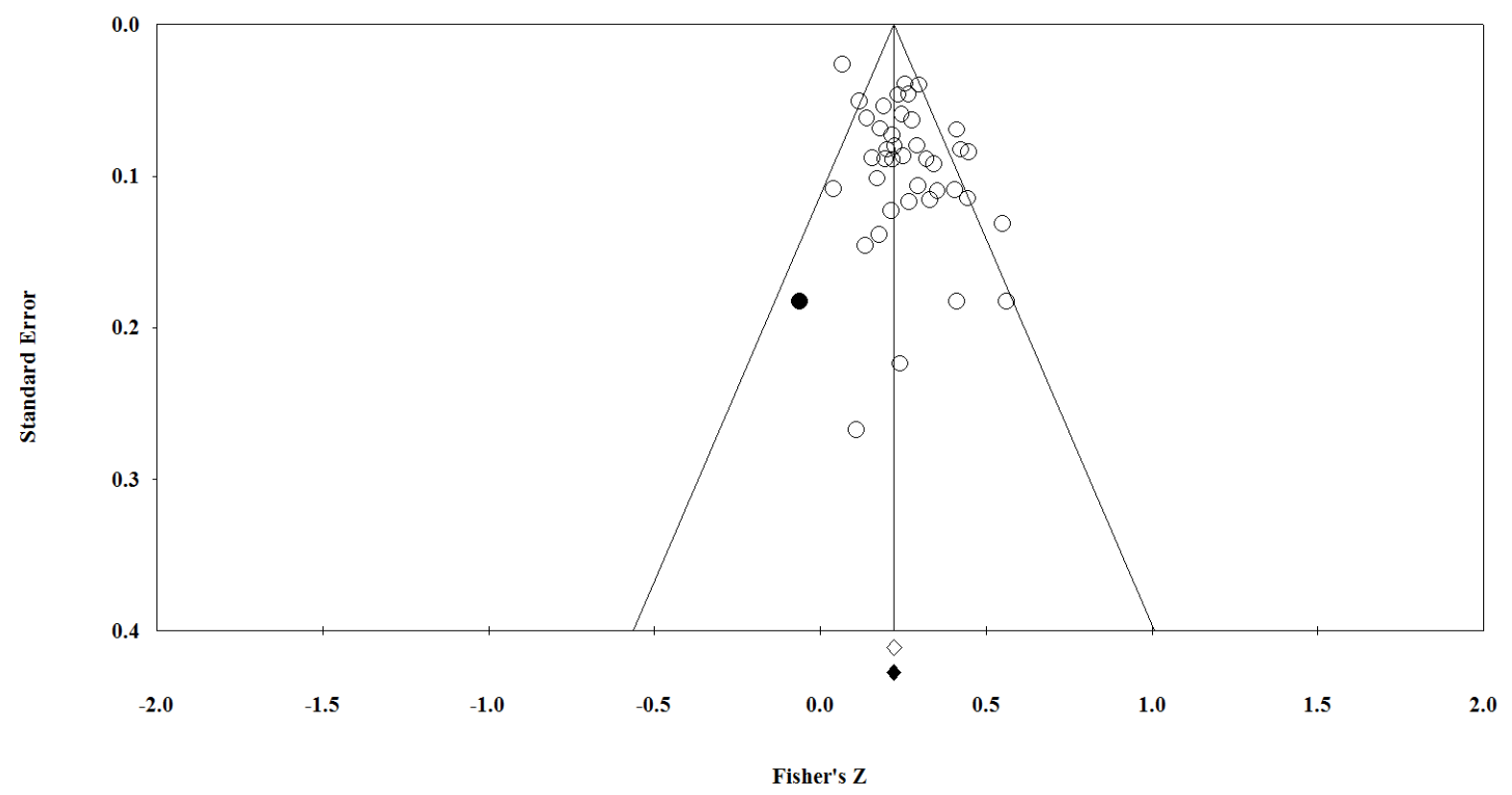

Figure 31. Funnel plot of the standard error by Fischer's $\mathrm{Z}$ for the association between negative perfectionism and suicidal thoughts and behaviour

Input of the individual study effect sizes into a funnel plot revealed significant asymmetry (Figure 31), suggesting publication bias. Specifically, there were a 
disproportionate number of studies that fell within the top right section of the funnel, suggesting a bias towards studies with larger samples and greater effect sizes. This was supported by Egger's regression intercept ( $p=.003)$, but not by Kendall's tau $(p=.65)$. Subsequently, Duval and Tweedies Trim and Fill method was used to assess how much influence the bias may have had on the calculated summary effect. This resulted in one trimmed studied and an adjusted effect size almost identical to the calculated effect $(r=.25$; $.21-.28,95 \% \mathrm{CI})$. In addition, Orwin's fail safe $\mathrm{N}$ indicated that a further 50 studies with negligible effect sizes would have to be identified in order to deem the summary effect also negligible. It is unlikely that further 50 studies exist, therefore it is reasonable to have confidence that the calculated effect size is not solely a product of selective publishing. Overall, this meta-analysis clearly demonstrates a moderate relationship between negative perfectionism and suicidality.

\section{Positive Perfectionism and Suicidal Thoughts and Behaviours}

Finally, the relationship between positive perfectionism and suicidal thoughts and behaviours was explored. Thirty one samples from 28 articles were included in this metaanalysis (Figure 32).

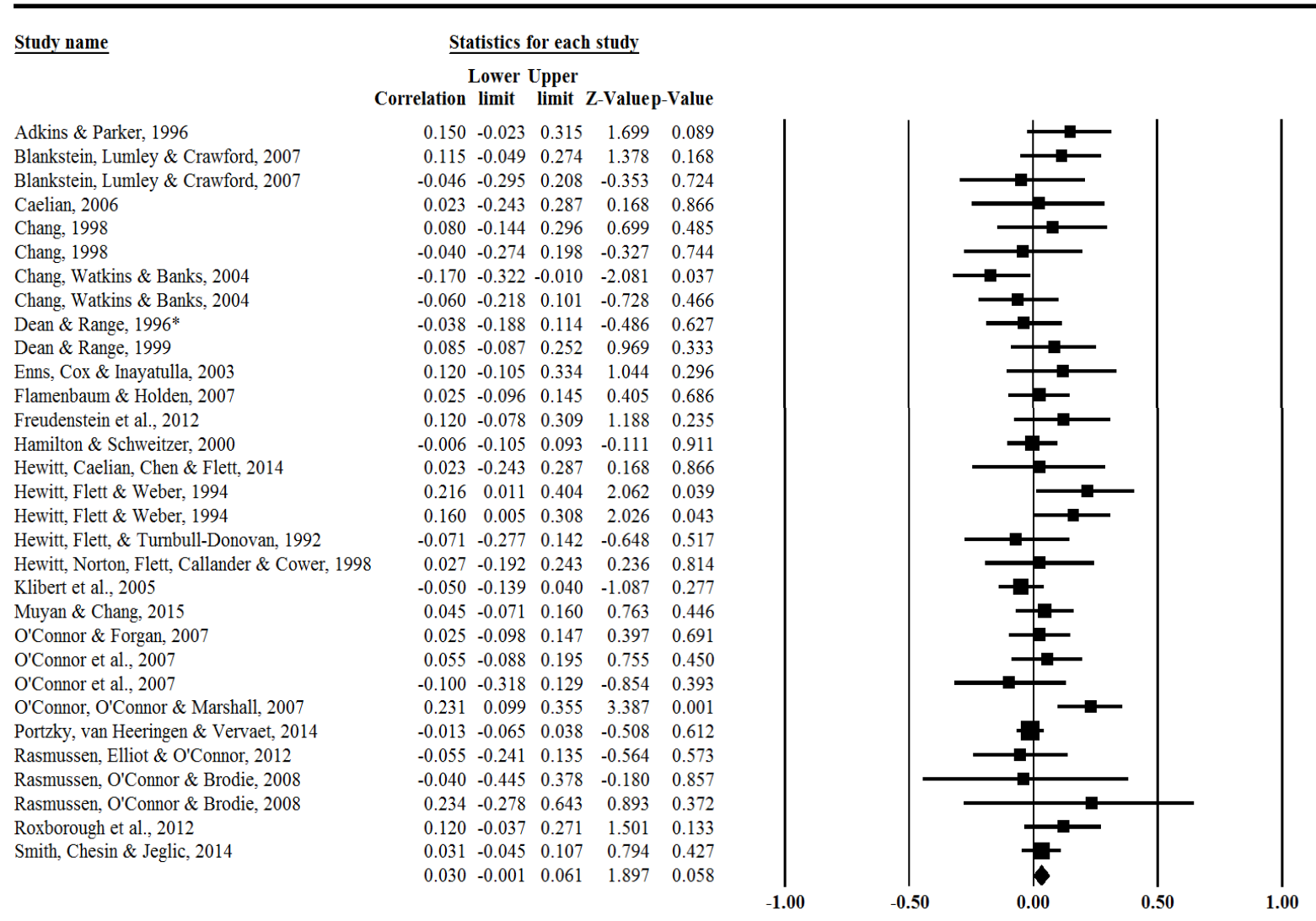

Figure 32. Forest plot with effect sizes for positive perfectionism and suicidal thoughts and behaviour 
Again, most samples were mixed sex samples and about half were clinical samples. Five consisted of only individuals with a previous suicide attempt. Again, the most reported facet of suicidal thoughts and behaviours was suicidal ideation. Similar to the relationship between positive perfectionism and self-injury, the summary effect for the relationship between positive perfectionism and suicide was very small and, by Cohen's guidelines (1978), would be considered negligible $(r=.03 ;-.00-.06,95 \% \mathrm{CI})$ and was non-significant $(p=06)$. Effect sizes ranged from -.17 (Chang et al., 2004) to .23 (Rasmussen et al., 2008). The Q-statistic indicated no significant heterogeneity between effect sizes $\left(Q(30)=39.00, p=.13 ; \mathrm{I}^{2}=\right.$ 23.05) and further analyses did not identify any significant moderators.

Analyses testing for publication bias did not indicate any bias in the studies included. Specifically, the included studies produced a symmetrical funnel plot (Figure 33) and both Egger's regression intercept and Kendall's tau were non-significant ( $p=.19$ and $p=.84$ respectively). Finally, despite a minimal summary effect, the classic fail safe $\mathrm{N}$ indicated that a further 11 studies with no relationship between the variables would need to be identified to bring the summary effect to 0 . Consistent with the overall self-injury-suicide meta-analysis this meta-analysis suggests that there is no significant direct relationship between positive perfectionism and suicidality.

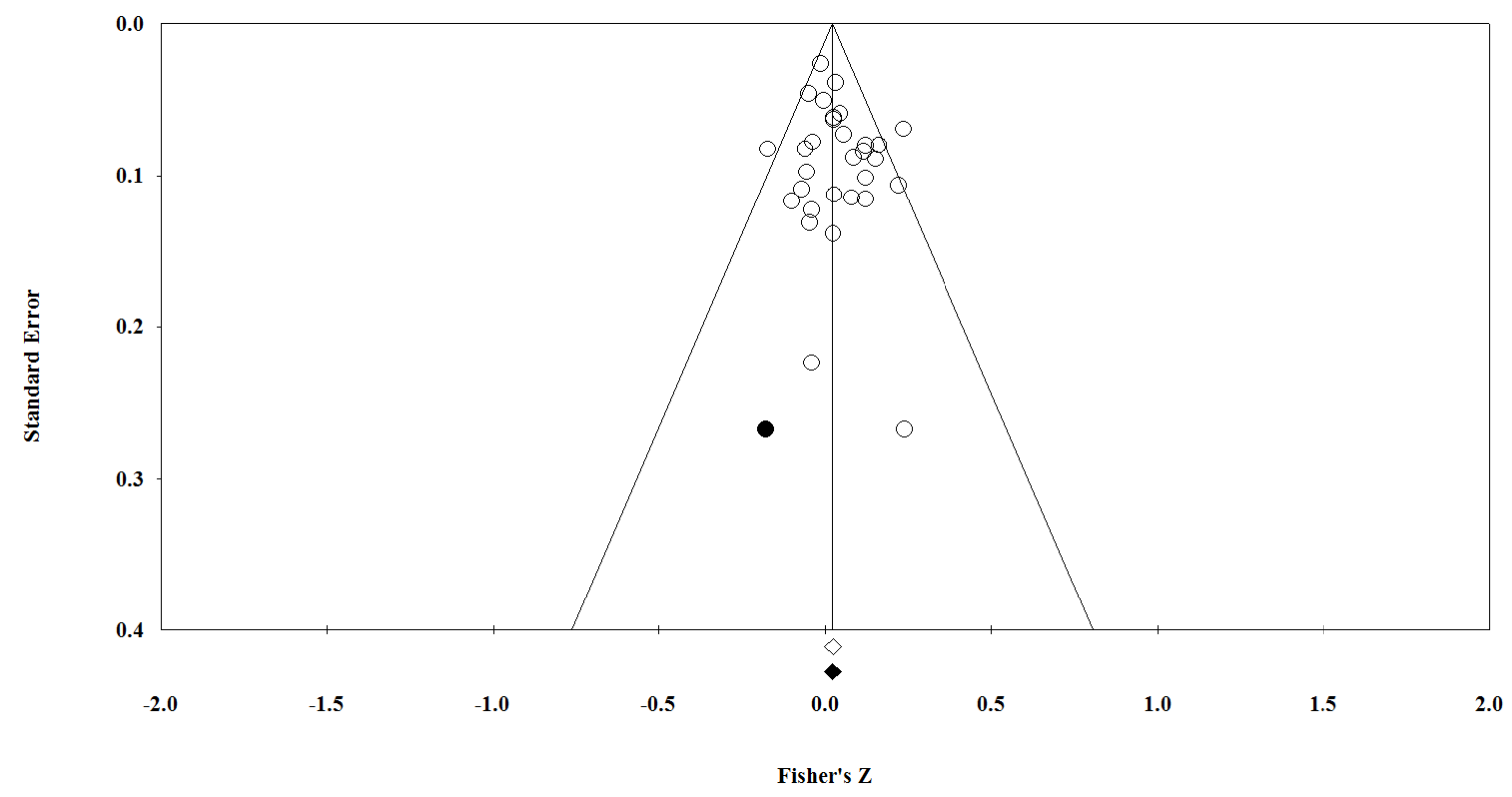

Figure 33: Funnel plot of the standard error by Fischer's Z for the association between positive perfectionism and suicidal thoughts and behaviour

\section{Summary and Conclusions}

The aim of Study $1 \mathrm{~b}$ was to fully explore and synthesise all existing literature on the 
relationship between perfectionism and self-injury. However, as this body of literature is very small, perfectionism-suicide research was also investigated with the aim of understanding the broader relationships between perfectionism and self-injurious and suicidal behaviours. Meta-analyses revealed that negative perfectionism is significantly associated with increased self-injurious and suicidal thoughts and behaviours. Consistent with hypotheses, and the results of Study 1a, these results suggest that negative perfectionism is indeed negative and moreover can predict risk for self-injury and suicide. Summary effects of the relationships between perfectionism and both self-injury and suicide were relatively statistically weak at .15 and .25 respectively. In other words, negative perfectionism contributes to approximately $2-6 \%$ of the variance associated with self-injury/suicide. To conclude, this suggests that, although negative perfectionism is consistently related to both self-injury and suicide (consistent with O'Connor's (2007) systematic review), it is likely that negative perfectionism is only one of multiple contributing factors in the progression to such maladaptive behaviours. Due to the serious nature of self-injury, and especially suicide these findings carry substantial clinical importance with regard to individual psychopathology and risk, regardless of their relatively weak statistical relationships.

Positive perfectionism was not-significantly associated with self-injury or suicidal thoughts and behaviours. These results suggest that positive perfectionism does not contribute to risk, nor protect against risk for self-injury or suicide. This conflicts with research suggesting that positive perfectionism may buffer against such outcomes (e.g., Chang et al., 2004; Flett et al., 2012; Hoff \& Muehlenkamp, 2009) and is inconsistent with Study 1a metaanalytic results, indicating that positive perfectionism is weakly associated with maladaptive behaviours or outcomes. However, the absence of significant effects is consistent with the mixed, and largely non-significant results, in O'Connor's (2007) systematic review of the relationship between perfectionism and suicide.

Based on the literature review, and inspection of individual study data, it was expected that the relationship between perfectionism and self-injury/suicide may differ according to demographic factors such as sex and sample type (clinical/non-clinical). Moderator analyses revealed that sex moderated the relationship between negative perfectionism and suicidal thoughts and behaviours. Specifically, the relationship between negative perfectionism and suicide was strongest for males, followed by female samples, and finally combined samples. However, as previously noted (see p. 111), there were only two male samples, which makes it difficult to determine whether this difference was due to sex or another unrelated study characteristic. In addition, age was a significant moderator of the relationship between 
negative perfectionism and self-injury. Specifically, analysis indicated that negative perfectionism was more strongly related to self-injury in older samples (university and adult samples) than it was in adolescents. This could suggest a maladaptive trajectory of perfectionism related risk across the life span. Further moderator analyses did not reveal any other systematic differences based on sample demographics. In summary, Study $1 \mathrm{~b}$ provides support for the detrimental nature of negative perfectionism specifically with regard to selfinjury and suicide risk. This could provide support for theories such as Baumeister's (1990) Escape from Self theory and the PSDM of suicide (Hewitt et al., 2006) which both propose that perfectionistic cognitions and behaviours can increase an individual's risk of suicide through perceived isolation, a sense of hopelessness, and a need to escape the associated distress. In addition, the results lead to further questioning of the true nature of positive perfectionism. Instead of buffering against the risk of maladaptive behaviours and outcomes, as commonly suggested in the early literature, or having a detrimental effect, as suggested by Study 1a meta-analyses, positive perfectionism seems to be largely unrelated to risk of selfinjury and suicide.

\section{Combined Study 1 Discussion}

Study 1 (1a and 1b) comprised the systematic reviews and meta-analytic summaries of existing perfectionism literature. This included both research that investigated perfectionism and broad mental health and wellbeing outcomes (however limited to research conducted with the FMPS; Study 1a), and research investigating the relationship between perfectionism and self-injurious and suicidal thoughts and behaviours (Study 1b).

To summarise, the meta-analyses demonstrated a robust relationship between negative perfectionism and poor mental health outcomes and maladaptive behaviours, including selfinjury and suicide. This suggests that negative perfectionism is inherently detrimental and is likely to have a negative effect on individual wellbeing. In contrast, according to these results, the prevailing conceptualisation of positive perfectionism as an adaptive trait may be somewhat unfounded. Instead, positive perfectionism appears to be associated with both positive (e.g., positive coping strategies and life satisfaction), and negative outcomes (e.g., $\mathrm{ED}, \mathrm{OCD})$, but is also often not significantly associated with the outcome in question.

In terms of self-injury and suicide, Study $1 b$ indicated that negative perfectionism is associated with increased risk of self-injury and even more so, increased risk of suicidal thoughts and behaviours. Whilst neither of these relationships would be considered strong at .15 and .25 for self-injury and suicide respectively, they are neither insignificant nor 
meaningless. Together, they suggest that negative perfectionism should be an important focus when considering an individual's risk of self-injurious and suicidal thoughts and behaviours.

\section{Strengths}

As previously outlined (see p. 51-53) meta-analytic techniques provide a method for summarising a large quantity of data in order to gain an overall understanding of the relationship between two variables. In this case, meta-analyses provided summary correlations for the relationships between negative perfectionism and multiple adaptive and maladaptive outcomes including self-injury and suicide. The equivalent was provided for relationships with positive perfectionism. Therefore, Study 1 provides easy access to a large sum of information that otherwise would have been comparatively inaccessible. Another strength of this Study is the ability to combine results from multiple studies in order to investigate systematic variance in relationships according to differing samples and methodology. As such, differences according to the measure of perfectionism, specific outcome variables, sex, age, and sample type were identified.

\section{Limitations}

It is important to note that these findings provide a strong basis for further research but are also limited in a number of ways. Recognition of these limitations will ensure that the findings are interpreted within reasonable limits and no unfounded claims are made. First, it is important to note that research of a cross-sectional, correlational nature, such as the research included in these meta-analyses, is often criticised due to its limitations regarding information about causality (Spector, 2013). That is, purely cross-sectional research does not allow for conclusions about which factor develops first and whether one factor is implicated in the development of the other. In this case, whether perfectionism increases risk of future selfinjury or suicide, or conversely, self-injury or suicide predicts subsequent increases in perfectionism. Therefore, although the findings provide a base understanding of the relationship between perfectionism and mental health outcomes, including self-injury and suicide, additional research is required. In particular, research investigating the mechanisms behind the association between negative perfectionism and self-injury, and longitudinal research exploring the causal nature of the relationships, will enable the findings to be more effectively used in self-injury intervention and prevention strategies.

Second, research using direct correlations is also limited as it does not allow for the consideration of potential mediators, and control of third variables. This is particularly problematic given the strong relationship between positive and negative perfectionism. With 
correlation methods used, it is impossible to determine the unique variance associated with positive or negative perfectionism with regard to the outcome in question. In future research, it would be beneficial to instead use partial correlations and recalculate each relationship whilst controlling for the countering form of perfectionism (e.g., controlling for positive perfectionism when examining the relationship between negative perfectionism and depression). Stoeber and Otto (2006) effectively used this method in their review of positive perfectionism and concluded that, when negative perfectionism is controlled for, positive perfectionism appears to be significantly more adaptive. The notion that the adaptive component of positive perfectionism is often masked by negative perfectionism was recently supported by some of the leading researchers in the field (for a comprehensive discussion on partialing out the effects of each form of perfectionism see Stoeber \& Gaudreau, 2017). Given that these meta-analyses were only one part of this thesis, it was deemed that further calculations, controlling for each form of perfectionism, were beyond the scope of this project.

\section{Conclusion}

In conclusion, these meta-analyses have successfully highlighted the differential relationships between negative and positive perfectionism and various mental health and wellbeing outcomes. Overall, the results suggest that negative perfectionism, rather than positive perfectionism, should be the focus in prevention and intervention efforts. Additional positive perfectionism research will be theoretically useful, however negative perfectionism research is likely to be more fruitful in terms of increasing the efficacy of clinical practice and promoting individual wellbeing.

Study 1 has effectively set the scene for the empirical component of this thesis, examining perfectionism and NSSI in NZ adolescents. It has also highlighted the limitations of direct correlational research especially with regard to inferring causality and understanding the likely competing influences of positive and negative perfectionism. As such, further studies in this thesis aim to investigate the intricate details of the association between perfectionism and NSSI, and if, as expected, the cross-sectional relationship is significant, further research will explore the mechanisms underlying the relationship between negative perfectionism and NSSI. 


\section{CHAPTER SIX: PERFECTIONISM IN A NEW ZEALAND ADOLESCENT SAMPLE}

This research will now progress to investigating the relationship between perfectionism and NSSI in a NZ community adolescent sample. Research thus far has indicated that both perfectionism and NSSI have robust associations with many indicators of poor mental health. Moreover, adolescents appear to be a particularly at-risk population, with higher rates of NSSI in adolescents than in adults (see p. 10-12; Swannell et al., 2014), and anecdotal reports that adolescents feel increasing pressure with regard to their appearance and academic, social and extra-curricular achievements. Therefore, it is reasonable to expect a concomitant a rise in adolescent perfectionism (refer to p. 31-35 for further discussion). Having conducted a thorough literature review and multiple meta-analytic studies, it is clear that there are still some clear gaps in the research regarding perfectionism and NSSI. For example, some previous studies have outlined the relationship between perfectionism and NSSI in large samples of adolescents, however few have investigated the relationship across different demographic groups (e.g., males versus females, samples outside of the USA and UK). In addition, although a fairly robust relationship between negative perfectionism and self-injury was identified meta-analytically, few studies have explored the possible mechanisms underpinning this relationship (see Claes et al., 2012 and O'Connor et al., 2010 for exceptions).

Therefore, Studies 2, 3, and 4 aim to address these gaps by first exploring appropriate assessment of perfectionism in NZ adolescents, followed by an investigation of the crosssectional relationships between perfectionism and NSSI behaviours and functions and, finally, exploration of the longitudinal nature of any significant relationships. In these empirical chapters, perfectionism will be measured using the Frost Multidimensional Perfectionism Scale (FMPS; Frost et al., 1990) and self-injury using the Deliberate Self-Harm Inventoryshort (DSHI-s, Lundh et al., 2007). Whilst the FMPS is the most widely used perfectionism scale (see Study 1a), there has been some debate about the structure of perfectionism in adolescent samples (e.g., Cox, Enns, \& Clara, 2002; Hawkins et al., 2006; Parker \& Stumpf, 1995). Therefore, Study 2 will focus on investigating the psychometric properties of the FMPS, and identifying the most appropriate conceptualisation of the underlying components for NZ adolescents.

\section{Study Two: Using the Frost Multidimensional Perfectionism Scale in a Sample of New Zealand Community Adolescents}

Study 2 will briefly highlight previous scales used to measure perfectionism, followed 
by an outline of the Frost Multidimensional Perfectionism Scale (FMPS, Frost et al., 1990) and the debated nature of its underlying factor structure. By clarifying the nature of perfectionism in adolescents Study 2 aims to assist other researchers' in their measurement and understanding of perfectionism in similar samples.

\section{Measuring Perfectionism}

Prior to the development of the FMPS, there was some recognition of the significant relationships between perfectionism and various negative outcomes (e.g., depression, OCD; Pacht, 1984 in Frost et al., 1990). However, the value of the research was limited due to a lack of consensus about the definition and nature of perfectionism (Enns \& Cox, 2002).

Additionally, there were no available measures that focused on all identified components and reliably captured perfectionism across diverse populations. Rather, existing measures such as the BPS (Burns, 1980), and subscales within the EDI (Garner et al., 1983), DAS (Weissman $\&$ Beck, 1978) and IBT (Jones, 1969), had different points of focus and varying conceptualisations of perfectionism. Frost and colleagues recognised this issue and subsequently consolidated the available literature and existing scales to form a more holistic picture of perfectionistic cognitions, traits and behaviours. In doing so they developed the FMPS, which has since become the most frequently used measure of perfectionism (Enns \& Cox, 2002; Flett \& Hewitt, 2015; also see Study 1a).

\section{The Frost Multidimensional Perfectionism Scale}

The FMPS is a 35-item self-report measure designed to comprehensively measure multiple dimensions of perfectionism (Frost et al., 1990). Original analyses with a sample of female, American undergraduates, identified six subscales (Concern over Mistakes [CM], Doubts about Actions [DA], Personal Standards [PS], Organisation, Parental Expectations [PE], and Parental Criticism [PC]) with Cronbach's alphas ranging from .77 to .90 indicating at least satisfactory internal reliability. Due to low inter-correlations with other subscales, Frost and colleagues recommended that the Organisation subscale should be excluded from the total perfectionism score. In the second part of their study, with another sample of female undergraduates, Frost and colleagues reported that the FMPS had better reliability than any of the previous scales (BPS; Burns, 1980) or subscales (EDI; Garner et al., 1983; IBT; Jones, 1969) used to measure perfectionism, as well as good convergent validity, and a replicable six-factor structure in the new sample. Finally, supporting early ideas that perfectionism may be related to negative outcomes, Frost and colleagues demonstrated that total perfectionism, $\mathrm{CM}$ and DA, were all significantly associated with a measure of depression. 
Following the development of the FMPS, there has been ongoing controversy regarding the FMPS's applicability to diverse populations. Although most researchers agree that perfectionism is multidimensional, there is little agreement on the most valid and theoretically useful conceptualisation of its underlying components. Subsequent researchers have proposed anywhere from Frost's and colleagues' original six-factor model, as described above (e.g., Parker \& Adkins, 1995; Parker \& Stumpf, 1995), right down to only two factors, representing positive and negative perfectionism (e.g., Chang et al., 2004; Cox et al., 2002; Khawaja \& Armstrong, 2005). Given the importance of appropriate measurement of perfectionism to this thesis, the various conceptualisations of the FMPS are briefly described and explored below.

Six factor structure. Early psychometric analyses of the FMPS looked promising with regard to Frost and colleagues' (1990) proposed six-factor structure. For example, Parker and Stumpf (1995) broadly replicated the six factors in a sample of academically talented children. Analyses revealed that Frost's six factor solution provided a moderate fit to the data $\left(\chi^{2}(545)=2117, \chi^{2} /\right.$ d.f. $=3.88$; Goodness of Fit Index $\left.[\mathrm{GFI}]=.86\right)$, and that slight variation could provide a better fit $\left(\chi^{2}(545)=1585, \chi^{2} /\right.$ d.f. $\left.=2.91 ; \mathrm{GFI}=.90\right)$. The final model retained six factors almost identical to Frost and colleagues' findings, except for two items (Items 4 and 26), which loaded on different factors. Internal reliabilities for the subscales and the overall perfectionism score ranged from .67 (DA) to .90 (Organisation); only slightly lower than those found in Frost's undergraduate sample. Parker and Stumpf also provided evidence for both an adaptive and a maladaptive element of perfectionism through their relation to key personality traits. Specifically, PS and Organisation were deemed adaptive through a positive association with conscientiousness while DA, CM and PC were deemed maladaptive due to a positive association with neuroticism.

Similarly, Parker and Adkins (1995) reported a six-factor structure in a sample comprised of undergraduate students. For five of the subscales they reported internal reliability scores similar to those found by Frost and colleagues $(.72$ - $.95 ; 1990)$. However, the internal reliability of the PE subscale was poor at only .57. Only one item did not load on its respective subscale (Item 15). Additionally, a number of items cross-loaded (above .40). Overall, Parker and Adkins decided to retain all the items in a six factor structure and concluded that the FMPS is "psychometrically sound" (p. 323) for use with undergraduate samples.

Five factor structure. Since Parker and colleagues' $(1995,1995)$ research, few other researchers have provided convincing support for the six factor structure. For example, both 
Cox, Enns and Clara (2002) and Stallman and Hurst (2011) have suggested that a lack of distinction between PE and PC calls for one combined subscale representing Parental Perceptions (PP). Thus, an argument for a five-factor model has arisen. Similar to Parker and Adkins (1995), in a sample of clinical out-patients, Cox and colleagues noted that many items load highly on more than one subscale. Consequently, after removing the cross-loading items, they proposed a 22-item modification of the scale (Brief FMPS; see Cox et al., 2002, p. 368). The Brief FMPS correlated highly ( $r$ 's from .86 to .97) with the original FMPS subscales and most subscales evidenced similar levels of internal reliability to previous studies (.63-.90). Further analyses indicated that a structure formed with the Brief FMPS and Brief HFMPS and representing the overarching positive and negative components of perfectionism (as proposed by Frost et al., 1993) demonstrated a good fit on three out of five Confirmatory Factor Analyses (CFA) fit indices for both the university and clinical samples (University: $\chi^{2}(457)=918.82$, Comparative Fit Index $[\mathrm{CFI}]=.91$; GFI $=.83$, Adjusted Goodness of Fit Index $[\mathrm{AGFI}]=.81$, Tucker Lewis Index $[\mathrm{TLI}]=.90$, Root Mean Square Error of Approximation $[\mathrm{RMSEA}]=.06$; Clinical: $\chi^{2}(457)=1075.18, \mathrm{CFI}=.91 ; \mathrm{GFI}=.85$, AGFI $=$ .83 , TLI $=.90$, RMSEA $=.06)$. In comparison, the equivalent model using the full FMPS and HFMPS did not demonstrate a good fit on any. Overall, Cox and colleagues (2002) supported a five-factor structure for the Brief FMPS, in which the five factors could be further groups into second-order factors corresponding to positive and negative perfectionism.

Finally, in an Australian undergraduate sample, Stallman and Hurst (2011) also reported a five-factor model whereby PE and PC were combined into 'Parenting'. Their model also proposed a smaller item set with the exclusion of six items due to low factor loadings (below 0.45, Items 4, 5, 16, 17, 18 and 26). Internal reliabilities of the five subscales ranged from .76 (DA) to .90 (Organisation, and Parenting) and CFA indicated a moderate fit $\left(\chi^{2}(356)=5018.68, p<.001 ; \mathrm{CFI}=.93 ; \mathrm{RMSEA}=.06\right)$.

Four factor structure. Some researchers have taken the five factor model and further reduced it to four (Hawkins et al., 2006; Stumpf \& Parker, 2000). This typically involves the amalgamation of CM and DA items. Stumpf and Parker (2000) first proposed four factors in a sample of academically talented children and subsequently replicated the model with undergraduate students. They reported that Concerns and Doubts (CMD; CM and DA combined) was positively associated with neuroticism and negatively associated with selfesteem, whereas PS and Organisation were positively associated with conscientiousness. These findings led them to explore second-order factors from which, consistent with Cox and colleagues (2002), they found support for higher healthy (positive; Organisation and PS) and 
unhealthy components (negative; $\mathrm{PE} / \mathrm{PC}$ and $\mathrm{CM} / \mathrm{DA}$ ) of perfectionism.

Hawkins and colleagues (2006) conducted some of the first research on the FMPS using community adolescents. In their all-female sample, they also broadly replicated the above four factors (Cronbach alphas ranging from .76 to .87) with only minor exclusions due to cross loadings (items 16 and 18). In addition, Hawkins and colleagues explored the possibility of two higher-order factors. They reported that PS correlated with all three other factors and, importantly, it correlated most strongly with CMD, rather than Organisation as would be expected if they were to make up a healthy perfectionism factor. This finding, along with low internal reliability scores (.45 for healthy perfectionism and .66 for unhealthy perfectionism), led Hawkins and colleagues to conclude that higher-order factors were not appropriate for their sample.

Three factor structure. Another less commonly proposed, although plausible, factor structure resulted from Purdon, Antony and Swinson's (1999) research with a sample of diagnosed anxiety disorder patients. Purdon and colleagues described a further-reduced three factor structure comprised of Fear of Mistakes (CM and DA), Goal Achievement Orientation (PS and Organisation), and Perceived Parental Pressure (PC and PE), with reliabilities of .91, .85 , and .91 respectively. They argue that, despite finding support for the original six factor structure, the last three factors of the six factor structure only accounted for minimal variance in the model suggesting the model was over-extracted, and a three factor was more statistically appropriate. However, it is important to note that, to my knowledge, the three factor structure has not been replicated in any other sample. Kantack (2014) calculated subscale scores based on Purdon and colleagues' proposed factor structure, but did not provide any psychometric analyses to support its use. Moreover, Gelabert and colleagues (2011) tested Purdon and colleagues' (1999) three factor structure along with the original six factor structure (Frost et al., 1990) and a four factor structure (Stoeber, 1998), and found that the six factor model demonstrated the best fit, followed by the four factor model and finally the three factor model.

Two factor structure. Finally, and arguably most importantly, many researchers have supported Frost and colleagues' (1993) argument for an overarching two-factor structure that represents positive and negative perfectionism. As evidenced above, the two factor structure is often hierarchical in nature with a more specific structure identifiable below (e.g., Cox et al., 2002; Stumpf \& Parker, 2000). Only Khawaja and Armstrong (2005) have conducted a first-order factor analysis of the individual scale items rather than of the predetermined subscales. In their sample of Australian undergraduates they found 17 items contributed to 
positive (Items 2, 7, 8, 27, 29, 31) or negative perfectionism (Items 4, 9, 12, 13, 14, 17, 18, $21,23,25,34)$ with internal consistency scores of .89 and .91 respectively.

Overall, the existence of positive and negative facets of perfectionism has been broadly supported in adult clinical (e.g., Cox et al., 2002), undergraduate samples (e.g., Frost et al., 1993; Stallman \& Hurst, 2011), adolescent non-clinical samples (e.g., Luyckx et al., 2008; Stumpf \& Parker, 2000), and samples of children (e.g., Parker \& Stumpf, 1995). Given that the focus of this thesis is adolescents, the following section will attend to evidence for or against the two factor model of perfectionism in adolescents.

\section{The Structure of Positive and Negative Perfectionism in Adolescents}

In a sample of Belgium adolescents and undergraduates, Luyckx and colleagues (2008) investigated perfectionism and identity exploration in relation to well-being, using the CM and DA subscales of the FMPS to represent negative perfectionism, and PS to represent positive perfectionism. Their model was subject to CFA and showed an adequate fit to the data (adolescent: $\chi^{2}(169)=489.36, p<.001 ; \mathrm{CFI}=.93$; RMSEA $=.07$; undergraduate: $\left.\chi^{2}(169)=456.65, p<.001 ; \mathrm{CFI}=.94 ; \mathrm{RMSEA}=.08\right)$. Moreover, they reported good internal consistency scores for both positive perfectionism $(\alpha$ 's $>=.75)$ and negative perfectionism (both $\alpha$ 's $=.87$ ). Luyckx and colleagues indicated that negative perfectionism predicted lower scores on positive facets of identity exploration (e.g., commitment making and identification with commitment) and predicted higher scores on negative facets of identity exploration (e.g., ruminative exploration). Conversely, positive perfectionism predicted lower scores on negative facets and higher scores on positive facets of identity exploration. Further, negative perfectionism was associated with more depressive symptoms and positive perfectionism was associated with greater academic adjustment.

In another study with adolescents, Thorpe and Nettlebeck (2014) first identified a four factor solution using Exploratory Factor Analysis (EFA), and subsequently conducted a second-order factor analysis resulting in two factors. Whilst Thorpe and Nettlebeck were confident with the reliability of positive perfectionism, they were less certain of the clarity of negative perfectionism in their sample. The main concerns were the strong negative loadings of PEC (PE + PC) items on positive perfectionism and CMD (CM + DA) items that loaded strongly and positively on both positive and negative perfectionism. However, the evidence supported a two-factor solution overall. Further, they reported that positive perfectionism was positively related to higher grades over and above intelligence and conscientiousness, and that negative perfectionism was associated with lower academic achievement. 
It is important to note that there is research to suggest that perfectionism operates and manifests differently in males and females (Blankstein et al., 2008; Blankstein \& Winkworth, 2004; Klibert et al., 2005 see p. 36-37), however there has not been any investigation of whether the construct and therefore whether the structure of the components of perfectionism differ for males and females. Thus far research on the FMPS has been conducted with mixed samples (e.g., Cox et al., 2002) and female only samples (e.g., Hawkins et al., 2006), however research has not yet been conducted with a male only sample. Moreover, no one has directly compared the structure of the FMPS across males and females.

Finally, investigation of the psychometric properties of the FMPS has been hindered by poor reporting of internal reliability scores. Specifically, Ha, Lee and Puig (2010) found that only $40 \%$ of the authors of 145 perfectionism studies reported overall, or subscale, coefficient alphas in their articles ${ }^{30}$. This concerning lack of internal reliability information raises questions as to whether alphas have been mistakenly unreported, or whether authors have chosen not include them due to misunderstanding of the importance of scale consistency or even due to undesirable values (Caruso, 2000). Unfortunately, this gap in information adds a further caveat to a significant amount of published research on perfectionism.

Against this background, Study 2 will aim to provide evidence for or against the use of the FMPS in a NZ community adolescent sample. The psychometric properties of the FMPS will be explored to identify the most appropriate factor structure for a NZ adolescent sample and investigate structural differences across males and females. Whilst it is difficult to predict which rudimentary factor structure will best suit the sample, it is expected that the resulting factors will fit into higher order components representing positive and negative perfectionism. Overall, this study aims to support valid measurement of perfectionism, and encourage valid research on the relationship between perfectionism and NSSI. It will also provide the basis for any future perfectionism research with $\mathrm{NZ}$ adolescents.

\section{Method}

The following research (Studies 2, 3, and 4) was conducted as part of the Youth Wellbeing Study (YWS). The YWS is a large scale longitudinal survey which primarily focuses on the nature of NSSI in young people. Some of the areas of interest include, why adolescents begin to injure themselves, what functions self-injury play for adolescents, why

\footnotetext{
${ }^{30}$ In a meta-analysis of those that have reported internal consistency, Doubts about Actions was reported as the least reliable subscale, while Organisation is consistently the most reliable. Ha and colleagues also report that higher internal reliabilities were associated with older samples, samples with higher proportions of 'White' and female participants, and greater standard deviations on the FMPS (Ha et al., 2010).
} 
adolescents stop engaging in self-injury, and how parents, school staff, and the wider community can support adolescents who may be at risk of poor outcomes. In addition to NSSI, the YWS also investigates other factors associated with youth wellbeing, including perfectionism, depression, anxiety, eating attitudes, self-esteem, and socio-economic factors. Some constructs (e.g., emotion regulation, depression, NSSI) were assessed at all three time points, while others (e.g., eating disordered attitudes, impulsivity and, importantly, perfectionism) were assessed intermittently.

In early 2011, 45 Wellington, Wairarapa and Kapiti Coast secondary schools were invited to participate in the YWS, of which 15 schools became involved ${ }^{31}$. Each year, students within these schools were provided with an information and consent pack, through which parent or caregiver consent was requested. Overall, caregiver consent was received for 1190 adolescents. A further 356 student's parents or guardians actively denied consent. Upon receiving parental consent (only required once across multiple survey administrations) students were invited to participate in the research. Student participation was voluntary.

\section{Participants}

Participants for Study 2 were 930 adolescents (78\% of the students for whom consent was received) from the Greater Wellington region. For some students this was their second time filling out the survey (the FMPS was first introduced in Wave 2 of the longitudinal study), whilst others were participating for the first time. The sample comprised of 381 males (41\%) and 543 females (59\%). A further three participants did not indicate their gender, and three endorsed trans-gender. Participants range in age from 13 years to 17 years $(M=14.50$ years, $S D=1.29$ ), however averaged 14 years old. The majority of the sample endorsed Pākehā (NZ/European; 72\%) as their main ethnic identification, followed by Māori (7\%) and Samoan (3\%). Nine percent of the sample endorsed 'Other' and 5\% endorsed more than one ethnicity and subsequently indicated that they could not choose a primary ethnicity. Overall, Māori (14\% of total NZ population) and Pacific ethnic groups (7\% of total NZ population) were particularly underrepresented according to national demographic information (2013 NZ Census, Statistics New Zealand, 2014).

\footnotetext{
${ }^{31}$ The schools that became involved are broadly representative of all of the schools in the Greater Wellington region. Both the group of schools that were involved, and the group of schools that declined involvement had average decile ratings of 7 .
} 


\begin{tabular}{|c|c|c|c|c|c|}
\hline $\begin{array}{l}\text { 13. Please read each statement, and select the option that best describes } \\
\text { how much you agree or disagree with each one. }\end{array}$ & 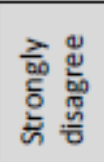 & 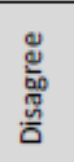 & 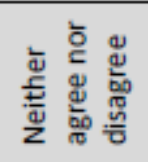 & $\stackrel{\Xi}{\sharp}$ & 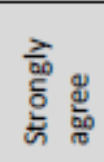 \\
\hline My parents/whänau set very high standards for me & $\square$ & $\square$ & $\square$ & $\square$ & $\square$ \\
\hline Organization is very important to me & $\square$ & $\square$ & $\square$ & $\square$ & $\square$ \\
\hline As a child, I was punished for doing things less than perfect & $\square$ & $\square$ & $\square$ & $\square$ & $\square$ \\
\hline $\begin{array}{l}\text { (Continued...) Please read each statement, and select the option that } \\
\text { best describes how much you agree or disagree with each one. }\end{array}$ & 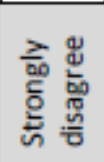 & 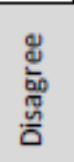 & 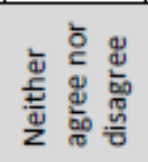 & $\stackrel{\Xi}{\&}$ & 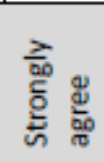 \\
\hline $\begin{array}{l}\text { If I do not set the highest standards for myself, I am likely to end up a } \\
\text { second-rate person }\end{array}$ & $\square$ & $\square$ & $\square$ & $\square$ & $\square$ \\
\hline My parents/whänau never try to understand my mistakes & $\square$ & $\square$ & $\square$ & $\square$ & $\square$ \\
\hline It is important to me that I am thoroughly competent in everything I do & $\square$ & $\square$ & $\square$ & $\square$ & $\square$ \\
\hline I am a tidy person & $\square$ & $\square$ & $\square$ & $\square$ & $\square$ \\
\hline I try to be an organized person & $\square$ & $\square$ & $\square$ & $\square$ & $\square$ \\
\hline If I fail at work/school, I am a failure as a person & $\square$ & $\square$ & $\square$ & $\square$ & $\square$ \\
\hline I should be upset if I make a mistake & $\square$ & $\square$ & $\square$ & $\square$ & $\square$ \\
\hline My parents/whänau want me to do the best at everything & $\square$ & $\square$ & $\square$ & $\square$ & $\square$ \\
\hline I set higher goals than most people & $\square$ & $\square$ & $\square$ & $\square$ & $\square$ \\
\hline $\begin{array}{l}\text { If someone does a task at work or school better than me, the } \\
\text { failed the whole task }\end{array}$ & $\square$ & $\square$ & $\square$ & $\square$ & $\square$ \\
\hline If I fail partly, it is as bad as being a complete failure & $\square$ & $\square$ & $\square$ & $\square$ & $\square$ \\
\hline Only outstanding performance is good enough in my family/whānau & $\square$ & $\square$ & $\square$ & $\square$ & $\square$ \\
\hline I am very good at focusing my efforts on attaining a goal & $\square$ & $\square$ & $\square$ & $\square$ & $\square$ \\
\hline $\begin{array}{l}\text { Even when I do something very carefully, I often feel that it is not quite } \\
\text { right }\end{array}$ & $\square$ & $\square$ & $\square$ & $\square$ & $\square$ \\
\hline I hate being less than the best at things & $\square$ & $\square$ & $\square$ & $\square$ & $\square$ \\
\hline I have extremely high goals & $\square$ & $\square$ & $\square$ & $\square$ & $\square$ \\
\hline My parents/whānau expect excellence from me & $\square$ & $\square$ & $\square$ & $\square$ & $\square$ \\
\hline People will probably think less of me if I make a mistake & $\square$ & $\square$ & $\square$ & $\square$ & $\square$ \\
\hline I never feel like I can meet my parents'/whānau's expectations & $\square$ & $\square$ & $\square$ & $\square$ & $\square$ \\
\hline $\begin{array}{l}\text { If I do not do as well as other people, it means I am an inferior human } \\
\text { being }\end{array}$ & $\square$ & $\square$ & $\square$ & $\square$ & $\square$ \\
\hline Other people seem to accept lower standards than I do & $\square$ & $\square$ & $\square$ & $\square$ & $\square$ \\
\hline If I do not do well all the time, people will not respect me & $\square$ & $\square$ & $\square$ & $\square$ & $\square$ \\
\hline $\begin{array}{l}\text { My parents/whänau have always had higher expectations for my future } \\
\text { than I have }\end{array}$ & $\square$ & $\square$ & $\square$ & $\square$ & $\square$ \\
\hline I try to be a neat person & $\square$ & $\square$ & $\square$ & $\square$ & $\square$ \\
\hline I usually have doubts about the simple everyday things I do & $\square$ & $\square$ & $\square$ & $\square$ & $\square$ \\
\hline Neatness is very important to me & $\square$ & $\square$ & $\square$ & $\square$ & $\square$ \\
\hline I expect higher performance in my daily tasks than most people & $\square$ & $\square$ & $\square$ & $\square$ & $\square$ \\
\hline I am an organized person & $\square$ & $\square$ & $\square$ & $\square$ & $\square$ \\
\hline I tend to get behind in my work because I repeat things over and over & $\square$ & $\square$ & $\square$ & $\square$ & $\square$ \\
\hline It takes me a long time to do something "right" & $\square$ & $\square$ & $\square$ & $\square$ & $\square$ \\
\hline The fewer mistakes I make, the more people will like me & $\square$ & $\square$ & $\square$ & $\square$ & $\square$ \\
\hline I never feel like I can meet my parents'/whänau's standards & $\square$ & $\square$ & $\square$ & $\square$ & $\square$ \\
\hline
\end{tabular}

Figure 34. The Frost Multidimensional Perfectionism Scale as presented in YWS Wave Two survey 


\section{Measures}

Frost Multidimensional Perfectionism Scale. Of the wide array of measures used in the YWS, this component of the research focuses on the FMPS (see Figure 34; Frost et al., 1990). As previously discussed, the FMPS was originally comprised of six dimensions, captured across 35 items measured on a 5 point Likert scale from 1 (Totally Disagree) to 5 (Totally Agree). The FMPS's six subscales, each representing a component of perfectionism, include; Concern over Mistakes (CM; e.g., "I should be upset if I make a mistake"), Doubts about Actions (DA; e.g., "Even when I do something very carefully, I often feel that it is not quite right”), Parental Expectations (PE; e.g., "My parents have expected excellence from me"), Parental Criticism (PC; e.g., "My parents never tried to understand my mistakes"), Personal Standards (PS; e.g., "It is important to me to be thoroughly competent in everything I do"), and Organisation (O; e.g., "I am an organised person"). In their seminal study, Frost and colleagues reported good internal reliability with Cronbach's alphas ranging from .77 to .93 for the subscales and an overall scale Cronbach alpha of .90. In this study, some items were slightly adapted to increase relevance to a NZ sample (e.g., 'My parents/whänau expect excellence from me'; see Figure 34).

Suicidal Behaviours Questionnaire - Revised. The Suicidal Behaviours Questionnaire - Revised (SBQ-R; Osman et al., 2001) was used to identify adolescents who may be at high risk of suicidal ideation and behaviour. The SBQ-R captures past and present suicidal thoughts and behaviours through four self-report questions. It also measures whether the individual has disclosed their behaviour or plans to any other person. The questions include "Have you ever thought about killing yourself, or attempted to kill yourself?" "How often have you thought about killing yourself in the past year?" "Have you ever told someone that you were going to commit suicide or that you might do it?" and "How likely is it that you will attempt suicide someday?" (see Figure 35). Osman and colleagues (2001) have demonstrated that the SBQ-R has acceptable internal reliability in an adolescent sample $(\alpha=$ 0.87). In combination with the SBQ-R, three additional questions were used to measure imminence/current presence of risk. These included, "In the last year have you made a plan about how you would kill yourself (attempt suicide)?" "In the last year have you tried to kill yourself (attempt suicide)?" and "In the last 2 weeks have you seriously thought about killing yourself (attempting suicide)?"

A wide array of demographic information (e.g., age, sex, and ethnicity) and other indicators of youth wellbeing were also gathered. 
Sometimes people try to hurt themselves on purpose, for lots of different reasons. Often, people who $d$ these kinds of things to themselves keep it a secret, for many different reasons. We want to know more about this so we can learn how to help people who do hurt themselves.

Please read the following statements carefully and answer honestly.

Tick the box next to the statement or phrase that best applies to you. Then follow the instructions of where to go next.

1. Have you ever thought about killing yourself, or attempted to kill yourself? (Please choose ONE)

\begin{tabular}{|c|c|}
\hline It was just a brief passing thought & \multirow{5}{*}{$\begin{array}{r}\text { boxes, please } \\
\text { TURN TO PAGE } 10\end{array}$} \\
\hline I have had a plan at least once to kill myself but did not try to do it & \\
\hline I have had a plan at least once to kill myself and really wanted to die & \\
\hline I have attempted to kill myself, but did not want to die & \\
\hline I have attempted to kill myself, and really hoped to die & \\
\hline
\end{tabular}

If you have NEVER thought about, or attempted to, kill yourself OR hurt yourself deliberately, please TURN TO PAGE 14.

2. How often have you thought about killing yourself in the past vear? (Please choose ONE)
$\square$ Never
$\square$ Rarely (1 time)
$\square$ Sometimes (2 times)
$\square$ Often (3-4 times)
$\square$ Very often (5 or more times)

3. Have you ever told someone that you were going to commit suicide, or that you might do it? (Please choose ONE)
$\square \quad$ No
$\square$ Yes, at one time, but I did not really want to die
$\square$ Yes, at one time, and I really wanted to do it
$\square$ Yes, more than once, but I did not want to do it
$\square$ Yes, more than once, and I really wanted to do it

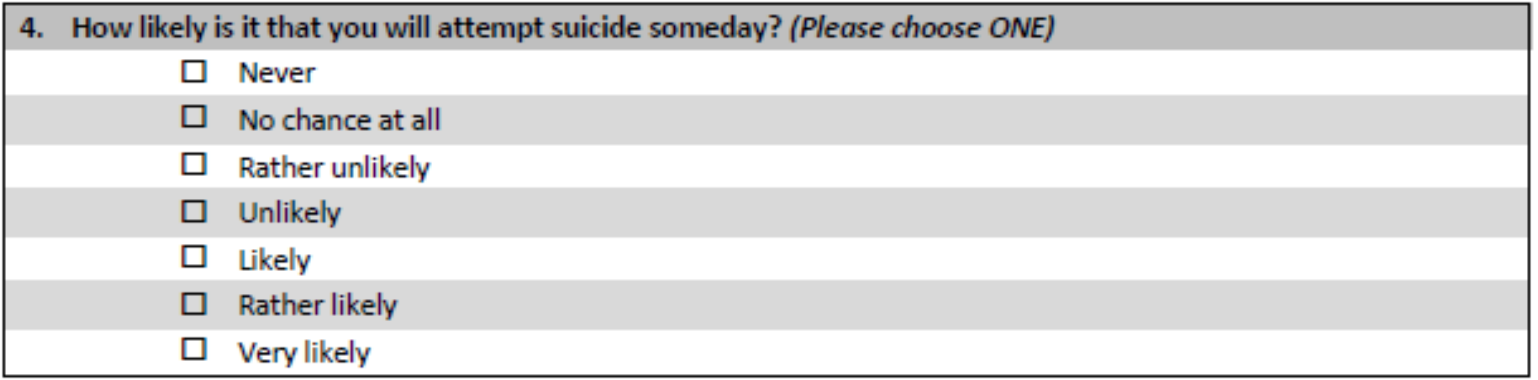
5. In the last year, have you ...
.. made a plan about how you would kill yourself (attempt suicide)?
$\square \quad$ No
$\square$ Yes ... tried to kill yourself (attempted suicide)?
$\square \quad$ No
$\square \quad$ Yes
6. In the last 2 weeks, have you seriously thought about killing yourself (attempting suicide)?

Figure 35. The Suicidal Behaviours Questionnaire as presented in YWS Wave Two survey 


\section{Procedure}

Ethical approval for this research was granted by the NZ National Health and Disability Ethics Committee (HDEC) in 2012 (approval number: 11/645). The YWS team, made up of Victoria University of Wellington post-graduate students and academic staff, and clinical psychologists, collected the data for Wave Two from March 2014 to November 2014. As previously mentioned, both school and parent/caregiver consent were sought prior to visiting the school to administer the survey. Students with parent/caregiver consent were then given the opportunity to participate in the survey during a school period within their regular class hours. Before conducting the survey a member of the research team briefed students on the survey's purpose and emphasised confidentiality and its voluntary nature. It was explained that if any students were suspected by the researcher team to represent an imminent risk of harm, the school counsellor would be advised to contact them. Students who decided to provide assent were advised that they were able to withdraw at any point during the survey. As alluded to above, most students were already aware of this process, both through provided information packs and having completed the Wave One survey approximately one year prior.

Students were typically allowed a full school period to complete the survey. However, on average it took 30 minutes. Students were asked to complete their survey in silence to ensure confidentiality. Each classroom had a researcher available to answer any questions the students had. On completion, students were debriefed, provided with a sheet of paper detailing services that they could contact for support, and given a chocolate bar for their participation. If any students had any immediate concerns, they were able to talk to one of the Clinical Psychologists within the research team who was on site for the duration of the surveying process.

Shortly after administration of the survey, members of the YWS team assessed each survey for indicators of risk. This involved checking the SBQ-R for any students who scored 7 or above on this scale. Members of the team paid particular attention to students who answered "Yes" to "In the last year, have you...made a plan about how you would kill yourself (attempt suicide)?” or “....tried to kill yourself (attempted suicide)?” Acute risk was also determined using the "In the last 2 weeks, have you seriously thought about killing yourself (attempting suicide)?'. If any students responded 'Yes' to this question or responded highly on the other questions from the SBQ-R they were considered high risk and within 
48hours their name was passed onto the school counsellor for follow-up ${ }^{32}$.

For ethical and safety reasons, students also reported their current mood (on a 5-point Likert scale, 1 = Very Unhappy, 5 = Very Happy) at both the beginning and end of the survey. The first administration of this survey in the wider longitudinal study showed students did not experience significant adverse effects on their mood as a result of completing the compilation of measures $(t(785)=.15, p=.88)$. This is consistent with the lack of evidence in previous research of any iatrogenic effect of explicitly investigating self-injury in adolescents (e.g., Muehlenkamp, Swenson, Batejan, \& Jarvi, 2014; Whitlock, Pietrusza, \& Purington, 2013).

Data was managed and analysed using Statistical Package for the Social Sciences (SPSS) version 23 statistical software package and AMOS version 22.

\section{Results}

\section{Confirmatory Factor Analysis}

Confirmatory Factor Analyses (CFA) were conducted to ascertain which, of the various proposed factor structures for the FMPS, best fit this sample of NZ adolescents. CFA enables the researcher to test the goodness-of-fit for existing models. Often Exploratory Factor Analysis (EFA), which determines the factor structure that best represents the data without consideration of previous models, is used to identify a data driven structure (Hair, Black, Babin, \& Anderson, 2009). However, given the plethora of existing factor structures, CFA was considered a more appropriate first step. In total, 14 different models were explored using AMOS 23, including Frost's (1990) original six-factor structure, other variations of the six-factor structure (Parker \& Stumpf, 1995), five-factor (Cox et al., 2002; Stallman \& Hurst, 2011), four-factor (Hawkins et al., 2006; Khawaja \& Armstrong, 2005; Stumpf \& Parker, 2000), a three-factor (Purdon et al., 1999), two-factor (Frost et al., 1993; Khawaja \& Armstrong, 2005; Luyckx et al., 2008) and hierarchical factor structures (Cox et al., 2002; Stumpf \& Parker, 2000). As shown in Table 10, each model was assessed using multiple fit indices. These included the Chi-Square $\left(\chi^{2}\right)$, Comparative Fit Index (CFI), and the Root Mean Square Error of Approximation (RMSEA) ${ }^{33}$.

As a guide, Tabachnick and Fidell (2013) suggest that a good fit is represented by a

\footnotetext{
$3219 \%$ of students scored above 7 on the SBQ-R, however further clinical judgment was used to identify those at risk and as a result approximately $10 \%$ of the total sample had their names provided to their school guidance counselling team for follow up.

${ }^{33}$ Multivariate outliers were identified and the 10 most extreme outliers removed. Further analysis showed that this had very little effect on the subsequent results, therefore all data was included in the final analyses.
} 
non-significant $\chi^{2}$, indicating that the model generated by the data is not significantly different to the model proposed. However, this statistic is particularly sensitive to large sample sizes, meaning our significant $\chi^{2}$ statistics provide little guidance for meaningful interpretation (MacCallum, 1990; Marsh, Balla, \& McDonald, 1988). Alternatively, it is suggested that $\chi^{2}$ divided by the degrees of freedom is a complementary measure to consider, where a $\chi^{2} / \mathrm{d} . f$. between 2 (Tabachnick \& Fidell, 2013) and 5 (Wheaton, Muthen, Alwin, \& Summers, 1977) indicates satisfactory fit relative to sample size and model complexity. According to these guidelines, Frost and colleagues' (1990) original factor structure and Parker and Stumpf's (1995) slight variation on the original structure were the closest to a good fit.

Table 10

Goodness of fit Statistics for Frost Multidimensional Perfectionism Scale Factor Structures

\begin{tabular}{|c|c|c|c|c|c|c|}
\hline & $\begin{array}{l}\text { Proposed } \\
\text { components }\end{array}$ & $\begin{array}{l}\text { Items } \\
\text { retained }\end{array}$ & $\chi^{2}$ (d.f.) & $\chi^{2} /$ d.f. & $\underline{\mathrm{CFI}}$ & RMSEA \\
\hline Frost et al., 1990 & 6 & 35 & $2971.58 *(545)$ & 5.45 & 0.86 & 0.07 \\
\hline Parker \& Stumpf, 1995 & 6 & 35 & $2726.36 *(545)$ & 5.00 & 0.87 & 0.07 \\
\hline Cox et al., 2002 & 5 & 22 & $1206.84 *(199)$ & 6.07 & 0.90 & 0.07 \\
\hline Cox et al., 2002 & $5-2$ & 22 & $1273.99 *(203)$ & 6.28 & 0.89 & 0.08 \\
\hline Stallman \& Hurst, 2011 & 5 & 29 & $2356.08 *(367)$ & 6.42 & 0.86 & 0.08 \\
\hline Stumpf \& Parker, 2000 & 4 & 35 & $3711.39 *(554)$ & 6.70 & 0.82 & 0.08 \\
\hline Stumpf \& Parker, 2000 & $4-2$ & 35 & $3719.56^{*}(555)$ & 6.70 & 0.82 & 0.08 \\
\hline Khawaja \& Armstrong, 2005 & 4 & 24 & $1544.38 *(224)$ & 6.90 & 0.86 & 0.08 \\
\hline Hawkins et al., 2006 & 4 & 33 & $3240.66 *(489)$ & 6.63 & 0.83 & 0.08 \\
\hline Purdon et al., 1999 & 3 & 35 & $5126.74 *(557)$ & 9.20 & 0.73 & 0.09 \\
\hline Khawaja \& Armstrong, 2005 & 2 & 17 & $835.34 *(118)$ & 7.08 & 0.91 & 0.08 \\
\hline Luyckx et al. 2008 & 2 & 35 & $1662.08 *(169)$ & 9.84 & 0.84 & 0.10 \\
\hline Frost et al., 1993 & 2 & 35 & $6029.19 *(559)$ & 10.79 & 0.68 & 0.10 \\
\hline One component solution & 1 & 35 & $9076.02 *(560)$ & 16.21 & 0.50 & 0.13 \\
\hline
\end{tabular}

$* p<.001$

Other goodness-of-fit statistics are broadly consistent with the $\chi^{2} /$ d.f. statistic and highlight large discrepancies between the proposed models and the model guided by this data. To illustrate, Tabachnick and Fidell (2013) state that the CFI should be greater than .95 (also see $\mathrm{Hu} \&$ Bentler, 1999). As seen in Table 10, according to this fit index, the data do not indicate good-fit with any of the previous models. However, Khawaja and Armstrong's (2005) two-factor model, and Cox and colleagues' (2002) five factor model appeared the closest to an acceptable fit with CFI statistics of 0.91 and 0.90 respectively.

The RMSEA was the final goodness-of-fit statistic considered. It too, raised questions 
about the validity of applying this data to previously proposed models. Hu and Bentler (1999) propose that RMSEA values less than 0.06 indicate a good-fit and values over 0.10 indicate a poor fit. According to this statistic, Frost and colleagues' (1990) original factor structure, Parker and Stumpf's (1995) slight modification, and Cox and colleagues' (2002) five-factor structure provided the best (although perhaps not good) fit. When combining all of the available fit indices, the statistics suggest that the data best fits Frost and colleague's original six factor structure $\left(\chi^{2}(545)=2971.58, p<.001 ; \chi^{2} /\right.$ d.f. $\left.=5.45 ; \mathrm{CFI}=.86 ; \mathrm{RMSEA}=.07\right)$, Parker and Stumpf's $(1995)$ variation $\left(\chi^{2}(545)=2726.36, p<.001 ; \chi^{2} /\right.$ d.f. $=5.00 ; \mathrm{CFI}=.87$; RMSEA = .07), and Cox and colleague's five factor structure $\left(\chi^{2}(199)=1206.84, p<.001\right.$; $\chi^{2} /$ d.f. $\left.=6.07 ; \mathrm{CFI}=.90 ; \mathrm{RMSEA}=.07\right)$. However, no combination of goodness-of-fit indices for the three models convincingly suggest that the data fit the models well.

\section{Exploratory Factor Analysis}

Consistent with other psychometric analyses (e.g., Choo, Walsh, Chinna, \& Tey, 2013; Cox et al., 2002; Moreira, Almeida, Pinto, Segarra, \& Barbosa, 2015), given that the CFAs conducted showed that the data from this NZ adolescent sample only marginally fit the previously proposed factor structures, exploratory Principle Components Analysis (PCA) with Varimax orthogonal rotation was employed to help determine the relationships between underlying variables and the individual items ${ }^{34}$. Traditional measures of suitability for factor analysis indicated that the data was suitable for PCA (Bartlett's Test of Sphericity $\chi^{2}(595)=$ 15771.70, $p<.001$; Keyser-Meyer-Olkin: .94).

The PCA conducted with the individual items (rather than the subscales) produced mixed results. Five components with eigenvalues greater than one accounted for $60.82 \%$ of the variance (Kaiser, 1974). However, Cattell's (1966) scree plot suggested either a two, or four, component solution. Given that Kaiser's criterion has often been critiqued for resulting in the retention of too many components (e.g., Pallant, 2013) and the ambiguity of the current scree plot, Horn's (1965) parallel analysis was conducted to help determine the appropriate number of components to retain.

Parallel analysis compares the eigenvalues produced with those from multiple randomly generated data sets. This enables the researcher to retain only components that have larger eigenvalues than those generated randomly. Many argue that this method is more accurate than either Kaiser's criterion or the use of a Catell's scree plot (e.g., Pallant, 2013;

\footnotetext{
34 PCA was also conducted using an oblique rotation and results did not significantly differ, therefore, the results of the orthogonal, Varimax rotation are reported.
} 
Velicer, Eaton, \& Fava, 2000). Parallel analysis resulted in the eigenvalues of four components exceeding those produced from a random data set (Table 11). Specifically, the fifth PCA component provided an eigenvalue of 1.05, below the 1.27 cut off recommended by parallel analysis. As a result, another PCA was conducted, this time limiting the number of components to four.

Table 11

Results of Parallel Analysis used to Determine the most Appropriate Number of Components, within the FMPS, for a sample of New Zealand Adolescents

\begin{tabular}{|c|c|c|c|}
\hline$\underline{\text { Component }}$ & PCA Eigenvalue & $\frac{\text { Parallel Analysis }}{\text { Criterion Value }}$ & $\underline{\text { Result }}$ \\
\hline 1 & 10.478 & 1.42 & Accept \\
\hline 2 & 5.495 & 1.37 & Accept \\
\hline 3 & 2.266 & 1.33 & Accept \\
\hline 4 & 2.001 & 1.30 & Accept \\
\hline 5 & 1.049 & 1.27 & Reject \\
\hline
\end{tabular}

In the resulting PCA, four components explained $57.83 \%$ of the variance. As can be seen in Table 12, the first component comprised of 13 items originally from CM and DA, plus two additional items (items 4 and 5) originally from the PS and PC factors respectively (Frost et al., 1990). The second and third components mirrored the original PS (6 items) and Organisation (6 items) factors respectively. Finally, the fourth component comprised of seven items from PE and PC. Item 3 was excluded due to low loadings on two components with neither loading significantly higher than the other (.42 on component $1 ; .40$ on component 4 ). The four components explained $29.94 \%, 15.70 \%, 6.47 \%$ and $5.72 \%$ of the variance respectively. They will be referred to as Concerns over Mistakes and Doubts (CMD), Personal Standards (PS), Organisation and Parental Pressure (PP) throughout. With regard to internal consistency, Bernardi (1994) suggests that a scale or subscale should have Cronbach's alpha of at least .70 to be considered adequate for use in a particular sample. As such, all four components displayed high internal consistency (CMD, $\alpha=.93$; PS, $\alpha=.84$; Organisation, $\alpha$ $=.91 ; \mathrm{PP}, \alpha=.83)$. 
Table 12

Result of FMPS PCA; Factor Structure and Item Loadings

Item (original factor from Frost et al., 1990)

$\underline{1}$

14. If I fail partly, it is as bad as being a complete failure (CM) $\quad .79$

23. If I do not do as well as other people, it means I am an inferior $\quad .78$

human being. (CM)

9. If I fail at school, I am a failure as a person. (CM) $\quad 76$

13. If someone does a task at work/school better than I, then I feel $\quad .74$

like I failed the whole task. $(\mathrm{CM})$

25. If I do not do well all the time, people will not respect me. (CM) $\quad .74$

21. People will probably think less of me if I make a mistake. (CM) $\quad .73$

28. I usually have doubts about the simple everyday things I do. (DA) $\quad .72$

34. The fewer mistakes I make, the more people will like me. (CM) $\quad .69$

10. I should be upset if I make a mistake. (CM)

17. Even when I do something very carefully, I often feel that it is not $\quad .65$ quite right. (DA)

33. It takes me a long time to do something "right." (DA) .64

18. I hate being less than the best at things. (CM) $\quad 62$

32. I tend to get behind in my work because I repeat things over and $\quad .59$

over. (DA)

4. If I do not set the highest standards for myself, I am likely to end $\quad .46$

up a second-rate person. (PS)

5. My parents never tried to understand my mistakes. (PC) $\quad .46$

31. I am an organized person. (O)

29. Neatness is very important to me. (O) $\quad .84$

7. I am a neat person. $(\mathrm{O}) \quad .83$

8. I try to be an organized person. $(\mathrm{O}) \quad .80$

27. I try to be a neat person. $(\mathrm{O}) \quad 80$

2. Organization is very important to me. $(\mathrm{O}) \quad .77$

12. I set higher goals than most people. (PS) $\quad .79$

19. I have extremely high goals. (PS) $\quad .76$

6. It is important to me that I be thoroughly competent in everything I

do. (PS)

24. Other people seem to accept lower standards from themselves than I do. (PS)

30. I expect higher performance in my daily tasks than most people.

(PS)

16. I am very good at focusing my efforts on attaining a goal. (PS)

26. My parents have always had higher expectations for my future than I have. (PE)

20. My parents have expected excellence from me. (PE)

35. I never felt like I could meet my parents' standards. (PC)

1. My parents set very high standards for me. (PE)

11. My parents wanted me to be the best at everything. (PE)

22. I never felt like I could meet my parents' expectations. (PC)

15. Only outstanding performance is good enough in my family. (PE) 
3. As a child I was punished for doing things less than perfect (PC) $)^{35}$

Note: Factor loadings less than .40 are not shown, to assist in interpretation.

As previously outlined, higher-order components are often created by combining correlated lower-level components (e.g., Cox et al., 2002; Hawkins et al., 2006). As such, an exploratory PCA with Varimax orthogonal rotation was undertaken to test whether the components combined to create two second-order components representing positive and negative perfectionism. The PCA produced two components with eigenvalues greater than one and accounted for $78.66 \%$ of the variance (Kaiser, 1974). Cattell's (1966) scree plot also supported a two component solution. As outlined in Table 13, CMD and PP combined to form a component representing negative perfectionism and Organisation and PS combined to form a component representing positive perfectionism.

\section{Table 13}

Result of FMPS Second-order PCA; Factor Structure and Item Loadings

\begin{tabular}{lcc}
\hline Subscale identified in first order & $\frac{\text { Negative Perfectionism }}{(\alpha=.89)^{\mathrm{a}}}$ & $\frac{\text { Positive Perfectionism }}{(\alpha=.93)^{\mathrm{a}}}$ \\
$\begin{array}{l}\text { factor analysis } \\
\text { Concerns and Doubts }\end{array}$ & .86 & \\
Parental Pressure & .87 & .93 \\
Organisation & & .75 \\
Personal Standards & & \\
${ }^{a}$ Cronbach's alpha representing internal reliability of all items within negative and positive perfectionism
\end{tabular}

\section{Model Fit across Sex}

Finally, the four component (CMD, PS, PP, Organisation), hierarchical (Positive, Negative) model was entered into AMOS for Multiple Indicators Multiple Causes (MIMIC) analyses (also known as CFA with covariates; Brown, 2006) ${ }^{36}$. First the model resulting from the preceding PCA was entered and refined according to modification indices. This involved allowing paths between item errors within the same overarching component to correlate (e.g., an extra path was added between the error for item 12 and the error for item 16 which are both items within the PS subscale). A simplified depiction of the model is presented in Figure 36 below. Before progressing it is important to note that these analyses are limited by two negative error variances (also known as Heywood cases, see Brown, 2006) on the PS and CMD latent variables. This is likely a result of the small number of latent variables (PS and

\footnotetext{
${ }^{35}$ Item 3 cross-loaded on CMD and PP, therefore was excluded in four factor solution but reinstated in the negative perfectionism component of the hierarchical solution

${ }^{36}$ All cases with any missing FMPS data points were removed for MIMIC analyses. This resulted in a sample of 781
} 
Organisation/CMD and PP) predicting the overarching positive and negative latent components. According to McDonald (1985), each common component should be defined by a minimum of three and preferably four variables that load heavily on the latent component. In this case only PS and Organisation load on positive perfectionism and only CMD and PP load on negative perfectionism, likely causing the negative error variances of PS ( $\delta=-1.83$ ) and $\mathrm{CMD}(\delta=-.01)$. One negative error variance can affect the reliability of other estimates therefore the variance of the errors in question were set to 0 to minimise impact. Despite this precaution, the results that follow should still be interpreted with care. Following the construction of the base model, MIMIC analyses were conducted. MIMIC analysis involves the introduction of a regression path to account for an additional variable. In this case, sex

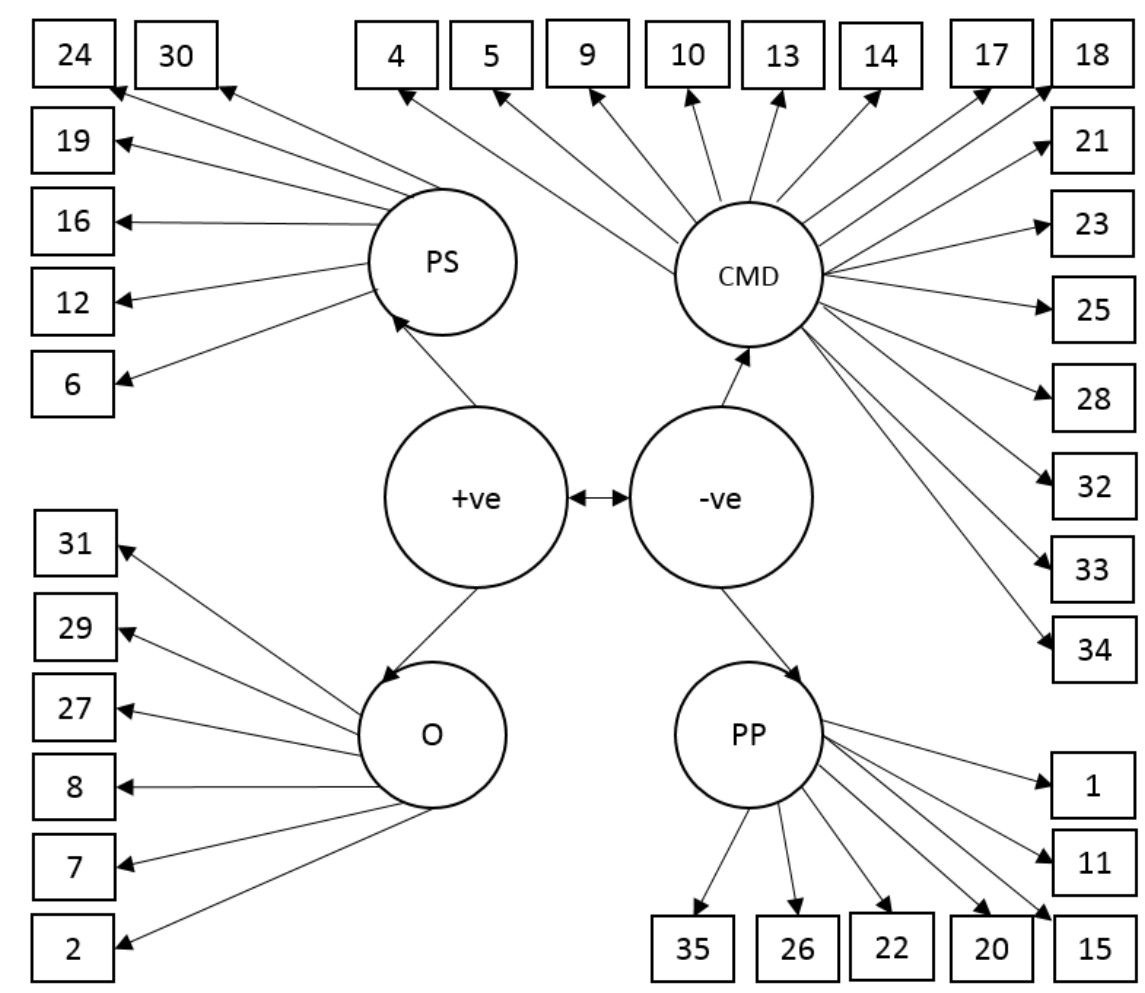

Figure 36. Four factor, hierarchical FMPS model resulting from PCA analyses

was introduced to the previously identified four-factor hierarchical FMPS model to test whether the model is approximately equally appropriate for both male and female adolescents (for comparison model see Figure 37 below). Initially the paths between sex and the two overarching components were examined. Significant path coefficients suggest heterogeneity, specifically that the means of one or both of the overarching subscales are different across sex. The results indicated that this was indeed the case with a significant sex-negative perfectionism path coefficient $(\beta=.17, p<.001)$. Given that males were coded 0 and females 
were coded 1, this suggests that females score significantly higher on negative perfectionism than males. This is a small but significant effect (Cohen, 1988).

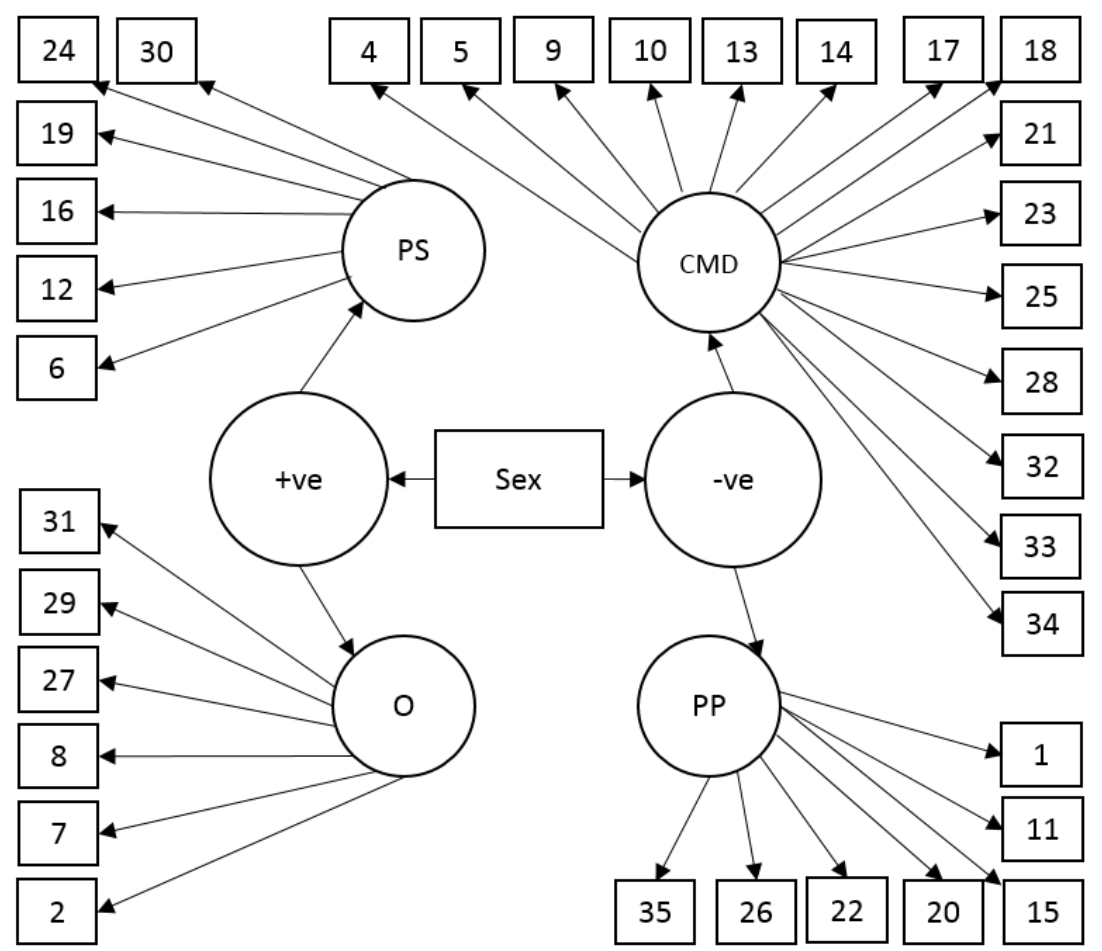

Figure 37. MIMIC analyses: Four factor, hierarchical FMPS model with sex as a covariate ${ }^{37}$

Next, fit indices for the base model (see Figure 36; $\chi^{2}(439)=1933.45, p<.001 ; \chi^{2} /$ d.f. $=4.40 ; \mathrm{CFI}=.90 ; \mathrm{RMSEA}=.07)$ were compared to the revised model including the sex covariate $\left(\right.$ see Figure $37 ; \chi^{2}(472)=2012.79, p<.001 ; \chi^{2} /$ d.f. $=4.26 ;$ CFI $=.90 ;$ RMSEA $=$ $.07 ; \Delta \chi^{2}=79.34, \Delta$ d.f. $\left.=33, p<.001\right)$. Analyses demonstrated that inclusion of the sex covariate significantly affected model fit $\left(\Delta \chi^{2}=79.34, \Delta\right.$ d.f. $\left.=33, p<.001\right)$. However, given that the chi-squared statistic is particularly sensitive to sample size (see discussion above p. 131-132), and this study has a relatively large sample $(n=781)$, it is more appropriate to assess the $\chi^{2} /$ d.f. In this case $\Delta \chi^{2} /$ d.f. was 2.40 which falls well within the 2-5 range suggested by Tabachnick and Fidell (2013) and Wheaton and colleagues (1977) for models with good fit. This suggests that although the model fit significantly changes with the introduction of a sex covariate, the significance of the change may be an artefact of sample size and instead suggests the model fit across both models is comparable. Moreover, regardless of whether model fit significantly changed, fit definitely did not improve. Overall, MIMIC analyses

${ }^{37}$ See Appendix D for a model containing path values 
suggest that sex does not have a substantial impact on the validity of the current structure of the FMPS with NZ adolescents, though it provided evidence that females may endorse negative perfectionism more than males.

\section{Summary and Conclusions}

This study investigated the psychometric properties of the FMPS (Frost et al., 1990) in a NZ adolescent sample. Given that there is wide debate regarding the FMPS factors, there was no specific prediction about which underlying factor structure would best fit this sample. However, due to the relatively consistent finding of two overarching factors, it was expected that positive and negative perfectionism would also be identified in this sample.

\section{First Level Structure}

Overall, CFA showed that none of the previously proposed models convincingly fit this sample well. However, of the 14 models tested, Frost and colleagues' (1990) original six factor structure was the closest-to-satisfactory fit. Subsequently, PCA was used to form a data-driven model specific to this sample. Parallel analysis supported four components, that subsequent PCA showed were broadly consistent with previously reported structures (e.g., Hawkins et al., 2006; Stumpf \& Parker, 2000; Thorpe \& Nettelbeck, 2014). Specifically, CM and DA are combined to form Concerns and Doubts and PC and PE were combined to form Parental Pressure. PS and Organisation remained their own components. Only one item was removed due to low and relatively equal loadings across two of the components.

The initial analyses lend some support to the factors proposed by Frost and colleagues (1990). However, in this younger sample it does raise questions about the validity of differentiating between CM and DA, and between PE and PC. It is possible that adolescents may have more difficulty than older samples in differentiating between these relatively similar constructs, or that they are not separable for younger adolescents. This is consistent with other research using the FMPS in child and adolescent samples which also found blurred divisions between some of the six originally proposed factors (Hawkins et al., 2006;

Kornblum \& Ainley, 2005; Stumpf \& Parker, 2000; Thorpe \& Nettelbeck, 2014). Moreover, a similar age effect has been demonstrated for other psychological constructs whereby the structure of a construct differs across age groups (e.g., alexithymia; Parker, Eastabrook, Keefer, \& Wood, 2010).

\section{Overarching Positive and Negative Components}

A second-order PCA supported combining CMD and PP, and combining PS and Organisation, to represent two overarching, positive and negative components. Therefore, this 
study lends support to the differentiation between positive and negative perfectionism in adolescents (Chang et al., 2004; Hawkins et al., 2006; Parker \& Stumpf, 1995; Stumpf \& Parker, 2000; Thorpe \& Nettelbeck, 2014). Both the resulting fundamental four components and the overarching two higher-order components had satisfactory internal consistency coefficients.

With regard to practical implications, this research supports the notion that perfectionism is comprised of both positive and negative components and therefore suggests that researchers should continue to use scales such as the FMPS that allow this differentiation. Failure to do so poses the risk that significant relationships with important outcomes could be masked through the cancelling out of each component's unique and often opposite effects.

In addition, clarifying the underlying nature of perfectionism in adolescents and providing a reliable measure of the components of perfectionism will allow researchers to develop a solid research base, with a common language, for the different facets of a perfectionistic presentation. This will then enable clinicians to have a better understanding of the correlates and risks associated with especially low or high positive and negative perfectionism.

\section{Strengths}

This study has multiple strengths. First, the aim was to comprehensively understand perfectionism in adolescents. Therefore, analysis was conducted with previously published models in mind, but also using a data-driven approach. Unlike studies that use only EFA, or that construct the scales based on the original factors, this multi-method approach limited the influence of any biases regarding the best model for this adolescent sample. Moreover, firstorder factor analyses were conducted using the original 35 items rather than assuming the validity of the subscales and progressing straight to a second-order factor analysis with Frost and colleagues' (1990) predetermined subscales. This allowed testing of Frost's underlying six-factor structure through giving freedom for the items to load on any factor, whether it be the factor proposed by Frost or a structure more suited to this sample. This approach also allowed for individual item analysis and the removal of items that cross-loaded or only loaded weakly on the scales.

A second strength is the analysis of differences in model fit across sex. Whilst many previous studies have only analysed their mixed-sex data as a whole (e.g., Cox et al., 2002, Parker \& Stumpf, 1995), or have only investigated the structure of the FMPS in females (e.g., Hawkins et al., 2006), this study used MIMIC analyses to investigate whether introducing the 
sex variable significantly impacted model fit. Overall, the addition of the sex covariate resulted in a slight drop in model fit, suggesting that sex is not a core determinant of how the factor structure operates. Further, detailed inspection of fit indices, path coefficients, and modification indices suggests that overall the factor structure is equally appropriate for the measurement of positive and negative perfectionism in both male and female adolescents.

Moreover, MIMIC analyses revealed a difference in the mean levels of perfectionism for males and females. Specifically, females scored significantly higher on negative perfectionism than males. Such differences on negative perfectionism are not commonly highlighted, however some researchers have previously demonstrated that females score higher on positive perfectionism (e.g., Parker \& Stumpf, 1995; Stallman \& Hurst, 2011). These analyses did not highlight a significant effect of sex on positive perfectionism in the MIMIC model.

\section{Limitations}

Despite the strengths outlined above, there are a number of limitations to consider in interpreting these results. First, this research is limited by the use of a relatively young group of adolescents. Although this provides information regarding perfectionism in a sample with a mean age of 14 years old, it does not shed light on how perfectionism may change over the course of adolescence, a period characterised by particularly frequent and sizable developmental changes. Thus, options for further research include a longitudinal investigation of perfectionism in adolescents to investigate how perfectionism develops and whether the underlying nature of the construct significantly changes. This could either provide support for one underlying model of perfectionism that is relevant throughout adolescence and young adult life or could shed some light on why there have been multiple conflicting models of perfectionism across different samples presented in published articles.

\section{Conclusions}

In summary, perfectionism researchers may benefit from careful consideration of which conceptualisation of perfectionism is most appropriate for samples of different age, sex and culture. It is possible that it is more appropriate for further research to adopt a four-factor structure rather than retain Frost and colleagues (1990) six-factor structure. At the very least, researchers must report the internal reliability of the FMPS subscales in their sample prior to using the data to make arguments about the relationship between perfectionism and other constructs. For the purpose of this research, Study 2 has demonstrated that a four component 
solution is appropriate for NZ adolescents. Research using this structure will allow for more accurate and streamlined interpretation of the data. 


\section{CHAPTER SEVEN: THE RELATIONSHIP BETWEEN NSSI AND PERFECTIONISM IN NEW ZEALAND ADOLESCENTS}

Previous chapters have shown that both perfectionism and NSSI are associated with detrimental wellbeing outcomes for adolescents. Thus, having systematically reviewed the relevant literature and established the most appropriate use of the FMPS for measuring perfectionism, the focus now moves to investigating the relationship between perfectionism and NSSI in NZ adolescents.

As a reminder, cross-sectional research on the direct relationship between perfectionism and NSSI in university and adult samples has demonstrated that negative perfectionism is typically associated with greater engagement in NSSI (e.g., Claes et al., 2012; Hoff \& Muehlenkamp, 2009; Izadi, 2014; also see Study 1b results). On the other hand, positive perfectionism is either highlighted as a slight buffer against NSSI risk (Hoff \& Muehlenkamp, 2009), or has been found have no significant relationship with self-injury (e.g., Claes et al., 2012; Kubal, 2005; Smith et al., 2014; Study 1b results).

Research specific to adolescents has shown that perfectionism, conceptualised as a unitary construct, is associated with greater engagement in NSSI in both community and psychiatric females (Luyckx et al., 2015). There is no available research looking at the relationship between a multidimensional measure of perfectionism and NSSI in both male and female adolescents. However, though conflating suicidal and non-suicidal behaviours, O'Connor et al., (2010) reported a significant relationship between negative perfectionism and DSH in their sample of Scottish adolescents. Based on their findings, it is reasonable to expect that a similar relationship will be identified in research specifically investigating NSSI, and in a NZ context.

In Study $1 \mathrm{~b}$ meta-analytic techniques were used to better understand the relationship between perfectionism and self-injury. Meta-analysis of all available perfectionism-selfinjury research produced a summary Pearson's correlation coefficient of .15 $(p<.001)$ for the relationship between negative perfectionism and self-injury. Conversely, the summary correlation coefficient for the relationship between positive perfectionism and self-injury was non-significant $(r=.00, p=.89)$.

To summarise, negative perfectionism appears to significantly contribute to risk of self-injury. However, further research is needed to clarify the nature of the relationship between negative perfectionism and self-injury in both male and female adolescents. This component of the thesis presents three studies intended to provide more detailed information regarding perfectionism and NSSI. It is intended that this research will help clinicians to 
identify at adolescents at risk of engaging in NSSI, and in particular adolescents who may be at increased risk due to high levels of negative perfectionism.

\section{Study Three: Non-Suicidal Self-Injury, its Functions, and Perfectionism in a Sample of New Zealand Adolescents}

First, Study $3 \mathrm{a}$ will involve examining the cross-sectional relationship between positive and negative perfectionism, and NSSI in male and female adolescents. Drawing on NSSI research with both adults, and adolescents, it is first expected that negative perfectionism will be associated with increased risk for NSSI, and second that positive perfectionism is unlikely to be associated with risk of NSSI, although the latter prediction is given with substantially less confidence.

Study $3 b$ will involve an investigation of the functions of NSSI in relation to perfectionism. In particular exploring whether perfectionistic adolescents who engage in NSSI endorse specific functions at different rates to non-perfectionistic adolescents. This component of the research aims to establish hypotheses about how perfectionism may be implicated in the development and maintenance of NSSI, and more specifically how understandings of perfectionism can be integrated with the dominant models of NSSI. Based on theoretical understandings of perfectionism (Flett et al., 2012), and the limited research that has investigated perfectionism and the functions of NSSI (Claes et al., 2012), it is predicted that negative perfectionism will be associated with NSSI for self-punishment.

Finally, Study 3c will investigate the two-component, 2x2, and tripartite models of perfectionism. According to these models it is expected that either adolescents low on positive perfectionism and high on negative perfectionism are at greatest risk for NSSI, thus supporting the 2x2 model (Gaudreau \& Thompson, 2010); or those high on positive and negative perfectionism are at greatest risk for NSSI, thus supporting the two-component and tripartite models (e.g., Alden et al., 2002; Rice \& Ashby, 2007; Stoeber \& Otto, 2006)

\section{Method for Study 3a, 3b and 3c}

\section{Participants}

Study 3 was conducted in conjunction with Study 2, thus also using Youth Wellbeing Study (YWS) Wave Two data. To briefly review, the sample comprised of 930 13-17 year old adolescents $(M=14.50$ years, $S D=1.29)$, with more females $(59 \%)$ than males $(41 \%)$ and was comprised of students from multiple ethnic groups (for more detail about participant demographics see p. 126). Three participants identified as trans-gender and three participants did not report their sex. Given that these six participants are only a very small proportion of 
the total sample, and analyses by sex will be conducted often, they were excluded from all further analyses. As a result, this study has a final sample of 924 adolescents.

\section{Measures}

As highlighted in Study 2, the FMPS (Frost et al., 1990) was used to measure perfectionism, and the SBQ-R (Osman et al., 2001) was used as a suicide risk screening tool (see p. 128-129 for SBQ-R information). Alongside the FMPS and SBQ-R, the Deliberate Self Harm Inventory-short (DSHI-s; Lundh et al., 2007), and the Inventory of Statements about Self-Injury (ISAS; Klonsky \& Glenn, 2009) were used to gain detailed information about the participants NSSI thoughts and behaviours.

Frost Multidimensional Perfectionism Scale. All 35 items of the FMPS (Frost et al., 1990) were administered to students. However, in accordance with Study 2 results (p. 131138) a four, rather than the six component structure was adopted. In addition, CMD and PP were combined to form negative perfectionism and PS and Organisation combined to form positive perfectionism. Item 3 ("as a child I was punished for doing things less than perfect") was excluded from the four-component solution due to high cross-loadings on CMD, and PP, however was reinstated for the two-component model as the cross loadings both occurred within the negative perfectionism component.

Deliberate Self-Harm Inventory-short version ${ }^{38}$. The DSHI-s is a self-report measure containing a behavioural checklist of common methods of intentional harm to one's own body (Lundh et al., 2007) and has been previously used in NSSI research with older adolescents and young adults in NZ (Garisch \& Wilson, 2015). The DSHI-s was chosen in light of research that has indicated that behavioural checklists provide more reliable data and capture a wider range of self-injurious behaviours than single item measures of self-injury (e.g., Heath, Toste, Nedecheva, \& Charlebois, 2008; Lundh et al., 2007; Muehlenkamp et al., 2012). Behavioural-checklists are also advantageous as they help counter any inconsistencies between the researcher's and the adolescent's conceptualisation of NSSI. Whilst this research uses the DSHI-s (rather than the DSHI; Gratz, 2001), originally modified for use in an adolescent sample (Lundh et al., 2007), the original DSHI leading question was retained to ensure that the scale was capturing NSSI rather than DSH. Therefore, this version of the scale asked "Have you ever deliberately (but without wanting to kill yourself) ....." followed by 14 different behaviours. For example, "cut your wrist, arms, or other areas of your body,"

\footnotetext{
${ }^{38}$ Although the name of this scale suggests the measurement of DSH (self-injury regardless of intent), the questions refer to NSSI throughout.
} 
"severely scratched yourself, to the extent that scarring or bleeding occurred" or "rubbed sand paper on your body" (see Figure 38 for the DSHI-s component of the survey). A slightly adapted 5-point scale from "I've never thought about doing this" to "I've done this many times" was used to capture participant's engagement in the self-injurious behaviours. This differs from the 4-point scale used in Lundh and colleagues study, such that instead of one item for participants who had never engaged in the behaviours ("I've never done this"), this option was divided based on whether the individual had had thoughts about self-injury ('I've never thought about doing this" and "I have thought about this but never done it"). This allowed a closer analysis of the transition between thinking about a behaviour and engaging in the behaviour and is in line with research that suggests thoughts about self-injury and engaging in self-injury are distinct and do not always occur together (e.g., Nock et al., 2009; O'Connor, Rasmussen, \& Hawton, 2012). Scores for NSSI were calculated by summing the rating for each item (I've never thought about doing this $=0$, I have thought about doing this but have never done it $=1$, I have done this once $=2$, I have done this a few times $=3$, I have done this many times $=4$ ) and dividing the sum score by 14 to get a mean item score (as recommended by Latimer et al., 2013). Participants who indicated no history of thoughts about self-injury or engagement in self-injury were assigned a score of 0.

In addition to the behavioural checklist, the DSHI-s included the question "how many times have you hurt yourself seriously enough to need medical treatment, or time in hospital?", however, responses to this item were included in analyses for this thesis. Finally, extra questions regarding how long since the individual had last engaged in self-injury, how many people knew about their self-injury, and who they knew that had self-injured were added to the survey (see the online supplementary material for a copy of the Wave Two survey).

Adequate psychometric properties have been demonstrated for the DSHI-s (Lundh et al., 2007). Whilst Lundh and colleagues shortened the DSHI for use with an adolescent sample, they did not report the psychometric properties. However, the DSHI-s has since been effectively used with other adolescent samples. For example, Hansen, Lundh, Homman and Wangby-Lundh (2009) investigated self-injury in a sample of over 200 Swedish school students and demonstrated that the DSHI-s had good internal reliability as indicated by a Cronbach's alpha of .82. In their study with NZ adolescents, Garisch and Wilson (2015) also reported a Cronbach's alpha greater than .70 for the DSHI-s. 


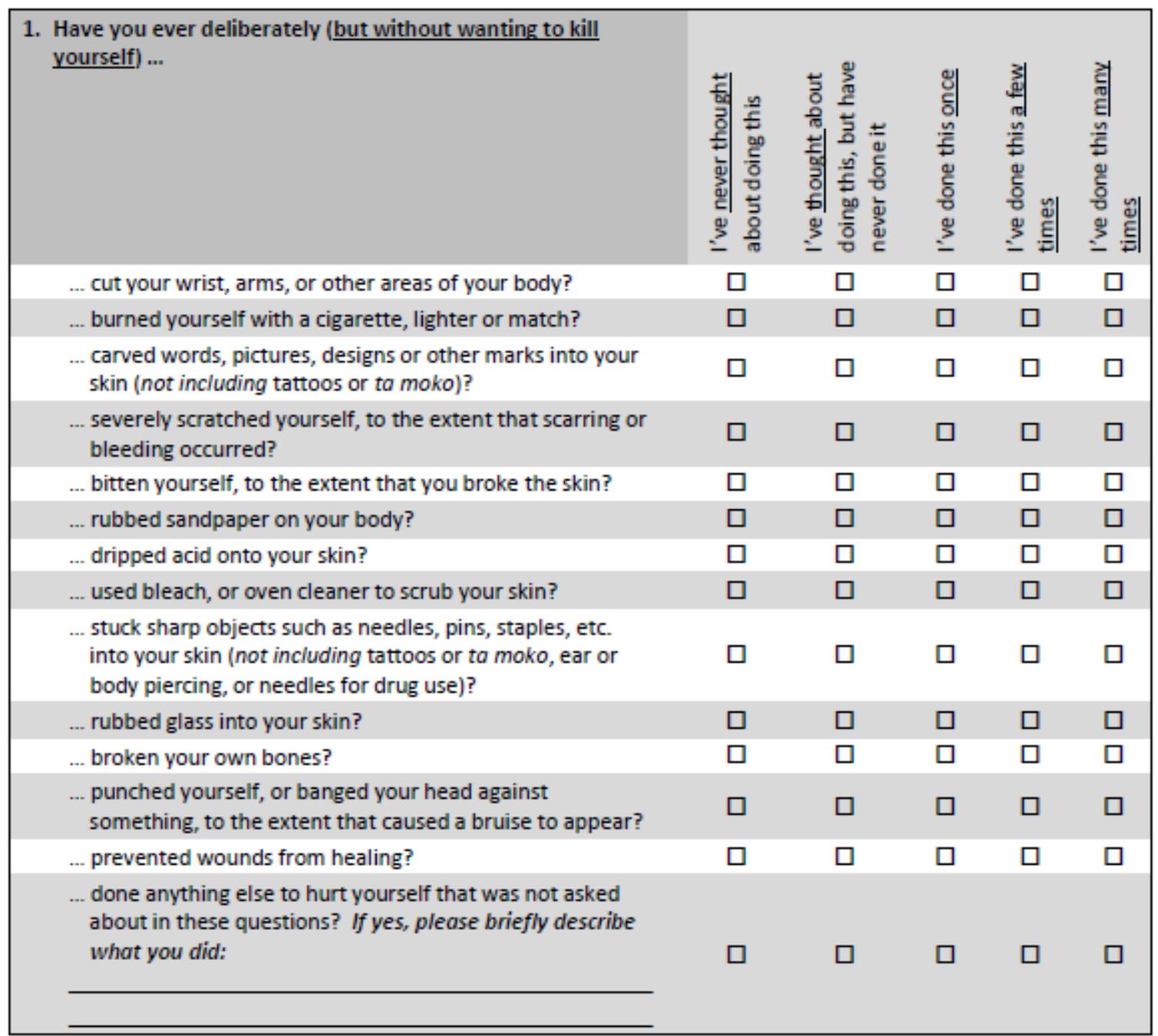

Figure 38. DSHI-s as presented in YWS Wave Two survey

Inventory of Statements About Self-injury. ${ }^{39}$ The DSHI-s was followed by the Inventory of Statements About Self-injury (ISAS; Klonsky \& Glenn, 2009). The ISAS contains two sections, the first assesses lifetime frequency of 12 specific NSSI behaviours (as the DSHI-s fulfils the same function, this section was not included in the YWS surveys), and the second is a self-report measure of 13 common functions of NSSI. The latter section is illustrated in Figures 39 and 40, and contains 39 items pertaining to the 13 common functions of NSSI. Each function is represented by three items (e.g., Affect Regulation: "When I selfharm I am calming myself down"; "releasing emotional pressure that has built up inside of me"; "reducing anxiety, frustration, anger, or other overwhelming emotions") to which participants respond on a 3-point Likert scale from 'Not relevant' to 'Very relevant'. The 13 functions include; affect regulation (e.g., 'calming myself down'), anti-dissociation (e.g.,

39 Only students who indicated that they had engaged in NSSI at least once were instructed to complete the ISAS 
'trying to feel something'), anti-suicide (e.g., 'putting a stop to suicidal thoughts'), autonomy (e.g., 'ensuring I am self-sufficient'), interpersonal boundaries (e.g., 'creating a boundary between myself and others'), interpersonal influence ('seeking care or help from others'), marking distress (e.g., 'creating a physical sign that I feel awful'), peer bonding (e.g., 'fitting in with others'), revenge (e.g., 'getting back at someone'), self-care (e.g., 'giving myself a way to care for myself'), self-punishment (e.g., 'punishing myself'), sensation seeking (e.g., 'doing something to generate excitement or exhilaration'), and toughness (e.g., 'seeing if I can stand the pain'). Each item is assigned a score of 0 (Not relevant), 1 (Somewhat relevant), or 2 (Very relevant). Subsequently, all three items for each function are summed and divided by three to get the mean function score.

People usually describe a range of reasons for deliberately hurting themselves. Some of these are described below. Please read each one, and tick the box that seems most true for you.

\begin{tabular}{|c|c|c|c|}
\hline 4. When I self-injure, I am ... & $\frac{\stackrel{ \pm}{\frac{\pi}{\pi}}}{\frac{3}{2}}$ & 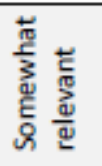 & 》 \\
\hline ... calming myself down & $\square$ & $\square$ & $\square$ \\
\hline ... creating a boundary between myself and others & $\square$ & $\square$ & $\square$ \\
\hline ... punishing myself & $\square$ & $\square$ & $\square$ \\
\hline ... giving myself a way to care for myself (by attending to the wound) & $\square$ & $\square$ & $\square$ \\
\hline ... causing pain so I will stop feeling numb & $\square$ & $\square$ & $\square$ \\
\hline ... avoiding the urge to attempt suicide & $\square$ & $\square$ & $\square$ \\
\hline ... doing something to feel excited (get a buzz) & $\square$ & $\square$ & $\square$ \\
\hline ... bonding with peers (to be close to other people) & $\square$ & $\square$ & $\square$ \\
\hline ... letting others know the extent of my emotional pain & $\square$ & $\square$ & $\square$ \\
\hline ... seeing if I can stand the pain & $\square$ & $\square$ & $\square$ \\
\hline ... creating a physical sign that I feel awful & $\square$ & $\square$ & $\square$ \\
\hline ... getting back at someone & $\square$ & $\square$ & $\square$ \\
\hline ... ensuring that I am self-sufficient (that I can do things for myself) & $\square$ & $\square$ & $\square$ \\
\hline ... releasing emotional pressure that has built up inside of me & $\square$ & $\square$ & $\square$ \\
\hline ... showing that I am separate from other people & $\square$ & $\square$ & $\square$ \\
\hline ... expressing anger towards myself for being worthless or stupid & $\square$ & $\square$ & $\square$ \\
\hline $\begin{array}{l}\text {... creating a physical injury that is easier to care for than my emotional } \\
\text { distress }\end{array}$ & $\square$ & $\square$ & $\square$ \\
\hline ... trying to feel something (as opposed to nothing) even if it is physical pain & $\square$ & $\square$ & $\square$ \\
\hline ... responding to suicidal thoughts without actually attempting suicide & $\square$ & $\square$ & $\square$ \\
\hline ... entertaining myself or others by doing something extreme & $\square$ & $\square$ & $\square$ \\
\hline ... fitting in with others & $\square$ & $\square$ & $\square$ \\
\hline ... seeking care or help from others & $\square$ & $\square$ & $\square$ \\
\hline ... showing I am tough or strong & $\square$ & $\square$ & $\square$ \\
\hline ... proving to myself that my emotional pain is real & $\square$ & $\square$ & $\square$ \\
\hline ... getting revenge against others & $\square$ & $\square$ & $\square$ \\
\hline ... showing that I do not need to rely on others for help & $\square$ & $\square$ & $\square$ \\
\hline$\ldots$... reducing anxiety, frustration, anger, or other overwhelming emotions & $\square$ & $\square$ & $\square$ \\
\hline
\end{tabular}

Figure 39. ISAS as presented in YWS Wave Two survey (Part one) 


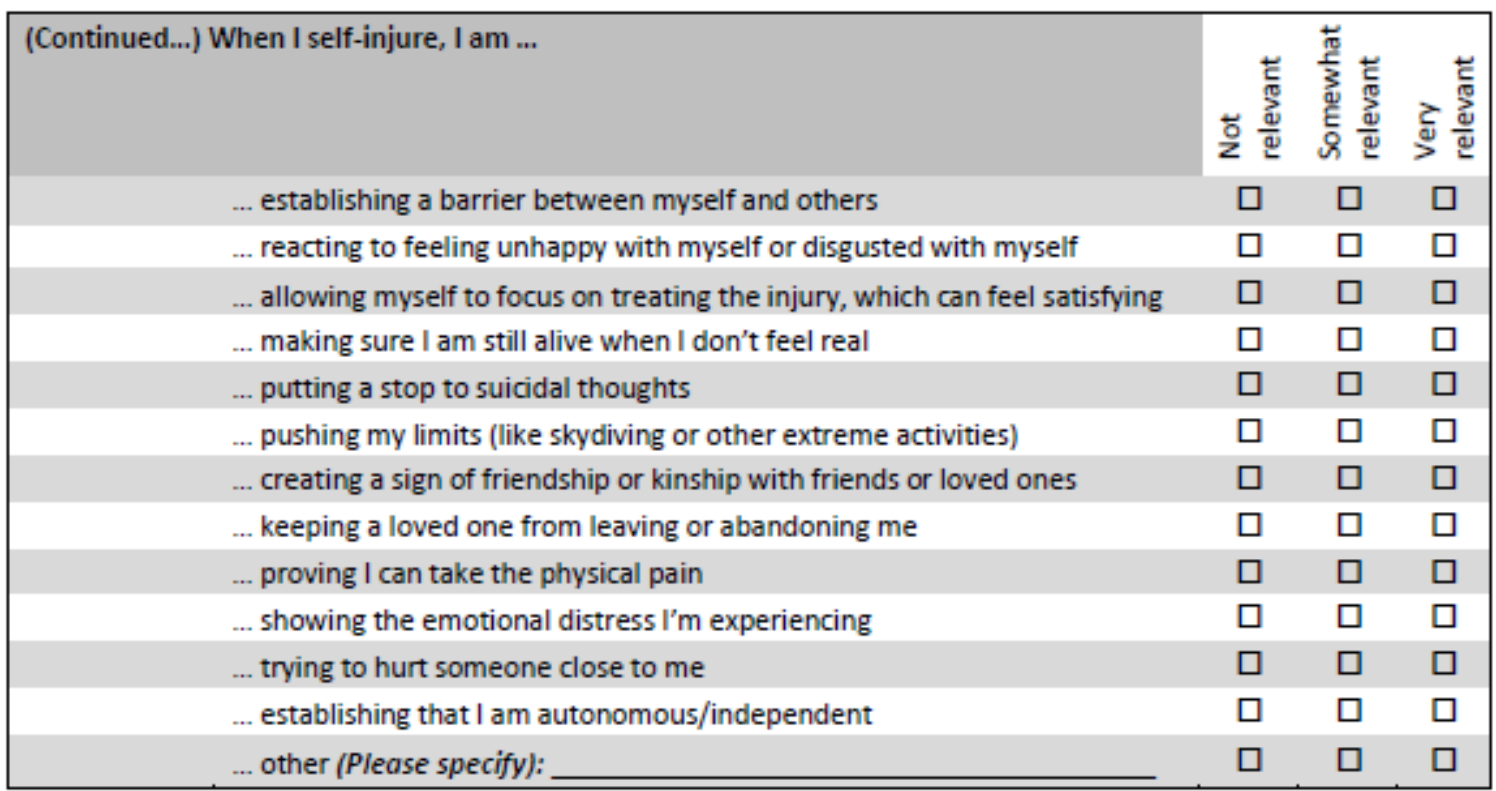

Figure 40. ISAS as presented in YWS Wave Two survey (Part two)

Klonsky and Glenn demonstrated that these functions could be categorised into overarching intrapersonal and interpersonal factors (see Table 14). Scores for these overarching intrapersonal and interpersonal factors are calculated by summing the scores from the relevant functions and dividing this by the number of functions in each overarching factor (5 intrapersonal functions; 8 interpersonal functions) to get a mean value.

Table 14

Two Factor Model of the Functions of NSSI

\begin{tabular}{ll}
\hline Intrapersonal & Interpersonal \\
Affect regulation & Autonomy \\
Anti-dissociation & Interpersonal boundaries \\
Anti-suicide & Interpersonal influence \\
Marking distress & Peer bonding \\
Self-punishment & Revenge \\
& Self-care \\
& Sensation seeking \\
& Toughness
\end{tabular}

In Klonsky and Glenn's (2009) sample of undergraduate students, Cronbach's alphas for the interpersonal and intrapersonal functional categories ranged from .80-.87. Moreover, in a later study with undergraduate students, Glenn and Klonsky (2011) demonstrated adequate test-retest reliability for these factors at .60 and .82 respectively. With regard to adolescents, Oktan (2014) used the ISAS in a sample of adolescents and demonstrated 
adequate Cronbach's alphas for both intrapersonal $(\alpha=.81)$ and interpersonal functions $(\alpha=$ $.86)$, and adequate test-retest reliability across two time points four weeks apart (.64).

Reliability analyses. Reliability analyses were conducted for the FMPS, DSHI-s and ISAS. As seen in Table 15, Cronbach's alphas for both total scores and subscales across the FMPS, DSHI-s, and the ISAS generally met the criteria or satisfactory internal reliability (.70). However, some alphas would be considered inadequate (ISAS - Affect Regulation, Interpersonal Boundaries, Sensation Seeking) and some fell well below adequacy (ISAS Revenge, Interpersonal Influence and Self-Care). Cronbach's alpha is very sensitive to the number of items in a scale or subscale, therefore, the low Cronbach alpha values for the ISAS subscales may have been partly due to each subscale only containing three items. Had there been additional items with identical inter-item correlations, Cronbach's alpha would have been higher. Giles (2002) suggests that in this case it is useful to look at the inter-item correlations, with correlations of .20 or above indicating adequate subscale properties. An investigation of the mean inter-item correlations for each subscale resulted in all subscales meeting Giles criteria of .20 (see Table 15).

Table 15

Cronbach's Alpha Reliability Coefficients and Mean Inter-Item Correlations for the FMPS, DSHI-s, and ISAS

\begin{tabular}{llll}
\hline \multicolumn{1}{c}{$\underline{\text { FMPS }}$} & $\underline{\alpha}$ & \multicolumn{1}{c}{${\underline{\text { ISAS }^{a}}}^{\text {a }}$} & $\frac{\alpha(M \text { inter-item }}{\text { correlation })}$ \\
Total & .93 & Affect Regulation & $.69(.43)$ \\
Concerns and Doubts & .93 & Anti-dissociation & $.74(.49)$ \\
Parental Pressure & .83 & Anti-suicide & $.82(.60)$ \\
Personal Standards & .84 & Marking distress & $.74(49)$ \\
Organisation & .91 & Self-punishment & $.79(.55)$ \\
Negative Perfectionism & .93 & Autonomy & $.70(.44)$ \\
Positive Perfectionism & .89 & Interpersonal boundaries & $.66(.41)$ \\
\multicolumn{1}{c}{$\underline{\text { DSHI-s }}$} & $\underline{ }$ & Interpersonal influence & $.51(.27)$ \\
DSHI-s & .84 & Peer bonding & $.82(.61)$ \\
& & Revenge & $.55(.30)$ \\
& & Self-care & $.53(.31)$ \\
& & Sensation seeking & $.62(.36)$ \\
& & Toughness & $.71(.45)$ \\
& & Intrapersonal functions & $.81(.46)$ \\
& & Interpersonal functions & $.82(.35)$ \\
\hline
\end{tabular}

${ }^{a}$ Only included students who had indicated engaging in self-injury at least once 


\section{Procedure}

For a detailed account of the procedure refer to pages 130-131. As a brief reminder, ethical approval for this research was granted by HDEC in 2011 (approval number: 11/645). In 2014, students were surveyed in their regular classrooms by members of the YWS team. Parent consent for each student was sought prior to visiting each school. Participation was voluntary. Each classroom had at least one member of the team present at all times and at least one YWS team clinical psychologist was on site to provide any extra support the students required. In addition, each student's response to the SBQ-R screening tool were checked within 24 hours and guidance counselors were notified of students at risk of suicide, who required additional follow-up and support.

With regard to the layout and length of the survey, some students were required to complete every scale presented, whilst others were able to skip scales that did not apply to them. For example, the survey was branched so that students who did not indicate any history of self-injury or suicidal ideation were not required to complete the full scale measures due to concerns raised by the responsible ethics committee. Specifically, students are asked one item from the SBQ-R ('Have you ever thought about killing yourself, or attempted to kill yourself?"), and a single item pertaining to self-injury ("Please indicate whether you have had thoughts about hurting yourself (but not actually done this), whether you have hurt yourself on purpose (e.g., cut, burnt, scratched or carved your skin, etc.), or whether you have never done this") (see Figure 41). If participants reported that they had 'Never' thought about, or

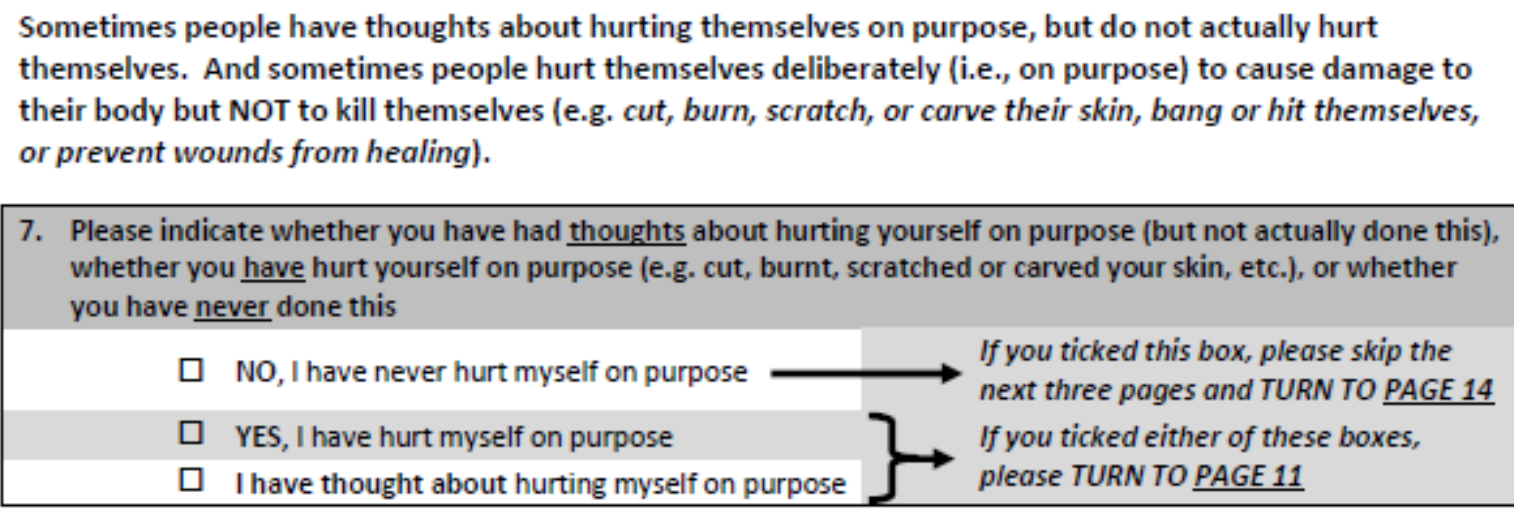

7. Please indicate whether you have had thoughts about hurting yourself on purpose (but not actually done this), whether you have hurt yourself on purpose (e.g. cut, burnt, scratched or carved your skin, etc.), or whether you have never done this

$\square \quad$ NO, I have never hurt myself on purpose If you ticked this box, please skip the next three pages and TURN TO PAGE 14

$\square$ YES, I have hurt myself on purpose If you ticked either of these boxes, $\square$ I have thought about hurting myself on purpose please TURN TO PAGE 11

Figure 41. Self-injury (DSHI-s) screening question, and branching instructions as presented in YWS Wave Two survey

attempted to kill themselves they did not complete the full SBQ-R, and similarly if participants indicated that they had not hurt themselves on purpose or had not thought about 
hurting themselves they were instructed to skip all following self-injury related questions (including DSHI-s and ISAS; see the supplementary online material for a copy of the T1 survey). As a result, some students skipped several pages of survey content and could complete the survey quickly, whereas others were required to complete most measures which on average took longer.

\section{Study 3a Results}

The aim of Study 3a was to investigate the cross-sectional relationship between positive and negative perfectionism, and NSSI in both male and female adolescents. Descriptive statistics outlining the profile of NSSI and perfectionism in this sample are reported prior to a report of the results for the relationship between NSSI and perfectionism.

\section{Descriptive Statistics}

Self-injury. Analysis of the data for the total sample indicated that $21.2 \%$ of the students had engaged in NSSI at some point in their lifetime (see Table 16 for all percentages, and means and standard deviations). A further $9.8 \%$ had thought about NSSI but had never tried it. Of those who have engaged in NSSI, $62.0 \%$ indicated at least one form of NSSI within the past six months, $22.2 \%$ within the past year, and $15.7 \%$ over a year ago. This suggests that, unlike some research with older samples (e.g., Whitlock, Prussien \& Pietrusza, 2015; who found that $58.4 \%$ of their self-injuring university sample had engaged in NSSI in the past 12 months), a large proportion of the NSSI captured in this study is current NSSI. The most endorsed form of NSSI was cutting (59.4\%), followed by severely scratching (41.8\%), and punching or banging one's head (32.8\%). Moreover, individuals with a history of NSSI indicated engaging in an average of three methods of self-injury $(M=2.67, S D=2.45)$.

Descriptive analyses showed key differences between males and females on the measure of NSSI engagement. A Chi-squared test for independence illustrated that sex was significantly related to NSSI history $\left(\chi^{2}(2, n=879)=78.32, p<.001\right)$ with males more likely to endorse 'No' and less likely to endorse 'Thought about it' and 'Yes' than expected. In contrast, females were more likely to endorse 'Yes' and 'Thought about it', and less likely to endorse 'No' than expected. Overall, $29.3 \%$ of females and $9.2 \%$ of males had engaged in NSSI at least once in their lifetime (see Table 16). Furthermore, when analysing the data by sex, the three most frequently endorsed methods of NSSI were broadly consistent across males (cutting, 45.9\%; scratching, 31.0\%; banging/punching, 30.0\%) and females (cutting, 63.1\%; scratching, 44.7\%; banging/punching, 33.6\%). However, Chi-squared analyses showed that females were significantly more likely, and males significantly less likely, to 
engage in cutting $\left(\chi^{2}(1, n=283)=5.84, p=.02\right)$, or carving $\left(\chi^{2}(1, n=274)=5.77, p=.02\right)$ methods of self-injury.

Table 16

NSSI Prevalence, Methods, and Functions Frequencies and Chi-Squared Analyses across Males and Females.

\begin{tabular}{|c|c|c|c|c|}
\hline DSHI-s $M(S D)$ & $\begin{array}{l}\underline{\text { Total }} \\
(n=867) \\
.23(.52)\end{array}$ & $\begin{array}{l}\underline{\text { Male }} \\
(n=354) \\
.10(.34)\end{array}$ & $\begin{array}{l}\underline{\text { Female }} \\
(n=513) \\
.32(.60)\end{array}$ & $\chi^{2}$ \\
\hline Prevalence $^{40}$ & & $\begin{array}{l}\% \text { / Actual } \\
\text { (Expected) }\end{array}$ & $\begin{array}{l}\% / \text { Actual } \\
\text { (Expected) }\end{array}$ & \\
\hline Yes & $21.2 \%$ & $9.2 / 33(76)$ & $29.3 / 153(111)$ & $78.32 * *$ \\
\hline Thought about it & $9.8 \%$ & $5.0 / 18(35)$ & $13.0 / 38(51)$ & \\
\hline No & $69.1 \%$ & $85.7 / 306$ (247) & $57.7301(360)$ & \\
\hline Methods $^{41}$ & & $\begin{array}{l}\% \text { / Actual } \\
\text { (Expected) }\end{array}$ & $\begin{array}{l}\% \text { / Actual } \\
\text { (Expected) }\end{array}$ & \\
\hline Cut & $59.4 \%$ & $45.9 / 28(36)$ & $63.1 / 140(132)$ & $5.84 *$ \\
\hline Scratched & $41.8 \%$ & $31.0 / 18(24)$ & 44.7/ 97 (91) & 3.51 \\
\hline $\begin{array}{l}\text { Punched/banged } \\
\text { head }\end{array}$ & $32.8 \%$ & $30.0 / 18(20)$ & $33.6 / 72(70)$ & .23 \\
\hline $\begin{array}{l}\text { Carved } \\
\text { markings into } \\
\text { skin }\end{array}$ & $28.1 \%$ & $15.5 / 9 \quad(16)$ & $31.5 / 68$ (61) & $5.77 *$ \\
\hline $\begin{array}{l}\text { Stuck sharp } \\
\text { objects into skin }\end{array}$ & $27.9 \%$ & 23.7/14 (17) & $29.1 / 62(60)$ & 42 \\
\hline $\begin{array}{l}\text { Prevented } \\
\text { wounds healing }\end{array}$ & $27.1 \%$ & $28.6 / 16(17)$ & 28.3/ 61 (60) & .05 \\
\hline Burned & $18.1 \%$ & $15.5 / 9(11)$ & $18.8 / 40(39)$ & .33 \\
\hline Bitten & $15.6 \%$ & $10.2 / 6(9)$ & 17.1/ 37 (34) & 1.70 \\
\hline Rubbed glass & $7.7 \%$ & $10.2 / 53(55)$ & 7.0/ 200 (199) & .67 \\
\hline $\begin{array}{l}\text { Rubbed } \\
\text { sandpaper }^{\mathrm{a}}\end{array}$ & $5.8 \%$ & $10.0 / 6(4)$ & 4.6/ $10(13)$ & 2.48 \\
\hline $\begin{array}{l}\text { Dripped acid } \\
\text { onto skin }^{\mathrm{a}}\end{array}$ & $2.2 \%$ & $5.1 / 3(1)$ & $1.4 / 3(5)$ & 2.94 \\
\hline $\begin{array}{l}\text { Bleach or oven } \\
\text { cleaner on skin }{ }^{\mathrm{a}}\end{array}$ & $0.7 \%$ & $0.0 / 0(0)$ & $0.9 / 2(2)$ & .53 \\
\hline Broke bones $^{\mathrm{a}}$ & $0.7 \%$ & $0.0 / 0(0)$ & $0.9 / 2(2)$ & .55 \\
\hline Other $^{\mathrm{a}}$ & $7.5 \%$ & $6.0 / 3(4)$ & $8.0 / 13(12)$ & .22 \\
\hline
\end{tabular}

$* p<.05 ; * * p<.001$

${ }^{40}$ Of total sample

${ }^{41}$ Of sample indicating that they have thought about or engaged in self-injury 
Perfectionism. A 2 (Sex: male, female) x 2 (Perfectionism: positive, negative) mixedfactor Analysis of Variance (ANOVA) was conducted to investigate the relationship between sex (between-subjects) and perfectionism (within-subjects). Mauchly's Test of Sphericity was significant $(p<.001)$ indicating that the assumption of sphericity was violated. Therefore, as recommended by Tabachnick and Fidell (2007) all analyses below have undergone the Huynh-Feldt correction. Overall, the analysis revealed a main effect of perfectionism $(F(1$, $\left.909)=657.98, p<.001 ; \eta^{\mathrm{p} 2}=.42\right)$ indicating that positive perfectionism was endorsed significantly more than negative perfectionism in both males and females (see Figure 42 and Table 17 for means and standard deviations). There was also a main effect of sex $(F(1,909)=$ $10.97, p=.01 ; \eta_{\mathrm{p}}{ }^{2}=.01$ ), illustrating that females scored significantly higher than males on both positive and negative perfectionism. The interaction between perfectionism and sex was non-significant $(F(1,909)=.26, p=.61)$, suggesting that the difference between positive and negative perfectionism was consistent across sex.

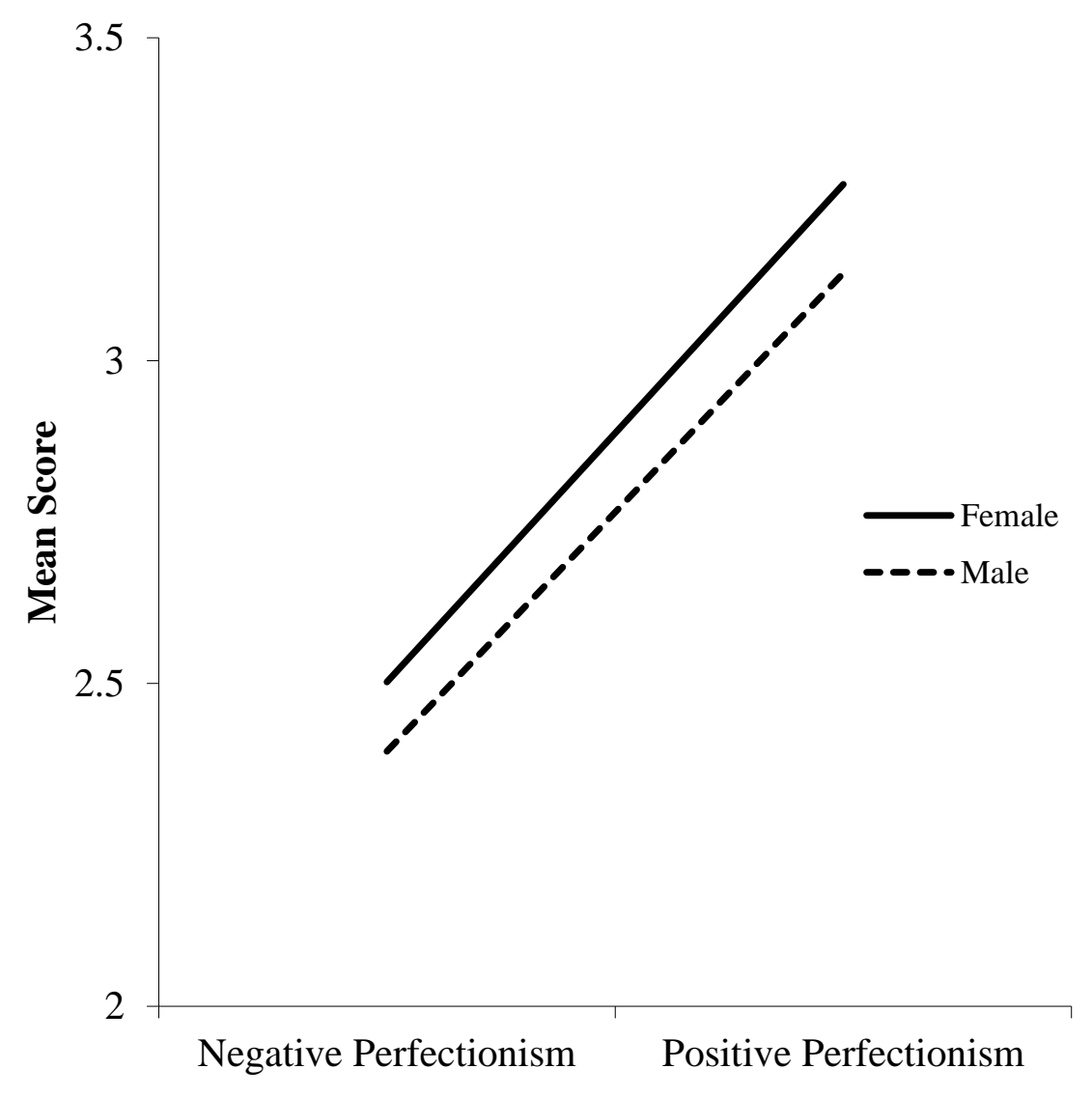

Figure 42. Mean FMPS negative and positive perfectionism scores among males and females

In addition, perfectionism was investigated according to the four rudimentary perfectionism subscales identified in Study 2 (CMD, PP, PS, Organisation). Perfectionism at the subscale level was examined using a 2 (between-subjects factor Sex: male, female) x 4 
(within-subjects factor Perfectionism: CMD, PP, PS, Organisation) mixed-factor ANOVA. Mauchly's Test of Sphericity was significant $(p<.001)$, again indicating that the sphericity assumption was violated. Therefore, as above, all analyses were Huynh-Feldt corrected. Similar to the results above, analyses revealed a main effect of perfectionism $(F(3,896)=$ $\left.277.03, p<.001 ; \eta_{\mathrm{p}}^{2}=.24\right)$, and a main effect of $\operatorname{sex}\left(F(1,896)=16.19, p<.001 ; \eta_{\mathrm{p}}{ }^{2}=.02\right)$. Overall, students appeared to score highest on the Organisation subscale, followed by PS, PP, and finally the CMD subscale (see Figure 43 and Table 17 for means and standard deviations). Post-hoc $t$-tests indicated that the difference between Organisation and PS was significant $(t(898)=12.41, p<.001)$, and the difference between CMD and PP was significant $(t(899)=-18.93, p<.001)$. Finally, there was also a significant difference between endorsement of PP and PS $(t(897)=-3.07, p=.01)$.

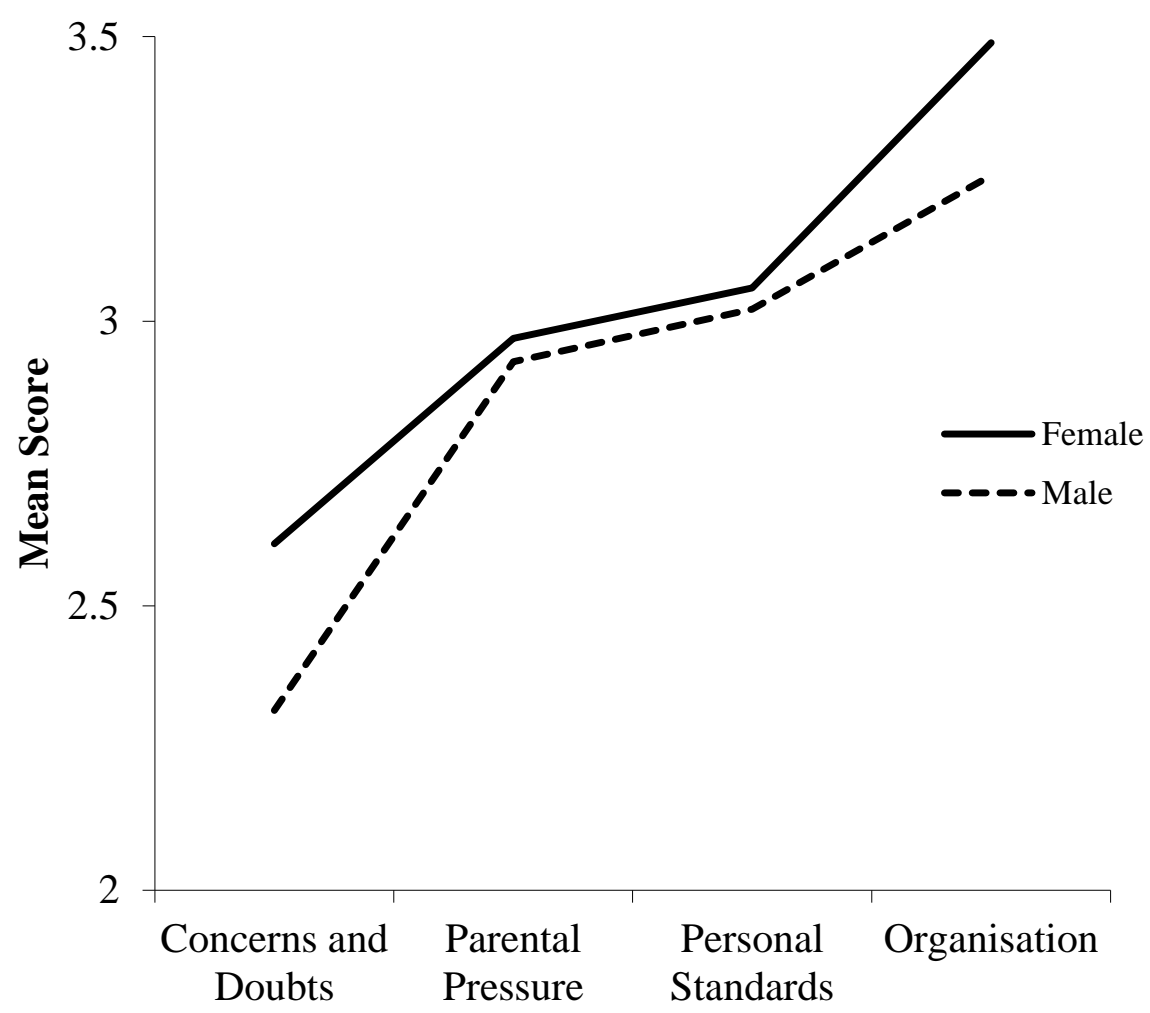

Figure 43. Mean FMPS subscale scores among males and females

Across all subscales females tended to score higher than males. The interaction between perfectionism and sex was also significant $\left(F(3,896)=8.47, p<.001 ; \eta_{\mathrm{p} 2}=.01\right)$, suggesting that differences between males and females on individual subscales scores differs depending on the subscale in question. Specifically, post-hoc $t$-tests revealed that females 
scored significantly higher than males on the CMD $(t(899)=-5.62, p<.001)$, and Organisation $(t(909)=-4.13, p<.001)$ subscales but not the PP $(t(908)=-.74, p=.46, n s)$ and PS $(t(897)=-.69, p=.49, n s)$ subscales. Pearson's correlations were used to examine the relationship between each subscale of the FMPS and positive and negative perfectionism. As expected, CMD and PP were highly correlated $(r(911)=.55, p<.001)$, as were PS and Organisation $(r(911)=.47, p<.001)$. The relationships were broadly consistent across males and females (see Table 18). In addition, these analyses illustrated a significant, positive correlation between positive and negative perfectionism, suggesting that an individual scoring high on negative perfectionism, is likely to also score high on positive perfectionism $(r(911)=.23, p<.001)$. Interestingly, this relationship appeared stronger in males $(r(367)=$ $.33, p<.001)$ than in females $(r(534)=.16, p<.001)$. Therefore, further analyses were conducted to test whether this difference was significant. First, the Pearson's correlation coefficients for both males and females are converted to $z$-values using a Fishers- $z$ transformation. Then, in combination with the respective sample sizes, the values were used to calculate an observed $z$-score and associated $p$-value. In this case, the significant $p$-value indicated that the relationship between positive perfectionism and negative perfectionism is significantly stronger for male adolescents than female adolescents $(z=2.67, p=.01)$.

\section{Perfectionism and NSSI}

Pearson's correlation coefficients were then calculated to quantify the relationships between the four perfectionism subscales, negative and positive perfectionism, and NSSI. These analyses and all analyses to follow only include data from participants that completed at least the first branching question of the DSHI-s and the FMPS $(n=858)$. Overall, three of the four perfectionism subscales were significantly associated with engagement in self-injury (see Table 18). Specifically, CMD, and PP were positively associated with engagement in NSSI at $.31(p<.001)$ and $.16(p<.001)$ respectively. Organisation was negatively associated with NSSI engagement $(r(858)=-.13, \mathrm{p}<.001)$. Analyses also revealed a significant correlation between negative perfectionism and NSSI $(r(858)=.26, p<.001)$, thus indicating that the more an individual endorses negative perfectionism, the more likely they are to report engaging in NSSI, while the reverse was true for positive perfectionism and $\operatorname{NSSI}(r(858)=-.11, p<.001)$.

These relationships were investigated further by separating the data by sex. Results illustrated that the relationship between negative perfectionism and NSSI remained significant for females $(r(509)=.31, p<.001)$, however the relationship was non-significant 
Table 17

Means, Standard Deviations, and Pearson's Correlation Coefficients for the Relationship between the Components of Perfectionism

\begin{tabular}{|c|c|c|c|c|c|c|c|c|c|}
\hline \multirow{9}{*}{$\begin{array}{l}\text { Total } \\
(n=911)\end{array}$} & & & \multirow{4}{*}{$\frac{S D}{.71}$} & \multicolumn{6}{|c|}{ Correlations } \\
\hline & & \multirow{3}{*}{$\frac{M}{3.22}$} & & \multirow{3}{*}{$\frac{\frac{\text { Positive }}{\text { Perfectionism }}}{-}$} & \multirow{3}{*}{ 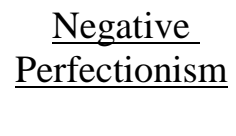 } & \multirow{3}{*}{$\frac{\text { Concerns and }}{\underline{\text { Doubts }}}$} & \multirow{3}{*}{$\frac{\text { Parental }}{\underline{\text { Pressure }}}$} & \multirow{3}{*}{$\underline{\underline{\text { Personal }}}$} & \multirow[t]{3}{*}{$\underline{\text { Organisation }}$} \\
\hline & & & & & & & & & \\
\hline & Positive perfectionism & & & & & & & & \\
\hline & Negative perfectionism & 2.46 & .69 & $.23 *$ & - & & & & \\
\hline & Concerns and Doubts & 2.49 & .78 & $.26^{*}$ & $.80^{*}$ & - & & & \\
\hline & Parental Pressure & 2.96 & .78 & $.22 *$ & $.81^{*}$ & $.55^{*}$ & - & & \\
\hline & Personal Standards & 3.04 & .79 & $.85^{*}$ & $.38^{*}$ & $.40^{*}$ & $.37 *$ & - & \\
\hline & Organisation & 3.39 & .86 & $.87 *$ & .03 & .06 & .02 & $.47 *$ & - \\
\hline \multirow{6}{*}{$\begin{array}{l}\text { Males } \\
(n=367)\end{array}$} & Positive perfectionism & 3.14 & .74 & - & & & & & \\
\hline & Negative perfectionism & 2.39 & .63 & $.33^{*}$ & - & & & & \\
\hline & Concerns and Doubts & 2.32 & .69 & $.35^{*}$ & $.77^{*}$ & - & & & \\
\hline & Parental Pressure & 2.93 & .70 & $.31 *$ & $.77^{*}$ & $.52^{*}$ & - & & \\
\hline & Personal Standards & 3.02 & .81 & $.85^{*}$ & $.44 *$ & $.47 *$ & $.42 *$ & - & \\
\hline & Organisation & 3.25 & .89 & $.88^{*}$ & $.14 *$ & $.16^{*}$ & $.14 *$ & $.50^{*}$ & - \\
\hline \multirow{6}{*}{$\begin{array}{l}\text { Females } \\
(n=534)\end{array}$} & Positive perfectionism & 3.27 & .69 & - & & & & & \\
\hline & Negative perfectionism & 2.50 & .73 & $.16^{*}$ & - & & & & \\
\hline & Concerns and Doubts & 2.61 & .82 & $.18^{*}$ & $.81^{*}$ & - & & & \\
\hline & Parental Pressure & 2.98 & .83 & $.16^{*}$ & $.83 *$ & $.58 *$ & - & & \\
\hline & Personal Standards & 3.06 & .78 & $.84^{*}$ & $.34^{*}$ & $.36^{*}$ & $.35^{*}$ & - & \\
\hline & Organisation & 3.49 & .83 & $.86^{*}$ & -.05 & -.04 & -.05 & $.45^{*}$ & - \\
\hline
\end{tabular}

Note: Post hoc Bonferroni correction was applied to address the issue of inflated family-wise error, the corrected $p$-value is .001 and significant correlations are indicated by $*$ 
for males $(r(349)=.08, p=.14, n s)$. The differences between males and females in the magnitude of the association between negative perfectionism and NSSI suggests that sex may moderate the relationship. In contrast, the relationship between positive perfectionism and NSSI remained consistent across sex $\left(r_{\text {Females }}(509)=-.14, p=.01 ; r_{\text {Males }}(349)=-.14, p=.01\right.$; see Table 18 for all relevant correlational statistics).

Table 18

Pearson's Correlation Coefficients for the Relationship between Perfectionism and NSSI

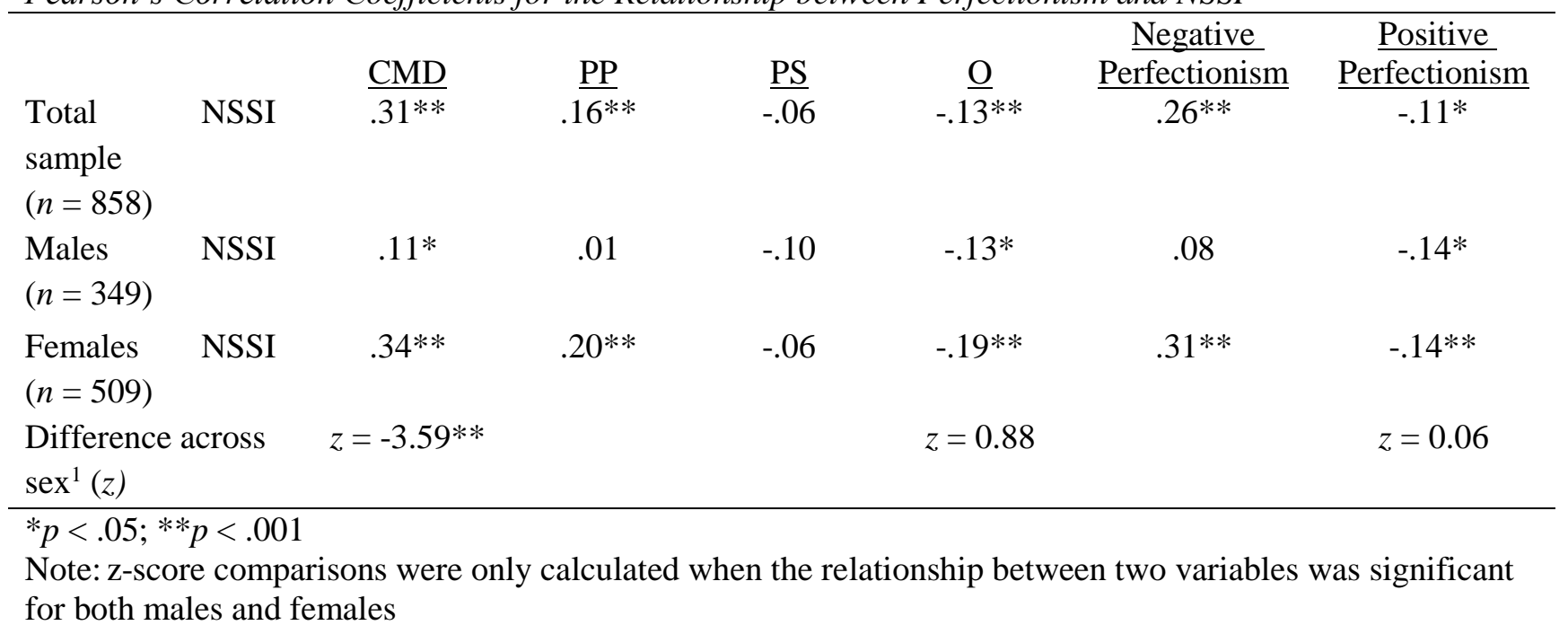

Given that the subscales of positive and negative perfectionism perform similarly to the overall positive and negative perfectionism scales, from here I will focus on positive and negative perfectionism, rather than referring to all four subscales. This will reduce the complexity of the results and allow greater depth of statistical analysis investigating the relationships between negative and positive perfectionism, and NSSI.

Moderator analyses. Based on the significant sex differences in the correlational relationships outlined above, I predicted that sex may significantly moderate the relationship between negative perfectionism and NSSI. Moderators are third variables that effect the size of the relationship between a predictor variable and an outcome variable (Baron \& Kenny, 1986; see graphical depiction in Figure 44 below). In other words, the strength of the relationship between two variables is dependent on the level of another moderating variable. Typically, moderator analyses utilise multiple regression statistical techniques which allow the simultaneous investigation of the relationship between continuous variables, such as perfectionism and self-injury, and a dichotomous variable, such as sex (Jose, 2013).

Following the guidelines originally set out by Baron and Kenny (1986), moderation analyses then extend beyond the simple multiple regression analyses, and investigate whether the two 
predictor variables interact to contribute further, unique, variance in predicting the outcome (Jose, 2013).

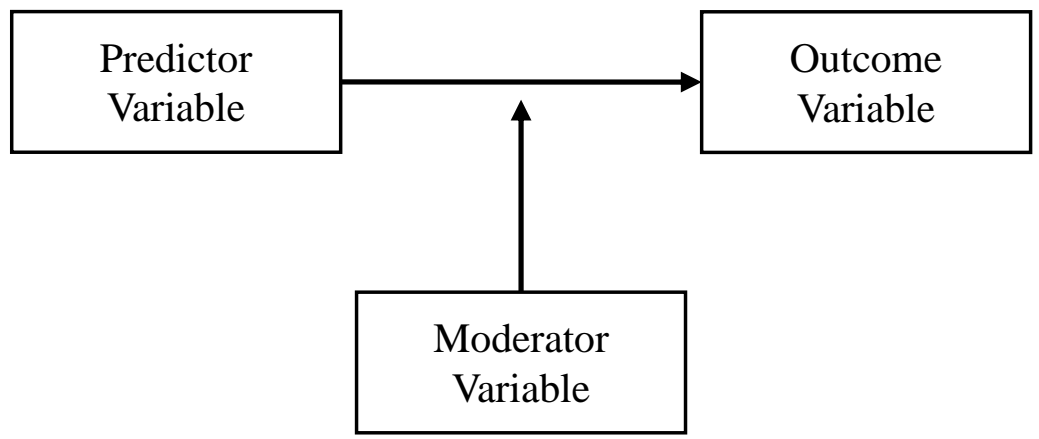

Figure 44. Graphical depiction of a moderation analysis

Therefore, to test whether sex significantly moderated the relationship between perfectionism and NSSI, and more specifically whether the interaction between negative perfectionism and sex predicted unique variance with regard to NSSI, a sex by negative perfectionism interaction variable was computed. ${ }^{42} \mathrm{Next}$, a hierarchical regression analysis was conducted, with negative perfectionism and sex in the first block, and negative perfectionism and sex, plus the sex by negative perfectionism interaction variable, in the second block. The hierarchical nature of the regression indicates whether the interaction variable (negative perfectionism x sex) significantly predicts the outcome variable (NSSI) over and above the additive effects of the original predictor variables (negative perfectionism and sex; Jose, 2013). In this case, the final model accounted for $12.0 \%$ (adjusted $R^{2}=.12$ ) of the variance in predicting $\operatorname{NSSI}(F(3,854)=38.67, p<.001)$, with the interaction variable accounted for significant unique variance beyond that accounted for by sex and negative perfectionism alone $\left(\Delta R^{2}=.02 ; \Delta F(1,854)=16.32, p<.001\right.$; see Table 19 below for the relevant statistics). Given that the sex by negative perfectionism interaction variable significantly predicted NSSI further investigation into the exact nature of this was warranted. The relevant statistics for the relationship between negative perfectionism and NSSI,

\footnotetext{
${ }^{42}$ Prior to computing the interaction variable, I dummy coded the sex variable. Put simply, this involved coding males as 0 and females as 1 . In addition, I considered whether negative perfectionism should first be centred based on claims that this reduces issues with multicollinearity. However, Jose (2013) suggests this is not the case and as suggests that centring variables is not necessary. Therefore, I have decided not to centre the variables prior to conducting moderator analyses.
} 
moderated by sex, are presented in Table 19. However, in order to meaningfully interpret the results, an online moderation tool was used to graph the findings (ModGraph; Jose, 2013).

Table 19

Summary of Multiple Regression of NSSI Predicted by Sex, Negative Perfectionism and SexNegative Perfectionism Interaction

\begin{tabular}{lccc} 
Sex & $\underline{\mathrm{B}}$ & $\underline{\beta}$ & $\Delta \underline{\mathrm{R}^{2}}$ \\
Negative perfectionism & $-.30^{*}$ & $-.29 *$ & - \\
$\begin{array}{l}\text { Sex x Negative } \\
\text { perfectionism interaction }\end{array}$ & .04 & .06 & $.10^{* *}$ \\
\hline${ }^{*} p<.05 ; * *<<.001$ & $.21^{* *}$ & $.54^{* *}$ & $.02^{* *}$ \\
\hline
\end{tabular}

As such, Figure 45, below, shows the graphical output of the interaction between negative perfectionism and sex. It is important to note that the gradient of the line is much greater for females than it is for males, indicating that the relationship between negative perfectionism and NSSI is stronger in females than it in males. Moreover, the largest difference between males and females was seen at high levels of negative perfectionism, such that high negative perfectionism was associated with much greater engagement in NSSI for females than for males.

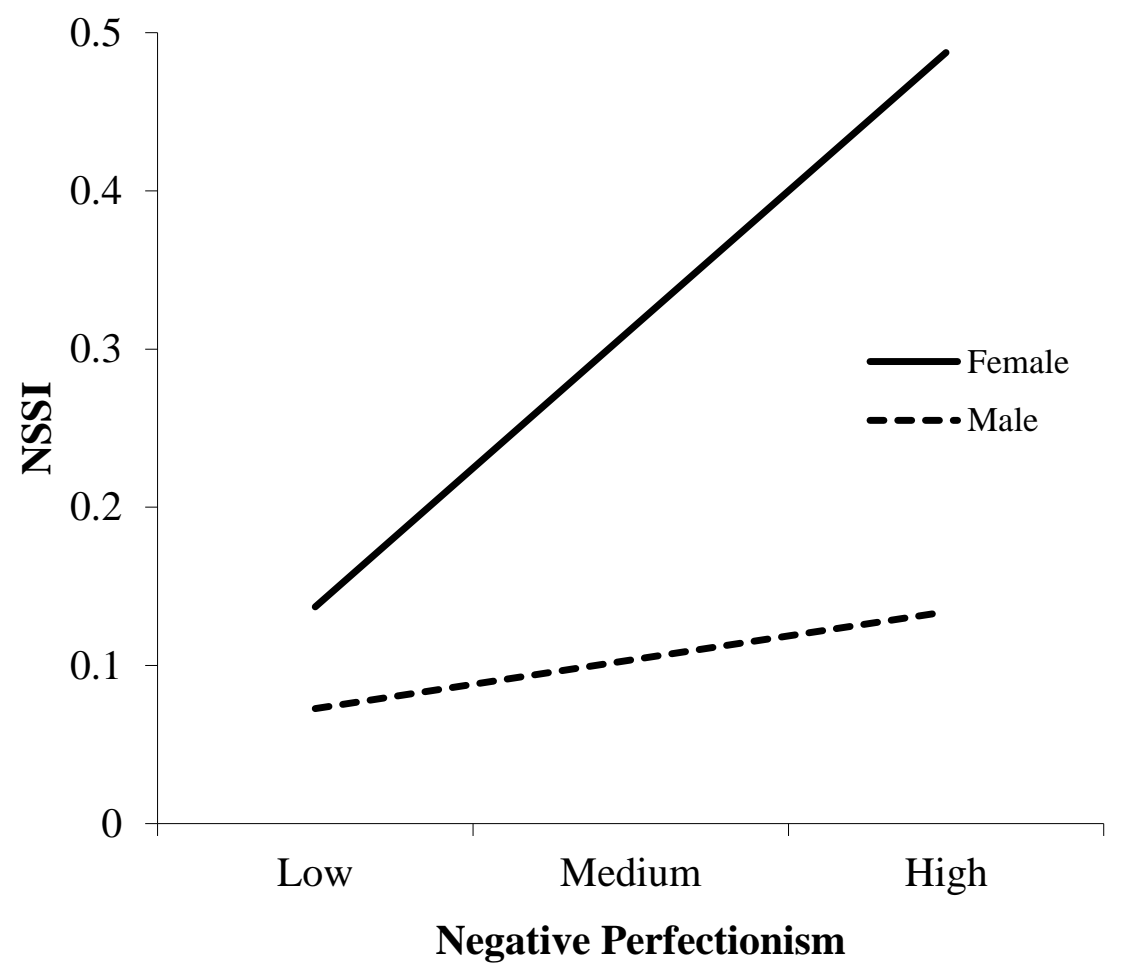

Figure 45. Moderation of negative perfectionism and sex against NSSI 
Finally, simple slope analyses were conducted to quantify the individual relationships between negative perfectionism and NSSI for females and males. To help with interpretation, larger simple slope values indicate stronger correlations between the predictor and outcome variables (Jose, 2013). First, simple slope analyses indicated that, for males, the slope was not significantly different to 0 (i.e., there is no significant relationship between negative perfectionism and NSSI in males; slope $=.04 ; t(858)=1.04, p=.30)$. Conversely, for females, the simple slope value was significant, indicating a moderate positive relationship between negative perfectionism and NSSI $($ slope $=.25 ; t(858)=8.49, p<.001)$.

A test for moderation of NSSI and positive perfectionism by sex accounted for $6.26 \%$ of the variance in predicting NSSI (adjusted $R^{2}=.06 ; F(3,854)=19.03, p<.001$ ), with the interaction variable not accounting for any significant unique variance beyond that accounted for by sex and positive perfectionism alone $\left(\Delta R^{2}=.00 ; \Delta F(1,854)=1.38, p=.24, n s\right.$; see Table 20). In other words the relationship between positive perfectionism and NSSI did not significantly differ across males and females.

Table 20

Summary of Multiple Regression of NSSI Predicted by Sex, Positive Perfectionism and SexPositive Perfectionism Interaction

\begin{tabular}{lccc}
\hline Sex & $\underline{\mathrm{B}}$ & $\underline{\beta}$ & $\Delta \underline{R^{2}}$ \\
Positive perfectionism & $.42^{*}$ & $.39 *$ & - \\
Sex x Positive perfectionism & -.06 & -.09 & $.06^{* *}$ \\
interaction & .06 & .19 & .00 \\
\hline$* p<.05 ; * *<.001$ & & &
\end{tabular}

\section{Summary of Results}

Analyses revealed that approximately one in five students reported having engaged in NSSI at least once, and of those $84 \%$ indicated that they had last self-injured within the last year. Females were more likely to report that they had ever thought about or engaged in selfinjury than males. Cutting was the most frequently reported method, followed by scratching, and punching and banging one's head. With regard to perfectionism, students scored significantly higher on positive perfectionism than negative perfectionism and overall females were more perfectionistic than males. Positive and negative perfectionism were significantly correlated in both males and females.

Moderator analyses demonstrated that sex moderated the relationship between negative perfectionism and NSSI such that negative perfectionism, in relation to engagement 
in NSSI, is particularly prominent and detrimental for females. Positive perfectionism showed a weak negative association with NSSI in both males and females. Sex did not further moderate this relationship.

\section{Study 3b: Examining the Relationship between Perfectionism and the Functions of NSSI}

The results above provide the basis for identifying perfectionistic individuals who are at greater risk of engaging in NSSI. However, once identified, they do not provide any guidance on intervention beyond addressing NSSI and perfectionism individually. With more information on specific details within the NSSI-perfectionism relationship researchers and clinicians can identify some precise targets for treatment.

When exploring the relationship between perfectionism and NSSI, it is important to recognise that almost all human behaviour serves a function for the individual engaging in it (Skinner, 1969), and NSSI is no exception (Klonsky, 2007). Therefore, investigating whether highly perfectionistic individuals engage in NSSI for a particular reason will be valuable in identifying the needs that are likely being satisfied with NSSI. Furthermore, identifying the needs fulfilled by NSSI is often considered the first step towards increasing appropriate adaptive coping strategies that can be used to substitute NSSI behaviours (Lloyd-Richardson, 2010; Stanley et al., 2014).

\section{Theoretical Predictions on the Relationship between Perfectionism and NSSI Functions}

As with the general relationship between NSSI and perfectionism, further research investigating the functions of NSSI in perfectionistic individuals is limited. Some researchers have proposed particular functions that may be more relevant to perfectionistic individuals but do not have the empirical data to confirm or disconfirm their hypotheses (e.g., Flett et al., 2012; Hoff \& Muehlenkamp, 2009). Others have investigated the functions but only reported broad findings or only assessed a limited range of possible functions of NSSI (e.g., Claes et al., 2012; Nock \& Prinstein, 2004). Such research on the functions of NSSI for perfectionistic individuals is outlined below.

Hoff and Muehlenkamp (2009) found that individuals scoring low on Organisation subscale of the FMPS are at more risk of engaging in NSSI, and hypothesised that these individuals may be using NSSI as a method of gaining control and to distract from their disorganisation. Unfortunately, Hoff and Muehlenkamp did not empirically test this theory and rather highlighted it as an area for future research. 
Flett and colleagues (2012) also considered the functions of DSH in perfectionistic individuals. Specifically they examined DSH with reference to a modified version of the selfpunitiveness model (Carver \& Ganellen, 1983). Flett and colleagues' version of the selfpunitiveness model denotes that highly perfectionistic individuals are especially prone to extreme self-criticism and experiences of shame. In turn, they may develop a desire to punish themselves for perceived short-comings. Further, Flett and colleagues proposed that one form of punishment may be DSH. They tested this model in a sample of male and female university students and found that, among men, perfectionism was mostly unrelated to DSH. Conversely, among women, PC and SPP were associated with DSH, suggesting that the interpersonal aspects of perfectionism are most detrimental. Wider components of the selfpunitiveness model, including self-criticism and shame, were also related to DSH in women. Flett and colleagues describe this as particularly worrying given that elevated shame and selfcriticism may further prevent women from seeking help when it is most needed. In addition, they describe a potentially concerning relationship between DSH and self-punishment where DSH is engaged in for self-punishment, however the act of DSH creates more shame, selfcriticism and reinforcement of negative perfectionism, which in turn is associated with greater DSH, thus capturing the individual in a dangerous and distressing cycle.

Consistent with Hoff and Muehlenkamp's (2009) work, Flett and colleagues' (2012) study is limited by the absence of direct measurements of the functions of self-injury. Although they infer that a self-punitive disposition could increase the risk of engaging in DSH for self-punishment, they do not have any empirical data to directly support this function over other common NSSI functions (e.g., emotion regulation, marking distress).

\section{Empirical Research on Perfectionism and NSSI Functions}

As briefly mentioned in Chapter 3, Nock and Prinstein (2005) conducted some of the first research directly assessing the behavioural functions of NSSI. Among a wide array of measures given to their sample of adolescent psychiatric inpatients, the Functional Assessment of Self-Mutilation (FASM; Lloyd et al., 1997) was used to assess for NSSI and its functions, and the Child and Adolescent Perfectionism Scale (CAPS; Flett et al., 1997) was used to measure perfectionistic tendencies. Within those who had engaged in NSSI, SPP was associated with both social negative reinforcement functions and social positive reinforcement functions. Although Nock and Prinstein (2005) did not report specific functions, they suggested that individuals high on SPP could be using NSSI as a method of 
either eliciting attention from others (social positive reinforcement), or to remove the perceived high expectations others may have for them (social negative reinforcement).

Claes and colleagues (2012) also investigated perfectionism and the functions of NSSI in a sample of 95 women from an eating disorder treatment unit. They administered the Self-Injury Questionnaire (Claes, Vandereycken, \& Vertommen, 2003) to assess for five different NSSI behaviours and their functions, and four subscales from the FMPS (CM, DA, PS, PC; Frost et al., 1990) to measure perfectionism. As a result, Claes and colleagues (2012) clearly illustrated that eating disordered patients who had engaged in NSSI reported significantly higher negative perfectionism (CM and DA) and PC than those with no history of NSSI. Further, consistent with both Nock and Prinstein's (2005) research and Flett and colleagues' (2012) theoretical hypotheses, negative perfectionism was significantly associated with self-punishment, self-torture and cry-for-help functions of NSSI. Additionally, PC was negatively associated with cry-for-help function. These results suggest that perfectionistic eating disorder patients are more likely to use NSSI as a method of punishment than less perfectionistic patients. It may be that NSSI is triggered by selfperceived failures thus resulting in self-punishment, or is a method of coping with distress, and/or seeking help for related negative emotions.

Unfortunately, Claes and colleagues (2012) only investigated the self-punishment, self-torture and cry-for-help functions of NSSI. As shown in previous research (e.g., Klonsky \& Glenn, 2009; Nock, 2009; Turner, Chapman, \& Layden, 2012) there are many more commonly endorsed functions than the three explored (e.g., affect regulation, antidissociation, anti-suicide, marking distress, peer bonding, autonomy). In particular, drawing on the hypotheses and findings of previous perfectionism-NSSI research (Hoff \& Muehlenkamp, 2009; Nock \& Prinstein, 2005) it may be especially important to investigate other functions such as interpersonal influence and those related to gaining a sense of selfcontrol (e.g., autonomy, self-care).

Against this background, Study $3 \mathrm{~b}$ aimed to provide empirical evidence that could either support or further question the relationships between perfectionism and NSSI functions explored above. It was expected that students scoring high on negative perfectionism would be more likely to use NSSI as a method of self-punishment, a method of gaining control, or as a way of distancing oneself from others perceived to hold very high expectations for their behaviour. In addition, affect regulation may be particularly important when the facing the distress associated with perceived failure and unrelenting expectations from the self and others. 


\section{Results}

Adolescents with a history of NSSI most frequently reported affect regulation $(91.5 \%$ indicated that at least one affect regulation item was relevant to their engagement in selfinjury, $\left.M_{\text {Affect Regulation }}=1.12, S D=.60\right)$, self-punishment $(81.8 \% ; M=.96, S D=.70)$, and anti-dissociation $(69.0 \% ; M=.65, S D=.64)$ functions for their self-injury (see Table 21 for all means and standard deviations). Overall, and consistent with previous research (e.g., Brown et al., 2002; Laye-Gindhu \& Schonert-Reichl, 2005; Nock \& Prinstein, 2004), intrapersonal functions $(M=.74, S D=.47)$ were endorsed significantly more than interpersonal functions $(M=.22, S D=.25 ; t(195)=-16.38 p<.001)$. With regard to differences in the functions of NSSI across sex, females were more likely to endorse intrapersonal functions than males $\left(M_{\text {Males }}=.52, S D=.47 ; M_{\text {Females }}=.80, S D=.45 ; t(199)=-\right.$ $3.39, p=.01)$. There was no significant sex difference for endorsement of interpersonal functions $\left(M_{\text {Males }}=.24, S D=.30 ; M_{\text {Females }}=.21, S D=.24 ; t(194)=.65, p=.51, n s\right)$. At the subscale level Chi-squared analyses showed that females were more likely, and males less likely to endorse affect regulation $\left(\chi^{2}(1, n=199)=13.78, p<.001\right)$, self-punishment $\left(\chi^{2}(1, n\right.$ $=198)=19.00, p<.001)$, anti-dissociation $\left(\chi^{2}(1, n=197)=7.95, p=.01\right)$, and marking distress $\left(\chi^{2}(1, n=197)=9.89, p=.01\right)$ functions than expected based on overall endorsement rates (see Table 21).

Correlations were used to both ascertain whether perfectionism is associated with any specific functions of NSSI, and to investigate the relationship between negative and positive perfectionism and the broad interpersonal/intrapersonal functional categories. Overall, negative perfectionism was significantly positively associated with nine of the 13 common functions of NSSI. In particular, there was a moderate positive correlation between negative perfectionism and self-punishment $(r(195)=.34, p<.001)$, suggesting that the more an individual endorsed negative perfectionism, the more likely they are to engage in NSSI to self-punish. Similarly, negative perfectionism was significantly associated with increased endorsement of autonomy, anti-suicide, self-care, interpersonal boundaries, and antidissociation (see Table 22). Negative perfectionism was associated with increased endorsement of both intrapersonal functions $(r(195)=.32, p<.001)$ and interpersonal functions $(r(193)=.22, p=.01)$, suggesting that perfectionistic adolescents may have both internal and external needs that can be temporarily remedied by NSSI.

With regard to positive perfectionism, analyses revealed a significant positive relationship only between positive perfectionism and autonomy $(r(193)=.19, p=.01)$. No significant associations were demonstrated with regard to positive perfectionism and the 
Table 21

NSSI Function Frequencies and Chi-Squared Analyses across Sex

\begin{tabular}{|c|c|c|c|c|}
\hline Functions & $\frac{\text { Total }}{(n=196)}$ & $\frac{\text { Male }}{(n=38)}$ & $\frac{\text { Female }}{(n=161)}$ & $\chi_{2}^{2}$ \\
\hline & $\%$ & $\begin{array}{l}\% \text { / Actual } \\
\text { (Expected) }\end{array}$ & $\%$ / Actual (Expected) & \\
\hline Affect regulation & $91.5 \%$ & $76.3 / 29(35)$ & $95.0 / 153(147)$ & $13.78 * *$ \\
\hline$M(S D)$ & $1.12(.60)$ & $0.82(.68)$ & $1.20(.56)$ & \\
\hline Self-punishment & $81.8 \%$ & $57.9 / 22(31)$ & $87.5 / 140(131)$ & $18.09 * *$ \\
\hline$M(S D)$ & $0.96(.70)$ & $0.58(.66)$ & $1.05(.68)$ & \\
\hline Anti-dissociation & $69.0 \%$ & $52.6 / 20(26)$ & $73.0 / 116(110)$ & $5.93^{*}$ \\
\hline$M(S D)$ & $0.65(.64)$ & $0.48(.64)$ & $0.69(.64)$ & \\
\hline Marking distress & $54.8 \%$ & $34.2 / 13(21)$ & $59.7 / 95(87)$ & $8.08^{*}$ \\
\hline$M(S D)$ & $0.46(.57)$ & $0.32(.57)$ & $0.50(.56)$ & \\
\hline Anti-suicide & $52.3 \%$ & $39.5 / 15(20)$ & $55.3 / 88(83)$ & 3.10 \\
\hline$M(S D)$ & $0.50(.62)$ & $0.39(.60)$ & $0.53(.62)$ & \\
\hline Toughness & $49.0 \%$ & $54.1 / 20(18)$ & $47.8 / 76(78)$ & 0.47 \\
\hline$M(S D)$ & $0.35(.48)$ & $0.43(.54)$ & $0.34(.46)$ & \\
\hline Interpersonal- & $47.4 \%$ & $43.2 / 16(18)$ & 48.4/ 77 (75) & 0.32 \\
\hline$M(S D)$ & $0.32(.45)$ & $0.27(.38)$ & $0.33(.46)$ & \\
\hline Self-care & $45.4 \%$ & $32.4 / 12(17)$ & 48.4/ 77 (72) & 3.10 \\
\hline$M(S D)$ & $0.30(.42)$ & $0.18(.34)$ & $0.32(.46)$ & \\
\hline Sensation seeking & $32.1 \%$ & $40.5 / 15(12)$ & $30.2 / 48(51)$ & 1.48 \\
\hline$M(S D)$ & $0.22(.40)$ & $0.35(.55)$ & $0.19(.35)$ & \\
\hline Interpersonal-influence & $30.1 \%$ & $32.4 / 12(11)$ & $29.6 / 47$ (48) & 0.12 \\
\hline$M(S D)$ & $0.16(.30)$ & $0.21(.41)$ & $0.15(.27)$ & \\
\hline Autonomy & $29.1 \%$ & $32.4 / 12(11)$ & $28.3 / 45(46)$ & 0.25 \\
\hline$M(S D)$ & $0.20(.39)$ & $0.18(.30)$ & $0.21(.41)$ & \\
\hline Revenge & $15.8 \%$ & $18.9 / 7(6)$ & $15.1 / 24(25)$ & 0.33 \\
\hline$M(S D)$ & $0.08(.22)$ & $0.11(.27)$ & $0.07(.20)$ & \\
\hline Peer-bonding & $14.3 \%$ & $24.3 / 9(5)$ & $11.9 / 19(23)$ & 3.75 \\
\hline$M(S D)$ & $0.11(.33)$ & $0.21(.46)$ & $0.09(.28)$ & \\
\hline Intrapersonal Functions & $0.69(.47)$ & $0.45(.47)$ & $0.76(.46)$ & \\
\hline Interpersonal Functions & $0.22(.26)$ & $0.22(.28)$ & $0.22(.25)$ & \\
\hline
\end{tabular}

$* p<.05 ; * * p<.001$ 
intrapersonal $(r(198)=.07, p=.34)$ and interpersonal $(r(193)=.13, p=.07)$ functional categories.

In light of previous results showing significant sex differences, analyses were also conducted separately for males and females. In females, the correlations between negative perfectionism and the thirteen functions broadly matched those seen in the total sample (see Table 22). However, for males, negative perfectionism was not significantly associated with the individual functions or the overarching interpersonal and intrapersonal functional categories. It is important to note that the male sample was smaller than the female sample, therefore there may not have been sufficient power to detect significant relationships. Positive perfectionism was not significantly associated with any of the individual functions, or the overarching functional categories for males or females.

Table 22

Pearson's Correlation Coefficients for NSSI Individual Functions, Functional Categories and Perfectionism

\begin{tabular}{|c|c|c|c|c|c|c|}
\hline \multirow[b]{2}{*}{$\underline{\text { NSSI Function }}$} & $\begin{array}{c}\text { Negative } \\
\text { Perfectionism }\end{array}$ & $\begin{array}{c}\text { Positive } \\
\text { Perfectionism }\end{array}$ & \multicolumn{2}{|c|}{$\begin{array}{c}\text { Negative } \\
\text { Perfectionism }\end{array}$} & \multicolumn{2}{|c|}{$\begin{array}{c}\text { Positive } \\
\text { Perfectionism }\end{array}$} \\
\hline & & $\frac{\mathrm{al}}{194)}$ & $\underline{\underline{\text { Males }}}$ & $\frac{\text { Females }}{(n=158)}$ & $\underline{\underline{\text { Males }}}$ & $\frac{\underline{\text { Females }}}{(n=158)}$ \\
\hline Affect regulation (Intra) & .14 & .03 & .03 & .15 & .20 & -.08 \\
\hline Self-punishment (Intra) & $.34 *$ & .05 & .20 & $.35 *$ & .25 & -.05 \\
\hline Anti-dissociation (Intra) & $.25^{*}$ & .03 & .29 & $.22 *$ & .25 & -.06 \\
\hline Marking distress (Intra) & .16 & .10 & .17 & .15 & .07 & .09 \\
\hline Anti-suicide (Intra) & $.30 *$ & .01 & .21 & $.31 *$ & .23 & -.06 \\
\hline Toughness (Inter) & .16 & .13 & .05 & $.21 *$ & .02 & .19 \\
\hline $\begin{array}{l}\text { Interpersonal boundaries } \\
\text { (Inter) }\end{array}$ & $.21 *$ & .06 & .29 & .19 & .21 & .02 \\
\hline Self-care (Inter) & $.20 *$ & .08 & .16 & .19 & .14 & .05 \\
\hline Sensation seeking (Inter) & .13 & .02 & .01 & .20 & -.09 & .10 \\
\hline $\begin{array}{l}\text { Interpersonal influence } \\
\text { (Inter) }\end{array}$ & -.05 & .13 & .17 & -.12 & .09 & .17 \\
\hline Autonomy (Inter) & $.30 *$ & $.19 *$ & .25 & $.31 *$ & .20 & .19 \\
\hline Revenge (Inter) & .07 & -.01 & .34 & .00 & .21 & -.08 \\
\hline Peer bonding (Inter) & .07 & .06 & .12 & .09 & .04 & .11 \\
\hline Intrapersonal functions & $.32 *$ & .07 & .23 & $.32 *$ & .27 & -.03 \\
\hline Interpersonal functions & $.22 *$ & .13 & .21 & $.25^{*}$ & .11 & .15 \\
\hline
\end{tabular}

Note: A post hoc Bonferroni correction was applied to address the issue of inflated family-wise error, the corrected $p$-value is .002 and significant correlations are indicated by $*$ 


\section{Summary of Results}

Overall, as expected negative perfectionism was significantly associated with the selfpunishment function of NSSI. In addition, greater endorsement of negative perfectionism was associated with NSSI for both interpersonal and intrapersonal needs. On the other hand, positive perfectionism was primarily associated with NSSI for interpersonal functions (interpersonal influence, toughness, and autonomy). On face value there appeared to be significant sex differences, however differences in sample size mean that comparisons should be made cautiously.

\section{Study 3c: Two-Component, 2x2, and Tripartite Models of Perfectionism}

The next component of this research moves beyond looking at the individual effects of positive perfectionism and negative perfectionism to consider how the two components of perfectionism interact in relation to NSSI. As outlined in Chapter 2, there are three competing models seeking to explain how negative and positive perfectionism combine to predict both adaptive and maladaptive outcomes. Central to these models is the argument that particular combinations of negative and positive perfectionism are more detrimental than others and are associated with poorer mental health and wellbeing outcomes. Whilst the $2 \times 2$ model (Gaudreau \& Thompson, 2010) proposes that high negative perfectionism and low positive perfectionism is the most maladaptive combination, both the tripartite (Rice \& Ashby, 2007; Stoeber \& Otto, 2006) and two-component (Alden et al., 2002) models propose that the combination of high negative perfectionism and high positive perfectionism is the most maladaptive (refer back to Figures 6, 7, and 8 for graphical summaries of the models).

Each of the models mentioned have been investigated in the context of various mental health outcomes with mixed results. For example, Smith, Saklofske, Yan and Sherry (2014) investigated the relationship between positive and negative perfectionism in relation to negative emotionality in Canadian and Chinese university students. In both samples they reported that the relationship between negative perfectionism and negative emotionality was strongest when students also scored high on positive perfectionism. Thus, the tripartite model was supported in this sample. In another study (Chan, 2012) cluster analyses were used to identify three groups based on perfectionism profiles. The resulting clusters also support the tripartite model with 'unhealthy' perfectionists (high on both positive and negative perfectionism) reporting less happiness and a more fixed mindset than 'healthy' perfectionists (high positive perfectionism, low negative perfectionism). 
Conversely, Gaudreau and Thompson (2010) provided evidence for their 2x2 model with regard to academic self-determination and adjustment, and positive and negative affect. Specifically, they found that a combination of high negative perfectionism and low positive perfectionism (evaluative concerns perfectionism) was associated with lower academic selfdetermination, academic satisfaction, less positive affect, and greater negative affect than those scoring low on positive and negative perfectionism (non-perfectionists). In addition, Damian, Stoeber, Negru and Baban (2014) provided support for the 2x2 model. Specifically, a combination of low positive perfectionism and high negative perfectionism (evaluative concerns perfectionism) was associated with lower levels of positive affect than all other perfectionism classifications (pure personal standards, mixed, non-perfectionism). Moreover, the combination of low positive perfectionism and high negative perfectionism was associated with higher levels of negative affect.

Finally, some research has negated all proposed models and instead indicated that positive and negative perfectionism operate in a parallel fashion, not significantly interacting with each other. For example, Douilliez and Lefevre (2011) set out to test the $2 \times 2$ model and found that both positive and negative perfectionism were positively associated with depression. However, there was no interactive effect of negative perfectionism and positive perfectionism.

Before advancing, it may have become apparent that in comparison to research on the tripartite and 2x2 models, there has been a lesser focus on the two-component model. This may partly be because of similarities with the tripartite model, and because the area in which the two-component and tripartite models differ (individuals with low positive perfectionism), is the combination considered to have the least influence on positive and negative outcomes. Therefore, in terms of theoretical utility and importance, testing and clarifying their differences does not appear to be an important focus of research. In summary, across all three models, there is agreement that high negative perfectionism is a key characteristic of an unhealthy or maladaptive perfectionist. The contention surrounds whether negative perfectionism combined with high positive perfectionism (tripartite and two-component models) or with low positive perfectionism ( $2 \times 2$ model) is associated with the most maladaptive outcomes. This will be explored in relation to NSSI.

\section{Results}

The results of Study 3a demonstrated that both positive perfectionism and negative perfectionism are significantly associated with frequency of NSSI, at least in female 
adolescents. Study $3 \mathrm{c}$ included further analyses to determine whether the interaction between positive and negative perfectionism accounted for any additional variance in predicting NSSI frequency. Following the moderation processes outlined in Study 3a, first, a positive perfectionism by negative perfectionism interaction variable was computed (Baron \& Kenny, 1986). Then, a hierarchical multiple regression analysis was conducted by entering positive perfectionism, negative perfectionism and the new interaction variable into a model predicting number of methods of NSSI and frequency of engagement in such NSSI behaviours. The resulting model accounted for $9.80 \%$ (adjusted $R^{2}=.10$ ) of the variance in predicting $\operatorname{NSSI}(F(3,857)=30.94, p<.001)$, but the interaction variable did not account for any significant unique variance beyond that accounted for by positive perfectionism and negative perfectionism alone $\left(\Delta R^{2}=.00 ; \Delta F(1,854)=.61, p=.43\right.$, ns; see Table 23 for relevant statistics). In combination with Study 3a results, this suggests that only negative perfectionism has a significant effect on the probability of engaging in NSSI. In other words, no particular combination of positive and negative perfectionism uniquely increases or decreases risk of NSSI, over and above the risk associated with negative perfectionism alone.

Table 23

Summary of Multiple Regression of NSSI Frequency Predicted by Positive Perfectionism, Negative Perfectionism and a Positive Perfectionism-Negative Perfectionism Interaction in the Total Sample

\begin{tabular}{lccc}
\hline Negative perfectionism & $\underline{B}$ & $\underline{\beta}$ & $\Delta \underline{R}^{2}$ \\
Positive perfectionism & $.30^{*}$ & $.40^{*}$ & - \\
$\begin{array}{l}\text { Negative perfectionism x } \\
\text { positive perfectionism }\end{array}$ & $-.07 \mathrm{~ns}$ & $-.10 \mathrm{~ns}$ & $.10^{* *}$ \\
interaction & $-.02 \mathrm{~ns}$ & $-.14 \mathrm{~ns}$ & $.01 \mathrm{~ns}$ \\
\hline${ }^{*} p<.05 ; * * p<.001$ & & &
\end{tabular}

However, given previous differences based on sex, the relationship between positive and negative perfectionism and the combined effect on NSSI frequency was explored in males and females separately. First an analysis of the male only data was conducted. Positive and negative perfectionism were entered in the first block, followed by the positive perfectionism-negative perfectionism interaction variable in the second block. The model accounted for $9.10 \%$ (adjusted $R^{2}=.08$ ) of the variance in predicting NSSI frequency in males $(F(3,345)=11.52, p<.001)$, with the negative perfectionism - positive perfectionism interaction variable accounting for significant unique variance beyond that of the individual 
perfectionism components $\left(\Delta R^{2}=.06 ; \Delta F(1,345)=20.95, p<.001\right.$; see Table 24 below for the relevant statistics).

Table 24

Summary of Multiple Regression of NSSI Frequency Predicted by Positive Perfectionism, Negative Perfectionism and a Positive Perfectionism-Negative Perfectionism Interaction in Males only

\begin{tabular}{|c|c|c|c|}
\hline & $\underline{B}$ & $\underline{\beta}$ & $\Delta \underline{R^{2}}$ \\
\hline Negative perfectionism & $-.41 * *$ & $-.74 * *$ & - \\
\hline Positive perfectionism & $-.44 * *$ & $-.96 * *$ & $.04 *$ \\
\hline $\begin{array}{l}\text { Negative perfectionism } \mathrm{x} \\
\text { positive perfectionism } \\
\text { interaction }\end{array}$ & $.15^{* *}$ & $1.38 * *$ & $.06^{* *}$ \\
\hline
\end{tabular}

The significant unique variance of the positive perfectionism - negative perfectionism interaction variable suggested that positive perfectionism could moderate the relationship between negative perfectionism and NSSI frequency in males. Subsequently, these results were graphed using ModGraph (Jose, 2013). Figure 46 below shows the graphical output produced.

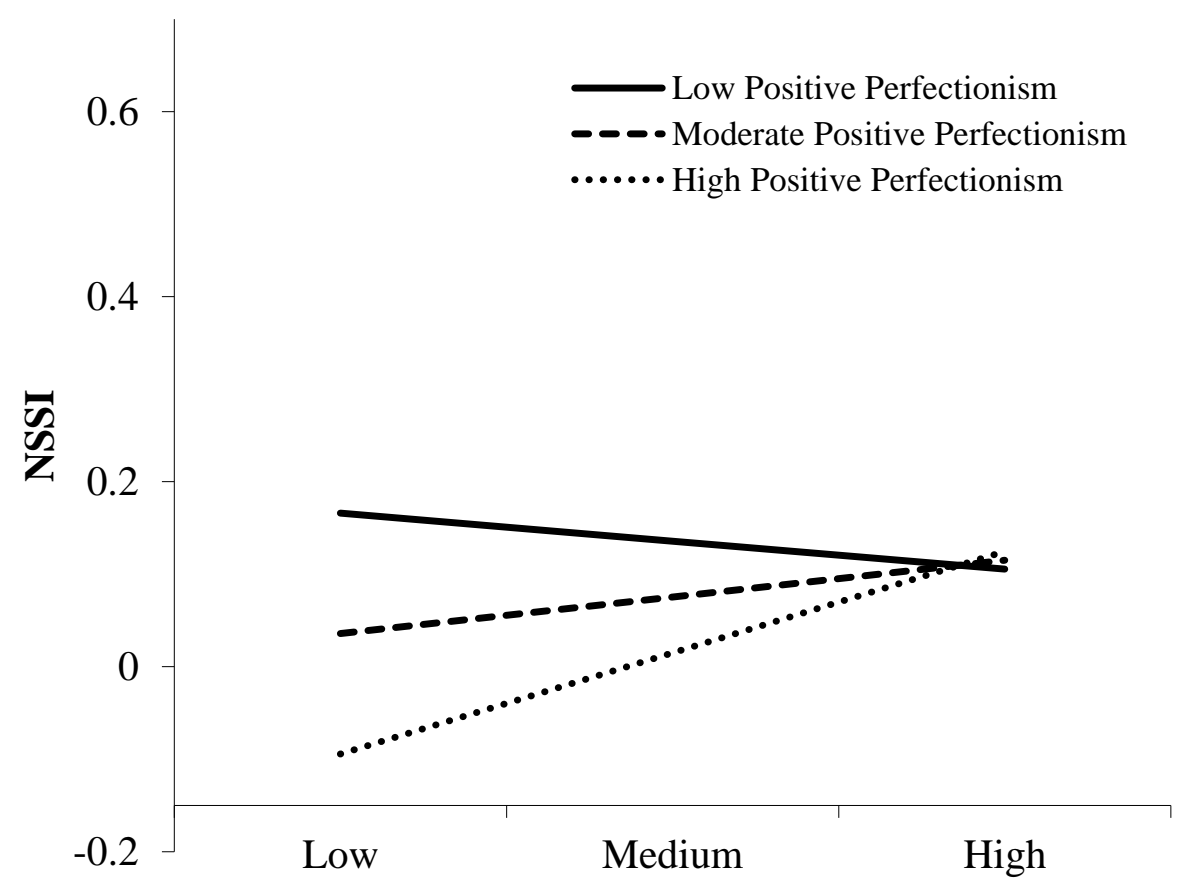

\section{Negative Perfectionism}

Figure 46. Moderation of negative perfectionism and positive perfectionism against NSSI for male participants. 
At a glance, Figure 46 suggests that at low and moderate endorsement of negative perfectionism, positive perfectionism significantly buffers against NSSI risk. Positive perfectionism appears to have no buffering effect when negative perfectionism is high. Visual interpretation of Figure 46 was supported by simple slope analyses. Specifically, analyses revealed that for males who score moderately on positive perfectionism there is a significant positive relationship between negative perfectionism and NSSI (slope $=.06 ; t(346)=2.11, p$ $=.04$ ), and for individuals high on positive perfectionism this relationship is significant and even stronger $($ slope $=.18 ; t(346)=4.77, p<.001)$. This indicates that positive perfectionism buffers against low or moderate negative perfectionism, but does not significantly combat high negative perfectionism in males. Similarly, the slope for males low on positive perfectionism was not significantly different from 0 (slope $=-.05 ; t(346)=-1.19, p=.23$ ), confirming no significant relationship between negative perfectionism and NSSI for the low positive perfectionism group.

Next, identical analyses were conducted with females. This model accounted for $14.24 \%$ (adjusted $\left.R^{2}=.14\right)$ of the variance in predicting NSSI in females $(F(3,505)=27.96, p$ $<.001$ ), which in line with previous findings is slightly more variance than accounted for in the model for males. Moreover, the negative perfectionism - positive perfectionism interaction variable accounted for significant unique variance beyond that of the individual perfectionism components alone $\left(\Delta R^{2}=.01 ; \Delta F(1,505)=6.17, p=.01\right.$; see Table 25 for relevant statistics). This suggests that for females, positive perfectionism could also moderate the negative perfectionism - NSSI frequency relationship. As above, ModGraph (Jose, 2013) was used to graph the results to aid in interpretation (see Figure 47).

Table 25

Summary of Multiple Regression of NSSI Predicted by Positive Perfectionism, Negative Perfectionism and a Positive Perfectionism-Negative Perfectionism Interaction in Females only

\begin{tabular}{|c|c|c|c|}
\hline & $\underline{B}$ & $\underline{\beta}$ & $\Delta \underline{R^{2}}$ \\
\hline Negative perfectionism & $.62 * *$ & $.76^{* *}$ & - \\
\hline Positive perfectionism & .10 & .11 & $.13^{* *}$ \\
\hline $\begin{array}{l}\text { Negative perfectionism } \mathrm{x} \\
\text { positive perfectionism } \\
\text { interaction }\end{array}$ & $-.11 *$ & $-.57^{*}$ & $.01 *$ \\
\hline
\end{tabular}

First, there was a significant relationship between negative perfectionism and NSSI at all levels of positive perfectionism. However, as evidenced by simple slope analyses the 
relationship between negative perfectionism and NSSI was strongest in females low on positive perfectionism $($ slope $=.35 ; t(506)=7.76, p<.001)$, followed by females scoring moderately on positive perfectionism ( weakest in those high on positive perfectionism (slope $=.20 ; t(506)=4.54, p<.001$ ). This suggests that positive perfectionism buffers against risk of NSSI at all levels of negative perfectionism, but most for females high on negative perfectionism.

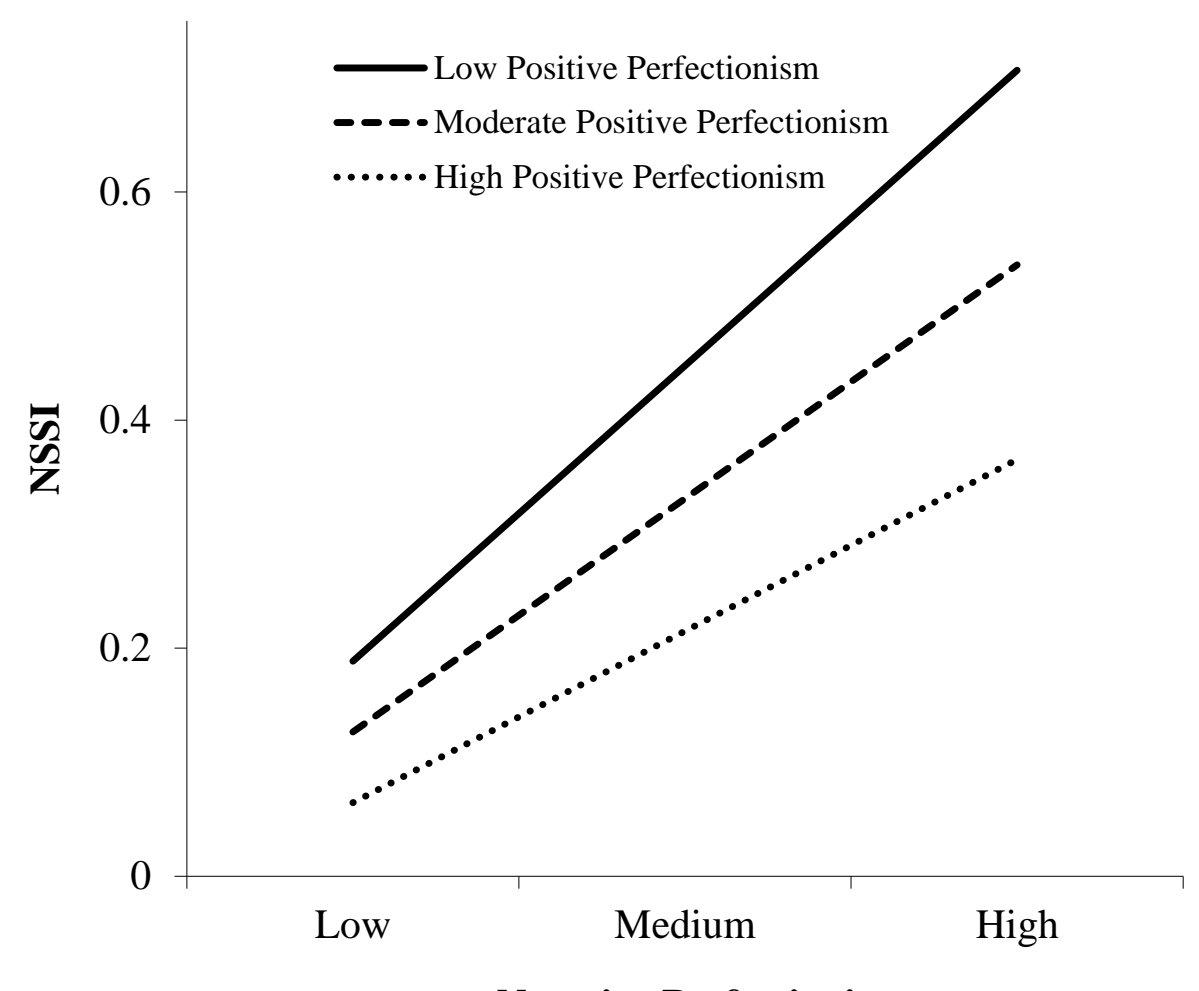

\section{Negative Perfectionism}

Figure 47. Moderation of negative perfectionism and positive perfectionism against NSSI for female participants

\section{Summary of Results}

Overall, Study $3 \mathrm{c}$ suggests that not only do positive and negative perfectionism contribute to risk of NSSI individually, their interaction also explains unique variance in predicting frequency of NSSI. Moreover, analyses revealed differing patterns for males and females. Whilst, negative perfectionism was only associated with NSSI when males scored moderate or high on positive perfectionism, negative perfectionism was associated with NSSI at all levels of positive perfectionism in females. Regardless of these sex differences, overall positive perfectionism either buffered against NSSI or made little difference to the 
relationship between negative perfectionism and NSSI. In other words, in no situation did positive perfectionism exacerbate risk of NSSI in the current sample of adolescents.

\section{Combined Study Three Summary}

Study 3 focused on the cross-sectional relationship between perfectionism and selfinjury in a local sample of NZ adolescents. Overall, $21.2 \%$ of the sample reported having engaging in NSSI at least once. This is broadly consistent with previous research in community adolescent samples (e.g., 21.5\% in Mohl \& Skandsen, 2012; 21.4\% in Muehlenkamp et al., 2009). Of those who reported NSSI, on average, each individual had engaged in three unique methods of NSSI. Cutting was the most frequently reported method, and affect regulation was the most endorsed function. Females (29\%) were more likely than males $(9 \%)$ to report a history of NSSI, and to report cutting or carving wounds into their skin. Overall adolescents scored higher on positive perfectionism than negative perfectionism, and females were in general more perfectionistic than males. Positive and negative perfectionism were significantly associated in both males and females, however this relationship was stronger for males than it was for females.

\section{Perfectionism and NSSI}

Study $3 \mathrm{a}$ focused on the relationship between negative and positive perfectionism, and NSSI across both males and females. In summary, negative perfectionism was significantly associated with NSSI. That is, individuals who scored high on negative perfectionism were more likely to report engagement in self-injury. Further, this relationship was moderated by sex such that the relationship between negative perfectionism and NSSI was significant for females, but non-significant for males. On the other hand, positive perfectionism was associated with less engagement in NSSI, and this relationship was not significantly influenced by sex. These results are consistent with previous research indicating a positive relationship between negative perfectionism and NSSI (e.g., Claes et al., 2012; Hoff \& Muehlenkamp, 2009; Izadi, 2014), and a negative relationship between positive perfectionism and NSSI (Hoff \& Muehlenkamp, 2009).

With regard to adolescents, this research supports Luyckx and colleagues' (2015) description of a significant positive relationship between perfectionism and NSSI in female clinical and community adolescents. However, it also builds on their research by investigating positive and negative perfectionism independent of each other, and extending the research with male adolescents. This resulted in significantly different relationships for positive and negative perfectionism, thus providing further justification for investigating 
perfectionism as a multidimensional, rather than a unidimensional phenomenon. In addition, this research highlights the importance of considering males and females separately rather than assuming results may be generalised to all adolescents. The absence of a significant relationship between negative perfectionism and NSSI in males supports Flett and colleagues (2014) similar lack of findings for the males within their sample.

This research was also broadly consistent with O'Connor and colleagues' (2010) work with community adolescents. Specifically, that negative perfectionism was associated with increased risk of self-injury. O'Connor and colleagues did not differentiate between selfinjury with and without suicide intent, therefore it remained unclear whether this relationship was driven by self-injury with suicide intent, NSSI, or both. However, taken together, the results reported by Luyckx et al., (2015) and Study 3a research suggest a robust relationship between negative perfectionism and self-injury that is specific to NSSI.

\section{Perfectionism and the Functions of Self-Injury}

Having established support for a significant relationship between negative perfectionism and NSSI in females, focus then shifted to the more intricate details of the relationship. In particular, Study $3 \mathrm{~b}$ investigated the relationship between positive and negative perfectionism, and the functions of NSSI. Analyses showed that for females, negative perfectionism was most strongly related with NSSI to punish oneself. This aligns with Flett and colleagues' (2012) adaptation of the self-punitiveness model, and provides additional empirical support for research suggesting that perfectionism is related to acts of self-punishment and self-torture (Claes et al., 2012). Specifically, Flett et al., (2012) suggested that perfectionism can trigger self-criticism and feelings of shame that the individual may attempt to remedy or relieve through acts of self-injury. Lynch and Cozza (2009) describe an alternative or complimentary perspective and suggest that engaging in NSSI to punish oneself is reinforcing because it verifies feelings of shame, and thoughts that one is bad and deserving of punishment. Moreover, drawing on Swann's (1983) selfverification theory, they argue that without a form of self-punishment, self-constructs of worthlessness are dis-confirmed and further negative emotion is triggered.

In addition, Study $3 \mathrm{~b}$ demonstrated an association between negative perfectionism and anti-suicide functions of NSSI that, again, was specific to females. With consideration of the wide body of literature suggesting that perfectionism increases risk for suicidal thoughts, and behaviours (see O'Connor, 2007 for a review of the perfectionism-suicide literature), this finding makes logical sense. That is, adolescents scoring high on negative perfectionism are 
at greater risk of suicidal thoughts and behaviours and therefore are more likely to adopt strategies such as self-injury that prevent them from acting on suicidal thoughts.

Moreover, negative perfectionism was also significantly related to a group of interpersonal functions (autonomy, interpersonal boundaries, self-care, sensation seeking and toughness). It is hypothesised that these functions are especially prominent in adolescents endorsing high negative perfectionism due to a desire to manage the high perceived expectations and critical stance of others close to them. They may do so by distancing themselves from others (interpersonal boundaries), and second by demonstrating that they can cope alone and look after themselves (autonomy, toughness, self-care). These findings are consistent with Nock and Prinstein's (2005) research in which they reported positive relationships between negative perfectionism and social negative and positive reinforcement functions.

Together, Study $3 \mathrm{a}$ and $3 \mathrm{~b}$ findings provide insight into how perfectionism may fit within the leading theoretical models of NSSI. For example, with regard to the ITMS (see Figure 4, p. 20), this research supports the notion that perfectionism could be an intrapersonal and an interpersonal risk factor for self-injury. As a reminder, NSSI functions often relate to and highlight underlying risk factors. For example, negative perfectionism was positively associated with both the intrapersonal and interpersonal overarching categories of NSSI functions, therefore suggesting that NSSI is used to meet some interpersonal or interpersonal need or deficit. Also consistent with the ITMS, these findings support Nock's selfpunishment hypothesis, whereby self-injury is selected over other coping strategies (e.g., substance use, risky behaviours), due to its particularly direct, harsh and immediately punitive nature.

It is comparatively harder to relate these findings to the EAM (Chapman at al., 2006). Whilst Study 3a suggests that for females negative perfectionism is in some way related to greater self-injurious thoughts and behaviours, these findings shed little light on where in the sequence of events, emotions, and behaviours, perfectionism is implicated. It is hypothesised that perfectionistic tendencies will be associated with specific interpretations of events, cognitions, or interpersonal experiences, which act as the stimulus for the EAM cycle. For example, a perfectionistic adolescent may receive a grade that is perceived as not good enough and a sign of failure thus triggering strong and overwhelming negative emotion. The adolescent may then use self-injury to temporarily manage this distress. Likewise, a perfectionistic adolescent may interpret feedback from a teacher or parent as more critical than their non-perfectionistic peers and therefore experience intense negative emotion that 
overwhelms their adaptive coping resources. Thus, the adolescent may again resort to selfinjury.

Perfectionism may also be implicated in the interpretation of the negative emotions that result from a particular trigger. That is, a perfectionist may view negative emotions as a sign of inadequacy in themselves, believing that instead they should be able to control their emotions. These beliefs may further exacerbate the negative emotion and trigger secondary emotions such as shame and guilt. Rimes and Chalder (2010) have provided some support for this hypothesis, reporting that negative perfectionism was strongly associated with beliefs that experiencing and expressing negative emotion is unacceptable $(r$ 's $=.38-.59)$. Both this hypothesis and the hypotheses regarding perfectionism as a trigger (outlined above) suggest that perfectionism precedes self-injury.

Alternatively, further down the EAM cycle, the act of self-injury may trigger reactions from others or self-critical judgments that motivate the individual to do better, or appear less imperfect in the future. Although conceptually and theoretically less likely than perfectionism preceding self-injury, it is possible that perfectionistic thoughts and behaviours could increase following self-injury. Therefore, perfectionism would be implicated at the end of the EAM cycle. Overall, further research is required to clarify the temporal nature of the relationship between negative perfectionism and NSSI in relation to Chapman and colleague's (2006) EAM.

\section{The Combined Effect of Positive and Negative Perfectionism in Relation to Self-Injury}

The final component of Study 3 involved simultaneously considering negative and positive perfectionism in relation to self-injurious thoughts and behaviours. Initially, hierarchical multiple regression analyses with positive perfectionism, negative perfectionism and a positive perfectionism-negative perfectionism interaction were conducted for the total sample. The interaction variable contributed no unique variance to risk of NSSI over and above positive and negative perfectionism alone.

Subsequently, identical analyses were conducted separately for both males and females. For males, negative perfectionism was significantly related to NSSI only when positive perfectionism was moderate or high such that negative perfectionism was associated with a slight increase in risk for NSSI. Conversely, when positive perfectionism was low, negative perfectionism had no additional effect on risk of NSSI. These results do not convincingly support any of the proposed perfectionism models. Rather they support some 
individual components from each model, and the notion that outcomes are dependent on the specific combination of both positive and negative perfectionism.

Specifically, Study $3 \mathrm{c}$ results for males suggest that high positive perfectionism and low negative perfectionism is the most adaptive combination of perfectionism. Thus, supporting all proposed models (e.g., healthy perfectionism, tripartite model; pure personal standards, 2x2 model; achievement orientation, two-component model). These results also support the notion of a single non-perfectionism group (characterised by low positive perfectionism, tripartite model) in which the outcomes for an individual low on positive perfectionism are not influenced by concurrent level of negative perfectionism. Finally, whilst there was a clear combination of perfectionism associated with the least risk of NSSI, there was no single combination associated with the greatest risk of NSSI. This is inconsistent with all models (i.e., the existence of evaluative concerns perfectionism, $2 \times 2$ model; unhealthy perfectionism, tripartite model; and pathological perfectionism, two component model).

Overall, although the relationship between negative perfectionism and NSSI in males was significant at moderate and high levels of positive perfectionism, it still remained relatively weak. In combination with the lack of significant findings in Study $3 a$ and Study $3 b$ this could suggest that negative perfectionism has very little impact on NSSI risk for male adolescents. As a result research with a male sample and using NSSI as the outcome variable could be a particularly poor test of the competing perfectionism models due to the inconsistent and weak relationship between the variables.

Conversely for females, negative perfectionism was moderately associated with risk of NSSI at all levels of positive perfectionism. Moreover, this relationship was strongest in females also low on positive perfectionism, and weakest in females high on positive perfectionism. Again, these results suggest that positive perfectionism buffers the relationship between negative perfectionism and NSSI. These results support all aspects of Gaudreau and Thompson's (2010) 2x2 model. For instance, high negative perfectionism and low positive perfectionism (evaluative concerns perfectionism) was associated with the greatest risk of NSSI, and high positive perfectionism and low negative perfectionism (pure personal standards) was associated with the least risk of NSSI.

With regard to the two component (Alden et al., 2002) and tripartite (Rice \& Ashby, 2007; Stoeber \& Otto, 2006) models of perfectionism these results support hypotheses that high positive perfectionism and low negative perfectionism (healthy perfectionism, tripartite model; achievement orientation, two-component model) is associated with the most adaptive 
outcomes. However, these results negate the proposed exacerbating effect of positive perfectionism such that individuals high on positive perfectionism (unhealthy perfectionism, tripartite model; pathological perfectionism, two-component model) experience worse outcomes compared to those low on positive perfectionism (non-perfectionism, tripartite model; self-deprecation, two-component model).

Taken together, and although slightly different across sex, these results suggest that, first, positive perfectionism is not detrimental; rather it either buffers against risk for NSSI or has no effect, and that the exact nature of this effect depends on concurrent levels of negative perfectionism. Moreover, of the three proposed models of perfectionism, the $2 \times 2$ model is most consistent with these results. The results for males were somewhat unclear with regard to the competing models, which could potentially be attributed to an almost negligible association between negative perfectionism and NSSI in males. However, the results for females were very clear in support for all components of Gaudreau and Thompson's (2010) model. To reiterate, the results indicated that perfectionism is especially detrimental to a female adolescent who scores high on negative perfectionism and low on positive perfectionism. That is, an adolescent who is experiencing all the maladaptive outcomes associated with negative perfectionism, who at the same time does not have the associated adaptive experiences or buffer of positive perfectionism.

\section{Strengths}

This study has provided an in depth summary of the nature of both perfectionism and self-injury in NZ adolescents. As will be discussed in the overall discussion, this research is particularly useful due to the specific focus on NSSI rather than DSH, and the investigation of perfectionism at both the subscale levels and at the higher levels of negative and positive perfectionism. In addition, the use of a community adolescent sample allowed for the investigation of relatively recent or even current (with the last 12 months) self-injurious thoughts and behaviours, rather than the investigation of mostly historical behaviours as is typically the case in older community samples. Moreover, a sufficiently detailed investigation of NSSI was carried out (e.g., behavioural checklist, assessing the functions of NSSI) allowing a comprehensive understanding of the nature of NSSI in NZ adolescents. This is in contrast to single item measures of self-injury previous used in NZ research, and in research investigating perfectionism and DSH (e.g., Lucassen et al., 2011; O'Connor et al., 2010). These strengths, and the resulting implications will be explored in more depth following the final study. 


\section{Limitations}

There are a few limitations in the methodology of this research that may impact upon the conclusions that can be drawn from these findings. First, these findings are based on cross-sectional data - unfortunately a significant relationship in cross-sectional research is insufficient to infer causality (Spector, 2013). That is, forgetting theoretical understandings of perfectionism and NSSI, there is no evidence to suggest that perfectionism precedes NSSI or vice versa, only evidence that they are in some way related. This is especially problematic when trying to place perfectionism within the ITMS (Nock, 2010) and EAM (Chapman et al., 2006) models of self-injury. In both models of self-injury perfectionism is best conceptualised as a distal (ITMS) or proximal (ITMS and EAM) risk factor that precedes the act of self-injury, however at this stage there is a lack of specific research supporting this temporal sequence.

Relatedly, the design of individual survey measures, and the survey as a whole, further limit the conclusions that can be made with regard to the temporal nature of these relationships. For example, the FMPS does not specify a time frame that the participant should consider when answering each item, rather implying that the participant should answer according to their current experience. Similarly the DSHI-s asks about lifetime history of each behaviour ("have you ever...."), rather than specifying a limited timeframe (e.g., within the last 12 months). Had both of these scales requested information about specific and different time periods, for example perfectionistic cognitions prior to beginning secondary school and past year history of NSSI, additional inferences about the temporal nature of the relationships could have been made. This is a common, but mostly overlooked, issue for survey based cross-sectional research.

Finally, there is some concern that the survey branching methods may have increased the likelihood that some individuals with a history of NSSI may have missed the full DSHI-s measure. Research has shown that the method of asking about self-injury can significantly impact reported self-injury rates (e.g., Heath et al., 2008; Lundh et al., 2007; Muehlenkamp et al., 2012). For example, prevalence rates are typically much higher when self-injury is measured using a behavioural checklists (e.g., DSHI-s), and lower with the use of a single item (e.g., "have you ever engaged in deliberate self-injury without wanting to die?"). Further research has suggested that when only a single item is used, there are often discrepancies between the participant's and the researchers' definitions of self-injury, and participants may report only cutting-related behaviours (Muehlenkamp et al., 2012). As such, individuals who engage in milder (e.g., scratching, picking at wounds) or more severe behaviours (e.g., 
burning, breaking bones) may not be accurately captured in the data. Moreover, males may be more likely to indicate that they have not engaged in self-injury, both because self-injury is often conceptualised as a behaviour particularly relevant to females (Swannell et al., 2014; Whitlock, Muehlenkamp, \& Eckenrode, 2008), and because previous research has shown that males can be more likely to engage NSSI methods, such as punching objects with the intent of hurting oneself, that are less often considered "self-injury" and may instead be considered general aggressive behaviour (Barrocas, Hankin, Young, \& Abela, 2012; Latimer et al., 2013; Whitlock et al., 2011).

This survey uses a single self-injury question from which any individual who indicates they have thought about or engaged in self-injury will go on to complete the full behavioural checklist of self-injuring behaviours. Those who initially indicated no history of self-injurious thoughts or behaviours are directed past the DSHI-s. Therefore, whilst the information collected through the behavioural checklist is likely to be relatively accurate, some individuals with a history of self-injury (as defined by the research team) may not have considered their behaviour as self-injurious and failed to complete the relevant measure (Bjärehed \& Lundh, 2008). As a result, the prevalence rates outlined above may be an underestimation of the true rates of self-injury in this adolescent sample.

\section{Conclusions}

This study adds to the literature by investigating the relationship between a multidimensional conceptualisation of perfectionism and NSSI, in a sample of both male and female community adolescents. Based on previous empirical work it was expected that adolescents who score high on negative perfectionism would have greater self-injurious thoughts, and engage in more self-injury than their comparatively less negatively perfectionistic peers. Previous research findings pertaining to positive perfectionism are mixed, therefore, there were no strong expectations for how positive perfectionism could be associated with self-injurious thoughts and behaviours.

Consistent with the predictions, the results of Study 3 suggest that negative perfectionism is associated with increased risk for self-injurious thoughts and behaviours, particularly for females, and positive perfectionism likely only minimally buffers self-injury risk. Negative perfectionism was particularly associated with self-injury to self-punish and/or regulate interpersonal situations. Finally, NSSI was most prominent for females low on positive perfectionism and high on negative perfectionism thus supporting the $2 \times 2$ model of perfectionism (Gaudreau \& Thompson, 2010). 
As mentioned above, it is currently impossible to conclude that negative perfectionism leads to self-injury, or positive perfectionism predicts less self-injury. Whilst theoretical understandings may lead researchers to believe that it is more likely that negative perfectionism leads to self-injury, rather than the other way around, this is yet to be supported by empirical research. 


\section{CHAPTER EIGHT: LONGITUDINAL INVESTIGATION OF PERFECTIONISM AND NON-SUICIDAL SELF-INJURY}

This research has demonstrated that negative perfectionism is associated with poor mental health and wellbeing outcomes, and that positive perfectionism demonstrates weak positive or negative, or non-significant, associations depending on various factors (e.g., outcome in question, sex, concurrent level of negative perfectionism, scale used for the measurement of positive perfectionism). Moreover, this research has demonstrated that in our sample of NZ female adolescents, negative perfectionism is associated with greater reported self-injury. Finally, positive perfectionism is associated with less reported self-injury across males and females; however this effect is demonstrably smaller than the opposite relationship with negative perfectionism.

Identification of significant relationships between negative and positive perfectionism, and NSSI is useful for identifying adolescents who may be at greater risk of using maladaptive coping strategies such self-injury. Knowledge of such relationships will also be useful in terms of selective promotion and fostering of particular qualities, skills, goals, and standards that lessen adolescents' risk of adverse outcomes. However, the crosssectional findings are somewhat limited. That is, at this stage we do not know if negative perfectionism is related to an increase in self-injury over time, or conversely whether selfinjury predicts a subsequent increase in negative perfectionism. Alternatively, negative perfectionism and self-injury could follow a similar pathway of development but not directly influence each other. In light of this, Study 4 will investigate the relationships between negative perfectionism, positive perfectionism, and self-injury over time. Prior to this, a review of longitudinal studies related to NSSI, and longitudinal research exploring perfectionism and various mental health outcomes will be highlighted.

\section{Longitudinal Research on Self-Injury}

Many researchers conceptualise self-injury as a strategy for managing distress. For example, in both the EAM (Chapman et al., 2006) and ITMS (Nock, 2010; see Figures 3 and 4, p. 19-20), self-injury is placed at the end of a sequence of risk factors and events thought to trigger strong emotional reactions. Therefore, these models inherently imply that self-injury is a result of a combination of specific risk factors (e.g., child sexual abuse, emotion dysregulation, poor distress tolerance), and is preceded by an adverse experience or experiences (e.g., bullying, interpersonal conflict).

Recently, Fox et al., (2015) conducted meta-analyses of research investigating 
prospective predictors of NSSI. In total they identified 20 published articles detailing a longitudinal relationship between NSSI and proposed risk factors. Overall, they summarised research for 25 different risk factors and identified 18 significant prospective predictors of NSSI. Of those, individuals who reported prior NSSI, or who demonstrated Cluster B personality disorders were almost six times more likely to engage in NSSI. Depression, suicidal thoughts and behaviours, eating disorder pathology, affect dysregulation, and abuse history were also among numerous other significant prospective predictors (see Fox et al., 2015 for all results).

Locally, Garisch and Wilson (2015) investigated NSSI in NZ adolescents across two time points approximately five months apart. Using cross-lagged panel correlations they identified that lower self-esteem, and greater depression and alexithymia, prospectively predicted greater engagement in self-injury. Conversely, engagement in self-injury predicted less resilience and less mindfulness five months later. Garisch and Wilson conclude that proximal factors such as depression and alexithymia may lead to NSSI which in turn is related to a decrease in adaptive coping (resilience and mindfulness). Therefore, it is incredibly important to intervene prior to reliance on NSSI as a coping strategy, and thus prior to a negative spiral of poor mental health and decreased resilience.

Within previous research there are a few stable, trait, temperamental and personality prospective predictors of NSSI that could function in a similar way to perfectionism and inform hypotheses for Study 4. As mentioned above, Cluster B personality disorders were shown to significantly predict NSSI, as were high levels of impulsivity (Fox et al., 2015). In addition to Fox and colleagues' research other researchers have demonstrated that having a negative attributional style predicts subsequent NSSI in psychiatric inpatient adolescents (Guerry \& Prinstein, 2010), and Chinese (Barrocas et al., 2014), and American community adolescents (Hankin \& Abela, 2011). Further research pertaining to impulsivity related personality traits has demonstrated that, amongst undergraduate students, negative urgency, and a lack of perseverance predicted both the onset and maintenance of NSSI respectively (Riley, Combs, Jordan, \& Smith, 2015).

Most of the studies described above investigated the effect of single NSSI risk factors. Other research has instead simultaneously considered multiple factors in the onset and exacerbation of NSSI thoughts and behaviours (e.g., Guerry \& Prinstein, 2010; Hasking, Whitlock, Voon, \& Rose, 2016). For example, Tatnell et al., (2014) considered the influence of both intrapersonal and interpersonal risk factors in the onset, maintenance, and cessation of NSSI. Tatnell and colleagues reported that, in their sample of almost 2,000 Australian 
adolescents, attachment anxiety prospectively predicted NSSI approximately 12 months later. However, this relationship was partially mediated by self-esteem, self-efficacy and cognitive reappraisal. That is, attachment anxiety was directly related to later NSSI, however it was also related to lower self-esteem, self-efficacy, and less cognitive reappraisal, all of which were in turn also related to greater NSSI. As previously alluded to this suggests that NSSI engagement is likely a product of a multitude of interacting intrapersonal and interpersonal risk factors.

Finally, there are some exceptions to this temporal sequence, with some research highlighting important consequences of self-injury. For example, research has shown that acts of self-injury can increase subsequent interpersonal conflict (e.g., Burke, Hamilton, Abramson, \& Alloy, 2015; Turner et al., 2012), or conversely increase interpersonal support (e.g., Hilt et al., 2008), and can lead to greater self-criticism (You et al., 2016). Some of the research alluded to above can be coupled with findings that many of these factors were also present before engagement in self-injury begun. Therefore, it is possible that a spiralling or negative cascade effect occurs, rather than self-injury strictly preceding the negative outcome. For example, self-criticism may increase risk for self-injury and, in turn, engagement in self-injury may then lead to further self-criticism, which subsequently increases risk of further NSSI.

\section{Longitudinal Research on Perfectionism}

Research on the longitudinal relationship between perfectionism and various mental health and wellbeing outcomes is still in its early stages. Multiple researchers have implicated negative perfectionism as a contributing factor in the development of poor mental health outcomes (e.g., Caro \& Blas, 2016; Chang \& Rand, 2000; Smith et al., 2014); however, some have also suggested that perfectionism and mental disorders are reciprocally related (e.g., they exacerbate one another; McGrath et al., 2012). Finally others have reported that some mental disorders give rise to an increase in perfectionistic tendencies (e.g., Gautreau, Sherry, Mushquash, \& Stewart, 2015).

For example, Soares and colleagues (2009) investigated the longitudinal relationship between perfectionism, and eating attitudes and behaviours in a sample of female university students. They found that positive perfectionism was positively associated with dieting concerns and students' total Eating Attitudes Test scores both one year and two years following the initial administration of tests. Moreover, negative perfectionism significantly predicted social pressure to eat one year and two years later, and bulimic behaviours one year 
later. Overall, these results suggest that perfectionism, whether positive or negative, can play a role in the development of disordered eating attitudes and behaviours.

Other research has suggested a reciprocal relationship whereby perfectionism influences later poor outcomes which then exacerbate perfectionistic tendencies. For example, McGrath and colleagues (2012) assessed perfectionism and depression once a week for four weeks and looked at the relationship between the two variables across time. Among their undergraduate sample they found that negative perfectionism both predicted and was exacerbated by depressive symptoms. Conversely, positive perfectionism protected against depressive symptoms. This suggests that negative perfectionism and depression may operate together and result in a spiral of increasingly poor mental health and greater negative perfectionism.

Finally, Gautreau and colleagues (2015) reported some interesting and somewhat unexpected results with regard to the longitudinal relationship between perfectionism and social anxiety. In particular, in a sample of undergraduate students, social anxiety predicted increases in negative perfectionism at both six months and twelve months following the initial administration of scales. The authors suggest that perfectionistic tendencies are adopted as coping strategies for socially anxious individuals. Specifically, these individuals may aim to appear perfect in order to avoid perceived negative evaluation from others. Whilst perfectionism did not seem to exacerbate social anxiety over time, the authors suggest that perhaps perfectionism has an effect on the development of social anxiety; however, once social anxiety is present, the exacerbating effects of negative perfectionism may dissipate and the relationship may be reversed. Alternatively, some research suggests that negative perfectionism may actually have no effect on the development of social anxiety. For example, Sherry et al., (2014) reported that over a 12 month period negative perfectionism predicted depression but not anxiety.

\section{Perfectionism and Self-Injurious and Suicidal Thoughts and Behaviours}

It has hopefully become apparent that cross-sectional research regarding both perfectionism and NSSI is relatively sparse (see Chapter 3). Therefore, it follows that longitudinal research focused on the two constructs is even more limited. As such, it is again useful to complement the existing perfectionism-self-injury research with longitudinal research of the relationship between perfectionism and suicide. Longitudinal perfectionismsuicide research will be discussed first, followed by a summary of the only known article addressing the longitudinal relationship between perfectionism and self-injury. 
Perfectionism and suicidal thoughts and behaviours. Beevers and Miller (2004) investigated perfectionism, cognitive bias and hopelessness in the context of suicidal ideation within a sample of individuals hospitalised for depression. Patients completed a battery of measures of psychological wellbeing, including the perfectionism subscale of the Dysfunctional Attitudes Scale (DAS; Weissman \& Beck, 1978), first during the first week of their hospitalisation and then again six months following discharge. During this time, most patients completed six months of outpatient treatment for their depression. Specifically relevant to this thesis, they reported that initial perfectionism was positively associated with suicidal ideation six months later. Moreover, the effect of perfectionism was notably stronger than the effect of initial suicidal ideation and depression, and the effect remained even with control of initial hopelessness and cognitive bias. The perfectionism subscale of the DAS is unidimensional and largely aligns with negative conceptualisations of perfectionism. Therefore, whilst these results indicate that negative perfectionism is implicated in future suicidal ideation, they did not investigate the role of positive perfectionism.

O'Connor and colleagues (2007) also explored the relationship between perfectionism and psychological wellbeing (hopelessness and suicidal thinking) in patients who presented to hospital following a suicide attempt, and who had a history of repetitive self-harm. They reported that individuals low on negative perfectionism, and high on positive future thinking scored lowest on measures of suicidal thinking and hopelessness two months following their initial presentation to the hospital. Conversely, those high on negative perfectionism scored significantly higher on both hopelessness and suicidal ideation at follow up. Overall, O'Connor and colleagues' research provides further empirical support for a relationship between negative perfectionism and later suicidal thoughts and behaviours.

Perfectionism and self-injurious thoughts and behaviours. To my knowledge there is only one study that has investigated the longitudinal relationship between perfectionism and self-injury (O'Connor et al., 2010). As previously reported in Chapter 3, O'Connor and colleagues investigated the role of perfectionism and acute life stress in predicting depression, anxiety and self-harm in a group of Scottish adolescents. They found that Time 1 depression, and anxiety, predicted self-harm, and acute life stress during the six months between survey administrations. In addition, the interaction between time one negative perfectionism and subsequent acute life stress also predicted self-harm. Specifically, in adolescents experiencing low acute life stress, the probability of engaging in DSH significantly increased with greater negative perfectionism. There was no effect of negative perfectionism on adolescents who experienced high acute life stress. Rather, the researchers 
suggested that these individuals already had a high probability of engaging in DSH. It is important to highlight that these results pertain to DSH rather than NSSI. That is, O'Connor and colleagues asked a single question ("have you ever deliberately taken an overdose (e.g., pills or other medication) or tried to harm yourself in some other way (such as cut yourself)?"') that did not differentiate acts of self-harm based on suicide intent from NSSI, and that reviews have shown produce lower reported rates of self-injury (Muehlenkamp et al., 2012; Swannell et al., 2014).

\section{Positive and Negative Perfectionism}

When considering the relationship between perfectionism and mental health and wellbeing outcomes it is often easy to overlook the moderate-strong relationship between positive and negative perfectionism. The robust relationship between positive and negative perfectionism has been convincingly demonstrated in both the adolescent sample used in this thesis (see p. 156), and in samples worldwide (see meta-analyses results p. 85-86). Whilst, this relationship is of a lesser focus than the relationship between perfectionism and selfinjury, it may still have important implications with regard to prevention and intervention strategies aimed at improving the mental health and wellbeing of our adolescents. For example, if positive and negative share a temporal relationship and therefore one predicts the other, indication of one form of perfectionism could be used to predict the course of that individual's perfectionism profile, and consequently, perfectionism-related outcomes.

However, there is a paucity of research investigating the longitudinal course of positive and negative perfectionism, and the relationship between the two quite different sets of traits. Despite this, some researchers have hypothesised that positive and negative perfectionism may be causally related. For example, Claes and colleagues (2012) have suggested that positive perfectionism may be related to an increase in negative perfectionism and subsequently an increase in NSSI. Therefore, they predicted that positive perfectionism may actually be indirectly related to risk of NSSI. Others have identified this as an area for future research (e.g., Bieling et al., 2004).

\section{Study Four: Examining the Longitudinal Relationship between Negative Perfectionism, Positive Perfectionism and NSSI in New Zealand Adolescents}

Study 4 will examine the relationship between negative perfectionism, positive perfectionism and NSSI over time. In light of both empirical research and theoretical understandings, it is expected that negative perfectionism will predict NSSI, or will at least travel alongside the development of NSSI in females. Due to the few findings regarding 
negative perfectionism and NSSI in males, no longitudinal relationships between negative perfectionism and NSSI are expected in the male subset of the sample. It is also expected that positive perfectionism may protect against future NSSI in both males and females. Finally, with regard to the relationship between positive and negative perfectionism it is expected that the two constructs will travel and develop together, rather than one predicting the other. This hypothesis is purely based on theoretical understandings of perfectionism rather than empirical research and therefore is very tentative.

\section{Method}

\section{Participants}

Study 4 is based on Wave Two (Time 1 of this research) and Wave Three (Time 2) of the YWS when students were on average 14 years old. Only participants who completed both Time 1 (T1) and Time 2 (T2) were included in this component of the research. Of the 930 students who completed T1, 608 (65\%) also completed T2 and provided the information required for their data to be matched successfully. Therefore, this sample comprises 212 males, 391 females, and 5 trans-gender adolescents. ${ }^{43}$ Participants within the matched sample ranged in age from 13 to $17(M=14.55, S D=.70)$ at T1. At T2 they were approximately one year older $(M=15.39, S D=.57$, range 14-17). Primary ethnic groups were proportionately similar to the ethnic composition of the total T1 sample. ${ }^{44}$ That is, $75 \%$ indicated Pākehā (NZ/European) as their main ethnic identification, followed by Māori (5\%) and Samoan $(3 \%) .{ }^{45}$ Nine percent of the sample endorsed 'Other', and a further 4\% reported more than one ethnicity and could not choose a primary ethnic group.

\section{Measures}

Study 4 focuses on responses to the DSHI-s (Lundh et al., 2007) and FMPS (Frost et al., 1990). Whilst the overall survey remained relatively consistent across T1 and T2, some changes were made to the length of the survey, with the aim of decreasing the time pressure for students. The most notable change impacting this research, was the change from the full 35-item FMPS to a shortened 22 item version (Cox et al., 2002).

FMPS. The FMPS was shortened using the results of Study 2. Specifically, the decision was based on the results from the CFA, which tested how well the data fit previous

\footnotetext{
43 At T1 only one adolescent endorsed transgender, whilst at T2 a further 4 students, who previously indicated male or female, also endorsed transgender. Due to the small sample size transgender students will be excluded from analyses split by sex.

44 Note: there is significant cross-over between this sample and the T1 only sample.

$452 \%$ or less endorsed Cook Island Maori, Tongan, Niuean, Chinese, or Indian. Some examples of other ethnicities include British, Filipino, American, Australian, South African, and Dutch
} 
versions of the FMPS, followed by consideration of the length of the various versions, and the results of the PCA analyses. As a result, Cox and colleagues' (2002) 22-item FMPS was chosen for use in the T2 survey (see Figure 48). With regard to the CFA, the FMPS-22 did not reach fit-indices values that represented a 'good-fit', however, the scale was the closest to a 'good-fit' out of all of the FMPS versions tested (see p. 131-132 for Study 2 CFA results). The original FMPS (Frost et al., 1990) was equally well fitting, however, given that the aim of this process was to reduce the number of items administered, the original version was excluded as an option. In addition, highest loading items on each of the four subscales that resulted from the PCA were broadly consistent with the items included in the FMPS- 22 .

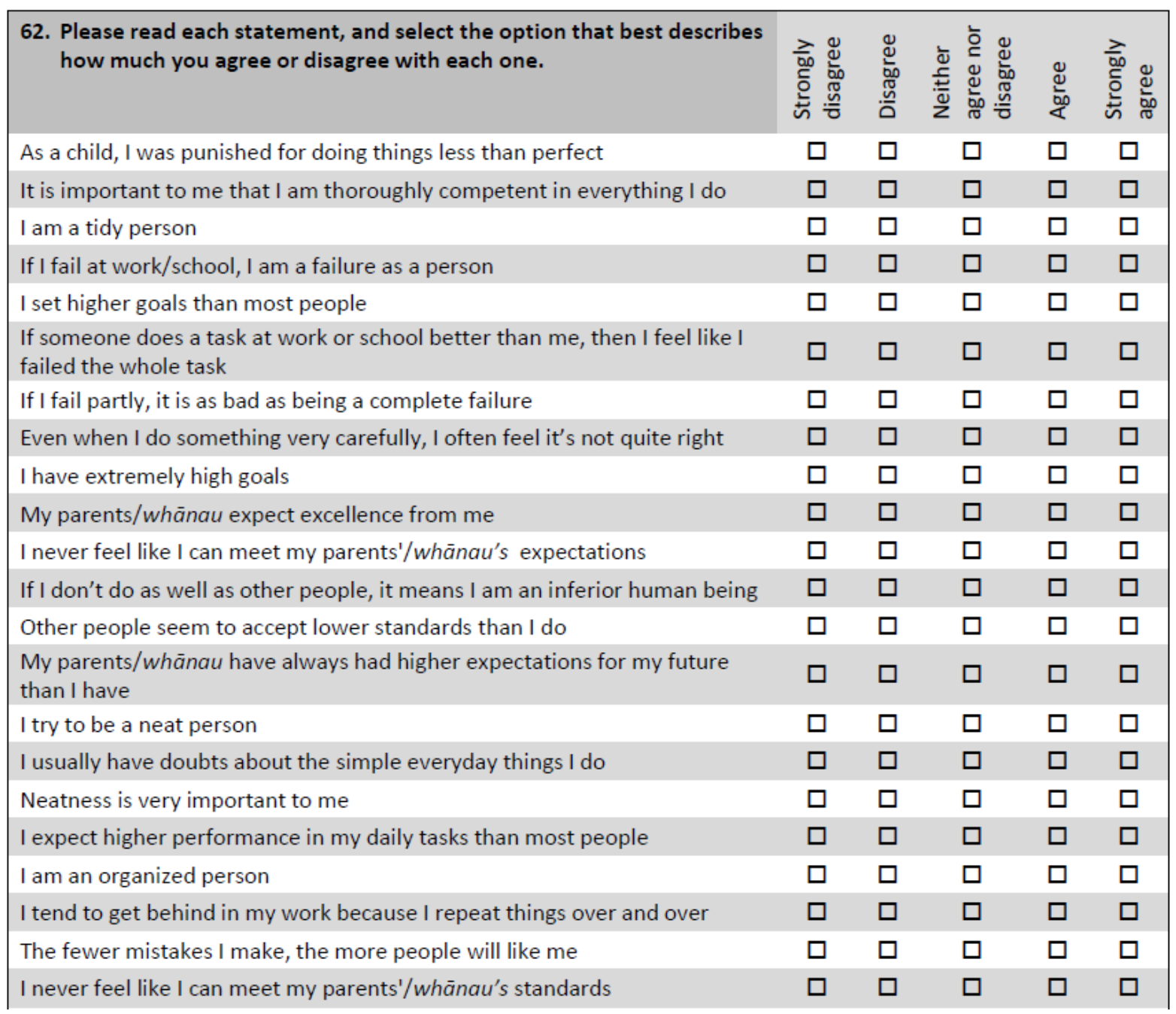

Figure 48. 22-item FMPS

Finally, although all 22 items were administered, item 3 was excluded from subscale analyses due to the ambiguity around which factor it is best suited to. Item 3 was reinstated for analyses using negative and positive perfectionism. In sum, Study 4 longitudinal analyses 
are conducted using the FMPS-22, with a hierarchical 4-2 factor structure for both T1 and T2 data. Accordingly, some T1 results reported in Study 4 may slightly differ from T1 results reported in Study 3 (for which the full scale and full sample was used).

Table 26

FMPS-22 Factor Structure based on Cox et al., 2002 and Results of Study 2

Item (Item number and original subscale)

\section{Concerns and Doubts (CMD)}

If I fail at work/school, I am a failure as a person. (Item 9, CM)

If someone does a task at work/school better than I, then I feel like I failed the whole task. (Item 13, CM)

If I fail partly, it is as bad as being a complete failure (Item 14, CM)

Even when I do something very carefully, I often feel that it is not quite right. (Item 17, DA)

If I do not do as well as other people, it means I am an inferior human being. (Item 23, CM)

I usually have doubts about the simple everyday things I do. (Item 28, DA)

I tend to get behind in my work because I repeat things over and over. (Item 32, DA)

The fewer mistakes I make, the more people will like me. (Item 34, CM)

\section{Parental Pressure (PP)}

My parents/whanau have always had higher expectations for my future than I have. (Item 26, PE)

My parents have expected excellence from me. (Item 20, PE)

I never felt like I could meet my parents' expectations. (Item 22, PC)

I never felt like I could meet my parents'/whanau's standards. (Item 35, PC)

As a child I was punished for doing things less than perfect (Item 3; cross-loaded on PP and CMD therefore excluded for subscale analyses but included in positive/negative perfectionism analyses)

\section{Personal Standards (PS)}

It is important to me that I be thoroughly competent in everything I do. (Item 6, PS)

I set higher goals than most people. (Item 12, PS)

I have extremely high goals. (Item 19, PS)

Other people seem to accept lower standards from themselves than I do. (Item 24, PS)

I expect higher performance in my daily tasks than most people. (Item 30, PS)

\section{Organisation}

I am a tidy person. (Item 7, O)

I try to be a neat person. (Item $27, \mathrm{O}$ )

Neatness is very important to me. (Item 29,0 )

I am an organized person. (Item 31, O)

DSHI-s. In addition to the FMPS-22, the DSHI-s was re-administered. This time, students were asked to complete the DSHI twice, once for their lifetime history of NSSI, and once for their engagement in NSSI since they had last completed the survey (approximately 1 year prior; see Figure 49 below for an image of how this was presented). As a reminder, the DSHI asks 'Have you ever deliberately, (but without wanting to kill yourself)...' followed by 14 items. The students then rate these items on a 5-point Likert scale from 'I've never thought about doing this', to 'I've done this many times'. Students' scores on their lifetime 
history of NSSI were used for T2 descriptive statistics, whereas measures last year engagement in NSSI were used for the longitudinal analyses.

\begin{tabular}{|c|c|c|c|c|c|c|c|c|c|c|}
\hline $\begin{array}{l}\text { 36. If you have EVER thought about or } \\
\text { deliberately hurt yourself, please } \\
\text { answer Part A. If also you took part } \\
\text { in this survey last year please } \\
\text { answer both Part A and Part B. }\end{array}$ & $\begin{array}{l}\text { tho } \\
\text { delibe } \\
\text { these }\end{array}$ & $\begin{array}{l}\quad \underline{P a} \\
\text { ght abcely d } \\
\text { hings, }\end{array}$ & $\begin{array}{l}\frac{\mathrm{t} A}{\mathrm{~A}} \\
\text { ut or } \\
\text { one } A \\
\text { VER? }\end{array}$ & NY of & & $\begin{array}{l}\text {.... tho } \\
\text { deliber } \\
\text { things, } \\
\text { THIS SI }\end{array}$ & $\begin{array}{r}\quad P \\
\text { ght abc } \\
\text { ately do } \\
\text { SINCEY } \\
\text { RVEY L } \\
\end{array}$ & $\begin{array}{l}\frac{\mathrm{B}}{\text { t or }} \\
\text { e AN } \\
U \mathrm{CO} \\
\mathrm{TSYE}\end{array}$ & $\begin{array}{l}\text { MPLE } \\
\text { MR? }\end{array}$ & $\begin{array}{l}\text { ese } \\
\text { TED } \\
\end{array}$ \\
\hline $\begin{array}{l}\text { Have you ever deliberately (but without } \\
\text { wanting to kill yourself) ... }\end{array}$ & 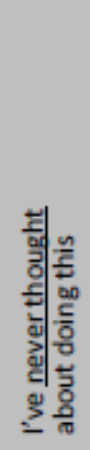 & 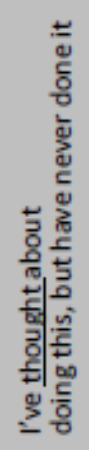 & 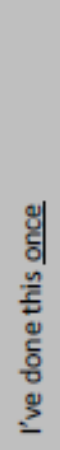 & 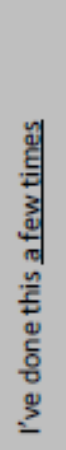 & 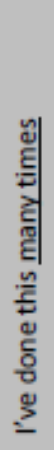 & 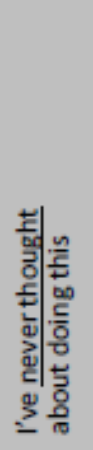 & 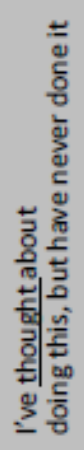 & 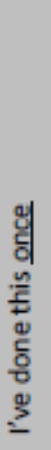 & 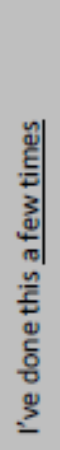 & 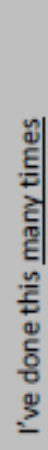 \\
\hline ... cut your wrist, arms, or other areas of your body? & $\square$ & $\square$ & $\square$ & $\square$ & $\square$ & $\square$ & $\square$ & $\square$ & $\square$ & $\square$ \\
\hline ... burned yourself with a cigarette, lighter or match? & $\square$ & $\square$ & $\square$ & $\square$ & $\square$ & $\square$ & $\square$ & $\square$ & $\square$ & $\square$ \\
\hline $\begin{array}{l}\text {... carved words, pictures, designs or other marks into } \\
\text { your skin (not including tattoos or ta moko)? }\end{array}$ & $\square$ & $\square$ & $\square$ & $\square$ & $\square$ & $\square$ & $\square$ & $\square$ & $\square$ & $\square$ \\
\hline $\begin{array}{l}\text {... severely scratched yourself, to the extent that } \\
\text { scarring or bleeding occurred? }\end{array}$ & $\square$ & $\square$ & $\square$ & $\square$ & $\square$ & $\square$ & $\square$ & $\square$ & $\square$ & $\square$ \\
\hline $\begin{array}{l}\text {... bitten yourself, to the extent that you broke the } \\
\text { skin? }\end{array}$ & $\square$ & $\square$ & $\square$ & $\square$ & $\square$ & $\square$ & $\square$ & $\square$ & $\square$ & $\square$ \\
\hline ... rubbed sandpaper on your body? & $\square$ & $\square$ & $\square$ & $\square$ & $\square$ & $\square$ & $\square$ & $\square$ & $\square$ & $\square$ \\
\hline ... dripped acid onto your skin? & $\square$ & $\square$ & $\square$ & $\square$ & $\square$ & $\square$ & $\square$ & $\square$ & $\square$ & $\square$ \\
\hline ... used bleach, or oven cleaner to scrub your skin? & $\square$ & $\square$ & $\square$ & $\square$ & $\square$ & $\square$ & $\square$ & $\square$ & $\square$ & $\square$ \\
\hline $\begin{array}{l}\text {... stuck sharp objects such as needles, pins, staples, } \\
\text { etc. into your skin (not including tattoos or ta moko, } \\
\text { ear or body piercing, or needles for drug use)? }\end{array}$ & $\square$ & $\square$ & $\square$ & $\square$ & $\square$ & $\square$ & $\square$ & $\square$ & $\square$ & $\square$ \\
\hline ... rubbed glass into your skin? & $\square$ & $\square$ & $\square$ & $\square$ & $\square$ & $\square$ & $\square$ & $\square$ & $\square$ & $\square$ \\
\hline ... broken your own bones? & $\square$ & $\square$ & $\square$ & $\square$ & $\square$ & $\square$ & $\square$ & $\square$ & $\square$ & $\square$ \\
\hline $\begin{array}{l}\text {... punched yourself, or banged your head against } \\
\text { something, to the extent that caused a bruise to } \\
\text { appear? }\end{array}$ & $\square$ & $\square$ & $\square$ & $\square$ & $\square$ & $\square$ & $\square$ & 口 & $\square$ & $\square$ \\
\hline ... prevented wounds from healing? & $\square$ & $\square$ & $\square$ & $\square$ & $\square$ & $\square$ & $\square$ & $\square$ & $\square$ & $\square$ \\
\hline $\begin{array}{l}\text {... done anything else to hurt yourself that was not } \\
\text { asked about in these questions? If yes, please } \\
\text { briefly describe what you did: }\end{array}$ & $\square$ & $\square$ & 口 & $\square$ & $\square$ & $\square$ & $\square$ & 口 & $\square$ & $\square$ \\
\hline
\end{tabular}

Figure 49. DSHI-s used in T2 survey 
ISAS. Again, the ISAS was administered to capture the functions of NSSI. The T2 presentation of this scale exactly mirrored the T1 presentation (refer to Figures 39 and 40).

Reliability analyses. Reliability analyses were conducted for the FMPS-22, DSHI-s, and the ISAS at T1 and T2 in the matched sample (see Table 27). All subscales on the FMPS22 and DSHI-s had adequate internal reliability, as evidenced by Cronbach's alphas greater than .70 (Bernardi, 1994). The FMPS-22, and the DSHI-s also demonstrated good test-retest scores in the longitudinal T1-T2 matched sample. Unfortunately the reliability of the ISAS subscales was relatively poor. In particular, Interpersonal Influence and Revenge fell well below adequate internal reliability. In some cases this can be partly attributed to the very few items in each subscale. However, Giles' (2002) method of examining the inter-item correlations also demonstrated that reliability was well below satisfactory. The low internal reliability of ISAS subscales is consistent with some other studies (e.g., Bildik, Somer, Kabukçu Başay, Başay, \& Özbaran, 2013; Langlands, 2012). Others have only reported the interpersonal and intrapersonal scores (e.g., Glenn \& Klonsky, 2009; Lindholm, Bjärehed, \& Lundh, 2011; Somer et al., 2015), perhaps due to similar issues with the reliability of the subscales. Despite inadequate internal reliability for the individual functions, the interpersonal and intrapersonal overarching subscales demonstrated good internal reliability with Cronbach's alphas of .77 and .81 respectively. Therefore, only the overarching subscales will be used in subsequent analyses.

Participant attrition. Analyses were conducted to investigate whether adolescents who completed both time points significantly differed to adolescents who only completed T1. Independent samples $t$-tests revealed that adolescents who dropped out of the study scored significantly higher on the negative perfectionism subscale $\left(M_{\text {Longitudinal }}=2.28, S D=.72\right.$; $\left.M_{\text {Time1Only }}=2.41, S D=.78 ; t(914)=-2.54, p=.01, d=.17\right)$ of the FMPS. Further investigation indicated that this is likely due to a difference in mean scores on the CMD subscales $\left(M_{\text {Longitudinal }}=2.34, S D=.83 ; M_{\text {Time1Only }}=2.51, S D=.89 ; t(901)=-2.06, p=.04, d\right.$ $=.14)$. However, this effect is only small (Cohen, 1988). Adolescents who dropped out did not significantly differ from adolescents who completed both time points on any of the other FMPS subscales, or the DSHI-s.

\section{Procedure}

The T2 survey was administered using the same procedure adopted at T1 (see p. 130131). Students who had not previously returned consent packs or who were new to the school were given another opportunity to gain parental consent, through a consent pack taken home. 
Students whose parents initially denied consent were not provided with a second opportunity to gain consent and were not invited to complete the survey. Once T2 data collection was complete it was matched with T1 data using the student's name or other identifying information. Each student was then assigned a unique identification code and their name and original survey were kept in a confidential file separate from the core data.

Table 27

Cronbach's Alpha Reliability Coefficients for the FMPS, DSHI-s, and ISAS across T1 and T2 for the Longitudinal Matched Sample only

\begin{tabular}{|c|c|c|c|}
\hline & \multicolumn{2}{|c|}{$\underline{\alpha}$} & \multirow[t]{2}{*}{${\underline{\text { Test-Retest }^{2}}}^{2}$} \\
\hline$\underline{\text { FMPS }}$ & $\underline{\mathrm{T} 1}$ & $\underline{\mathrm{T} 2}$ & \\
\hline Concerns and Doubts & .89 & .88 & .68 \\
\hline Parental Pressure & .81 & .82 & .57 \\
\hline Personal Standards & .83 & .83 & .63 \\
\hline Organisation & .88 & .82 & .70 \\
\hline Negative Perfectionism & .89 & .89 & .71 \\
\hline Positive Perfectionism & .83 & .82 & .69 \\
\hline \multicolumn{4}{|l|}{$\underline{\text { DSHI-s }}$} \\
\hline DSHI-s & .83 & .83 & .61 \\
\hline \multicolumn{4}{|c|}{$\alpha(M$ inter-item correlation $)$} \\
\hline$\underline{\text { ISAS }^{1}}$ & & & \\
\hline Affect regulation & $.70(.43)$ & $.73(.48)$ & .51 \\
\hline Anti-dissociation & $.70(.45)$ & $.66(.44)$ & .41 \\
\hline Anti-suicide & $.82(.60)$ & $.86(.67)$ & .61 \\
\hline Marking distress & $.73(.47)$ & $.70(.44)$ & .53 \\
\hline Self-punishment & $.79(.56)$ & $.80(.58)$ & .52 \\
\hline Autonomy & $.74(.49)$ & $.76(.51)$ & .82 \\
\hline $\begin{array}{l}\text { Interpersonal } \\
\text { boundaries }\end{array}$ & $.63(.37)$ & $.75(.53)$ & .53 \\
\hline Interpersonal influence & $.49(.24)$ & $.37(.34)$ & .48 \\
\hline Peer bonding & $.81(.59)$ & $.76(.51)$ & .56 \\
\hline Revenge & $.31(.11)$ & $.82(.65)$ & .23 \\
\hline Self-care & $.56(.35)$ & $.60(.37)$ & .59 \\
\hline Sensation seeking & $.47(.25)$ & $.58(.33)$ & .46 \\
\hline Toughness & $.69(.43)$ & $.74(.50)$ & .70 \\
\hline Intrapersonal functions & .81 & .74 & .55 \\
\hline Interpersonal functions & .77 & .79 & .54 \\
\hline
\end{tabular}

${ }^{1}$ Only included students who had indicated engaging in self-injury at least once

${ }^{2}$ All $r$ 's significant at $p<.001$ 


\section{Results}

\section{Descriptive Statistics for the Longitudinal Matched Sample}

In total, 608 participants completed the survey at both T1 and T2. Subsequently, data from $\mathrm{T} 1$ and $\mathrm{T} 2$ were matched to give data for negative perfectionism, positive perfectionism and NSSI at two time points approximately one year apart. This data was then used in longitudinal analyses of the relationship between perfectionism and NSSI as outlined below. All results below pertain to only the matched subset of the total sample (i.e., only those who completed the survey at both $\mathrm{T} 1$ and $\mathrm{T} 2$ and provided sufficient identifying information to match their data). Therefore, the results for T1 may slightly differ to those reported in Studies 2 and 3.

Self-injury. At T1, 20\% of adolescents reported engaging in NSSI at least once (see Table 28 for all percentages). A further $12 \%$ reported thinking about NSSI, and $68 \%$ reported never having thought or engaged in NSSI. At T2, 22\% of the matched sample having engaged in NSSI, whilst $8 \%$ had thought about NSSI but had never tried it. Finally, $70 \%$ reported never having thought about or engaged in NSSI. T2 prevalence data is slightly different to the T1 results. First, the proportion of students indicating that they had thought about NSSI slightly decreased (T1: 12\%, T2: $\%$ ), and the proportion indicating that they had engaged in NSSI slightly increased (T1: 20\%, T2: 22\%). This suggests that over the 12 months separating T1 and T2, some of the students who endorsed 'Thought about it' in T1 survey likely had tried self-injury by T2. Further chi-squared analyses showed that endorsement of NSSI across T1 and T2 were significantly different to what would be expected by chance $\left(\chi^{2}(4, n=547)=342.02, p<.001\right.$; see Table 28$)$. They also highlighted some inconsistency in self-report amongst this sample. Namely, those who reported selfinjury at T1, at T2 15\% subsequently reported no history of self-injury, and a further $6 \%$ reported only thinking about self-injury. Similarly, of those who reported thoughts about selfinjury at T1, 40\% reported no thoughts or engagement in self-injury at T2 (see Table 28). This is not practically possible and suggests that there is an error in self-report at some stage of the process. ${ }^{46}$ Inconsistencies in report of self-injurious and suicidal thoughts and behaviours have been highlighted as relatively common (e.g., Hart, Musci, Ialongo, Ballard, \& Wilcox, 2013; Mars et al., 2016). For example, Mars and colleagues (2016) investigated

\footnotetext{
46 It is possible that some students who initially indicated that they had thought about NSSI, subsequently reported no thoughts about NSSI at T2 due to a misunderstanding of the time period that they were considering (i.e., some may have thought that they were reporting based on the time since the last survey). Alternatively, some students may have endorsed 'No' rather than 'Thought about it' as it allowed them to skip a large portion of the survey and complete the task quicker.
} 
whether self-reported lifetime history of self-harm at age 16, was consistent with the adolescents report, two years later at age 18. They reported that $35 \%$ of the participants who indicated a history of self-harm when surveyed at 16 years old, no longer indicated having ever engaged in self-harm when surveyed at 18 years old.

Table 28

Change in NSSI Prevalence for T1 and T2

\begin{tabular}{|c|c|c|c|c|}
\hline \multirow{3}{*}{$\frac{\text { Prevalence T1 }}{\text { Yes }}$} & \multicolumn{3}{|c|}{ Prevalence T2 - \% / Actual (Expected) } & \multirow[t]{2}{*}{$\chi^{2}$} \\
\hline & Yes & Thought about it & No & \\
\hline & $78.8 / 89(25)$ & $6.2 / 7(10)$ & $15.0 / 17(77)$ & $342.02 * *$ \\
\hline Thought about it & $29.4 / 20(15)$ & $30.9 / 21(6)$ & $39.7 / 27(6)$ & \\
\hline No & $3.8 / 14$ (82) & $5.5 / 20(32)$ & $90.7 / 332(252)$ & \\
\hline
\end{tabular}

In addition, mean scores on the DSHI-s, thus the severity of NSSI was compared across time points (see Table 29). Paired samples $t$-test showed no significant difference in NSSI severity across $\mathrm{T} 1$ and $\mathrm{T} 2\left(M_{\mathrm{T} 1}=.22, S D=.49 ; M_{\mathrm{T} 2}=.24, S D=.51, t(529)=-.81, p=\right.$ $.42, n s)$. That is, although slightly more adolescents reported having engaged in NSSI, as evidenced by the prevalence rates above, thoughts about NSSI, and engagement in NSSI did not appear to be any more severe at T2 than at T1. Moreover, the number of different methods of self-injury did not significantly differ across $\mathrm{T} 1$ and $\mathrm{T} 2\left(M_{\mathrm{T} 1}=3.51, S D=2.32\right.$; $\left.M_{\mathrm{T} 2}=3.77, S D=2.40, t(88)=-1.11, p=.27, n s\right)$. Analyses were also conducted for males and females separately, however no significant differences were revealed (see Table 29).

At T2 students who indicated that they had thought about self-injury or had engaged in self-injury ( $n=171)$ were also asked "how many times have you hurt yourself since the last survey?" Sixteen percent indicated hurting themselves once, 33\% indicated hurting themselves a few times, and finally $15 \%$ indicated hurting themselves many times. Others reported no self-injury since the last survey (36\%). Of those who had hurt themselves within the last year $(n=114), 17 \%$ had self-injured within the last week, $20 \%$ within the last month, $24 \%$ within the last three months, $23 \%$ within the last six months, and $17 \%$ over six months ago.

Functions of self-injury were examined across time points. However, given the poor internal reliability of the 13 subscales of the ISAS in this sample, only the overarching intrapersonal and interpersonal functional categories are reported. Paired samples $t$-tests revealed that endorsement of intrapersonal functions significantly increased $\left(M_{\mathrm{T} 1}=.70, S D=\right.$ 
$\left..45 ; M_{\mathrm{T} 2}=.80, S D=.46, t(99)=-2.27, p=.03\right)$, whilst endorsement of interpersonal functions remained stable $\left(M_{\mathrm{T} 1}=.19, S D=.23 ; M_{\mathrm{T} 2}=.22, S D=.30, t(99)=-1.25, p=.21\right.$, $n s)$.

Table 29

NSSI Prevalence and Chi-Squared Analyses across Sex for T1 and T2

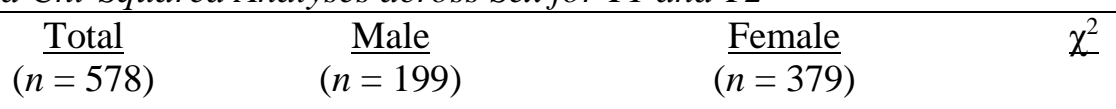

\begin{tabular}{|c|c|c|c|c|}
\hline Prevalence T1 & & \% / Actual (Expected) & \% / Actual (Expected) & \\
\hline Yes & $20.4 \%$ & $9.5 / 19(41)$ & $26 / 99(77)$ & $38.03 *$ \\
\hline Thought about it & $12.1 \%$ & $6.5 / 13(24)$ & $15.0 / 57(46)$ & \\
\hline No & $67.5 \%$ & $83.9 / 167$ (134) & $18.8 / 223(256)$ & \\
\hline & $\frac{\text { Total }}{(n=569)}$ & $\underset{(n=205)}{\text { Male }}$ & $\frac{\text { Female }}{(n=364)}$ & $\chi^{2}$ \\
\hline$\underline{\text { Prevalence T2 }}$ & & \% / Actual (Expected) & \% / Actual (Expected) & \\
\hline Yes & $21.5 \%$ & $10.2 / 21(44)$ & $28.0 / 102(78)$ & $37.11 *$ \\
\hline Thought about it & $8.4 \%$ & $4.4 / 9$ (17) & $10.7 / 39(31)$ & \\
\hline No & $70.1 \%$ & $85.4 / 176(144)$ & $61.3 / 223(255)$ & \\
\hline
\end{tabular}

Perfectionism. Next, analyses were conducted to investigate variability in perfectionism scores across the two time points. A repeated measures ANOVA highlighted a significant effect for perfectionism $\left(F(3,1740)=184.59, p<.001 ; \eta_{\mathrm{p}}^{2}=.24\right)$, thus indicating significant differences across the perfectionism subscales ${ }^{47}$. In addition, the analyses showed a significant effect for the interaction between perfectionism and time $(F(3,1740)=9.30, p<$ $.001 ; \eta_{\mathrm{p}}^{2}=.02$ ). This suggests that the difference between scores on the perfectionism subscales is not uniform over both time points.

Post-hoc $t$-tests showed that whilst PP significantly increased $\left(M_{\mathrm{T} 1}=2.61, S D=.91\right.$; $\left.M_{\mathrm{T} 2}=2.73, S D=.97, t(580)=-3.32, p=.01\right)$ and Organisation significantly decreased $\left(M_{\mathrm{T} 1}\right.$ $\left.=3.30, S D=.89 ; M_{\mathrm{T} 2}=3.21, S D=.87, t(584)=3.05, p=.01\right)$, there was no significant

\footnotetext{
${ }^{47}$ Mauchly's Test of Sphericity was significant $(p<.001)$, thus indicating that the sphericity assumption was violated. Therefore, all analyses were Huynh-Feldt corrected.
} 
Table 30

Endorsement of Perfectionism, NSSI, and Functions at T1 and T2 - total and Split by Sex

\begin{tabular}{|c|c|c|c|c|}
\hline \multirow{4}{*}{ DSHI-s } & $\underline{\text { Sex }}$ & $\mathrm{T} 1 M(S D)$ & $\underline{\mathrm{T} 2 M(S D)}$ & $\underline{t}$ \\
\hline & Total & $.22(.49)$ & $.24(.51)$ & -.81 \\
\hline & Male & $.11(.35)$ & $.12(.41)$ & -.38 \\
\hline & Female & $.29(.54)$ & $.30(.55)$ & -.71 \\
\hline \multirow{3}{*}{$\begin{array}{l}\text { Interpersonal } \\
\text { Functions (ISAS) }\end{array}$} & Total & $.19(.23)$ & $.22(.30)$ & -1.25 \\
\hline & Male & $.18(.30)$ & $.23(.42)$ & -.43 \\
\hline & Female & $.19(.21)$ & $.22(.27)$ & -1.38 \\
\hline \multirow{3}{*}{$\begin{array}{l}\text { Intrapersonal } \\
\text { Functions (ISAS) }\end{array}$} & Total & $.70(.45)$ & $.80(.46)$ & $-2.27 *$ \\
\hline & Male & $.44(.38)$ & $.60(.45)$ & -1.48 \\
\hline & Female & $.76(.45)$ & $.85(.46)$ & -1.82 \\
\hline \multirow{3}{*}{$\begin{array}{l}\text { Concerns and } \\
\text { Doubts (FMPS) }\end{array}$} & Total & $2.39(.83)$ & $2.40(.83)$ & -.75 \\
\hline & Male & $2.17(.70)$ & $2.22(.73)$ & -.95 \\
\hline & Female & $2.50(.87)$ & $2.51(.87)$ & -.30 \\
\hline \multirow{3}{*}{$\begin{array}{l}\text { Parental Pressure } \\
\text { (FMPS) }\end{array}$} & Total & $2.61(.91)$ & $2.73(.97)$ & $-3.32 *$ \\
\hline & Male & $2.57(.81)$ & $2.70(.87)$ & $-2.06^{*}$ \\
\hline & Female & $2.63(.96)$ & $2.75(1.02)$ & $-2.68 *$ \\
\hline \multirow{3}{*}{$\begin{array}{l}\text { Personal Standards } \\
\text { (FMPS) }\end{array}$} & Total & $3.01(.82)$ & $3.02(.86)$ & -.46 \\
\hline & Male & $2.98(.83)$ & $3.01(.85)$ & -.78 \\
\hline & Female & $3.03(.81)$ & $3.04(.87)$ & -.08 \\
\hline \multirow{3}{*}{$\begin{array}{l}\text { Organisation } \\
\text { (FMPS) }\end{array}$} & Total & $3.30(.89)$ & $3.21(.87)$ & $3.05^{*}$ \\
\hline & Male & $3.12(.91)$ & $2.99(.92)$ & $2.52 *$ \\
\hline & Female & $3.39(.87)$ & $3.32(.83)$ & 1.91 \\
\hline \multirow{3}{*}{$\begin{array}{l}\text { Positive } \\
\text { Perfectionism } \\
\text { (FMPS) }\end{array}$} & Total & $3.16(.69)$ & $3.12(.70)$ & 1.63 \\
\hline & Male & $3.05(.70)$ & $3.00(.72)$ & 1.13 \\
\hline & Female & $3.21(.68)$ & $3.17(.68)$ & 1.14 \\
\hline \multirow{3}{*}{$\begin{array}{l}\text { Negative } \\
\text { Perfectionism } \\
\text { (FMPS) }\end{array}$} & Total & $2.28(.72)$ & $2.30(.75)$ & -.83 \\
\hline & Male & $2.20(.65)$ & $2.21(.64)$ & -.46 \\
\hline & Female & $2.32(.75)$ & $2.34(.80)$ & -.81 \\
\hline
\end{tabular}

$* p<.05 ; * * p<.001$

change in reported CMD, PS, overarching positive perfectionism, or negative perfectionism across the two time points (see Table 30). Mean scores for all perfectionism subscales across $\mathrm{T} 1$ and $\mathrm{T} 2$ were also examined by sex. These analyses showed that while both sexes reported greater PP $\left(M_{\mathrm{T} 1 \text { - Males }}=2.57, S D=.81 ; M_{\mathrm{T} 2}=2.70, S D=.87, t(200)=-2.06, p=.04 ; M_{\mathrm{T} 1-}\right.$ Females $\left.=2.63, S D=.96 ; M_{\mathrm{T} 2}=2.75, S D=1.02, t(378)=-2.68, p=.01\right)$, only males reported less Organisation $\left(M_{\mathrm{T} 1}\right.$ - Males $=3.12, S D=.91 ; M_{\mathrm{T} 2}=2.99, S D=.92, t(202)=2.52, p=.01$; see Table 30). 


\section{Perfectionism and NSSI across T1 and T2}

Correlations for T1 and T2 variables are shown in Table 31. Relationships between the variables remained relatively stable across T1 and T2. However, there was one exception to this. Namely, that the relationship between positive perfectionism and negative perfectionism was significantly stronger at T2 than at T1 $(z=-2.22, p=.01)$. Further analyses split by sex, demonstrated that this change in relationship only occurred in females $(z=-2.75$, $p=.01$ ), thus suggesting that the result mentioned above was driven by the female subset of the total sample.

\section{Table 31}

Pearson's Correlation Coefficients for the Relationship between Positive Perfectionism, Negative Perfectionism, and NSSI at T1 and T2 for both Males and Females

\begin{tabular}{|c|c|c|c|c|}
\hline \multirow{5}{*}{$\begin{array}{l}\text { Total sample } \\
(n=599)\end{array}$} & & $\underline{\mathrm{DSHI}}$ & Positive & Negative \\
\hline & & & Perfectionism & $\underline{\text { Perfectionism }}$ \\
\hline & DSHI & - & .01 & $.26^{* *}$ \\
\hline & Positive perfectionism & -.03 & - & $.20 * *$ \\
\hline & $\begin{array}{l}\text { Negative } \\
\text { perfectionism }\end{array}$ & $.27 * *$ & $.32 * *$ & -- \\
\hline \multirow{3}{*}{$\begin{array}{l}\text { Males } \\
(n=211)\end{array}$} & DSHI & - & -.06 & .14 \\
\hline & Positive perfectionism & $.22 * *$ & - & $.26^{* *}$ \\
\hline & $\begin{array}{l}\text { Negative } \\
\text { perfectionism }\end{array}$ & $.14^{*}$ & $.25^{* *}$ & - \\
\hline \multirow{3}{*}{$\begin{array}{l}\text { Females } \\
(n=387)\end{array}$} & DSHI & - & .01 & $.29 * *$ \\
\hline & Positive perfectionism & .02 & - & $.16^{*}$ \\
\hline & $\begin{array}{l}\text { Negative } \\
\text { perfectionism }\end{array}$ & $.30 * *$ & $.34 * *$ & - \\
\hline
\end{tabular}

Following an investigation of the strength of relationships between the variables within each time point, the correlational relationships across time points were explored (see Table 32). To note, most variables were relatively stable over time (test-retest coefficients $>$.50). However, for males only, NSSI endorsement ( $r=.40)$, interpersonal functions $(r=.19)$ and intrapersonal functions $(r=.45)$ correlated only weakly or moderately across the two time points. This is somewhat consistent with previous research that has demonstrated common inconsistencies in self-report of self-injurious thoughts and behaviours across time (see Mars et al., 2016). 


\section{Table 32}

Correlations between Perfectionism and NSSI at T1 and T2

\begin{tabular}{|c|c|c|c|c|c|c|c|c|c|c|c|}
\hline & & $\underline{\mathrm{TS} 2}$ & $\frac{\frac{\mathrm{T} 2}{\mathrm{DSHI}}}{\underline{\text { Last }}}$ & $\begin{array}{l}\text { Intrapersonal } \\
\text { Functions T2 }\end{array}$ & $\begin{array}{l}\text { Interpersonal } \\
\text { Functions T2 }\end{array}$ & $\frac{\text { T2 Positive }}{\text { Perfectionism }}$ & $\frac{\text { T2 Negative }}{\text { Perfectionism }}$ & $\frac{\text { T2 Concerns }}{\text { and Doubts }}$ & $\frac{\underline{\mathrm{T} 2}}{\underline{\text { Parental }}} \underline{\underline{\text { Pressure }}}$ & $\begin{array}{c}\underline{\mathrm{T} 2} \\
\underline{\text { Personal }} \\
\underline{\text { Standards }}\end{array}$ & $\frac{\underline{\mathrm{T} 2}}{\underline{\text { Organis }}}$ \\
\hline \multirow{9}{*}{$\begin{array}{l}\text { Total } \\
\text { sample } \\
(n=586)\end{array}$} & T1 DSHI & $.61 *$ & $\frac{\text { Year }}{.39 *}$ & .30 & .14 & -.00 & $.22 *$ & $.23^{*}$ & .14 & -.02 & -.01 \\
\hline & $\begin{array}{l}\text { Intrapersonal } \\
\text { Functions } \mathrm{T} 1\end{array}$ & $.37 *$ & .25 & $.54^{*}$ & .22 & .18 & .06 & 20 & -.01 & .14 & .16 \\
\hline & $\begin{array}{l}\text { Interpersonal } \\
\text { Functions } \mathrm{T} 1\end{array}$ & .17 & .31 & $.41 *$ & $.55^{*}$ & $.34 *$ & .28 & $.36^{*}$ & .09 & $.31 *$ & .24 \\
\hline & $\begin{array}{l}\text { T1 Positive } \\
\text { perfectionism }\end{array}$ & -.07 & .03 & .09 & -.04 & $.69 *$ & $.21 *$ & $.26^{*}$ & .13 & $.54^{*}$ & $.57 *$ \\
\hline & $\begin{array}{l}\text { T1 Negative } \\
\text { perfectionism }\end{array}$ & $.21 *$ & .17 & .23 & .08 & $.19 *$ & $.71 *$ & $.59 *$ & $.55^{*}$ & $.29 *$ & .01 \\
\hline & $\begin{array}{l}\text { T1 Concerns } \\
\text { and Doubts }\end{array}$ & $.23^{*}$ & .14 & .26 & .04 & $.25^{*}$ & $.58 *$ & $.68^{*}$ & $.37 *$ & $.32 *$ & .08 \\
\hline & $\begin{array}{l}\text { T1 Parental } \\
\text { Pressure }\end{array}$ & $.17 *$ & .11 & .10 & .05 & .08 & $.59 *$ & $.37 *$ & $.57 *$ & $.18^{*}$ & -.06 \\
\hline & $\begin{array}{l}\text { T1 Personal } \\
\text { Standards }\end{array}$ & -.00 & .04 & .07 & -.02 & $.51 *$ & $.34^{*}$ & $.35^{*}$ & $.24^{*}$ & $.63^{*}$ & $.20 *$ \\
\hline & $\begin{array}{l}\mathrm{T} 1 \\
\text { Organisation }\end{array}$ & -.11 & .01 & .07 & -.05 & $.59 *$ & .01 & .07 & -.02 & $.26^{*}$ & $.70 *$ \\
\hline \multirow{3}{*}{$\begin{array}{l}\text { Males } \\
(n=202)\end{array}$} & T1 DSHI & $.40^{*}$ & .19 & .24 & .06 & -.10 & -.11 & .08 & .08 & -.15 & -.02 \\
\hline & $\begin{array}{l}\text { Intrapersonal } \\
\text { Functions } \mathrm{T} 1\end{array}$ & .12 & .11 & .45 & .16 & .12 & -.15 & .19 & -.07 & .05 & .14 \\
\hline & $\begin{array}{l}\text { Interpersonal } \\
\text { Functions } \mathrm{T} 1\end{array}$ & -.25 & .18 & .05 & .19 & .36 & .27 & .40 & .20 & .32 & .30 \\
\hline
\end{tabular}




\section{Table 32}

Correlations between Perfectionism and NSSI at T1 and T2

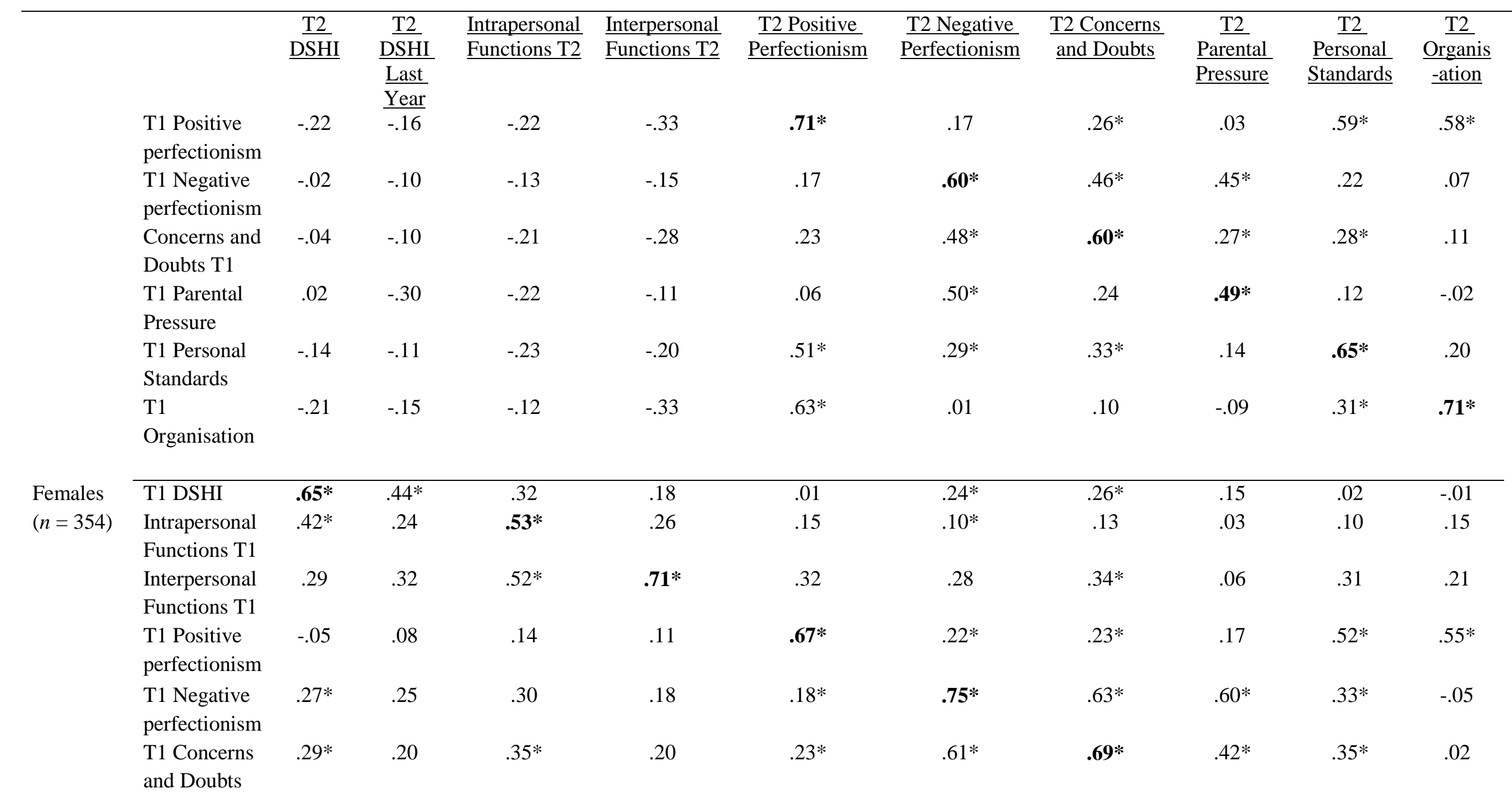




\section{Table 32}

Correlations between Perfectionism and NSSI at T1 and T2

\begin{tabular}{|c|c|c|c|c|c|c|c|c|c|c|}
\hline & $\underline{\mathrm{T} 2}$ & $\frac{\frac{\mathrm{T} 2}{\mathrm{DSHI}}}{\underline{\underline{\text { Last }}}}$ & $\frac{\text { Intrapersonal }}{\text { Functions T2 }}$ & $\begin{array}{l}\text { Interpersonal } \\
\text { Functions T2 }\end{array}$ & $\frac{\text { T2 Positive }}{\text { Perfectionism }}$ & $\frac{\text { T2 Negative }}{\underline{\text { Perfectionism }}}$ & $\frac{\text { T2 Concerns }}{\underline{\text { and Doubts }}}$ & $\frac{\underline{\mathrm{T} 2}}{\underline{\text { Parental }}} \underline{\underline{\text { Pressure }}}$ & $\begin{array}{c}\underline{\mathrm{T} 2} \\
\underline{\text { Personal }} \\
\underline{\text { Standards }}\end{array}$ & $\frac{\underline{\mathrm{T} 2}}{\underline{\text { Organis }}}$ \\
\hline $\begin{array}{l}\text { T1 Parental } \\
\text { Pressure }\end{array}$ & $.22 *$ & .19 & .17 & .12 & .08 & $.62 *$ & $.42 *$ & $.60 *$ & $.21 *$ & -.09 \\
\hline $\begin{array}{l}\text { T1 Personal } \\
\text { Standards }\end{array}$ & .05 & .08 & .14 & .07 & $.52 *$ & $.37 *$ & $.36 *$ & $.28 *$ & $.62 *$ & $.20 *$ \\
\hline $\begin{array}{l}\mathrm{T} 1 \\
\text { Organisation }\end{array}$ & -.11 & .05 & .09 & .11 & $.56^{*}$ & -.00 & .03 & -.00 & $.24 *$ & $.68^{*}$ \\
\hline
\end{tabular}

Note: A post hoc Bonferroni correction was applied to address the issue of inflated family-wise error, the corrected $p$-value is .0005 and significant correlations are indicated by *; autocorrelations are highlighted in bold. 
With regard to inter-variable correlations, in females, T1 negative perfectionism was associated with T2 NSSI $(r=.27)$. Conversely, T1 NSSI history was also associated with T2 negative perfectionism $(r=.24)$. Positive perfectionism and NSSI were not significantly associated across T1 and T2. For males, negative perfectionism and NSSI were not associated across time points, nor were positive perfectionism and NSSI. Finally, positive and negative perfectionism were significantly associated with each other across both time points for females only (see Table 31).

\section{Cross-Lagged Panel Correlations}

Finally, Cross Lagged Panel Correlations (CLPC) were used to test for the association between multiple variables at multiple time points (Kenny, 1975). For example, the most simple model would include two variables (X, Y), measured at two time points (X1, Y1, X2, Y2). A key assumption of CLPC is that "cause precedes effect" (Shingles, 1976, p. 97). In other words, because they came first, T1 data could have influenced T2 data. But the T1 data could not have been caused by the T2 data. Therefore, if there is a significant relationship between $\mathrm{X} 1$ and $\mathrm{Y} 2$, it is possible that X1 caused Y2 but not that Y2 caused X1. Moreover, at its heart, CLPC is a test for spuriousness (Kenny, 1975). In other words, a test of the possibility that the relationship between variables across time is not indicative of causality, and instead should be attributed to a common third variable or other related factor (Shingles, 1976). Thus, non-significant crosslagged correlations signify spuriousness, whilst significant cross-lagged correlations provide evidence that the association between the variables of interest may indeed be causal in nature (Kenny, 1975).

With regard to this research, T1 positive perfectionism, T1 negative perfectionism and lifetime history of NSSI were entered into a model (in AMOS 23) with T2 positive perfectionism, T2 negative perfectionism, and last year NSSI (see base model in Figure 50; direct correlations presented in Table 31). Within this model, there is control for the crosssectional relationships (e.g., relationships between positive perfectionism, negative perfectionism and NSSI at T1) and the stability coefficients for each variable (e.g., the relationship between T1 negative perfectionism and $\mathrm{T} 2$ negative perfectionism). Therefore, the resulting model has six cross-lagged correlation paths (see Figure 50). Considering the previous differences across sex, these longitudinal relationships were tested first with the total sample and second individually by sex. 


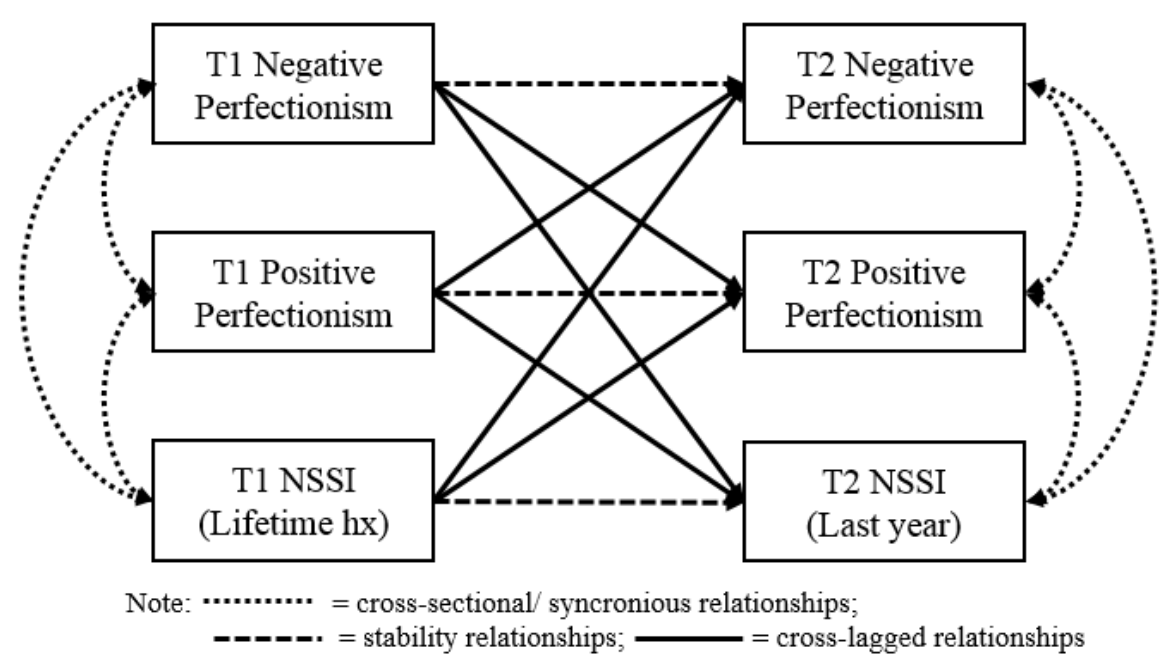

Figure 50. Proposed cross lagged panel correlation model to test for the relationship between perfectionism and NSSI over time

The CLPC for the total sample revealed that negative perfectionism at T1 significantly predicted NSSI at $\mathrm{T} 2(\beta=.12, p<.05)$, and $\mathrm{T} 1$ positive perfectionism significantly predicted $\mathrm{T} 2$ negative perfectionism $(\beta=.07, p<.001)$. In addition, all variables were relatively stable over time (correlation coefficients ranged from .53-.68 and were all significant; see Figure 51).

In the male sample there were no significant cross-lagged correlations (Figure 51). This suggests that spuriousness exists in these relationships and there is no evidence of causal relationships between the variables. Similar to the analyses with the total sample, all three variables were significantly stable across time (.36-.71). NSSI was least stable (.36).

Finally, the model was tested with the female subset of the sample (Figure 51). Analyses uncovered results very similar to those found for the total sample. That is, T1 negative perfectionism predicted T2 NSSI $(\beta=.16, p<.001)$ and T1 positive perfectionism predicted T2 negative perfectionism $(\beta=.09, p<.05)$. Again, all three variables were significantly correlated 

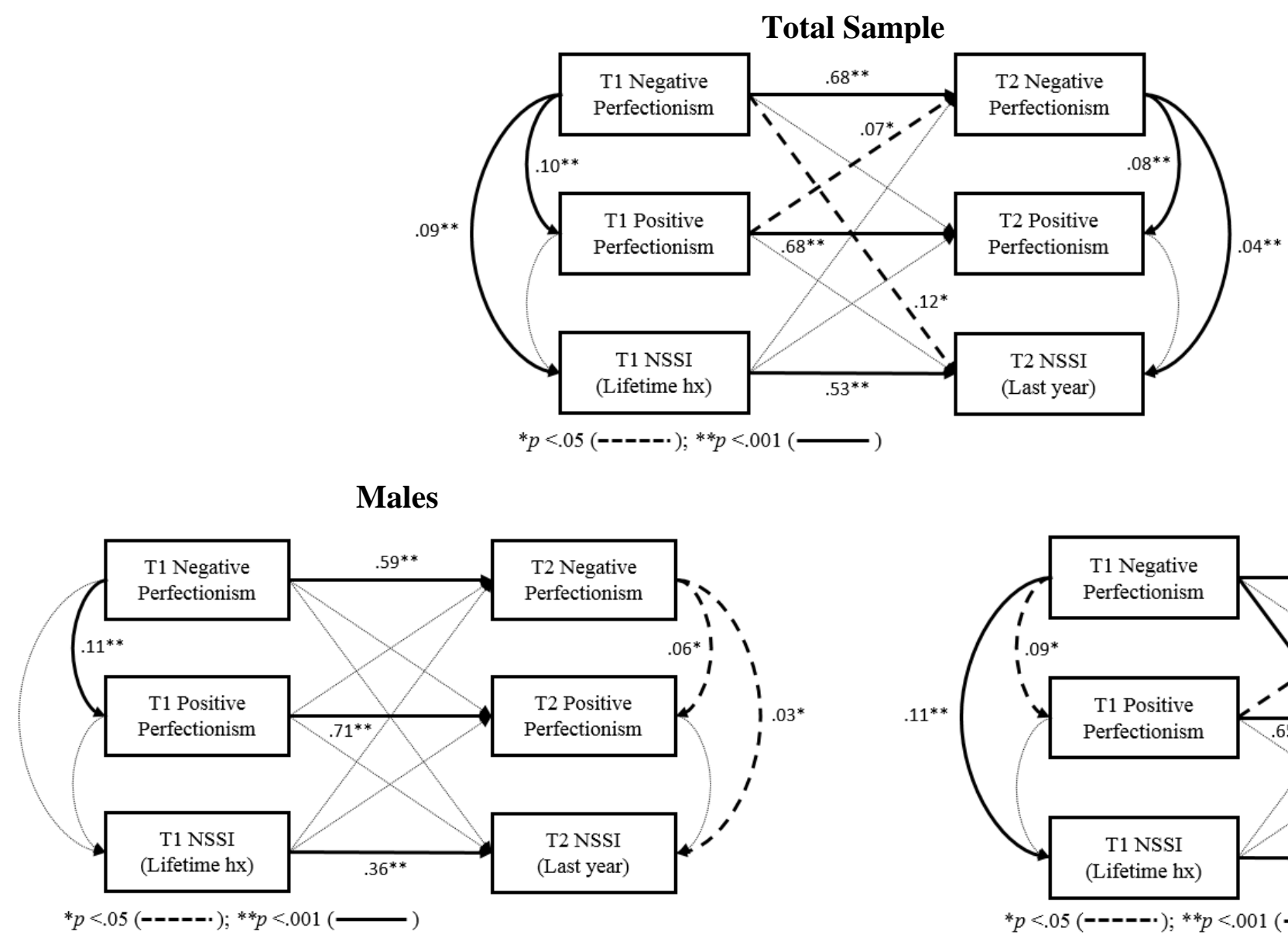

Females

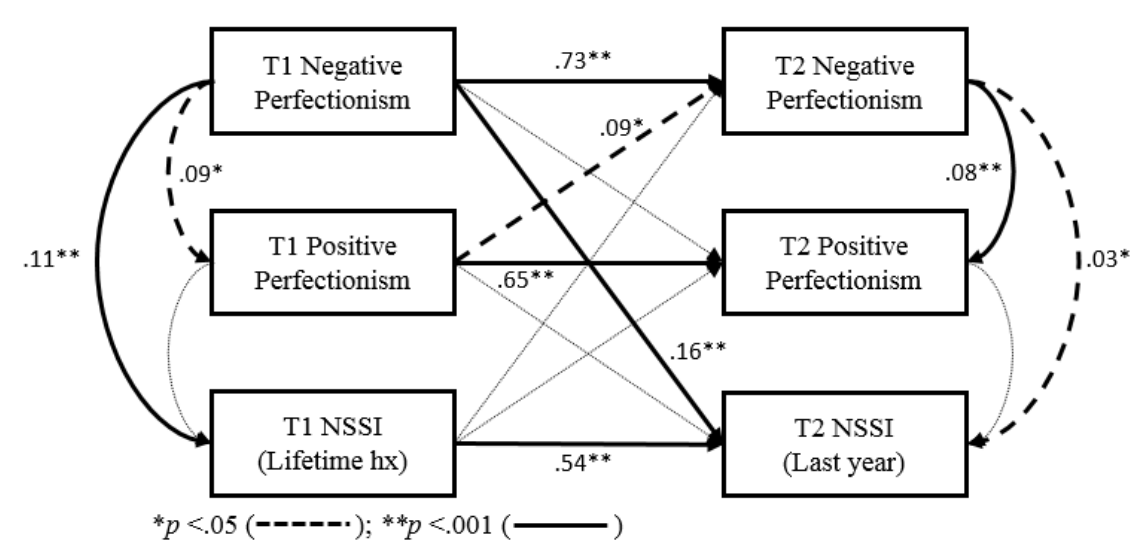

Figure 51. Cross lagged panel correlation models testing for significant relationships between positive perfectionism, negative perfectionism and NSSI over time 
over time (.54-.73). This suggests that the results for the total sample are being driven by females within the total sample.

These results indicate that negative perfectionism is a prospective risk factor for NSSI in females. Moreover, positive perfectionism appears to precede an increase in negative perfectionism, again only in females. Taken together, this could suggest a pathway that begins with positive perfectionism, which promotes the development of negative perfectionism and then increases risk for NSSI.

\section{Summary and Conclusions}

Study 4 builds on the previous studies in this thesis by investigating the relationship between perfectionism and NSSI over time. The T2 surveys were administered approximately one year following T1. In total, 608 students completed both surveys allowing their data to be matched and analysed longitudinally. It was hypothesised that negative perfectionism would predict an increase in self-injurious thoughts and behaviours, especially for females, and that positive perfectionism may buffer against NSSI. Overall, this study showed that as predicted, in females, negative perfectionism at T1 predicted self-injury at T2. There was no equivalent relationship for males. No significant relationships were demonstrated between positive perfectionism and NSSI over time. In addition, positive perfectionism predicted an increase in negative perfectionism in females only.

\section{Negative Perfectionism and NSSI}

Longitudinal research findings such as these allow researchers to make claims about causality with greater confidence than would be appropriate based solely on cross-sectional analyses. Compared to cross-sectional research, within which a significant relationship sheds little light on the direction of that relationship, longitudinal research lets the researcher be certain that data collected at $\mathrm{T} 1$ came before the data at $\mathrm{T} 2$, and therefore where there is a significant relationship, the variables at $\mathrm{T} 1$ are more likely to have influenced the outcome at $\mathrm{T} 2$, than the other way around. Therefore, from these results it can be concluded that for females' negative perfectionism effectively predicts future self-injurious thoughts and behaviours.

To my knowledge only one other study has examined the longitudinal relationship between perfectionism and self-injury. O'Connor and colleagues (2010) reported that negative perfectionism significantly increased the probability of an adolescent engaging in self-harm in response to a negative life event. Therefore, their research suggests that either adolescents high on negative perfectionism experience significantly more distress when faced with 
adverse life events, and/or are particularly likely to use self-harm as a coping strategy. This research effectively builds on O'Connor and colleagues' both by investigating NSSI (as opposed to DSH), and looking at the relationships across sex.

\section{Positive Perfectionism and NSSI}

Unexpectedly, positive perfectionism did not protect against NSSI for males or females. This may be due to the majority of the variance being attributed to variables in the model that more strongly associated with each other (all stability relationships (autocorrelations), for example, negative perfectionism at $\mathrm{T} 1$ with negative perfectionism at $\mathrm{T} 2$; in addition to strong relationships between other variables, for example negative perfectionism and positive perfectionism). Therefore, once the variance associated with the stability relationships, and with relationships between negative perfectionism and NSSI, and negative perfectionism and positive perfectionism, have been partialed out, the remaining variance that could be attributed to positive perfectionism is negligible.

In addition, it is possible that positive perfectionism may only be adaptive in the shortterm. In other words, positive perfectionism may only be beneficial prior to an associated increase in negative perfectionism. This is consistent with the significant relationship between positive perfectionism and NSSI demonstrated in the cross-sectional research (Study 3) but the absence of such a relationship longitudinally. If this is the case, it could be possible that longitudinal research with shorter and more frequent time lags will show a diminishing inverse relationship between positive perfectionism and NSSI over time. Continuing to research and clarify this longitudinal relationship will be an important area of focus for future research in this field.

Finally, it is important to note that there were some differences in the relationship between positive perfectionism and NSSI at T1 and T2 across the cross-sectional and longitudinal studies. Specifically, at T1in the longitudinal sub-sample (Study 4) there was no significant relationship between positive perfectionism and NSSI in males or females. This is contrary to the results of Study 3 in which positive perfectionism was associated with less NSSI in both males and females. The change in measure across studies (full scale FMPS to FMPS-22), or the decrease in sample size could partially account for the differences.

However, there was also a significant difference between the relationships with positive perfectionism at $\mathrm{T} 1$ and the relationships at $\mathrm{T} 2$ in the same longitudinal sample. This is more 
difficult to explain ${ }^{48}$. It does however fit with variability of positive perfectionism research findings and its relatively inconsistent nature.

\section{Positive and Negative Perfectionism}

As mentioned above, positive perfectionism at T1 predicted an increase in females' negative perfectionism at $\mathrm{T} 2$. This is an especially important and novel finding with regard to the development of perfectionism. As far as I am aware, this relationship has not previously been studied longitudinally. This suggests that although seemingly benign or even adaptive, positive perfectionism may actually be a warning sign or foundation stone for the onset of problematic levels of negative perfectionism. It is hard to identify the exact point at which combinations of positive and negative perfectionism begin to lead to problematic outcomes however it is useful to know that the trajectory of perfectionism in females appears to be increasingly negative regardless of whether the individual is primarily negatively or positively perfectionistic.

These empirical findings support Claes and colleagues (2012) hypothesis that positive perfectionism could be related to later increases in negative perfectionism. Claes et al., also suggested that positive perfectionism may predict an increase in negative perfectionism which then predicts subsequent self-injury. Although there are only two waves of data in this research, a combination of the finding that positive perfectionism predicted negative perfectionism, and negative perfectionism predicted NSSI leads to a hypothesis that, had there been a third wave of data, Claes et al.,'s (2012) hypothesis would likely be supported. That is, negative perfectionism longitudinally mediates a relationship between positive perfectionism and NSSI.

Given that this is the last study in this thesis, and many of the limitations and strengths relevant here are also relevant to other research components, an in depth discussion of these has been left to the final discussion. However, very briefly the causal claims resulting from this study are limited by the use of only two waves of data, and the relatively long period between data collections (Kenny, 2005; Singer \& Willett, 2003). This will be discussed in more detail shortly.

\section{Conclusion}

In summary, this study makes a significant contribution to existing empirical evidence regarding the relationship between perfectionism and NSSI, and individually to both the

\footnotetext{
${ }^{48}$ Further analyses revealed that the T1 relationship between positive perfectionism and NSSI was only significant in the portion of the sample that subsequently dropped out.
} 
perfectionism and NSSI fields of research. The findings further suggest that negative perfectionism could contribute to an increase in detrimental outcomes for adolescents, especially females. In particular, negative perfectionism appears to place female adolescents at significantly higher risk of engaging in self-injury. These findings are valuable with regard to clinical practice and consideration of school wide intervention and prevention strategies. Specifically, they suggest that it is important to address perfectionism, whether it be positive or negative perfectionism, to prevent or reduce the impact of poor mental health outcomes and maladaptive behaviours (such as self-injury). 


\section{CHAPTER NINE: DISCUSSION AND CONCLUSIONS}

\section{Summary of the Research}

In this thesis I have sought to provide understanding of the role of perfectionism in the development and maintenance of NSSI in adolescents. The initial contribution was to systematically review and conduct a meta-analyses of the relationship between positive and negative perfectionism and multiple mental health and wellbeing outcomes (Study 1a). This was followed by a meta-analysis of all research investigating perfectionism and self-injurious and suicidal thoughts and behaviours (Study 1b). Together, these meta-analyses set the scene for the empirical research that followed. Next, a psychometric analysis of the FMPS was conducted (Study 2) to determine an appropriate conceptualisation of perfectionism in this NZ adolescent sample. Subsequently, the cross-sectional relationship between perfectionism and NSSI was explored (Study 3), followed by an investigation of the longitudinal relationship between the positive perfectionism, negative perfectionism and NSSI (Study 4). This discussion summarises the research findings in the context of the research questions, and in relation to the existing literature. As such, to aid in bringing the results together and forming theoretical links with previous findings, theories of perfectionism and NSSI will be referenced throughout.

\section{Positive and Negative Perfectionism and Mental Health and Wellbeing}

The results of Study 1a highlighted a robust relationship between negative perfectionism, as measured by the FMPS, and various poor mental health outcomes (depression, anxiety, OCD, ED, stress, psychological distress, negative coping skills; see Table 3, p. 69). However, the relationship between FMPS positive perfectionism and mental health outcomes was less consistent. Specifically, positive perfectionism is associated with some positive outcomes (e.g., life satisfaction) but also some negative outcomes (e.g., depression, OCD, ED). However, positive perfectionism is typically only weakly associated with such variables, and demonstrated no significant relationship with other mental health outcomes tested (see Table 4, p. 73).

Study $1 \mathrm{~b}$ detailed the meta-analytic summary correlations for the relationships between positive and negative perfectionism, and self-injurious and suicidal thoughts and behaviours. The results supported Study 1a and the conceptualisation of negative perfectionism as maladaptive, with summary effects indicating significant relationships between negative perfectionism and self-injurious $(r=.15)$ and suicidal thoughts and behaviours ( $r=.25$; see Table 9, p. 104). Conversely, positive perfectionism was not 
associated with either self-injury or suicide. Again, this highlights the clearly detrimental nature of negative perfectionism and further questions whether positive perfectionism is as adaptive as many have claimed.

Further questioning the nature of perfectionism. Study 1 findings are consistent with other research reviews and theoretical papers arguing that negative perfectionism is maladaptive, but question the proposed adaptive nature of positive perfectionism (Hill, Witcher, Gotwals, \& Leyland, 2015; O’Connor, 2007; Shafran et al., 2002). Overall, there appears to be support for an adaptive form of perfectionism, at least when compared to the clearly detrimental nature of negative perfectionism. However, the extent to which positive perfectionism affects individual outcomes remains unclear. Multiple hypotheses about the true nature of positive perfectionism and possible explanations for the lack of clarity in the positive perfectionism literature are briefly summarised below.

First, the research findings specific to positive perfectionism may have been clouded due to inconsistent use of subscales, and the varied conceptualisations of positive perfectionism captured with each individual scale or subscale. For example, in Study 1a the relationship between positive perfectionism and negative outcomes was trending negative when Organisation was included in the subscale score, but was positive and significant when not included (e.g., subscale scores of only PS or PPS; see supplementary online material for detailed results of the moderator analyses). This suggests that Organisation may be the more adaptive component of positive perfectionism. Moreover, it highlights the importance of careful consideration of the subscale both when designing research and interpreting others research results.

Other researchers have also noted similar issues relating to the composition of subscales argued to measure positive and negative perfectionism. For example, O'Connor (2007) suggested that the relationship between positive perfectionism and suicide is likely unclear because SOP (HFMPS) is comprised of items corresponding to both adaptive and maladaptive components of perfectionism. Whilst O'Connor did not give specific examples of the components of SOP thought to be negative, it is reasonable to suggest that he could have been referring to items such as "it is very important that I am perfect in everything I attempt" and "when I am working on something, I cannot relax until it is perfect". In addition, with regard to the PS sub scale of the FMPS, a revised sub scale, namely Pure Personal Standards (PPS; PS excluding items 4 and 6) is often used in an attempt to partial out some negative items (e.g., DiBartolo et al., 2007, 2004; Wimberley \& Stasio, 2013). This typically results in stronger positive correlations with adaptive outcomes, and stronger negative correlations with 
maladaptive outcomes (supported by Study 1a meta-analyses). In this research, psychometric analyses demonstrated that one of the items previously excluded from PPS (Item 4) loaded on CMD rather than PS. Thus, suggesting that the item is more appropriate for the measurement of negative perfectionism and supporting O'Connor's claim of contaminated subscales.

Second, any adaptive effects of positive perfectionism may have been masked by cooccurring negative perfectionism. This research, and the existing literature, has indicated that if an individual scores high on positive perfectionism, they are likely to also score high on negative perfectionism. Given, the proposed opposing effects of positive and negative perfectionism, it is highly likely that the detrimental effects of negative perfectionism could mask any adaptive effects of positive perfectionism. It was not possible to account for this in the meta-analytic studies. However, previous research has looked at the effect of positive perfectionism with the concurrent effects of negative perfectionism partialed out, and has demonstrated that, without negative perfectionism, positive perfectionism has a greater adaptive effect on wellbeing (for a review on partialing negative perfectionism see Stoeber \& Gaudreau, 2017).

Third, when predicting outcomes at an individual level, the relative levels and the interaction between positive and negative perfectionism is arguably more informative than the effects of each alone. As discussed throughout this thesis, three opposing models of the interaction between positive and negative perfectionism have been proposed; the twocomponent, tripartite, and $2 \times 2$ models of perfectionism. In the context of self-injury, this research provides support for Gaudreau and Thompson's (2010) '2x2' model, whereby, positive perfectionism buffers against the effects of negative perfectionism. Thus, an individual scoring high on negative perfectionism and low on positive perfectionism is at the greatest risk for poor outcomes, including self-injury.

To summarise the points above, when investigating positive perfectionism, consideration should be given to the subscales used, and the concurrent effects of negative perfectionism. Further, considering perfectionism at a holistic level, that is investigating the interacting effects of positive and negative perfectionism, will allow more accurate predictions of the association with mental health and wellbeing outcomes.

\section{Perfectionism in NZ Adolescents}

As the most widely used perfectionism scale, and the focus of Study 1a, the FMPS (Frost et al., 1990) was chosen to measure perfectionism in NZ adolescents. CFA and PCA statistical procedures identified a four component solution comprising Concerns and Doubts 
(CMD), Parental Pressure (PP), Personal Standards (PS), and Organisation (O), followed by higher order positive and negative perfectionism components. Moreover, MIMIC analyses indicated this structure was appropriate for both males and females, while also demonstrating that females score higher on some subscales than males. These results reiterate the importance of viewing perfectionism as a multidimensional, rather than unitary, construct. It also clearly provides support for the differing characteristics and thus effects of positive and negative perfectionism demonstrated in the Study 1 meta-analyses.

Turning now to Study 3 which explored the characteristics of perfectionism in NZ adolescents. Analysis of the FMPS demonstrated that on average, NZ adolescents endorse greater positive perfectionism than negative perfectionism. In light of the findings of Study 1 , and an understanding of the combined effects of positive and negative perfectionism (e.g., 2x2 model; Gaudreau \& Thompson, 2010) this initially appears to be an encouraging finding. This raises hope that for many adolescents positive perfectionism is buffering against concurrent negative perfectionism and thus protecting them against poor mental health outcomes. However, in the context of the final study, which demonstrated positive perfectionism predicts increases in negative perfectionism, the long term outcomes may not be so positive. This finding will be discussed in more detail shortly.

This research demonstrated that females score higher than males on both positive and negative perfectionism. Study 3 further highlighted that this difference lay in CMD, rather than PP, and in Organisation, rather than PS. Since there is limited research documenting a sex difference in mean levels of perfectionism, I will draw on other areas of research to hypothesise why females report greater perfectionism. Overall, it is possible that differences in the performance related attributions made by males and females, as well as differing socialisation processes may contribute to the development of perfectionism in females. For example, Dweck and colleagues (1980, 1980, 2005) have presented a large body of research demonstrating that compared to males, females perceive failure as fixed and internal (e.g., based on ability), and therefore failure is likely to significantly impact upon their sense of self-worth and self-efficacy. In contrast, males are more likely to externalise responsibility for poor outcomes (e.g., attributing it to an ineffective teacher, harsh evaluator, or luck) or attribute them to dynamic factors (e.g., lack of effort or motivation). As a result, males are more likely to preserve their sense of self-worth in the face of poor performance (see also Beyer, 1998; Flett et al., 1998; Stipek \& Gralinski, 1991).

This effect has been demonstrated with NZ adolescents. To illustrate, in their sample of NZ 15 year olds, McClure et al., (2011) reported that males were more likely than females 
to attribute their worst marks to 'bad luck'. Moreover, females were more likely than males to attribute their best marks to effort and their worst marks to poor ability. As a result, McClure and colleagues also suggested that NZ female adolescents are more likely than males to experience significant distress in the face of perceived failure. Overall, the processes outlined above closely resemble the strong link between performance and sense of self-worth that characterises negative perfectionism, and may help to explain why females are more concerned about failure and how they will be evaluated by others.

On a similar note, research has demonstrated a sex difference in the weight placed on the importance of achievement. For example, Pomerantz, Altermatt and Saxon (2002) investigated academic performance and distress in a sample of children, with a mean age of 11. They found that despite achieving to a higher level than males, females experienced more achievement-related distress. Moreover, this distress was exacerbated by worsening grades. Beyer (1998) similarly demonstrated that females experienced greater emotional reactions to success and failure than males. Specifically, females reported greater happiness when imagining success, and a stronger sense of personal failure when imagining getting an $\mathrm{F}$ on an exam. Overall, consistent with perfectionistic tendencies, these researchers concluded that, compared to males, females are more likely to place great importance on achievement, negatively evaluate themselves, and as a result worry about their performance.

Finally, some research suggests that within the classroom females engage in more achievement and academic related interactions with their teachers, whereas males engage in more behaviour related interactions. This in turn could indicate, or even lead to females placing greater importance on achieving to a high standard than their male peers (see Beaman, Wheldall \& Kemp, 2006 for a review).

Consistent with previous research (e.g., Aldea \& Rice, 2006; O’Connor et al., 2009; Sironic \& Reeve, 2015) positive perfectionism and negative perfectionism were both relatively stable across time. However, at the subscale level, PP significantly increased across $\mathrm{T} 1$ and $\mathrm{T} 2$. This increase in perceived expectations and criticism may have coincided with the transition to adolescent's first year of formal examination (NCEA Level 1, usually completed at age 15-16). With upcoming exams that impact upon their school progression and eligibility for tertiary education, it is not surprising that adolescents may feel more pressure to achieve high grades. Moreover, some perfectionism research suggests that perceived pressure from parents may lead to a subsequent increase in other forms of negative perfectionism (Damian et al., 2013). Therefore, had the research spanned another year, a subsequent increase in CMD 
is hypothesised. In addition, Organisation significantly decreased from T1 to T2, for males only.

Positive perfectionism as a warning sign for negative perfectionism. This research is one of very few studies to investigate the relationship between positive and negative perfectionism over time. As a result, a core contribution of this research lies in the finding that, for females positive perfectionism prospectively predicted negative perfectionism. It can then be argued that due to the robust association between negative perfectionism and psychological distress the individual will consequently be at a greater risk for poor mental health outcomes.

Empirical findings regarding perfectionism across time naturally lead to predictions about why positive perfectionism longitudinally increases risk for negative perfectionism. In particular, cognitive behavioural models of perfectionism (e.g., Fursland, Raykos, \& Steele, 2009; Shafran et al., 2016; also refer to Figure 5, p. 28) can inform predictions about how positive perfectionism increases risk for negative perfectionism. One possibility is that achievement is originally reinforced with a sense of satisfaction, and praise from important others (e.g., teachers, parents). However, over time, without continually increasing standards, reinforcement from others could stabilise or even decrease. Subsequently, to maintain explicit recognition the individual must achieve to higher levels. Unfortunately, as perfectionists set higher standards for themselves, they become more likely to 'fail' (Egan, Dick, \& Allen, 2012; Shafran et al., 2016), and consequently, will doubt their ability and spend longer checking and completing their work. In addition, decreased praise from others may be interpreted as a sign of inadequacy, which is compounded by hypervigilance to any criticism or perceived negative evaluation. Overall, the individual who used to strive for goals to experience satisfaction, now tries to meet their high standards to avoid negative evaluation. Whilst this process seems plausible, there is currently little empirical evidence to elucidate such hypotheses. Therefore, examining the mechanisms involved in the longitudinal relationship between positive and negative perfectionism is a particularly important area for future research. This point will be further highlighted shortly.

\section{Non-Suicidal Self-Injury in NZ Adolescents}

As a reminder, at $\mathrm{T}^{1}, 20 \%$ of $\mathrm{NZ}$ adolescents reported at least one previous episode of self-injury. At T2 this increased to a $22 \%$ lifetime prevalence rate, which is largely

\footnotetext{
${ }^{1}$ Based on data from the longitudinal sample. NSSI prevalence rates for T1 cross-sectional sample were comparable (see p. 152)
} 
consistent with previous research with adolescents (e.g., Muehlenkamp et al., 2012; Swannell et al., 2014). However, it is worth noting the inconsistency with Garisch's (2010) research (almost $50 \%$ of the NZ adolescent sample reported a history of self-injury) that may reflect the younger age of the sample, or the method of eliciting NSSI history. To further explain, research shows that single-item measures of NSSI typically produce lower rates of reported NSSI, and it may be that the 'screening question' used in this research effectively reduced the 14-item DSHI-s to a single item measure for some participants.

On a similar note, my own research demonstrated a significant difference in NSSI prevalence rates for males and females. Although this fits the common stereotype that females are more likely to engage in self-injury than males (e.g., noted by Garisch \& Wilson, 2010; Muehlenkamp \& Gutierrez, 2004), it is inconsistent with research that suggests that gender has only a small effect (e.g., a meta-analysis of 120 articles reporting the prevalence rates of self-injury across males and females found female were 1.5 times more likely to report a history of self-injury; Bresin \& Schoenleber, 2015). As discussed in the limitations of Study 3 , the branching method used in the survey may have biased the survey to capture more females than males given that some less stereotypical forms of self-injury, often typical to males (e.g., using sandpaper, bleach or acid to self-injure; Garisch, 2010), may not have been considered when answering this question.

Finally, research has shown that males tend to begin engaging in NSSI at a later age than females (e.g., Andover et al., 2010; Hawton, Bergen, et al., 2012; Nixon et al., 2002). Therefore, it is possible that over time the difference in prevalence rates between males and females will decrease or completely dissipate. Accordingly, research that has not found a difference in prevalence across sex, or demonstrated only a small difference have often comprised of older adolescent or university samples (e.g., Garisch, 2010; Heath et al., 2008). In my investigation, whilst it did not appear that this difference was decreasing across the two years examined, at T2 the adolescents were on average still only 15 years old, and therefore were just mid-way through their adolescence. A similar sex difference has been demonstrated in multiple samples of approximately the same age (e.g., Bjärehed \& Lundh, 2008; Muehlenkamp et al., 2009), therefore it is likely that this data is a reasonably accurate representation of self-injury prevalence in this age group.

The most common methods of NSSI (e.g., cutting and carving marks into skin), as well as functions associated with NSSI (e.g., affect regulation specifically, and intrapersonal functions generally) are consistent both with international literature (e.g., Gratz, 2001; Muehlenkamp \& Gutierrez, 2004) and the EAM (Chapman et al., 2006). Finally, consistent 
with previous research (Muehlenkamp, Brausch, Quigley, \& Whitlock, 2013) whilst endorsement of interpersonal functions remained stable across the two time points, endorsement of intrapersonal functions significantly increased. At a broad level, this suggests that intervention strategies should place a greater focus on intrapersonal functions than interpersonal functions.

\section{Empirical Findings for the Relationship between Perfectionism and NSSI in NZ Adolescents}

Study 3 demonstrated a significant relationship between negative perfectionism and NSSI in NZ female adolescents. Moreover, this relationship was moderated by positive perfectionism which buffered against the detrimental effects of negative perfectionism (see Figure 47, p. 173) thus supporting the $2 \times 2$ model of perfectionism. In addition, again for females, Study 4 illustrated that negative perfectionism prospectively predicts an increase in self-injurious thoughts and behaviours approximately one year later. In combination, this suggests that a young woman who scores high on negative perfectionism and low on positive perfectionism is at the greatest risk of self-injury.

The relationship between perfectionism and NSSI in male adolescents is less clear. On first examination, it appeared that negative perfectionism was not significantly associated with NSSI. However, when also considering positive perfectionism, analyses demonstrated a significant relationship between negative perfectionism and NSSI when positive perfectionism was moderate or high. Thus, it appears that positive perfectionism also crosssectionally buffers against the risk associated with negative perfectionism (refer to Figure 46, p. 171) for males. This also supports the $2 \times 2$ model of perfectionism and suggests, under certain conditions, negative perfectionism in males is weakly associated to NSSI. However, unlike for females, neither negative perfectionism nor positive perfectionism prospectively predicted NSSI in male adolescents.

Overall, the significant relationship between negative perfectionism and self-injury is consistent with the notion of negative perfectionism as maladaptive. In addition, the inverse relationship between positive perfectionism and self-injury, albeit smaller and only significant cross-sectionally, lends some support to positive perfectionism as relatively adaptive. This is further supported by the buffering effect of positive perfectionism in the relationship between negative perfectionism and NSSI. For simplicity, from here on, unless otherwise stated, use of the term perfectionists (for whom the most maladaptive outcomes are expected), refers to individuals high on negative perfectionism and low on positive perfectionism. 


\section{Perfectionism and the Theoretical Models of NSSI}

This research has demonstrated that negative perfectionism prospectively predicts selfinjury in females. This finding is consistent with both the EAM (Chapman et al., 2006) and ITMS (Nock, 2010) models of self-injury within which self-injury is featured at the end of the chain of vulnerability factors and subjectively adverse events. This research suggests that perfectionism is one such contributing factor. Therefore, the specific details of this relationship will now be outlined in relation to the ITMS and EAM. Due to its inclusion of distal vulnerability factors, the ITMS will be addressed first. Hypotheses will then be made with regard to the EAM. Given that the relationship between negative perfectionism and selfinjury in males was minimal, this section will primarily draw on the results for females.

With regard to the ITMS (Nock, 2010; refer to Figure 4, p. 20), this research suggests that perfectionism should be categorised as one of Nock's proposed vulnerability factors, thus predisposing an individual to self-injury. However, exactly where perfectionism is implicated remains unclear. Perfectionism may contribute to risk as a interpersonal, or intrapersonal vulnerability factor, and is also likely to be related to the self-punishment, NSSI-specific vulnerability factor. Each of these possibilities will be explored below.

Perfectionism as an ITMS intrapersonal vulnerability factor. Previous research has shown that taking a functional approach to self-injury can assist in identifying risk factors that are particularly relevant to a specific individual (e.g., Glenn \& Klonsky, 2009; Nock \& Cha, 2009; Nock \& Prinstein, 2005). Thus, identifying the functions of self-injury for perfectionistic individuals can highlight where their vulnerabilities lie. As such, results from the functions analyses suggest that perfectionism could fit within the class of intrapersonal vulnerability factors. Specifically, perfectionism was significantly associated with self-injury for Self-Punishment and Anti-Suicide $(r>30)$ functions. A weaker, but significant association was also discovered between negative perfectionism and Anti-Dissociation function.

Perfectionism is characterised by a particularly self-critical and self-depreciating cognitive style that can be highly aversive for the individual, and can lead to a desire to punish oneself. Thus, it was unsurprising that negative perfectionism was associated with Self-Punishment functions of NSSI. To further explain, Lynch and Cozza (2009) propose that perfectionistic cognitions are likely to trigger the onset of negative emotion and distress, and punishing oneself may provide an effective outlet for the feelings of shame, anger, guilt and sadness, typically associated with perceived inadequacy and low self-worth. 
In addition, negative perfectionism was also associated with the Anti-Suicide function of self-injury. This association between negative perfectionism and Anti-Suicide is alarming. It suggests that not only are perfectionists at higher risk for self-injury than non-perfectionists, perfectionists are disproportionately likely to be using NSSI to prevent suicide. In combination with the significant summary correlation between negative perfectionism and suicidal thoughts and behaviours (Study 1b, $r=.25$ ), this suggests that the combination of perfectionism and self-injury should be considered a warning for heightened risk of suicide. While Glenn and Klonsky (2009) conceptualise the Anti-Suicide function as intrapersonal, in the case of perfectionism it is also likely that the desire to attempt suicide is linked with interpersonal vulnerability factors. Therefore, Anti-Suicide functions will be discussed in more detail below.

Perfectionism as an ITMS interpersonal vulnerability factor. This research demonstrated a significant relationship between negative perfectionism and multiple interpersonal functions of self-injury in female adolescents. Thus, suggesting that perfectionists may struggle to manage their interpersonal experience in an adaptive manner. Specifically, negative perfectionism was associated with the endorsement of NSSI for Autonomy and Toughness functions.

Consistent with Nock and Prinstein's (2005) hypothesis, these results suggest that perfectionists may be using self-injury as a mechanism to cognitively and emotionally distance themselves from others (Autonomy). This distancing may provide subjective escape from the perceived expectations of others and allow the individual to avoid negative evaluation (also see Hewitt et al., 2006).

Unfortunately, whilst interpersonal distancing may give temporary relief, researchers have highlighted worrying long-term consequences of such separation from others. To illustrate, Hewitt et al., (2006) proposed the PSDM of suicide (refer to Figure 24, p. 94) whereby perfectionists experience both subjective and objective social disconnection. Hewitt and colleagues then propose that this disconnection places an individual at significantly increased risk of suicide. Again, in this research the meta-analytic results, and the significant relationship between negative perfectionism and Anti-Suicide, suggest that perfectionistic adolescents are especially at risk of suicidal thoughts and behaviours.

Therefore, with reference to the PSDM (Hewitt et al., 2006), this research suggests that perfectionistic adolescents appear to use self-injury to distance and protect themselves from others. As a result, they are likely to be socially isolated, and thus will not have access to 
the social support networks that others might use to manage distress. Consequently, interpersonal hopelessness may arise, and the individual becomes at greater risk of suicide.

On a similar note, it is hypothesised that the low self-worth and low self-esteem associated with perfectionism may lead perfectionists to feel as though they are a burden to their family and friends and as such they will distance themselves not only for their own benefit (i.e., avoidance of negative evaluation) but for the benefit of those they care about. Worryingly, this perceived burdensomeness (originally hypothesised by Joiner, 2005) has been shown to mediate the relationship between perfectionism and suicidality (Rasmussen, Slish, Wingate, Davidson \& Grant, 2012), again suggesting that perfectionists who rely on self-injury to manage their social situation, and perhaps feelings of burdensomeness, could be at increased risk of suicidal behaviours. Together, these hypotheses can be linked back to the significant relationship between negative perfectionism and the intrapersonal function, AntiSuicide. To explain, it is predicted that interpersonal vulnerabilities (as described above), result in a lack of social support which in turn can place an individual at greater risk for suicide. Therefore, perfectionists may need to rely on maladaptive strategies such as selfinjury to prevent them from acting on such suicidal thoughts (Anti-Suicide).

Finally, with regard to interpersonal functions, these results suggest that perfectionistic adolescents also self-injure to demonstrate that they can cope alone, are capable of brave acts, and can manage their own pain (Toughness). As such, self-injury could be a mechanism for reinstating a sense of self-efficacy, and demonstrating to themselves and others that they are worthy.

Perfectionism as an ITMS NSSI-specific vulnerability. As the last component of the ITMS, Nock (2010) hypothesises multiple NSSI-specific vulnerability factors which seek to explain why an individual engages in self-injury rather than other maladaptive coping strategies (e.g., substance use, risky behaviours). Particularly relevant to this research, Nock proposes that self-injury is an especially effective coping strategy for individuals with a desire to self-punish. This research suggests that perfectionists are especially motivated by selfpunishment, and therefore according to Nock's hypothesis are at a greater risk of choosing self-injury over alternative coping strategies. Whilst I do not have data to investigate the use of other coping strategies (for example, substance use), I suspect that due to the particularly punitive and secretive nature of self-injury, NSSI is likely a particularly effective strategy for meeting the short-term, self-punishing needs of perfectionists.

Perfectionism in the context of the EAM. The ITMS (Nock, 2010) simply acknowledges that self-injury is reinforced through modification of one's affective or social 
experience. In comparison, the EAM (Chapman et al., 2006) addresses this part of the cycle in much more depth. Chapman and colleagues (2006) argue that self-injury is a method of experiential avoidance particularly in the context of emotional distress. Figure 52 builds on the discussion in Chapter 7 (see p. 174-177) and presents a modified EAM illustrating the proposed influence of perfectionism. As illustrated, there are multiple places within the EAM that perfectionism could be implicated. Each of the noted hypotheses are explained below.

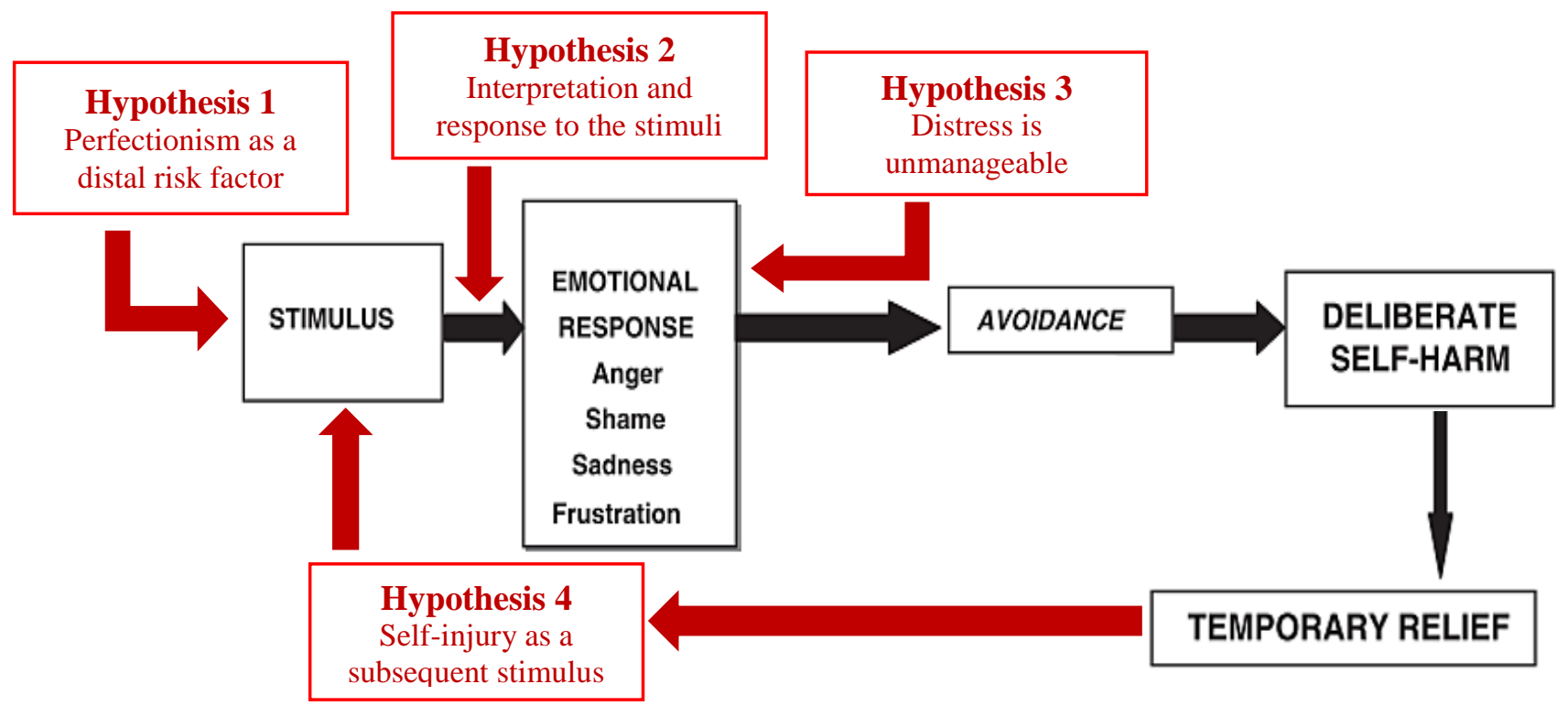

Figure 52. Experiential Avoidance Model (EAM; adapted from Chapman et al., 2006, p. 373) modified to include the hypothesised influence of negative perfectionism

Hypothesis One (see Figure 52) suggests perfectionism is a distal risk factor that increases risk of experiencing a stimulus that will trigger the self-injury cycle. To explain, perfectionists, by their very nature, set excessively high standards for performance (Burns, 1980; Frost et al., 1990) and are hypervigilant to their mistakes and signs of inadequacy (Menatti, Weeks, Levinson, \& McGowan, 2013; Powers, 2005). Accordingly, they are typically reluctant to mobilise social support networks out of fear of negative evaluation (Hewitt et al., 2006). Therefore, perfectionists are more likely to fail to reach their standards, to notice mistakes, and receive less social support when distressed, all of which can be considered stimuli that could trigger the process preceding self-injury.

Second, perfectionism is characterised by a highly critical view of one's performance and behaviour, and biased interpretations of interactions with others. Therefore, Hypothesis Two (see Figure 52) denotes that perfectionism is implicated in the biased interpretations of stimuli, thus resulting in significant distress. For example, Chester and colleagues (2015) 
reported that negative perfectionism was associated with aggression towards oneself (using a Voodoo doll task as a proxy for self-injury) in response to negative feedback about a recent task. This suggests that perfectionism influences how a stimulus (in this case negative feedback) is interpreted and responded to. Relatedly, research also supports the link between negative perfectionism and emotional distress in response to daily events. For example, although not specific to self-injury, Milyavskaya et al., (2014) identified a significant positive relationship between negative perfectionism and negative affect at stressful times of the university year. This again suggests that perfectionism influences how an individual responds to life stressors, and indicates that perfectionistic interpretations of stimuli are typically associated with greater emotional distress.

Similarly, Hypothesis Three (see Figure 52) proposes that perfectionistic interpretations of events are first experienced as especially unmanageable due to a perfectionist's hypothesised poor emotion regulation skills, and second a tendency to make negative judgments about the experience of strong emotion. To explain, research suggests that perfectionists are more likely to have poor emotion regulation skills than non-perfectionists (see for example Brand et al., 2015; Hill \& Davis, 2014; Richardson, Rice, \& Devine, 2014; Rudolph, Flett, \& Hewitt, 2007) thus suggesting that they are more likely to experience emotion and unmanageable and turn to maladaptive coping strategies such as self-injury. Second, it is also hypothesised that perfectionists are more likely to make negative judgments about the experience of distressing emotion (i.e. non-acceptance of strong emotion and selfcriticism regarding their inability to manage the distress), and therefore will in turn be vulnerable to further perceived inadequacy and distress.

It is important to note that in light of this it could be expected that negative perfectionism should also be associated with the Affect Regulation function of NSSI but this was not the case. Subsequent examination of the items pertaining to Self-Punishment suggest that although seeming more cognitively focused, Self-Punishment is also inherently an emotion regulation function (e.g., "expressing anger towards myself for being worthless or stupid", "reacting to feeling unhappy with myself, or disgusted with myself", "punishing myself"). Indeed, Lynch and Cozza (2009) argue that self-punishment can be conceptualised as an emotion regulation strategy specifically targeting self-depreciating emotions such as embarrassment, shame and guilt. Therefore, results pertaining to Self-Punishment can be used to support Hypothesis Three and supports the interpretation of results within the emotionally focused EAM. 
Both models also imply that the regulation of the individual's experience is typically short-lived. Hypothesis Four (see Figure 52) suggests that this relief may be particularly short-lived for perfectionistic adolescents as the act of self-injury could become yet another perceived failure. Consequently, another negative emotional response may be triggered, thus increasing risk for further self-injury. Similarly, external to the individual, research has shown that the reactions of other people finding out about one's self-injury can cause significant distress for the individual (Chandler, 2016). For perfectionists, who are especially sensitive to feedback from others, revealing self-injury, whether disclosure is intentional or unintentional, is likely to be especially anxiety provoking (Chester et al., 2015). Therefore, self-injury may trigger interpersonal situations that in turn trigger increase risk for further self-injury.

In sum, it is likely that perfectionism is both an interpersonal and intrapersonal vulnerability for NSSI. Results suggest that this vulnerability is often driven by a need to selfpunish for one's perceived shortcomings or as a way of preventing suicidal behaviours. Moreover, perfectionism appears to hinder an adolescents ability to meet their interpersonal needs in an adaptive manner. As such, NSSI provides perfectionistic adolescents with a much needed, albeit maladaptive, short term coping strategy.

\section{Sex Differences in the Relationship between Perfectionism and NSSI}

This research has uncovered a longitudinal relationship between negative perfectionism and NSSI in females but not in males. Positive perfectionism was associated with decreased risk of NSSI in both males and females cross-sectionally, however this relationship did not hold in the longitudinal data. Finally positive perfectionism prospectively predicted negative perfectionism in females only.

First it is important to note that these results are consistent with research investigating perfectionism and disordered eating attitudes in male and female athletes (e.g., Hopkinson \& Lock, 2004; Shanmugam \& Davies, 2015). For example, Hopkinson and Lock (2004) reported that among university age athletes, perfectionism was associated with disordered eating in females only. Moreover, Shanmugam and Davies (2015) also investigated EDs and NSSI in a sample of competitive athletes and reported that perfectionism predicted disordered eating in female athletes.

The results above suggest that the sex difference observed in this research is not an anomaly. Therefore, it is important to consider what else may be contributing to this effect. First, when examining both perfectionism and NSSI individually, this research has shown that both constructs differ across males and females on multiple aspects. Notably, both 
perfectionism and NSSI were more prevalent in female adolescents. Thus, females are more likely to experience the distress associated with negative perfectionism, and also appear to be more likely to choose NSSI as a coping strategy.

Alternatively, there may be systematic differences in male and female responses to perfectionistic cognitions that can help explain differences in the relationship with NSSI. For example, substantial research has demonstrated that females are more likely to both ruminate and co-ruminate (e.g., Lyubomirsky, Layous, Chancellor, \& Nelson, 2015; Rose, 2002; Rose, Schwartz-Mette, Glick, Smith, \& Luebbe, 2014; Stone \& Gibb, 2015). In other words, females are more prone to dwelling on worries either alone or with family and peers. Therefore, perfectionistic females could be more likely than perfectionistic males to spend time agonising over their perceived short-comings, and worrying about negative evaluation from their parents, teachers, and peers. In turn, this results in greater or more frequent emotional distress and significantly contributes to the progression from negative perfectionism to self-injury. Moreover, co-rumination can provide the perfect opportunity for females to both compare their achievement to those around them, and receive feedback from others that they perceive as critical. Thus, exacerbating negative emotion and their risk of engaging in maladaptive coping strategies such as self-injury.

Second, there is a body of research that suggests differences in emotion socialisation across males and females (e.g., Chaplin, Cole, \& Zahn-Waxler, 2005; Kring \& Gordon, 1998; Miller-Slough \& Dunsmore, 2016). In their review of this research Miller-Slough and Dunsmore (2016) explained that parents talk about emotion with their daughters more than with their sons. As a result, females develop an understanding of their emotions earlier than males and are more willing to discuss their emotions with others. This may mean that females have a more nuanced understanding of their emotional experience and therefore are able to effectively identify the source of distress. As such, they are more likely to attribute shame and guilt to failure or perceived negative evaluation. With reference to Nock's (2010) ITMS selfpunishment hypothesis, awareness of the source (e.g., perceived failure) of their negative emotions (e.g., shame and guilt) could lead them to adopt self-punishing strategies, such as self-injury to manage the distress. Males, on the other hand, may experience similar distress but have less understanding of its source or its exact nature. Therefore, they will likely choose general coping strategies such as distraction (Trives, Bravo, Postigo, Segura, \& Watkins, 2016) and behavioural acting out (Chaplin \& Aldao, 2013).

Finally, other research has demonstrated that females rely on social support as a core method of coping more than their male counterparts (Day \& Livingstone, 2003; Landman- 
Peeters et al., 2005; Nolen-Hoeksema \& Aldao, 2011). Therefore, reluctance to seek support from others out of a fear of criticism will have a particularly detrimental impact on a female's capacity to cope with distress.

\section{Other Contributing Factors}

In this research the relationship between negative perfectionism and NSSI in NZ adolescents ranged from .14 to .30. By statistical standards this would be considered small to moderate (Cohen, 1988). However, there will be a raft of other factors involved in this relationship, and therefore multiple indirect paths between negative perfectionism and NSSI that may contribute to an overall greater sum effect. Some other psychological factors that could be important to consider are discussed below.

Eating disorders. Study 1a demonstrated a robust relationship between negative perfectionism and eating disorders (summary correlation coefficient $=.41$ ). In addition, the perfectionism subscale of the EDI suggests that perfectionism is a core contributing factor for the development of ED psychopathology, if not a core component of EDs itself (Garner, 1991). With regard to the relationship between EDs and NSSI, researchers have suggested that eating disordered behaviours such as binging and purging fulfil a similar function to selfinjurious behaviours (see Wedig, 2014). Thus, EDs and self-injury are frequently correlated, especially in females (e.g., Bjärehed \& Lundh, 2008; Jacobson \& Luik, 2014). Consequently, one might speculate that shared relationships with EDs could significantly mediate the relationship between negative perfectionism and NSSI. Alternatively, given that they fulfil similar functions, self-injury and disordered eating behaviours may alternate with one another, each in turn providing a similar function.

Depression and anxiety. Study 1a demonstrated a significant relationship between negative perfectionism and depression ( $r=.45$; also see Smith et al., 2016 for a meta-analysis showing that perfectionism longitudinally predicts depression), and negative perfectionism and anxiety $(r=.42)$. Moreover, depression is commonly diagnosed in individuals who selfinjure (e.g., Tuisku et al., 2014; Wolff et al., 2014), as are anxiety disorders (e.g., Bentley et al., 2015; Esposito-Smythers et al., 2010). As such, it is suspected that perfectionism is involved in the development of anxiety and depressive disorders for some, which in turn can lead to the individual engaging in self-injury as a mechanism for coping with the distress.

Emotion regulation. As previously mentioned, emotion dysregulation has been associated with negative perfectionism (see Brand et al., 2015; Hill \& Davis, 2014; Richardson et al., 2014; Rudolph et al., 2007), and is commonly accepted as a driving factor 
for self-injurious behaviours (e.g., Chapman et al., 2006; Nock, 2010). Therefore, based on theory and empirical findings, it is expected that perfectionistic individuals who have an emotion regulation skill deficit, who experience emotions as especially intense, or have poor distress tolerance, are especially likely to adopt maladaptive coping strategies such as selfinjury. As such, emotion regulation proficiency is expected to mediate the relationship between negative perfectionism and self-injury.

Body regard. Recent research has suggested the importance of considering body regard, that is how an individual experiences and cares for their body, in the development of self-injury (Armey et al., 2012; Muehlenkamp, Bagge, Tull, \& Gratz, 2013). Specifically, body regard can help determine whether the individual chooses self-injury over another coping strategy (similar to a NSSI-specific vulnerability factors, Nock, 2010). Muehlenkamp and colleagues (e.g., Armey et al., 2012; Muehlenkamp, et al., 2013; Walsh, 2006) argue that an individual is more likely to inflict direct harm to their body if they have low regard for their body.

It makes intuitive sense that perfectionistic individuals will be highly critical not only of their performance, but also of their appearance. Therefore they are likely to experience greater body-dissatisfaction, poorer body-esteem, and overall lower body regard than nonperfectionistic individuals. This is supported by Davis (1997) who reported that negatively perfectionistic ED patients scored significantly lower on body-esteem than positive perfectionists. In addition, longitudinal research has shown that perfectionism, in combination with body dissatisfaction can predict an increase in EDs (Boone, Soenens, \& Luyten, 2014). Thus, given that self-injury and ED behaviours are often considered as two sides of the same coin (e.g., Wedig, 2014), it is likely that the combination of perfectionism and low body regard would predict self-injury.

Overall, it is important to note that many of these variables are more relevant to females than males. For example, Bjarehed and Lundh (2008) demonstrated a significant negative relationship between DSH and body-esteem in their sample of 14 year old Swedish females, however this relationship was not significant for males. Accordingly, it is worth considering that the difference in the relationship between negative perfectionism and NSSI is partly accounted for by sex differences in vulnerability factors such as depression, anxiety, and eating disorders, all of which are all more common in adolescent females than adolescent males (see DSM-5 prevalence rates; APA, 2013). In sum, whilst, these additional variables were not examined in the current research, they provide an important direction for future 
research aimed at an even more comprehensive understanding of the relationship between perfectionism and self-injury in NZ adolescents.

\section{Summary of Theoretical Implications}

This research has convincingly highlighted perfectionism as a prospective risk factor for NSSI in female adolescents. Furthermore, investigation of the relationship between perfectionism and the functions of NSSI indicated that perfectionistic females are especially likely to use self-injury as a method of punishment or to prevent themselves from engaging in suicidal acts. At an interpersonal level, this research suggests that perfectionism is used to distance oneself from others and that this is likely motivated by the avoidance of negative evaluation from others. As such, perfectionism operates as both an intrapersonal and an interpersonal vulnerability factor, whilst also increasing an individual's propensity to choose self-injury as a method for coping with their distress. Another interesting but largely unforeseen finding is that positive perfectionism prospectively predicted negative perfectionism (in females), something that has not previously been demonstrated, and therefore provides a new and interesting avenue for future research.

\section{Clinical Implications}

First and foremost, this research has shown that over 1 in 5 of $\mathrm{NZ}$ adolescents, with an average age of 15 , have intentionally hurt themselves at least once. Of these, many are likely to be engaging in NSSI on a relatively frequent basis. Therefore, adults responsible for the wellbeing of adolescents (i.e. caregivers, teachers) should be vigilant for NSSI, as well as be aware of signs of distress, and how to respond appropriately to the discovery that an individual in their care is engaging in self-injury (see Muehlenkamp, Brausch, et al., 2013). Moreover, knowledge of factors commonly associated with self-injury, and the many reasons why an adolescent may engage in NSSI will be important in dispelling myths about attention seeking, reducing stigma, and increasing understanding and empathy for the adolescent.

Further, this research indicates that perfectionistic adolescents, particularly females, and those exhibiting negative perfectionism in the relative absence of positive perfectionism, are at increased risk for many poor mental health outcomes. These adolescents are at risk for self-injury and, through common association, suicide. Therefore, researchers, clinicians, parents and school staff should be aware of the signs of adolescent perfectionism, and understand how their own actions may contribute to its development in the adolescents they work with. Moreover, they should have an idea of what can be done to prevent perfectionism, and how to intervene when it is identified. 
The results presented in this thesis and the existing empirical evidence suggest that perfectionistic adolescents should be recognised so that they can be provided with the appropriate support in home, school, and professional health care settings. The combination of significant relationships between perfectionism, self-injury (including the relationship with anti-suicide functions) and suicide, plus the abundance of evidence suggesting that perfectionistic individuals are reluctant to disclose and seek help for their difficulties, places these adolescents in a highly vulnerable position. This vulnerability has been highlighted in the many anecdotal and empirical reports of perfectionists who have suicided without anyone being aware that they were distressed (e.g., Aldea \& Rice, 2006; Kiamanesh et al., 2014). Thus, given the findings of this research and theories connecting NSSI and suicide, a perfectionistic adolescent who is either currently engaging in NSSI or has a history of NSSI, should be considered at particularly high risk of continued NSSI or worse, of suicidal behaviour. As such, it is recommended that clinicians thoroughly screen for perfectionistic traits, cognitions, and behaviours in their assessment of adolescents presenting to mental health services.

\section{Applications of the Research}

The primary aim of this research was to identify factors that increase risk of self-injury in $\mathrm{NZ}$ adolescents in order to identify specific targets for treatment and intervention. This research has identified a significant longitudinal relationship between positive perfectionism and negative perfectionism, and negative perfectionism and NSSI in young women. Therefore, the most important application of this research is that by limiting the development of both positive and negative perfectionism, risk of NSSI is likely to also decrease.

First, due to the strong influence of a perfectionist's social environment, interventions for perfectionism must involve caregivers, and wider social network on at least some level. Perhaps the most influential starting point could be to work with parents and teachers on how to prevent the development of perfectionism (see Flett et al, 2002). This could include psycho-education about the importance of how adults frame expectations for their young people, and how they can model acceptance of making mistakes or falling short of their goals. Likewise, it is necessary to highlight the importance of providing consistent praise for effort rather than an exclusive focus on achievement, and making sure that praise is non-conditional.

In addition to strategies aimed at caregivers and teachers, there are multiple structured prevention strategies that directly target the individual's perfectionism. For example, Nehmy and Wade (2015) have developed an eight-session school-based programme ('Healthy 
Minds'), designed to help students identify and challenge their perfectionistic behaviours and cognitions. An evaluation of this programme in Australia has demonstrated that its introduction significantly decreased perfectionism and self-criticism in adolescents (Nehmy \& Wade, 2015). Other prevention programmes include the 'Minding Young Minds' programme (Fairweather-Schmidt \& Wade, 2015), and a perfectionism-focused school based program designed to prevent EDs (Wilksch et al., 2008). Such school based programmes are likely to be an effective method of reaching large groups of adolescents.

Also with prevention strategies in mind, this research has highlighted a possible causal link between positive perfectionism and negative perfectionism in female adolescents. As such, it is important to emphasise the difference between positive and negative perfectionism, and the importance of paying attention to the transition between the two. In particular, attending to adolescent's goal-setting and their motivations for achievement are likely to provide clues about whether positive or negative perfectionism is dominant (Slade \& Owens, 1998). Therefore, monitoring the individual's motivations for achievement and looking for a transition from intrinsically motivated action to extrinsically motivated action could be particularly informative with regard to intervening before negative perfectionism has substantial negative impacts.

Unfortunately, more often than not, intervention is not concerned with prevention and is rather focused on how to reduce perfectionistic tendencies in already distressed adolescents. In this case, individual therapy has been shown to be effective (e.g., see Shafran et al., 2016 for a review). Very briefly, this may involve psychoeducation, investigating the pros and cons of perfectionism, challenging self-critical cognitions, and encouraging appropriate goalsetting. Moreover, focus can be placed on recognising and celebrating successes that perfectionists are unlikely to consider worthy of praise (see for example Fairweather-Schmidt $\&$ Wade, 2015). Overall, perfectionism interventions have been shown to decrease a broad range of psychopathology (Shafran et al., 2016) and, therefore, it would make sense that targeting perfectionism will also lead to reduced engagement in NSSI.

On a slightly different note, a particular challenge in identifying and working with perfectionistic adolescents will be their reluctance to seek help from others (e.g., Flett \& Hewitt, 2014; Kiamanesh et al., 2014; Rice \& Aldea, 2006). Rice and Aldea (2006) appropriately describe perfectionists as at significant risk of "silently suffering" (p. 211). Given that perfectionistic individuals are less likely to show their distress, it is especially important for those close to them to be able to recognise signs of perfectionism. Shafran and colleagues (2016) highlight perfectionistic behaviours such as excessive checking and 
excessive time spent on tasks, avoiding trying anything new, and procrastination that may be of use in identifying adolescents struggling with perfectionism.

Finally, it is worth again highlighting the relationship between perfectionism and suicide. The meta-analyses conducted point to a very concerning relationship. The gravity of this relationship has been recognised by leading perfectionism researchers who, in their summary article on the current state of the field of perfectionism, stated "we are especially troubled by the suicides of young perfectionists that occur seemingly without warning" (Flett $\&$ Hewitt, 2016, p. 616). Suicide is the worst possible outcome of negative perfectionism and therefore this area of research should not be overlooked. Whilst perfectionism-suicide research requires a specific focus on the relationship between the two constructs, self-injury research, and recognising self-injury in clinical practice may provide an intermediary source of information. That is, negative perfectionism combined with NSSI should be a serious warning sign for hidden distress and increased risk of suicide.

\section{Strengths}

This research was informed by particularly in-depth understanding of the existing literature. The meta-analyses of the perfectionism literature, conducted prior to the local empirical components, laid an important foundation for this research. Specifically, the metaanalytic process provided the opportunity to gain insight into the challenges associated with measuring and conceptualising both positive and negative perfectionism. Moreover, it allowed exposure to a wide range of perspectives on perfectionism and substantial empirical data demonstrating associations with a raft of adaptive and maladaptive outcomes. In addition, Study $1 \mathrm{~b}$ was guided by a systematic review process, thus enabling the identification of less accessible self-injury and suicide research that may not have otherwise been uncovered.

Another strength of this research was the psychometric analysis of the FMPS (Frost et al., 1990) prior to its use with NZ adolescents. Study 2 highlighted that the original conceptualisation of the FMPS (6 factors) did not reliably capture the different components of perfectionism in this sample. Therefore, the psychometric analyses allowed for the development of a four factor structure appropriate for the studies that followed.

This research specifically investigated NSSI rather than self-injury regardless of suicidal intent. As alluded to throughout this work, this distinction is increasingly being recognised as imperative given the different cognitions, motivations and functions associated with NSSI (e.g., Joiner et al., 2012; Muehlenkamp \& Kerr, 2010). Therefore, research of this 
kind is essential to inform specialised, effective assessment strategies and intervention programmes for those engaging in NSSI (Jacobson et al., 2008; Muehlenkamp, 2014).

Similarly, unlike the majority of self-injury research, ambiguity in the results was further minimised by the use of a checklist of NSSI behaviours, rather than a single item measure (Muehlenkamp et al., 2012). Keeping in mind the limitations associated with a single screening question, those who completed the DSHI-s were likely to provide more valid results than they would have if the results were based on the screening question alone. In particular, behavioural-checklists are advantageous as they help counter any inconsistencies between the researcher's and the adolescent's conceptualisation of NSSI. Moreover, the survey included additional questions regarding self-injury, allowing an in-depth understanding of the common self-injury profiles of $\mathrm{NZ}$ adolescents.

Finally, there are a number of strengths associated with conducting research in this sample. First and foremost, this is one of very few studies to investigate NSSI in a NZ adolescent sample. Moreover, it is the only NZ-based research on perfectionism. This is important with regard to understanding culturally, and nationally-based nuances in the relationships investigated. Therefore, this research will be an important resource for informing the development of local programmes aimed at enhancing adolescent wellbeing.

Moreover, as opposed to research using convenience samples (e.g., undergraduate psychology students, inpatients), this research was deliberately targeted at a sample of community adolescents. Whilst this raised some initial ethical and practical concerns; for example hesitation from schools, teachers and parents, persistence to gain such a sample was beneficial in many ways. For one, of the NSSI reported, a high proportion was current (NSSI within the past 12 months). As such, adolescents' recall about their NSSI behaviours is likely to be more accurate than if we asked them about their self-injury following cessation of the behaviour. In addition, research with such a sample has produced findings that are directly relevant to adolescents nationally, a sample that can be argued as the most in need of selfinjury and suicide related intervention (Ministry of Health, 2015).

Lastly, this research included males, and females at a wide range of secondary schools; from private and public schools, single sex and co-education schools, and religious and non-religious schools. Moreover, schools were spread out across the wider Wellington region (including Kapiti and Wairarapa), and therefore captured multiple urban and rural communities that vary on their demographic characteristics and socio-economic position. As such, this research broadly represents the heterogeneous nature of $\mathrm{NZ}$ adolescents. 


\section{Limitations of this Research}

As with any investigation of this type there are a number of limiting factors and caveats to consider when interpreting the results. First, this research is potentially limited by the focus on the FMPS (Frost et al., 1990) as the only scale to measure perfectionism. Specifically, both the meta-analyses in Study 1a, and the local empirical components in Studies 2, 3 and 4, focused solely on data that had been collected using the FMPS. In Study 1 this can be considered a strength given that it effectively eliminated variation associated with the use of different scales. Likewise, in the following studies, as a result of remaining with the FMPS, the data could be more easily compared to the meta-analytic results. However, such a focus on a single scale means that the results are rooted in the conceptualisation of perfectionism that underlies the FMPS. Recently, Flett and Hewitt (2016) have noted that researchers should not only be questioning the underlying nature of perfectionism but also the measures we use to measure perfectionism. They argue that due to the seemingly unquestioned dominance of the two multidimensional perfectionism measures (Frost et al., 1990; Hewitt \& Flett, 1991), over time perfectionism has become defined by the items within the scales. As a result, some researchers have suggested that current conceptualisations of negative perfectionism (since the introduction of the FMPS and HFMPS in the early 1990's) differ to those proposed by early researchers in the field (see Shafran \& Mansell, 2001; Stoeber \& Otto, 2006).

Another challenge in this research was ensuring that the data collected was as valid and reliable as possible. In general, self-report data is a combination of both objective experience and subjective interpretation and recall. Therefore, it is never going to be completely equivalent to a clinical researcher's interpretation of the behaviours, emotions and cognitions investigated. Moreover, self-reported data is vulnerable to biased reporting. This is particularly relevant to perfectionistic individuals who, due to the nature of perfectionism, may have a natural tendency to under-report on factors that could show imperfections, and over-report on aspects that will reflect positively on them (Fairburn \& Beglin, 1990; Roxborough et al., 2012). In other words, perfectionism is often related to higher scores on measures of social desirability (e.g. Craddock et al., 2010, 2009). As a result, if highly perfectionistic individuals are under-reporting NSSI as suspected, the relationship between perfectionism and NSSI in this population may be understated. In addition, regardless of whether an individual is perfectionistic or not, self-reported data on self-injury is likely to be under-reported due to the stigma associated with engaging in such behaviours (Mars et al., 2016; Miller, Muehlenkamp, \& Jacobson, 2008). 
To minimise this impact, confidential self-report surveys were chosen over face-toface interviews, parent or teacher report, or focus groups. Further, the surveys were administered in as close to 'test' conditions as possible to allow adolescents the privacy required for them to feel comfortable to answer the questions honestly. However, even if the adolescents answered the questions honestly, it is still reasonable to question the reliability of self-report data on self-injury (see Mars et al., 2016; Muehlenkamp et al., 2012). For example, Latimer et al., (2013) describes complications in accurately capturing intent, noting that the individual is not always aware of their intentions when self-injuring and will be even less able to reliably reflect on their intentions days, weeks or months, after the behaviour. Likewise, self-report methods rely on the adolescent's memory of when the behaviour occurred and what exactly they did. Overall, the data in this research suggests that adolescents' selfreported account of self-injury is somewhat inconsistent. However, this is similar to the findings of other self-injury research (see Mars et al., 2016 for a review).

As outlined in Study 3 conclusions, accuracy of the self-injury data collected may have been further limited by the use of a single self-injury screening question. Specifically, I speculate that the single screening question may not have been explicit enough about the definition of NSSI used in this research, for the adolescent to reliably complete the question. In other words, some adolescents may have held differing understandings of what is and isn't considered self-injury. Therefore, this study may actually underestimate self-injury prevalence rates.

In addition, the generalisability of these results is subject to certain limitations. First, the sample consisted of only students who were still attending school, were present on the day of data collection, and whose parents had provided consent. Research suggests that adolescents that have already dropped out of school or who engage in truancy have significantly higher levels of psychopathology than those who remain in education (Egger, Costello, \& Angold, 2003). Therefore, it could be possible that students who have higher levels of psychopathology, who, based on previous research, we might expect to have the highest level of self-injury, may not be well represented in this sample. Second, in comparison to national demographic information, Māori and Pacific Islanders were particularly underrepresented in this sample. Therefore, it is important to remain cautious when generalising these finding to these groups. ${ }^{49}$

\footnotetext{
${ }^{49}$ Discussed in more detail on p. 13
} 
Finally, this research could have benefited from an additional wave of data. Some authors argue that whilst two time points allows for some causal claims, three (and more) time points lend to more methodologically sound research and inferences (Kenny, 2005; Ployhart \& Vandenberg, 2010; Singer \& Willett, 2003). Therefore, this research would have benefitted from the addition of at least one more time point. This would have allowed an investigation of whether positive perfectionism leads to negative perfectionism and then to NSSI as suggested by Claes and colleagues (2012). Relatedly, other researchers have suggested that reduced times between data collection may lead to stronger, and more robust causal findings (Fox et al., 2015). Specifically, the longer the period in between data collection, the more time there is for the influence of third variables (for a discussion of possible third variables see p. 225226). Therefore, had it been viable it would have been beneficial to survey the students at shorter intervals (e.g., 6-monthly) and on more occasions. In general, it is important to highlight that although longitudinal research allows the researcher to make claims about potential causality, these claims are by no means irrefutable (see Selig \& Little, 2012).

\section{Future Directions}

First, it is imperative that future research distinguishes between NSSI and DSH or suicidal behaviours, recognises both positive and negative perfectionism, and at the very least accounts for negative perfectionism when investigating the role of positive perfectionism. It is also important that researchers explore whether there are any differences in relationships across males and females. Such use of accurate terms (NSSI vs DSH) and definitions (positive perfectionism vs negative perfectionism) is especially important for the translation of research to clinical practice. Specifically, when clinicians identify self-injurious or suicidal behaviours, or positive or negative perfectionism in their clients, it is important for them to have a specific research base that can inform their understanding of factors contributing to their client's behaviour, and what subsequent behaviours or outcomes their client may be at risk for (Nock, 2009).

Further, research regarding the relationship between perfectionism and self-injury, but using an alternative measure of perfectionism will be worthwhile. As noted in the limitations section above, these results are based on Frost and colleagues (1990) conceptualisation and operationalisation of perfectionism. Thus, the adolescent's reports of perceived criticism and expectations were based solely on those attributed to their parents. Previous research has demonstrated that whilst parental pressures are important, perceived expectations are likely to come from multiple sources (e.g., peers, teachers, society; Perera \& Chang, 2015). Therefore, 
this research is likely to have only captured the tip of the iceberg with regard to the external pressures that NZ adolescents experience. Moreover, the advent and particularly fast development of social media, and changes in schooling systems, may mean that the nature of perfectionism in adolescents and young adults has substantially changed since the development of measures such as the FMPS (Frost et al., 1990) and the HFMPS (Hewitt \& Flett, 1991). Thus, future research may benefit from the adaption of these scales to better suit the current social environment.

This research uncovered a plethora of research that has investigated the role of negative perfectionism in the development of maladaptive outcomes. Whilst it is easy to recognise the motivations for focusing on the negative, a more complete picture will be formed with more research on the arguably more benign, positive perfectionism. This is especially so in light of the finding that positive perfectionism predicts negative perfectionism. As such, research on positive perfectionism should focus on if and how individuals, and the people around them can promote positive perfectionism, without subsequently increasing negative perfectionism.

Considering self-injury, there is substantial research demonstrating significant differences in psychological characteristics of those who self-injure once compared to those who engage in self-injury multiple times (e.g., Aldridge et al., 2014; Howe-Martin et al., 2012; Manca et al., 2014). Unfortunately, the DSHI-s score used to capture self-injurious behaviours did not allow for identifying the specific number of self-injury episodes each individual had engaged in. Therefore, it was hard to determine whether perfectionists are briefly experimenting with self-injury or whether they rely on it as one of their main coping strategies. As such, further research comparing perfectionists who engage in repetitive selfinjury to those who have only tried it once or a couple of times would contribute to our understanding of the relationship between perfectionism and self-injury.

Finally, this research has highlighted a clinically important relationship between perfectionism and self-injury in adolescents. It has also summarised existing international research that has detailed the relationship between perfectionism and suicidal behaviours and explored further research examining the link between self-injury and suicidality. Therefore, the missing piece of this research is exploration of the relationship between perfectionism and suicide in NZ adolescents. It is hypothesised that such research would demonstrate a relationship between perfectionism and suicidal thoughts and behaviours that is equal if not stronger than the equivalent relationship with self-injury. In a clinical setting this is extremely valuable information in terms of predicting risk and ensuring the safety of our adolescents. 
Regardless, further work to identify what can be done to prevent suicide in perfectionistic adolescents is of great importance.

\section{Finally}

This research has demonstrated a significant longitudinal relationship between negative perfectionism and self-injury in NZ female adolescents. Therefore, suggesting that perfectionistic females are experiencing considerable distress related to their perfectionistic cognitions and behaviours. Unfortunately, for many of these adolescents social support networks will be perceived as inaccessible, or will be avoided due to fear of negative evaluation. Consequently, perfectionists are forced to develop alternative coping strategies, one of which is NSSI.

Alongside these findings, it is important to be continually reminded that adolescents are facing multi-modal, and multifaceted demands and pressures, some of which are unique only to their generation. For one, technology and social media are moving at a faster pace than the research investigating their effects on adolescent psychological health and wellbeing. In addition, school achievement systems are placing a greater emphasis on grades, and adolescents are reporting considerable pressure to gain entrance to university or obtain a job.

The New Zealand Educational Review Office has recently argued that in order to increase engagement and achievement at school, and therefore young people's career prospects, focus must first be on the student's overall wellbeing (ERO, 2014). This makes intuitive and logical sense, however in practice it remains difficult to argue for a greater focus on student wellbeing at the expense of time and resources currently assigned to increasing achievement. Perhaps an intermediary step aimed at the prevention of perfectionism, could include encouraging students to view themselves as worthy, to practice self-compassion and to be confident in accessing support if needed.

It is reasonable to expect the lay person to question why a perfectionist, someone who is striving to maintain perfect presentation and do their best at all times, would intentionally engage in such a stigmatised behaviour. Moreover, why would a perfectionist self-injure when the behaviour temporarily, if not indefinitely, places their goal of perfection further out of reach? This research has suggested that, despite fears of social stigmatisation, self-injury remains an especially effective method of punishing oneself for 'failures', distancing from others or warding off suicidal thoughts and behaviours. As surprising as it may be, for perfectionists self-injury may be perceived as the best way to right wrong doings and continue to function on a day to day basis. 
This research was a necessary and valuable step in understanding the relationship between perfectionism and NSSI in adolescents. Research such as this, reiterates the importance of understanding adolescent wellbeing, and further suggests that at a societal and policy level more can be done to prevent significant distress in our adolescents. Moreover, due to concealment of distress and limited help-seeking behaviours, there seems to be a lack of common awareness that many perfectionists face substantial distress, and are in turn placed in a particularly vulnerable and isolated position. Thus, researchers should continue to investigate how best to limit the development of perfectionism, identify perfectionists, and provide appropriate support aimed at decreasing distress and thus reducing risk of NSSI. 


\section{REFERENCES}

*(indicates publications included in Study 1 meta-analyses)

*Adkins, K. K., \& Parker, W. (1996). Perfectionism and suicidal preoccupation. Journal of Personality, 64(2), 529-43. doi:10.1111/j.1467-6494.1996.tb00520.x

Aldea, M. a., \& Rice, K. G. (2006). The role of emotional dysregulation in perfectionism and psychological distress. Journal of Counseling Psychology, 53(4), 498-510. doi:10.1037/0022-0167.53.4.498

Alden, L. E., Ryder, A. G., \& Mellings, T. M. B. (2002). Perfectionism in the context of social fears: Toward a two-component model. In G. L. Flett \& P. L. Hewitt (Eds.), Perfectionism: Theory, research, and treatment (pp. 373-391). Washington DC: American Psychological Association.

Aldridge, D., Antoniewicz, T., Gebhardt, M., Juzwin, K., Mitckess, D., Rudolph, J., ... Yourek, A. (2014). Self-injury: Simple answers to complex questions. Hoffman Estates, IL: Alexian Brothers Behavioural Health Hospital.

American Psychiatric Association. (2000). Diagnostic and statistical manual of mental disorders (4th ed). Washington, DC: American Psychiatric Association.

American Psychiatric Association. (2013). The diagnostic and statistical manual of mental disorders (5th ed.). Arlington, VA: American Psychiatric Association.

Andover, M. S., Primack, J. M., Gibb, B. E., \& Pepper, C. M. (2010). An examination of non-suicidal self-injury in men: Do men differ from women in basic NSSI characteristics? Archives of Suicide Research, 14(1), 79-88. doi:10.1080/13811110903479086

*Andrews, L. M., Bullock-Yowell, E., Dahlen, E. R., \& Nicholson, B. C. (2014). Can perfectionism affect career development? Exploring career thoughts and self-efficacy. Journal of Counseling and Development, 92(3), 270-279. doi:10.1002/j.15566676.2014.00155.x

Andrews, T., Martin, G., Hasking, P., \& Page, A. (2013). Predictors of onset for non-suicidal self-injury within a school-based sample of adolescents. Prevention Science, 15, 1-10. doi:10.1007/s11121-013-0412-8

*Ansell, E. B., Pinto, A., Crosby, R. D., Becker, D. F., Anez, L. M., Paris, M., \& Grilo, C. M. (2010). The prevalence and structure of obsessive-compulsive personality disorder in Hispanic psychiatric outpatients. Journal of Behavior Therapy and Experimental Psychiatry, 41(3), 275-281. doi:10.1016/j.jbtep.2010.02.005 
Antony, M. M., Purdon, C. L., Huta, V., Swinson, R. P., Richard, P. S., Swinson, R. P., \& Richard, P. S. (1998). Dimensions of perfectionism across the anxiety disorders. Behaviour Research and Therapy, 36(12), 1143-1154. doi:10.1016/S00057967(98)00083-7

Appleton, P. R., Hall, H. K., \& Hill, A. P. (2010). Family patterns of perfectionism: An examination of elite junior athletes and their parents. Psychology of Sport and Exercise, 11(5), 363-371. doi:10.1016/j.psychsport.2010.04.005

Arbuthnott, A. E., \& Lewis, S. P. (2015). Parents of youth who self-injure: A review of the literature and implications for mental health professionals. Child and Adolescent Psychiatry and Mental Health, 9(35), 1-20. doi:10.1186/s13034-015-0066-3

Armey, M. F., Andover, M. S., Muehlenkamp, J. J., Washburn, J. J., Gebhardt, M., Styer, D. M., ... Tull, M. T. (2012). Body regard in nonsuicidal self-injury: Theoretical explanations and treatment directions. Journal of Cognitive Psychotherapy, 26(4), 331347. doi:10.1891/0889-8391.26.4.331

Asarnow, J. R., Porta, G., Spirito, A., Emslie, G., Clarke, G., Wagner, K. D., .. Brent, D. (2011). Suicide attempts and nonsuicidal self-injury in the treatment of resistant depression in adolescents: Findings from the TORDIA study. Journal of the American Academy of Child and Adolescent Psychiatry, 50(8), 772-781. doi:10.1016/j.jaac.2011.04.003

Ashby, J. S. (1995). An investigation of perfectionism in a university sample of adult children of alcoholics. Journal of College Student Development, 36(5), 452-456. Retrieved from: https://muse.jhu.edu/journal/238

Ashby, J. S., \& Kottman, T. (1996). Inferiority as a distinction between normal and neurotic perfectionism. Individual Psychology, 52(3), 237-245. Retrieved from https://utpress.utexas.edu/journals/journal-of-individual-psychology

Asseraf, M., \& Vaillancourt, T. (2014). Longitudinal links between perfectionism and depression in children. Journal of Abnormal Child Psychology, 43(5), 895-908. doi:10.1007/s10802-014-9947-9

Bardone-Cone, A. M., Wonderlich, S. A., Frost, R. O., Bulik, C. M., Mitchell, J. E., Uppala, S., \& Simonich, H. (2007). Perfectionism and eating disorders: Current status and future directions. Clinical Psychology Review, 27(3), 384-405. doi:10.1016/j.cpr.2006.12.005

Baron, R. M., \& Kenny, D. A. (1986). The moderator-mediator variable distinction in social psychological research: Conceptual, strategic, and statistical considerations. Journal of Personality and Social Psychology, 51(6), 1173-1182. doi:10.1037/0022- 
3514.51.6.1173

Barrocas, A. L., Giletta, M., Hankin, B. L., Prinstein, M. J., \& Abela, J. R. Z. (2014).

Nonsuicidal self-injury in adolescence: Longitudinal course, trajectories, and intrapersonal predictors. Journal of Abnormal Child Psychology, 43(2), 369-380. doi:10.1007/s10802-014-9895-4

Barrocas, A. L., Hankin, B. L., Young, J. F., \& Abela, J. R. Z. (2012). Rates of nonsuicidal self-injury in youth: Age, sex, and behavioral methods in a community sample. Pediatrics, 130(1), 39-45. doi:10.1542/peds.2011-2094

Batejan, K. L., Jarvi, S. M., \& Swenson, L. P. (2015). Sexual orientation and non-suicidal self-injury: A meta-analytic review. Archives of Suicide Research, 19(2), 131-150. doi:10.1080/13811118.2014.957450

Baumeister, R. F. (1990). Suicide as escape from self. Psychological Review, 97(1), 90-113. doi:10.1080/00224498809551444

Beaman, R., Wheldall, K. \& Kemp, C. (2006). Differential teacher attention to boys and girls in the classroom. Educational Review, 58(3), 339-366. doi: $10.1080 / 00131910600748406$

*Beevers, C. G., \& Miller, I. W. (2004). Perfectionism, cognitive bias, and hopelessness as prospective predictors of suicidal ideation. Suicide \& Life-Threatening Behavior, 34(2), 126-137. doi:10.1521/suli.34.2.126.32791

Begg, C. B., \& Mazumdar, M. (1994). Operating characteristics of a rank correlation test for publication bias. Biometrics, 50(4), 1088-1101. doi: 10.2307/2533446

Bentley, K. H., Cassiello-Robbins, C. F., Vittorio, L., Sauer-Zavala, S., \& Barlow, D. H. (2015). The association between nonsuicidal self-injury and the emotional disorders: A meta-analytic review. Clinical Psychology Review, 37(April), 72-88. doi:10.1016/j.cpr.2015.02.006

Bernardi, R. (1994). Validating research results when cronbach's alpha is below .70: A methodological procedure. Educational and Psychological Measurement, 54(3), 766775. doi:10.1177/0013164494054003023

Besharat, M. A. (2004). An investigation of the relationship between perfectionism and selfesteem in pre-college students. Journal of Iranian Psychologists, 1(1), 21-34.

Beyer, S. (1998). Gender differences in causal attributions by college students of performance on course examinations. Current Psychology, 17(4), 346-358. doi:10.1007/s12144-998-1016-5

Bhui, K., McKenzie, K., \& Rasul, F. (2007). Rates, risk factors \& methods of self harm 
among minority ethnic groups in the UK: A systematic review. BMC Public Health, 7, 336-350. doi:10.1186/1471-2458-7-336

Bieling, P. J., Israeli, A. L., \& Antony, M. M. (2004). Is perfectionism good, bad, or both? Examining models of the perfectionism construct. Personality and Individual Differences, 36(6), 1373-1385. doi:10.1016/S0191-8869(03)00235-6

Bildik, T., Somer, O., Kabukçu Başay, B., Başay, Ö., \& Özbaran, B. (2013). The validity and reliability of the Turkish version of the Inventory of Statements About Self-injury. Turkish Journal of Psychiatry, 24(1), 41-49. doi:10.5080/u6901

Bjärehed, J., \& Lundh, L.G. (2008). Deliberate self-harm in 14-year-old adolescents: How frequent is it, and how is it associated with psychopathology, relationship variables, and styles of emotional regulation? Cognitive Behaviour Therapy, 37(1), 26-37. doi:10.1080/16506070701778951

Blankstein, K. R., Dunkley, D. M., \& Wilson, J. (2008). Evaluative concerns and personal standards perfectionism: Self-esteem as a mediator and moderator of relations with personal and academic needs and estimated GPA. Current Psychology, 27(1), 29-61. doi:10.1007/s12144-008-9022-1

*Blankstein, K. R., Lumley, C. H., \& Crawford, A. (2007). Perfectionism, hopelessness, and suicide ideation: Revisions to diathesis-stress and specific vulnerability models. Journal of Rational - Emotive and Cognitive - Behavior Therapy, 25(4), 279-319. doi:10.1007/s10942-007-0053-6

Blankstein, K. R., \& Winkworth, G. R. (2004). Dimensions of perfectionism and levels of attributions for grades: Relations with dysphoria and academic performance. Journal of Rational-Emotive and Cognitive-Behavior Therapy, 22(4), 267-295. doi:10.1023/B:JORE.0000047312.20212.30

Bong, M., Hwang, A., Noh, A., \& Kim, S. (2014). Perfectionism and motivation of adolescents in academic contexts. Journal of Educational Psychology, 106(3), 711-729. doi:10.1037/a0035836

*Boone, L., Braet, C., Vandereycken, W., \& Claes, L. (2013). Are maladaptive schema domains and perfectionism related to body image concerns in eating disorder patients? European Eating Disorders Review, 21(1), 45-51. doi:10.1002/erv.2175

*Boone, L., Claes, L., \& Luyten, P. (2014). Too strict or too loose? Perfectionism and impulsivity: The relation with eating disorder symptoms using a person-centered approach. Eating Behaviors, 15(1), 17-23. doi:10.1016/j.eatbeh.2013.10.013

*Boone, L., \& Soenens, B. (2015). In double trouble for eating pathology? An experimental 
study on the combined role of perfectionism and body dissatisfaction. Journal of Behavior Therapy and Experimental Psychiatry, 47(3), 77-83.

doi:10.1016/j.jbtep.2014.11.005

*Boone, L., Soenens, B., \& Braet, C. (2011). Perfectionism, body dissatisfaction, and bulimic symptoms: The intervening role of perceived pressure to be thin and thin ideal internalization. Journal of Social and Clinical Psychology, 30(10), 1043-1068. doi:10.1521/jscp.2011.30.10.1043

*Boone, L., Soenens, B., Braet, C., \& Goossens, L. (2010). An empirical typology of perfectionism in early-to-mid adolescents and its relation with eating disorder symptoms. Behaviour Research and Therapy, 48(7), 686-691. doi:10.1016/j.brat.2010.03.022

*Boone, L., Soenens, B., \& Luyten, P. (2014). When or why does perfectionism translate into eating disorder pathology? A longitudinal examination of the moderating and mediating role of body dissatisfaction. Journal of Abnormal Psychology, 123(2), 412-418. doi:10.1037/a0036254

*Boone, L., Soenens, B., Mouratidis, A., Vansteenkiste, M., Verstuyf, J., \& Braet, C. (2012). Daily fluctuations in perfectionism dimensions and their relation to eating disorder symptoms. Journal of Research in Personality, 46(6), 678-687. doi:10.1016/j.jrp.2012.08.001

*Boone, L., Soenens, B., Vansteenkiste, M., \& Braet, C. (2012). Is there a perfectionist in each of us? An experimental study on perfectionism and eating disorder symptoms. Appetite, 59(2), 531-540. doi:10.1016/j.appet.2012.06.015

*Boone, L., Vansteenkiste, M., Soenens, B., Van der Kaap-Deeder, J., \& Verstuyf, J. (2014). Self-critical perfectionism and binge eating symptoms: A longitudinal test of the intervening role of psychological need frustration. Journal of Counseling Psychology, 61(3), 363-73. doi:10.1037/a0036418

Borenstein, M., Hedges, L. V, Higgins, J. P. T., \& Rothstein, H. (2009). Introduction to metaanalysis. West Sussex, UK: John Wiley \& Sons Ltd.

Borenstein, M., Hedges, L. V, Higgins, J., \& Rothstein, H. (2005). Comprehensive MetaAnalysis Version 2. Englewood, New Jersey: Biostat.

Brand, S., Kirov, R., Kalak, N., Gerber, M., Puhse, U., Lemola, S., ... Trachsler, E. H. (2015). Perfectionism related to self-reported insomnia severity, but not when controlled for stress and emotion regulation. Neuropsychiatric Disease and Treatment, 11, 263271. doi:10.2147/NDT.S74905 
Bresin, K., \& Schoenleber, M. (2015). Gender differences in the prevalence of nonsuicidal self-injury: A meta-analysis. Clinical Psychology Review, 38, 55-64. doi:10.1016/j.cpr.2015.02.009

Briere, J., \& Gil, E. (1998). Self-mutilation in clinical and general population samples: Prevalence, correlates, and functions. American Journal of Orthopsychiatry, 68(4), 609620. doi:10.1037/h0080369

Brown, E. (2015). What is the nature of the relationship between bullying and NSSI in NZ adolescents. (Unpublished doctoral thesis). Victoria University of Wellington, Wellington, New Zealand.

Brown, G. P., \& Beck, A. T. (2002). Dysfunctional attitudes, perfectionism, and models of vulnerability to depression. In G. L. Flett \& P. L. Hewitt (Eds.), Perfectionism: Theory, research and treatment (pp. 231-251). Washington, DC: American Psychological Association.

*Brown, J. R., \& Kocovski, N. L. (2014). Perfectionism as a predictor of post-event rumination in a socially anxious sample. Journal of Rational-Emotive \& CognitiveBehavior Therapy, 32(2), 150-163. doi:10.1007/s10942-013-0175-y

Brown, M. Z., Comtois, K. A., \& Linehan, M. M. (2002). Reasons for suicide attempts and non-suicidal self-injury in women with borderline personality disorder. Journal of Abnormal Psychology, 111(1), 198-202. doi:10. 10,371/002 I -843X. I I 1. 1. I 98

Brown, T. A. (2006). Confirmatory factor analysis for applied research. New York, NY: Guilford Press.

Brunner, R., Kaess, M., Parzer, P., Fischer, G., Carli, V., Hoven, C. W., ... Wasserman, D. (2014). Life-time prevalence and psychosocial correlates of adolescent direct selfinjurious behavior: A comparative study of findings in 11 European countries. Journal of Child Psychology and Psychiatry and Allied Disciplines, 55(4), 337-348. doi:10.1111/jcpp.12166

Bureau, J. F., Martin, J., Freynet, N., Poirier, A. A., Lafontaine, M. F., \& Cloutier, P. (2010). Perceived dimensions of parenting and non-suicidal self-injury in young adults. Journal of Youth and Adolescence, 39(5), 484-494. doi:10.1007/s10964-009-9470-4

Burke, T. A., Hamilton, J. L., Abramson, L. Y., \& Alloy, L. B. (2015). Non-suicidal selfinjury prospectively predicts interpersonal stressful life events and depressive symptoms among adolescent girls. Psychiatry Research, 228(3), 416-424. doi:10.1016/j.psychres.2015.06.021

Burns, D. (1980). The perfectionist's script for self-defeat. Psychology Today, 14(6), 34-52. 
Retrieved from https://www.yumpu.com/en/document/view/10716810/theperfectionists-script-for-self-defeat-university-of-colorado-

*Burton, D., Gillham, A., \& Glenn, S. (2013). The forgotten self-talk attribute: Examining perceived effectiveness profiles and patterns of elite female junior soccer players. International Journal of Sports Science and Coaching, 8(2), 357-372. doi:10.1260/1747-9541.8.2.357

Byrne, S., Morgan, S., Fitzpatrick, C., Boylan, C., Crowley, S., Gahan, H., ... Guerin, S. (2008). Deliberate self-harm in children and adolescents: A qualitative study exploring the needs of parents and carers. Clinical Child Psychology and Psychiatry, 13(4), 493504. doi:10.1177/1359104508096765

*Caelian, C. F. (2005). The role of perfectionism and stress in the suicidal behaviour of depressed adolescents. (Unpublished doctoral thesis). University of British Columbia, Canada.

Card, N. (2012). Applied meta-analysis for social science research. New York, NY: The Guilford Press.

Caro, E. F. De, \& Blas, L. Di. (2016). A prospective study on the reciprocal influence between personality and attitudes, behaviors, and psychological characteristics salient in eating disorders in a sample of non-clinical adolescents. Eating Disorders, 24(5), 453468. doi:10.1080/10640266.2016.1207454

Caruso, J. C. (2000). Reliability generalization of the NEO personality scales. Educational and Psychological Measurement, 60(2), 236-254. doi:10.1177/00131640021970484

Carver, C. S., \& Ganellen, R. J. (1983). Depression and components of self-punitiveness: high standards, self-criticism, and overgeneralization. Journal of Abnormal Psychology, 92(3), 330-337. doi:10.1037/0021-843X.92.3.330

Casale, S., Fioravanti, G., Flett, G. L., \& Hewitt, P. L. (2014). From socially prescribed perfectionism to problematic use of internet communicative services: The mediating roles of perceived social support and the fear of negative evaluation. Addictive Behaviors, 39(12), 1816-1822. doi:10.1016/j.addbeh.2014.06.006

Castro, J. R., \& Rice, K. G. (2003). Perfectionism and ethnicity: Implications for depressive symptoms and self-reported academic achievement. Cultural Diversity \& Ethnic Minority Psychology, 9(1), 64-78. doi:10.1037/1099-9809.9.1.64

Cattell, R. B. (1966). The scree test for number of factors. Multivariate Behavioral Research, 1(2), 245-276. doi:10.1207/s15327906mbr0102_10

Chan, D. W. (2012). Life satisfaction, happiness, and the growth mindset of healthy and 
unhealthy perfectionists among Hong Kong Chinese gifted students. Roeper Review, 34(4), 224-233. doi:10.1080/02783193.2012.715333

Chandler, A. (2016). Constructing and situating an embodied, sociological account of selfinjury. Self-injury, medicine and society: Authentic bodies. London, UK: Macmillan Publishers Ltd.

*Chang, E. C. (1998). Cultural differences, perfectionism, and suicidal risk in a college population: Does social problem solving still matter? Cognitive Therapy and Research, 22(3), 237-254. doi:10.1023/A:1018792709351

Chang, E. C. (2000). Perfectionism as a predictor of positive and negative psychological outcomes: Examining a mediation model in younger and older adults. Journal of Counseling Psychology, 47(1), 18. doi:10.1037/0022-0167.47.1.18

*Chang, E. C. (2002). Examining the link between perfectionism and psychological maladjustment: Social problem solving as a buffer. Cognitive Therapy and Research, 26(5), 581-595. doi:10.1023/A:1020329625158

*Chang, E. C., Banks, K. H., \& Watkins, A. (2004). How adaptive and maladaptive perfectionism relate to positive and negative psychological functioning: Testing a stressmediation model in black and white female college students. Journal of Counseling Psychology, 51(1), 93-103. doi:10.1037/0022-0167.51.1.93

Chang, E. C., \& Rand, K. L. (2000). Perfectionism as a predictor of subsequent adjustment: Evidence for a specific diathesis-stress mechanism among college students. Journal of Counseling Psychology, 47(1), 129-137. doi:10.1037/0022-0167.47.1.129

Chang, E. C., \& Sanna, L. J. (2001). Negative attributional style as a moderator of the link between perfectionism and depressive symptoms: Preliminary evidence for an integrative model. Journal of Counseling Psychology, 48(4), 490-495. doi:10.1037/0022-0167.48.4.490

Chang, E. C., Yu, E. A., \& Lin, E. Y. (2014). An examination of ethnic variations in perfectionism and interpersonal influences as predictors of eating disturbances: A look at Asian and European American females. Asian American Journal of Psychology, 5(3), 243-251. doi:10.1037/a0034621

Chaplin, T. M., \& Aldao, A. (2013). Gender differences in emotion expression in children: A meta-analytic review. Psychological Bulletin, 139(4), 735-765. doi:10.1037/a0030737

Chaplin, T. M., Cole, P. M., \& Zahn-Waxler, C. (2005). Parental socialization of emotion expression: Gender differences and relations to child adjustment. Emotion, 5(1), 80-88. doi:10.1037/1528-3542.5.1.80 
Chapman, A. L., Gratz, K. L., \& Brown, M. Z. (2006). Solving the puzzle of deliberate selfharm: The experiential avoidance model. Behaviour Research and Therapy, 44(3), 371394. doi:10.1016/j.brat.2005.03.005

Chapman, A. L., Specht, M. W., \& Cellucci, T. (2005). Borderline personality disorder and deliberate self-harm: Does experiential avoidance play a role? Suicide \& LifeThreatening Behavior, 35(August), 388-399. doi:10.1521/suli.2005.35.4.388

Chen, C., Hewitt, P. L., \& Flett, G. L. (2015). Preoccupied attachment, need to belong, shame, and interpersonal perfectionism: An investigation of the Perfectionism Social Disconnection Model. Personality and Individual Differences, 76, 177-182. doi:10.1016/j.paid.2014.12.001

*Cheng, S. K. (2001). Life stress, problem solving, perfectionism, and depressive symptoms in Chinese. Cognitive Therapy and Research, 25(3), 303-310. doi:10.1023/A:1010788513083

*Chester, D. S., Merwin, L. M., \& Dewall, C. N. (2015). Maladaptive perfectionism's link to aggression and self-harm: Emotion regulation as a mechanism. Aggressive Behavior, 41(5), 443-454. doi:10.1002/AB.21578

Choo, W. Y., Walsh, K., Chinna, K., \& Tey, N. P. (2013). Teacher Reporting Attitudes Scale (TRAS): Confirmatory and exploratory factor analyses with a Malaysian sample. Journal of Interpersonal Violence, 28(2), 231-53. doi:10.1177/0886260512454720

Claes, L., Luyckx, K., Baetens, I., Van de Ven, M., \& Witteman, C. (2015). Bullying and victimization, depressive mood, and non-suicidal self-injury in adolescents: The moderating role of parental support. Journal of Child and Family Studies, 24(11), 33633371. doi:10.1007/s10826-015-0138-2

*Claes, L., Soenens, B., Vansteenkiste, M., \& Vandereycken, W. (2012). The scars of the inner critic: Perfectionism and nonsuicidal self-injury in eating disorders. European Eating Disorders Review, 20, 196-202. doi:10.1002/erv.1158

Claes, L., \& Vandereycken, W. (2007). Self-injurious behavior: Differential diagnosis and functional differentiation. Comprehensive Psychiatry, 48, 137-144. doi:10.1016/j.comppsych.2006.10.009

Claes, L., Vandereycken, W., \& Vertommen, H. (2003). Eating-disordered patients with and without self-injurious behaviours: A comparison of psychopathological features. European Eating Disorders Review, 11, 379-396. doi:10.1002/erv.510

*Clark, S., \& Coker, S. (2009). Perfectionism, self-criticism and maternal criticism: A study of mothers and their children. Personality and Individual Differences, 47(4), 321-325. 
doi:10.1016/j.paid.2009.03.020

Cohen, J. (1988). Statistical power analysis for the behavioral sciences (2nd ed.). Hillsdale, NJ: Lawrence Earlbaum Associates.

Cooper, H., \& Hedges, L. (2009). Potentials and limitations. In H. Cooper, L. Hedges, \& J. C. Valentine (Eds.), The handbook of research synthesis and meta-analysis (pp. 561-572). New York, NY: Russel Sage Foundation.

Cox, B. J., Enns, M. W., \& Clara, I. P. (2002). The multidimensional structure of perfectionism in clinically distressed and college student samples. Psychological Assessment, 14(3), 365-373. doi:10.1037/1040-3590.14.3.365

*Craddock, A. E., Church, W., Harrison, F., \& Sands, A. (2010). Family of origin qualities as predictors of religious dysfunctional perfectionism. Journal of Psychology \& Theology, 38(3), 205-214. doi:0091-6471/410-730

*Craddock, A. E., Church, W., \& Sands, A. (2009). Family of origin characteristics as predictors of perfectionism. Australian Journal of Psychology, 61(3), 136-144. doi:10.1080/00049530802239326

Cucchi, A., Ryan, D., Konstantakopoulos, G., Stroumpa, S., Kaçar, A. Ş., \& Renshaw, S. (2016). Lifetime prevalence of non-suicidal self-injury in patients with eating disorders: A systematic review and meta-analysis. Psychological Medicine, 46(7), 1345-1358. doi:10.1017/S0033291716000027

Cwik, M. F., Barlow, A., Tingey, L., Larzelere-Hinton, F., Goklish, N., \& Walkup, J. T. (2011). Nonsuicidal self-injury in an American Indian reservation community: Results from the White Mountain Apache surveillance system, 2007-2008. Journal of the American Academy of Child and Adolescent Psychiatry, 50(9), 860-869. doi:10.1016/j.jaac.2011.06.007

*Dakanalis, A., Carra, G., Calogero, R., Zanetti, M. A., Gaudio, S., Caccialanza, R., ... Clerici, M. (2015). Testing the cognitive-behavioural maintenance models across DSM5 bulimic-type eating disorder diagnostic groups: A multi-centre study. European Archives of Psychiatry and Clinical Neuroscience, 265(8), 663-676. doi:10.1007/s00406-014-0560-2

*Dakanalis, A., Timko, C. A., Clerici, M., Zanetti, M. A., \& Riva, G. (2014). Comprehensive examination of the trans-diagnostic cognitive behavioral model of eating disorders in males. Eating Behaviors, 15(1), 63-67. doi:10.1016/j.eatbeh.2013.10.003

*Dakanalis, A., Timko, C. A., Zanetti, M. A., Rinaldi, L., Prunas, A., Carrà, G., ... Clerici, M. (2014). Attachment insecurities, maladaptive perfectionism, and eating disorder 
symptoms: A latent mediated and moderated structural equation modeling analysis across diagnostic groups. Psychiatry Research, 215(1), 176-184.

doi:10.1016/j.psychres.2013.10.039

Damian, L. E., Stoeber, J., Negru, O., \& Băban, A. (2014). Positive and negative affect in adolescents: An investigation of the $2 \times 2$ model of perfectionism. Cognition, Brain, Behavior. An Interdisciplinary Jounal, 18(1), 1-16. Retrieved from https://kar.kent.ac.uk/id/eprint/38155

Damian, L. E., Stoeber, J., Negru, O., Băban, A., Băban, A., \& Băban, A. (2013). On the development of perfectionism in adolescence: Perceived parental expectations predict longitudinal increases in socially prescribed perfectionism. Personality and Individual Differences, 55(6), 688-693. doi:10.1016/j.paid.2013.05.021

Davis, C. (1997). Normal and neuroting perfectionsm in eating disorders: An interactive model. International Journal of Eating Disorders, 22(4), 421-426. doi:10.1002/(sici)1098-108x(199712)22:4\%3C421::aid-eat7\%3E3.0.co;2-o

Day, A. L., \& Livingstone, H. A. (2003). Gender differences in perceptions of stressors and utilization of social support among university students. Canadian Journal of Behavioural Science, 35(2), 73-83. doi:10.1037/h0087190

*Dean, P. J., \& Range, L. M. (1996). The escape theory of suicide and perfectionism in college students. Death Studies, 20(4), 415-424. doi:10.1080/07481189608252790

*Dean, P. J., \& Range, L. M. (1999). Testing the escape theory of suicide in an outpatient clinical population. Cognitive Therapy and Research, 23(6), 561-572. doi: 10.1023/A: 1018728606568

De Petrillo, L., Kaufman, K., Glass, C., \& Arnkoff, D. (2009). Mindfullness for long distance runners: An open trial using Mindful Sport Performance Enhancement (MSPE). Journal of Clinical Sport Psychology, 3(4), 357-376. doi:10.1037/t11612-000

Di Pierro, R., Sarno, I., Perego, S., Gallucci, M., \& Madeddu, F. (2012). Adolescent nonsuicidal self-injury: The effects of personality traits, family relationships and maltreatment on the presence and severity of behaviours. European Child and Adolescent Psychiatry, 21, 511-520. doi:10.1007/s00787-012-0289-2

*Di Schiena, R., Luminet, O., Philippot, P., \& Douilliez, C. (2012). Adaptive and maladaptive perfectionism in depression: Preliminary evidence on the role of adaptive and maladaptive rumination. Personality and Individual Differences, 53(6), 774-778. doi:10.1016/j.paid.2012.05.017

DiBartolo, P. M., Frost, R. O., Chang, P., LaSota, M., \& Grills, A. E. (2004). Shedding light 
on the relationship between personal standards and psychopathology: The case for contingent self-worth. Journal of Rational-Emotive and Cognitive-Behavior Therapy, 22(4), 241-254. doi:10.1023/B:JORE.0000047310.94044.ac

*DiBartolo, P. M., Li, C. Y., Averett, S., Skotheim, S., Smith, L. M., Raney, C., \& McMillen, C. (2007). The relationship of perfectionism to judgmental bias and psychopathology. Cognitive Therapy and Research, 31(5), 573-587. doi:10.1007/s10608-006-9112-z

*DiBartolo, P. M., Li, C. Y., \& Frost, R. O. (2008). How do the dimensions of perfectionism relate to mental health? Cognitive Therapy and Research, 32(3), 401-417. doi:10.1007/s10608-007-9157-7

DiBartolo, P. M., \& Rendón, M. J. (2012). A critical examination of the construct of perfectionism and its relationship to mental health in Asian and African Americans using a cross-cultural framework. Clinical Psychology Review, 32(3), 139-152. doi:10.1016/j.cpr.2011.09.007

*Dickie, L., Wilson, M., McDowall, J., \& Surgenor, L. J. (2012). What components of perfectionism predict drive for thinness? Eating Disorders: The Journal of Treatment and Prevention, 20(3), 232-247. doi:10.1080/10640266.2012.668484

Diener, C. I., \& Dweck, C. S. (1978). An analysis of learned helplessness: Continuous changes in performance, strategy, and achievement cognitions following failure. Journal of Personality and Social Psychology, 36(5), 451-462. doi:10.1037/0022-3514.36.5.451

Douilliez, C., \& Lefevre, F. (2011). Interactive effect of perfectionism dimensions on depressive symptoms: A reply to Gaudreau and Thompson (2010). Personality and Individual Differences, 50(7), 1147-1151. doi:10.1016/j.paid.2011.02.005

Downey, C. A., Reinking, K. R., Gibson, J. M., Cloud, J. A., \& Chang, E. C. (2014). Perfectionistic cognitions and eating disturbance: Distinct mediational models for males and females. Eating Behaviors, 15(3), 419-426. doi:10.1016/j.eatbeh.2014.04.020

Dunkley, D. M., Blankstein, K. R., Halsall, J., Williams, M., \& Winkworth, G. (2000). The relation between perfectionism and distress: Hassles, coping, and perceived social support as mediators and moderators. Journal of Counseling Psychology, 47(4), 437453. doi:10.1037/0022-0167.47.4.437

Dunkley, D. M., Sanislow, C. A., Grilo, C. M., \& McGlashan, T. H. (2006). Perfectionism and depressive symptoms 3 years later: Negative social interactions, avoidant coping, and perceived social support as mediators. Comprehensive Psychiatry, 47(2), 106-115. doi:10.1016/j.comppsych.2005.06.003

Duval, S., \& Tweedie, R. (2000). A nonparametric “ Trim and Fill” method of accounting 
for publication bias in meta-analysis. Journal of the American Statistical Society, 95(449), 89-98. doi:10.2307/2669529

Dweck, C. S., Goetz, T., \& Strauss, N (1980). Sex differences in learned helplessness: An experimental and naturalistic study of failure generalization and its mediators. Journal of Personality and Social Psychology, 38(3), 441-452. doi: 10.1037/00223514.38.3.441

Dweck, C., \& Licht, B. (1980). Learned helplessness and intellectual achievement. In J. Garber \& M. Seligman (Eds.), Human helplessness: Theory and applications. New York, NY: Academic Press.

Dweck, C., \& Molden, D. (2005). Self-theories: Their impact on competence motivation and acquisition. In A. J. Elliot \& C. S. Dweck (Eds.), Handbook of competence and motivation (pp. 122-140). New York, NY: The Guilford Press.

Dweck, C. S. (2007). The perils and promises of praise. Educational Leadership, 65(2), 3439. doi:10.1037//0022-3514.75.1.33

Education Review Office. (2015). Wellbeing for young people's success at secondary school. Wellington, New Zealand: Education Evaluation Reports. Retrieved from http://www.ero.govt.nz/assets/Uploads/ERO-Wellbeing-SecondSchools-web.pdf

Egan, S. J., Dick, M., \& Allen, P. J. (2012). An experimental investigation of standard setting in clinical perfectionism. Behaviour Change, 29(3), 183-195. doi:10.1017/bec.2012.16

Egan, S. J., Wade, T. T. D., \& Shafran, R. (2011). Perfectionism as a transdiagnostic process: A clinical review. Clinical Psychology Review, 31(2), 203-212. doi:10.1016/j.cpr.2010.04.009

Egger, H. L., Costello, J. E., \& Angold, A. (2003). School refusal and psychiatric disorders: A community study. Journal of the American Academy of Child \& Adolescent Psychiatry, 42(7), 797-807. doi:10.1097/01.CHI.0000046865.56865.79

*Ellickson-Larew, S., Naragon-Gainey, K., \& Watson, D. (2013). Pathological eating behaviors, BMI, and facet-level traits: The roles of conscientiousness, neuroticism, and impulsivity. Eating Behaviors, 14(4), 428-431. doi:10.1016/j.eatbeh.2013.06.015

*Engel, S. G., Corneliussen, S. J., Wonderlich, S. A., Crosby, R. D., Le Grange, D., Crow, S., ... Steiger, H. (2005). Impulsivity and compulsivity in bulimia nervosa. International Journal of Eating Disorders, 38(3), 244-251. doi:10.1002/eat.20169

Enns, M. W., \& Cox, B. J. (2002). The nature and assessment of perfectionism: A critical analysis. In G. L. Flett \& P. L. Hewitt (Eds.), Perfectionism: Theory, research and treatment (pp. 33-62). Washington DC: American Psychological Association. 
*Enns, M. W., Cox, B. J., \& Inayatulla, M. (2003). Personality predictors of outcome for adolescents hospitalized for suicidal ideation. Journal of the American Academy of Child and Adolescent Psychiatry, 42(6), 720-727. doi:10.1097/01.CHI.0000046847.56865.B0

Esposito-Smythers, C., Goldstein, T., Birmaher, B., Goldstein, B., Hunt, J., Ryan, N., ... Keller, M. (2010). Clinical and psychosocial correlates of non-suicidal self-injury within a sample of children and adolescents with bipolar disorder. Journal of Affective Disorders, 125(1-3), 89-97. doi:10.1016/j.jad.2009.12.029

Essau, C. A., Leung, P. W. L., Conradt, J., Cheng, H., \& Wong, T. (2008). Anxiety symptoms in Chinese and German adolescents: Their relationship with early learning experiences, perfectionism, and learning motivation. Depression and Anxiety, 25(9), 801-810. doi:10.1002/da.20334

Fairburn, C. G., \& Beglin, S. J. (1990). Studies of the epidemiology of bulimia nervosa. The American Journal of Psychiatry, 147(4), 401-408. doi:10.1176/ajp.147.4.401

Fairweather-Schmidt, A. K., \& Wade, T. D. (2015). Piloting a perfectionism intervention for pre-adolescent children. Behaviour Research and Therapy, 73, 67-73. doi:10.1016/j.brat.2015.07.004

Farstad, S. M., McGeown, L., \& von Ranson, K. M. (2016). Eating disorders and personality, 2004-2016: A systematic review and meta-analysis. Clinical Psychology Review, 46, 91-105. doi:10.1016/j.cpr.2016.04.005

Fedewa, B. A., Burns, L. R., \& Gomez, A. A. (2005). Positive and negative perfectionism and the shame/guilt distinction: Adaptive and maladaptive characteristics. Personality and Individual Differences, 38(7), 1609-1619. doi:10.1016/j.paid.2004.09.026

Ferreiro, F., Seoane, G., \& Senra, C. (2012). Gender-related risk and protective factors for depressive symptoms and disordered eating in adolescence: A 4-year longitudinal study. Journal of Youth and Adolescence, 41(5), 607-622. doi:10.1007/s10964-011-9718-7

Field, A. P., \& Gillett, R. (2010). How to do a meta-analysis. British Journal of Mathematical \& Statistical Psychology, 63(3), 665-694. doi:10.1348/000711010x502733

Fitzgerald, J., \& Curtis, C. (2015). Prevalence and characteristics of nonsuicidal self-injury in a NZ university sample. Paper presented at the NZPsS Annual Conference, Hamilton, New Zealand.

*Flamenbaum, R., \& Holden, R. R. (2007). Psychache as a mediator in the relationship between perfectionism and suicidality. Journal of Counseling Psychology, 54(1), 51-61. doi:10.1037/0022-0167.54.1.51

Flett, G. L., Coulter, L.-M., Hewitt, P. L., \& Nepon, T. (2011). Perfectionism, rumination, 
worry, and depressive symptoms in early adolescents. Canadian Journal of School Psychology, 26(3), 159-176. doi:10.1177/0829573511422039

*Flett, G. L., Goldstein, A. L., Hewitt, P. L., \& Wekerle, C. (2012). Predictors of deliberate self-harm behavior among emerging adolescents: An initial test of a self-punitiveness model. Current Psychology, 31(1), 49-64. doi:10.1007/s12144-012-9130-9

Flett, G. L., \& Hewitt, P. L. (2014). A proposed framework for preventing perfectionism and promoting resilience and mental health among vulnerable children and adolescents. Psychology in the Schools, 51(9), 899-912. doi:10.1002/pits

Flett, G. L., \& Hewitt, P. L. (2016). Still measuring perfectionism after all these years: Reflections and an introduction to the special issue on advances in the assessment of perfectionism. Journal of Psychoeducational Assessment, 34(7), 1-5. doi:10.1177/0734282916651540

Flett, G. L., Hewitt, P. L., Blankstein, K., \& O’Brien, S. (1991). Perfectionism and learned resourcefulness in depression and self-esteem. Personality and Individual Differences, 12(I), 61-68. doi:10.1016/0191-8869(91)90132-U

Flett, G. L., Hewitt, P. L., Blankstein, K. R., \& Pickering, D. (1998). Perfectionism in relation to attributions for success or failure. Current Psychology, 17(2-3), 249-262. doi:10.1007/s12144-998-1010-y

Flett, G. L., Hewitt, P. L., Boucher, D., Davidson, L., \& Munro, Y. (2000). The ChildAdolescent Perfectionism Scale: Development, validation, and association with adjustment. Unpublished manuscript, York University, Toronto, Ontario, Canada.

Flett, G. L., Hewitt, P. L., \& De Rosa, T. (1996). Dimensions of perfectionism, psychosocial adjustment, and social skills. Personality and Individual Differences, 20(2), 143-150. doi:10.1016/0191-8869(95)00170-0

Flett, G. L., Hewitt, P. L., \& Heisel, M. J. (2014). The destructiveness of perfectionism revisited : Implications for the assessment of suicide risk and the prevention of suicide. Review of General Psychology, 18(3), 156-172. doi:10.1037/gpr0000011

Flett, G. L., Hewitt, P. L., Oliver, J. J., \& Macdonald, S. (2002). Perfectionism in children and their parents: A developmental analysis. In G. L. Flett \& P. L. Hewitt (Eds.), Perfectionism: Theory, research, and treatment (pp. 89-132). Washington: American Psychological Association.

Fortune, S., Seymour, F., \& Lambie, I. (2005). Suicide behaviour in a clinical sample of children and adolescents in New Zealand. New Zealand Journal of Psychology, 34(3), 164-170. Retrieved from http://www.psychology.org.nz/wp-content/uploads/NZJP- 
Vol343-2005-4-Fortune.pdf

Fox, K. R., Franklin, J. C., Ribeiro, J. D., Kleiman, E. M., Bentley, K. H., \& Nock, M. K. (2015). Meta-analysis of risk factors for nonsuicidal self-injury. Clinical Psychology Review, 42, 156-167. doi:10.1016/j.cpr.2015.09.002

Franklin, J. C., Ribeiro, J. D., Fox, K. R., Bentley, K. H., Kleiman, E. M., Huang, X., ... Nock, M. K. (2016). Risk factors for suicidal thoughts and behaviors: A meta-analysis of 50 years of research. Psychological Bulletin. doi:10.1037/bul0000084

*Freudenstein, O., Valevski, A., Apter, A., Zohar, A., Shoval, G., Nahshoni, E., ... Zalsman, G. (2012). Perfectionism, narcissism, and depression in suicidal and nonsuicidal adolescent inpatients. Comprehensive Psychiatry, 53(6), 746-752. doi:10.1016/j.comppsych.2011.08.011

Frost, R. O., Heimberg, R. G., Holt, C. S., Mattia, J. I., \& Neubauer, A. L. (1993). A comparison of two measures of perfectionism. Personality and Individual Differences, 14(1), 119-126. doi:10.1016/0191-8869(93)90181-2

Frost, R. O., Lahart, C. M., \& Rosenblate, R. (1991). The development of perfectionism: A study of daughters and their parents. Cognitive Therapy and Research, 15(6), 469-489. doi:10.1007/BF01175730

Frost, R. O., Marten, P., Lahart, C., \& Rosenblate, R. (1990). The dimensions of perfectionism. Cognitive Therapy and Research, 14(5), 449-468. doi:10.1007/BF01172967

*Fujimori, A., Wada, Y., Yamashita, T., Choi, H., Nishizawa, S., Yamamoto, H., \& Fukui, K. (2011). Parental bonding in patients with eating disorders and self-injurious behavior. Psychiatry and Clinical Neurosciences, 65(3), 272-279. doi:10.1111/j.14401819.2011.02192.x

Fursland, A., Raykos, B. C., \& Steele, A. L. (2009). Module 3: What keeps perfectionism going? In Perfectionism in perspective (pp. 1-11). Perth, Western Australia: Centre for Clinical Intervention.

Garisch, J. A. (2010). Youth deliberate self-harm : Interpersonal and intrapersonal vulnerability factors, and constructions and attitudes within the social environment. (Unpublished doctoral thesis). Victoria University of Wellington, Wellington, New Zealand.

Garisch, J. A., \& Wilson, M. S. (2010). Vulnerabilities to deliberate self-harm among adolescents: The role of alexithymia and victimization. The British Journal of Clinical Psychology, 49(2), 151-162. doi:10.1348/014466509X441709 
Garisch, J. A., \& Wilson, M. S. (2015). Prevalence, correlates, and prospective predictors of non-suicidal self-injury among New Zealand adolescents: Cross-sectional and longitudinal survey data. Child and Adolescent Psychiatry and Mental Health, 9(1), 28. doi:10.1186/s13034-015-0055-6

Garner, D. M. (1991). Eating Disorder Inventory-2: Professional manual. Odessa, FL: Psychological Assessment Resources.

Garner, D. M., Olmsted, M. P., Polivy, J., Olmstead, M. P., \& Polivy, J. (1983). Development and validation of a multidimensional eating disorder inventory for anorexia nervosa and bulimia. International Journal of Eating Disorders, 2(2), 15-34. doi:10.1002/1098108X(198321)2:2<15::AID-EAT2260020203>3.0.CO;2-6

Gaudreau, P., \& Thompson, A. (2010). Testing a $2 \times 2$ model of dispositional perfectionism. Personality and Individual Differences, 48(5), 532-537. doi:10.1016/j.paid.2009.11.031

Gautreau, C. M., Sherry, S. B., Mushquash, A. R., \& Stewart, S. H. (2015). Is self-critical perfectionism an antecedent of or a consequence of social anxiety, or both ? A 12month , three-wave longitudinal study. Personality and Individual Differences, 82, 125 130. doi:10.1016/j.paid.2015.03.005

Gelabert, E., García-Esteve, L., Martín-Santos, R., Gutiérrez, F., Torres, A., \& Subirà, S. (2011). Psychometric properties of the Spanish version of the Frost Multidimensional Perfectionism Scale in women. Psicothema, 23(1), 133-139. Retrieved from https://www.ncbi.nlm.nih.gov/pubmed/21266154

Giles, D. (2002). Advanced research methods in psychology. New York, NY: Routledge. Glassman, L. H., Weierich, M. R., Hooley, J. M., Deliberto, T. L., \& Nock, M. K. (2007). Child maltreatment, non-suicidal self-injury, and the mediating role of self-criticism. Behaviour Research and Therapy, 45(10), 2483-2490. doi:10.1016/j.brat.2007.04.002

Glenn, C. R., \& Klonsky, E. D. (2009). Social context during non-suicidal self-injury indicates suicide risk. Personality and Individual Differences, 46(1), 25-29. doi:10.1016/j.paid.2008.08.020

Glenn, C. R., \& Klonsky, E. D. (2011). Prospective prediction of nonsuicidal self-injury: A 1year longitudinal study in young adults. Behavior Therapy, 42(4), 751-762. doi:10.1016/j.beth.2011.04.005

Glenn, C. R., \& Klonsky, E. D. (2013). Nonsuicidal self-injury disorder: An empirical investigation in adolescent psychiatric patients. Journal of Clinical Child \& Adolescent Psychiatry, 42(4), 496-507. doi:10.1080/15374416.2013.794699

Gotwals, J. K., Stoeber, J., Dunn, J. G. H., \& Stoll, O. (2012). Are perfectionistic strivings in 
sport adaptive? A systematic review of confirmatory, contradictory, and mixed evidence. Canadian Psychology, 53(4), 263-279. doi:10.1037/a0030288

Gratz, K. L. (2001). Measurement of deliberate self-harm: Preliminary data on the Deliberate Self-Harm Inventory. Journal of Psychopathology and Behavioral Assessment, 23(4), 253-263. doi:10.1023/A:1012779403943

Gratz, K. L., Conrad, S. D., \& Roemer, L. (2002). Risk factors for deliberate self-harm among college students. The American Journal of Orthopsychiatry, 72(1), 128-140. doi:10.1037//0002-9432.72.1.128

Groschwitz, R. C., Plener, P. L., Kaess, M., Schumacher, T., Stoehr, R., \& Boege, I. (2015). The situation of former adolescent self-injurers as young adults: A follow-up study. BMC Psychiatry, 15(1), 160-169. doi:10.1186/s12888-015-0555-1

Guerry, J. D., \& Prinstein, M. J. (2010). Longitudinal prediction of adolescent nonsuicidal self-injury: Examination of a cognitive vulnerability-stress model. Journal of Clinical Child \& Adolescent Psychology, 39(1), 77-89. doi:10.1080/15374410903401195

Ha, J. H., Lee, S. M., \& Puig, A. (2010). A reliability generalization study of the Frost Multidimensional Perfectionism Scale (F-MPS). Psychological Reports, 107, 95-112. doi:10.2466/03.09.20.PR0.107.4.95-112

Haimovitz, K., \& Dweck, C. S. (2016). What predicts children's fixed and growth intelligence mind-sets? Not their parents' views of intelligence but their parents' views of failure. Psychological Science, 27(6), 859-869. doi:10.1177/0956797616639727

Hair, J. F., Black, W. C., Babin, B. J., \& Anderson, R. E. (2009). Multivariate data analysis (7th ed.). New York: Prentice Hall/Pearson Education.

*Halpin, K., \& Lester, D. (2001). Hopelessness and perfectionism. Psychological Reports, 88(1), 252. doi:10.2466/pr0.88.1.252-252

Hamachek, D. E. (1978). Psychodynamics of normal and neurotic perfectionism. Psychology: A Journal of Human Behavior, 15(1), 27-33.

*Hamilton, T. K., \& Schweitzer, R. D. (2000). The cost of being perfect: Perfectionism and suicide ideation in university students. Australian and New Zealand Journal of Psychiatry, 34, 829-835. doi:10.1046/j.1440-1614.2000.00801.x

Hamza, C. A., Stewart, S. L., \& Willoughby, T. (2012). Examining the link between nonsuicidal self-injury and suicidal behavior: A review of the literature and an integrated model. Clinical Psychology Review, 32(6), 482-495. doi:10.1016/j.cpr.2012.05.003

Hamza, C. A., \& Willoughby, T. (2016). Nonsuicidal self-injury and suicidal risk among emerging adults. Journal of Adolescent Health, 59(4), 1-5. 
doi:10.1016/j.jadohealth.2016.05.019

Handley, A. K., Egan, S. J., Kane, R. T., \& Rees, C. S. (2014). The relationships between perfectionism, pathological worry and generalised anxiety disorder. BMC Psychiatry, 14(1), 98-106. doi:10.1186/1471-244X-14-98

Hankin, B. L., \& Abela, J. R. Z. (2011). Nonsuicidal self-injury in adolescence: Prospective rates and risk factors in a 21/2 year longitudinal study. Psychiatry Research, 186(1), 6570. doi:10.1016/j.psychres.2010.07.056

Hansen, E., Lundh, L.-G., Homman, A., \& Wångby-Lundh, M. (2009). Measuring mindfulness: Pilot studies with the Swedish versions of the Mindful Attention Awareness Scale and the Kentucky Inventory of Mindfulness Skills. Cognitive Behaviour Therapy, 38(1), 2-15. doi:10.1080/16506070802383230

*Harris, P. W., Pepper, C. M., \& Maack, D. J. (2008). The relationship between maladaptive perfectionism and depressive symptoms: The mediating role of rumination. Personality and Individual Differences, 44(1), 150-160. doi:10.1016/j.paid.2007.07.011

Hart, S. R., Musci, R. J., Ialongo, N., Ballard, E. D., \& Wilcox, H. C. (2013). Demographic and clinical characteristics of consistent and inconsistent longitudinal reporters of lifetime suicide attempts in adolescence through young adulthood. Depression and Anxiety, 30(10), 997-1004. doi:10.1002/da.22135

Hasking, P. A., Coric, S. J., Swannell, S., Martin, G., Thompson, H. K., \& Frost, A. D. J. (2010). Brief report: Emotion regulation and coping as moderators in the relationship between personality and self-injury. Journal of Adolescence, 33(5), 767-773. doi:10.1016/j.adolescence.2009.12.006

Hasking, P., Whitlock, J., Voon, D., \& Rose, A. (2016). A cognitive-emotional model of NSSI: Using emotion regulation and cognitive processes to explain why people selfinjure. Cognition and Emotion, 30(8), 1-14. doi:10.1080/02699931.2016.1241219

Hassan, S., Flett, G. L., Ganguli, R., \& Hewitt, P. L. (2014). Perfectionistic self-presentation and suicide in a young woman with major depression and psychotic features. Case Reports in Psychiatry, 2014, 1-6. doi:10.1155/2014/901981

Hatcher, S., Sharon, C., \& Collins, N. (2009). Epidemiology of intentional self-harm presenting to four district health boards in New Zealand over 12 months, and comparison with official data. Australian an New Zealand Journal of Psychiatry, 43(7), 659-665. doi:10.1080/00048670902970833

Hawkins, C. C., Watt, H. M. G., \& Sinclair, K. E. (2006). Psychometric properties of the Frost Multidimensional Perfectionism Scale with Australian adolescent girls 
clarification of multidimensionality and perfectionist typology. Educational and Psychological Measurement, 66(6), 1001-1022. doi:10.1177/0013164405285909

Hawton, K., Bergen, H., Kapur, N., Cooper, J., Steeg, S., Ness, J., \& Waters, K. (2012). Repetition of self-harm and suicide following self-harm in children and adolescents: Findings from the Multicentre Study of Self-harm in England. Journal of Child Psychology and Psychiatry and Allied Disciplines, 53(12), 1212-1219. doi:10.1111/j.1469-7610.2012.02559.x

Hawton, K., \& James, A. (2005). Suicide and deliberate self harm in young people. BMJ (Clinical Research Ed.), 330(7496), 891-4. doi:10.1136/bmj.330.7496.891

Hawton, K., Saunders, K. E., \& O’Connor, R. C. (2012). Self-harm and suicide in adolescents. The Lancet, 379(9834), 2373-2382. doi:10.1016/S0140-6736(12)60322-5

Headmasters' and Headmistresses' Conference. (2015). First data on mental health trends in independent schools shows pupils are kinder to each other but harder on themselves. Headmasters' and Headmistresses' Conference: Leading Independent Schools.

Heath, N. L., Toste, J. R., \& MacPhee, S. (2014). Prevention of nonsuicidal self-injury. In M. K. Nock (Ed.), The oxford handbook of suicide and self-injury (pp. 397-408). New York, NY: Oxford University Press.

Heath, N. L., Toste, J. R., Nedecheva, T., \& Charlebois, A. (2008). An examination of nonsuicidal self-injury among college students. Journal of Mental Health Counseling, 30(2), 137-156. doi:10.17744/mehc.30.2.8p879p3443514678

Hellmann, E. (2016). Keeping up appearances: Perfectionism and perfectionistic selfpresentation on social media. (Unpublished doctoral thesis). DePauw University, Greencastle, Indiana, USA.

Henrich, J., Heine, S. J., \& Norenzayan, A. (2010). The weirdest people in the world? The Behavioral and Brain Sciences, 33(2-3), 61-83-135. doi:10.1017/S0140525X0999152X

*Hewitt, P. L., Caelian, C. F., Chen, C., \& Flett, G. L. (2014). Perfectionism, stress, daily hassles, hopelessness, and suicide potential in depressed psychiatric adolescents. Journal of Psychopathology and Behavioral Assessment, 36(4), 663-674. doi:10.1007/s10862014-9427-0

Hewitt, P. L., Caelian, C. F., Flett, G. L., Sherry, S. B., Collins, L., \& Flynn, C. A. (2002). Perfectionism in children: Associations with depression, anxiety, and anger. Personality and Individual Differences, 32(6), 1049-1061. doi:10.1016/S0191-8869(01)00109-X

Hewitt, P. L., \& Flett, G. L. (1991). Perfectionism in the self and social contexts: Conceptualization, assessment, and association with psychopathology. Journal of 
Personality and Social Psychology, 60(3), 456-470. doi:10.1037/0022-3514.60.3.456

Hewitt, P. L., Flett, G. L., \& Ediger, E. (1995). Perfectionism traits and perfectionistic selfpresentation in eating disorder attitudes, characteristics, and symptoms. International Journal of Eating Disorders, 18(4), 317-326. doi:10.1002/1098108X(199512)18:4<317::AID-EAT2260180404>3.0.CO;2-2

Hewitt, P. L., Flett, G. L., Sherry, S. B., \& Caelian, C. (2006). Trait perfectionism dimensions and suicide behavior. In T. Ellis (Ed.), Cognition and suicide: Theory, research, and therapy (pp. 215-235). Washington, DC: American Psychological Association.

*Hewitt, P. L., Flett, G. L., \& Turnbull-Donovan, W. (1992). Perfectionism and suicide potential. British Journal of Clinical Psychology, 31(2), 181-190. doi:10.1111/j.20448260.1992.tb00982.x

*Hewitt, P. L., Flett, G. L., \& Weber, C. (1994). Dimensions of perfectionism and suicide ideation. Cognitive Therapy and Research, 18(5), 439-460. doi:10.1007/BF02357753

*Hewitt, P. L., Newton, J., Flett, G. L., \& Callander, L. (1997). Perfectionism and suicide ideation in adolescent psychiatric patients. Journal of Abnormal Child Psychology, 25(2), 95-101. doi:10.1023/A:1025723327188

*Hewitt, P. L., Norton, G. R., Flett, G. L., Callander, L., \& Cowan, T. (1998). Dimensions of perfectionism, hopelessness, and attempted suicide in a sample of alcoholics. Suicide and Life-Threatening Behavior, 28(4), 395-406. doi:10.1111/j.1943278X.1998.tb00975.x

Hill, A. P., \& Davis, P. A. (2014). Perfectionism and emotion regulation in coaches: A test of the 2x2 model of dispositional perfectionism. Motivation and Emotion, 38(5), 715-726. doi:10.1007/s11031-014-9404-7

Hill, A. P., Witcher, C. S. G., Gotwals, J. K., \& Leyland, A. F. (2015). The predictive ability of perfectionistic traits and self-presentational styles in relation to exercise dependence. Personality and Individual Differences, 86, 176-183. doi:10.1016/j.paid.2015.06.015

Hill, R. W., Huelsman, T., Furr, M., Kibler, J., Vicente, B., \& Kennedy, C. (2004). A new measure of perfectionism: The Perfectionism Inventory. Journal of Personality Assessment, 82(1), 80-91. doi:10.1207/s15327752jpa8201_13

Hilt, L. M., Nock, M. K., Lloyd-Richardson, E. E., \& Prinstein, M. J. (2008). Longitudinal study of nonsuicidal self-injury among young adolescents: Rates, correlates, and preliminary test of an interpersonal model. The Journal of Early Adolescence, 28(3), 455-469. doi:10.1177/0272431608316604

*Hoff, E. R., \& Muehlenkamp, J. J. (2009). Nonsuicidal self-injury in college students: The 
role of perfectionism and rumination. Suicide and Life-Threatening Behavior, 39(6), 576-587. doi:10.1521/suli.2009.39.6.576

Holding, T. A., Buglass, D., Duffy, J. C., \& Kreitman, N. (1977). Parasuicide in Edinburg: A seven-year review 1968-74. The British Journal of Psychiatry, 130(6), 534-543. doi:10.1192/bjp.130.6.534

Hopkinson, R. A., \& Lock, J. (2004). Athletics, perfectionism, and disordered eating. Eating and Weight Disorders, 9(2), 99-106. doi:10.1007/BF03325052

Howe-Martin, L. S., Murrell, A. R., \& Guarnaccia, C. A. (2012). Repetitive nonsuicidal selfinjury as experiential avoidance among a community sample of adolescents. Journal of Clinical Psychology, 68(7), 809-829. doi:10.1002/jclp.21868

Hu, L., \& Bentler, P. M. (1999). Cutoff criteria for fit indexes in covariance structure analysis: Conventional criteria versus new alternatives. Structural Equation Modeling: A Multidisciplinary Journal, 6(1), 1-55. doi:10.1080/10705519909540118

Huedo-Medina, T. B., Sánchez-Meca, J., Marín-Martínez, F., \& Botella, J. (2006). Assessing heterogeneity in meta-analysis: Q statistic or I2 index? Psychological Methods, 11(2), 193-206. doi:10.1037/1082-989X.11.2.193

Huggins, L., Davis, M., Rooney, R., \& Kane, R. (2008). Socially prescribed and self-oriented perfectionism as predictors of depressive diagnosis in preadolescents. Australian Journal of Guidance \& Counselling, 18(2), 182-194. doi:10.1375/ajgc.18.2.182

*Hunter, E. C., \& O’Connor, R. C. (2003). Hopelessness and future thinking in parasuicide: The role of perfectionism. The British Journal of Clinical Psychology, 42(4), 355-365. doi:10.1348/014466503322528900

In-Albon, T., Burli, M., Ruf, C., \& Schmid, M. (2013). Non-suicidal self-injury and emotion regulation: A review on facial emotion recognition and facial mimicry. Child and Adolescent Psychiatry and Mental Health, 7(1), 1-11. doi:10.1186/1753-2000-7-5

International Society for the Study of Self-Injury. (2007). Fast Facts. Retrieved from http://itriples.org/redesadmin15/fast-facts/

*Izadi, M. (2014). Perfectionism, shame and non-suicidal self-injury. (Unpublished doctoral thesis). California School of Professional Psychology, San Diego, California, USA.

Jacobson, C. M., \& Gould, M. (2007). The epidemiology and phenomenology of non-suicidal self-injurious behavior among adolescents: A critical review of the literature. Archives of Suicide Research, 11(2), 129-147. doi:10.1080/13811110701247602

Jacobson, C. M., \& Luik, C. (2014). Epidemiology and sociocultural aspects of non-suicidal self-injury and eating disorders. In J. J. Muehlenkamp \& L. Claes (Eds.), Non-suicidal 
self-injury in eating disorders (pp. 19-35). Heidelberg: Springer Berlin Heidelberg. Jacobson, C. M., Muehlenkamp, J. J., Miller, A. L., \& Turner, J. B. (2008). Psychiatric impairment among adolescents engaging in different types of deliberate self-harm. Journal of Clinical Child \& Adolescent Psychology, 37(2), 363-375. doi:10.1080/15374410801955771

*James, K., Verplanken, B., \& Rimes, K. A. (2015). Self-criticism as a mediator in the relationship between unhealthy perfectionism and distress. Personality and Individual Differences, 79, 123-128. doi:10.1016/j.paid.2015.01.030

*Jeglic, E. L., Pepper, C. M., Vanderhoff, H. A., \& Ryabchenko, K. A. (2007). An analysis of suicidal ideation in a college sample. Archives of Suicide Research, 11(1), 41-56. doi:10.1080/13811110600897176

Jeppson, J., Richards, P. S., Hardman, R., \& Granley, H. (2003). Binge and purge processes in bulimia nervosa: A qualitative investigation. Eating Disorders, 11(2), 115-128. doi:10.1080/10640260390199307

Johnson, J., Panagioti, M., Bass, J., Ramsey, L., \& Harrison, R. (2017). Resilience to emotional distress in response to failure, error or mistakes: A systematic review. Clinical Psychology Review, 52, 19-42. doi:10.1016/j.cpr.2016.11.007

Joiner, T. E. (2005). Why people die by suicide. Cambridge, MA: Harvard University Press.

Joiner, T. E., Ribeiro, J. D., \& Silva, C. (2012). Nonsuicidal self-injury, suicidal behavior, and their co-occurrence as viewed through the lens of the interpersonal theory of suicide. Current Directions in Psychological Science, 21(5), 342-347. doi:10.1177/0963721412454873

Joiner, T. E., \& Schmidt, N. B. (1995). Dimensions of perfectionism, life stress, and depressed and anxious symptoms: Prospective support for diathesis-stress but not specific vulnerability among male undergraduates. Journal of Social and Clinical Psychology, 14(2), 165-183. doi:10.1521/jscp.1995.14.2.165

Jones, R. G. (1969). A factored measure of Ellis' irrational belief system with personality and maladjustment correlates. (Unpublished doctoral thesis). Texas Technological College, Texas, USA.

Jose, P. E. (2013). ModGraph: A programme to compute cell means for the graphical display of moderational analyses. Wellington: Victoria University of Wellington. Retrieved from http://pavlov.psyc.vuw.ac.nz/paul-jose/modgraph/

*Jung, J. Y. (2013). The cognitive processes associated with occupational/career indecision: A model for gifted adolescents. Journal for the Education of the Gifted, 36(4), 433-460. 
doi:10.1177/0162353213506067

Kaiser, H. (1974). An index of factorial simplicity. Psychometrika, 39(1), 31-36. doi:10.1007/BF02291575

Kantack, W. (2014). The relationships between alexythymia, perfectionism, self esteem, ethnicity and body image disturbance. (Unpublished doctoral thesis). University of Detroit Mercy, Detroit, Michigan, USA.

*Kawamura, K. Y., \& Frost, R. O. (2004). Self-concealment as a mediator in the relationship between perfectionism and psychological distress. Cognitive Therapy and Research, 28(2), 183-191. doi:10.1023/b:cotr.0000021539.48926.c1

*Kawamura, K. Y., Hunt, S. L., Frost, R. O., \& DiBartolo, P. M. (2001). Perfectionism, anxiety, and depression: Are the relationships independent? Cognitive Therapy and Research, 25(3), 291-301. doi:10.1023/A:1010736529013

Kelly, J. D. (2015). Your best life: Perfectionism, the bane of happiness. Clinical Orthopaedics and Related Research, 473(10), 3108-3111. doi:10.1007/s11999-0154279-9

Kenny, D. (1975). Cross-lagged panel correlation: A test for spuriousness. Psychological Bulletin, 82(6), 887-903. doi:10.1037/0033-2909.82.6.887

Kenny, D. A. (2005). Cross-lagged panel design. Encyclopedia of Statistics in Behavioral Science, 1, 450-451. doi:10.1002/0470013192.bsa156

*Khawaja, N. G., \& Armstrong, K. a. (2005). Factor structure and psychometric properties of the Frost Multidimensional Perfectionism Scale: Developing shorter versions using an Australian sample. Australian Journal of Psychology, 57(2), 129-138. doi:10.1080/10519990500048611

Kiamanesh, P., Dyregrov, K., Haavind, H., \& Dieserud, G. (2014). Suicide and perfectionism: A psychological autopsy study of non-clinical suicides. Omega, 69(4), 381-99. doi:10.2190/OM.69.4.c

Kingi, T. (2015). Understanding self-injury in rangatahi Māori. Paper presented at the 19th Biennial conference of the Australasian Human Development Association. Wellington, New Zealand.

*Kirtley, O., O’Connor, R. C., \& O’Carroll, R. (2015). Hurting inside and out? Emotional and physical pain in self-harm ideation and enactment. International Journal of Cognitive Therapy, 8(2), 156-171. doi:10.1521/ijct.2015.8.2.156

Klibert, J. J., Lamis, D. a., Naufel, K., Yancey, C. T., \& Lohr, S. (2015). Associations between perfectionism and generalized anxiety: Examining cognitive schemas and 
gender. Journal of Rational-Emotive \& Cognitive-Behavior Therapy, 33(2), 160-178. doi:10.1007/s10942-015-0208-9

*Klibert, J. J., Langhinrichsen-Rohling, J., \& Saito, M. (2005). Adaptive and maladaptive aspects of self-oriented versus socially prescribed perfectionism. Journal of College Student Development, 46(2), 141-156. doi:10.1353/csd.2005.0017

Klonsky, E. D. (2007). The functions of deliberate self-injury: A review of the evidence. Clinical Psychology Review, 27(2), 226-239. doi:10.1016/j.cpr.2006.08.002

Klonsky, E. D., \& Glenn, C. R. (2009). Assessing the functions of non-suicidal self-injury: Psychometric properties of the Inventory of Statements about Self-injury (ISAS). Journal of Psychopathology and Behavioral Assessment, 31(3), 215-219. doi:10.1007/s10862-008-9107-z

Klonsky, E. D., May, A. M., \& Glenn, C. R. (2013). The relationship between nonsuicidal self-injury and attempted suicide: Converging evidence from four samples. Journal of Abnormal Psychology, 122(1), 231-237. doi:10.1037/a0030278

Klonsky, E. D., \& Muehlenkamp, J. J. (2007). Self-injury: A research review for the practitioner. Journal of Clinical Psychology, 63(11), 1045-1056. doi:10.1002/jclp.20412

Klonsky, E. D., Victor, S. E., \& Saffer, B. (2007). Nonsuicidal self-injury: What we know and what we need to know. The Canadian Journal of Psychiatry, 52(11), 275-276. doi:10.1177/070674371405901101

Kornblum, M., \& Ainley, M. (2005). Perfectionism and the gifted: A study of an Australian school sample. International Education Journal, 6(2), 232-239. Retrieved from http://files.eric.ed.gov/fulltext/EJ854975.pdf

Kring, A. M., \& Gordon, A. H. (1998). Sex differences in emotion: Expression, experience, and physiology. Journal of Personality and Social Psychology, 74(3), 686-703. doi:10.1037//0022-3514.74.3.686

*Kubal, A. (2005). Perfectionism among women seeking help for deliberate self-harm and/or eating disorders: A comparitive study. (Unpublished doctoral thesis). Michigan State University, Michigan, USA. Retrieved from http://journals.cambridge.org/abstract_S0165115300023299

Kumar, G., Pepe, D., \& Steer, R. A. (2004). Adolescent psychiatric inpatients self-reported reasons for cutting themselves. The Journal of Nervous and Mental Disease, 192(12), 830-836. doi:10.1097/01.nmd.0000146737.18053.d2

*Laird, R. D. (2011). Correlates and antecedents of parental psychological control in early 
adolescence. Parenting: Science and Practice, 11(1), 72-86.

doi:10.1080/15295192.2011.539510

Landman-Peeters, K. M. C., Hartman, C. A., Van Der Pompe, G., Den Boer, J. A., Minderaa, R. B., \& Ormel, J. (2005). Gender differences in the relation between social support, problems in parent-offspring communication, and depression and anxiety. Social Science and Medicine, 60(11), 2549-2559. doi:10.1016/j.socscimed.2004.10.024

Langlands, R. L. (2012). Does non-suicidal self-injury function primarily as an experientially avoidant behaviour within Aotearoa New Zealand? (Unpublished doctoral thesis).Victoria University of Wellington, Wellington, New Zealand. Retrieved from http://researcharchive.vuw.ac.nz/handle/10063/2153\%5Cnhttp://researcharchive.vuw.ac. nz/xmlui/bitstream/handle/10063/2153/thesis.pdf?sequence=2

Latimer, S., Meade, T., \& Tennant, A. (2013). Measuring engagement in deliberate self-harm behaviours: Psychometric evaluation of six scales. BMC Psychiatry, 13(4), 1-11. doi:10.1186/1471-244X-13-4

*Lavender, J. M., Wonderlich, S. A., Crosby, R. D., Engel, S. G., Mitchell, J. E., Crow, S. J., ... Le Grange, D. (2013). Personality-based subtypes of anorexia nervosa: Examining validity and utility using baseline clinical variables and ecological momentary assessment. Behaviour Research and Therapy, 51(8), 512-517. doi:10.1016/j.brat.2013.05.007

Laye-Gindhu, A., \& Schonert-Reichl, K. a. (2005). Nonsuicidal self-harm among community adolescents: Understanding the "whats" and "whys" of self-harm. Journal of Youth and Adolescence, 34(5), 447-457. doi:10.1007/s10964-005-7262-z

Lee, D., \& Park, H. (2011). Cross-cultural validity of the Frost Multidimensional Perfectionism Scale in Korea. The Counseling Psychologist, 39(2), 320-345. doi:10.1177/0011000010365910

*Lehmann, I. S., \& Konstam, V. (2011). Growing up perfect: Perfectionism, problematic internet use, and career indecision in emerging adults. Journal of Counseling \& Development, 89(2), 155-162. doi:10.1002/j.1556-6678.2011.tb00073.x

Leong, C. H., Wu, A. M. S., \& Poon, M. M. (2014). Measurement of perceived functions of non-suicidal self-injury for Chinese adolescents. Archives of Suicide Research, 18(2), 37-41. doi:10.1080/13811118.2013.824828

*Levinson, C. a., Rodebaugh, T. L., Shumaker, E. a., Menatti, A. R., Weeks, J. W., White, E. K., ... Liebowitz, M. R. (2015). Perception matters for clinical perfectionism and social anxiety. Journal of Anxiety Disorders, 29, 61-71. doi:10.1016/j.janxdis.2014.11.002 
*Levinson, C. a., Rodebaugh, T. L., White, E. K., Menatti, A. R., Weeks, J. W., Iacovino, J. M., \& Warren, C. S. (2013). Social appearance anxiety, perfectionism, and fear of negative evaluation. Distinct or shared risk factors for social anxiety and eating disorders? Appetite, 67, 125-133. doi:10.1016/j.appet.2013.04.002

Lewis, S., \& Clarke, M. (2001). Forest plots: trying to see the wood and the trees. BMJ, 322(73), 1479-1480. doi:10.1136/bmj.322.7300.1479

Lewis, S. P., \& Arbuthnott, A. E. (2012). Nonsuicidal self-injury: Characteristics, functions, and strategies. Journal of College Student Psychotherapy, 26(3), 185-200. doi:10.1080/87568225.2012.685853

Libby, S., Reynolds, S., Derisley, J., \& Clark, S. (2004). Cognitive appraisals in young people with obsessive-compulsive disorder. Journal of Child Psychology and Psychiatry and Allied Disciplines, 45(6), 1076-1084. doi:10.1111/j.1469-7610.2004.t01-1-00300.x

Lindholm, T., Bjärehed, J., \& Lundh, L.-G. (2011). Functions of nonsuicidal self-injury among young women in residential care: A pilot study with the Swedish version of the Inventory of Statements about Self-Injury. Cognitive Behaviour Therapy, 40(3), 183189. doi:10.1080/16506073.2011.565791

Lloyd-Richardson, E. E. (2010). Non-suicidal self-injury in adolescents. The Prevention Researcher, 17(1), 3-7. doi:10.1037/e515372010-001

Lloyd-Richardson, E. E., Lewis, S. P., Whitlock, J., Rodham, K., \& Schatten, H. T. (2015).

Research with adolescents who engage in non-suicidal self-injury: Ethical considerations and challenges. Child and Adolescent Psychiatry and Mental Health, 9(1), 37-51. doi:10.1186/s13034-015-0071-6

Lloyd-Richardson, E. E., Nock, M. K., \& Prinstein, M. J. (2009). Functions of adolescent nonsuicidal self-injury. In M. Nixon \& N. Heath (Eds.), Self-injury in youth: The essential guide to assessment and intervention (pp. 29-41). New York, NY: Routledge.

Lloyd-Richardson, E. E., Perrine, N., Dierker, L., \& Kelley, M. L. (2007). Characteristics and functions of non-suicidal self-injury in a community sample of adolescents. Psychological Medicine, 37(8), 1183-1192. doi:10.1017/S003329170700027X

Lloyd, E., Kelly, M., \& Hope, T. (1997). Self-mutilation in a community sample of adolescents: Descriptive characteristics and provisional prevalence rates. Paper presented at the Annual Meeting of the Society for Behavioral Medicine. New Orleans, LA.

*Lombardo, C., Mallia, L., Battagliese, G., Grano, C., \& Violani, C. (2013). Perfectionism mediates the relationship between insomnia and depressive symptoms. Sleep and 
Biological Rhythms, 11(2), 90-98. doi:10.1111/sbr.12009

Longbottom, J. L., Robert Grove, J., \& Dimmock, J. A. (2010). An examination of perfectionism traits and physical activity motivation. Psychology of Sport and Exercise, 11(6), 574-581. doi:10.1016/j.psychsport.2010.06.007

Lucassen, M. F. G., Merry, S. N., Robinson, E. M., Denny, S., Clark, T., Ameratunga, S., ... Rossen, F. V. (2011). Sexual attraction, depression, self-harm, suicidality and helpseeking behaviour in New Zealand secondary school students. The Australian and New Zealand Journal of Psychiatry, 45(5), 376-383. doi:10.3109/00048674.2011.559635

Lundh, L. G., Wångby-Lundh, M., \& Bjärehed, J. (2011). Deliberate self-harm and psychological problems in young adolescents: Evidence of a bidirectional relationship in girls. Scandinavian Journal of Psychology, 52(5), 476-483. doi:10.1111/j.14679450.2011.00894.x

Lundh, L., Karim, J., \& Quilisch, E. V. a. (2007). Deliberate self-harm in 15-year-old adolescents: A pilot study with a modified version of the Deliberate Self-Harm Inventory. Scandinavian Journal of Psychology, 48(1), 33-41. doi:10.1111/j.14679450.2006.00567.x

*Luyckx, K., Gandhi, A., Bijttebier, P., \& Claes, L. (2015). Non-suicidal self-injury in female adolescents and psychiatric patients: A replication and extension of the role of identity formation. Personality and Individual Differences, 77, 91-96. doi:10.1016/j.paid.2014.12.057

*Luyckx, K., Soenens, B., Goossens, L., Beckx, K., \& Wouters, S. (2008). Identity exploration and commitment in late adolescence: Correlates of perfectionism and mediating mechanisms on the pathway to well-being. Journal of Social and Clinical Psychology, 27(4), 336-361. doi:10.1521/jscp.2008.27.4.336

Lynch, T., \& Cozza, C. (2009). Behvaiour therapy for nonsuicidal self-injury. In M. K. Nock (Ed.), Understanding nonsuicidal self-injury: Origins, assessment, and treatment (pp. 221-250). Washington, DC: American Psychological Association.

*Lynd-Stevenson, R. M., \& Hearne, C. (1999). Perfectionism and depressive affect: The pros and cons of being a perfectionist. Personality and Individual Differences, 26(3), 549562. doi:10.1016/S0191-8869(98)00170-6

Lyubomirsky, S., Layous, K., Chancellor, J., \& Nelson, S. K. (2015). Thinking about rumination: The scholarly contributions and intellectual legacy of Susan NolenHoeksema. Annual Review of Clinical Psychology, 11(1), 1-22. doi:10.1146/annurevclinpsy-032814-112733 
MacCallum, R. (1990). The need for alternative measures of fit in covariance structure modeling. Multivariate Behavioral Research, 25(2), 157-162. doi:10.1207/s15327906mbr2502_2

*Malkina-Pykh, I. G. (2012). Effectiveness of rhythmic movement therapy for disordered eating behaviors and obesity. The Spanish Journal of Psychology, 15(3), 1371-1387. doi:10.5209/rev_SJOP.2012.v15.n3.39422

Manca, M., Presaghi, F., \& Cerutti, R. (2014). Clinical specificity of acute versus chronic self-injury: Measurement and evaluation of repetitive non-suicidal self-injury. Psychiatry Research, 215, 111-119. doi:10.1016/j.psychres.2013.10.010

Mars, B., Cornish, R., Heron, J., Boyd, A., Crane, C., Hawton, K., ... Gunnell, D. (2016). Using data linkage to investigate inconsistent reporting of self-harm and questionnaire non-response. Archives of Suicide Research, 20(2), 1-29. doi:10.1080/13811118.2015.1033121

Marsh, H. W., Balla, J. R., \& McDonald, R. P. (1988). Goodness-of-fit indexes in confirmatory factor analysis: The effect of sample size. Psychological Bulletin, 103(3), 391. doi:10.1037/0033-2909.103.3.391

Marshall, S. K., Tilton-Weaver, L. C., \& Stattin, H. (2013). Non-suicidal self-injury and depressive symptoms during middle adolescence: A longitudinal analysis. Journal of Youth and Adolescence, 42(8), 1234-1242. doi:10.1007/s10964-013-9919-3

Mazur, J. E. (2002). Learning and behavior. New Jersey: Prentice Hall/Pearson Education. McAllister, M. (2003). Multiple meanings of self harm: A critical review. International Journal of Mental Health Nursing, 12(3), 177-185. doi:10.1046/j.14400979.2003.00287.x

McAuliffe, C., Corcoran, P., Keeley, H. S., Arensman, E., Bille-Brahe, U., De Leo, D., ... Wasserman, D. (2005). Problem-solving ability and repetition of deliberate self-harm: A multicentre study. Psychological Medicine, 36(1), 45-55. doi:10.1017/S0033291705005945

McClure, J., Meyer, L. H., Garisch, J., Fischer, R., Weir, K. F., \& Walkey, F. H. (2011). Students' attributions for their best and worst marks: Do they relate to achievement? Contemporary Educational Psychology, 36(2), 71-81. doi:10.1016/j.cedpsych.2010.11.001

McDonald, R. (1985). Factor analysis and related methods. Hillsdale, NJ: Erlbaum.

McGrath, D. S., Sherry, S. B., Stewart, S. H., Mushquash, A. R., Allen, S. L., Nealis, L. J., \& Sherry, D. L. (2012). Reciprocal relations between self-critical perfectionism and 
depressive symptoms: Evidence from a short-term, four-wave longitudinal study. Canadian Journal of Behavioural Science, 44(3), 169-181. doi:10.1037/a0027764

*Menatti, A. R., Weeks, J. W., Levinson, C., \& McGowan, M. M. (2013). Exploring the relationship between social anxiety and bulimic symptoms: Mediational effects of perfectionism among females. Cognitive Therapy and Research, 37(5), 914-922. doi:10.1007/s10608-013-9521-8

Miller-Slough, R. L., \& Dunsmore, J. C. (2016). Parent and friend emotion socialization in adolescence: Associations with psychological adjustment. Adolescent Research Review, l(4), 287-305. doi:10.1007/s40894-016-0026-z

Miller, A. L., Muehlenkamp, J. J., \& Jacobson, C. M. (2008). Fact or fiction: Diagnosing borderline personality disorder in adolescents. Clinical Psychology Review, 28(6), 969981. doi:10.1016/j.cpr.2008.02.004

Miller, A. L., Muehlenkamp, J. J., \& Jacobson, C. M. (2009). Special issues in treating adolescent self-injury. In M. K. Nock (Ed.), Understanding nonsuicidal self-injury: Origins, assessment, and treatment (pp. 251-271). Washington, DC: American Psychological Association.

Milyavskaya, M., Harvey, B., Koestner, R., Powers, T., Rosenbaum, J., Ianakieva, I., \& Prior, A. (2014). Affect across the year: How perfectionism influences the pattern of university students' affect across the calendar year. Journal of Social \& Clinical Psychology, 33(2), 124-142. doi:10.1521/jscp.2014.33.2.124

Ministry of Health. (2015). Suicide facts: Deaths and intentional self-harm hospitalisations 2012. Wellington: Ministry of Health. Retrieved from http://www.health.govt.nz/publication/suicide-facts-deaths-and-intentional-self-harmhospitalisations-2012

*Miskey, H. M., Hill, R. W., \& Heulsman, T. (2012). Big five traits and perfectionism are risk factors for nonsuicidal cutting. Psychology and Behavioral Sciences, 1(1), 1-7. doi:10.11648/j.pbs.20120101.11

Mitzman, S. F., Slade, P., \& Dewey, M. E. (1994). Preliminary development of a questionnaire designed to measure neurotic perfectionism in the eating disorders. Journal of Clinical Psychology, 50(4), 516-22. doi:10.1002/10974679(199407)50:4<516::AID-JCLP2270500406>3.0.CO;2-0

Moher, D., Liberati, A., Tetzlaff, J., \& Altman, D. G. (2009). Preferred reporting items for systematic reviews and meta-analyses: The PRISMA statement. Physical Therapy, 89(9), 873-880. doi:10.1136/bmj.b2535 
Mohl, B., \& Skandsen, A. (2012). The prevalence and distribution of self-harm among Danish high school students. Personality and Mental Health, 6(2), 147-155. doi:10.1002/pmh.191

Moreira, D., Almeida, F., Pinto, M., Segarra, P., \& Barbosa, F. (2015). Data concerning the psychometric properties of the Behavioral Inhibition/Behavioral Activation Scales for the Portuguese population. Psychological Assessment, 27(3), 1117-1122. doi:10.1037/pas0000108

*Moretz, M. W., \& McKay, D. (2009). The role of perfectionism in obsessive-compulsive symptoms: "Not just right" experiences and checking compulsions. Journal of Anxiety Disorders, 23(5), 640-644. doi:10.1016/j.janxdis.2009.01.015

Moroz, M., \& Dunkley, D. M. (2015). Self-critical perfectionism and depressive symptoms: Low self-esteem and experiential avoidance as mediators. Personality and Individual Differences, 87, 174-179. doi:10.1016/j.paid.2015.07.044

Morris, L., \& Lomax, C. (2014). Review: Assessment, development, and treatment of childhood perfectionism: A systematic review. Child and Adolescent Mental Health, 19(4), 225-234. doi:10.1111/camh.12067

Muehlenkamp, J. J. (2006). Empirically supported treatments and general therapy guidelines for non-suicidal self-injury. Journal of Mental Health Counseling, 28(2), 166-185. doi:10.17744/mehc.28.2.6w61cut21xjdg3m7

Muehlenkamp, J. J. (2014). Distinguishing between suicidal and nonsuicidal self-injury. In M. K. Nock (Ed.), The oxford handbook of suicide and self-injury (pp. 23-46). New York, NY: Oxford University Press.

Muehlenkamp, J. J., Bagge, C. L., Tull, M. T., \& Gratz, K. L. (2013). Body regard as a moderator of the relation between emotion dysregulation and nonsuicidal self-injury. Suicide and Life-Threatening Behavior, 43(5), 479-493. doi:10.1111/sltb.12032

Muehlenkamp, J. J., \& Brausch, A. M. (2016). Reconsidering criterion A for the diagnosis of Non-Suicidal Self-Injury Disorder. Journal of Psychopathology and Behavioral Assessment, 38(4), 1-12. doi:10.1007/s10862-016-9543-0

Muehlenkamp, J. J., Brausch, A., Quigley, K., \& Whitlock, J. (2013). Interpersonal features and functions of nonsuicidal self-injury. Suicide and Life-Threatening Behavior, 43(1), 67-80. doi:10.1111/j.1943-278X.2012.00128.x

Muehlenkamp, J. J., Claes, L., Havertape, L., \& Plener, P. L. (2012). International prevalence of adolescent non-suicidal self-injury and deliberate self-harm. Child and Adolescent Psychiatry and Mental Health, 6(10), 1-9. doi:10.1186/1753-2000-6-10 
Muehlenkamp, J. J., \& Gutierrez, P. M. (2004). An investigation of differences between selfinjurious behavior and suicide attempts in a sample of adolescents. Suicide \& LifeThreatening Behavior, 34(1), 12-23. doi:10.1521/suli.34.1.12.27769

Muehlenkamp, J. J., \& Kerr, P. L. (2010). Untangling a complex web : How non-suicidal self-injury and suicide attempts differ. The Prevention Researcher, 17(1), 8-11. doi:10.1037/e515372010-002

Muehlenkamp, J. J., Swenson, L. P., Batejan, K. L., \& Jarvi, S. M. (2014). Emotional and behavioral effects of participating in an online study of nonsuicidal self-injury: An experimental analysis. Clinical Psychological Science, 3(1), 26-37. doi: $10.1177 / 2167702614531579$

Muehlenkamp, J. J., Williams, K. L., Gutierrez, P. M., \& Claes, L. (2009). Rates of nonsuicidal self-injury in high school students across five years. Archives of Suicide Research, 13(3), 317-329. doi:10.1080/13811110903266368

*Muyan, M., \& Chang, E. C. (2015). Perfectionism as a predictor of suicidal risk in Turkish college students: Does loneliness contribute to further risk? Cognitive Therapy and Research, 39(6), 776-784. doi:10.1007/s10608-015-9711-7

Nada-Raja, S., Skegg, K., Langley, J., Morrison, D., \& Sowerby, P. (2004). Self-harmful behaviors in a population-based sample of young adults. Suicide and Life-Threatening Behavior, 34(2), 177-186. doi:10.1521/suli.34.2.177.32781

Nehmy, T. J., \& Wade, T. D. (2015). Reducing the onset of negative affect in adolescents: Evaluation of a perfectionism program in a universal prevention setting. Behaviour Research and Therapy, 67, 55-63. doi:10.1016/j.brat.2015.02.007

Nepon, T., Flett, G. L., \& Hewitt, P. L. (2016). Self-image goals in trait perfectionism and perfectionistic self-presentation: Toward a broader understanding of the drives and motives of perfectionists. Self and Identity, 15(6), 683-706. doi:10.1080/15298868.2016.1197847

Neumeister, K. L. S., Williams, K. K., \& Cross, T. L. (2009). Gifted high school students' perspectives on the development of perfectionism. Roeper Review, 31(4), 198-206. doi:10.1080/02783190903177564

Nilsson, K., Sundbom, E., \& Hagglof, B. (2008). A longitudinal study of perfectionism in adolescent onset anorexia nervosa-restricting type. European Eating Disorders Review, 16(5), 386-394. doi:10.1002/erv.850

Nixon, M. K., Cloutier, P. F., \& Aggarwal, S. (2002). Affect regulation and addictive aspects of repetitive self-injury in hospitalized adolescents. Journal of the American Academy of 
Child and Adolescent Psychiatry, 41(11), 1333-1341. doi:10.1097/00004583200211000-00015

Nock, M. K. (2009). Why do people hurt themselves?: New insights into the nature and functions of self-injury. Current Directions in Psychological Science, 18(2), 78-83. doi:10.1111/j.1467-8721.2009.01613.x

Nock, M. K. (2010). Self-injury. Annual Review of Clinical Psychology, 6(15), 339-363. doi:0.1146/annurev.clinpsy.121208.131258

Nock, M. K., \& Cha, C. B. (2009). Psychological models of non-suicidal self-injury. In M. K. Nock (Ed.), Understanding nonsuicidal self-injury: Origins, assessment, and treatment (pp. 37-77). Washington: American Psychological Association.

Nock, M. K., Cha, C. B., \& Dour, H. J. (2011). Disorders of impulse control and self-harm. In D. H. Barlow (Ed.), The oxford handbook of clinical psychology (pp. 504-529). New York: Oxford University Press.

Nock, M. K., \& Favazza, A. (2009). Non-suicidal self-injury: Definition and classification. In M. K. Nock (Ed.), Understanding nonsuicidal self-injury: Origins, assessment, and treatment (pp. 9-18). Washington, DC: American Psychological Association.

Nock, M. K., Joiner, T. E., Gordon, K. H., Lloyd-Richardson, E., \& Prinstein, M. J. (2006). Non-suicidal self-injury among adolescents: Diagnostic correlates and relation to suicide attempts. Psychiatry Research, 144(1), 65-72. doi:10.1016/j.psychres.2006.05.010

Nock, M. K., \& Mendes, W. B. (2008). Physiological arousal, distress tolerance, and social problem-solving deficits among adolescent self-injurers. Journal of Consulting and Clinical Psychology, 76(1), 28-38. doi:10.1037/0022-006X.76.1.28

Nock, M. K., \& Prinstein, M. J. (2004). A functional approach to the assessment of selfmutilative behavior. Journal of Consulting and Clinical Psychology, 72(5), 885-890. doi:10.1037/0022-006X.72.5.885

Nock, M. K., \& Prinstein, M. J. (2005). Contextual features and behavioral functions of selfmutilation among adolescents. Journal of Abnormal Psychology, 114(1), 140-146. doi:10.1037/0021-843X.114.1.140

Nock, M. K., Prinstein, M. J., \& Sterba, S. K. (2009). Revealing the form and function of self-injurious thoughts and behaviors: A real-time ecological assessment study among adolescents and young adults. Journal of Abnormal Psychology, 118(4), 816-827. doi:10.1037/a0016948

Nock, M. K., Wedig, M. M., Holmberg, E. B., \& Hooley, J. M. (2008). The Emotion Reactivity Scale: Development, evaluation, and relation to self-injurious thoughts and 
behaviors. Behavior Therapy, 39(2), 107-116. doi:10.1016/j.beth.2007.05.005

Nolen-Hoeksema, S., \& Aldao, A. (2011). Gender and age differences in emotion regulation strategies and their relationship to depressive symptoms. Personality and Individual Differences, 51(6), 704-708. doi:10.1016/j.paid.2011.06.012

*Norman, R. M. G., Davies, F., Nicholson, I. R., Cortese, L., \& Malla, A. K. (1998). The relationship of two aspects of perfectionism with symptoms in a psychiatric outpatient population. Journal of Social and Clinical Psychology, 17(1), 50-68. doi:10.1521/jscp.1998.17.1.50

*O'Connor, D. B., O’Connor, R. C., \& Marshall, R. (2007). Perfectionism and psychological distress: Evidence of the mediating effects of rumination. European Journal of Personality, 21(4), 429-452. doi:10.1002/per.616

O'Connor, R. C. (2007). The relations between perfectionism and suicidality: A systematic review. Suicide \& Life-Threatening Behavior, 37(6), 698-714. doi:10.1521/suli.2007.37.6.698

O’Connor, R. C., Dixon, D., \& Rasmussen, S. (2009). The structure and temporal stability of the Child and Adolescent Perfectionism Scale. Psychological Assessment, 21(3), 437443. doi:10.1037/a0016264

*O'Connor, R. C., \& Forgan, G. (2007). Suicidal thinking and perfectionism: The role of goal adjustment and Behavioral Inhibition/Activation Systems (BIS/BAS). Journal of Rational - Emotive and Cognitive - Behavior Therapy, 25(4), 321-341. doi:10.1007/s10942-007-0057-2

O’Connor, R. C., Rasmussen, S., \& Hawton, K. (2009). Predicting deliberate self-harm in adolescents: A six month prospective study. Suicide \& Life-Threatening Behavior, 39(4), 364-375. doi:10.1521/suli.2009.39.4.364

*O'Connor, R. C., Rasmussen, S., \& Hawton, K. (2010). Predicting depression, anxiety and self-harm in adolescents: The role of perfectionism and acute life stress. Behaviour Research and Therapy, 48(1), 52-59. doi:10.1016/j.brat.2009.09.008

O’Connor, R. C., Rasmussen, S., \& Hawton, K. (2012). Distinguishing adolescents who think about self-harm from those who engage in self-harm. British Journal of Psychiatry, 200(4), 330-335. doi:10.1192/bjp.bp.111.097808

*O'Connor, R. C., Rasmussen, S., \& Hawton, K. (2014). Adolescent self-harm: A schoolbased study in Northern Ireland. Journal of Affective Disorders, 159, 46-52. doi:10.1016/j.jad.2014.02.015

O’Connor, R. C., Rasmussen, S., Miles, J., \& Hawton, K. (2009). Self-harm in adolescents: 
Self-report survey in schools in Scotland. British Journal of Psychiatry, 194(1), 68-72. doi:10.1192/bjp.bp.107.047704

O’Connor, R. C., Whyte, M. C., Fraser, L., Masterton, G., Miles, J., \& MacHale, S. (2007).

Predicting short-term outcome in well-being following suicidal behaviour: The conjoint effects of social perfectionism and positive future thinking. Behaviour Research and Therapy, 45(7), 1543-1555. doi:10.1016/j.brat.2006.11.006

O’Dea, D., \& Tucker, S. (2005). The cost of suicide to society. Wellington: Ministry of Health. Retrieved from http://www.health.govt.nz/publication/cost-suicide-society *O'Riley, A. A. (2010). The role of autonomy in suicidal ideation among older adults. (Unpublished doctoral thesis). West Virginia University, West Virginia, USA.

*O'Riley, A. A., \& Fiske, A. (2012). Emphasis on autonomy and propensity for suicidal behavior in younger and older adults. Suicide and Life-Threatening Behavior, 42(4), 394-404. doi:10.1111/j.1943-278X.2012.00098.x

Oktan, V. (2014). A characterization of self-injurious behavior among Turkish adolescents. Psychological Reports, 115(3), 645-654. doi:10.2466/16.02.PR0.115c25z5

Oliver, C., \& Richards, C. (2010). Self-injurious behaviour in people with intellectual disability. Current Opinion in Psychiatry, 23(5), 421-426. doi:10.1111/j.13652788.2012.01553.x

*Ommundsen, Y., Roberts, G. C., Lemyre, P.-N., \& Miller, B. W. (2005). Peer relationships in adolescent competitive soccer: Associations to perceived motivational climate, achievement goals and perfectionism. Journal of Sports Sciences, 23(9), 977-989. doi:10.1080/02640410500127975

Orwin, R. G. (1983). A fail-safe N for effect size in meta-analysis. Journal of Educational Statistics, 8(2), 157-159. doi:10.2307/1164923.

Osman, A., Bagge, C. L., Gutierrez, P. M., Konick, L. C., Kopper, B. A, \& Barrios, F. X. (2001). The Suicidal Behaviors Questionnaire-Revised (SBQ-R): Validation with clinical and nonclinical samples. Assessment, 8(4), 443-454. doi:10.1177/107319110100800409

Owens, R. G., \& Slade, P. D. (1987). Running and anorexia nervosa: An empirical study. International Journal of Eating Disorders, 6(6), 771-775. doi:10.1002/1098108X(198711)6:6<771::AID-EAT2260060612>3.0.CO;2-V

Page, A., Lewis, G., Kidger, J., Heron, J., Chittleborough, C., Evans, J., \& Gunnell, D. (2014). Parental socio-economic position during childhood as a determinant of self-harm in adolescence. Social Psychiatry and Psychiatric Epidemiology, 49(2), 193-203. 
doi:10.1007/s00127-013-0722-y

Pallant, J. (2013). SPSS survival manual: A step by step guide to data analysis using IBM SPSS (5th ed.). Sydney: Allen and Dunwin.

Parker, W. D. (1997). An empirical typology of perfectionism in academically talented children. American Educational Research Journal, 34(3), 545-562. doi:10.3102/00028312034003545

Parker, W. D., \& Adkins, K. K. (1995). A psychometric examination of the multidimensional perfectionism scale. Journal of Psychopathology and Behavioral Assessment, 17(4), 323-334. doi:10.1007/BF02229054

Parker, W. D., \& Stumpf, H. (1995). An examination of the multidimensional perfectionism scale with a sample of academically talented children. Journal of Psychoeducational Assessment, 13(4), 372-383. doi:10.1177/073428299501300404

Parker, Eastabrook, J. M., Keefer, K. V, \& Wood, L. M. (2010). Can alexithymia be assessed in adolescents? Psychometric properties of the 20 -item Toronto Alexithymia Scale in younger, middle, and older adolescents. Psychological Assessment, 22(4), 798-808. doi:10.1037/a0020256

Perera, M. J., \& Chang, E. C. (2015). Ethnic variations between Asian and European Americans in interpersonal sources of socially prescribed perfectionism: It's not just about parents! Asian American Journal of Psychology, 6(1), 31-37. doi:10.1037/a0036175

*Peyerl, N. (2010). A comparison of the functions of eating disorder behaviors to nonsuicidal self-injury. (Unpublished doctoral thesis). University of North Dakota, North Dakota, USA.

Pfeffer, C. R., Jiang, H., \& Kakuma, T. (2000). Child-Adolescent Suicidal Potential Index (CASPI): A screen for risk for early onset suicidal behavior. Psychological Assessment, 12(3), 304-318. doi:10.1037/1040-3590.12.3.304

Plener, P. L., Libal, G., Keller, F., Fegert, J. M., \& Muehlenkamp, J. J. (2009). An international comparison of adolescent non-suicidal self-injury (NSSI) and suicide attempts: Germany and the USA. Psychological Medicine, 39(9), 1549-1558. doi:10.1017/S0033291708005114

Ployhart, R., \& Vandenberg, R. (2010). Longitudinal research: The theory, design, and analysis of change. Journal of Management, 36(1), 94-120. doi:10.1177/0149206309352110

Pomerantz, E. M., Altermatt, E. R., \& Saxon, J. L. (2002). Making the grade but feeling 
distressed: Gender differences in academic performance and internal distress. Journal of Educational Psychology, 94(2), 396-404. doi:10.1037/0022-0663.94.2.396

Portesova, S., \& Urbanek, T. (2013). Typology of perfectionism in a group of mathematically gifted Czech adolescents over one decade. The Journal of Early Adolescence, 33(8), 1116-1144. doi:10.1177/0272431613487603

*Portzky, G., van Heeringen, K., \& Vervaet, M. (2014). Attempted suicide in patients with eating disorders. Crisis, 35(6), 378-387. doi:10.1027/0227-5910/a000275

Powers, T. A. (2005). Implementation intentions, perfectionism, and goal progress: Perhaps the road to hell is paved with good intentions. Personality and Social Psychology Bulletin, 31(7), 902-912. doi:10.1177/0146167204272311

Prinstein, M. J. (2008). Introduction to the special section on suicide and nonsuicidal selfinjury: A review of unique challenges and important directions for self-injury science. Journal of Consulting and Clinical Psychology, 76(1), 1-8. doi:10.1037/0022006X.76.1.1

Prinstein, M. J., Nock, M. K., Simon, V., Aikins, J. W., Cheah, C. S. L., \& Spirito, A. (2008). Longitudinal trajectories and predictors of adolescent suicidal ideation and attempts following inpatient hospitalization. Journal of Consulting and Clinical Psychology, 76(1), 92-103. doi:10.1037/0022-006X.76.1.92

*Pulford, B. D., \& Sohal, H. (2006). The influence of personality on HE students' confidence in their academic abilities. Personality and Individual Differences, 41(8), 1409-1419. doi:10.1016/j.paid.2006.05.010

Purdon, C., Antony, M. M., \& Swinson, R. P. (1999). Psychometric properties of the Frost Multidimensional Perfectionism Scale in a clinical anxiety disorders sample. Journal of Clinical Psychology, 55(10), 1271-1286. doi:10.1002/(SICI)10974679(199910)55:10<1271::AID-JCLP8>3.0.CO;2-A

Putwain, D. (2008). Examination stress and test anxiety. British Psychological Society, 21(12), 1026-1030. Retrieved from http://scholar.google.com/scholar?hl=en\&btnG=Search\&q=intitle:Examination+stress+a nd+test+anxiety\#0

Rankin, J. L., Lane, D. J., Gibbons, F. X., \& Gerrard, M. (2004). Adolescent selfconsciousness: Longitudinal age changes and gender differences in two cohorts. Journal of Research on Adolescence, 14(1), 1-21. doi:10.1111/j.1532-7795.2004.01401001.x

*Rasmussen, K. A., Slish, M. L., Wingate, L. R. R., Davidson, C. L., \& Grant, D. M. M. (2012). Can perceived burdensomeness explain the relationship between suicide and 
perfectionism? Suicide and Life-Threatening Behavior, 42(2), 121-128. doi:10.1111/j.1943-278X.2011.00074.X

*Rasmussen, S., Elliott, M. A., \& O’Connor, R. C. (2012). Psychological distress and perfectionism in recent suicide attempters: The role of behavioural inhibition and activation. Personality and Individual Differences, 52(6), 680-685. doi:10.1016/j.paid.2011.12.011

*Rasmussen, S., O’Connor, R. C., \& Brodie, D. (2008). The role of perfectionism and autobiographical memory in a sample of parasuicide patients an exploratory study. Crisis, 29(2), 64-72. doi:10.1027/0227-5910.29.2.64

Rice, K. G., \& Aldea, M. a. (2006). State dependence and trait stability of perfectionism: A short-term longitudinal study. Journal of Counseling Psychology, 53(2), 205-213. doi:10.1037/0022-0167.53.2.205

Rice, K. G., \& Ashby, J. S. (2007). An efficient method for classifying perfectionists. Journal of Counseling Psychology, 54(1), 72-85. doi:10.1037/0022-0167.54.1.72

Rice, K. G., \& Mirzadeh, S. A. (2000). Perfectionism, attachment, and adjustment. Journal of Counseling Psychology, 47(2), 238-250. doi:10.1037/0022-0167.47.2.238

Richardson, C. M. E., \& Rice, K. G. (2015). Self-critical perfectionism, daily stress, and disclosure of daily emotional events. Journal of Counseling Psychology, 62(4), 694702. doi:10.1037/cou0000100

Richardson, C. M. E., Rice, K. G., \& Devine, D. P. (2014). Perfectionism, emotion regulation, and the cortisol stress response. Journal of Counseling Psychology, 61(1), 110-8. doi:10.1037/a0034446

Riley, C., Lee, M., Cooper, Z., Fairburn, C. G., \& Shafran, R. (2007). A randomised controlled trial of cognitive-behaviour therapy for clinical perfectionism: A preliminary study. Behaviour Research and Therapy, 45(9), 2221-2231. doi:10.1016/j.brat.2006.12.003

Riley, E. N., Combs, J. L., Jordan, C. E., \& Smith, G. T. (2015). Negative urgency and lack of perseverance: Identification of differential pathways of onset and maintenance risk in the longitudinal prediction of nonsuicidal self-injury. Behavior Therapy, 46(4), 439448. doi:10.1016/j.beth.2015.03.002

Rimes, K. A., \& Chalder, T. (2010). The Beliefs about Emotions Scale: Validity, reliability and sensitivity to change. Journal of Psychosomatic Research, 68(3), 285-292. doi:10.1016/j.jpsychores.2009.09.014

Robertson, C. D., Miskey, H., Mitchell, J., \& Nelson-Gray, R. (2013). Variety of self-injury: 
Is the number of different methods of non-suicidal self-injury related to personality, psychopathology, or functions of self-injury. Archives of Suicide Research, 17(1), 3340. doi:10.1080/13811118.2013.748410

Robins, C., Ladd, J., Welkowitz, J., Blaney, P., Diaz, R., \& Kutcher, G. (1994). The personal style inventory: Preliminary validation studies of new measures of sociotropy and autonomy. Journal of Psychopathology and Behavioral Assessment, 16(4), 277-300. doi:10.1007/bf02239408

Rodham, K., \& Hawton, K. (2009). Epidemiology and phenomonology of nonsuicidal selfinjury. In M. K. Nock (Ed.), Understanding nonsuicidal self-injury: Origins, assessment, and treatment (pp. 37-63). Washington, DC: American Psychological Association.

Rose, A. J. (2002). Co-rumination in the friendships of girls and boys. Child Development, 73(6), 1830-1843. doi:10.1111/1467-8624.00509

Rose, A. J., Schwartz-Mette, R. A., Glick, G. C., Smith, R. L., \& Luebbe, A. M. (2014). Developmental psychology an observational study of co-rumination in adolescent friendships an observational study of co-rumination in adolescent friendships. Developmental Psychology, 50(9), 2199-2209. doi:10.1037/a0037465

Rosenthal, R. (1979). The file drawer problem and tolerance for null results. Psychological Bulletin, 86(3), 638-641. doi:10.1037/0033-2909.86.3.638

*Roxborough, H. M., Hewitt, P. L., Kaldas, J., Flett, G. L., Caelian, C. M., Sherry, S., \& Sherry, D. L. (2012). Perfectionistic self-presentation, socially prescribed perfectionism, and suicide in youth: A test of the perfectionism social disconnection model. Suicide and Life-Threatening Behavior, 42(2), 217-233. doi:10.1111/j.1943-278X.2012.00084.X

Rudolph, S. G., Flett, G. L., \& Hewitt, P. L. (2007). Perfectionism and deficits in cognitive emotion regulation. Journal of Rational - Emotive and Cognitive - Behavior Therapy, 25(4), 343-357. doi:10.1007/s10942-007-0056-3

*Santanello, A. W., \& Gardner, F. L. (2007). The role of experiential avoidance in the relationship between maladaptive perfectionism and worry. Cognitive Therapy and Research, 31(3), 319-332. doi:10.1007/s10608-006-9000-6

*Scott, J. H., Yap, K., Francis, A. J. P., \& Schuster, S. (2014). Perfectionism and its relationship with anticipatory processing in social anxiety. Australian Journal of Psychology, 66(3), 187-196. doi:10.1111/ajpy.12045

Selby, E. a., Bender, T. W., Gordon, K. H., Nock, M. K., \& Joiner, T. E. (2012). Non-suicidal self-injury (NSSI) disorder: A preliminary study. Personality Disorders: Theory, 
Research, and Treatment, 3(2), 167-175. doi:10.1037/a0024405

Selig, J., \& Little, T. D. (2012). Autoregressive an cross-lagged panel analysis for longituinal data. In B. Laursen, T. Little, \& N. Card (Eds.), Handbook of developmental research methods. (pp. 265-278). Washington, DC: The Guildford Press.

Shafran, R., Cooper, Z., \& Fairburn, C. G. (2002). Clinical perfectionism: A cognitivebehavioural analysis. Behaviour Research and Therapy, 40(7), 773-791. doi:10.1016/S0005-7967(01)00059-6

Shafran, R., Coughtrey, A., \& Kothari, R. (2016). New frontiers in the treatment of perfectionism. International Journal of Cognitive Therapy, 9(2), 156-170. doi:10.1521/ijct.2016.9.2.156

Shafran, R., \& Mansell, W. (2001). Perfectionism and psychopathology: A review of research and treatment. Clinical Psychology Review, 21(6), 879-906. doi:10.1016/S02727358(00)00072-6

Shanmugam, V., \& Davies, B. (2015). Clinical perfectionism and eating psychopathology in athletes: The role of gender. Personality and Individual Differences, 74, 99-105. doi:10.1016/j.paid.2014.09.047

Shea, B. J., Hamel, C., Wells, G. A., Bouter, L. M., Kristjansson, E., Grimshaw, J., ... Boers, M. (2009). AMSTAR is a reliable and valid measurement tool to assess the methodological quality of systematic reviews. Journal of Clinical Epidemiology, 62(10), 1013-1020. doi:10.1016/j.jclinepi.2008.10.009

Sher, L., \& Stanley, B. H. (2009). Biological models of non-suicidal self-injury. In M. K. Nock (Ed.), Understanding nonsuicidal self-injury: Origins, assessment, and treatment (pp. 99-116). Washington, DC: American Psychological Association.

Sherry, S. B., Sabourin, B. C., Hall, P. A., Hewitt, P. L., Flett, G. L., \& Gralnick, T. M. (2014). The perfectionism model of binge eating: Testing unique contributions, mediating mechanisms, and cross-cultural similarities using a daily diary methodology. Psychology of Addictive Behaviors, 28(4), 1230-1239. doi:10.1037/a0037939

*Shih, S. S. (2011). Perfectionism, implicit theories of intelligence, and Taiwanese eighthgrade students' academic engagement. The Journal of Educational Research, 104(2), 131-142. doi:10.1080/00220670903570368

Shingles, R. D. (1976). Causal inference in cross-lagged panel analysis. Political Methodology, 3(1), 95-133. Retrieved from http://www.jstor.org/stable/25791444?seq=1\#page_scan_tab_contents

Short, M. M., \& Mazmanian, D. (2013). Perfectionism and negative repetitive thoughts: 
Examining a multiple mediator model in relation to mindfulness. Personality and Individual Differences, 55(6), 716-721. doi:10.1016/j.paid.2013.05.026

*Shumaker, E. a, \& Rodebaugh, T. L. (2009). Perfectionism and social anxiety: Rethinking the role of high standards. Journal of Behavior Therapy and Experimental Psychiatry, 40(3), 423-33. doi:10.1016/j.jbtep.2009.04.002

Singer, J., \& Willett, J. (2003). Applied longitudinal data analysis: Modeling change and event occurrence. New York, NY: Oxford University Press.

Sironic, A., \& Reeve, R. A. (2015). A combined analysis of the Frost Multidimensional Perfectionism Scale (FMPS), Child and Adolescent Perfectionism Scale (CAPS), and Almost Perfect Scale - Revised (APS-R): Different perfectionist profiles in adolescent high school students. Psychological Assessment, 27(4), 1471-1483. doi:10.1037/pas0000137

Skinner, B. F. (1969). Contingencies of reinforcement: A theoretical analysis. New York, NY: Meredith Corporation.

Slade, P. D. (1982). Towards a functional analysis of anorexia nervosa and bulimia nervosa. British Journal of Clinical Psychology, 21(3), 167-179. doi:10.1111/j.20448260.1982.tb00549.x

Slade, P. D., \& Owens, R. G. (1998). A dual process model of perfectionism based on reinforcement theory. Behavior Modification, 22(3), 372-390. doi:10.1177/01454455980223010

Slaney, R. B., Rice, K., Mobley, M., Trippi, J., \& Ashby, J. S. (2001). The revised Almost Perfect Scale. Measurement and Evaluation in Counseling and Development, 34, 130 145. Retrieved from http://connection.ebscohost.com/c/articles/5541978/revised-almostperfect-scale

*Smith, K. M., Chesin, M. S., \& Jeglic, E. L. (2014). Minority college student mental health: Does majority status matter? Implications for college counseling services. Journal of Multicultural Counseling and Development, 42(2), 77-92. doi:10.1002/j.21611912.2014.00046.x

Smith, M. M., Saklofske, D. H., Yan, G., \& Sherry, S. B. (2014). Perfectionistic strivings and perfectionistic concerns interact to predict negative emotionality: Support for the tripartite model of perfectionism in Canadian and Chinese university students. Personality and Individual Differences, 81, 141-147. doi:10.1016/j.paid.2014.09.006

Soares, M. J., Macedo, A., Bos, S. C., Marques, M., Maia, B., Pereira, A. T., ... Azevedo, M. H. (2009). Perfectionism and eating attitudes in Portuguese students: A longitudinal 
study. European Eating Disorders Review, 17(5), 390-398. doi:10.1002/erv.926

*Soenens, B., Luyckx, K., Vansteenkiste, M., Luyten, P., Duriez, B., \& Goossens, L. (2008). Maladaptive perfectionism as an intervening variable between psychological control and adolescent depressive symptoms: A three-wave longitudinal study. Journal of Family Psychology, 22(3), 465-474. doi:10.1037/0893-3200.22.3.465

*Soenens, B., Vansteenkiste, M., Duriez, B., \& Goossens, L. (2006). In search of the sources of psychologically controlling parenting: The role of parental separation anxiety and parental maladaptive perfectionism. Journal of Research on Adolescence, 16(4), 539559. doi:10.1111/j.1532-7795.2006.00507.x

*Soenens, B., Vansteenkiste, M., \& Luyten, P. (2010). Toward a domain-specific approach to the study of parental psychological control: Distinguishing between dependencyoriented and achievement-oriented psychological control. Journal of Personality, 78(1), 217-256. doi:10.1111/j.1467-6494.2009.00614.x

*Soenens, B., Vansteenkiste, M., Luyten, P., Duriez, B., \& Goossens, L. (2005). Maladaptive perfectionistic self-representations: The mediational link between psychological control and adjustment. Personality and Individual Differences, 38(2), 487-498. doi:10.1016/j.paid.2004.05.008

*Soenens, B., Vansteenkiste, M., Vandereycken, W., Luyten, P., Sierens, E., \& Goossens, L. (2008). Perceived parental psychological control and eating-disordered symptoms. The Journal of Nervous and Mental Disease, 196(2), 144-152. doi:10.1097/NMD.0b013e318162aabf

Somer, O., Bildik, T., Kabukçu-Başay, B., Güngör, D., Başay, Ö., \& Farmer, R. F. (2015). Prevalence of non-suicidal self-injury and distinct groups of self-injurers in a community sample of adolescents. Social Psychiatry and Psychiatric Epidemiology, 50(7), 1163-1171. doi:10.1007/s00127-015-1060-z

Soreni, N., Streiner, D., McCabe, R., Bullard, C., Swinson, R., Greco, A., ... Szatmari, P. (2014). Dimensions of perfectionism in children and adolescents with obsessivecompulsive disorder. Journal of the Canadian Academy of Child and Adolescent Psychiatry, 23(2), 136-141. Retrieved from https://www.ncbi.nlm.nih.gov/pubmed/24872829

*Soysa, C. K., \& Weiss, A. (2014). Mediating perceived parenting styles-test anxiety relationships: Academic procrastination and maladaptive perfectionism. Learning and Individual Differences, 34, 77-85. doi:10.1016/j.lindif.2014.05.004

Spector, P. (2013). Survey design and measure development. In T. D. Little (Ed.), The oxford 
handbook of quantitative methods: Volume 1, foundations (pp. 170-188). New York, NY: Oxford University Press.

Spence, J., \& Robbins, A. (1992). Workaholism: Definition, measurement, and preliminary results. Journal of Personality Assessment, 58(1), 160-178. doi:10.1207/s15327752jpa5801_15

Stairs, A. M., Smith, G. T., Zapolski, T. C. B., Combs, J. L., \& Settles, R. E. (2012). Clarifying the construct of perfectionism. Assessment, 19(2), 146-166. doi:10.1177/1073191111411663

Stallman, H. M., \& Hurst, C. P. (2011). The factor structure of the Frost Multidimensional Perfectionism Scale in university students. Australian Psychologist, 46(4), 229-236. doi:10.1111/j.1742-9544.2010.00010.x

Stanley, B., Fineran, V., \& Brodsky, B. (2014). Psychological treatments for non-suicidal self-injury. In M. K. Nock (Ed.), The oxford handbook of suicide and self-injury (pp. 409-418). New York, NY: Oxford University Press.

Statistics New Zealand. (2014). 2013 Census - Major Ethnic Groups in New Zealand. Wellington : Statistics NZ. Retrieved from http://www.stats.govt.nz/Census/2013census/profile-and-summary-reports/infographic-culture-identity.aspx

*Steffen, P. R. (2014). Perfectionism and life aspirations in intrinsically and extrinsically religious individuals. Journal of Religion and Health, 53(4), 945-58. doi:10.1007/s10943-013-9692-3

Steinberg, L., \& Morris, A. S. (2001). Adolescent development. Annual Review of Psychology, 52, 83-110. doi:10.1146/annurev.psych.52.1.83

Sterne, J., Egger, M., \& Moher, D. (2008). Addressing reporting biases. In J. Higgins \& S. Green (Eds.), Cochrane handbook for systematic reviews of interventions (pp. 297-333). West Sussex: The Cochrane Collaboration and John Wiley and Sons.

Sterne, J., Gavaghan, D., \& Egger, M. (2000). Publication and related bias in meta-analysis: Power of statistical tests and prevalence in the literature. Journal of Clinical Epidemiology, 53(11), 1119-1129. doi:10.1016/S0895-4356(00)00242-0

Sterne, J., \& Harbord, R. (2004). Funnel plots in meta-analysis. Stata Journal, 4(2), 127-141. Retrieved from http://ideas.repec.org/a/tsj/stataj/v7y2007i4p465-506.html

Stipek, D. J., \& Gralinski, J. H. (1991). Gender differences in children's achievement-related beliefs and emotional responses to success and failure in mathematics. Journal of Educational Psychology, 83(3), 361-371. doi:10.1037/0022-0663.83.3.361

Stoeber, J. (1998). The Frost Multidimensional Perfectionism Scale revisited: More perfect 
with four (instead of six) dimensions. Personality and Individual Differences, 24(4), 481-491. doi:10.1016/S0191-8869(97)00232-8

Stoeber, J., \& Gaudreau, P. (2017). The advantages of partialling perfectionistic strivings and perfectionistic concerns: Critical issues and recommendations. Personality and Individual Differences, 104, 379-386. doi:10.1016/j.paid.2016.08.039

*Stoeber, J., \& Joormann, J. (2001). Worry, procrastination, and perfectionism: Differentiating amount of worry, pathological worry, anxiety, and depression. Cognitive Therapy and Research, 25(1), 49-60. doi:10.1023/A:1026474715384

Stoeber, J., \& Otto, K. (2006). Positive conceptions of perfectionism: Approaches, evidence, challenges. Personality and Social Psychology Review, 10(4), 295-319. doi:10.1207/s15327957pspr1004_2

Stoeber, J., \& Rambow, A. (2007). Perfectionism in adolescent school students: Relations with motivation, achievement, and well-being. Personality and Individual Differences, 42(7), 1379-1389. doi:10.1016/j.paid.2006.10.015

Stone, L. B., \& Gibb, B. E. (2015). Brief report: Preliminary evidence that co-rumination fosters adolescents' depression risk by increasing rumination. Journal of Adolescence, 38, 1-4. doi:10.1016/j.adolescence.2014.10.008

*Street, M., \& Lester, D. (2000). Manic depressive tendencies, suicidality, and perfectionism. Psychological Reports, 86(1), 142. doi:10.2466/pr0.86.1.142-142

*Stumpf, H., \& Parker, W. D. (2000). A hierarchical structural analysis of perfectionism and its relation to other personality characteristics. Personality and Individual Differences, 28(5), 837-852. doi:10.1016/S0191-8869(99)00141-5

Swann, W. (1983). Self-verification: Bringing social reality into harmony with the self. In J. Suls \& A. Greenwald (Eds.), Social psychological perspectives on the self (pp. 33-66). Hillsdale, NJ: Erlbaum.

Swannell, S. V., Martin, G. E., Page, A., Hasking, P., \& St John, N. J. (2014). Prevalence of nonsuicidal self-injury in nonclinical samples: Systematic review, meta-analysis and meta-regression. Suicide and Life-Threatening Behavior, 44(3), 273-303. doi:10.1111/sltb. 12070

Tabachnick, B., \& Fidell, L. (2013). Using multivariate statistics (6th ed.). Boston: Pearson Education.

Tachi, T., Kusano-Schwarz, M., Murakami, K., Kobayashi, Y., \& Miyake, Y. (2007). Usefulness of the Eating Disorder Inventory-2 Japanese version in patients with eating disorders. Tokai Journal of Experimental Clinical Medicine, 32(3), 78-82. Retrieved 
from https://www.ncbi.nlm.nih.gov/pubmed/21318941

Taliaferro, L. A., \& Muehlenkamp, J. J. (2014). Risk and protective factors that distinguish adolescents who attempt suicide from those who only consider suicide in the past year. Suicide and Life-Threatening Behavior, 44(1), 6-22. doi:10.1111/sltb.12046

Tang, J., Yu, Y., Wu, Y., Du, Y., Ma, Y., Zhu, H., ... Liu, Z. (2011). Association between non-suicidal self-injuries and suicide attempts in Chinese adolescents and college students: A cross-section study. PLoS ONE, 6(4). doi:10.1371/journal.pone.0017977

Tangney, J. P. (2002). Perfectionism and the self-consious emotions: Shame, guilt, embarrassment, and pride. In G. L. Flett \& P. L. Hewitt (Eds.), Perfectionism: Theory, research and treatment (pp. 199-215). Washington, DC: American Psychological Association.

Tanner, A. K., Hasking, P., \& Martin, G. (2013). Effects of rumination and optimism on the relationship between psychological distress and non-suicidal self-injury. Prevention Science, 15(6), 860-868. doi:10.1007/s11121-013-0444-0

Tatnell, R., Kelada, L., Hasking, P., \& Martin, G. (2014). Longitudinal analysis of adolescent NSSI: The role of intrapersonal and interpersonal factors. Journal of Abnormal Child Psychology, 42, 885-896. doi:10.1007/s10802-013-9837-6

Taylor, B. (2003). Exploring the perspectives of men who self-harm. Learning in Health and Social Care, 2(2), 83-91. doi:10.1046/j.1473-6861.2003.00042.x

Terry-Short, L. A., Owens, R. G. G., Slade, P. D. D., \& Dewey, M. E. E. (1995). Positive and negative perfectionism. Personality and Individual Differences, 18(5), 663-668. doi:10.1016/0191-8869(94)00192-U

Thompson, T. (2004). Failure-avoidance: Parenting, the achievement environment of the home and strategies for reduction. Learning and Instruction, 14(1), 3-26. doi:10.1016/j.learninstruc.2003.10.005

Thorpe, E., \& Nettelbeck, T. (2014). Testing if healthy perfectionism enhances academic achievement in Australian secondary school students. Journal of Educational and Developmental Psychology, 4(2), 1-9. doi:10.5539/jedp.v4n2p1

Trives, R., Bravo, B., Postigo, J., Segura, L., \& Watkins, E. (2016). Age and gender differences in emotion regulation strategies: Autobiographical memory, rumination, problem solving and distraction. The Spanish Journal of Psychology, 19(43), 1-9. doi:10.1017/sjp.2016.46

Tuisku, V., Kiviruusu, O., Pelkonen, M., Karlsson, L., Strandholm, T., \& Marttunen, M. 
(2014). Depressed adolescents as young adults - Predictors of suicide attempt and nonsuicidal self-injury during an 8-year follow-up. Journal of Affective Disorders, 152, 313-319. doi:10.1016/j.jad.2013.09.031

Turner, B. J., Chapman, A. L., \& Layden, B. K. (2012). Intrapersonal and interpersonal functions of non suicidal self-injury: Associations with emotional and social functioning. Suicide and Life-Threatening Behavior, 42(1), 36-55. doi:10.1111/j.1943278X.2011.00069.X

*Turner, L. A., \& Turner, P. E. (2011). The relation of behavioral inhibition and perceived parenting to maladaptive perfectionism in college students. Personality and Individual Differences, 50(6), 840-844. doi:10.1016/j.paid.2011.01.006

*Valero, S., Sáez-Francàs, N., Calvo, N., Alegre, J., \& Casas, M. (2013). The role of neuroticism, perfectionism and depression in chronic fatigue syndrome. A structural equation modeling approach. Comprehensive Psychiatry, 54(7), 1061-1067. doi:10.1016/j.comppsych.2013.04.015

*Vansteenkiste, M., Smeets, S., Soenens, B., Lens, W., Matos, L., \& Deci, E. L. (2010). Autonomous and controlled regulation of performance-approach goals: Their relations to perfectionism and educational outcomes. Motivation and Emotion, 34(4), 333-353. doi:10.1007/s11031-010-9188-3

*Vartanian, L. R., \& Grisham, J. R. (2011). Obsessive compulsive symptoms and body checking in women and men. Cognitive Therapy and Research, 36(4), 367-374. doi:10.1007/s10608-011-9356-0

Velicer, W. F., Eaton, C., \& Fava, J. L. (2000). Construct explication through factor or component analysis: A review and evaluation of alternative procedures for determining the number of factors or components. In R. Goffin \& E. Helmes (Eds.), Problems and solutions in human assessment. Boston: Wolters Kluwer.

Victor, S. E., \& Klonsky, E. D. (2014). Correlates of suicide attempts among self-injurers: A meta-analysis. Clinical Psychology Review, 34(4), 282-297. doi:10.1016/j.cpr.2014.03.005

Vieira, A. I., Ramalho, S., Brandão, I., Saraiva, J., \& Gonçalves, S. (2016). Adversity, emotion regulation, and non-suicidal self-injury in eating disorders. Eating Disorders: The Journal of Treatment and Prevention, 24(5), 1-13. doi:10.1080/10640266.2016.1198205

Vieth, A., \& Trull, T. (1999). Family patterns of perfectionism: An examination of college students and their parents. Journal of Personality Assessment, 72(1), 49-67. 
doi:10.1207/s15327752jpa7201_3 To

Voon, D., Hasking, P., \& Martin, G. (2014). The roles of emotion regulation and ruminative thoughts in non-suicidal self-injury. British Journal of Clinical Psychology, 53(1), 95113. doi:10.1111/bjc. 12030

*Wallack, C. (2007). Factors associated with suicidal ideation among American college students: A re-examination of the escape theory of suicide. (Unpublished doctoral thesis). University of Florida, FL, USA.

Walsh, B. W. (2012). Treating self-injury: A pratical guide (2nd ed.). New York, NY: Guilford Press.

*Wang, K. T., Wong, Y. J., \& Fu, C.-C. (2013). Moderation effects of perfectionism and discrimination on interpersonal factors and suicide ideation. Journal of Counseling Psychology, 60(3), 367-378. doi:10.1037/a0032551

Wedig, M. M. (2014). Psychological meanings and functions of non-suicidal self-injury and eating disorders. In L. Claes \& J. J. Muehlenkamp (Eds.), Non-suicidal self-injury in eating disorders (pp. 73-87). Heidelberg: Springer Berlin Heidelberg.

*Weiner, B. A., \& Carton, J. S. (2012). Avoidant coping: A mediator of maladaptive perfectionism and test anxiety. Personality and Individual Differences, 52(5), 632-636. doi:10.1016/j.paid.2011.12.009

Weissman, A. N., \& Beck, A. T. (1978). Development and validation of the Dysfunctional Attidute Scale: A preliminary investigation. Paper presente at the Annual Meeting of the American Educational Research Association, Toronto, Canada.

Wheaton, B., Muthen, B., Alwin, D., \& Summers, G. (1977). Assessing reliability and stability in panel models. Sociological Methodology, 8, 84-136. doi:10.2307/270754

Whitlock, J., Muehlenkamp, J. J., \& Eckenrode, J. (2008). Variation in nonsuicidal selfinjury: identification and features of latent classes in a college population of emerging adults. Journal of Clinical Child \& Adolescent Psychology, 37(4), 725-735. doi:10.1080/15374410802359734

Whitlock, J., Muehlenkamp, J. J., Eckenrode, J., Purington, A., Baral Abrams, G., Barreira, P., \& Kress, V. (2013). Nonsuicidal self-injury as a gateway to suicide in young adults. Journal of Adolescent Health, 52(4), 486-492. doi:10.1016/j.jadohealth.2012.09.010

Whitlock, J., Muehlenkamp, J. J., Purington, A., Eckenrode, J., Barreira, P., Baral Abrams, G., ... Knox, K. (2011). Nonsuicidal self-injury in a college population: General trends and sex differences. Journal of American College Health, 59(8), 691-698.

doi:10.1080/07448481.2010.529626 
Whitlock, J., Pietrusza, C., \& Purington, A. (2013). Young adult respondent experiences of disclosing self-injury, suicide-related behavior, and psychological distress in a webbased survey. Archives of Suicide Research, 17(1), 20-32. doi:10.1080/13811118.2013.748405

Whitlock, J., Prussien, K., \& Pietrusza, C. (2015). Predictors of self-injury cessation and subsequent psychological growth: Results of a probability sample survey of students in eight universities and colleges. Child and Adolescent Psychiatry and Mental Health, 9(19), 1-12. doi:10.1186/s13034-015-0048-5

Whitlock, J., \& Selekman, M. (2014). Nonsuicidal self-injury across the life span. In M. K. Nock (Ed.), The oxford handbook of suicide and self-injury (pp. 133-151). New York, NY: Oxford University Press.

Wichstrøm, L. (2009). Predictors of non-suicidal self-injury versus attempted suicide: Similar or different? Archives of Suicide Research, 13(2), 105-122. doi:10.1080/13811110902834992

*Wielkiewicz, R., \& Wonderlich, S. (2006). Correlations between perfectionism and coping strategies in response to researcher-selected vignettes or participant selected events. Psychological Reports, 98, 745-755. doi:10.2466RR0.98.3.745-755

*Wigert, B., Reiter-Palmon, R., Kaufman, J. C., \& Silvia, P. J. (2012). Perfectionism: The good, the bad, and the creative. Journal of Research in Personality, 46(6), 775-779. doi:10.1016/j.jrp.2012.08.007

Wilkinson, P. (2013). Non-suicidal self-injury. European Child \& Adolescent Psychiatry, 22(1), 75-79. doi:10.1007/s00787-012-0365-7

Wilkinson, P., Kelvin, R., Roberts, C., Dubicka, B., \& Goodyer, I. (2011). Clinical and psychosocial predictors of suicide attempts and nonsuicidal self-injury in the Adolescent Depression Antidepressants and Psychotherapy Trial (ADAPT). American Journal of Psychiatry, 168(5), 495-501. doi:10.1176/appi.ajp.2010.10050718

Wilksch, S. M., Durbridge, M. R., \& Wade, T. D. (2008). A preliminary controlled comparison of programs designed to reduce risk of eating disorders targeting perfectionism and media literacy. Journal of the American Academy of Child and Adolescent Psychiatry, 47(8), 937-947. doi:10.1097/CHI.0b013e3181799f4a

*Williams, C. J., \& Cropley, M. (2014). The relationship between perfectionism and engagement in preventive health behaviours: The mediating role of self-concealment. Journal of Health Psychology, 19(10), 1211-1221. doi:10.1177/1359105313488971

*Wimberley, T. E., \& Stasio, M. J. (2013). Perfectionistic thoughts, personal standards, and 
evaluative concerns: Further investigating relationships to psychological distress. Cognitive Therapy and Research, 37(2), 277-283. doi:10.1007/s10608-012-9462-7

*Wirtz, P. H., Ehlert, U., Kottwitz, M. U., La Marca, R., \& Semmer, N. K. (2013). Occupational role stress is associated with higher cortisol reactivity to acute stress. Journal of Occupational Health Psychology, 18(2), 121-131. doi:10.1037/a0031802

*Wirtz, P. H., Elsenbruch, S., Emini, L., Rüdisüli, K., Groessbauer, S., \& Ehlert, U. (2007). Perfectionism and the cortisol response to psychosocial stress in men. Psychosomatic Medicine, 69(3), 249-255. doi:10.1097/PSY.0b013e318042589e

*Wirtz, P. H., Siegrist, J., Rimmele, U., \& Ehlert, U. (2008). Higher overcommitment to work is associated with lower norepinephrine secretion before and after acute psychosocial stress in men. Psychoneuroendocrinology, 33(1), 92-99. doi:10.1016/j.psyneuen.2007.10.003

Wolff, J. C., Frazier, E., Esposito-Smythers, C., Becker, S. J., Burke, T., Cataldo, A., \& Spirito, A. (2014). Negative cognitive style and perceived social support mediate the relationship between aggression and NSSI in hospitalized adolescents. Journal of Adolescence, 37(4), 483-491. doi:10.1016/j.adolescence.2014.03.016

*Wu, K. D., \& Cortesi, G. T. (2009). Relations between perfectionism and obsessivecompulsive symptoms: Examination of specificity among the dimensions. Journal of Anxiety Disorders, 23(3), 393-400. doi:10.1016/j.janxdis.2008.11.006

*Yamaguchi, N., Kobayashi, J., Tachikawa, H., Sato, S., Hori, M., Suzuki, T., \& Shiraishi, H. (2000). Parental representation in eating disorder patients with suicide. Journal of Psychosomatic Research, 49(2), 131-136. doi:10.1016/S0022-3999(00)00146-X

*Yates, T. M., Tracy, A. J., \& Luthar, S. S. (2008). Nonsuicidal self-injury among “privileged” youths: Longitudinal and cross-sectional approaches to developmental process. Journal of Consulting and Clinical Psychology, 76(1), 52-62. doi:10.1037/0022-006X.76.1.52

Yeager, D. S., \& Dweck, C. S. (2012). Mindsets that promote resilience: When students believe that personal characteristics can be developed. Educational Psychologist, 47(4), 302-314. doi:10.1080/00461520.2012.722805

*Yoon, J., \& Lau, A. S. (2008). Maladaptive perfectionism and depressive symptoms among Asian American college students: contributions of interdependence and parental relations. Cultural Diversity \& Ethnic Minority Psychology, 14(2), 92-101. doi:10.1037/1099-9809.14.2.92

You, J., Jiang, Y., Zhang, M., Du, C., Lin, M.-P., \& Leung, F. (2016). Perceived parental 
control, self-criticism, and nonsuicidal self-injury among adolescents: Testing the reciprocal relationships by a three-wave cross-lag model. Archives of Suicide Research, 1118(1), 1-13. doi:10.1080/13811118.2016.1199989

Youth Wellbeing Study. (2015). Guidance Counsellor Discussion. Facilitated discussion at the Youth Wellbeing Study Annual Guidance Counsellor Workshop. Wellington, New Zealand.

Zanarini, M., Frankenburg, F., Sickel, A., \& Yong, L. (1996). The diagnostic interview for DSM-IV personality disorders (DIPD-IV). Belmont, MA: McLean Hospital.

*Zeigler-Hill, V., \& Terry, C. (2007). Perfectionism and explicit self-esteem: The moderating role of implicit self-esteem. Self and Identity, 6(2-3), 137-153. doi:10.1080/15298860601118850

Zetterqvist, M. (2015). The DSM-5 diagnosis of nonsuicidal self-injury disorder: A review of the empirical literature. Child and Adolescent Psychiatry and Mental Health, 9(31), 113. doi:10.1186/s13034-015-0062-7

Zetterqvist, M., Lundh, L. G., Dahlström, Ö., \& Svedin, C. G. (2013). Prevalence and function of non-suicidal self-injury (NSSI) in a community sample of adolescents, using suggested DSM-5 criteria for a potential NSSI disorder. Journal of Abnormal Child Psychology, 41(5), 759-773. doi:10.1007/s10802-013-9712-5

Zhang, B., \& Cai, T. (2012). Using SEM to examine the dimensions of perfectionism and investigate the mediating role of self-esteem between perfectionism and depression in China. Australian Journal of Guidance and Counselling, 22(1), 44-57. doi:10.1017/jgc.2012.3 


\section{APPENDIX A}

\section{Acronym Glossary}

ANOVA - Analysis of Variance

APS-R - Almost Perfect Scale -Revised

BMI - Body Mass Index

BPD - Borderline Personality Disorder

BPS - Burns Perfectionism Scale

CAPS - Child and Adolescent Perfectionism Scale

CASPI - Child and Adolescent Suicide Potential Index

CEM-NSSI - Cognitive Emotion Model of NSSI

CFA - Confirmatory Factor Analysis

CFI - Comparative Fit Index

CI - Confidence Interval

CLPC - Cross Lagged Panel Correlations

$\mathrm{CM}$ - Concerns over Mistakes (subscale of the FMPS)

CMA - Comprehensive Meta-Analysis

CMD - Concerns and Doubts (subscales of the FMPS)

CPQ - Clinical Perfectionism Questionnaire

DA - Doubts about Actions (subscale of the FMPS)

DAS - Dysfunctional Attitudes Scale

DEQ - Depressive Experiences Questionnaire

DIPV-IV - Diagnostic Interview for DSM-IV Personality Disorders

DSH - Deliberate Self-Harm

DSHI - Deliberate Self-Harm Inventory

DSHI-s - Deliberate Self-Harm Inventory-short

DSM-IV - Diagnostic and Statistical Manual of Mental Disorders - Fourth Edition

DSM-5 - Diagnostic and Statistical Manual of Mental Disorders - Fifth Edition

EAM - Experiential Avoidance Model of DSH

ED - Eating Disorder

EDI - Eating Disorders Inventory

EFA - Exploratory Factor Analysis

ERO - Education Review Office 
FAPS - Family Almost Perfect Scale

FASM - Functional Assessment of Self-Mutilation

FFM - Four Function Model of NSSI

FMPS - Frost Multidimensional Perfectionism Scale

GFI - Goodness of Fit Index

HDEC - Health and Disability Ethics Committee

HFMPS - Hewitt and Flett Multidimensional Perfectionism Scale

HMC - Headmasters' and Headmistresses' Conference

ISAS - Inventory of Statements About Self-injury

IBT - Irrational Beliefs Test

ITMS - Integrated Theoretical Model of Self-injury

ISAS - Inventory of Statements About Self-injury

MIMIC - Multiple Indicators Multiple Causes Analysis

NS-cutting - Nonsuicidal cutting

NSSI - Non-Suicidal Self -Injury

NPQ - Neurotic Perfectionism Questionnaire

NZ - New Zealand

OCD - Obssessive Compulsive Disorder

OOP - Other-Orientated Perfectionism (subscale of the HFMPS)

$\mathrm{O}$ - Organisation (subscale of the FMPS)

PC - Parental Criticism (subscale of the FMPS)

PCA - Principal Components Analysis

PCI - Perfectionistic Cognitions Inventory

PE - Parental Expectations (subscale of the FMPS)

PEC - Parental Expectations and Criticism (subscales of the FMPS)

PI - Perfectionism Inventory

PP - Parental Pressure (PE + PC; subscales of the FMPS)

PPS - Pure Personal Standards (PS, excluding two items)

PRISMA - Preferred Reporting Items for Systematic reviews and Meta-Analyses

PS - Personal Standards (subscale of the FMPS)

PSDM - Perfectionism Social Disconnection Model

PSI-II - Personal Style Inventory-II

PSPS - Perfectionistic Self-Presentation Scale

RMSEA - Root Mean Square Error of Approximation 
SBQ-R - Suicidal Behaviors Questionnaire-Revised

SIQ- TR - Self-Injurious Questionnaire-Treatment Related

SOP - Self-Orientated Perfectionism (subscale of the HFMPS or CAPS)

SPP - Socially-Prescribed Perfectionism (subscale of the HFMPS or CAPS)

TLI - Tucker Lewis Index

YWS - Youth Wellbeing Study 


\section{APPENDIX B}

\section{Study name}

Brown \& Kocovski, 2014

Burton et al., 2013

DiBartolo et al., 2007

DiBartolo et al., 2008

Kawamura et al., 2001

Khawaja \& Armstrong, 2005

Laird, 2011

Levinson et al., 2013

Levinson et al., 2013

Levinson et al., 2015

Levinson et al., 2015

Menatti et al., 2013

Moretz \& McKay, 2009

Norman et al., 1998

Santanello \& Gardener, 2007

Scott et al., 2014

Shumaker et al., 2009

Soenens et al., 2006

Soenens et al., 2006

Soenens et al., 2010

Soenens et al., 2010

Soysa \& Weiss, 2014

Steffen, 2014

Steffen, 2014

Stoeber \& Joormann, 2001

Vansteenkiste et al., 2010

Weiner \& Carton, 2012

Wimberley \& Stasio, 2013

Wirtz et al., 2007

\section{Statistics for each study}

Lower Upper

Correlation limit limit Z-Value p-Value

$\begin{array}{lllll}0.34 & 0.16 & 0.50 & 3.51 & 0.000\end{array}$

$\begin{array}{lllll}0.35 & 0.22 & 0.46 & 5.23 & 0.000\end{array}$

$\begin{array}{lllll}0.45 & 0.36 & 0.53 & 8.93 & 0.000\end{array}$

$\begin{array}{lllll}0.47 & 0.38 & 0.56 & 8.48 & 0.000\end{array}$

$\begin{array}{lllll}0.45 & 0.34 & 0.55 & 7.01 & 0.000\end{array}$

$\begin{array}{lllll}0.61 & 0.53 & 0.68 & 11.67 & 0.000\end{array}$

$\begin{array}{lllll}0.36 & 0.24 & 0.47 & 5.53 & 0.000\end{array}$

$\begin{array}{lllll}0.35 & 0.23 & 0.46 & 5.58 & 0.000\end{array}$

$\begin{array}{lllll}0.40 & 0.26 & 0.53 & 5.26 & 0.000\end{array}$

$\begin{array}{lllll}0.30 & 0.16 & 0.42 & 4.05 & 0.000\end{array}$

$\begin{array}{lllll}0.29 & 0.13 & 0.44 & 3.42 & 0.001\end{array}$

$\begin{array}{lllll}0.25 & 0.10 & 0.39 & 3.27 & 0.001\end{array}$

$\begin{array}{lllll}0.48 & 0.36 & 0.58 & 7.13 & 0.000\end{array}$

$\begin{array}{lllll}0.27 & 0.10 & 0.43 & 3.08 & 0.002\end{array}$

$\begin{array}{lllll}0.29 & 0.12 & 0.44 & 3.30 & 0.001\end{array}$

$\begin{array}{llllll}0.45 & 0.34 & 0.55 & 7.46 & 0.000\end{array}$

$\begin{array}{lllll}0.33 & 0.16 & 0.47 & 3.79 & 0.000\end{array}$

$\begin{array}{lllll}0.33 & 0.25 & 0.41 & 7.43 & 0.000\end{array}$

$\begin{array}{lllll}0.37 & 0.29 & 0.44 & 9.00 & 0.000\end{array}$

$\begin{array}{lllll}0.34 & 0.24 & 0.43 & 6.32 & 0.000\end{array}$

$\begin{array}{lllll}0.39 & 0.29 & 0.48 & 7.35 & 0.000\end{array}$

$\begin{array}{lllll}0.31 & 0.18 & 0.43 & 4.57 & 0.000\end{array}$

$\begin{array}{lllll}0.61 & 0.55 & 0.66 & 16.43 & 0.000\end{array}$

$\begin{array}{lllll}0.59 & 0.53 & 0.65 & 14.88 & 0.000\end{array}$

$\begin{array}{lllll}0.51 & 0.40 & 0.61 & 7.55 & 0.000\end{array}$

$\begin{array}{lllll}0.57 & 0.47 & 0.66 & 8.85 & 0.000\end{array}$

$\begin{array}{lllll}0.42 & 0.29 & 0.54 & 5.82 & 0.000\end{array}$

$\begin{array}{lllll}0.52 & 0.37 & 0.64 & 6.04 & 0.000\end{array}$

$\begin{array}{lllll}0.45 & 0.20 & 0.65 & 3.34 & 0.001\end{array}$

$\begin{array}{lllll}0.43 & 0.41 & 0.45 & 38.37 & 0.000\end{array}$

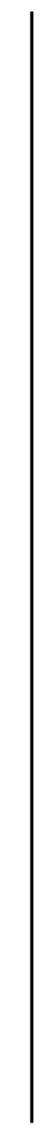

$-1.00$
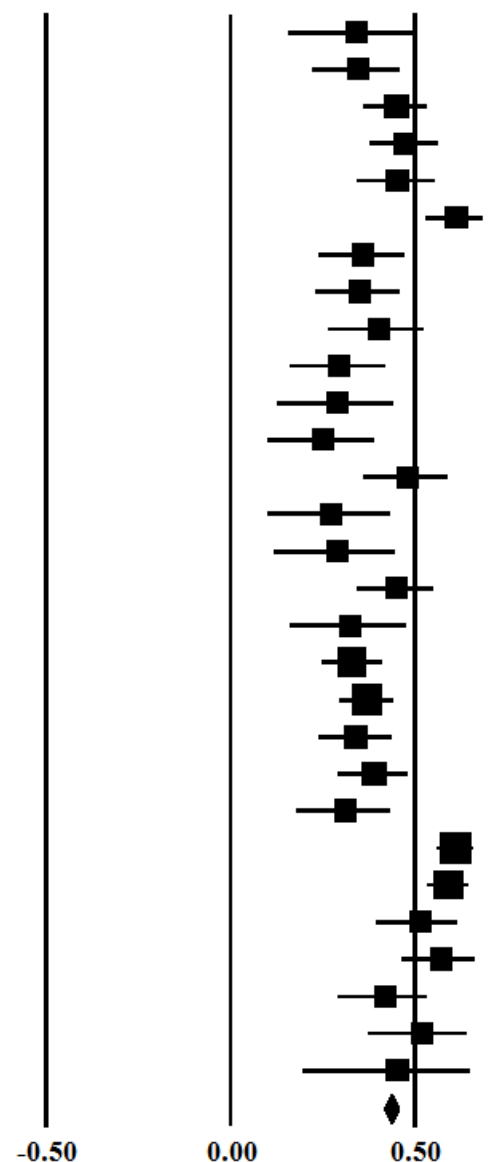

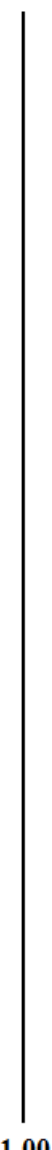

Figure 1. Forest plot with effect sizes for negative perfectionism and anxiety 
Study name

Statistics for each study

$\begin{array}{rrrrr}\text { Correlation } & \begin{array}{c}\text { Lower } \\ \text { limit }\end{array} & \begin{array}{c}\text { Upper } \\ \text { limit }\end{array} & \text { Z-Value } & \text { p-Value } \\ 0.45 & 0.19 & 0.65 & 3.23 & 0.001 \\ 0.33 & 0.26 & 0.40 & 8.48 & 0.000 \\ 0.36 & 0.18 & 0.52 & 3.72 & 0.000 \\ 0.50 & 0.32 & 0.64 & 5.06 & 0.000 \\ 0.37 & 0.28 & 0.44 & 8.25 & 0.000 \\ 0.21 & 0.00 & 0.40 & 1.99 & 0.047 \\ 0.59 & 0.53 & 0.63 & 17.43 & 0.000 \\ 0.53 & 0.47 & 0.58 & 14.48 & 0.000 \\ 0.55 & 0.48 & 0.61 & 12.37 & 0.000 \\ 0.28 & 0.16 & 0.38 & 4.66 & 0.000 \\ 0.46 & 0.32 & 0.58 & 5.78 & 0.000 \\ 0.50 & 0.21 & 0.71 & 3.20 & 0.001 \\ 0.41 & 0.30 & 0.50 & 7.27 & 0.000 \\ 0.34 & 0.22 & 0.45 & 5.40 & 0.000 \\ 0.22 & 0.07 & 0.36 & 2.77 & 0.006 \\ 0.42 & 0.25 & 0.57 & 4.51 & 0.000 \\ 0.43 & 0.29 & 0.54 & 5.82 & 0.000 \\ 0.17 & -0.09 & 0.40 & 1.26 & 0.208 \\ 0.45 & 0.26 & 0.61 & 4.40 & 0.000 \\ 0.37 & 0.21 & 0.50 & 4.51 & 0.000 \\ 0.28 & 0.14 & 0.41 & 3.81 & 0.000 \\ 0.40 & 0.34 & 0.45 & 12.33 & 0.000\end{array}$
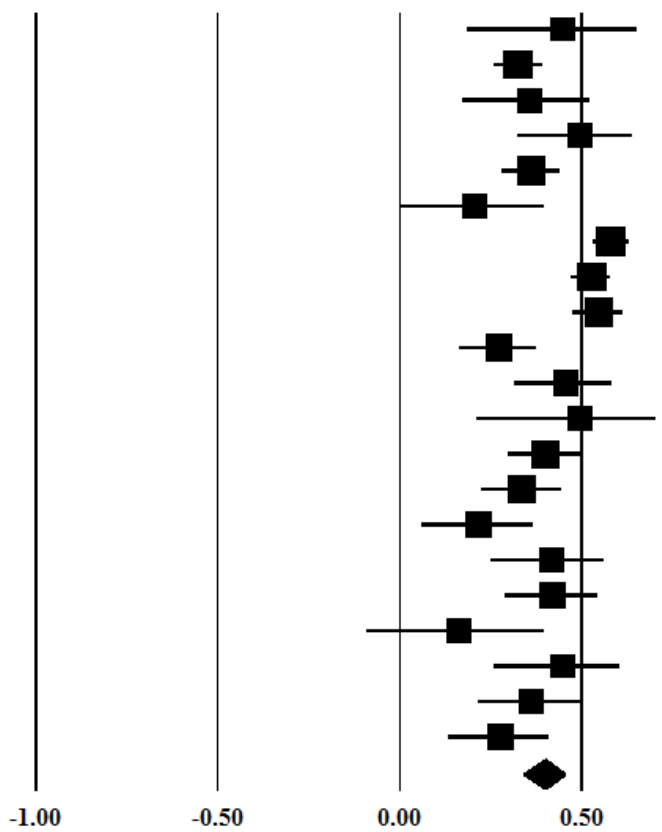

Boone \& Soenens, 2015

Boone et al., 2010/Boone et al., 2011

Boone et al., 2012

Boone et al., 2013

Boone et al., 2014*

Claes et al., 2012

Dakanalis et al., 2015

Dakanalis, Timko, Clerici et al., 2014

Dakanalis, Timko, Zanetti et al., 201

DiBartolo et al., 2008

Dickie et al., 2012

Dickie et al., 2012

Ellickson-Larew et al., 2013

Levinson et al., 2013

Levinson et al., 2013

Soenens, Vansteenkiste et al., 2008

Vartanian \& Grisham, 2012

Vartanian \& Grisham, 2012

Figure 2. Forest plot with effect sizes for negative perfectionism and eating disorders

\begin{tabular}{|c|c|c|c|c|}
\hline \multicolumn{5}{|c|}{ Lower Upper } \\
\hline Correlation & limit & limit & Z-Valuep & p-Value \\
\hline 0.19 & 0.06 & 0.32 & 2.76 & 0.006 \\
\hline 0.34 & 0.23 & 0.44 & 5.80 & 0.000 \\
\hline 0.44 & 0.32 & 0.55 & 6.50 & 0.000 \\
\hline 0.47 & 0.32 & 0.59 & 5.53 & 0.000 \\
\hline 0.48 & 0.34 & 0.60 & 6.17 & 0.000 \\
\hline 0.50 & 0.38 & 0.60 & 7.27 & 0.000 \\
\hline 0.41 & 0.33 & 0.48 & 9.18 & 0.000 \\
\hline 0.40 & 0.33 & 0.47 & 9.44 & 0.000 \\
\hline
\end{tabular}
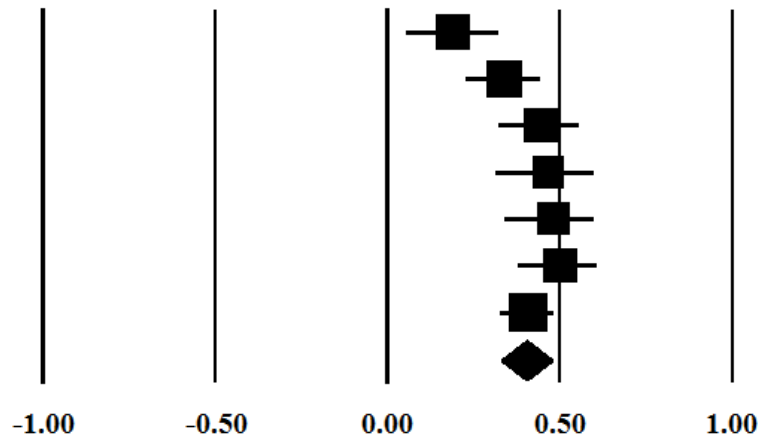

Kawamura et al., 2001

Khawaja \& Armstrong, 2005

Moretz \& McKay, 2009

Norman et al., 1998

Vartanian \& Grisham, 2012

Vartanian \& Grisham, 2012

Wu \& Cortesi, 2009

Figure 3. Forest plot with effect sizes for negative perfectionism and obsessive compulsive disorder 
$\underline{\text { Study name }}$

\begin{tabular}{lrrrrrr} 
& \multicolumn{6}{c}{ Lower Upper } \\
& Correlation & \multicolumn{1}{l}{ limit } & \multicolumn{1}{l}{ limit } & Z-Valuep-Value \\
Burton et al., 2013 & -0.13 & -0.26 & 0.00 & -1.90 & 0.058 \\
Harris et al., 2008 & 0.30 & 0.11 & 0.47 & 2.98 & 0.003 \\
Weiner \& Carton, 2012 & 0.00 & -0.15 & 0.15 & 0.03 & 0.979 \\
Wielkiewicz \& Wonderlich, 2006 & -0.04 & -0.14 & 0.06 & -0.72 & 0.470 \\
& 0.02 & -0.13 & 0.16 & 0.25 & 0.803
\end{tabular}

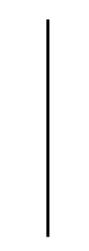

$-1.00$
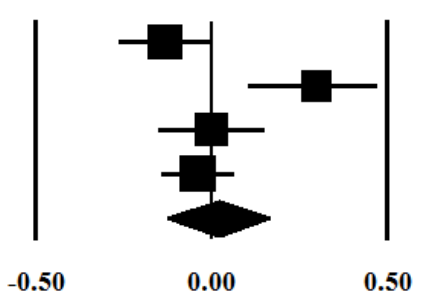

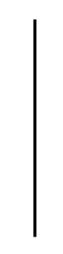

1.00

Figure 4. Forest plot with effect sizes for negative perfectionism and positive coping strategies

Study name

Brown \& Kocovski, 2014

Burton et al., 2013

Cheng, 2001

Di Schiena et al., 2012

Harris et al., 2008

Norman et al., 1998

Santanello \& Gardener, 2007

Weiner \& Carton, 2012

Wielkiewicz \& Wonderlich, 2006

\section{Statistics for each study}

Lower Upper

Correlation limit limit Z-Valuep-Value

$\begin{array}{rrrrr}0.37 & 0.19 & 0.53 & 3.82 & 0.000 \\ 0.31 & 0.18 & 0.43 & 4.66 & 0.000 \\ 0.30 & 0.14 & 0.44 & 3.57 & 0.000 \\ 0.18 & 0.03 & 0.33 & 2.30 & 0.021 \\ 0.56 & 0.40 & 0.68 & 6.06 & 0.000 \\ 0.57 & 0.44 & 0.68 & 7.09 & 0.000 \\ 0.37 & 0.21 & 0.51 & 4.29 & 0.000 \\ 0.33 & 0.19 & 0.46 & 4.46 & 0.000 \\ 0.02 & -0.08 & 0.12 & 0.39 & 0.700 \\ 0.34 & 0.21 & 0.45 & 4.90 & 0.000\end{array}$

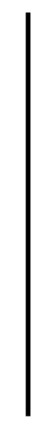

$-1.00$
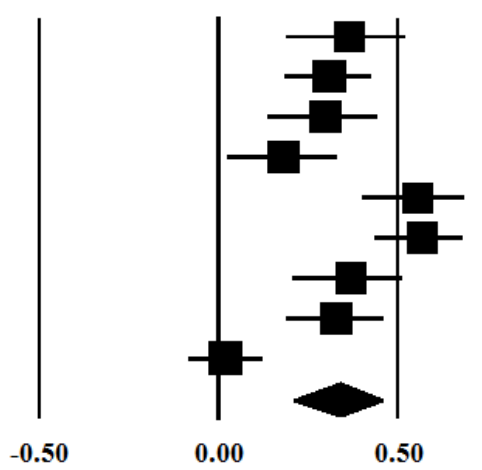

Figure 5. Forest plot with effect sizes for negative perfectionism and negative coping strategies

Study name

\section{$\underline{\text { Statistics for each study }}$}

Lower Upper

Correlation limit limit Z-Valuep-Value

James et al., 2015

Kawamura \& Frost, 2004

Khawaja \& Armstrong, 2005

Luyckx et al., 2008

Stumpf \& Parker, 2000

Vartanian \& Grisham, 2012

Vartanian \& Grisham, 2012

Williams \& Cropley, 2014

$\begin{array}{rrrrr}0.56 & 0.49 & 0.63 & 12.30 & 0.000 \\ 0.46 & 0.32 & 0.58 & 5.93 & 0.000 \\ 0.65 & 0.58 & 0.71 & 12.69 & 0.000 \\ 0.24 & 0.10 & 0.37 & 3.37 & 0.001 \\ 0.29 & 0.19 & 0.39 & 5.42 & 0.000 \\ 0.57 & 0.45 & 0.67 & 7.63 & 0.000 \\ 0.41 & 0.28 & 0.53 & 5.76 & 0.000 \\ 0.49 & 0.40 & 0.56 & 10.18 & 0.000 \\ 0.47 & 0.36 & 0.56 & 7.85 & 0.000\end{array}$

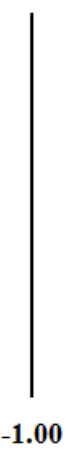

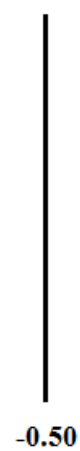

$-\mathbf{0 . 5 0}$

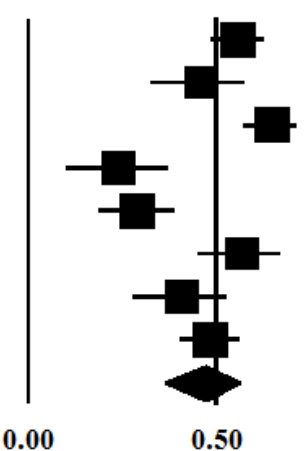

Figure 6. Forest plot with effect sizes for negative perfectionism and psychological distress 
Correlation limit limit Z-Valuep-Value

Chang et al., 2004

Chang et al., 2004

Cheng, 2001

DiBartolo et al., 2007

Khawaja \& Armstrong, 2005

Lynd-Stevenson \& Hearne, 1999

Wirtz et al., 2013

$\begin{array}{rrrrr}0.47 & 0.33 & 0.59 & 6.18 & 0.000 \\ 0.49 & 0.36 & 0.60 & 6.50 & 0.000 \\ 0.20 & 0.03 & 0.35 & 2.31 & 0.021 \\ 0.57 & 0.49 & 0.64 & 11.92 & 0.000 \\ 0.66 & 0.59 & 0.72 & 12.98 & 0.000 \\ 0.55 & 0.42 & 0.66 & 7.29 & 0.000 \\ 0.22 & -0.09 & 0.49 & 1.41 & 0.157 \\ 0.48 & 0.36 & 0.59 & 6.79 & 0.000\end{array}$
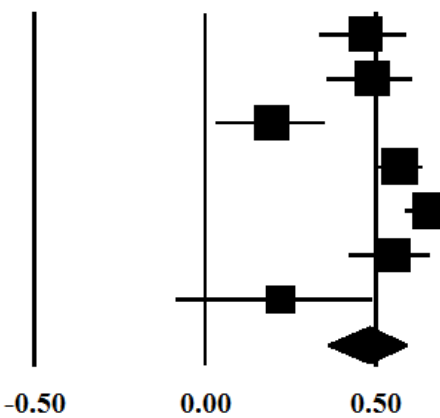

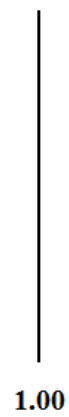

Figure 7. Forest plot with effect sizes for negative perfectionism and stress

\section{Study name}

\begin{tabular}{lrrrrr} 
Study name & \multicolumn{5}{c}{$\begin{array}{c}\text { Statistics for each study } \\
\text { Lower }\end{array}$} \\
& $\begin{array}{c}\text { Upper } \\
\text { Correlation }\end{array}$ & $\begin{array}{l}\text { limit } \\
\text { limit }\end{array}$ & Z-Value p-Value \\
Dakanalis et al., 2015 & -0.30 & -0.37 & -0.23 & -8.05 & 0.000 \\
Dakanalis, Timko, Clerici et al., 2014 & -0.26 & -0.33 & -0.18 & -6.53 & 0.000 \\
Pulford \& Sohal, 2006 & -0.60 & -0.71 & -0.46 & -6.88 & 0.000 \\
Soenens et al., 2005 & -0.50 & -0.58 & -0.42 & -10.02 & 0.000 \\
Soenens et al., 2005 & -0.35 & -0.44 & -0.25 & -6.69 & 0.000 \\
Stumpf \& Parker, 2000 & -0.49 & -0.54 & -0.44 & -15.10 & 0.000 \\
Zeiger-Hill \& Terry, 2007 & -0.20 & -0.28 & -0.12 & -4.80 & 0.000 \\
& -0.39 & -0.48 & -0.28 & -6.84 & 0.000
\end{tabular}
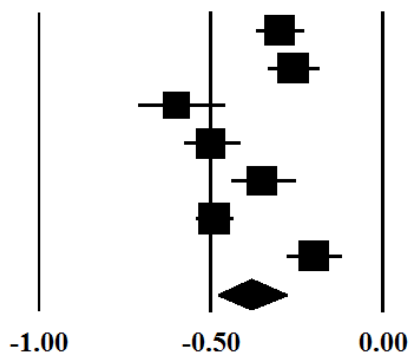

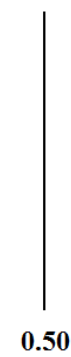

0.50

Figure 8. Forest plot with effect sizes for negative perfectionism and self-esteem

Study name

Statistics for each study

Lower Upper

Correlation limit limit Z-Valuep-Value

Chang et al., 2004

Chang et al., 2004

Steffen, 2014

Steffen, 2014

Williams \& Cropley, 2014

$$
\begin{array}{rrrrr}
-0.08 & -0.24 & 0.08 & -0.97 & 0.331 \\
-0.42 & -0.54 & -0.28 & -5.43 & 0.000 \\
-0.44 & -0.51 & -0.37 & -10.94 & 0.000 \\
-0.43 & -0.50 & -0.35 & -10.10 & 0.000 \\
-0.38 & -0.46 & -0.29 & -7.66 & 0.000 \\
-0.37 & -0.46 & -0.27 & -6.80 & 0.000
\end{array}
$$
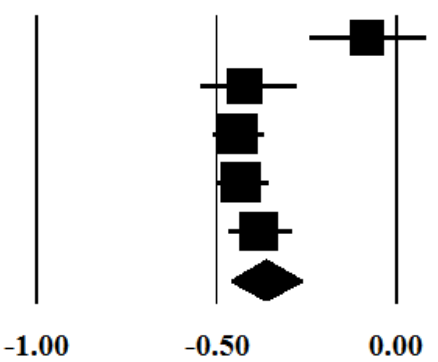

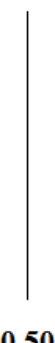

0.50

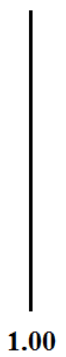

1.00

Figure 9. Forest plot with effect sizes for negative perfectionism and life satisfaction 
Study name

Statistics for each study

Lower Upper
Correlation limit limit Z-Valuep-Value

DiBartolo et al., 2007

DiBartolo et al., 2008

Kawamura et al., 2001

Khawaja \& Armstrong, 2005

Levinson et al., 2013

Levinson et al., 2013

Levinson et al., 2015

Levinson et al., 2015

Menatti et al., 2013

Norman et al., 1998

Santanello \& Gardener, 2007

Shumaker et al., 2009

Steffen, 2014

Steffen, 2014

Stoeber \& Joormann, 2001

Vansteenkiste et al., 2010

Weiner \& Carton, 2012

Wimberley \& Stasio, 2013

$\begin{array}{rrrrr}0.10 & -0.01 & 0.20 & 1.85 & 0.065 \\ 0.23 & 0.11 & 0.34 & 3.82 & 0.000 \\ 0.16 & 0.02 & 0.29 & 2.27 & 0.023 \\ 0.30 & 0.18 & 0.40 & 4.98 & 0.000 \\ -0.02 & -0.14 & 0.11 & -0.26 & 0.798 \\ -0.01 & -0.17 & 0.14 & -0.17 & 0.869 \\ -0.05 & -0.20 & 0.10 & -0.67 & 0.504 \\ 0.10 & -0.07 & 0.27 & 1.15 & 0.251 \\ -0.09 & -0.24 & 0.06 & -1.16 & 0.248 \\ -0.09 & -0.26 & 0.09 & -0.99 & 0.323 \\ 0.12 & -0.06 & 0.28 & 1.28 & 0.202 \\ 0.08 & -0.09 & 0.25 & 0.93 & 0.350 \\ -0.14 & -0.22 & -0.06 & -3.27 & 0.001 \\ -0.18 & -0.26 & -0.09 & -4.00 & 0.000 \\ 0.15 & 0.00 & 0.29 & 1.97 & 0.049 \\ 0.26 & 0.12 & 0.39 & 3.64 & 0.000 \\ -0.16 & -0.30 & -0.01 & -2.10 & 0.036 \\ 0.09 & -0.10 & 0.27 & 0.95 & 0.344 \\ 0.05 & -0.03 & 0.12 & 1.17 & 0.242\end{array}$
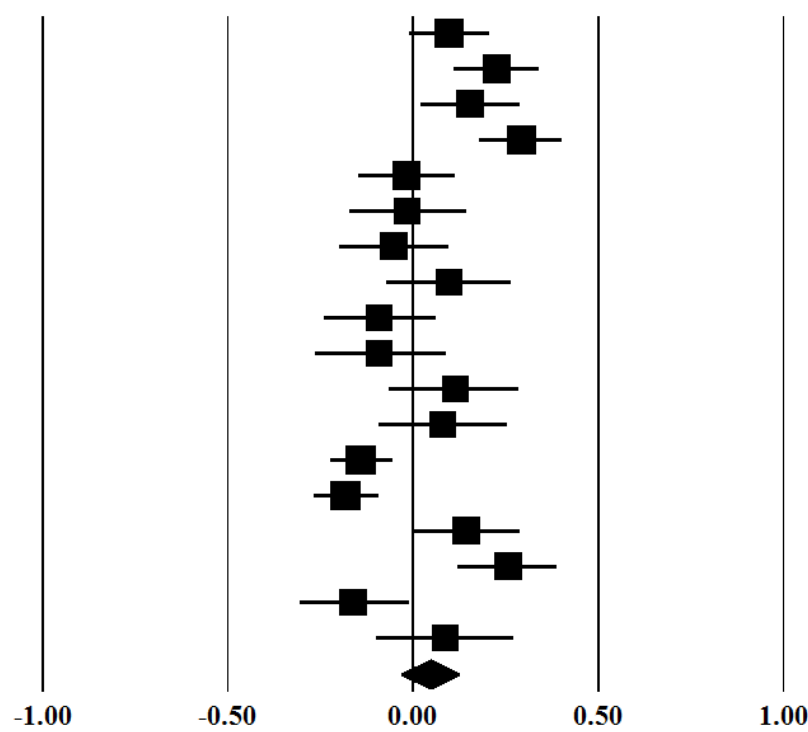

Figure 10. Forest plot with effect sizes for positive perfectionism and anxiety

Study name

\begin{tabular}{|c|c|c|c|c|c|}
\hline & \\
\hline & \multicolumn{4}{|c|}{ Lower Upper } & \multirow[b]{2}{*}{ p-Value } \\
\hline & Correlation & & limit $\mathrm{Z}$ & Z-Valuep & \\
\hline Boone \& Soenens, 2015 & 0.20 & -0.10 & 0.46 & 1.32 & 0.186 \\
\hline Boone et al., 2010/Boone et al., 2011 & 0.15 & 0.07 & 0.23 & 3.73 & 0.000 \\
\hline Boone et al., 2012 & 0.28 & 0.09 & 0.45 & 2.87 & 0.004 \\
\hline Boone et al., 2013 & 0.34 & 0.14 & 0.51 & 3.26 & 0.001 \\
\hline Boone et al., 2014* & 0.17 & 0.08 & 0.25 & 3.56 & 0.000 \\
\hline Claes et al., 2012 & -0.06 & -0.27 & 0.15 & -0.58 & 0.563 \\
\hline DiBartolo et al., 2008 & 0.05 & -0.07 & 0.16 & 0.77 & 0.439 \\
\hline Dickie et al., 2012 & 0.17 & 0.00 & 0.33 & 1.99 & 0.046 \\
\hline Dickie et al., 2012 & 0.32 & -0.00 & 0.58 & 1.93 & 0.053 \\
\hline Levinson et al., 2013 & 0.11 & -0.02 & 0.23 & 1.69 & 0.092 \\
\hline Levinson et al., 2013 & -0.09 & -0.24 & 0.07 & -1.12 & 0.264 \\
\hline Malkina-Pykh, 2012 & 0.30 & 0.12 & 0.47 & 3.13 & 0.002 \\
\hline Menatti et al., 2013 & 0.13 & -0.03 & 0.27 & 1.61 & 0.107 \\
\hline Soenens, Vansteenkiste et al., 2008 & -0.02 & -0.28 & 0.23 & -0.18 & 0.860 \\
\hline \multirow[t]{2}{*}{ Soenens, Vansteenkiste et al., 2008} & 0.12 & -0.10 & 0.32 & 1.06 & 0.287 \\
\hline & 0.13 & 0.08 & 0.19 & 4.55 & 0.00 \\
\hline
\end{tabular}

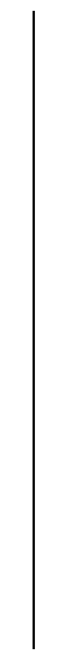

$-1.00$
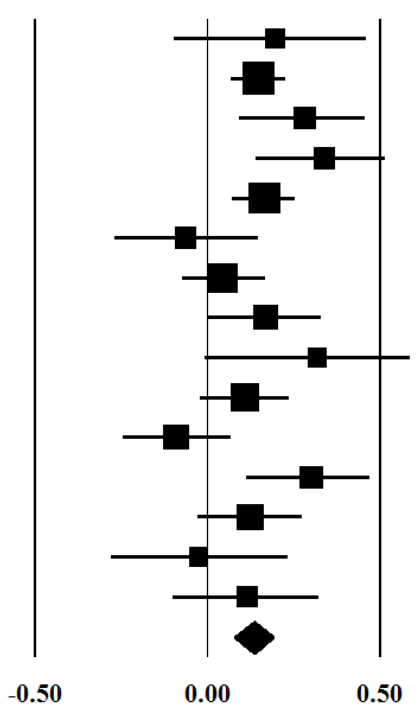

Figure 11. Forest plot with effect sizes for positive perfectionism and eating disorders 


\begin{tabular}{|c|c|c|c|c|}
\hline \multicolumn{5}{|c|}{ Lower Upper } \\
\hline Correlation & limit & limit & Z-Value p & -Value \\
\hline 0.15 & 0.01 & 0.28 & 2.13 & 0.034 \\
\hline 0.17 & 0.05 & 0.28 & 2.81 & 0.005 \\
\hline 0.21 & 0.03 & 0.37 & 2.34 & 0.019 \\
\hline 0.18 & 0.09 & 0.27 & 3.93 & 0.000 \\
\hline 0.18 & 0.12 & 0.23 & 5.74 & 0.00 \\
\hline
\end{tabular}

Kawamura et al., 2001

Khawaja \& Armstrong, 2005

Norman et al., 1998

Wu \& Cortesi, 2009

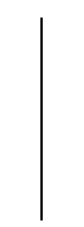

$-1.00$
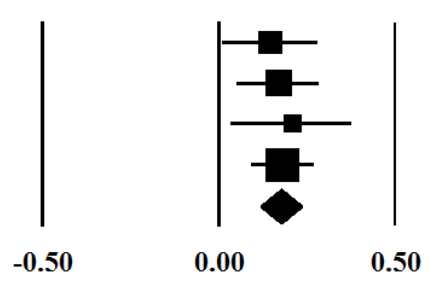

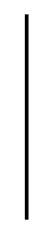

1.00

Figure 12. Forest plot with effect sizes for positive perfectionism and obsessive compulsive disorder

\begin{tabular}{rrrrrr}
\multicolumn{8}{c}{$\begin{array}{c}\text { Lower } \\
\text { Correlation }\end{array}$} & limit & \multicolumn{3}{c}{ limit } & \multicolumn{2}{c}{ Z-Valuep-Value } \\
& & & & & \\
0.13 & -0.08 & 0.32 & 1.21 & 0.225 \\
0.14 & -0.04 & 0.31 & 1.56 & 0.120 \\
-0.23 & -0.37 & -0.08 & -3.04 & 0.002 \\
6 & -0.04 & -0.14 & 0.06 & -0.79 & 0.429 \\
& -0.01 & -0.17 & 0.15 & -0.15 & 0.884
\end{tabular}

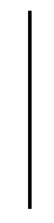

$-1.00$

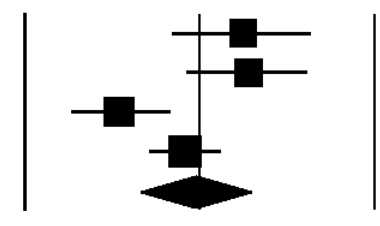

$-0.50$

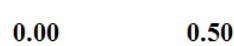

0.50

1.00

Figure 13. Forest plot with effect sizes for positive perfectionism and negative coping strategies

Study name

\section{$\underline{\text { Statistics for each study }}$}

\begin{tabular}{|c|c|c|c|c|}
\hline \multicolumn{5}{|c|}{ Lower Upper } \\
\hline orrelation & limit & limit & Z-Value & -Value \\
\hline 0.06 & -0.10 & 0.22 & 0.72 & 0.474 \\
\hline 0.25 & 0.13 & 0.36 & 4.18 & 0.000 \\
\hline 0.06 & -0.08 & 0.20 & 0.83 & 0.408 \\
\hline-0.11 & -0.22 & -0.00 & -2.00 & 0.045 \\
\hline 0.07 & -0.10 & 0.23 & 0.76 & 0.447 \\
\hline
\end{tabular}

Kawamura \& Frost, 2004

Khawaja \& Armstrong, 2005

Luyckx et al., 2008

Stumpf \& Parker, 2000

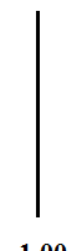

$-1.00$
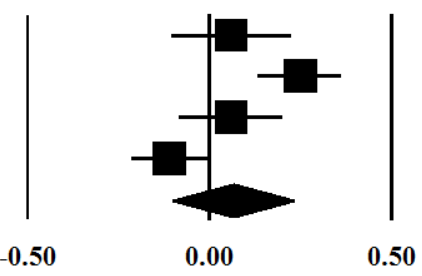

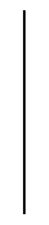

1.00

Figure 14. Forest plot with effect sizes for positive perfectionism and psychological distress 
Study name

Chang et al., 2004

Chang et al., 2004

DiBartolo et al., 2007

Khawaja \& Armstrong, 2005

\section{Statistics for each study}

\section{Lower Upper}

Correlation limit limit Z-Valuep-Value

$\begin{array}{rrrrr}-0.07 & -0.23 & 0.09 & -0.85 & 0.395 \\ -0.11 & -0.27 & 0.05 & -1.34 & 0.181 \\ 0.23 & 0.13 & 0.33 & 4.31 & 0.000 \\ 0.25 & 0.13 & 0.36 & 4.18 & 0.000 \\ 0.09 & -0.10 & 0.26 & 0.92 & 0.359\end{array}$

$\mid$

$-1.00$
$-0.50$

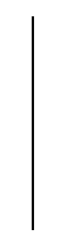

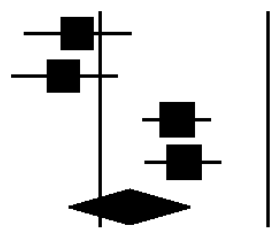

0.00 0.50

1.00

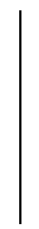

Figure 15. Forest plot with effect sizes for positive perfectionism and stress

$\underline{\text { Study name }}$

$\underline{\text { Statistics for each study }}$

\section{Lower Upper}

Correlation limit limit Z-Value p-Value

Pulford \& Sohal, 2006

Soenens et al., 2005

Soenens et al., 2005

Stumpf \& Parker, 2000

Zeiger-Hill \& Terry, 2007

$\begin{array}{rrrrr}-0.20 & -0.38 & -0.01 & -2.02 & 0.044 \\ -0.11 & -0.21 & -0.00 & -2.02 & 0.044 \\ 0.03 & -0.08 & 0.14 & 0.55 & 0.583 \\ 0.29 & 0.23 & 0.35 & 8.41 & 0.000 \\ 0.09 & 0.01 & 0.17 & 2.14 & 0.033 \\ 0.03 & -0.14 & 0.20 & 0.37 & 0.711\end{array}$

00
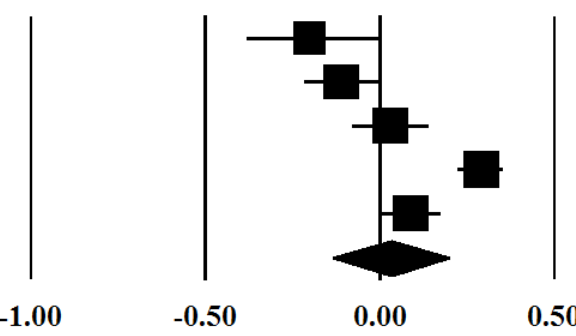

0.50

Figure 16. Forest plot with effect sizes for positive perfectionism and self-esteem

Study name

Statistics for each study

\section{Lower Upper}

Correlation limit limit Z-Value p-Value

$\begin{array}{lrrrrr}\text { Chang et al., 2004 } & 0.04 & -0.12 & 0.20 & 0.49 & 0.628 \\ \text { Chang et al., 2004 } & 0.22 & 0.06 & 0.37 & 2.71 & 0.007 \\ \text { Steffen, 2014 } & 0.19 & 0.11 & 0.27 & 4.46 & 0.000 \\ \text { Steffen, 2014 } & 0.24 & 0.15 & 0.32 & 5.37 & 0.000 \\ & 0.19 & 0.12 & 0.26 & 5.12 & 0.000\end{array}$

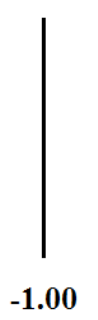

$-1.00$
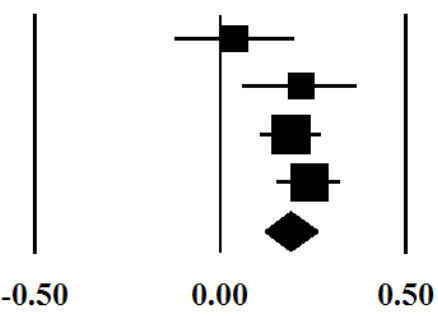

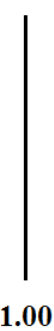

1.00

Figure 17. Forest plot with effect sizes for positive perfectionism and life satisfaction 
Figure 18. Forest plot with effect sizes for negative perfectionism and age
Study name

Statistics for each study

Lower Upper

\section{Correlation limit limit Z-Value p-Value}

*Boone et al., 2014

Boone et al., 2011

$\begin{array}{rrrrr}0.147 & 0.013 & 0.276 & 2.143 & 0.032 \\ 0.070 & -0.013 & 0.152 & 1.653 & 0.098 \\ -0.140 & -0.324 & 0.054 & -1.416 & 0.157 \\ -0.012 & -0.206 & 0.183 & -0.119 & 0.905 \\ 0.070 & -0.235 & 0.363 & 0.443 & 0.657 \\ 0.057 & -0.005 & 0.118 & 1.811 & 0.070\end{array}$

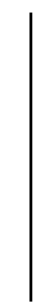

$-1.00$

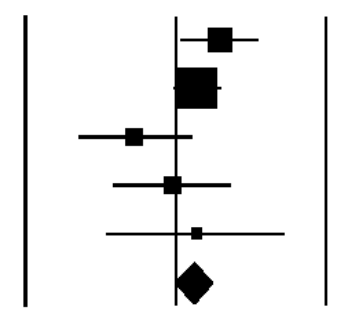

0.00

0.50

1.00

\begin{tabular}{lrrrrr} 
Study name & \multicolumn{5}{c}{ Statistics for each study } \\
\cline { 2 - 6 } & $\begin{array}{c}\text { Lower Upper } \\
\text { Correlation }\end{array}$ & limit & limit & Z-Value p-Value \\
Craddock et al., 2010 & -0.120 & -0.238 & 0.002 & -1.933 & 0.053 \\
Lehmann \& Konstam, 2011 & 0.140 & 0.052 & 0.226 & 3.097 & 0.002 \\
Pulford \& Sohal, 2006 & 0.121 & -0.075 & 0.308 & 1.210 & 0.226 \\
Vartanian \& Grisham, 2012 & -0.075 & -0.182 & 0.035 & -1.338 & 0.181 \\
& 0.013 & -0.127 & 0.152 & 0.177 & 0.859
\end{tabular}

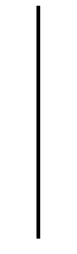

$-1.00$

\section{Correlation and $95 \% \mathrm{CI}$}
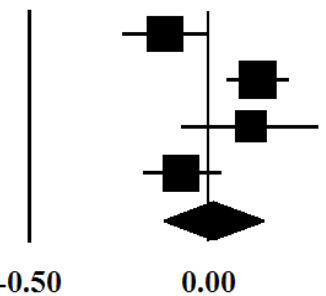

0.00

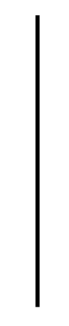

Figure 19. Forest plot with effect sizes for negative perfectionism and sex

Study name

$\underline{\text { Statistics for each study }}$

Lower Upper

Correlation limit limit Z-Value p-Value

*Boone et al., 2014

Boone et al., 2011

Malkina-Pykh, 2012

Pulford \& Sohal, 2006

$\begin{array}{rrrrr}0.125 & -0.029 & 0.273 & 1.590 & 0.112 \\ 0.040 & -0.043 & 0.123 & 0.944 & 0.345 \\ 0.100 & -0.094 & 0.287 & 1.008 & 0.313 \\ -0.026 & -0.219 & 0.169 & -0.259 & 0.796 \\ 0.054 & -0.010 & 0.119 & 1.649 & 0.099\end{array}$

\section{Correlation and $95 \% \mathrm{CI}$}
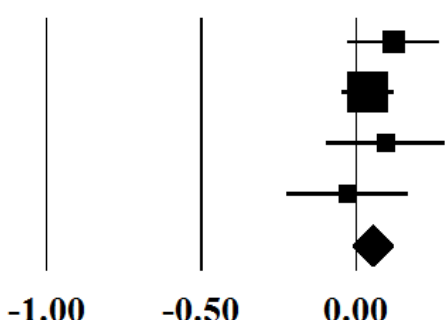

0.00

0.50

$$
-1.00-0.50
$$

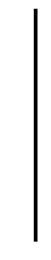

1.00

Figure 20. Forest plot with effect sizes for positive perfectionism and age 
Study name

Boone et al., 2014

Craddock et al., 2010

Lehmann \& Konstam, 2011

Pulford \& Sohal, 2006

Wielkiewicz \& Wonderlich, 2006
Statistics for each study

Lower Upper

Correlation limit limit Z-Value p-Value

$\begin{array}{rrrrr}0.150 & 0.060 & 0.237 & 3.256 & 0.001 \\ -0.240 & -0.351 & -0.122 & -3.924 & 0.000 \\ 0.090 & 0.001 & 0.178 & 1.983 & 0.047 \\ 0.121 & -0.075 & 0.308 & 1.210 & 0.226 \\ -0.162 & -0.258 & -0.062 & -3.168 & 0.002 \\ 0.001 & -0.047 & 0.049 & 0.033 & 0.974\end{array}$

\section{Correlation and $95 \% \mathrm{CI}$}

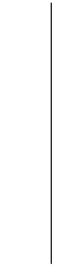

$-1.00$

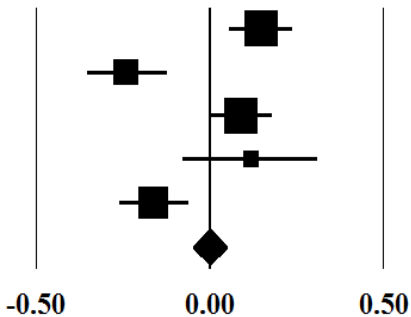

0.50

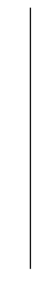

1.00

Figure 21. Forest plot with effect sizes for positive perfectionism and sex 
APPENDIX C

Study name

Adkins \& Parker, 1996

Ansell et al., 2010

Beevers \& Miller, 2004

Blankstein, Lumley \& Crawford, 2007

Blankstein, Lumley \& Crawford, 2007

Caelian, 2006

Chang, 1998

Chang, 1998

Chang, Watkins \& Banks, 2004

Chang, Watkins \& Banks, 2004

Chester, Merwin \& DeWall, 2015

Claes et al., 2012

Dean \& Range, 1996*

Dean \& Range, 1999

Enns, Cox \& Inayatulla, 2003

Flamenbaum \& Holden, 2007

Flett, Goldstein, Hewitt \& Wekerle, 2012

Flett, Goldstein, Hewitt \& Wekerle, 2012

Freudenstein et al., 2012

Hamilton \& Schweitzer, 2000

Hewitt, Caelian, Chen \& Flett, 2014

Hewitt, Flett \& Weber, 1994

Hewitt, Flett \& Weber, 1994

Hewitt, Flett, \& Turnbull-Donovan, 1992

Hewitt, Newton, Flett \& Callander, 1997

Hewitt, Newton, Flett \& Callander, 1997

Hewitt, Norton, Flett, Callander \& Cower, 1998

Hoff \& Muehlenkamp, 2009

Hunter \& O'Connor, 2003

Izadi, 2014

Kirtley, O'Connor \& Carroll, 2015

Klibert et al., 2005

Kubal, 2005

Miskey, Hill \& Huelsman, 2012

Muyan \& Chang, 2015

O'Connor \& Forgan, 2007

O'Connor et al., 2007

O'Connor et al., 2007

O'Connor et al., 2007

O'Connor, O'Connor \& Marshall, 2007

O'Connor, Rasmussen \& Hawton, 2010

O'Connor, Rasmussen \& Hawton, 2014

O'Riley \& Fiske, 2012

O'Riley, 2011

Peyeri, 2011

Portzky, van Heeringen \& Vervaet, 2014

Rasmussen et al., 2012

Rasmussen, Elliot \& O'Connor, 2012

Rasmussen, O'Connor \& Brodie, 2008

Rasmussen, O'Connor \& Brodie, 2008

Roxborough et al., 2012

Smith, Chesin \& Jeglic, 2014

Wang, Wong \& Fu, 2013

Yates, Tracy \& Luthar, 2008

Yates, Tracy \& Luthar, 2008

\section{Statistics for each study}

\section{Lower Upper}

Correlation limit limit Z-Valuep-Value

\begin{tabular}{|c|c|c|c|c|}
\hline & & & & \\
\hline 210 & 0.146 & & 612 & \\
\hline & 0.161 & & & \\
\hline 420 & 0.275 & 46 & & 000 \\
\hline & 284 & & & \\
\hline & -0.094 & & & 0 \\
\hline & 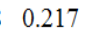 & & & \\
\hline & -0.0 & & 33 & \\
\hline & .0 & 349 & & \\
\hline & 2 & & & \\
\hline & 1 & & & \\
\hline & -0.0 & & & \\
\hline & $0.0^{\circ}$ & 97 & -1 & \\
\hline & -0.0 & 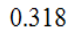 & 1.700 & \\
\hline & 0.1 & & 2.872 & \\
\hline & 0.0 & & & \\
\hline & 0 & & & \\
\hline & -0.1 & 87 & P & \\
\hline & & & & \\
\hline & 0.0 & & 22 & \\
\hline & $x^{2}$ & & & \\
\hline & & & & \\
\hline & & 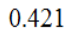 & 34 & \\
\hline & & & & \\
\hline & 00 & 17 & & \\
\hline & 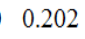 & & .082 & \\
\hline & 01 & & 21 & \\
\hline & 0.3 & & 2 & \\
\hline & 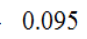 & & 6 & \\
\hline & & & & \\
\hline & & & & \\
\hline & $0.1^{\prime}$ & 2 & 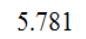 & \\
\hline & -0.0 & & & \\
\hline & & 4 & & \\
\hline & & & & \\
\hline & 0.1 & & & \\
\hline & $-0.0^{\prime}$ & & 972 & \\
\hline & & & & \\
\hline 2 & ? & 0 & 2 & \\
\hline & & & & \\
\hline & & & 521 & \\
\hline & & & & \\
\hline & & & & \\
\hline & -0 . & 7 & .369 & \\
\hline & & & & \\
\hline & $0 \Omega$ & 8 & & \\
\hline & 0.0 & & & \\
\hline & & & & \\
\hline & -0.19 & & & \\
\hline & & & & \\
\hline & & & & \\
\hline & 01 & r & $=-7$ & $0 \cap 0$ \\
\hline & 0.143 & & & \\
\hline & 0.026 & 0.193 & 2555 & 0.011 \\
\hline & & & & \\
\hline & 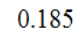 & 0.250 & 11.4 & 0.00 \\
\hline
\end{tabular}

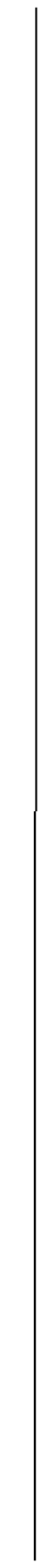

$-1.00$
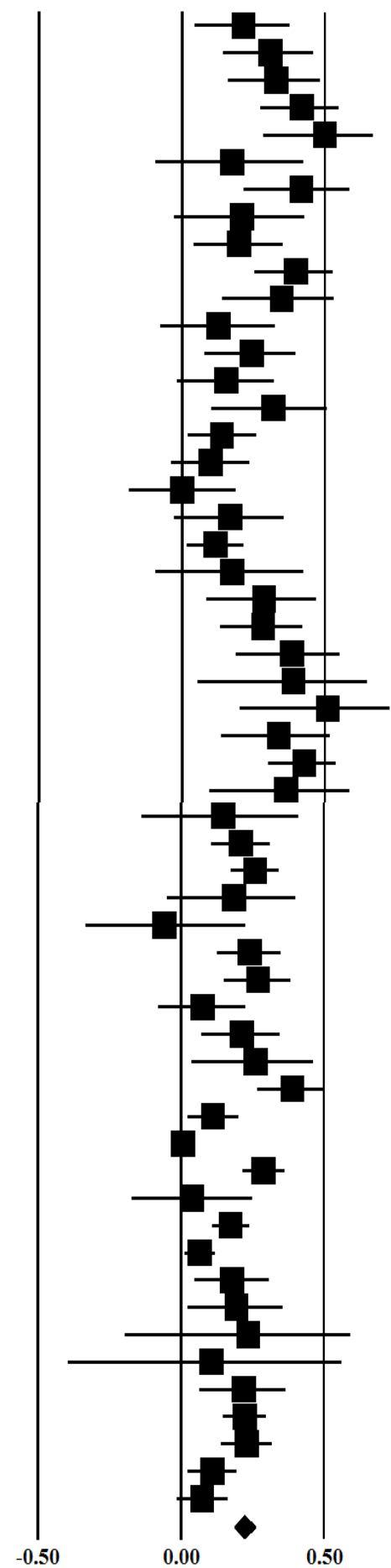

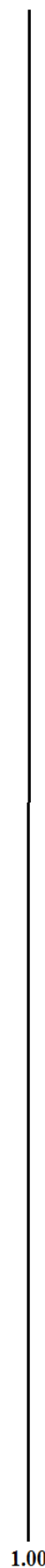

Figure 1. Forest plot with effect sizes for negative perfectionism and self-injury/suicide 
Study name

Adkins \& Parker, 1996

Blankstein, Lumley \& Crawford, 2007

Blankstein, Lumley \& Crawford, 2007

Caelian, 2006

Chang, 1998

Chang, 1998

Chang, Watkins \& Banks, 2004

Chang, Watkins \& Banks, 2004

Chester, Merwin \& DeWall, 2015

Claes et al., 2012

Dean \& Range, 1996*

Dean \& Range, 1999

Enns, Cox \& Inayatulla, 2003

Flamenbaum \& Holden, 2007

Flett, Goldstein, Hewitt \& Wekerle, 2012

Flett, Goldstein, Hewitt \& Wekerle, 2012

Freudenstein et al., 2012

Hamilton \& Schweitzer, 2000

Hewitt, Caelian, Chen \& Flett, 2014

Hewitt, Flett \& Weber, 1994

Hewitt, Flett \& Weber, 1994

Hewitt, Flett, \& Turnbull-Donovan, 1992

Hewitt, Norton, Flett, Callander \& Cower, 1998

Hoff \& Muehlenkamp, 2009

Hunter \& O'Connor, 2003

Klibert et al., 2005

Kubal, 2005

Miskey, Hill \& Huelsman, 2012

Muyan \& Chang, 2015

O'Connor \& Forgan, 2007

O'Connor et al., 2007

O'Connor et al., 2007

O'Connor et al., 2007

O'Connor, O'Connor \& Marshall, 2007

O'Connor, Rasmussen \& Hawton, 2010

O'Connor, Rasmussen \& Hawton, 2014

Portzky, van Heeringen \& Vervaet, 2014

Rasmussen, Elliot \& O'Connor, 2012

Rasmussen, O'Connor \& Brodie, 2008

Rasmussen, O'Connor \& Brodie, 2008

Roxborough et al., 2012

Smith, Chesin \& Jeglic, 2014

\section{Statistics for each study}

Lower Upper

Correlation limit limit Z-Valuep-Value

$\begin{array}{lllll}0.150 & -0.023 & 0.315 & 1.699 & 0.089\end{array}$

$\begin{array}{llllll}0.115 & -0.049 & 0.274 & 1.378 & 0.168\end{array}$

$\begin{array}{lllll}-0.046 & -0.295 & 0.208 & -0.353 & 0.724\end{array}$

$\begin{array}{llllll}0.023 & -0.243 & 0.287 & 0.168 & 0.866\end{array}$

$\begin{array}{lllll}0.080 & -0.144 & 0.296 & 0.699 & 0.485\end{array}$

$\begin{array}{lllll}-0.040 & -0.274 & 0.198 & -0.327 & 0.744\end{array}$

$\begin{array}{lllll}-0.170 & -0.322 & -0.010 & -2.081 & 0.037\end{array}$

$\begin{array}{lllll}-0.060 & -0.218 & 0.101 & -0.728 & 0.466\end{array}$

$\begin{array}{lllll}0.161 & -0.061 & 0.368 & 1.428 & 0.153\end{array}$

$\begin{array}{llllll}-0.007 & -0.208 & 0.195 & -0.067 & 0.946\end{array}$

$\begin{array}{llllll}-0.038 & -0.188 & 0.114 & -0.486 & 0.627\end{array}$

$\begin{array}{llllll}0.085 & -0.087 & 0.252 & 0.969 & 0.333\end{array}$

$\begin{array}{lllll}0.120 & -0.105 & 0.334 & 1.044 & 0.296\end{array}$

$\begin{array}{lllll}0.025 & -0.096 & 0.145 & 0.405 & 0.686\end{array}$

$\begin{array}{llllll}-0.007 & -0.143 & 0.130 & -0.095 & 0.924\end{array}$

$\begin{array}{llllll}-0.183 & -0.356 & 0.003 & -1.930 & 0.054\end{array}$

$\begin{array}{lllll}0.120 & -0.078 & 0.309 & 1.188 & 0.235\end{array}$

$\begin{array}{lllll}-0.006 & -0.105 & 0.093 & -0.111 & 0.911\end{array}$

$\begin{array}{lllll}0.023 & -0.243 & 0.287 & 0.168 & 0.866\end{array}$

$\begin{array}{llllll}0.216 & 0.011 & 0.404 & 2.062 & 0.039\end{array}$

$\begin{array}{llllll}0.160 & 0.005 & 0.308 & 2.026 & 0.043\end{array}$

$\begin{array}{llllll}-0.071 & -0.277 & 0.142 & -0.648 & 0.517\end{array}$

$\begin{array}{lllll}0.027 & -0.192 & 0.243 & 0.236 & 0.814\end{array}$

$\begin{array}{lllll}-0.216 & -0.354 & -0.069 & -2.860 & 0.004\end{array}$

$\begin{array}{lllll}-0.010 & -0.296 & 0.278 & -0.066 & 0.947\end{array}$

$\begin{array}{llllll}-0.050 & -0.139 & 0.040 & -1.087 & 0.277\end{array}$

$\begin{array}{llllll}0.017 & -0.217 & 0.249 & 0.137 & 0.891\end{array}$

$\begin{array}{lllll}0.109 & -0.175 & 0.376 & 0.748 & 0.454\end{array}$

$\begin{array}{llllll}0.045 & -0.071 & 0.160 & 0.763 & 0.446\end{array}$

$\begin{array}{llllll}0.025 & -0.098 & 0.147 & 0.397 & 0.691\end{array}$

$\begin{array}{llllll}0.028 & -0.124 & 0.179 & 0.358 & 0.720\end{array}$

$\begin{array}{lllll}0.055 & -0.088 & 0.195 & 0.755 & 0.450\end{array}$

$\begin{array}{lllll}-0.100 & -0.318 & 0.129 & -0.854 & 0.393\end{array}$

$\begin{array}{llllll}0.231 & 0.099 & 0.355 & 3.387 & 0.001\end{array}$

$\begin{array}{llllll}0.012 & -0.076 & 0.100 & 0.268 & 0.789\end{array}$

$\begin{array}{llllll}0.041 & 0.008 & 0.074 & 2.434 & 0.015\end{array}$

$\begin{array}{lllll}-0.013 & -0.065 & 0.038 & -0.508 & 0.612\end{array}$

$\begin{array}{lllll}-0.055 & -0.241 & 0.135 & -0.564 & 0.573\end{array}$

$\begin{array}{llllll}-0.040 & -0.445 & 0.378 & -0.180 & 0.857\end{array}$

$\begin{array}{lllll}0.234 & -0.278 & 0.643 & 0.893 & 0.372\end{array}$

$\begin{array}{lllll}0.120 & -0.037 & 0.271 & 1.501 & 0.133\end{array}$

$\begin{array}{lllll}0.039 & -0.038 & 0.116 & 0.989 & 0.323\end{array}$

$\begin{array}{lllll}0.021 & -0.005 & 0.048 & 1.584 & 0.113\end{array}$
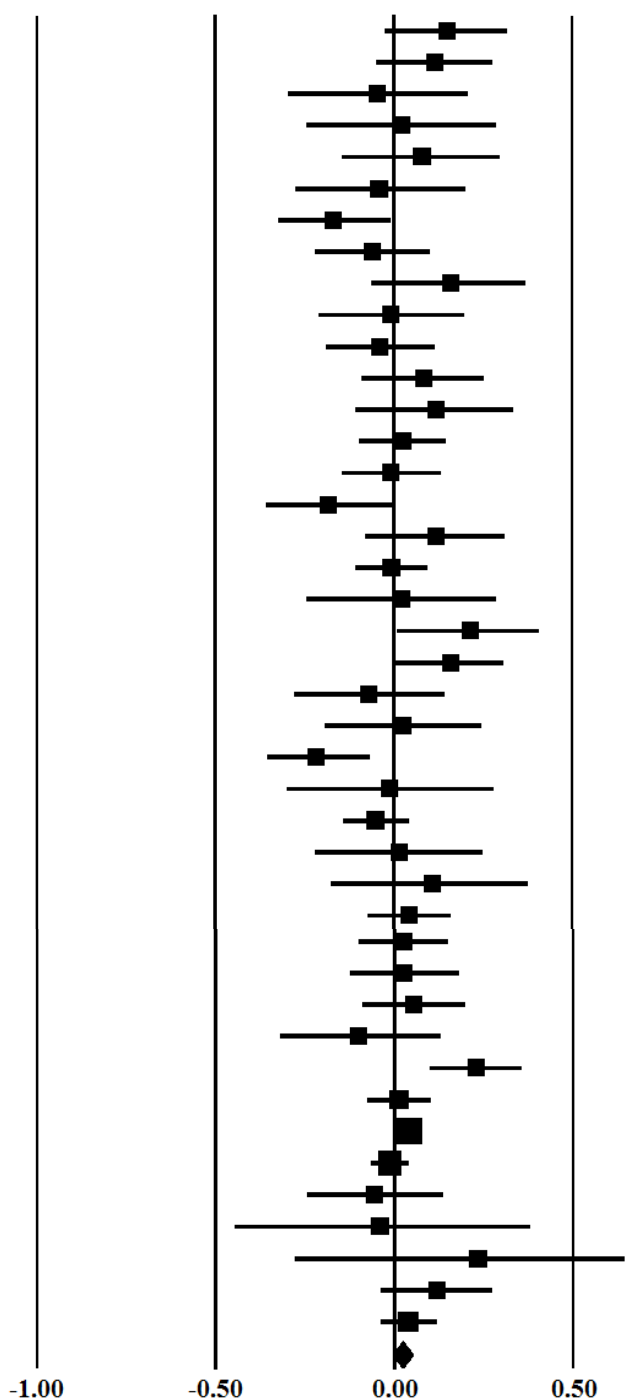

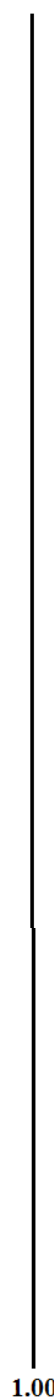

Figure 2. Forest plot with effect sizes for positive perfectionism and self-injury/suicide 
APPENDIX D

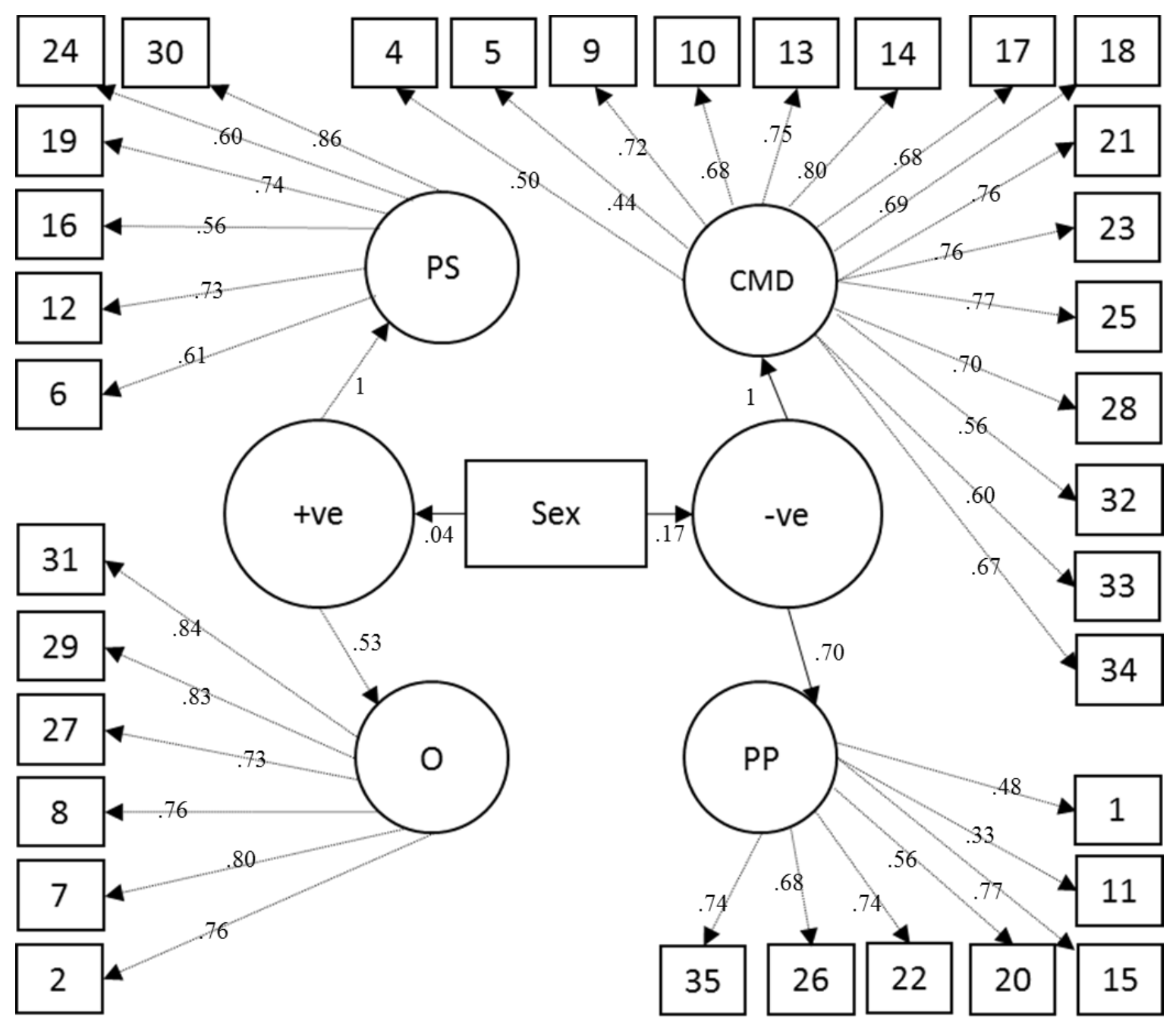

Figure 1. MIMIC analyses: Four factor, hierarchical FMPS model with sex as a covariate, complete model with standardised regression path values 